\title{
Pottery Presentation
}

\section{Catalogue}

\subsection{Explanatory Notes for the Catalogue}

Construction/Techniques

Wheel-made (1) - simple wheel

Wheel-made (2)_compound wheel

Handmade (1) — paddle-and-ground and beating method

Handmade (2) — coiling method

Handmade (3) - moulding method

Handmade — unrecognised technique

\section{State of Preservation}

Complete: when a vessel survives intact.

Complete or almost complete, restored from sherds: when a vessel was found in pieces but restored and all fragments survive.

Incomplete: when a few parts of the vessel are missing, but the vessel is almost complete.

Fragmentary: when only part of a vessel survives, without a complete profile and/or more than a few parts of the vessel are missing.

\section{Measurements}

$\mathrm{Dr}=$ rim diameter

$\mathrm{D}_{\mathrm{b}}=$ base diameter

$\mathrm{H}=$ height

${ }_{\mathrm{p}} \mathrm{H}=$ preserved height

Surface Treatment

Burnish descriptions (for cups I, bowls II, cooking pots IV-VI):

Burnish 1 = Dense, applied with overlapping strokes; the whole surface is smooth, wheel marks scarcely visible.

Burnish 2 = Medium dense, without overlap of strokes of the burnishing tool; gaps between burnishing marks narrower than the strokes and each one of the same width.

Burnish 3 = Thin/rare, with narrow burnishing marks, sometimes with wavy lines which look like decoration of some kind; gaps between marks are wider than in the above groups.

Burnish can be carefully or carelessly executed.
Polish - a glossy lustre on the surface, executed by rubbing surface with yielding tool.

Slipped and burnished on both surfaces-describes burnish and slip applied to both the external and internal surface.

Smoothed surface-when the surface is carefully smoothed.

The hardness of the vessels is expressed in terms of the Mohs scale (see Glossary, this volume):

2 soft - surface can be scratched by a fingernail;

3 medium hard - surface easily scratched by a copper wire;

4 hard - surface easily scratched by a knife.

\section{Illustrations}

Red slip is represented in grey; two different shades of grey denote two different shades of slip: light and dark red.

White/cream slip is described as such.

The colour of the internal and external surface, slip and fresh breaks/cores are described using Munsell Colour Charts (The Munsell Soil Color Book 2013), "deep black" is added to the description of the core, to describe black similar to the coal colour (Munsell Colour Charts).

The term "not possible to determine" is used:

- in relation to the fabric and colour of a break when a vessel is complete;

- in relation to the fabric and colour of a break when a vessel is almost complete, any losses in the vessel are unclean and it was not possible to make a fresh break;

- in relation to an external or internal surface which is poorly preserved, abraded and dirty, making it impossible to describe the colour. 


\section{Cups Type I.1}

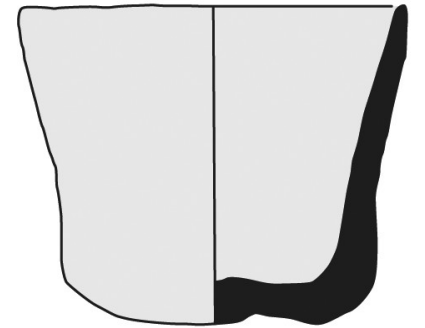

Cat. 1

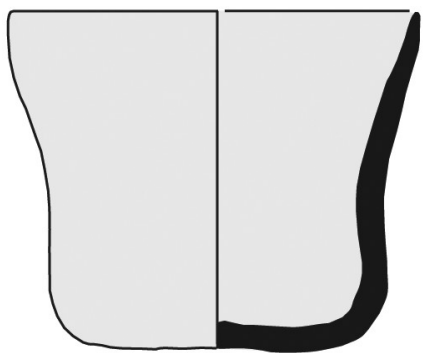

Cat. 4

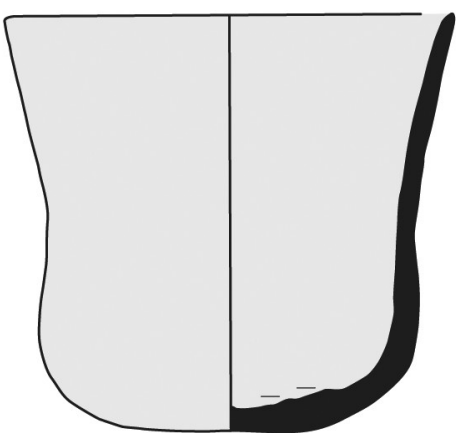

Cat. 2
Z26/47

Z15/49
Z26/15

$Z 15 / 46$

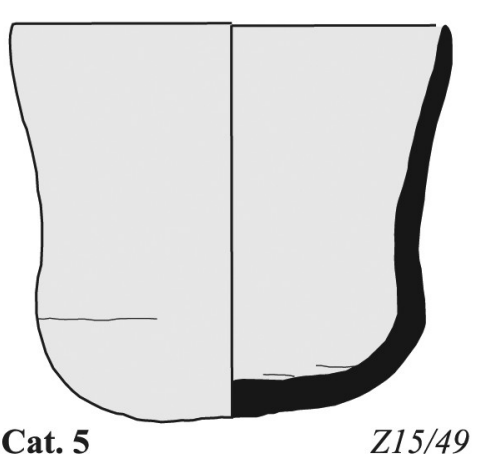

Cat. 5

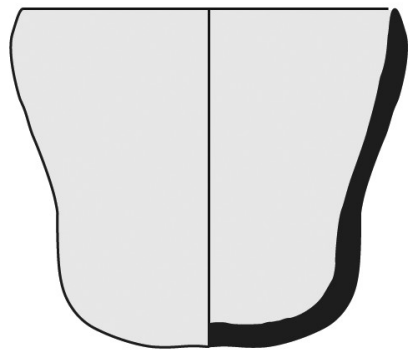

Cat. 3

Z4/198

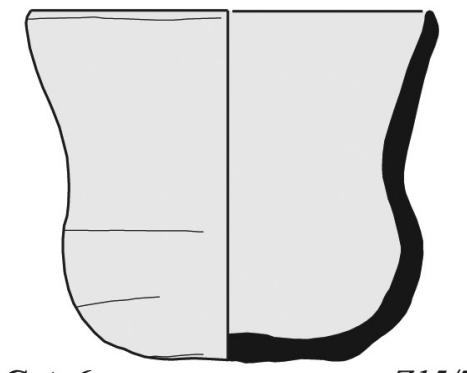

Cat. 6

FIGURE 7.1 Cups of type I.1 with clearly visible S-shaped profile

2 Group I-Cups

2.1 Cups Type I.1

Cat. 1

Cup

Inv. no: $\mathbf{Z 2 6 / 1 5}$

Provenance: Tumulus 26, chamber 1, eastern part

Construction: wheel-made (1)

State of preservation: incomplete, $92 \%$ of rim

Measurements: $\mathrm{Dr}=9 \mathrm{~cm} ; \mathrm{H}=7 \cdot 5 \mathrm{~cm}$

Fabric: not possible to determine, group ZF1

Hardness: 3

Firing: oxidized

Surface treatment: slipped and burnished on both surfaces; external-burnish 2, carefully executed; internalburnish 1, carefully executed

Surface colour: external, slip-10R $5 / 6$ red; internal, slip10R $4 / 6$ red

Colour of break: not possible to determine

Cat. 2

Cup

Inv. no: Z26/47

Provenance: Tumulus 26, chamber 1, central part
Construction: wheel-made (1)

State of preservation: incomplete, $86 \%$ of rim

[Figs 7.86-7.87] Measurements: $\mathrm{Dr}=10.5 \mathrm{~cm} ; \mathrm{H}=9.7 \mathrm{~cm}$

Fabric: not possible to determine, group ZF1

Hardness: 3

Firing: reduced

Surface treatment: slipped and burnished on both surfaces; external-burnish 1, carefully executed; internalburnish 1, carefully executed in the upper part, less carefully executed in the lower part

Surface colour: external, slip-10R 4/4 weak red; internal, slip-1oR $4 / 4$ weak red

Colour of break: 5 YR $5 / 6$ yellowish red

Cat. 3

Cup

Inv. no: $\mathrm{Z} 4 / 198$

Provenance: Tumulus 4, fill of E tunnel

Construction: wheel-made (1)

State of preservation: fragmentary, $16 \%$ of rim, body fragments missing

Measurements: $\mathrm{Dr}=9.5 \mathrm{~cm} ; \mathrm{H}=7.9 \mathrm{~cm}$

Fabric: ZF2

Hardness: 3 
Firing: oxidized

Surface treatment: slipped and burnished on both surfaces; external-burnish 1, carefully executed; internalburnish 2, less carefully executed

Surface colour: external, slip-2.5YR 4/4 reddish brown; internal, slip-1oR 3/4 dusky red

Colour of break: 5YR 5/6 yellowish red

\section{Cat. 4}

Cup

Inv. no: $\mathbf{Z 1 5} / 46$

Provenance: Tumulus 15, main burial chamber, inside vessel $\mathrm{Z}_{15} / 45$

Construction: wheel-made (1)

State of preservation: complete

Measurements: $\mathrm{Dr}=9 \cdot 5^{-9} .8 \mathrm{~cm} ; \mathrm{H}=8 \mathrm{~cm}$

Fabric: not possible to determine

Hardness: $3 / 4$

Firing: not possible to determine

Surface treatment: slipped and burnished on both surfaces; external-burnish 1, carefully executed; internalburnish 1 on the upper part, burnish 2 on the lower part, carefully executed

Surface colour: external, slip_-10R 4/4 weak red; internal, slip-10R 4/4 weak red

Colour of break: not possible to determine

\section{Cat. 5}

Cup

Inv. no: $\mathrm{Z}_{15} / 49$

Provenance: Tumulus 15, main burial chamber, eastern part

Construction: wheel-made (1)

State of preservation: complete

Measurements: $\mathrm{Dr}=10.3 \mathrm{~cm} ; \mathrm{H}=9.2 \mathrm{~cm}$

Fabric: not possible to determine

Hardness: $3 / 4$

Firing: not possible to determine

Surface treatment: slipped and burnished on both surfaces; external-burnish 1, carefully executed; internalburnish 1 on the upper part, burnish 3 on the lower part, carelessly executed

Surface colour: external, slip-1oR 4/4 weak red; internal, slip-10R 4/4 weak red

Colour of break: not possible to determine

Cat. 6

Cup

Inv. no: $\mathrm{Z}_{15} / 7$

Provenance: Tumulus 15, shaft, southern part, close to blocking wall of main burial chamber
Construction: wheel-made (1)

State of preservation: incomplete, $58 \%$ of rim

Measurements: $\mathrm{Dr}=9.2-9.5 \mathrm{~cm} ; \mathrm{H}=8.2 \mathrm{~cm}$

Fabric: ZF2

Hardness: 3

Firing: oxidized

Surface treatment: slipped and burnished on both surfaces; external-burnish 1, carefully executed; internalburnish 1, carefully executed

Surface colour: external, slip-10R 4/6 red; internal, slip1oR $4 / 6$ red

Colour of break: 1OYR 5/4 yellowish brown

References: (Czyżewska-Zalewska 2016, 727-728)

Cat. 7

Cup

Inv. no: $\mathbf{Z 1 5} / \mathbf{2 2}$

Provenance: Tumulus 15, shaft, southern part, close to blocking wall of main burial chamber

Construction: wheel-made (1)

State of preservation: incomplete, $87 \%$ of rim

Measurements: $\mathrm{Dr}=9.7 \mathrm{~cm} ; \mathrm{H}=8.5 \mathrm{~cm}$

Fabric: ZF2

Hardness: $3 / 4$

Firing: oxidized

Surface treatment: slipped and burnished on both surfaces; external-burnish 1, carefully executed; internalburnish 1 on the upper part, burnish 2 on the lower part, carefully executed

Surface colour: external, slip_—10R 4/6 red; internal, slip1oR $4 / 4$ weak red

Colour of break: 10YR 5/4 yellowish brown

Cat. 8

Cup

Inv. no: $\mathrm{Z25} / 5$

Provenance: Tumulus 25, chamber 1, central part

Construction: wheel-made (1)

State of preservation: incomplete, $70 \%$ of rim, wall fragments missing

Measurements: $\mathrm{Dr}=9.2 \mathrm{~cm} ; \mathrm{H}=8.3 \mathrm{~cm}$

Fabric: ZF2

Hardness: $3 / 4$

Firing: reduced

Surface treatment: slipped and burnished on both surfaces; external-burnish 2, less carefully executed; internalburnish 1 , less carefully executed

Surface colour: external, slip_-10R 4/6 red; internal, slip1oR $4 / 6$ red

Colour of break: 7.5YR 3/3 dark brown

References: (Klimaszewska-Drabot 2010, 48o-481) 
Cups Type I.1

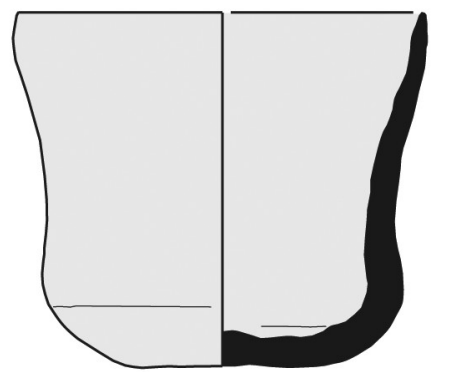

Cat. 7

$Z 15 / 22$

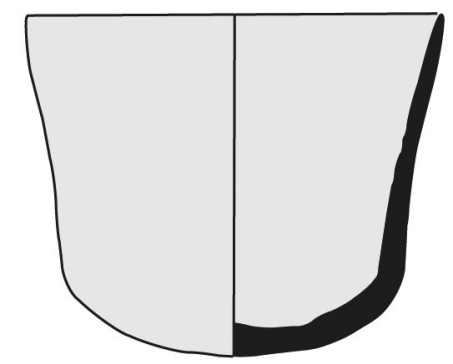

Cat. 10

Z23/35

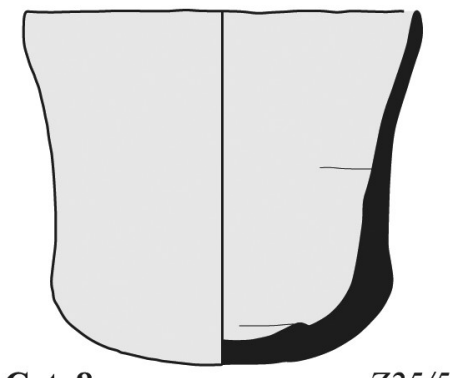

Cat. 8

$Z 25 / 5$

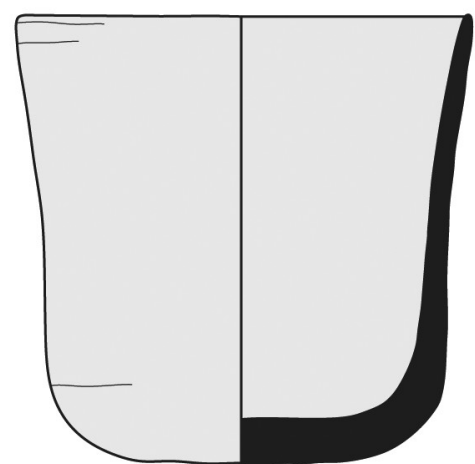

Cat. 11

$Z 14 / 24$

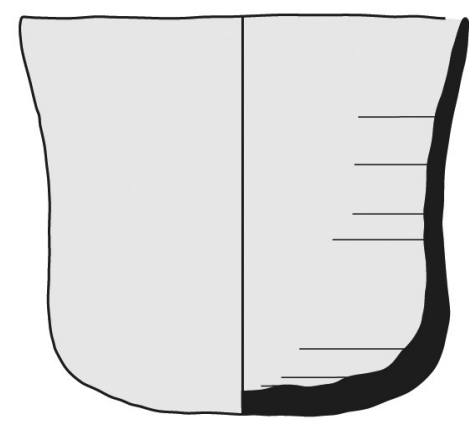

Cat. 9

$Z 13 / 20$

FIGURE 7.2 Cups of type I.1 with clearly visible S-shaped profile

Cat. 9

Cup

Inv. no: Z13/2o

Provenance: Tumulus 13, chamber 2, northern part, found on the rim of bottle $\mathrm{Z}_{13} / 19$

Construction: wheel-made (1)

State of preservation: incomplete, $69 \%$ of rim, wall fragments missing

Measurements: $\mathrm{Dr}=10.5 \mathrm{~cm} ; \mathrm{H}=9.4 \mathrm{~cm}$

Fabric: ZF2

Hardness: 3

Firing: oxidized

Surface treatment: slipped and burnished on both surfaces; external-burnish 1, carefully executed; internalburnish 1 in the upper part, carefully executed, burnish 2 in the lower part, less carefully executed

Surface colour: external, slip—1oR 4/6 red; internal, slip— $10 \mathrm{R} 4 / 6$ red

Colour of break: 5YR 5/4 reddish brown

Cat. 10

Cup

Inv. no: $\mathrm{Z23} / 35$
Provenance: Tumulus 23, chamber 1

Construction: wheel-made (1)

State of preservation: complete

Measurements: $\mathrm{Dr}=9.6 \mathrm{~cm} ; \mathrm{H}=8 \mathrm{~cm}$

Fabric: not possible to determine

Hardness: $3 / 4$

Firing: not possible to determine

Surface treatment: slipped and burnished on both surfaces; external-burnish 1, carefully executed; internalburnish 1 , carefully executed

Surface colour: external, slip—10R 5/6 red; internal, slip1oR $4 / 4$ weak red

Colour of break: not possible to determine

References: (Klimaszewska-Drabot and Mahmoud ElTayeb 2014, 10-13)

Cat. 11

Cup

Inv. no: Z14/24

Provenance: Tumulus 14, chamber 2, central part

Construction: wheel-made (1)

State of preservation: incomplete, $88 \%$ of rim, wall fragments missing 


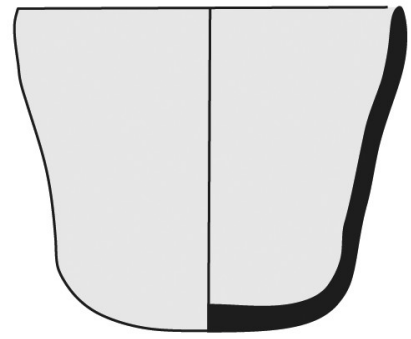

Cat. 13 $Z 2 / 27$

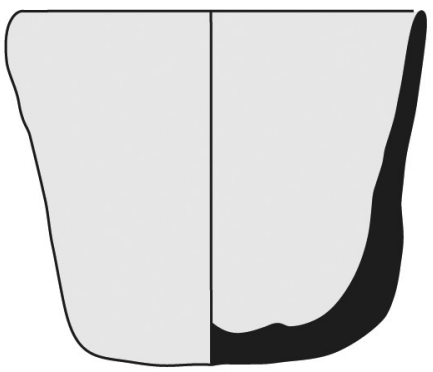

Cat. 14

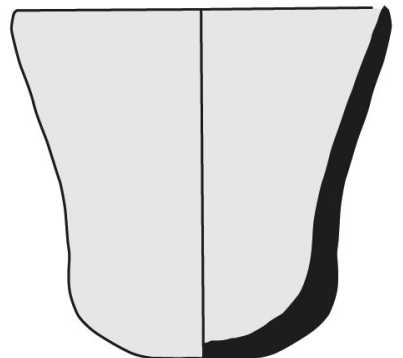

Cat. 17

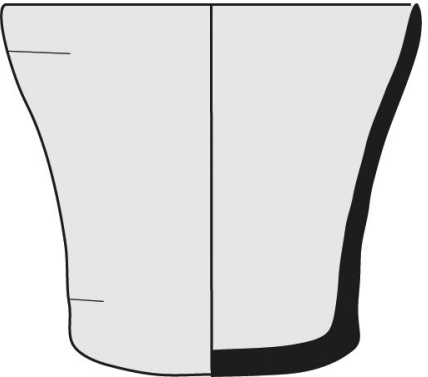

Cat. 15

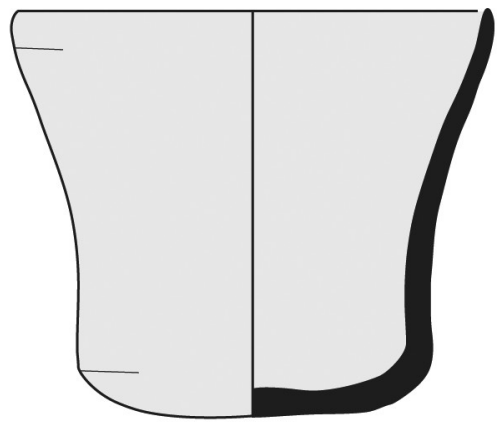

Cat. 16
Z24/37

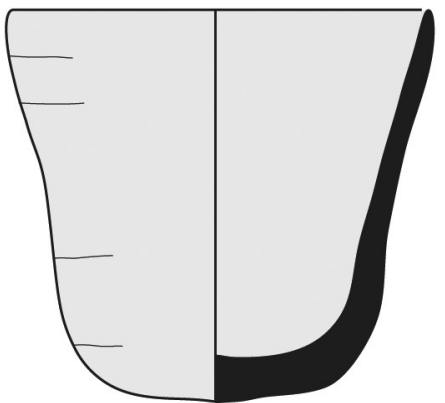

Cat. 18

FIGURE 7.3 Cups of type I.1 with less pronounced S-shaped profile

Measurements: $\mathrm{Dr}=10.5 \mathrm{~cm} ; \mathrm{H}=10.5 \mathrm{~cm}$

Fabric: ZF1

Hardness: $3 / 4$

Firing: oxidized

Surface treatment: slipped and burnished on both surfaces; burnish 1, carefully executed, on both surfaces

Surface colour: external, slip—-1oR 4/6 red; internal, slip$10 \mathrm{R} 4 / 6$ red

Colour of break: 5 YR 4/6 yellowish red

Cat. 12

Cup

Inv. no: $\mathbf{Z 2 6} / 37$

Provenance: Tumulus 26, chamber 1, central part

Construction: wheel-made (1)

State of preservation: incomplete, $86 \%$ of rim, wall fragments missing

Measurements: $\mathrm{Dr}=8.9 \mathrm{~cm} ; \mathrm{H}=7.5 \mathrm{~cm}$

Fabric: ZF3

Hardness: $3 / 4$

Firing: reduced
Surface treatment: slipped and burnished on both surfaces; burnish 1, carefully executed, on both surfaces

Surface colour: external, slip- $10 \mathrm{R} 5 / 6$ red; internal, slip$10 \mathrm{R} 4 / 6 \mathrm{red}$

Colour of break: 1OYR $3 / 3$ dark brown

Cat. 13

Cup

Inv. no: $\mathbf{Z} 2 / 27$

Provenance: Tumulus 2, chamber 2

Construction: wheel-made (1)

State of preservation: complete

Measurements: $\mathrm{Dr}=9 \mathrm{~cm} ; \mathrm{H}=7.5 \mathrm{~cm}$

Fabric: not possible to determine

Hardness: $3 / 4$

Firing: not possible to determine

Surface treatment: slipped and burnished on both surfaces; burnish 2, carefully executed, on both surfaces

Surface colour: external, slip—-1oR 5/6 red; internal, slip$10 \mathrm{R} 4 / 6 \mathrm{red}$

Colour of break: not possible to determine 
Cat. 14

Cup

Inv. no: Z23/39

Provenance: Tumulus 23, chamber 2

Construction: wheel-made (1)

State of preservation: incomplete, $95 \%$ of rim

Measurements: $\mathrm{Dr}=10.5 \mathrm{~cm} ; \mathrm{H}=8.4 \mathrm{~cm}$

Fabric: not possible to determine

Hardness: $3 / 4$

Firing: not possible to determine

Surface treatment: slipped and burnished on both surfaces; burnish 1, carefully executed, on both surfaces

Surface colour: external, slip-2.5YR 6/4 light yellowbrown; internal, slip-2.5YR 5/6 red

Colour of break: not possible to determine

\section{Cat. 15}

Cup

Inv. no: $\mathrm{Z} 24 / 53$

Provenance: Tumulus 24, chamber 1

Construction: wheel-made (1)

State of preservation: incomplete, restored from sherds, $95 \%$ of rim, body fragments missing

Measurements: $\mathrm{Dr}=9.5 \mathrm{~cm} ; \mathrm{H}=8.5 \mathrm{~cm}$

Fabric: ZF3

Hardness: 3

Firing: reduced

Surface treatment: slipped and burnished on both surfaces; burnish 1 , carefully executed, on both surfaces

Surface colour: external, slip — $10 \mathrm{R} 5 / 6$ red; internal, slip10R $4 / 6$ red

Colour of break: ${ }_{5} \mathrm{Y}$ 2.5/1 black

Cat. 16

Cup

Inv. no: Z24/37

Provenance: Tumulus 24, chamber 3

Construction: wheel-made (1)

State of preservation: incomplete, body fragments missing

Measurements: $\mathrm{Dr}=11.3 \mathrm{~cm} ; \mathrm{H}=9.5 \mathrm{~cm}$

Fabric: $\mathrm{ZF} 3$

Hardness: $3 / 4$

Firing: reduced

Surface treatment: slipped and burnished on both surfaces; burnish 1, carefully executed, on both surfaces

Surface colour: external, slip_-10R 4/6 red; internal, slip1oR $4 / 4$ weak red

Colour of break: $2.5 \mathrm{Y}$ 3/1 very dark grey
Cat. 17

Cup

Inv. no: Z26/71.1

Provenance: Tumulus 26, fill of shaft

Construction: wheel-made (1)

State of preservation: fragmentary, $34 \%$ of rim, body fragments missing

Measurements: $\mathrm{Dr}=9 \mathrm{~cm} ; \mathrm{H}=8.1 \mathrm{~cm}$

Fabric: $\mathrm{ZF} 3$

Hardness: $3 / 4$

Firing: oxidized

Surface treatment: slipped and burnished on both surfaces; burnish 1, carefully executed, on both surfaces

Surface colour: external, slip—-10R 4/6 red; internal, slip$10 \mathrm{R} 4 / 6 \mathrm{red}$

Colour of break: 1OYR 4/2 dark greyish brown

Cat. 18

Cup

Inv. no: Z14/17

Provenance: Tumulus 14, chamber 1, central part

Construction: wheel-made (1)

State of preservation: incomplete, $50 \%$ of rim

Measurements: $\mathrm{Dr}=9.8 \mathrm{~cm} ; \mathrm{H}=9.2 \mathrm{~cm}$

Fabric: $\mathrm{ZF} 3$

Hardness: 3

Firing: oxidized

Surface treatment: slipped and burnished on both surfaces; external-burnish 1, carefully executed; internalburnish 1 on the upper part, burnish 2 on the lower part, carefully executed

Surface colour: external, slip_—1OR 4/6 red; internal, slip10R $4 / 6$ red

Colour of break: $10 \mathrm{R} 5 / 3$ weak red

Variant I.1a

Cat. 19

Cup

Inv. no: $\mathbf{Z} \mathbf{2 1} / 6$

Provenance: Tumulus 21, shaft, central part

Construction: wheel-made (1)

State of preservation: complete

Measurements: $\mathrm{Dr}=6.8 \mathrm{~cm} ; \mathrm{H}=8.2 \mathrm{~cm}$

Fabric: not possible to determine

Hardness: $3 / 4$

Firing: oxidized

Surface treatment: slipped and burnished on both surfaces; internal-burnish 3 on the upper part, carelessly executed

Surface colour: external-5YR 5/6 yellowish red, slip10R 4/6 red; internal— 5 YR 5/6 yellowish red, slip$10 \mathrm{R}_{4} / 6 \mathrm{red}$ 


\section{Cups Type I.1}

variant I.1a

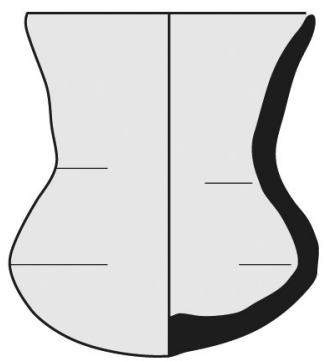

Cat. 19

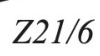

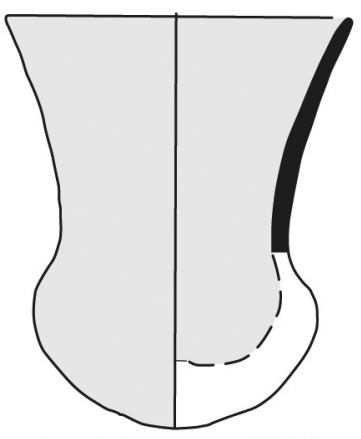

Cat. 20
Z20/2 variant $I .1 b$

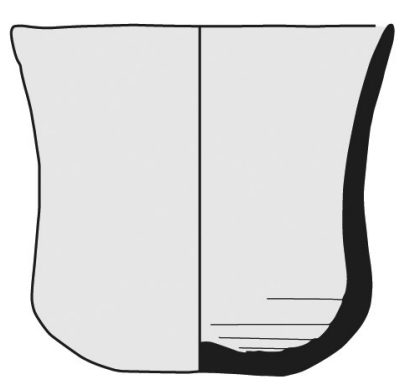

Cat. 21
Z25/21

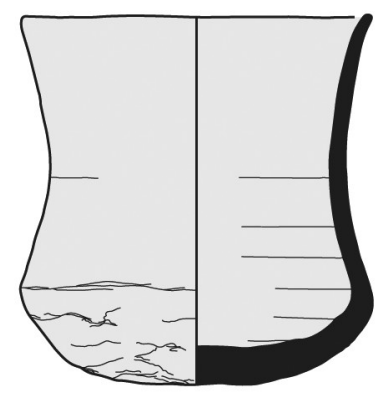

Cat. 22 $5 \mathrm{~cm}$

FIGURE 7.4 Cups of type I.1 with less pronounced S-shaped profile with two variants: variant I.1a and $\mathrm{I} .1 \mathrm{~b}$

Colour of break: not possible to determine

Notes: external surface rubbed off, slip fragmentary, burnish not possible to determine

References: (Czyżewska-Zalewska 2016, 727-728)

Cat. 20

Cup

Inv. no: $\mathbf{Z 2 O} / \mathbf{2}$

Provenance: Tumulus 20, shaft, south-western part

Construction: wheel-made (1)

State of preservation: incomplete, $55 \%$ of rim

Measurements: $\mathrm{Dr}=8 \mathrm{~cm} ; \mathrm{H}=9.7 \mathrm{~cm}$

Fabric: ZF1

Hardness: $3 / 4$

Firing: oxidized

Surface treatment: slipped and burnished on both surfaces; external-burnish 2, carelessly executed, internalburnish 3 on the upper part, carelessly executed

Surface colour: external-5YR 5/6 yellowish red, slip$10 \mathrm{R} 4 / 6$ red; internal -5 YR $5 / 6$ yellowish red, slip$10 \mathrm{R} 4 / 6$ red

Colour of break: $10 \mathrm{R} 5 / 3$ weak red

References: (Mahmoud El-Tayeb, Juszczyk-Futkowska, and Czyżewska 2014, 367, 369-371)

Variant I.1b

Cat. 21

Cup

Inv. no: Z25/21

Provenance: Tumulus 25, chamber 3

Construction: wheel-made (1)

State of preservation: complete
Measurements: $\mathrm{Dr}=8.9 \mathrm{~cm} ; \mathrm{H}=8.2 \mathrm{~cm}$

Fabric: not possible to determine

Hardness: 4

Firing: not possible to determine

Surface treatment: slipped and polished on both surfaces

Surface colour: external, slip—10R 4/6 red; internal, slip$10 \mathrm{R} 4 / 6 \mathrm{red}$

Colour of break: not possible to determine

References: (Klimaszewska-Drabot 2010, 480-481)

Cat. 22

Cup

Inv. no: Z1o/9

Provenance: Tumulus 10, burial chamber, southern part

Construction: wheel-made (1)

State of preservation: incomplete, $75 \%$ of rim

Measurements: $\mathrm{Dr}=7.8-8.1 \mathrm{~cm} ; \mathrm{H}=8.7 \mathrm{~cm}$

Fabric: ZF2

Hardness: $3 / 4$

Firing: oxidized

Surface treatment: slipped and polished on both surfaces

Surface colour: external, slip $-{ }_{5} \mathrm{R}$ 4/8 red; internal, slip$7.5 \mathrm{R} 4 / 4$ weak red

Colour of break: $2.5 \mathrm{Y} 3 / 2$ very dark greyish brown

2.2 Cups Type 1.2

[Figs 7.87-7.89]

Cat. 23

Cup

Inv. no: Z22/2

Provenance: Tumulus 22, burial chamber

Construction: wheel-made (1)

State of preservation: incomplete, $87 \%$ of rim 


\section{Cups Type I.2}

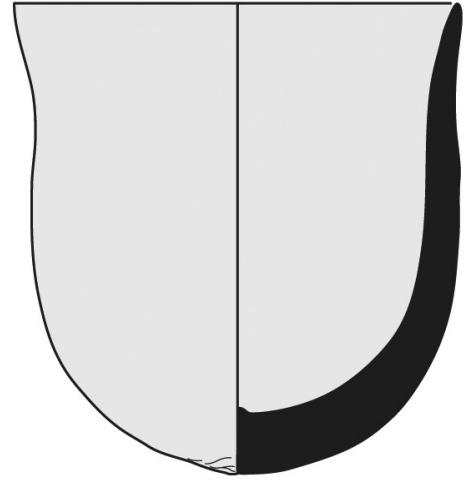

Cat. 23

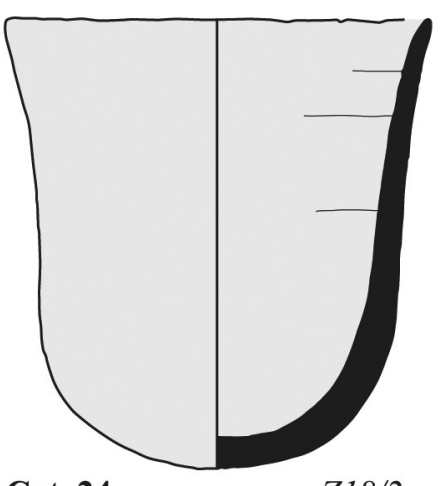

Cat. 24

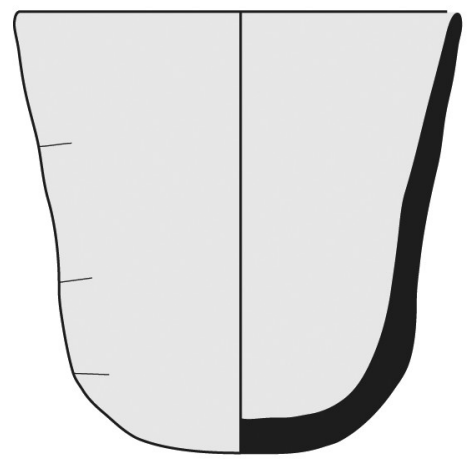

Cat. 25

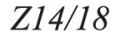

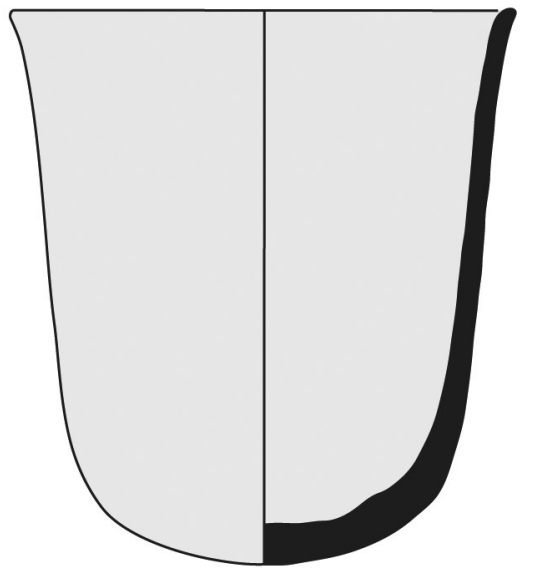

Cat. 26
$Z 12 / 110$

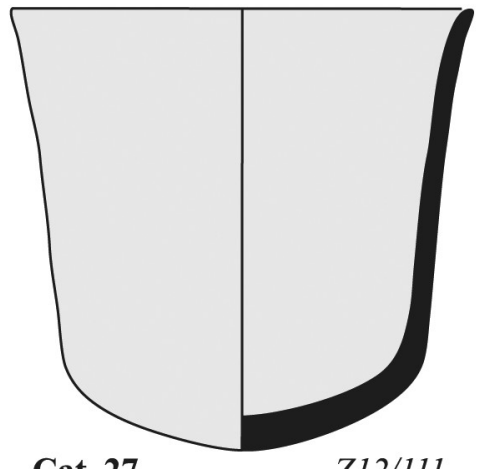

Cat. 27

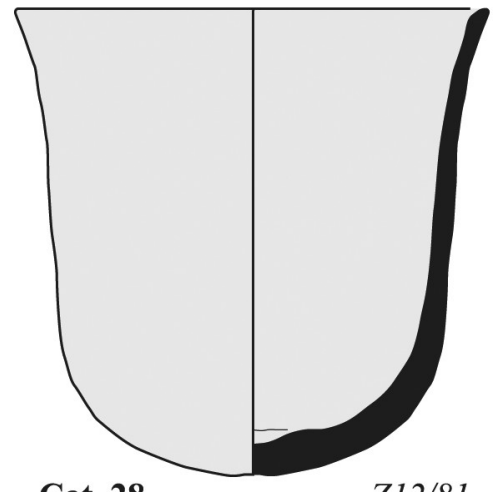

Cat. 28 $5 \mathrm{~cm}$

FIGURE 7.5 Cups of type I.2 with a slightly pointed base and flared walls

Measurements: $\mathrm{Dr}=10.4 \mathrm{~cm} ; \mathrm{H}=11 \mathrm{~cm}$

Fabric: ZF1

Hardness: $3 / 4$

Firing: oxidized

Surface treatment: slipped and burnished on both surfaces; external-burnish 2, carefully executed; internalburnish 1, carefully executed

Surface colour: external, slip—1oR 4/8 red; internal, slip$10 \mathrm{R} 4 / 6$ red

Colour of break: $10 \mathrm{R} 4 / 8$ red

References: (Klimaszewska-Drabot and Mahmoud El-Tayeb 2014, 10-13)

\section{Cat. 24}

Cup

Inv. no: $\mathbf{Z 1 8} / \mathbf{2}$

Provenance: Tumulus 18, burial chamber, central part
Construction: wheel-made (1)

State of preservation: incomplete, $59 \%$ of rim, wall fragments missing

Measurements: $\mathrm{Dr}=10 \mathrm{~cm} ; \mathrm{H}=10.6 \mathrm{~cm}$

Fabric: ZF1

Hardness: 3

Firing: oxidized

Surface treatment: slipped and burnished on both surfaces; burnish 2, carefully executed on both surfaces

Surface colour: external, slip-10R $5 / 6$ red; internal, slip$10 \mathrm{R} 5 / 6$ red

Colour of break: 10YR 4/2 dark greyish brown

References: (Klimaszewska-Drabot 2010, 480-481)

Cat. 25

Cup

Inv. no: Z14/18 
Provenance: Tumulus 14, chamber 1, central part

Construction: wheel-made (1)

State of preservation: incomplete, $97 \%$ of rim

Measurements: $\mathrm{Dr}=10.4 \mathrm{~cm} ; \mathrm{H}=10.3 \mathrm{~cm}$

Fabric: ZF1

Hardness: 4

Firing: oxidized

Surface treatment: slipped and burnished on both surfaces; external-burnish 2, carefully executed on the upper part, less carefully executed on the lower part; internal-burnish 1 on the upper part, burnish 2 on the lower part, carefully executed

Surface colour: external, slip—-10R 5/6 red; internal, slip$10 \mathrm{R} 5 / 6$ red

Colour of break: 2.5YR 6/6 light red

\section{Cat. 26}

Cup

Inv. no: $\mathbf{Z 1 2} / \mathbf{1 1 0}$

Provenance: Tumulus 12, chamber 3

Construction: wheel-made (1)

State of preservation: incomplete, $66 \%$ of rim, wall fragments missing

Measurements: $\mathrm{Dr}=12 \mathrm{~cm} ; \mathrm{H}=13 \mathrm{~cm}$

Fabric: ZF1

Hardness: $3 / 4$

Firing: oxidized

Surface treatment: slipped and burnished on both surfaces; external-burnish 2, carefully executed; internalburnish 3, carelessly executed

Surface colour: external—1oR 5/6 red, slip—-10R 4/4 weak red; internal—oR 4/6 red, slip-1oR 4/4 weak red

Colour of break: 7.5 YR $4 / 3$ brown

\section{Cat. 27}

Cup

Inv. no: Z12/111

Provenance: Tumulus 12, chamber 3

Construction: wheel-made (1)

State of preservation: complete

Measurements: $\mathrm{Dr}=10.8 \mathrm{~cm} ; \mathrm{H}=10.5 \mathrm{~cm}$

Fabric: not possible to determine

Hardness: 3

Firing: oxidized

Surface treatment: slipped and burnished on both surfaces; external—burnish 2, less carefully executed; internalburnish 3 , less carefully executed

Surface colour: external, slip— $\mathrm{OR}_{5} / 6$ red; internal, slip$10 \mathrm{R}_{5} / 6$ red

Colour of break: not possible to determine
Cat. 28

Cup

Inv. no: $\mathbf{Z 1 2} / 81$

Provenance: Tumulus 12, chamber 1, eastern part

Construction: wheel-made (1)

State of preservation: fragmentary, restored from sherds, $11 \%$ of rim, base complete, body fragments missing

Measurements: $\mathrm{Dr}=11 \mathrm{~cm} ; \mathrm{H}=10.5 \mathrm{~cm}$

Fabric: ZF3

Hardness: 4

Firing: oxidized

Surface treatment: slipped and burnished on both surfaces; external-burnish 3 , less carefully executed; internalburnish 2, less carefully executed

Surface colour: external-2.5YR 5/6 red, slip-2.5YR 4/4 reddish brown; internal -2.5 YR $5 / 6$ red, slip -5 YR $3 / 3$ dark reddish brown

Colour of break: 7.5YR 4/4 dark brown

Cat. 29

Cup

Inv. no: $\mathrm{Z}_{\mathbf{5}} / \mathbf{1}$

Provenance: Tumulus 5, superstructure

Construction: wheel-made (1)

State of preservation: fragmentary, restored from sherds, $2 \%$ of rim, $50 \%$ of base, body fragments missing

Measurements: $\mathrm{Dr}=8.2 \mathrm{~cm} ; \mathrm{H}=8.7 \mathrm{~cm}$

Fabric: $\mathrm{zF} 3$

Hardness: $3 / 4$

Firing: oxidized

Surface treatment: slipped and burnished on both surfaces; external-burnish 3 , less carefully executed; internalburnish 2, less carefully executed

Surface colour: external-5YR 5/4 reddish brown, slip10R 5/4 weak red; internal, slip-10R 4/6 red

Colour of break: 5 YR 6/6 reddish yellow

Cat. $3^{\circ}$

Cup

Inv. no: $\mathrm{Z}_{5} / 4$

Provenance: Tumulus 5, chamber 1, western part

Construction: wheel-made (1)

State of preservation: fragmentary, restored from sherds, $6 \%$ of rim, body fragments missing

Measurements: $\mathrm{Dr}=10 \mathrm{~cm} ; \mathrm{H}=8.2 \mathrm{~cm}$

Fabric: $\mathrm{ZF} 3$

Hardness: 3

Firing: oxidized

Surface treatment: slipped and burnished on both surfaces; burnish 2, less carefully executed on both surfaces 


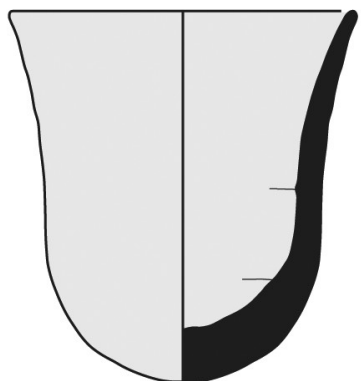

Cat. 29
Z5/1

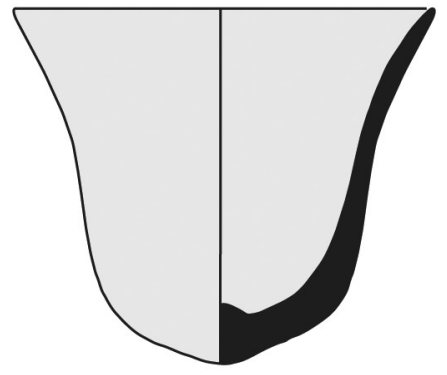

Cat. 30
$Z 5 / 4$

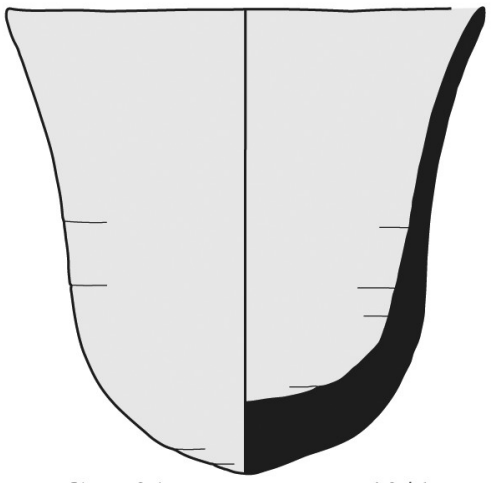

Cat. 31

Z19/6

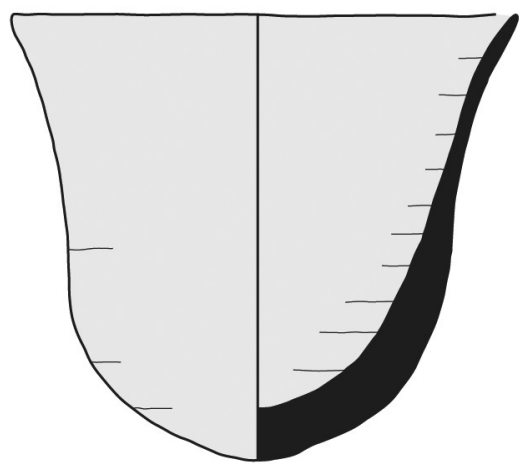

Cat. 32

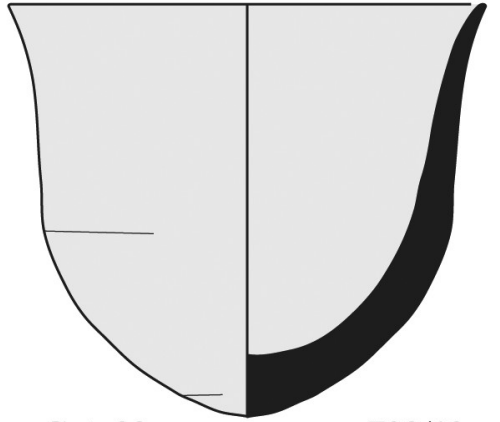

Cat. 33
$Z 28 / 10$

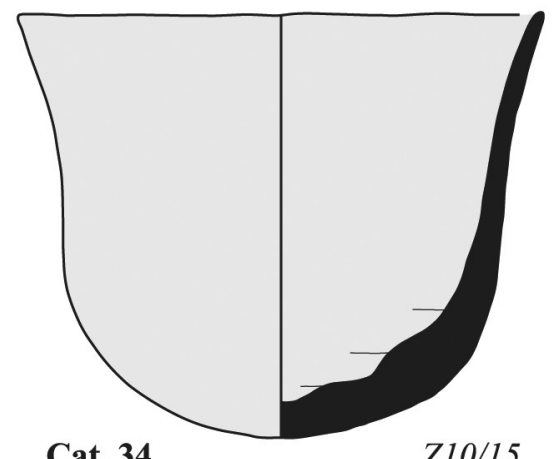

Cat. 34
Z10/15

FIGURE 7.6 Cups of type I.2 with a slightly pointed base and flared walls

Surface colour: external, slip-7.5R 5/4 weak red; internal, slip-10R $4 / 4$ weak red

Colour of break: 7.5YR 4/4 dark brown

References: (Klimaszewska-Drabot 2010, 480-481)

Cat. 31

Cup

Inv. no: Z19/6

Provenance: Tumulus 19, burial chamber

Construction: wheel-made (1)

State of preservation: incomplete, $44 \%$ of rim

Measurements: $\mathrm{Dr}=11.2 \mathrm{~cm} ; \mathrm{H}=10.8 \mathrm{~cm}$

Fabric: ZF3

Hardness: 3

Firing: oxidized

Surface treatment: slipped and burnished on both surfaces; burnish 2, carefully executed on both surfaces

Surface colour: external, slip—10R 4/6 red; internal, slip$10 \mathrm{R}_{4} / 6$ red

Colour of break: 7.5 YR $5 / 6$ strong brown

References: (Klimaszewska-Drabot 2010, 480-481)
Cat. $3^{2}$

Cup

Inv. no: $\mathrm{Z18} / 4$

Provenance: Tumulus 16, burial chamber, found on rim of bottle $\mathrm{Z} 18 / 6$

Construction: wheel-made (1)

State of preservation: complete

Measurements: $\mathrm{Dr}=11.3 \mathrm{~cm} ; \mathrm{H}=10.4 \mathrm{~cm}$

Fabric: not possible to determine

Hardness: 3

Firing: oxidized?

Surface treatment: slipped and burnished on both surfaces; external—surface dirty and burnish difficult to determine; internal-burnish 2, carefully executed

Surface colour: external, slip— $10 \mathrm{R} 5 / 6$ red; internal, slip$10 \mathrm{R} 4 / 6$ red

Colour of break: not possible to determine

References: (Klimaszewska-Drabot 2010, 480-481) 
Cat. 33

Cup

Inv. no: $\mathrm{Z28/10}$

Provenance: Tumulus 28, burial chamber, southern part

Construction: wheel-made (1)

State of preservation: incomplete, $61 \%$ of rim, wall fragments missing

Measurements: $\mathrm{Dr}=11 \mathrm{~cm} ; \mathrm{H}=9.7 \mathrm{~cm}$

Fabric: $\mathrm{ZF} 3$

Hardness: 3

Firing: oxidized

Surface treatment: slipped and burnished on both surfaces; external-burnish 2, carefully executed; internalburnish 2 on the upper part, burnish 3 on the lower part, carelessly executed

Surface colour: external, slip-7.5YR 5/6 strong brown; internal, slip- 7.5 YR $5 / 6$ strong brown

Colour of break: 1OYR 3/2 very dark greyish brown

Cat. 34

Cup

Inv. no: $\mathrm{Z1o} / \mathbf{1 5}$

Provenance: Tumulus 10, burial chamber, southern part

Construction: wheel-made (1)

State of preservation: incomplete, $85 \%$ of rim

Measurements: $\mathrm{Dr}=13 \mathrm{~cm} ; \mathrm{H}=10 \mathrm{~cm}$

Fabric: ZF3

Hardness: 3

Firing: oxidized

Surface treatment: slipped and burnished on both surfaces; burnish 2, carefully executed on both surfaces

Surface colour: external, slip-7.5YR 5/6 strong brown; internal, slip-7.5YR 5/6 strong brown

Colour of break: 1OYR 3/2 very dark greyish brown

References: (Klimaszewska-Drabot 2010, 48o-481)

Cat. 35

Cup

Inv. no: Z12/95

Provenance: Tumulus 12, chamber 3, western part

Construction: wheel-made (1)

State of preservation: incomplete, $75 \%$ of rim

Measurements: $\mathrm{Dr}=10.3 \mathrm{~cm} ; \mathrm{H}=10.3 \mathrm{~cm}$

Fabric: ZF3

Hardness: 4

Firing: oxidized

Surface treatment: slipped and burnished on both surfaces; external-burnish 2, less carefully executed; internalburnish 3 , less carefully executed

Surface colour: external-2.5YR 6/8 light red, slip2.5YR 5/6 red; internal-2.5YR 6/8 light red, slip2.5 YR $5 / 6$ red
Colour of break: 7.5YR 5/6 strong brown

References: (Czyżewska-Zalewska 2016, 727-728)

Cat. 36

Cup

Inv. no: Z12/92

Provenance: Tumulus 12, chamber 3, western part

Construction: wheel-made (1)

State of preservation: incomplete, $93 \%$ of rim

Measurements: $\mathrm{Dr}=12 \mathrm{~cm} ; \mathrm{H}=12 \mathrm{~cm}$

Fabric: ZF3

Hardness: 4

Firing: oxidized

Surface treatment: slipped and burnished on both surfaces; burnish 2, carefully executed on both surfaces

Surface colour: external-2.5YR 5/8 red, slip $-10 \mathrm{R}$ 5/6 red; internal-2.5YR $5 / 8$ red, slip- $10 \mathrm{R} 5 / 4$ weak red

Colour of break: 7.5YR 6/4 light brown

References: (Czyżewska-Zalewska 2016, 727-728)

Cat. 37

Cup

Inv. no: $\mathrm{Z6} / \mathbf{1 5}+\mathrm{Z6} / \mathbf{1 8}$

Provenance: Tumulus 6, tunnel, western part

Construction: wheel-made (1)

State of preservation: incomplete, chipped rim, $91 \%$ of rim

Measurements: $\mathrm{Dr}=11 \mathrm{~cm} ; \mathrm{H}=10.4 \mathrm{~cm}$

Fabric: ZF3

Hardness: 3

Firing: not possible to determine

Surface treatment: slipped and burnished on both surfaces; burnish 2, carefully executed on both surfaces

Surface colour: external, slip-7.5 R $5 / 8$ red; internal, slip2.5YR 5/6 red

Colour of break: 7.5YR 6/4 light brown

Cat. 38

Cup

Inv. no: $\mathrm{Z11} / \mathbf{1 1}$

Provenance: Tumulus 11, chamber 1, eastern part

Construction: wheel-made (1)

State of preservation: complete

Measurements: $\mathrm{Dr}=11 \mathrm{~cm} ; \mathrm{H}=10.1 \mathrm{~cm}$

Fabric: not possible to determine

Hardness: 3

Firing: not possible to determine

Surface treatment: slipped and burnished on both surfaces; burnish 2, carefully executed on both surfaces

Surface colour: external, slip-10R $5 / 8$ red; internal, slip1oR $5 / 6$ red

Colour of break: not possible to determine 


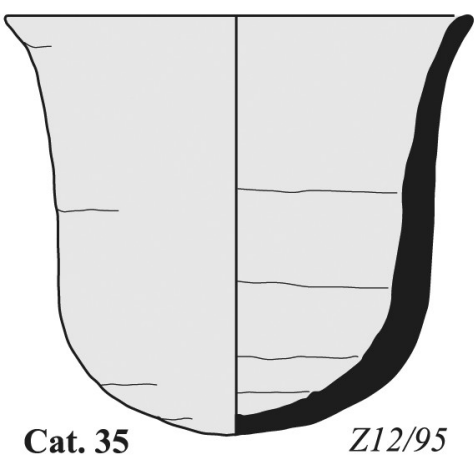

Z12/95

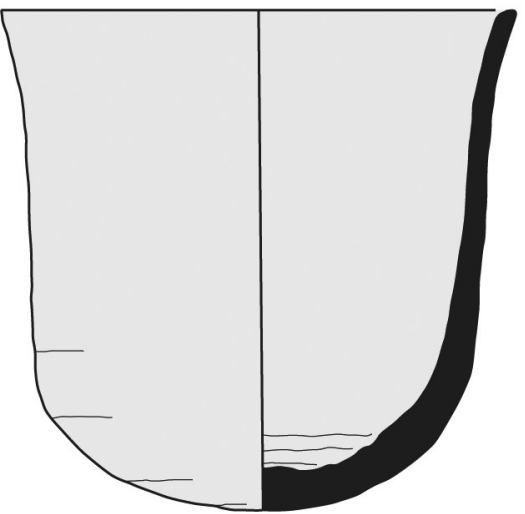

Cat. 36

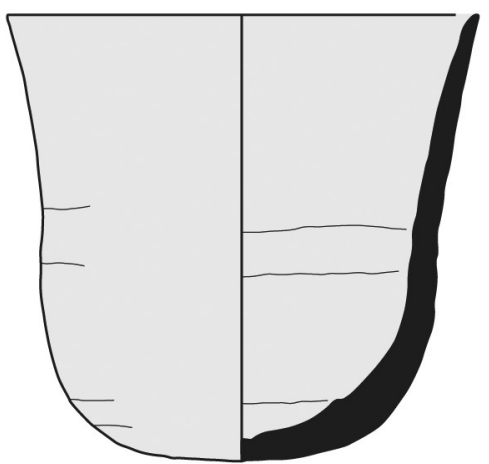

Cat. 37

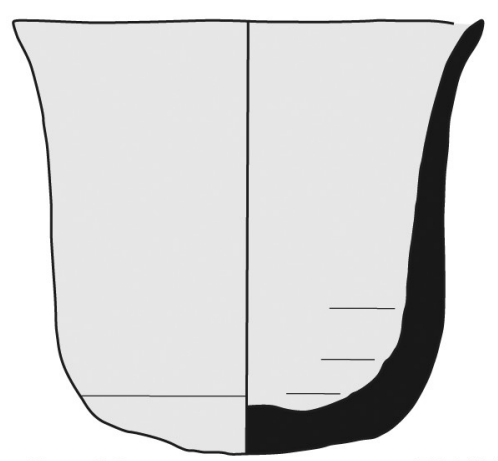

Cat. 38

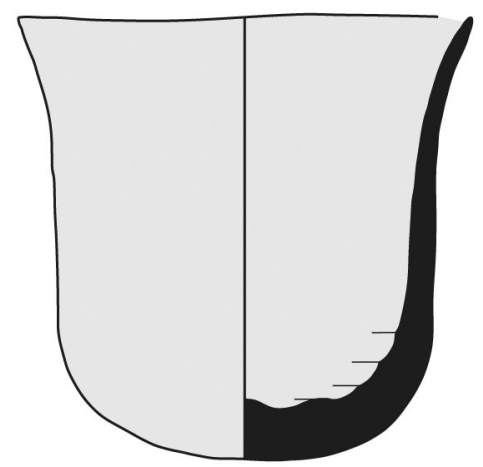

Cat. 39
Z11/14

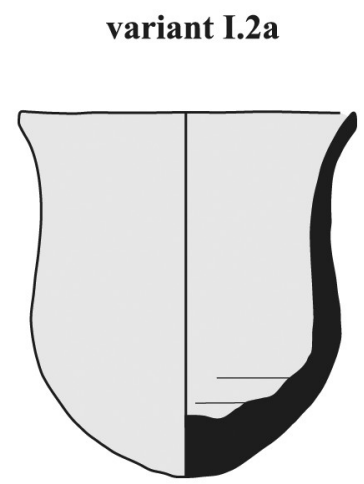

Cat. 40

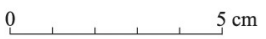

FIGURE 7.7 Cups of type I.2 with a slightly pointed base and flared walls

References: (Klimaszewska-Drabot and Czyżewska 2012, 368-372)

Cat. 39

Cup

Inv. no: $\mathrm{Z11} / 14$

Provenance: Tumulus 11, chamber 1, eastern part

Construction: wheel-made (1)

State of preservation: incomplete, $51 \%$ of rim

Measurements: $\mathrm{Dr}=10.7 \mathrm{~cm} ; \mathrm{H}=10.3 \mathrm{~cm}$

Fabric: ZF1

Hardness: 3

Firing: reduced

Surface treatment: slipped and burnished on both surfaces; burnish 2, carefully executed on both surfaces

Surface colour: external, slip-10R $5 / 6$ red; internal, slip2.5 YR $5 / 6$ red
Colour of break: 5 YR 4/4 reddish brown

References: (Klimaszewska-Drabot and Czyżewska 2012, 368-372)

Variant I.2a

Cat. 40

Cup

Inv. no: Z11/19

Provenance: Tumulus 11, chamber 2, central part

Construction: wheel-made (1)

State of preservation: incomplete, $97 \%$ of rim

Measurements: $\mathrm{Dr}=7.9-8.4 \mathrm{~cm} ; \mathrm{H}=8.6 \mathrm{~cm}$

Fabric: $\mathrm{ZF} 4$

Hardness: 4

Firing: oxidized?

Surface treatment: slipped and polished on both surfaces Surface colour: external, slip—1oR 4/6 red; internal, slip$10 \mathrm{R}_{4} / 6 \mathrm{red}$ 


\section{Cups Type I.3}

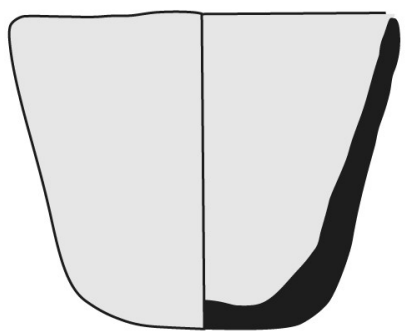

Cat. 41
Z23/7

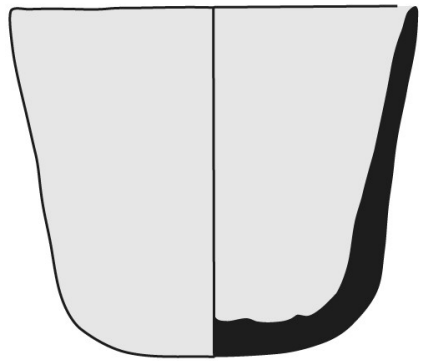

Cat. 42
Z23/8

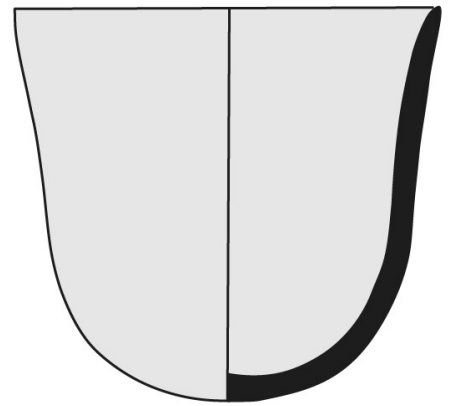

Cat. 45
Z22/1

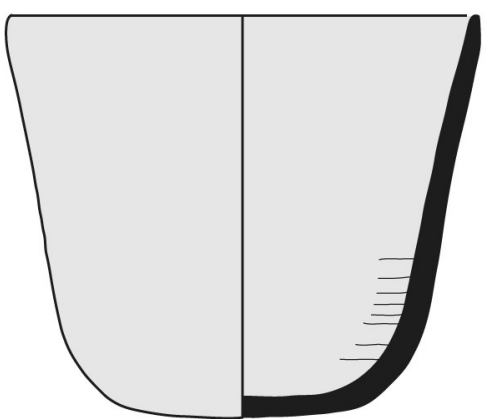

Cat. 43
Z14/12

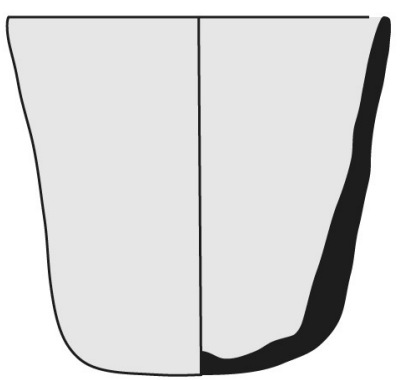

Cat. 44

$Z 23 / 2$

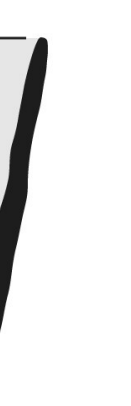

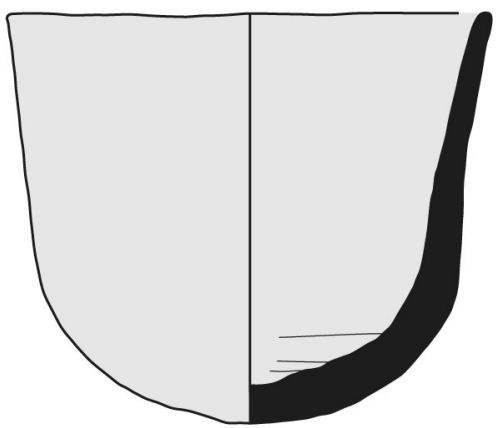

Cat. 46

FIGURE 7.8 Tulip-shaped cups of type I.3 with flared walls

Colour of break: 2.5 YR $5 / 6$ red

References: (Klimaszewska-Drabot and Czyżewska 2012, $368-372)$

\subsection{Cups Type I.3}

Cat. 41

Cup

Inv. no: $\mathrm{Z} 23 / 7$

Provenance: Tumulus 23, chamber 1

Construction: wheel-made (1)

State of preservation: complete

Measurements: $\mathrm{Dr}=9 \mathrm{~cm} ; \mathrm{H}=7.4 \mathrm{~cm}$

Fabric: not possible to determine

Hardness: $3 / 4$

Firing: not possible to determine

Surface treatment: slipped and burnished on both surfaces; external-burnish 1, carefully executed; internalburnish 2, less carefully executed

Surface colour: external, slip-10R 4/4 weak red; internal, slip-10R 4/6 red

Colour of break: not possible to determine
Cat. 42

Cup Inv. no: $\mathrm{Z} 23 / 8$

Provenance: Tumulus 23, chamber 2

[Figs 7.89-7.90] Construction: wheel-made (1)

State of preservation: complete

Measurements: $\mathrm{Dr}=9.5 \mathrm{~cm} ; \mathrm{H}=8.1 \mathrm{~cm}$

Fabric: not possible to determine

Hardness: $3 / 4$

Firing: not possible to determine

Surface treatment: slipped and burnished on both surfaces; burnish 1, carefully executed on both surfaces

Surface colour: external, slip-2.5YR 4/6 red; internal, slip-2.5YR 4/4 reddish brown

Colour of break: not possible to determine

Cat. 43

Cup

Inv. no: $\mathrm{Z14} / 12$

Provenance: Tumulus 14, chamber 3, central part

Construction: wheel-made (1) 
State of preservation: complete

Measurements: $\mathrm{Dr}=11 \mathrm{~cm} ; \mathrm{H}=9.3 \mathrm{~cm}$

Fabric: not possible to determine

Hardness: 4

Firing: not possible to determine

Surface treatment: slipped and burnished on both surfaces; external-burnish 3 , less carefully executed; internalburnish 1, carefully executed

Surface colour: external, slip-10R 5/6 red; internal, slip1oR $5 / 6$ red

Colour of break: not possible to determine

\section{Cat. 44}

Cup

Inv. no: $\mathrm{Z23} / \mathbf{2}$

Provenance: Tumulus 23, chamber 1

Construction: wheel-made (1)

State of preservation: complete

Measurements: $\mathrm{Dr}=8.9 \mathrm{~cm} ; \mathrm{H}=8.3 \mathrm{~cm}$

Fabric: not possible to determine

Hardness: $3 / 4$

Firing: not possible to determine

Surface treatment: slipped and burnished on both surfaces; external-burnish 1, carefully executed; internalburnish 1 on the upper part, burnish 2 on the lower part, carefully executed

Surface colour: external, slip_-10R 5/6 red; internal, slip10R $4 / 6$ red

Colour of break: not possible to determine

\section{Cat. 45}

Cup

Inv. no: $\mathrm{Z22} / 1$

Provenance: Tumulus 22, burial chamber

Construction: wheel-made (1)

State of preservation: complete

Measurements: $\mathrm{Dr}=10 \mathrm{~cm} ; \mathrm{H}=9 \mathrm{~cm}$

Fabric: not possible to determine

Hardness: $3 / 4$

Firing: not possible to determine

Surface treatment: slipped and burnished on both surfaces; external-burnish 3, carefully executed; internalburnish 2, less carefully executed

Surface colour: external, slip_-10R 4/6 red; internal, slip$10 \mathrm{R}_{4} / 6$ red

Colour of break: not possible to determine

References: (Klimaszewska-Drabot and Mahmoud ElTayeb 2014, 10-13)
Cat. 46

Cup

Inv. no: $\mathrm{Z} 27 / 3$

Provenance: Tumulus 27, burial chamber, southern corner Construction: wheel-made (1)

State of preservation: incomplete, $83 \%$ of rim

Measurements: $\mathrm{Dr}=11.2 \mathrm{~cm} ; \mathrm{H}=9.5 \mathrm{~cm}$

Fabric: ZF2

Hardness: 3

Firing: oxidized

Surface treatment: slipped and burnished on both surfaces; burnish 2, carelessly executed on both surfaces

Surface colour: external, slip-10R $5 / 6$ red; internal, slip10R $5 / 6$ red

Colour of break: 5 YR $5 / 6$ yellowish red

References: (Klimaszewska-Drabot and Czyżewska 2012, 364)

Cat. 47

Cup

Inv. no: Z12/9o

Provenance: Tumulus 12, chamber 3, western part

Construction: wheel-made (1)

State of preservation: fragmentary, restored from sherds, $43 \%$ of rim, body fragments missing

Measurements: $\mathrm{Dr}=12 \mathrm{~cm} ; \mathrm{H}=10.5 \mathrm{~cm}$

Fabric: $\mathrm{ZF} 3$

Hardness: $3 / 4$

Firing: oxidized

Surface treatment: slipped and burnished on both surfaces; burnish 3 , less carefully executed on both surfaces

Surface colour: external, slip-10R $5 / 6$ red; internal, slip10R $5 / 6$ red

Colour of break: 10YR $5 / 4$ yellowish brown

References: (Czyżewska-Zalewska 2016, 727-728)

Cat. 48

Cup

Inv. no: $\mathbf{Z 1 2} / 124$

Provenance: Tumulus 12, chamber 3

Construction: wheel-made (1)

State of preservation: complete

Measurements: $\mathrm{Dr}=11.7-12 \mathrm{~cm} ; \mathrm{H}=11 \mathrm{~cm}$

Fabric: not possible to determine

Hardness: $3 / 4$

Firing: not possible to determine

Surface treatment: slipped and burnished on both surfaces; burnish 3 , less carefully executed on both surfaces

Surface colour: external, slip-10R $5 / 6$ red; internal, slip1oR 5/8 red

Colour of break: not possible to determine 


\section{Cups Type I.3}

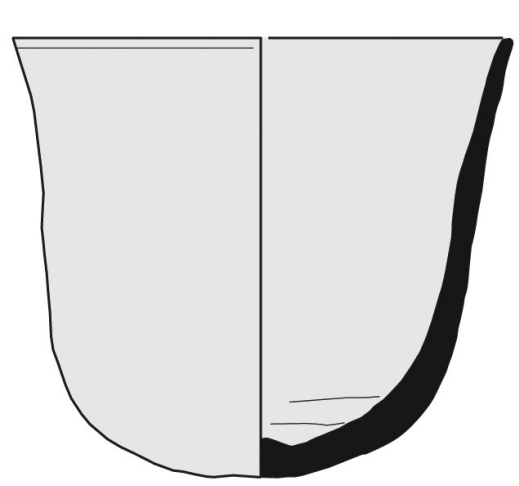

Cat. 47

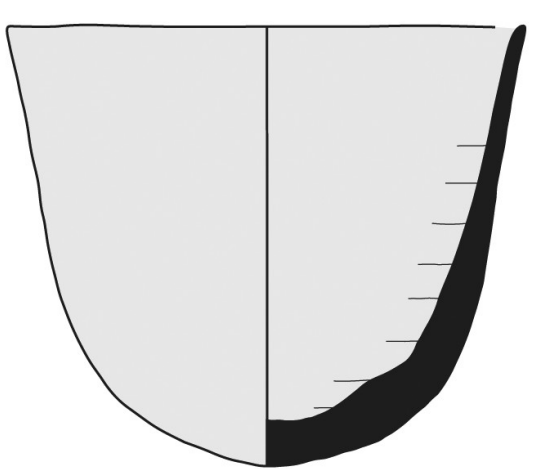

Cat. 50
Z12/90

Z10/5

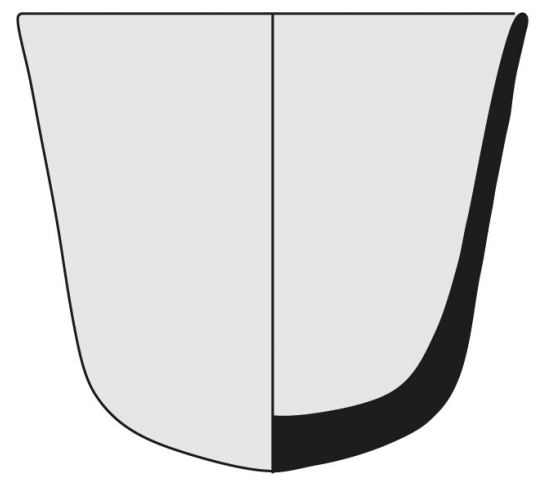

$Z 12 / 124$

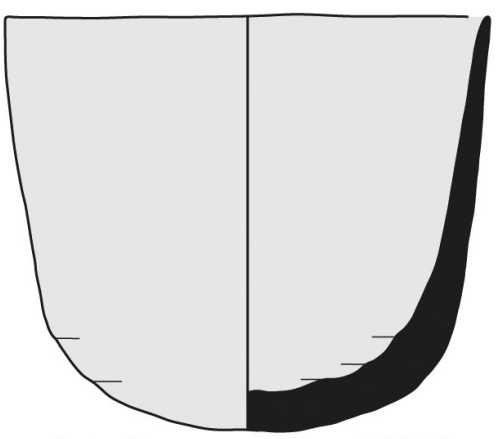

Cat. 49

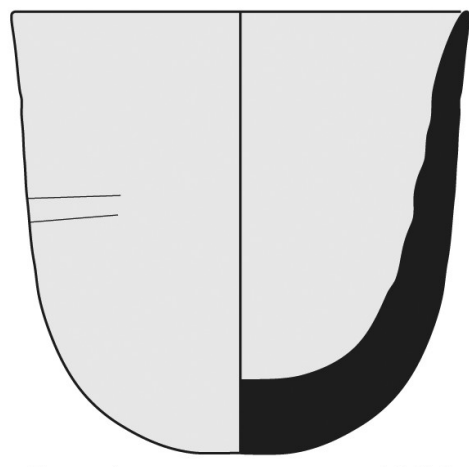

Cat. 51
$Z 28 / 12$

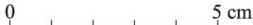

FIGURE 7.9 Tulip-shaped cups of type I.3 with flared walls

Cat. 49

Cup

Inv. no: $\mathbf{Z 1 1} / \mathbf{2 8}$

Provenance: Tumulus 11, chamber 2, southern part

Construction: wheel-made (1)

State of preservation: incomplete, $82 \%$ of rim

Measurements: $\mathrm{Dr}=11.5 \mathrm{~cm} ; \mathrm{H}=9.8 \mathrm{~cm}$

Fabric: $\mathrm{ZF} 3$

Hardness: $2 / 3$

Firing: oxidized

Surface treatment: slipped and burnished on both surfaces; external-burnish 3, carelessly executed; internalburnish 2, less carefully executed

Surface colour: external, slip-10R 4/6 red; internal, slip10R $4 / 6$ red

Colour of break: 5YR 4/6 yellowish red

References: (Klimaszewska-Drabot and Czyżewska 2012, $368-372)$
Cat. $5^{\circ}$

Cup

Inv. no: $\mathbf{Z 1 o} / 5$

Provenance: Tumulus 10, burial chamber, southern part

Construction: wheel-made (1)

State of preservation: incomplete, $95 \%$ of rim

Measurements: $\mathrm{Dr}=12.1 \mathrm{~cm} ; \mathrm{H}=10.5 \mathrm{~cm}$

Fabric: not possible to determine

Hardness: 3

Firing: oxidized?

Surface treatment: slipped and burnished on both surfaces; burnish 1, carefully executed on both surfaces

Surface colour: external, slip-7.5R 4/4 weak red; internal, slip- $7.5^{\mathrm{R}} 4 / 3$ weak red

Colour of break: 7.5YR 5/6 strong brown

References: (Klimaszewska-Drabot 2010, 480-481)

Cat. $5^{1}$

Cup

Inv. no: $\mathbf{Z 2 8 / 1 2}$

Provenance: Tumulus 28, burial chamber, southern part 


\section{Cups Type I.4}

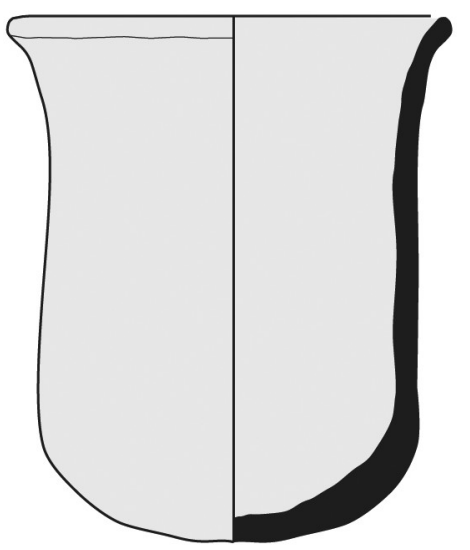

Cat. 52

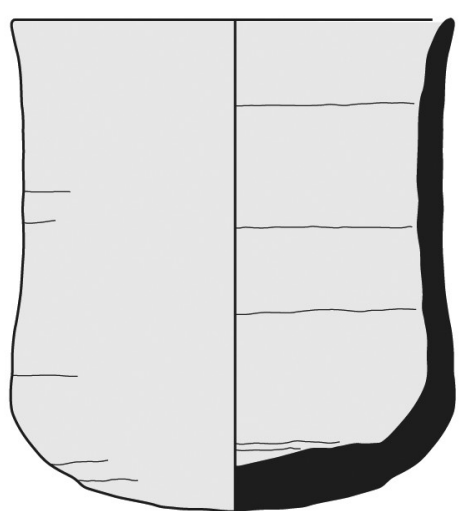

Cat. 53
Z12/102

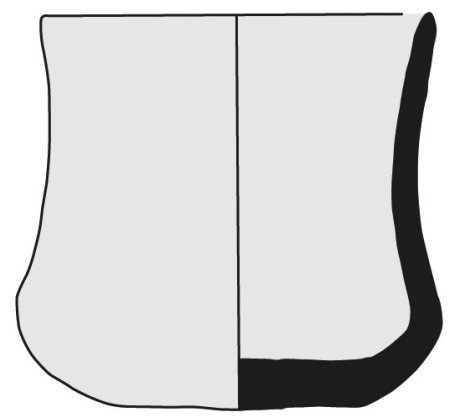

Cat. 54
Z26/51

0 $5 \mathrm{~cm}$

FIGURE 7.10 Slender cups of type I.4 with straight or concave walls

Construction: wheel-made (1)

State of preservation: incomplete, $88 \%$ of rim

Measurements: $\mathrm{Dr}=10.5 \mathrm{~cm} ; \mathrm{H}=10.5 \mathrm{~cm}$

Fabric: $\mathrm{ZF} 3$

Hardness: 3

Firing: oxidized

Surface treatment: slipped and burnished on both surfaces; external-burnish 2, carefully executed, internalburnish 1, carefully executed

Surface colour: external, slip_-10R $5 / 6$ red; internal, slip$10 \mathrm{R} 5 / 6$ red

Colour of break: 7.5YR 5/6 strong brown

\subsection{Cups Type 1.4}

Cat. $5^{2}$

Cup

Inv. no: $\mathrm{Z12} / 115+\mathrm{Z12} / 98$

Provenance: Tumulus 12, chamber 3, western part

Construction: wheel-made (1)

State of preservation: fragmentary, restored from sherds, $43 \%$ of rim, body sherds missing

Measurements: $\mathrm{Dr}=10 \mathrm{~cm} ; \mathrm{H}=12 \mathrm{~cm}$

Fabric: ZF3

Hardness: 3

Firing: oxidized

Surface treatment: slipped and burnished on both surfaces; burnish 3 , less carefully executed on both surfaces

Surface colour: external, slip-10R $5 / 6$ red; internal, slip1oR $5 / 6$ red

Colour of break: 7.5YR 5/4 brown

References: (Czyżewska-Zalewska 2016, 727-728)
Cat. 53

Cup

Inv. no: $\mathrm{Z12} / 102$

Provenance: Tumulus 12, chamber 3

Construction: wheel-made (1)

State of preservation: incomplete, $62 \%$ of rim

Measurements: $\mathrm{Dr}=10 \mathrm{~cm} ; \mathrm{H}=11 \mathrm{~cm}$

Fabric: $\mathrm{ZF} 3$

Hardness: 3

Firing: oxidized

Surface treatment: slipped and burnished on both surfaces; burnish 3 , less carefully executed on both surfaces

Surface colour: external-1oR 5/8 red, slip-10R 4/4 weak red; internal—2.5YR 5/8 red, slip-10R 4/4 weak red

Colour of break: 7.5YR 5/4 brown

References: (Czyżewska-Zalewska 2016, 727-728)

Cat. 54

Cup

Inv. no: $\mathbf{Z 2 6} / 51$

Provenance: Tumulus 26, chamber 1, southern part

Construction: wheel-made (1)

State of preservation: incomplete, chipped rim, $96 \%$ of rim Measurements: $\mathrm{Dr}=9 \mathrm{~cm} ; \mathrm{H}=9 \mathrm{~cm}$

Fabric: ZF1

Hardness: 3

Firing: reduced

Surface treatment: slipped and burnished on both surfaces; burnish 2, carefully executed on both surfaces

Surface colour: external, slip—1oR 4/4 weak red; internal, slip_-10R 3/4 dusky red

Colour of break: 7.5YR 5/6 strong brown 


\section{Cups Type I.5}

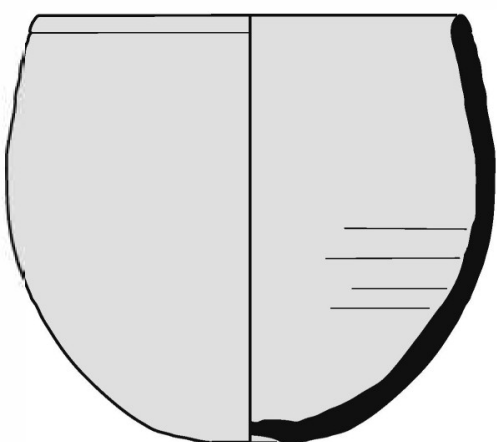

Cat. 55

Z11/6

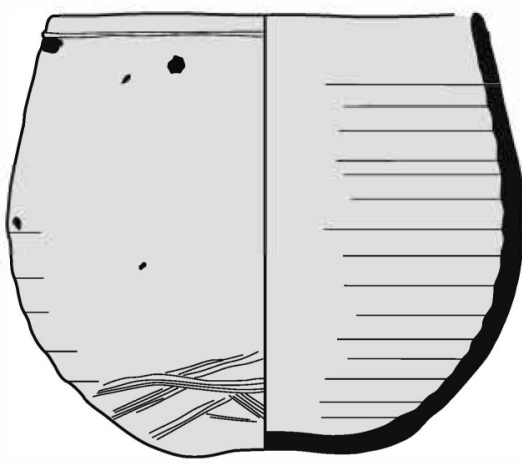

Cat. 56
$Z 11 / 10$

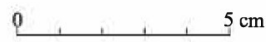
$5 \mathrm{~cm}$

FIGURE 7.11 Globular cups of type I.5

\subsection{Cups Type I.5}

Cat. 55

Cup

Inv. no: $\mathrm{Zu1} / 6$

Provenance: Tumulus 11, chamber 1, eastern part

Construction: wheel-made (2)

State of preservation: almost complete, $97 \%$ of rim

Measurements: $\mathrm{Dr}=10 \mathrm{~cm} ; \mathrm{H}=10 \mathrm{~cm}$

Fabric: difficult to determine, probably ZF4

Hardness: $3 / 4$

Firing: oxidized

Surface treatment: slipped on both surfaces

Surface colour: external, slip-10R $5 / 8$ red; internal, slip1oR $5 / 8$ red

Colour of break: $10 \mathrm{R} 5 / 8$ red

References: (Klimaszewska-Drabot and Czyżewska 2012, 368-372)

\section{Cat. $5^{6}$}

Cup

Inv. no: Z11/1o

Provenance: Tumulus 11, chamber 1, eastern part

Construction: wheel-made (2)

State of preservation: complete

Measurements: $\mathrm{Dr}=10-10.2 \mathrm{~cm} ; \mathrm{H}=10.2 \mathrm{~cm}$

Fabric: not possible to determine

Hardness: $3 / 4$

Firing: not possible to determine

Surface treatment: slipped on both surfaces

Surface colour: external, slip-1oR $5 / 6$ red; internal, slip$10 \mathrm{R}_{5} / 6$ red

Colour of break: not possible to determine

References: (Klimaszewska-Drabot and Czyżewska 2012, 368-372; Mahmoud El-Tayeb and Czyżewska 2011, $115-117$ )
[Fig. 7.90] 2.6 Cups Varia

Cat. 57

Cup

Inv. no: Z14/14

Provenance: Tumulus 14, chamber 1, south-eastern corner Construction: wheel made (2)

State of preservation: incomplete, $19 \%$ of rim, wall fragment missing, complete profile

Measurements: $\mathrm{Dr}=9.2 \mathrm{~cm}, \mathrm{H}=10 \mathrm{~cm}$

Fabric: Lower Nubian?

Hardness: 4

Firing: oxidized

Surface treatment: slipped on both surfaces

Surface colour: external, slip-7.5YR 7/3 pink; internal, slip- 7.5 Y $8 / 2$ pinkish white

Colour of break: 7.5YR 5/4 brown

Decoration: painted and grooved decoration on external surface; grooves on body forming border for painted decoration of brown diagonally crossed lines-5YR 3/3 dark reddish brown

Cat. 58

Cup

Inv. no: $\mathrm{Z26} / 5^{2}$

Provenance: Tumulus 26, chamber 1, central part

Construction: wheel-made (2)

State of preservation: complete

Measurements: $\mathrm{Dr}=9 \mathrm{~cm} ; \mathrm{H}=8.9 \mathrm{~cm}$

Fabric: not possible to determine

Hardness: 3

Firing: not possible to determine

Surface treatment: uncoated

Surface colour: external-5YR 5/6 yellowish red; internal- 5 Y $4 / 3$ reddish brown

Colour of break: not possible to determine 
Cat. 59

Cup

Inv. no: $\mathrm{Z14} / 8$

Provenance: Tumulus 14, chamber 3, western part

Construction: wheel-made (2)

State of preservation: complete

Measurements: $\mathrm{Dr}=10.5 \mathrm{~cm} ; \mathrm{H}=9.5 \mathrm{~cm}$

Fabric: not possible to determine

Hardness: $2 / 3$

Firing: not possible to determine

Surface treatment: uncoated

Surface colour: external-5YR 5/4 reddish brown; internal-5YR $5 / 4$ reddish brown

Colour of break: not possible to determine

Cat. 60

Cup

Inv. no: $\mathrm{Z14} / \mathbf{1 1}$

Provenance: Tumulus 14, chamber 3 , central part

Construction: wheel-made (2)

State of preservation: complete

Measurements: $\mathrm{Dr}=9.3 \mathrm{~cm} ; \mathrm{H}=10 \mathrm{~cm}$

Fabric: not possible to determine

Hardness: $2 / 3$

Firing: not possible to determine

Surface treatment: uncoated

Surface colour: external—5YR 6/4 light reddish brown; internal-5YR 6/4 light reddish brown

Colour of break: not possible to determine

Cat. 61

Cup

Inv. no: Z14/5

Provenance: Tumulus 14, chamber 3, western part

Construction: wheel-made (2)

State of preservation: complete

Measurements: $\mathrm{Dr}=11.3 \mathrm{~cm} ; \mathrm{D}_{\mathrm{b}}=4 \mathrm{~cm} ; \mathrm{H}=7.6 \mathrm{~cm}$

Fabric: not possible to determine

Hardness: 3

Firing: not possible to determine

Surface treatment: uncoated

Surface colour: external-5YR 5/4 reddish brown; internal -5 YR $5 / 4$ reddish brown

Colour of break: not possible to determine

Cat. 62

Cup (similar to type I.3)

Inv. no: $\mathrm{Z12} / 68$

Provenance: Tumulus 12, fill of shaft

Construction: wheel-made (1)

State of preservation: complete
Measurements: $\mathrm{Dr}=9.3 \mathrm{~cm} ; \mathrm{H}=7.5 \mathrm{~cm}$

Fabric: not possible to determine

Hardness: $3 / 4$

Firing: reduced

Surface treatment: slipped on both surfaces

Surface colour: external-5YR 5/6 yellowish red, slip1OR 4/4 weak red; internal-5YR 5/6 yellowish red, slip-10R $4 / 6$ red

Colour of break: not possible to determine

Cat. 63

Cup

Inv. no: $\mathrm{Z}_{7} / \mathbf{2 o}$

Provenance: Tumulus 7, topsoil, probably modern dump

Construction: wheel-made (2)

State of preservation: fragmentary, $50 \%$ of rim, base almost complete, body sherds missing

Measurements: $\mathrm{Dr}=9 \mathrm{~cm} ;{ }_{\mathrm{p}} \mathrm{H}=c .9 \mathrm{~cm}$

Fabric: ZF4

Hardness: 3

Firing: oxidized

Surface treatment: slipped and polished on both surfaces

Surface colour: slip_-10R 5/6 red on both surfaces

Colour of break: $10 \mathrm{R} 5 / 6$ red

\section{Cat. 64}

Cup

Inv. no: $\mathrm{Z} \mathbf{9} / \mathbf{2}$

Provenance: Tumulus 9, fill of shaft

Construction: wheel-made (2)

State of preservation: incomplete, $61 \%$ of rim, body fragment missing

Measurements: $\mathrm{Dr}=9.5 \mathrm{~cm} ; \mathrm{H}=7.2 \mathrm{~cm}$

Fabric: $\mathrm{ZF} 4$

Hardness: 3

Firing: oxidized

Surface treatment: slipped and polished on both surfaces

Surface colour: slip-10R 4/6 red, on both surfaces

Colour of break: $10 \mathrm{R} 4 / 6$ red

Decoration: on the external surface; incised-grooves on whole external surface

References: (Mahmoud El-Tayeb, Juszczyk-Futkowska, and Czyżewska 2014, 367, 369-371) 


\section{Cups Varia}

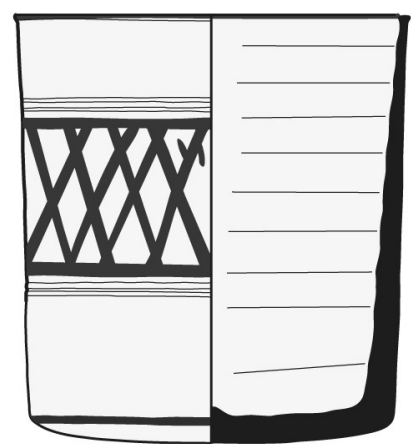

Cat. 57

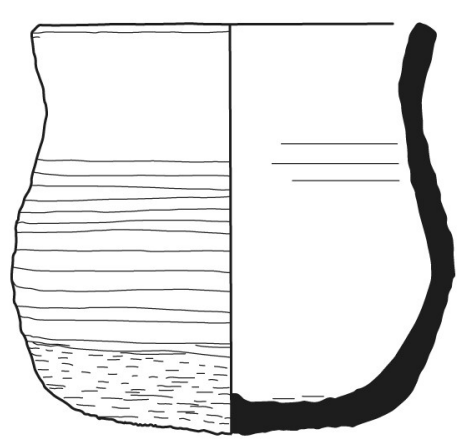

Cat. 58
Z26/52

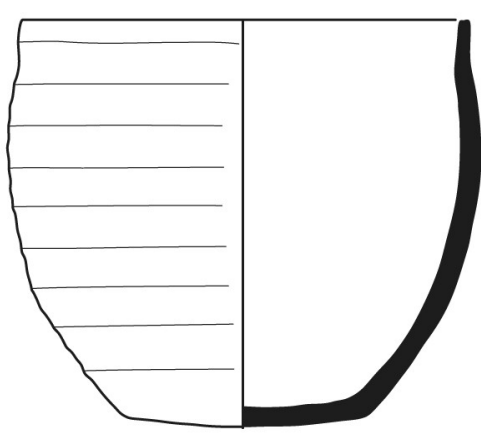

Cat. 59

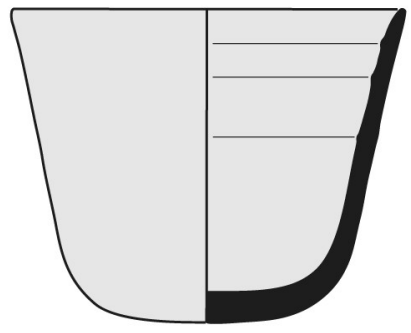

Cat. 62

Z12/68

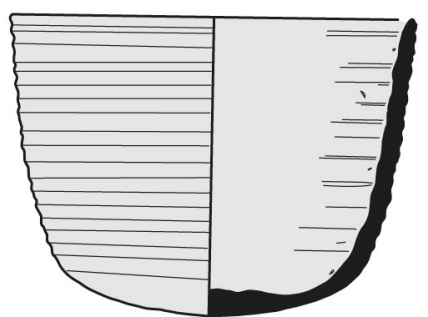

Cat. 64

$Z 9 / 2$

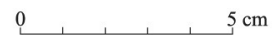

Cat. 63

$Z 7 / 20$

FIGURE 7.12 Cups of different shapes

3

Group II-Bowls

3.1 Bowls Type II.1

Variant II.1a

Cat. 65

Bowl

Inv. no: Z19/5

Provenance: Tumulus 19, burial chamber

Construction: wheel-made (1)

State of preservation: incomplete, body fragment missing Measurements: $\mathrm{Dr}=16 \mathrm{~cm} ; \mathrm{H}=11.1 \mathrm{~cm}$

Fabric: ZF3?
Hardness: 3

Firing: oxidized

[Fig. 7.92] Surface treatment: slipped on both surfaces; externalburnish 2, carelessly executed; internal-burnish 2, carefully executed

Surface colour: external natural surface-5YR 6/3 light reddish brown, slip $-7.5^{\mathrm{R}} 4 / 6$ red; internal, slip $-7.5^{\mathrm{R}} 4 / 6$ red

Colour of break: 7.5YR 5/4 brown

References: (Klimaszewska-Drabot 2010, 481) 


\section{Bowls Type II.1}

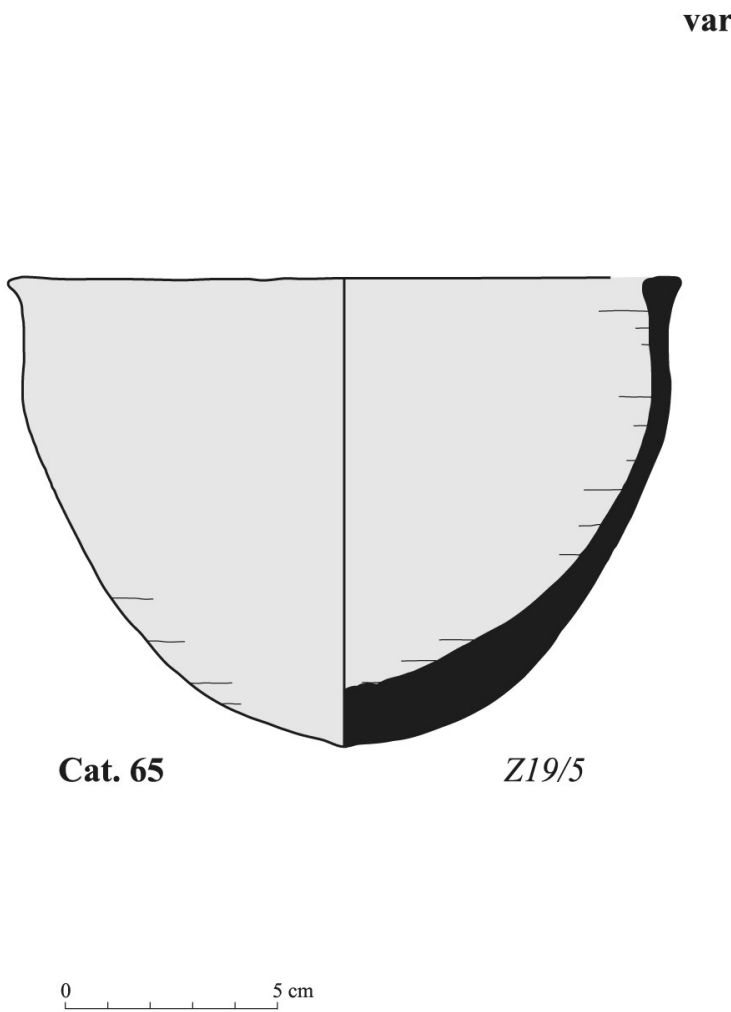

\section{ariant II.1a}

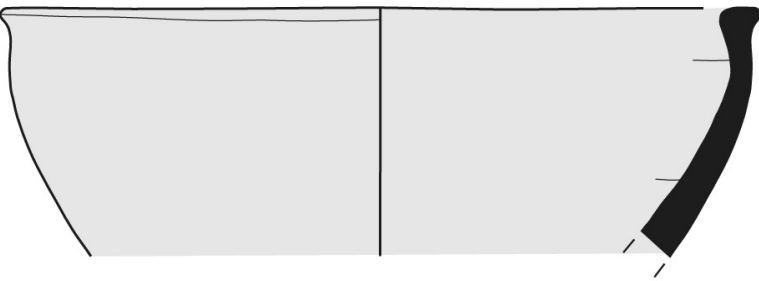

Cat. 66

FIGURE 7.13 External ledge-rimmed bowls of type II.1, variant a

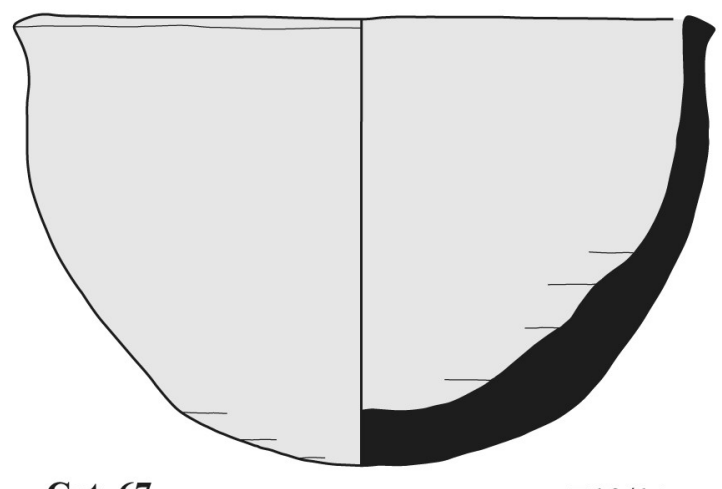

Cat. 67

Z10/14

Cat. 66

Bowl

Inv. no: Z19/2

Provenance: Tumulus 19, fill of shaft

Construction: wheel-made (1)

State of preservation: fragmentary, $9 \%$ of rim

Measurements: $\mathrm{Dr}=18 \mathrm{~cm} ;{ }_{\mathrm{p}} \mathrm{H}=5.8 \mathrm{~cm}$

Fabric: $\mathrm{ZF} 3$

Hardness: $3 / 4$

Firing: oxidized

Surface treatment: slipped on both surfaces; externalburnish 2, carefully executed; internal-surface corroded, burnish not possible to determine

Surface colour: external, slip-10R $5 / 6$ red; internal natural surface-7.5YR 6/4 light brown, slip-10R 6/6 light red Colour of break: 1OYR 4/2 dark greyish brown

\section{Cat. 67}

Bowl

Inv. no: $\mathrm{Z1O} / 14$

Provenance: Tumulus 10, burial chamber, northern part

Construction: wheel-made (1)

State of preservation: incomplete, $82 \%$ of rim
Measurements: $\mathrm{Dr}=16.5 \mathrm{~cm} ; \mathrm{H}=10.6 \mathrm{~cm}$

Fabric: $\mathrm{ZF} 3$

Hardness: 3

Firing: oxidized

Surface treatment: slipped on both surfaces; burnish 2, carefully executed on both surfaces

Surface colour: external, slip-7.5R 4/6 red; internal, slip-7.5R 4/6 red

Colour of break: 7.5YR 5/4 brown

References: (Klimaszewska-Drabot 2010, 481)

\section{Cat. 68}

Bowl

Inv. no: Z1o/18

Provenance: Tumulus 10, burial chamber, southern part

Construction: wheel-made (1)

State of preservation: complete

Measurements: $\mathrm{Dr}=18 \mathrm{~cm} ; \mathrm{H}=12.2 \mathrm{~cm}$

Fabric: not possible to determine

Hardness: 4

Firing: not possible to determine

Surface treatment: slipped on both surfaces; burnish 1, carefully executed on both surfaces 


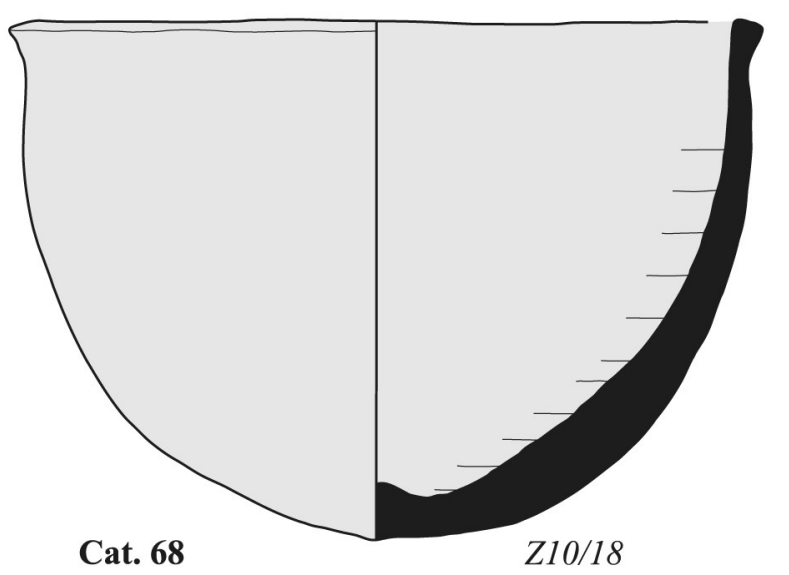

0 $5 \mathrm{~cm}$

FIGURE 7.14 External ledge-rimmed bowls of type II.1, variant a

Surface colour: external, slip—1oR 4/6 red; internal, slip$10 \mathrm{R} 4 / 6$ red

Colour of break: not possible to determine

References: (Klimaszewska-Drabot 2010, 481)

\section{Cat. 69}

Bowl

Inv. no: Z11/29

Provenance: Tumulus 11, chamber 2, southern part

Construction: wheel-made (1)

State of preservation: complete

Measurements: $\mathrm{Dr}=13 \mathrm{~cm} ; \mathrm{H}=9 \mathrm{~cm}$

Fabric: not possible to determine

Hardness: $3 / 4$

Firing: not possible to determine

Surface treatment: slipped on both surfaces; externalburnish 2 , carefully executed; internal—burnish 2 , carelessly executed

Surface colour: external, slip-1oR 5/6 red; internal, slip$10 \mathrm{R} 5 / 6$ red

Colour of break: not possible to determine

References: (Klimaszewska-Drabot and Czyżewska 2012, 368-372)

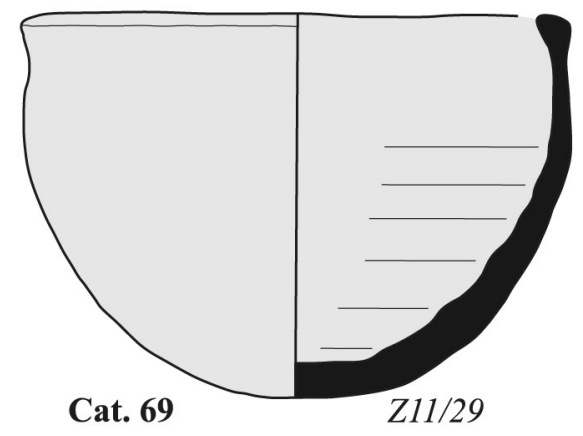

Bowls variant II.1a1

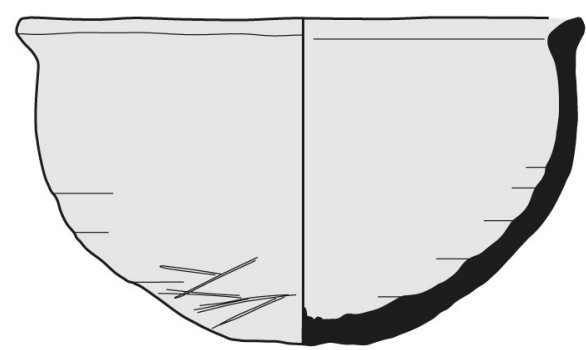

Cat. 70
Variant II.1a

Cat. 70

Bowl

Inv. no: Z11/17

Provenance: Tumulus 11, chamber 2

Construction: wheel-made (1)

State of preservation: complete

Measurements: $\mathrm{Dr}=13.5 \mathrm{~cm} ; \mathrm{H}=7.7 \mathrm{~cm}$

Fabric: not possible to determine

Hardness: medium hard (3/4)

Firing: not possible to determine

Surface treatment: slipped on both surfaces

Surface colour: slip-10R $4 / 6$ red, on both surfaces

Colour of break: not possible to determine

References: (Klimaszewska-Drabot and Czyżewska 2012, 368-372; Mahmoud El-Tayeb and Czyżewska 2011, $115^{-117)}$

\section{Variant II.1b}

Cat. 71

Bowl

Inv. no: Z6/16+Z6/20.1

Provenance: Tumulus 6, Eastern tunnel, near entrance to tunnel

Construction: wheel-made (1)

State of preservation: incomplete, $80 \%$ of rim, body fragment missing 


\section{variant II.1b}

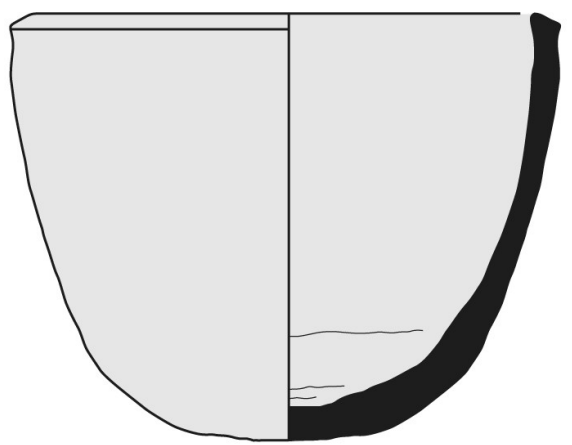

Cat. 71

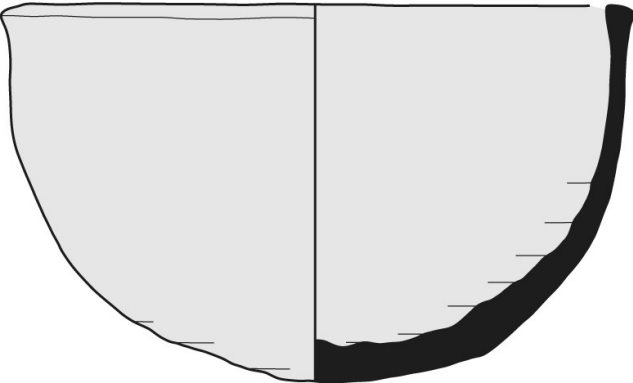

Cat. 72

$Z 11 / 22$

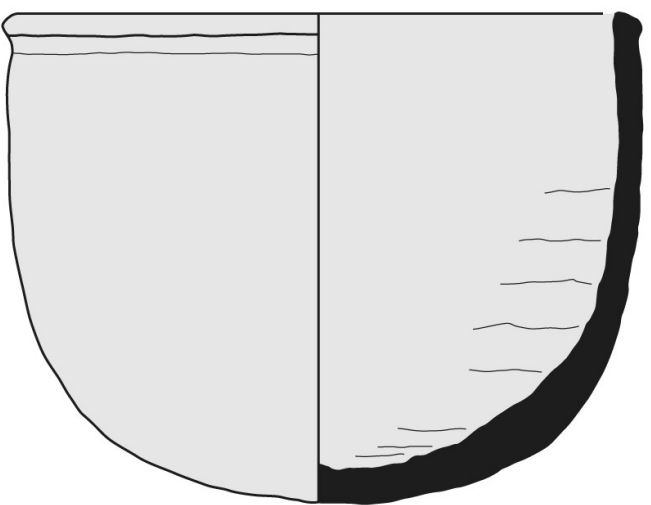

Cat. 73 $5 \mathrm{~cm}$

FIGURE 7.15 External ledge-rimmed bowls of type II.1, variant b

Measurements: $\mathrm{Dr}=12 \mathrm{~cm} ; \mathrm{H}=10.3 \mathrm{~cm}$

Fabric: $\mathrm{ZF} 3$

Hardness: $3 / 4$

Firing: oxidized

Surface treatment: slipped on both surfaces; externalburnish 3 , carefully executed; internal-burnish 2 , carefully executed

Surface colour: external, slip $-7.5 \mathrm{R} 5 / 8$ red; internal, slip$7 \cdot 5 \mathrm{R} 5 / 8$ red

Colour of break: $7 \cdot 5$ YR 4/6 strong brown

Cat. 72

Bowl

Inv. no: Z11/22

Provenance: Tumulus 11, chamber 2, central part, near blocking wall of chamber

Construction: wheel-made (1)

State of preservation: complete

Measurements: $\mathrm{Dr}=14.5^{-15} \cdot 3 \mathrm{~cm} ; \mathrm{H}=9 \mathrm{~cm}$

Fabric: not possible to determine

Hardness: 3
Firing: not possible to determine

Surface treatment: slipped on both surfaces, burnish 2, carelessly executed on both surfaces

Surface colour: external, slip-2.5YR 5/6 red; internal, slip-2.5YR $5 / 6$ red

Colour of break: not possible to determine

References: (Klimaszewska-Drabot and Czyżewska 2012, $368-372)$

Cat. 73

Bowl

Inv. no: Z12/129

Provenance: Tumulus 12, chamber 2, southern part

Construction: wheel-made (1)

State of preservation: complete

Measurements: $\mathrm{Dr}=14.5^{-15} \mathrm{~cm} ; \mathrm{H}=12 \mathrm{~cm}$

Fabric: not possible to determine

Hardness: $3 / 4$

Firing: not possible to determine

Surface treatment: slipped on both surfaces; externalburnish 2 on upper part and burnish 1 on lower 
part, carefully executed; internal-burnish 2, carefully executed

Surface colour: external natural surface-2.5YR 5/6 red, slip $-10 R$ 5/6 red; internal, slip $-10 R$ R/6 red

Colour of break: not possible to determine

\subsection{Bowls Type II.2}

Variant II.2a

Cat. 74

Bowl

Inv. no: $\mathrm{Z26} / 75$

Provenance: Tumulus 26, chamber 2, northern part

Construction: wheel-made (1)

State of preservation: complete

Measurements: $\mathrm{Dr}=11 \mathrm{~cm} ; \mathrm{H}=5.7 \mathrm{~cm}$

Fabric: not possible to determine

Hardness: 3

Firing: not possible to determine

Surface treatment: slipped and burnished on both surfaces; external-burnish 2, carefully executed; internalburnish 1, carefully executed

Surface colour: external, slip—-10R 4/6 red; internal, slip1oR $4 / 4$ weak red

Colour of break: not possible to determine

Cat. 75

Bowl

Inv. no: $\mathrm{Z}_{\mathbf{2 3}} / \mathbf{1 6}$

Provenance: Tumulus 23, chamber 2

Construction: wheel-made (1)

State of preservation: incomplete, chipped rim, $94 \%$ of rim

Measurements: $\mathrm{Dr}=11.8 \mathrm{~cm} ; \mathrm{H}=7 \mathrm{~cm}$

Fabric: not possible to determine

Hardness: $3 / 4$

Firing: oxidized

Surface treatment: slipped and burnished on both surfaces; burnish 1 on both surfaces, carefully executed

Surface colour: external, slip-10R $5 / 6$ red; internal, slip1oR $4 / 6$ weak red

Colour of break: 2.5 YR $5 / 4$ reddish brown

Cat. 76

Bowl

Inv. no: Z26/8o

Provenance: Tumulus 26, chamber 4, southern part

Construction: wheel-made (1)

State of preservation: complete

Measurements: $\mathrm{Dr}=13 \mathrm{~cm} ; \mathrm{H}=7.4 \mathrm{~cm}$

Fabric: not possible to determine

Hardness: $3 / 4$

Firing: not possible to determine
Surface treatment: slipped and burnished on both surfaces; burnish 1 on both surfaces, carefully executed

Surface colour: external, slip-10R $5 / 8$ red; internal, slip10R 2.5/1 reddish black

Colour of break: not possible to determine

Cat. 77

Bowl

Inv. no: $\mathrm{Z26} / 43$

Provenance: Tumulus 26, chamber 2, northern part

Construction: wheel-made (1)

State of preservation: incomplete, $94 \%$ of rim

Measurements: $\mathrm{Dr}=10 \mathrm{~cm} ; \mathrm{H}=6.5 \mathrm{~cm}$

Fabric: not possible to determine

Hardness: 3

Firing: oxidized

Surface treatment: slipped and burnished on both surfaces; external-burnish 2 less carefully executed; internalburnish 2, less carefully executed on the upper part, carelessly executed on the lower part

Surface colour: external, slip—1oR 5/6 red; internal, slip10R 4/8 red

Colour of break: 1OYR 5/3 brown

Cat. 78

Bowl

Inv. no: $\mathrm{Z12} / 46$

Provenance: Tumulus 12, fill of shaft, south-eastern part

Construction: wheel-made (1)

State of preservation: incomplete, restored from sherds, $66 \%$ of rim, body sherds missing

Measurements: $\mathrm{Dr}=12.6 \mathrm{~cm} ; \mathrm{H}=8.5 \mathrm{~cm}$

Fabric: ZF2

Hardness: $3 / 4$

Firing: oxidized

Surface treatment: slipped and burnished on both surfaces; burnish 1 on both surfaces, carefully executed

Surface colour: external natural surface-2.5YR 5/8 red, slip-10YR $5 / 3$ brown; internal, slip-10R 4/6 red

Colour of break: 1 OYR $5 / 3$ brown

Cat. 79

Bowl

Inv. no: Z12/119

Provenance: Tumulus 12, chamber 3

Construction: wheel-made (1)

State of preservation: incomplete, $97 \%$ of rim

Measurements: $\mathrm{Dr}=10.5 \mathrm{~cm} ; \mathrm{H}=7 \mathrm{~cm}$

Fabric: not possible to determine

Hardness: $3 / 4$ 


\section{Bowls Type II.2}

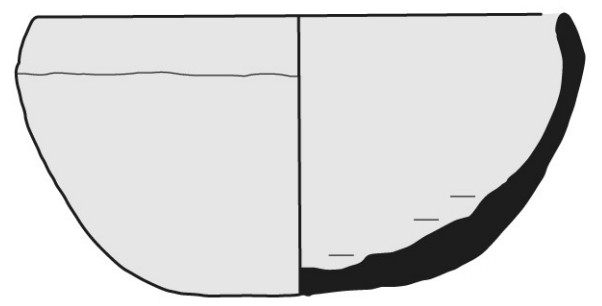

Cat. 74

$Z 26 / 75$

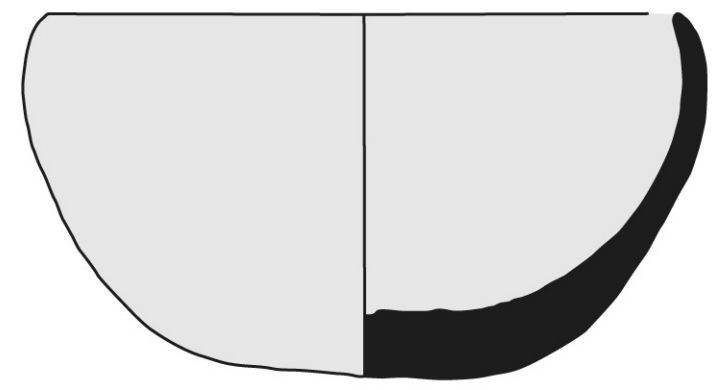

Cat. 76

$Z 26 / 80$

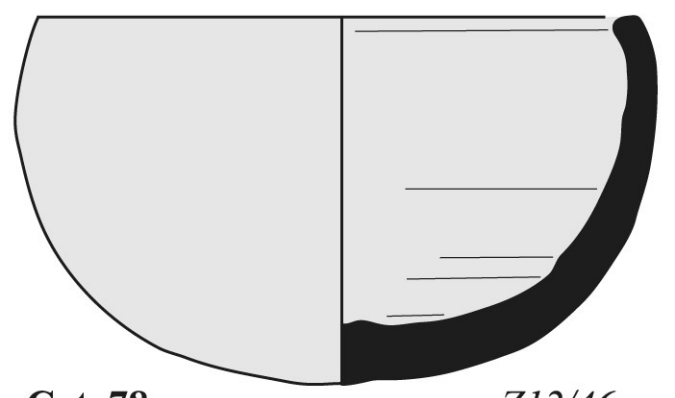

Cat. 78

Z12/46

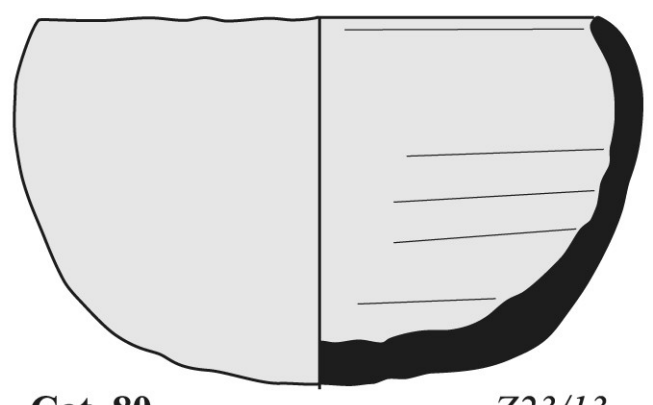

Cat. 80

$Z 23 / 13$ variant II.2a

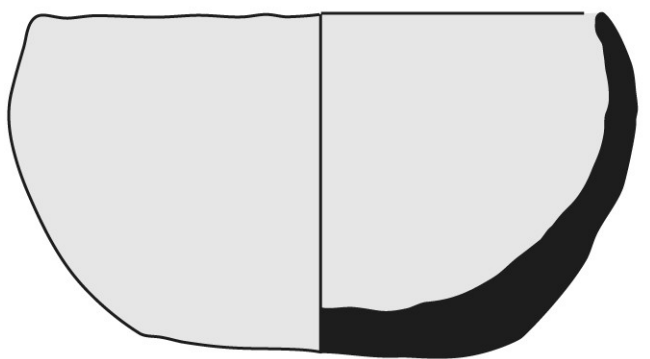

Cat. 75

Z23/16

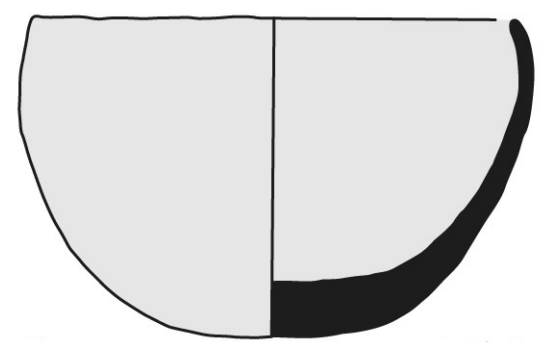

Cat. 77
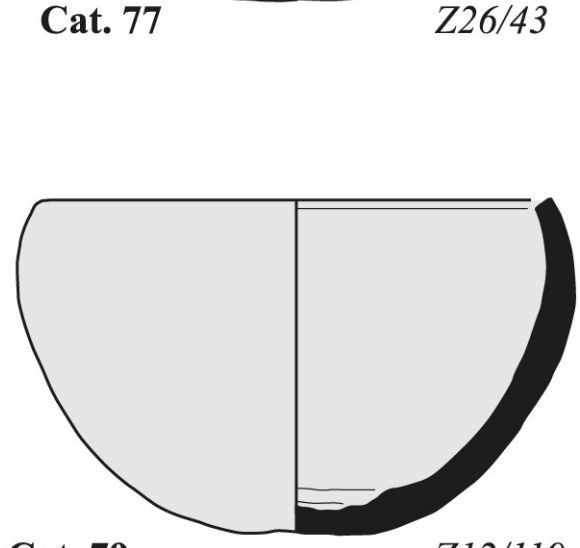

Cat. 79
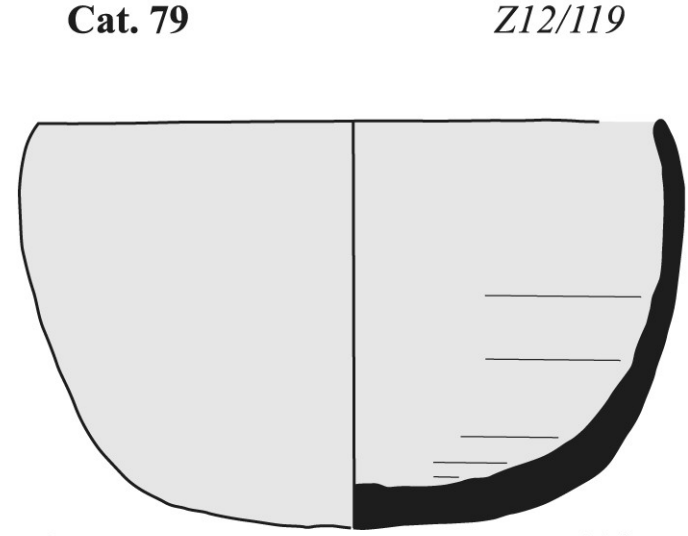

Cat. 81

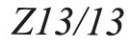

0

FIGURE 7.16 Hemispherical bowls of type II.2 with incurved walls, variant a 
Firing: oxidized

Surface treatment: slipped and burnished on both surfaces; burnish 1 on both surfaces, carefully executed

Surface colour: external, slip_-10R $5 / 6$ red; internal, slip1oR $5 / 6$ red

Colour of break: not possible to determine

Cat. 8o

Bowl

Inv. no: $\mathrm{Z}_{23} / \mathbf{1 3}$

Provenance: Tumulus 23, chamber 2

Construction: wheel-made (1)

State of preservation: incomplete, chipped rim, $94 \%$ of rim

Measurements: $\mathrm{Dr}=11 \mathrm{~cm} ; \mathrm{H}=7.7 \mathrm{~cm}$

Fabric: not possible to determine

Hardness: $3 / 4$

Firing: oxidized

Surface treatment: slipped and burnished on both surfaces; external-burnish 1, carefully executed; internalburnish 2, carefully executed

Surface colour: external, slip-7.5YR 4/4 dark brown; internal, slip-7.5YR 4/6 strong brown

Colour of break: not possible to determine

References: (Klimaszewska-Drabot and Mahmoud ElTayeb 2014, 10-13)

Cat. 81

Bowl

Inv. no: $\mathrm{Z}_{13} / \mathbf{1 3}$

Provenance: Tumulus 13, chamber 4, central part

Construction: wheel-made (1)

State of preservation: complete

Measurements: $\mathrm{Dr}=13.2 \mathrm{~cm} ; \mathrm{H}=8.3 \mathrm{~cm}$

Fabric: not possible to determine

Hardness: $3 / 4$

Firing: not possible to determine

Surface treatment: slipped and burnished on both surfaces; external-burnish 2, carefully executed; internalburnish 1, carefully executed

Surface colour: external, slip_-10R 4/6 red; internal, slip10R $4 / 6$ red

Colour of break: not possible to determine

Cat. 82

Bowl

Inv. no: $\mathrm{Z26} / 5^{\mathrm{O}}+\mathrm{Z26} / 83.2$

Provenance: Tumulus 26, chamber 2, southern part

Construction: wheel-made (1)

State of preservation: complete

Measurements: $\mathrm{Dr}=10.5 \mathrm{~cm} ; \mathrm{H}=7 \mathrm{~cm}$

Fabric: not possible to determine
Hardness: 3

Firing: not possible to determine

Surface treatment: slipped and burnished on both surfaces; burnish 2 on both surfaces, carefully executed

Surface colour: external natural surface-7.5Y $5 / 4$ brown, slip-10R 4/6 red; internal, slip-2.5YR 4/4 reddish brown

Colour of break: not possible to determine

Cat. 83

Bowl

Inv. no: Z14/13

Provenance: Tumulus 14, chamber 3, north-western corner Construction: wheel-made (1)

State of preservation: complete

Measurements: $\mathrm{Dr}=12 \mathrm{~cm} ; \mathrm{H}=7.1 \mathrm{~cm}$

Fabric: not possible to determine

Hardness: $3 / 4$

Firing: not possible to determine

Surface treatment: slipped and burnished on both surfaces; burnish 2 on both surfaces, carefully executed

Surface colour: external, slip_-10R 5/6 red; internal, slip1oR $5 / 6$ red

Colour of break: not possible to determine

\section{Cat. 84}

Bowl

Inv. no: Z24/19

Provenance: Tumulus 24, chamber 1, eastern part

Construction: wheel-made (1)

State of preservation: fragmentary, restored from sherds, $88 \%$ of rim, body-sherd fragments missing

Measurements: $\mathrm{Dr}=11.8 \mathrm{~cm} ; \mathrm{H}=7.2 \mathrm{~cm}$

Fabric: $\mathrm{ZF} 3$

Hardness: 3

Firing: reduced

Surface treatment: slipped and burnished on both surfaces; external-burnish 2, carefully executed; internalburnish 1, carefully executed

Surface colour: external, slip-1oR 4/4 weak red; internal, slip-10R 4/6 red

Colour of break: 2.5YR 3/2 dusky red

Cat. 85

Bowl

Inv. no: $\mathrm{Z14} / 10$

Provenance: Tumulus 14, chamber 3, central part

Construction: wheel-made (1)

State of preservation: complete

Measurements: $\mathrm{Dr}=13 \mathrm{~cm} ; \mathrm{H}=9 \mathrm{~cm}$

Fabric: not possible to determine 
variant II.2a

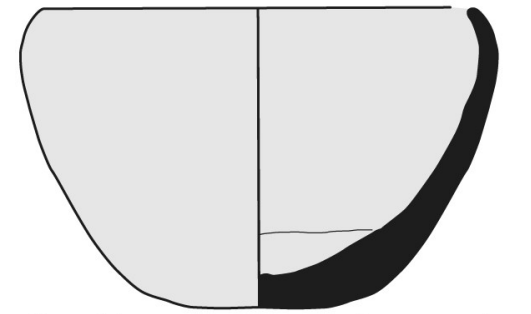

Cat. 82

$Z 26 / 50+Z 26 / 83.2$

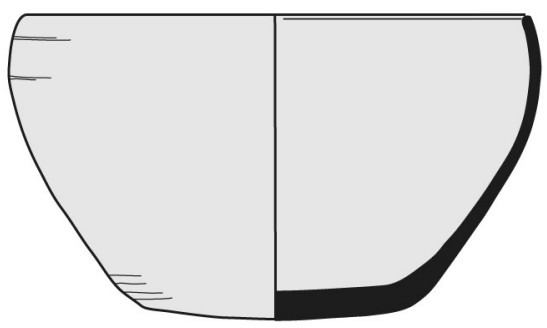

Cat. 84

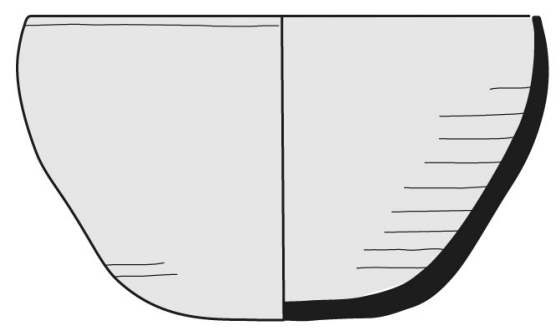

Cat. 83

Z14/13

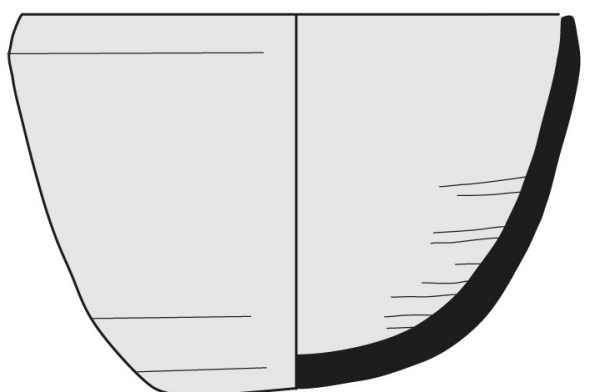

Cat. 85

Z14/10

0 $5 \mathrm{~cm}$

FIGURE 7.17 Hemispherical bowls of type II.2 with incurved walls, variant a

\section{Hardness: $3 / 4$}

Firing: not possible to determine

Surface treatment: slipped and burnished on both surfaces; external-burnish 1, carefully executed; internalburnish 2, carelessly executed

Surface colour: external, slip_-10R $5 / 6$ red; internal, slip1oR $5 / 6$ red

Colour of break: not possible to determine

Variant II.2b

Cat. 86

Bowl

Inv. no: $\mathrm{Z12} / 35$

Provenance: Tumulus 12, fill of shaft, south-eastern part

Construction: wheel-made (1)

State of preservation: incomplete, body-sherd fragments missing

Measurements: $\mathrm{Dr}=10.2 \mathrm{~cm} ; \mathrm{H}=6.8 \mathrm{~cm}$

Fabric: ZF1

Hardness: $3 / 4$

Firing: oxidized

Surface treatment: slipped and burnished on both surfaces; burnish 2 on both surfaces, carefully executed

Surface colour: external, slip_-10R 4/6 red; internal, slip$10 \mathrm{R} 5 / 6$ red

Colour of break: 5YR 4/6 yellowish red

\section{Cat. 87}

Bowl

Inv. no: Z12/97

Provenance: Tumulus 12, chamber 3, western part

Construction: wheel-made (1)

State of preservation: complete

Measurements: $\mathrm{Dr}=12.3 \mathrm{~cm} ; \mathrm{H}=8 \mathrm{~cm}$

Fabric: not possible to determine

Hardness: $3 / 4$

Firing: not possible to determine

Surface treatment: slipped and burnished on both surfaces; burnish 1 on both surfaces, carefully executed

Surface colour: external, slip-10R $5 / 6$ red; internal, slip1oR $5 / 6$ red

Colour of break: not possible to determine

\section{Cat. 88}

Bowl

Inv. no: Z12/123

Provenance: Tumulus 12, chamber 3

Construction: wheel-made (1)

State of preservation: complete

Measurements: $\mathrm{Dr}=16.2-16.5 \mathrm{~cm} ; \mathrm{H}=10.5 \mathrm{~cm}$

Fabric: not possible to determine

Hardness: $3 / 4$

Firing: not possible to determine 


\section{variant II.2b}

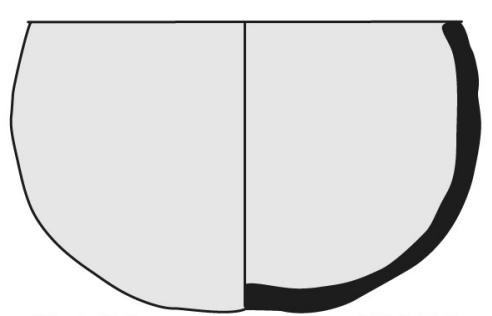

Cat. 86 Z12/35

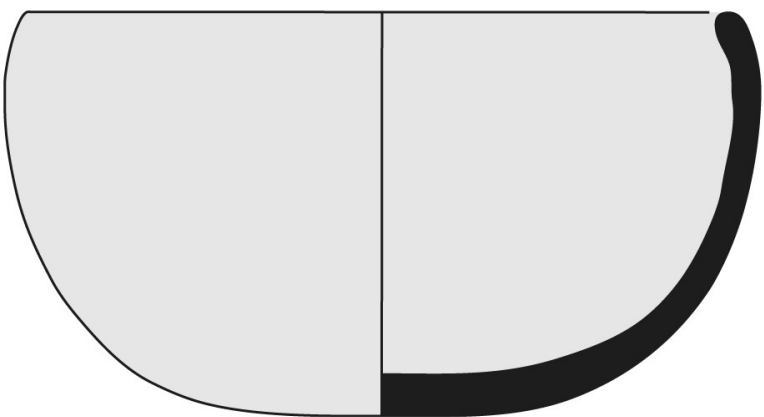

Cat. 88

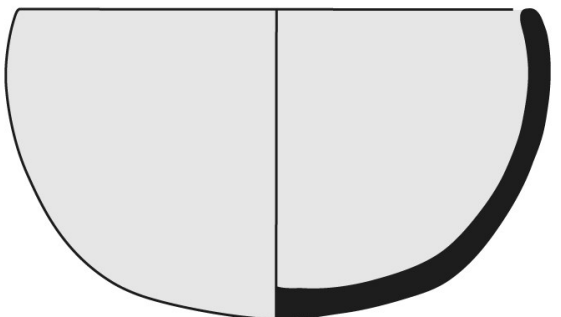

Cat. 87

Z12/97

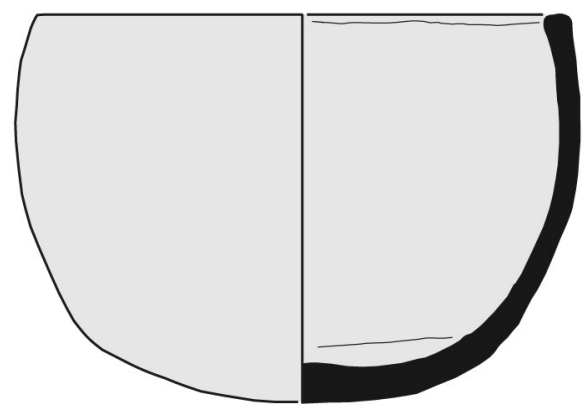

Cat. 89

Z12/14

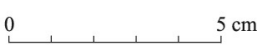

FIGURE 7.18 Hemispherical bowls of type II.2 with incurved walls and maximum diameter at mid-H, variant b

Surface treatment: slipped and burnished on both surfaces; external-burnish 1, carefully executed; internalburnish 1 on the upper part, burnish 2 on the lower part, carefully executed

Surface colour: external, slip—1oR 5/4 weak red; internal, slip- $10 R 5 / 4$ weak red

Colour of break: not possible to determine

\section{Cat. 89}

Bowl

Inv. no: $\mathrm{Z12} / 14$

Provenance: Tumulus 12, fill of shaft, south-eastern part

Construction: wheel-made (1)

State of preservation: incomplete, body-sherd fragments missing

Measurements: $\mathrm{Dr}=12.8 \mathrm{~cm} ; \mathrm{H}=9.2 \mathrm{~cm}$

Fabric: ZF1

Hardness: $3 / 4$

Firing: oxidized

Surface treatment: slipped and burnished on both surfaces; burnish 2 on both surfaces, less carefully executed

Surface colour: external, slip—-1oR 4/8 red; internal, slip2.5 Y $5 / 6$ red

Colour of break: $7.5^{Y R} 5 / 4$ brown

References: (Czyżewska-Zalewska 2016, 728-730)
Cat. 9o

Bowl

Inv. no: Z12/44

Provenance: Tumulus 12, fill of shaft, south-eastern part

Construction: wheel-made (1)

State of preservation: incomplete, 9o \% of rim, body-sherd fragments missing

Measurements: $\mathrm{Dr}=12 \mathrm{~cm} ; \mathrm{H}=9.5 \mathrm{~cm}$

Fabric: ZF2

Hardness: $3 / 4$

Firing: oxidized

Surface treatment: slipped and burnished on both surfaces; burnish 2 on both surfaces, carefully executed

Surface colour: external natural surface -7.5 YR $5 / 6$ strong brown, slip-2.5YR 4/4 reddish brown; internal, slip2.5 Y $5 / 6$ red

Colour of break: 5YR 4/6 yellowish red

Cat. 91

Bowl

Inv. no: Z12/106

Provenance: Tumulus 12, chamber 3

Construction: wheel-made (1)

State of preservation: incomplete, $91 \%$ of rim, body-sherd fragments missing 


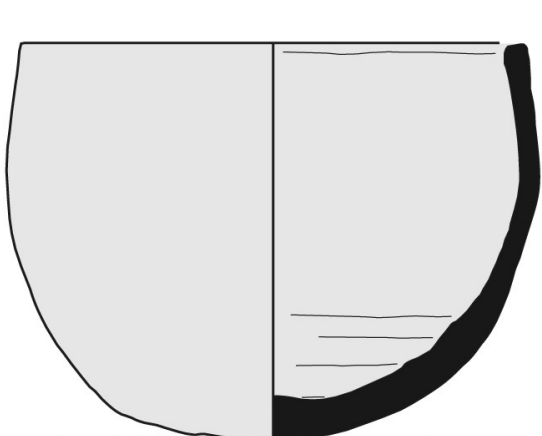

Cat. 90

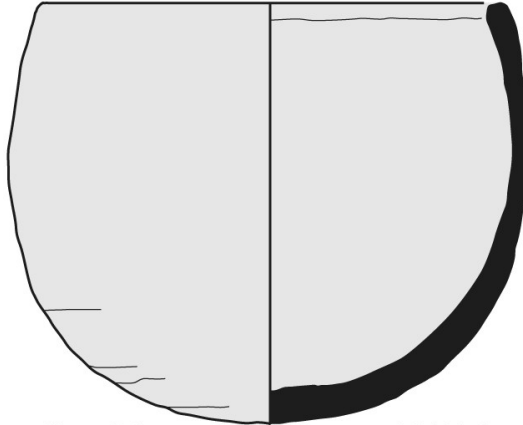

Cat. 91
Z12/106

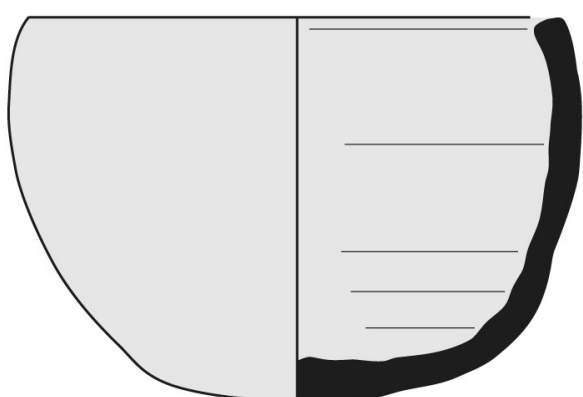

Cat. 92

0 $5 \mathrm{~cm}$

FIGURE 7.19 Hemispherical bowls of type II.2 with straight walls and maximum diameter at mid-H, variant b

Measurements: $\mathrm{Dr}=11 \mathrm{~cm} ; \mathrm{H}=9.8 \mathrm{~cm}$

Fabric: not possible to determine

Hardness: $3 / 4$

Firing: oxidized

Surface treatment: slipped and burnished on both surfaces; burnish 3 on both surfaces, carefully executed

Surface colour: external, slip_-10R 5/4 weak red; internal, slip-10R 4/4 weak red

Colour of break: 7.5YR 5/4 brown

References: (Czyżewska-Zalewska 2016, 728-730)

Variant II.2b

Cat. 92

Bowl

Inv. no: $\mathbf{Z 2 1} / 7$

Provenance: Tumulus 21, burial chamber

Construction: wheel-made (1)

State of preservation: almost complete, $92 \%$ of rim, lip chipped off

Measurements: $\mathrm{Dr}=13 \mathrm{~cm} ; \mathrm{H}=9.2 \mathrm{~cm}$

Fabric: $\mathrm{ZF} 3$

Hardness: $3 / 4$

Firing: reduced

Surface treatment: slipped on both surfaces

Surface colour: external natural surface-7.5YR 6/4 light brown, slip-10R 5/6 red; internal, slip-10R 4/6 red

Colour of break: Gley2 3/5P B very dark bluish grey

Variant II.2C

Cat. 93

Bowl

Inv. no: $\mathrm{Z12} / 41$

Provenance: Tumulus 12, fill of shaft, south-eastern part
Construction: wheel-made (1)

State of preservation: incomplete, $55 \%$ of rim, body-sherd fragments missing

Measurements: $\mathrm{Dr}=11.5 \mathrm{~cm} ; \mathrm{H}=5.9 \mathrm{~cm}$

Fabric: ZF2

Hardness: 3

Firing: reduced

Surface treatment: slipped and burnished on both surfaces; burnish 1 on both surfaces, carefully executed

Surface colour: external natural surface-10R 4/8 red, slip-10R 4/4 weak red; internal, slip-1oR 4/6 red

Colour of break: 7.5YR 3/1 very dark grey

Cat. 94

Bowl

Inv. no: Z12/15

Provenance: Tumulus 12, chamber 2, southern part

Construction: wheel-made (1)

State of preservation: complete

Measurements: $\mathrm{Dr}=12.8 \mathrm{~cm} ; \mathrm{H}=6.8 \mathrm{~cm}$

Fabric: not possible to determine

Hardness: $3 / 4$

Firing: not possible to determine

Surface treatment: slipped and burnished on both surfaces; external-burnish 1, carefully executed; internalburnish 2-less carefully executed

Surface colour: external, slip—10R 4/6 red; internal, slip10R $4 / 6$ red

Colour of break: not possible to determine

References: (Czyżewska-Zalewska 2016, 728-730) 


\section{variant II.2c}
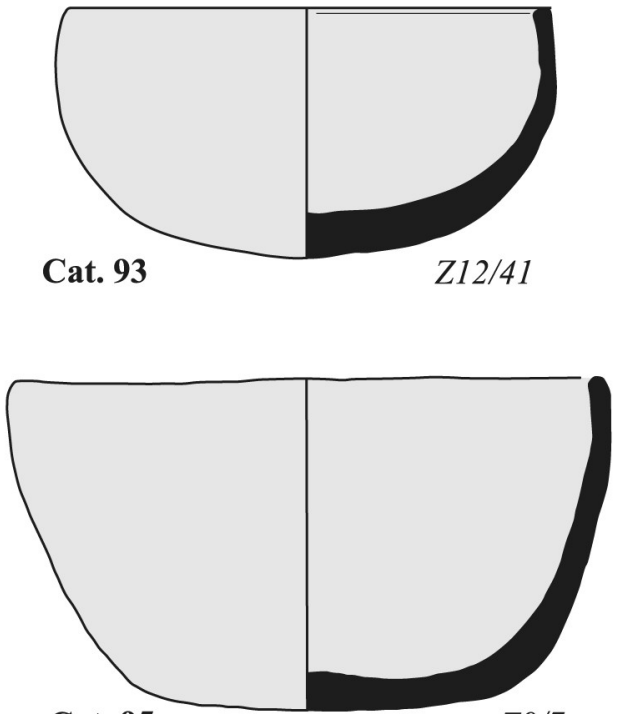

Cat. 95 $Z 9 / 7$

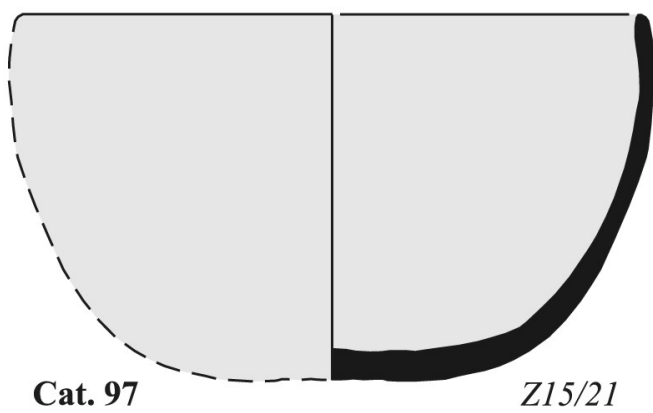

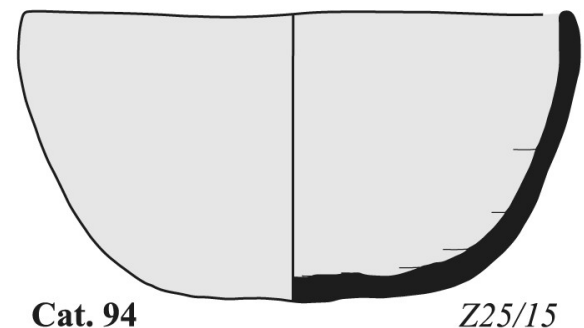

$Z 25 / 15$

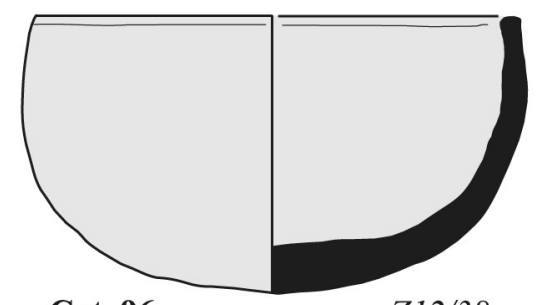

Cat. 96
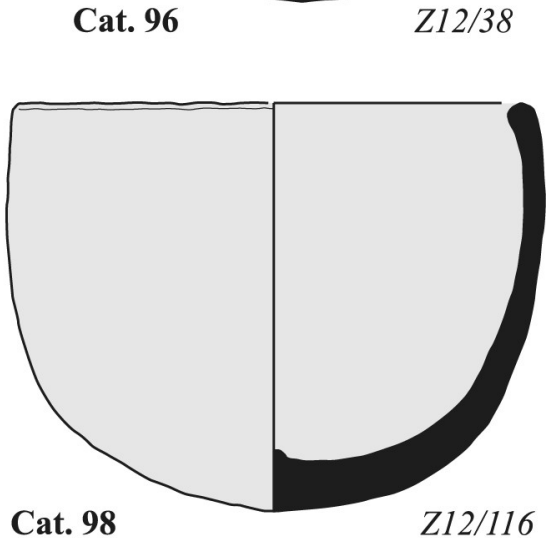

0 $5 \mathrm{~cm}$

Cat. 95

Bowl

Inv. no: $\mathbf{Z 9} / 7$

Provenance: Tumulus 9, chamber 1, central part

Construction: wheel-made (1)

State of preservation: complete

Measurements: $\mathrm{Dr}=14 \mathrm{~cm} ; \mathrm{H}=7.8 \mathrm{~cm}$

Fabric: not possible to determine

Hardness: $3 / 4$

Firing: not possible to determine

Surface treatment: slipped and burnished on both surfaces; external-burnish 2, carefully executed; internalburnish 2-less carefully executed

Surface colour: external, slip_-10R 4/6 red; internal, slip10R 4/6 red

Colour of break: not possible to determine
Cat. 96

Bowl

Inv. no: $\mathbf{Z 1 2} / 38$

Provenance: Tumulus 12, fill of shaft, south-eastern part

Construction: wheel-made (1)

State of preservation: incomplete, $47 \%$ of rim, body-sherd fragments missing

Measurements: $\mathrm{Dr}=11.5 \mathrm{~cm} ; \mathrm{H}=6.8 \mathrm{~cm}$

Fabric: ZF1

Hardness: $3 / 4$

Firing: oxidized

Surface treatment: slipped and burnished on both surfaces; burnish 1 on both surfaces, carefully executed

Surface colour: external, slip-10R 5/6 red; internal, slip10R $4 / 6$ red

Colour of break: 1OYR 5/4 yellowish brown 
Cat. 97

Bowl

Inv. no: $\mathbf{Z} 15 / 21$

Provenance: Tumulus 15, fill of shaft, southern part, near blocking wall of main burial chamber

Construction: wheel-made (1)

State of preservation: incomplete, $37 \%$ of rim, body-sherd fragments missing

Measurements: $\mathrm{Dr}=15 \mathrm{~cm} ; \mathrm{H}=8.4 \mathrm{~cm}$

Fabric: $\mathrm{ZF} 5$

Hardness: 3

Firing: oxidized

Surface treatment: slipped and burnished on both surfaces; burnish 1 on both surfaces, carefully executed

Surface colour: external, slip-10R 4/6 red; internal, slip$10 \mathrm{R}_{4} / 6$ red

Colour of break: 1OYR 5/4 yellowish brown

Cat. 98

Bowl

Inv. no: $\mathrm{Z12} / \mathbf{1 1 6}$

Provenance: Tumulus 12, chamber 3

Construction: wheel-made (1)

State of preservation: complete

Measurements: $\mathrm{Dr}=12 \mathrm{~cm} ; \mathrm{H}=9.7 \mathrm{~cm}$

Fabric: not possible to determine

Hardness: $3 / 4$

Firing: not possible to determine

Surface treatment: slipped and burnished on both surfaces; burnish 3 on both surfaces, carelessly executed

Surface colour: external natural surface-2.5YR 5/8 red, slip-10R 5/6 red; internal, slip-2.5YR 5/4 reddish brown

Colour of break: not possible to determine

Variant II.2d

Cat. 99

Bowl

Inv. no: $\mathrm{Z}_{2} 6 / 85+\mathrm{Z}_{2} 6 / 86$

Provenance: Tumulus 26 , chamber 4 , central part

Construction: wheel-made (1)

State of preservation: complete, restored from sherds

Measurements: $\mathrm{Dr}=11 \mathrm{~cm} ; \mathrm{H}=6.6 \mathrm{~cm}$

Fabric: ZF1

Hardness: $3 / 4$

Firing: reduced

Surface treatment: slipped and burnished on both surfaces; burnish 2 on both surfaces, carefully executed

Surface colour: external, slip-5YR 5/4 reddish brown; internal, slip- 5 YR $4 / 3$ reddish brown

Colour of break: 2.5Y 2.5/1 black
Cat. 100

Bowl

Inv. no: $\mathrm{Z12} / 101$

Provenance: Tumulus 12, chamber 3

Construction: wheel-made (1)

State of preservation: complete

Measurements: $\mathrm{Dr}=13 \mathrm{~cm} ; \mathrm{H}=7.5 \mathrm{~cm}$

Fabric: not possible to determine

Hardness: 3

Firing: not possible to determine

Surface treatment: slipped and burnished on both surfaces; external-burnish 2, carefully executed; internalburnish 1, less carefully executed

Surface colour: external natural surface-2.5YR 5/8 red, slip_-1OYR 4/4 weak red; internal, slip-1oR 4/4 weak red

Colour of break: not possible to determine

Cat. 101

Bowl

Inv. no: $\mathbf{Z 1 2} / \mathbf{1 2} 6$

Provenance: Tumulus 12, chamber 3

Construction: wheel-made (1)

State of preservation: complete

Measurements: $\mathrm{Dr}=13 \mathrm{~cm} ; \mathrm{H}=8.5 \mathrm{~cm}$

Fabric: not possible to determine

Hardness: $3 / 4$

Firing: not possible to determine

Surface treatment: slipped and burnished on both surfaces; external-burnish 2, carefully executed; internalburnish 1, carefully executed

Surface colour: external natural surface-10R 5/6 red, slip_-10R 4/6 red; internal, slip_-10R 4/4 weak red

Colour of break: not possible to determine

Notes: bowl much heavier than other vessels of this type

Cat. 102

Bowl

Inv. no: $\mathrm{Z} 14 / 7$

Provenance: Tumulus 14, chamber 3, western part

Construction: wheel-made (1)

State of preservation: complete

Measurements: $\mathrm{Dr}=14 \mathrm{~cm} ; \mathrm{H}=8.2 \mathrm{~cm}$

Fabric: not possible to determine

Hardness: $3 / 4$

Firing: not possible to determine

Surface treatment: slipped and burnished on both surfaces; external-burnish 1, carefully executed; internalburnish 2, carefully executed

Surface colour: external, slip-10R 4/6 red; internal, slip1oR 4/6 red

Colour of break: not possible to determine 
variant II.2d

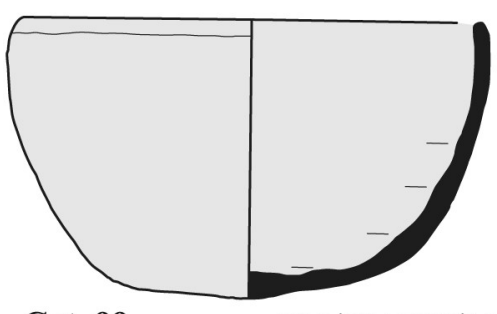

Cat. 99

$Z 26 / 85+Z 26 / 86$

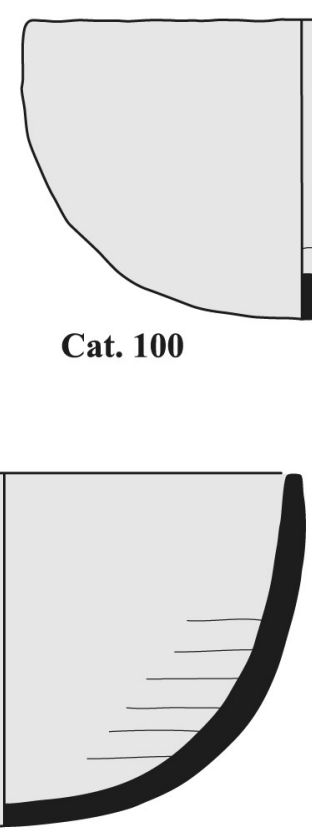

Z14/7

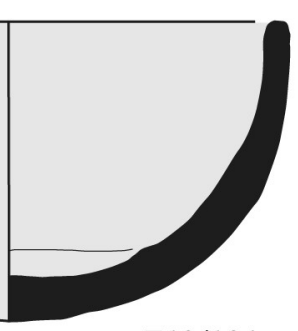

Z12/101

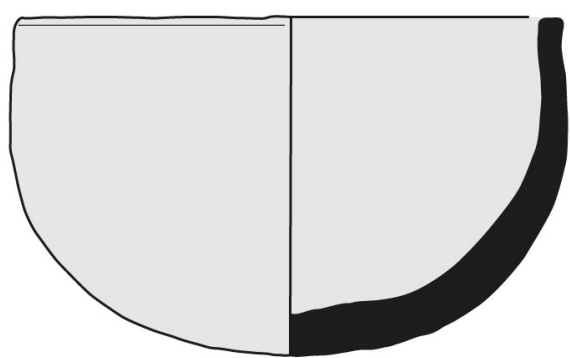

Cat. 101

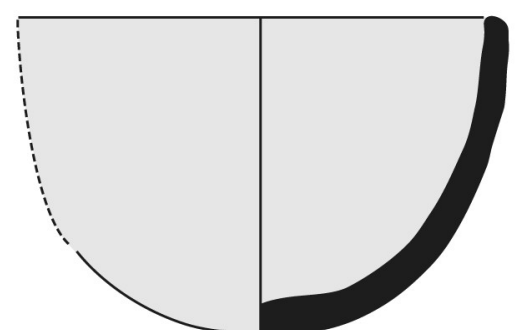

Cat. 103
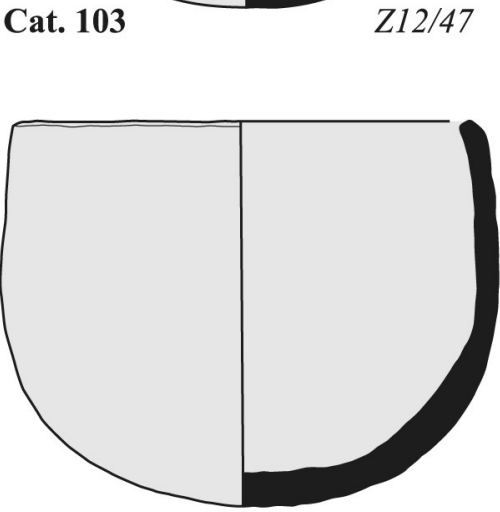

Cat. 105

\section{Z12/108}

Cat. 104

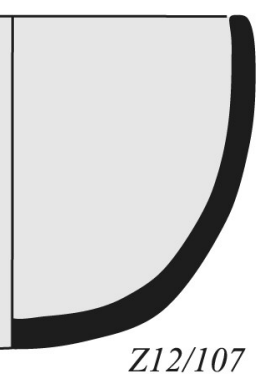

0 $5 \mathrm{~cm}$

FIGURE 7.21 Deep and medium-deep hemispherical bowls of type II.2 with straight walls, variant d

\section{Cat. 103}

Bowl

Inv. no: $\mathbf{Z 1 2} / 47$

Provenance: Tumulus 12, fill of shaft, south-eastern part

Construction: wheel-made (1)

State of preservation: fragmentary, restored from sherds, $40 \%$ of rim, body-sherd fragments missing

Measurements: $\mathrm{Dr}=11.4 \mathrm{~cm} ; \mathrm{H}=8.4 \mathrm{~cm}$

Fabric: ZF1

Hardness: $3 / 4$

Firing: oxidized

Surface treatment: slipped and burnished on both surfaces; burnish 2 on both surfaces, less carefully executed

Surface colour: external, slip-1oR 4/4 weak red; internal, slip-2.5YR $5 / 4$ reddish brown

Colour of break: 7.5YR 4/4 dark brown
Cat. 104

Bowl

Inv. no: $\mathbf{Z 1 2} / 107$

Provenance: Tumulus 12, chamber 3

Construction: wheel-made (1)

State of preservation: complete

Measurements: $\mathrm{Dr}=11 \mathrm{~cm} ; \mathrm{H}=8 \mathrm{~cm}$

Fabric: not possible to determine

Hardness: $3 / 4$

Firing: not possible to determine

Surface treatment: slipped and burnished on both surfaces; external-burnish 1, carefully executed; internalburnish 2, carefully executed

Surface colour: external, slip-10R $5 / 6$ red; internal, slip$10 \mathrm{R} 4 / 4$ weak red

Colour of break: not possible to determine 


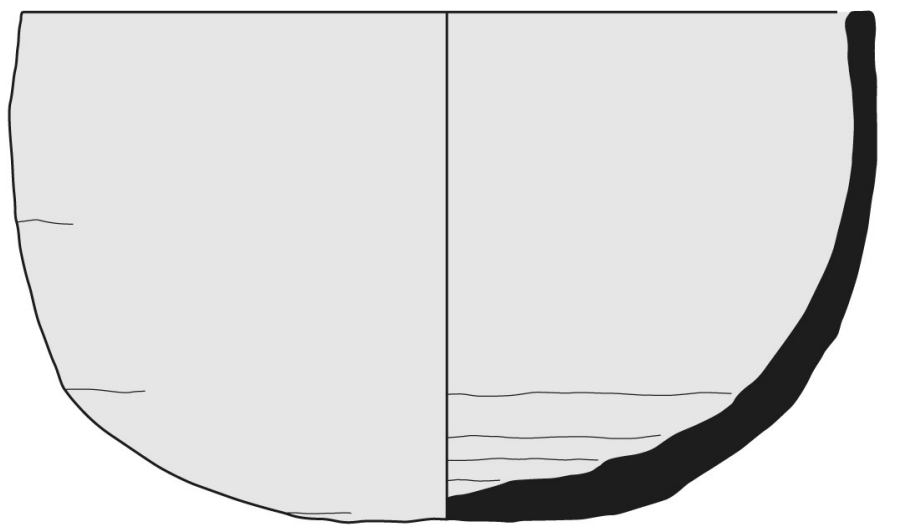
Cat. 106
Z12/86
0 $5 \mathrm{~cm}$

FIGURE 7.22 Deep hemispherical bowl of type II.2 with straight walls, variant d

Cat. 105

Bowl

Inv. no: $\mathrm{Z12} / 108$

Provenance: Tumulus 12, chamber 3

Construction: wheel-made (1)

State of preservation: incomplete, chipped rim, $97 \%$ of rim

Measurements: $\mathrm{Dr}=11 \mathrm{~cm} ; \mathrm{H}=9 \mathrm{~cm}$

Fabric: not possible to determine

Hardness: 4

Firing: not possible to determine

Surface treatment: slipped and burnished on both surfaces; external-burnish 3 , less carefully executed; internalburnish 3 , carelessly executed

Surface colour: external, slip — $10 \mathrm{R}$ 5/6 red; internal, slip10R $4 / 6$ red

Colour of break: not possible to determine

Cat. 106

Bowl

Inv. no: $\mathrm{Z12} / 86$

Provenance: Tumulus 12, chamber 3

Construction: wheel-made (1)

State of preservation: incomplete, $88 \%$ of rim

Measurements: $\mathrm{Dr}=20 \mathrm{~cm} ; \mathrm{H}=12 \mathrm{~cm}$

Fabric: ZF3

Hardness: $3 / 4$

Firing: oxidized

Surface treatment: slipped and burnished on both surfaces; external-burnish 1, carefully executed; internalburnish 2, carefully executed

Surface colour: external, slip-10R 4/4 weak red; internal, slip-10R 4/4 weak red

Colour of break: 1OYR 4/1 dark grey

References: (Czyżewska-Zalewska 2016, 728-730)
Variant II.2e

Cat. 107

Bowl

Inv. no: $\mathbf{Z} 25 / 11$

Provenance: Tumulus 25, chamber 2, southern part

Construction: wheel-made (1)

State of preservation: incomplete, chipped rim, $91 \%$ of rim Measurements: $\mathrm{Dr}=11.4 \mathrm{~cm} ; \mathrm{H}=6.8 \mathrm{~cm}$

Fabric: not possible to determine

Hardness: $3 / 4$

Firing: oxidized

Surface treatment: slipped and burnished on both surfaces; external-burnish 1, carefully executed; internalburnish 2, carelessly executed

Surface colour: external, slip-10R $5 / 6$ red; internal, slip10R $5 / 6$ red

Colour of break: 5 YR $5 / 6$ yellowish red

References: (Klimaszewska-Drabot 2010, 481)

Cat. 108

Bowl

Inv. no: $\mathbf{Z 1 2} / 37$

Provenance: Tumulus 12, fill of shaft, south-eastern part

Construction: wheel-made (1)

State of preservation: incomplete, $84 \%$ of rim, body sherds missing

Measurements: $\mathrm{Dr}=11.6 \mathrm{~cm} ; \mathrm{H}=6.5 \mathrm{~cm}$

Fabric: ZF3

Hardness: 4

Firing: oxidized

Surface treatment: slipped and burnished on both surfaces; burnish 1, carefully executed on both surfaces

Surface colour: external, slip-10R 5/6 red; internal, slip1oR $4 / 6$ red

Colour of break: 10YR 5/4 yellowish brown 


\section{variant II.2e}

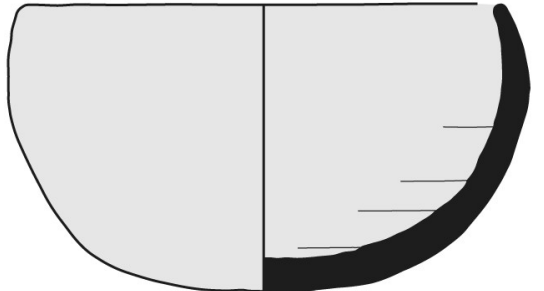

Cat. 107

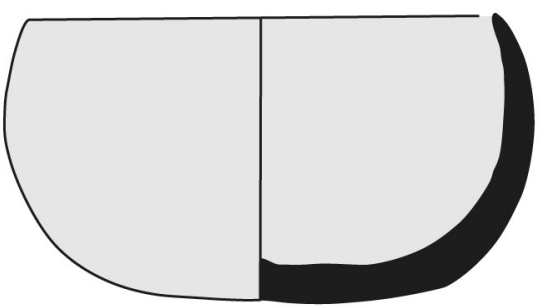

Cat. 109

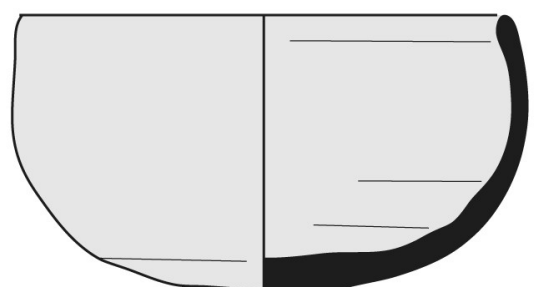

Z12/37

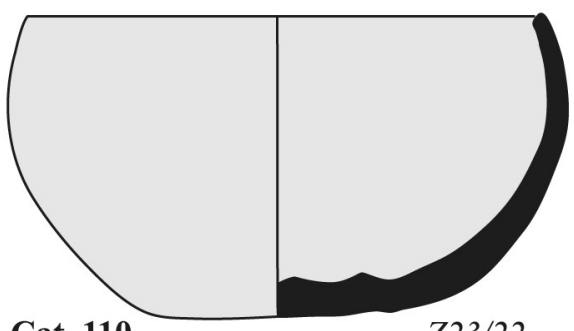

Cat. 110
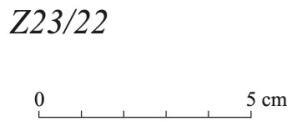

FIGURE 7.23 Low hemispherical bowls of type II.2 with incurved walls and maximum diameter at mid-H, variant e

Cat. 109

Bowl

Inv. no: $\mathbf{Z 2 3} / 29$

Provenance: Tumulus 23, chamber 2

Construction: wheel-made (1)

State of preservation: complete

Measurements: $\mathrm{Dr}=11-11.5 \mathrm{~cm} ; \mathrm{H}=6.7 \mathrm{~cm}$

Fabric: not possible to determine

Hardness: $3 / 4$

Firing: not possible to determine

Surface treatment: slipped and burnished on both surfaces; burnish 1, carefully executed on both surfaces

Surface colour: external, slip_-10R 5/6 red; internal, slip1oR $4 / 4$ weak red

Colour of break: not possible to determine

\section{Cat. 110}

Bowl

Inv. no: $\mathbf{Z 2 3} / 22$

Provenance: Tumulus 23, chamber 2

Construction: wheel-made (1)

State of preservation: complete

Measurements: $\mathrm{Dr}=12.4 \mathrm{~cm} ; \mathrm{H}=7.2 \mathrm{~cm}$

Fabric: not possible to determine

Hardness: $3 / 4$

Firing: not possible to determine

Surface treatment: slipped and burnished on both surfaces; external-burnish 1, carefully executed; internalburnish 1, less carefully executed

Surface colour: external, slip—1oR 5/4 weak red; internal, slip-10R 4/6 red

Colour of break: not possible to determine

3.3 Bowls Type II.3

Variant II.3a

Cat. 111

Bowl

Inv. no: $\mathbf{Z 1 2} / \mathbf{1 8}$

Provenance: Tumulus 12, fill of shaft

Construction: wheel-made (2)

State of preservation: complete

Measurements: $\mathrm{Dr}=10.5 \mathrm{~cm} ; \mathrm{H}=5.6 \mathrm{~cm}$

Fabric: not possible to determine

Hardness: 4

Firing: not possible to determine

Surface treatment: slipped on both surfaces

Surface colour: external, slip-1oR 4/4 weak red; internal, slip-10R 4/6 red

Colour of break: not possible to determine

References: (Czyżewska-Zalewska 2016, 728-73o)

Cat. 112

Bowl

Inv. no: Z12/17 
Provenance: Tumulus 12, fill of shaft

Construction: wheel-made (2)

State of preservation: complete

Measurements: $\mathrm{Dr}=11.5 \mathrm{~cm} ; \mathrm{H}=5.5 \mathrm{~cm}$

Fabric: not possible to determine

Hardness: 4

Firing: not possible to determine

Surface treatment: slipped on both surfaces

Surface colour: external, slip-2.5YR 4/4 reddish brown; internal, slip -5 YR $4 / 3$ reddish brown

Colour of break: not possible to determine

References: (Czyżewska-Zalewska 2016, 728-730)

Cat. 113

Bowl

Inv. no: $\mathbf{Z 1 2} / 42$

Provenance: Tumulus 12, fill of shaft

Construction: wheel-made (2)

State of preservation: incomplete, $65 \%$ of rim, body sherds missing

Measurements: $\mathrm{Dr}=10.8 \mathrm{~cm} ; \mathrm{H}=6 \mathrm{~cm}$

Fabric: $\mathrm{ZF} 4$

Hardness: 4

Firing: oxidized

Surface treatment: slipped on both surfaces

Surface colour: external, slip_-10R 4/4 weak red; internal, slip-10R $4 / 6$ red

Colour of break: 5 YR $5 / 6$ yellowish red

\section{Cat. 114}

Bowl

Inv. no: $\mathrm{Z1o} / 1$

Provenance: Tumulus 10, burial chamber, southern part

Construction: wheel-made (2)

State of preservation: complete

Measurements: $\mathrm{Dr}=10.6 \mathrm{~cm} ; \mathrm{H}=6.7 \mathrm{~cm}$

Fabric: not possible to determine

Hardness: 4

Firing: not possible to determine

Surface treatment: slipped on both surfaces

Surface colour: external natural surface-2.5YR 5/6 red, slip-7.5YR 4/6 strong brown; internal, slip-7.5R 4/6 red

Colour of break: not possible to determine

References: (Klimaszewska-Drabot 2010, 481)

\section{Cat. 115}

Bowl

Inv. no: $\mathrm{Z} 10 / 6$

Provenance: Tumulus 10, burial chamber, central part

Construction: wheel-made (2)
State of preservation: complete but broken

Measurements: $\mathrm{Dr}=14 \mathrm{~cm} ; \mathrm{H}=8.5 \mathrm{~cm}$

Fabric: not possible to determine

Hardness: 4

Firing: oxidized

Surface treatment: slipped on both surfaces

Surface colour: external, slip_-10R 4/6 red; internal, slip10R 4/6 red

Colour of break: 2.5 YR $5 / 6$ red

References: (Klimaszewska-Drabot 2010, 481)

Cat. 116

Bowl

Inv. no: $\mathrm{Z12} / 117$

Provenance: Tumulus 12, chamber 3

Construction: wheel-made (2)

State of preservation: complete

Measurements: $\mathrm{Dr}=14.4 \mathrm{~cm} ; \mathrm{H}=8.5 \mathrm{~cm}$

Fabric: not possible to determine

Hardness: $3 / 4$

Firing: not possible to determine

Surface treatment: slipped on both surfaces

Surface colour: slip_-10R 5/6 red on both surfaces

Colour of break: not possible to determine

Cat. 117

Bowl

Inv. no: $\mathbf{Z 1 1} / 25$

Provenance: Tumulus 11, chamber 2, central part

Construction: wheel-made (2)

State of preservation: complete

Measurements: $\mathrm{Dr}=14 \mathrm{~cm} ; \mathrm{H}=8.4 \mathrm{~cm}$

Fabric: not possible to determine

Hardness: $3 / 4$

Firing: not possible to determine

Surface treatment: slipped and polished on both surfaces

Surface colour: slip-10R 5/8 red on both surfaces

Colour of break: not possible to determine

References: (Klimaszewska-Drabot and Czyżewska 2012, 368-372)

Cat. 118

Bowl

Inv. no: Zu1/27

Provenance: Tumulus 11, chamber 2, central part

Construction: wheel-made (2)

State of preservation: complete

Measurements: $\mathrm{Dr}=12.2 \mathrm{~cm} ; \mathrm{H}=7.6 \mathrm{~cm}$

Fabric: not possible to determine

Hardness: $3 / 4$

Firing: not possible to determine 


\section{Bowls Type II.3 \\ variant II.3a}

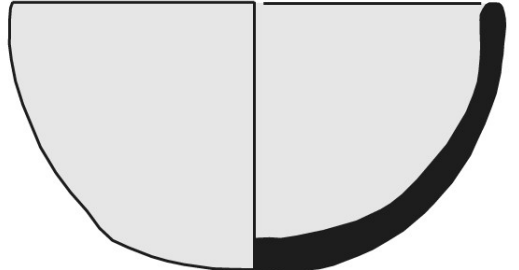

Cat. 111
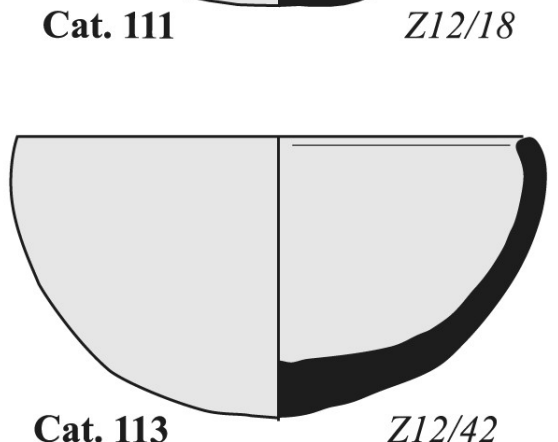

Cat. 113

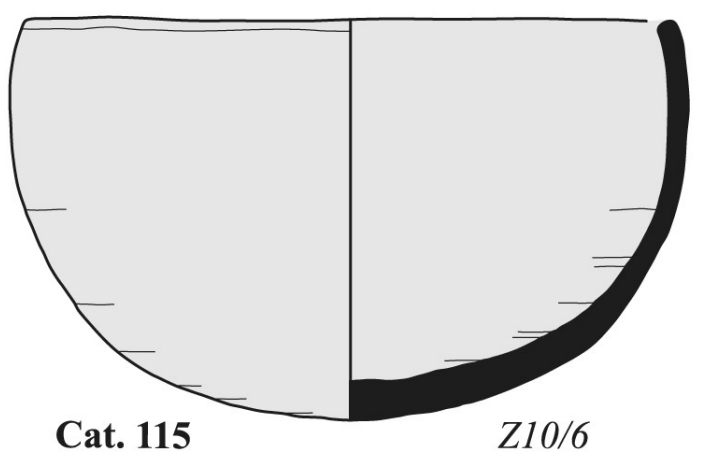

Cat. 115

\section{$Z 10 / 6$}

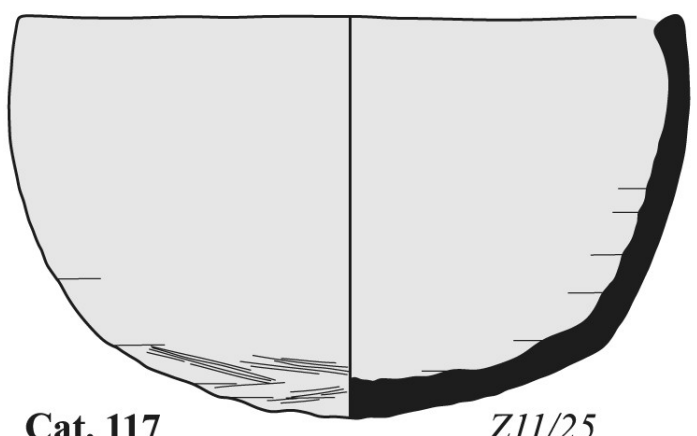

Cat. 117

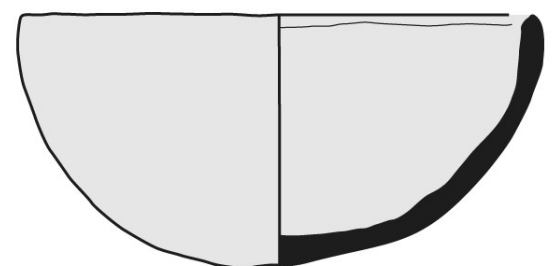

Cat. 112

Z12/17

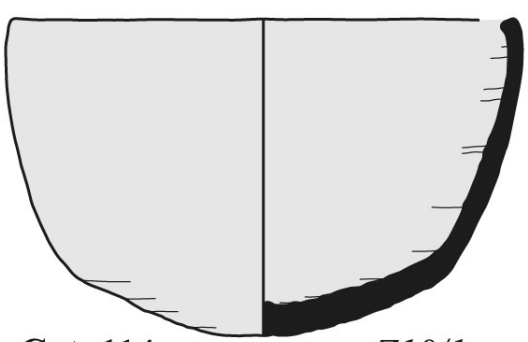

Cat. 114

Z10/1

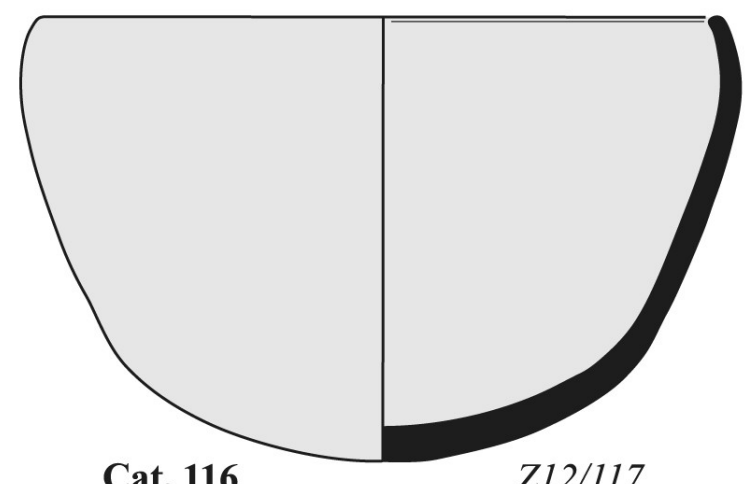

Cat. 116

Z12/117

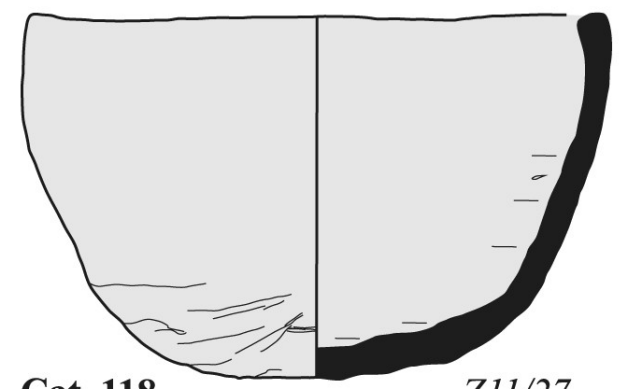

Cat. 118

0

FIGURE 7.24 Hemispherical bowls of type II.3 with incurved walls, variant a

Surface treatment: slipped and polished on both surfaces Surface colour: external, slip—-10R $5 / 6$ red; internal, slip1OYR $5 / 6$ yellowish brown

Colour of break: not possible to determine
Variant II.3b

Cat. 119

Bowl

Inv. no: Z12/39

Provenance: Tumulus 12, fill of shaft 


\section{variant II.3b}

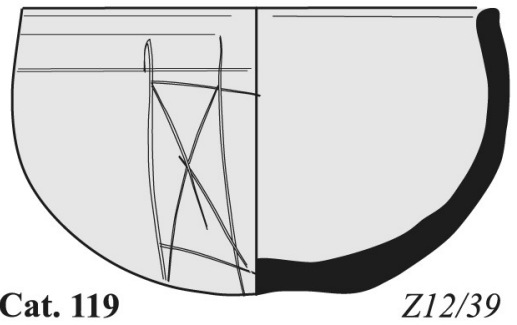

Cat. 119 Z12/39

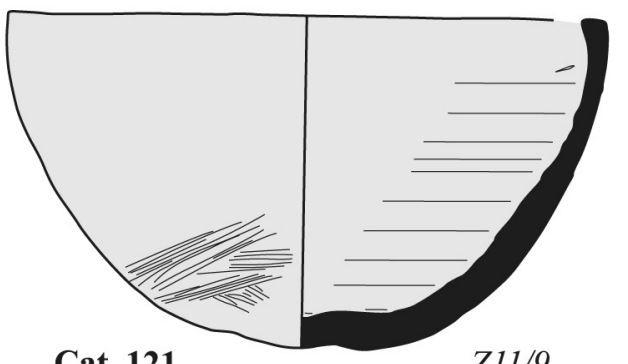

Cat. 121

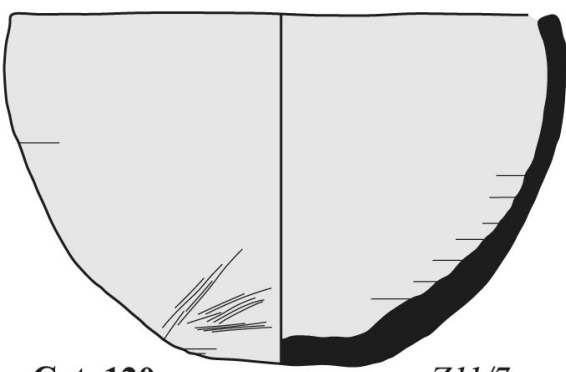

Cat. 120
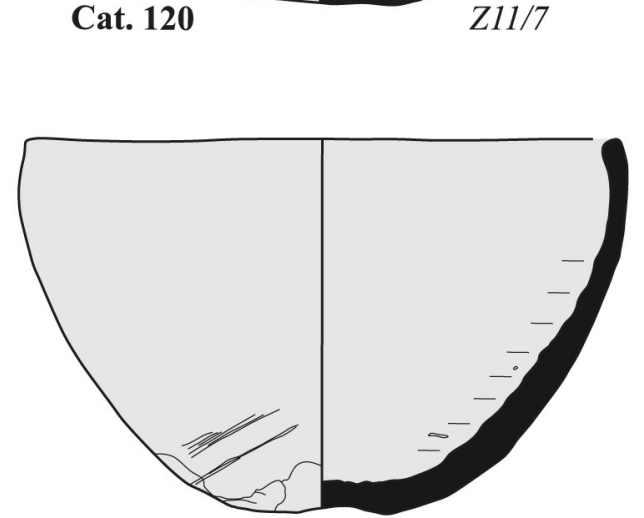

Cat. 122

$Z 11 / 13$

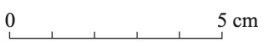

FIGURE 7.25 Hemispherical bowls of type II.3 with incurved walls, variant b

Construction: wheel-made (2)

State of preservation: fragmentary, $43 \%$ of rim, lower part complete

Measurements: $\mathrm{Dr}=11.2 \mathrm{~cm} ; \mathrm{H}=6.8 \mathrm{~cm}$

Fabric: $\mathrm{ZF1}$

Hardness: 4

Firing: oxidized

Surface treatment: slipped and polished on both surfaces

Surface colour: external, slip — $10 \mathrm{R} 5 / 6$ red; internal, slip$10 \mathrm{R}_{4} / 6$ red

Colour of break: 7.5YR 5/1 grey

Cat. 120

Bowl

Inv. no: $\mathrm{Zu1} / 7$

Provenance: Tumulus 11, chamber 1, eastern part

Construction: wheel-made (2)

State of preservation: incomplete, $97 \%$ of rim, lip chipped off

Measurements: $\mathrm{Dr}=12.9 \mathrm{~cm} ; \mathrm{H}=8.3 \mathrm{~cm}$

Fabric: not possible to determine

Hardness: $3 / 4$

Firing: oxidized

Surface treatment: slipped and polished on both surfaces Surface colour: slip-10R 5/6 red on both surfaces

Colour of break: not possible to determine
References: (Klimaszewska-Drabot and Czyżewska 2012, 368-372)

Cat. 121

Bowl

Inv. no: Z11/9

Provenance: Tumulus 11, chamber 1, eastern part

Construction: wheel-made (2)

State of preservation: incomplete, $88 \%$ of rim, body fragment missing

Measurements: $\mathrm{Dr}=14 \mathrm{~cm} ; \mathrm{H}=8 \mathrm{~cm}$

Fabric: ZF4

Hardness: 4

Firing: oxidized

Surface treatment: slipped and polished on both surfaces

Surface colour: slip_-10R $5 / 8$ red on both surfaces

Colour of break: $10 \mathrm{R} 5 / 8$ red

References: (Klimaszewska-Drabot and Czyżewska 2012, 368-372)

Cat. 122

Bowl

Inv. no: $\mathrm{Z11} / \mathbf{1 3}$

Provenance: Tumulus 11, chamber 1, eastern part

Construction: wheel-made (2)

State of preservation: complete 
Measurements: $\mathrm{Dr}=14 \mathrm{~cm} ; \mathrm{H}=8.8 \mathrm{~cm}$

Fabric: not possible to determine

Hardness: 4

Firing: not possible to determine

Surface treatment: slipped and polished on both surfaces

Surface colour: slip-10R $5 / 8$ red on both surfaces

Colour of break: not possible to determine

References: (Klimaszewska-Drabot and Czyżewska 2012, 368-372; Mahmoud El-Tayeb and Czyżewska 2011, 115117)

\subsection{Bowls Type II.4}

Variant II.4a

Cat. 123

Bowl

Inv. no: $\mathrm{Z} 4 / 254$

Provenance: Tumulus 4, E tunnel, southern part

Construction: wheel-made (2)

State of preservation: fragmentary, restored from sherds, $17 \%$ of rim, wall fragments survive

Measurements: $\mathrm{Dr}=12.6 \mathrm{~cm} ; \mathrm{H}=5.2 \mathrm{~cm}$

Fabric: $\mathrm{ZF} 3$

Hardness: 4

Firing: oxidized

Surface treatment: slipped and polished on both surfaces

Surface colour: slip_-10R 4/6 red on both surfaces

Colour of break: 2.5 YR $5 / 6$ red

Decoration: incised-one groove below rim

Cat. 124

Bowl

Inv. no: $\mathrm{Z14} / 6$

Provenance: Tumulus 14, chamber, western part

Construction: wheel-made (2)

State of preservation: complete

Measurements: $\mathrm{Dr}=12.5 \mathrm{~cm} ; \mathrm{H}=7.6 \mathrm{~cm}$

Fabric: not possible to determine

Hardness: 4

Firing: not possible to determine

Surface treatment: slipped and polished on both surfaces

Surface colour: slip_-10R 4/6 red on both surfaces

Colour of break: not possible to determine

Decoration: incised—one to two spiral grooves below rim

\section{Cat. 125}

Bowl

Inv. no: $\mathrm{Z}_{4} / \mathbf{1 3 4}+\mathrm{Z}_{4} / 34$

Provenance: Tumulus 4, W tunnel

Construction: wheel-made (2)

State of preservation: complete

Measurements: $\mathrm{Dr}=11.6 \mathrm{~cm} ; \mathrm{H}=7.3 \mathrm{~cm}$
Fabric: not possible to determine

Hardness: 4

Firing: not possible to determine

Surface treatment: slipped and polished on both surfaces

Surface colour: slip_-10R 4/6 red on both surfaces

Colour of break: not possible to determine

Decoration: incised-two to three spiral grooves below rim

Cat. 126

Bowl

Inv. no: $\mathrm{Z2} / 24$

Provenance: Tumulus 2, chamber 2

Construction: wheel-made (2)

State of preservation: incomplete, body sherd missing

Measurements: $\mathrm{Dr}=13.4 \mathrm{~cm} ; \mathrm{H}=7.5 \mathrm{~cm}$

Fabric: not possible to determine

Hardness: 4

Firing: not possible to determine

Surface treatment: slipped and polished on both surfaces

Surface colour: slip_-1oR 4/8 red on both surfaces

Colour of break: not possible to determine

Decoration: incised - two grooves below rim

Cat. 127

Bowl

Inv. no: $\mathrm{Z}_{7} / 46$

Provenance: Tumulus 7, sherds scattered in different places, context 2, fill of shaft

Construction: wheel-made (2)

State of preservation: incomplete, $87 \%$ of rim, base fragment missing

Measurements: $\mathrm{Dr}=12.2 \mathrm{~cm} ; \mathrm{H}=8 \mathrm{~cm}$

Fabric: ZF1

Hardness: 4

Firing: oxidized

Surface treatment: slipped and polished on both surfaces

Surface colour: slip-2.5YR $5 / 6$ red on both surfaces

Colour of break: 2.5 YR $5 / 8$ red

Decoration: incised-three grooves below rim

Cat. 128

Bowl

Inv. no: $\mathrm{Z} 1 / \mathbf{1}$

Provenance: Tumulus 1, trench 2, tunnel entrance, eastern side

Construction: wheel-made (2)

State of preservation: fragmentary, $25 \%$ of rim, body sherds missing

Measurements: $\mathrm{Dr}=10.5 \mathrm{~cm} ; \mathrm{H}=6 \mathrm{~cm}$

Fabric: $\mathrm{ZF} 3$

Hardness: 4 


\section{Bowls Type II.4}

\section{variant II.4a}

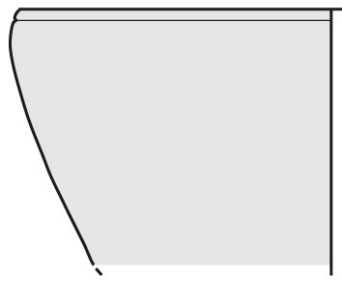

Cat. 123

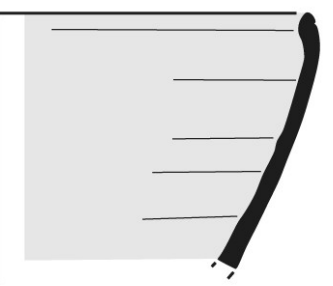

Z4/254

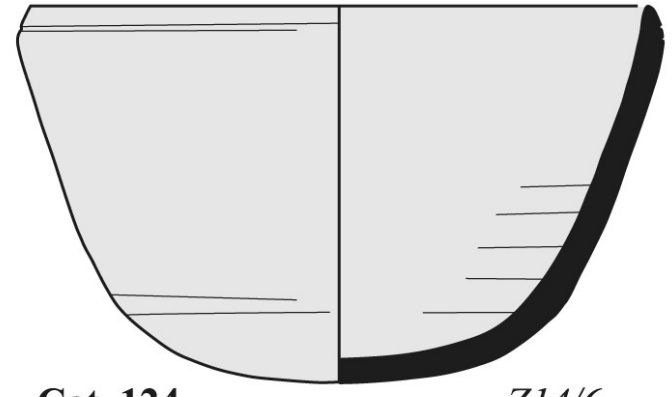

Cat. 124

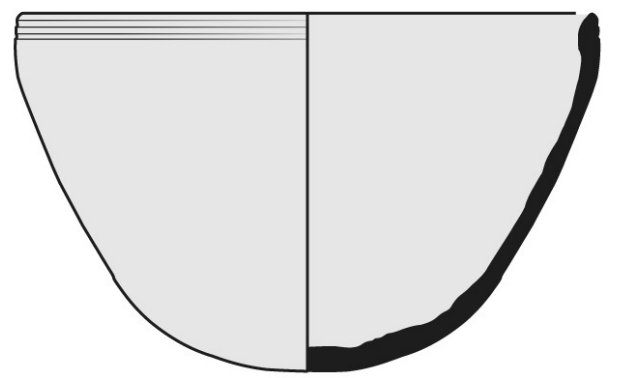

Cat. 125

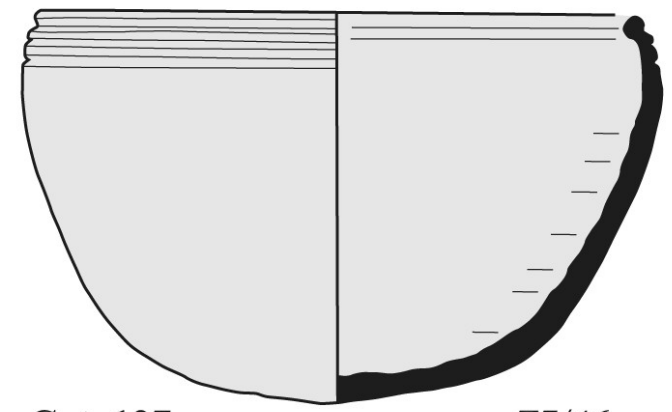

Cat. 127

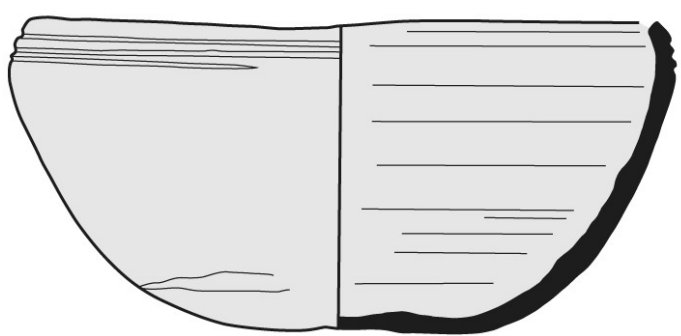

Cat. 129
$Z 4 / 34+Z 4 / 134$

Z7/46

Z9/1

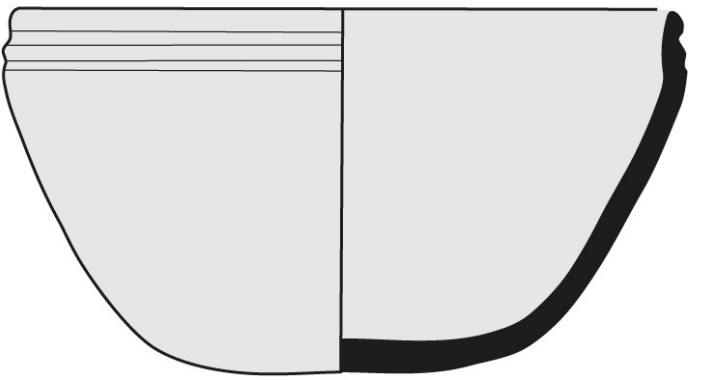

$Z 2 / 24$

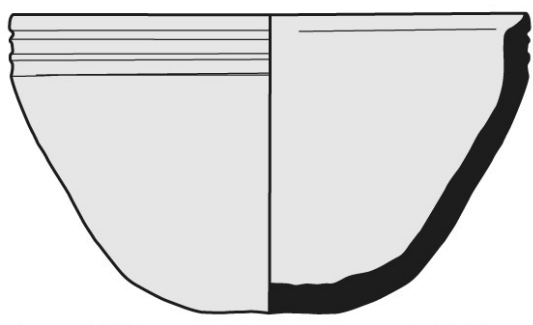

Cat. 128

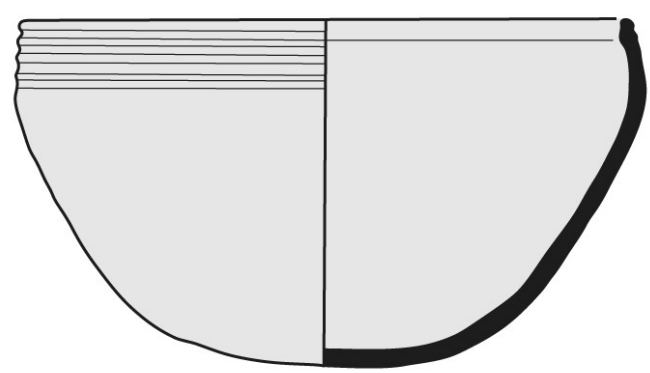

Cat. 130

0 $5 \mathrm{~cm}$ FIGURE 7.26 Conical bowls of type II.4 with incurved walls and grooves below the rim, variant a 
Firing: oxidized

Surface treatment: slipped and polished on both surfaces

Surface colour: slip-10R $5 / 6$ red on both surfaces

Colour of break: $7 \cdot 5$ YR $5 / 3$ brown

Decoration: incised-three grooves below rim

\section{Cat. 129}

Bowl

Inv. no: $\mathrm{Z} \mathbf{9} / \mathbf{1}$

Provenance: Tumulus 9, bottom of shaft, southern part

Construction: wheel-made (2)

State of preservation: complete

Measurements: $\mathrm{Dr}=12.5 \mathrm{~cm} ; \mathrm{H}=6.3 \mathrm{~cm}$

Fabric: not possible to determine

Hardness: 4

Firing: not possible to determine

Surface treatment: slipped and polished on both surfaces

Surface colour: slip_-10R 4/6 red on both surfaces

Colour of break: not possible to determine

Decoration: incised-three to four spiral grooves below rim

References: (Mahmoud El-Tayeb, Juszczyk-Futkowska, and Czyżewska 2014, 367, 369-371)

\section{Cat. 130}

Bowl

Inv. no: $\mathrm{Z} 2 / 26$

Provenance: Tumulus 2, chamber 2

Construction: wheel-made (2)

State of preservation: complete

Measurements: $\mathrm{Dr}=12.2 \mathrm{~cm} ; \mathrm{H}=7 \mathrm{~cm}$

Fabric: not possible to determine

Hardness: 4

Firing: not possible to determine

Surface treatment: slipped and polished on both surfaces

Surface colour: slip—-10R 4/8 red on both surfaces

Colour of break: not possible to determine

Decoration: incised-four to five spiral grooves below rim

Cat. 131

Bowl

Inv. no: $\mathbf{Z 2} / 4$

Provenance: Tumulus 2, chamber 3

Construction: wheel-made (2)

State of preservation: complete

Measurements: $\mathrm{Dr}=11 \mathrm{~cm} ; \mathrm{H}=7.5 \mathrm{~cm}$

Fabric: not possible to determine

Hardness: 4

Firing: not possible to determine

Surface treatment: slipped and polished on both surfaces

Surface colour: slip-10R 4/8 red, on both surfaces
Colour of break: not possible to determine

Decoration: incised - two grooves below rim, one groove at mid-H

Cat. 132

Bowl

Inv. no: $\mathbf{Z 2 3} / 26$

Provenance: Tumulus 23, chamber 2

Construction: wheel-made (2)

State of preservation: incomplete, $94 \%$ of rim

Measurements: $\mathrm{Dr}=12.1 \mathrm{~cm} ; \mathrm{H}=8.2 \mathrm{~cm}$

Fabric: not possible to determine

Hardness: $3 / 4$

Firing: not possible to determine

Surface treatment: slipped and polished on both surfaces

Surface colour: external, slip_-10R $5 / 8$ red; internal, slip10R $5 / 6$ red

Colour of break: $10 \mathrm{R} 5 / 8$ red

Decoration: incised-two grooves below rim, one groove at mid-H

Cat. 133

Bowl

Inv. no: $\mathrm{Z} 4 / 35$

Provenance: Tumulus 4, W tunnel

Construction: wheel-made (2)

State of preservation: incomplete, restored from sherds, $97 \%$ of rim, body-sherd fragments missing

Measurements: $\mathrm{Dr}=12.6 \mathrm{~cm} ; \mathrm{H}=7.2 \mathrm{~cm}$

Fabric: $\mathrm{ZF} 3$

Hardness: $3 / 4$

Firing: oxidized

Surface treatment: slipped and polished on both surfaces

Surface colour: external, slip—10R 4/6 red; internal, slip$7 \cdot 5 \mathrm{R}_{4} / 6$ red

Colour of break: 2.5 YR $5 / 6$ red

Decoration: incised-two grooves below rim, one groove at mid-H

Cat. 134

Bowl

Inv. no: Z2/19

Provenance: Tumulus 2, chamber 2

Construction: wheel-made (2)

State of preservation: complete

Measurements: $\mathrm{Dr}=10.6 \mathrm{~cm} ; \mathrm{H}=7.5 \mathrm{~cm}$

Fabric: not possible to determine

Hardness: 4

Firing: not possible to determine

Surface treatment: slipped and polished on both surfaces

Surface colour: slip_-1oR 4/8 red, on both surfaces 
Colour of break: not possible to determine

Decoration: incised-two to three grooves below rim, two grooves at mid-H

Cat. 135

Bowl

Inv. no: $\mathbf{Z}_{4} / \mathbf{2} 01$

Provenance: Tumulus 4, E tunnel

Construction: wheel-made (2)

State of preservation: fragmentary, $58 \%$ of rim, body-sherd fragments missing

Measurements: $\mathrm{Dr}=12 \mathrm{~cm} ; \mathrm{H}=7.6 \mathrm{~cm}$

Fabric: ZF1

Hardness: $3 / 4$

Firing: oxidized

Surface treatment: slipped and polished on both surfaces

Surface colour: external, slip—1oR 3/6 dark red; internal, slip-10R $4 / 6$ red

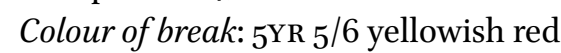

Decoration: incised-two to three grooves below rim, one groove at mid-H

Cat. 136

Bowl

Inv. no: $\mathbf{Z} 2 / 28$

Provenance: Tumulus 2, chamber 2

Construction: wheel-made (2)

State of preservation: complete

Measurements: $\mathrm{Dr}=12.2 \mathrm{~cm} ; \mathrm{H}=7.3 \mathrm{~cm}$

Fabric: not possible to determine

Hardness: 4

Firing: not possible to determine

Surface treatment: slipped and polished on both surfaces

Surface colour: slip—1oR 4/8 red, on both surfaces

Colour of break: not possible to determine

Decoration: incised-three grooves below rim, two grooves at mid-H

\section{Cat. 137}

Bowl

Inv. no: $\mathbf{Z} 2 / 5$

Provenance: Tumulus 2, chamber 3

Construction: wheel-made (2)

State of preservation: complete

Measurements: $\mathrm{Dr}=11.5 \mathrm{~cm} ; \mathrm{H}=7 \mathrm{~cm}$

Fabric: not possible to determine

Hardness: 4

Firing: not possible to determine

Surface treatment: slipped and polished on both surfaces

Surface colour: slip-10R 4/8 red, on both surfaces

Colour of break: not possible to determine
Decoration: incised-three to four spiral grooves below rim, two grooves at mid-H

Cat. 138

Bowl

Inv. no: $\mathbf{Z 2} / \mathbf{2 2}$

Provenance: Tumulus 2, chamber 2

Construction: wheel-made (2)

State of preservation: complete

Measurements: $\mathrm{Dr}=10.6 \mathrm{~cm} ; \mathrm{H}=6.5 \mathrm{~cm}$

Fabric: not possible to determine

Hardness: 4

Firing: not possible to determine

Surface treatment: slipped and polished on both surfaces

Surface colour: external, slip $-10 \mathrm{R} 5 / 8$ red; internal, $10 \mathrm{R}$ $4 / 8$ red

Colour of break: not possible to determine

Decoration: incised-four grooves below rim, two grooves at mid-H

References: (Klimaszewska-Drabot and Mahmoud ElTayeb 2014, 10-13)

Variant II.4b

Cat. 139

Bowl

Inv. no: $\mathbf{Z} 4 / 14.3 \mathrm{a}$

Provenance: Tumulus 4, tunnel

Construction: wheel-made (2)

State of preservation: fragmentary, $51 \%$ of rim, body sherds and base missing

Measurements: $\mathrm{Dr}=11 \mathrm{~cm} ; \mathrm{H}=8.2 \mathrm{~cm}$

Fabric: ZF1

Hardness: $3 / 4$

Firing: oxidized

Surface treatment: slipped and polished on both surfaces

Surface colour: slip-1oR 4/6 red on both surfaces

Colour of break: 5YR $5 / 6$ yellowish red

Decoration: incised-two to three spiral grooves below rim;

painted—sets of white bands with four black dots on rim (two sets extant)

Cat. 140

Bowl

Inv. no: Z25/19

Provenance: Tumulus 25, chamber 3, central part

Construction: wheel-made (2)

State of preservation: complete

Measurements: $\mathrm{Dr}=13.5 \mathrm{~cm} ; \mathrm{H}=8.3 \mathrm{~cm}$

Fabric: not possible to determine

Hardness: 4 
variant II.4a
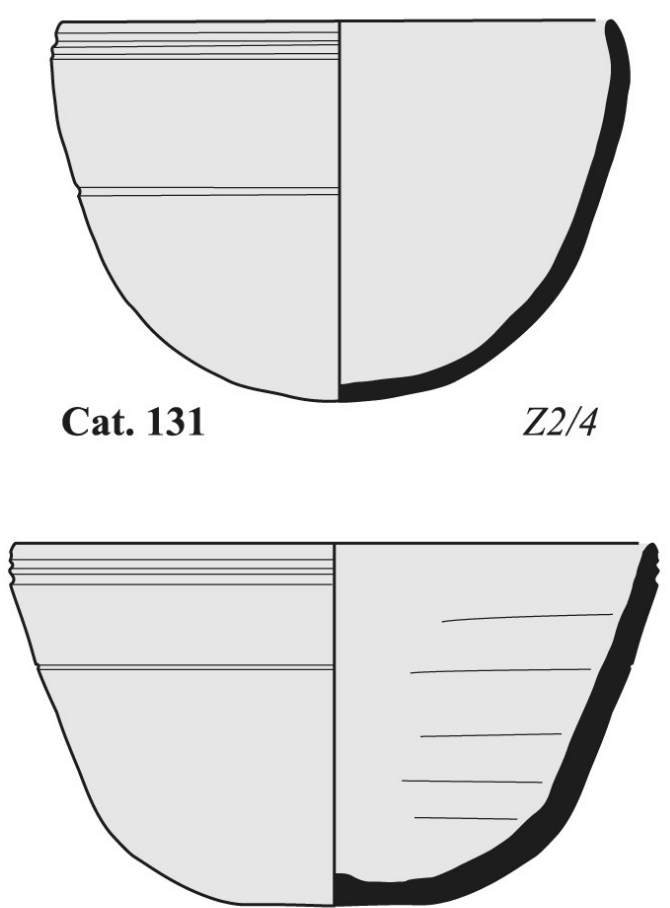

Cat. 133

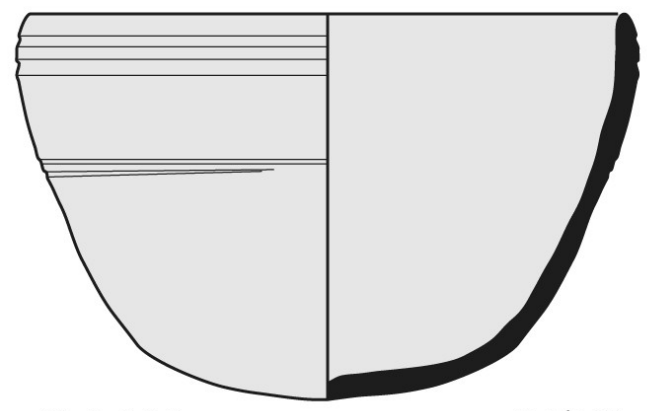

Cat. 135
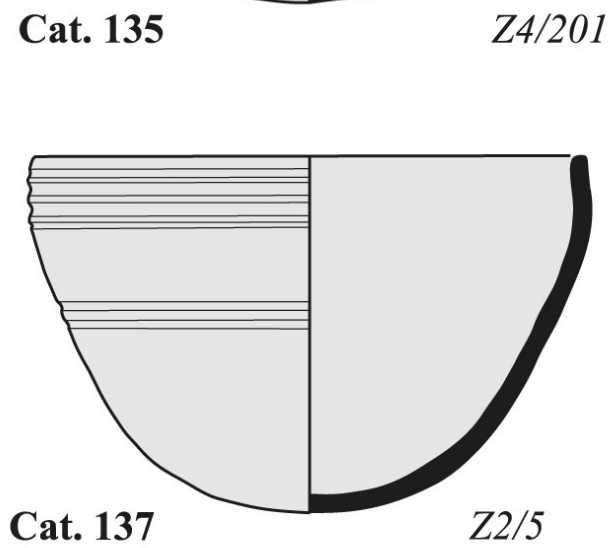

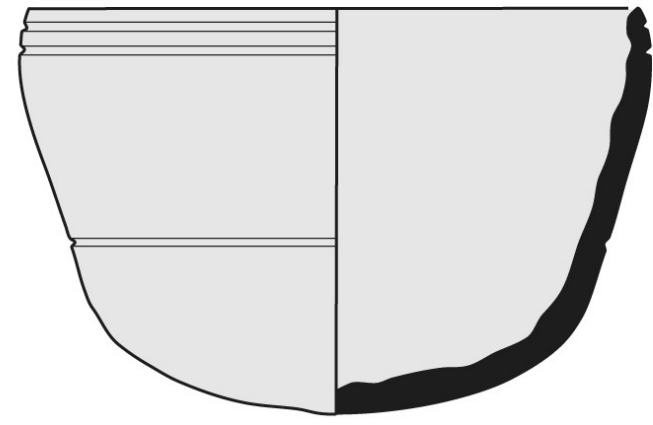

Cat. 132

Z23/26

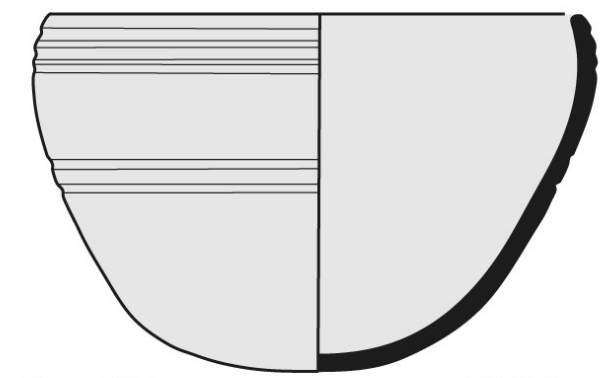

Cat. 134

Z2/19

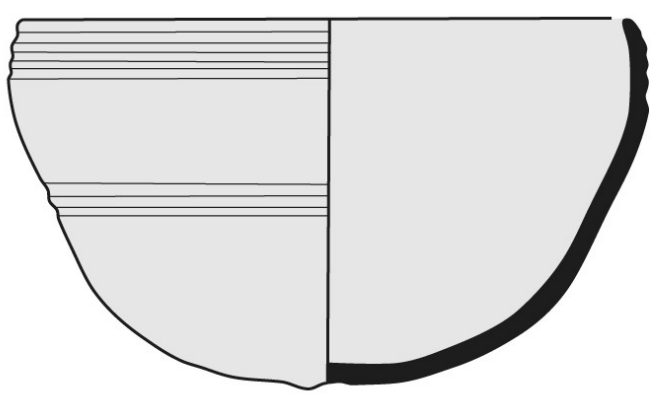

Cat. 136

$Z 2 / 28$

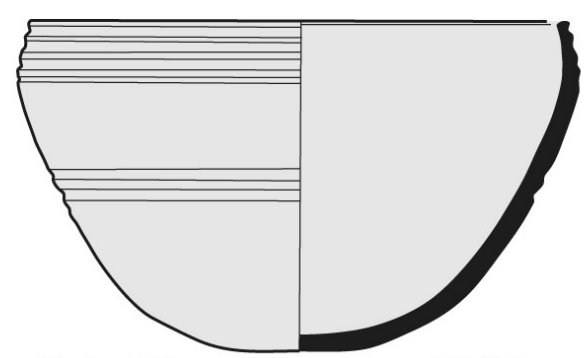

Cat. 138

0 $5 \mathrm{~cm}$ FIGURE 7.27 Conical bowls of type II.4 with incurved walls and grooves below the rim, variant a 
variant II.4b
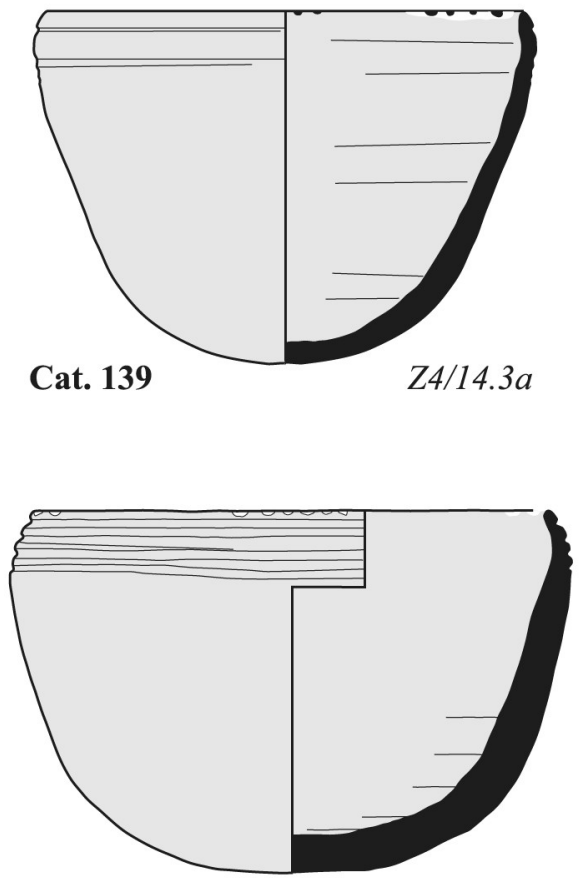

Cat. 141
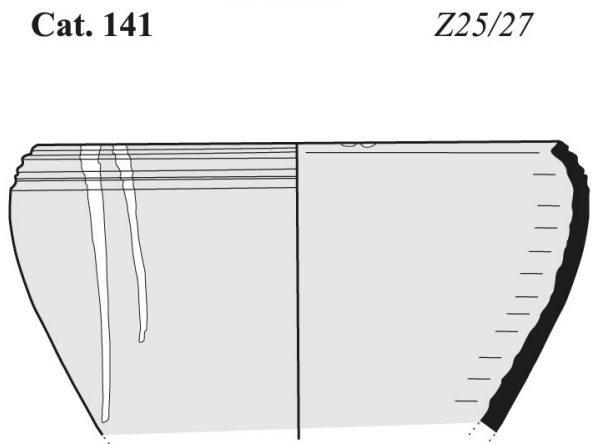

Cat. 143

Z7/27

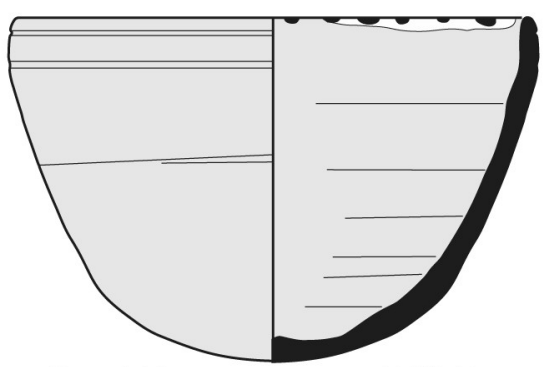

Cat. 145

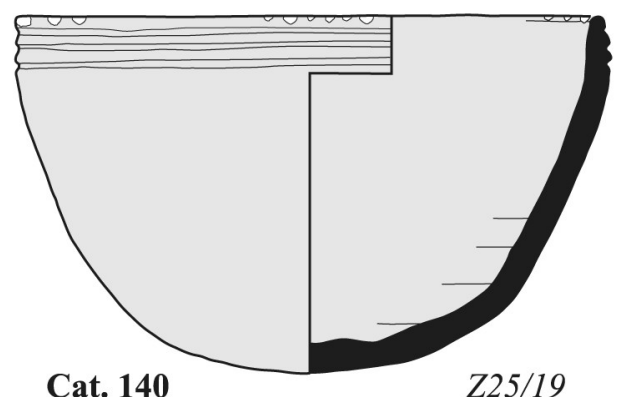

Z25/19

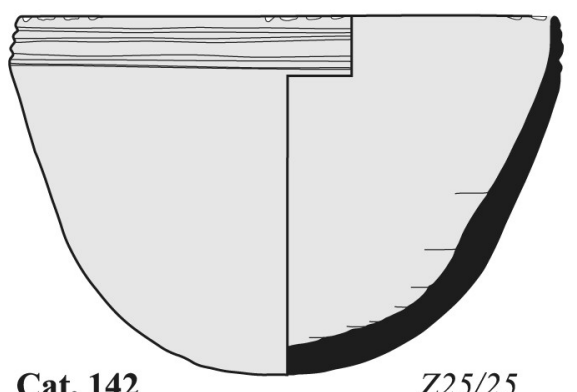

Cat. 142

$Z 25 / 25$

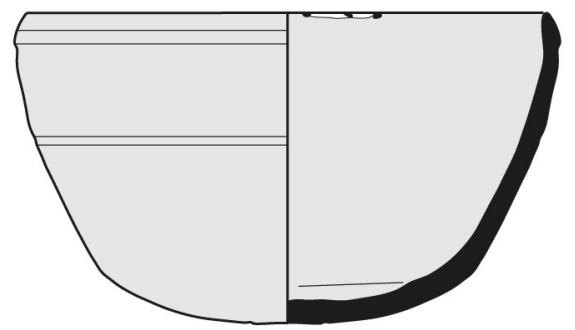

Cat. 144

FIGURE 7.28 Conical bowls of type II.4 with incurved walls, grooves below the rim and painted decoration, variant $b$ 
Firing: not possible to determine

Surface treatment: slipped and polished on both surfaces

Surface colour: external, slip_-1oR 4/4 weak red; internal, slip-10R 4/6 red

Colour of break: not possible to determine

Decoration: incised - three grooves below rim;

painted - three sets of five white dots, and one set of six white dots on rim

References: (Klimaszewska-Drabot 2010, 481)

Cat. 141

Bowl

Inv. no: $\mathrm{Z25/27}$

Provenance: Tumulus 25, chamber 3, southern part

Construction: wheel-made (2)

State of preservation: complete

Measurements: $\mathrm{Dr}=12.3 \mathrm{~cm} ; \mathrm{H}=8.4 \mathrm{~cm}$

Fabric: not possible to determine

Hardness: 4

Firing: not possible to determine

Surface treatment: slipped and polished on both surfaces

Surface colour: external, slip—-10R $5 / 6$ red; internal, slip1oR $4 / 6$ red

Colour of break: not possible to determine

Decoration: incised - four to five spiral grooves below rim; painted-four sets of five white dots on rim

References: (Klimaszewska-Drabot 2010, 481; Czyżewska-

Zalewska 2016, 728-730)

Cat. 142

Bowl

Inv. no: $\mathrm{Z25} / 25$

Provenance: Tumulus 25, chamber 3, central part

Construction: wheel-made (2)

State of preservation: complete

Measurements: $\mathrm{Dr}=12.4 \mathrm{~cm} ; \mathrm{H}=8.3 \mathrm{~cm}$

Fabric: not possible to determine

Hardness: 4

Firing: not possible to determine

Surface treatment: slipped and polished on both surfaces

Surface colour: slip—1oR 4/6 red on both surfaces

Colour of break: not possible to determine

Decoration: incised-three to four spiral grooves below rim;

painted-four sets of six white dots on rim

References: (Klimaszewska-Drabot 2010, 481)

Cat. 143

Bowl

Inv. no: $\mathrm{Z}_{7} / \mathbf{2 7}$

Provenance: Tumulus 7, context 1, fill of shaft
Construction: wheel-made (2)

State of preservation: fragmentary, $58 \%$ of rim, bottom part and body sherds missing

Measurements: $\mathrm{Dr}=12 \mathrm{~cm} ; \mathrm{H}=6.7 \mathrm{~cm}$

Fabric: ZF4

Hardness: 4

Firing: oxidized

Surface treatment: slipped and polished on both surfaces

Surface colour: external, slip-1oR 4/4 weak red; internal, slip-10R $4 / 6$ red

Colour of break: 7.5YR 5/4 brown

Decoration: incised-three to four spiral grooves below rim;

painted - two sets of two white lines extending downwards from rim

Cat. 144

Bowl

Inv. no: $\mathrm{Z}_{4} / \mathbf{1 4} \cdot \mathbf{3} \mathrm{d}$

Provenance: Tumulus 4, tunnel

Construction: wheel-made (2)

State of preservation: fragmentary, $20 \%$ of rim, body-sherd fragments missing

Measurements: $\mathrm{Dr}=12 \mathrm{~cm} ; \mathrm{H}=7.3 \mathrm{~cm}$

Fabric: $\mathrm{ZF} 4$

Hardness: $3 / 4$

Firing: oxidized

Surface treatment: slipped and polished on both surfaces Surface colour: slip_-10R 4/4 weak red, on both surfaces Colour of break: 5 YR $4 / 4$ reddish brown

Decoration: incised-two grooves below rim, one groove at mid-H;

painted - remnants of two sets of white bands with black dots extant on rim

Cat. 145

Bowl

Inv. no: $\mathrm{Z} 4 / 240$

Provenance: Tumulus 4, E tunnel

Construction: wheel-made (2)

State of preservation: fragmentary, $71 \%$ of rim, body-sherd and base fragments missing

Measurements: $\mathrm{Dr}=12 \mathrm{~cm} ; \mathrm{H}=8.1 \mathrm{~cm}$

Fabric: $\mathrm{ZF} 3$

Hardness: $3 / 4$

Firing: oxidized

Surface treatment: slipped and polished on both surfaces

Surface colour: slip-10R 4/6 red, on both surfaces

Colour of break: 5 YR $4 / 6$ yellowish red

Decoration: incised-two to three spiral grooves below rim, one to two spiral grooves at mid-H; painted- 


\section{Bowls Type II.5}

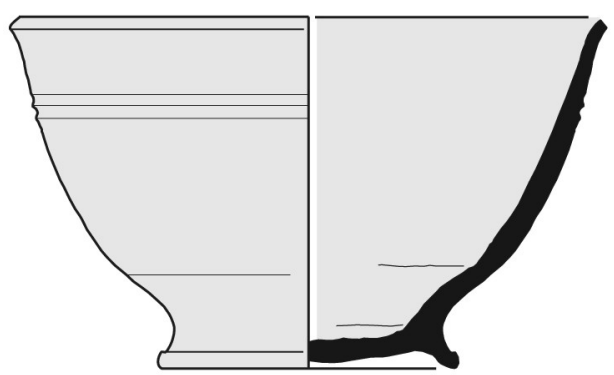

Cat. 146

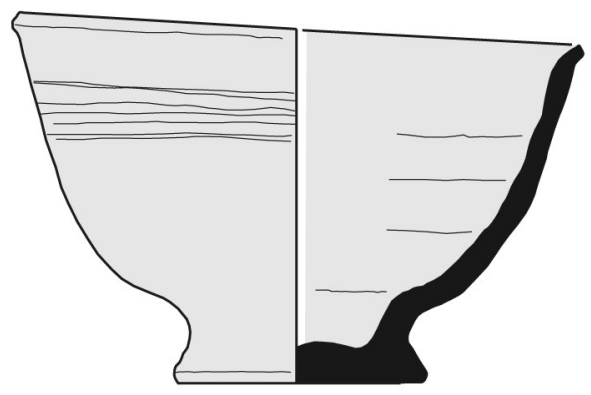

Cat. 148

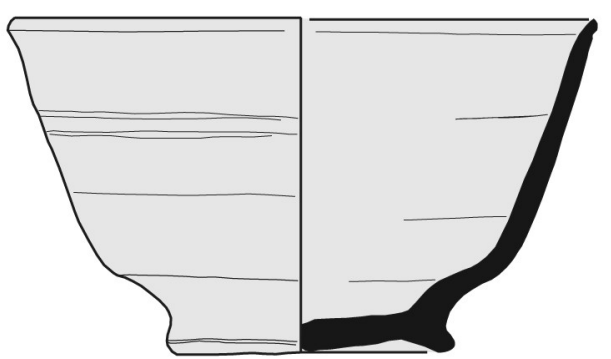

Cat. 147

$Z 15 / 20$

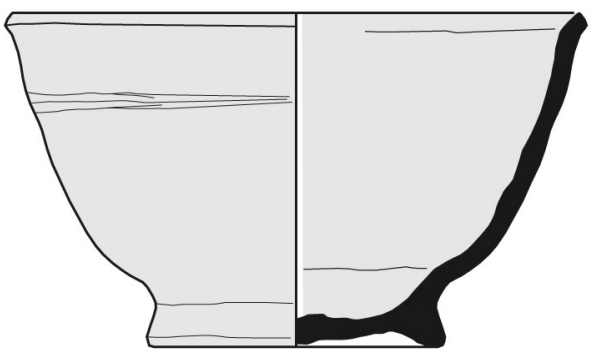

Cat. 149

Z15/61

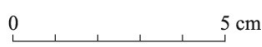

FIGURE 7.29 Footed bowls of type II.5 with flared walls and grooved decoration

fragments of two sets of white bands with six and seven black dots extant on rim

\subsection{Bowls Type II.5}

Cat. 146

Bowl

Inv. no: $\mathrm{Z}_{15} / 19$

Provenance: Tumulus 15, shaft (southern part), near blocking wall of main burial chamber

Construction: wheel-made (2)

State of preservation: fragmentary, rim and wall fragments missing, $28 \%$ of rim, almost complete base

Measurements: $\mathrm{Dr}=14 \mathrm{~cm} ; \mathrm{H}=8.2 \mathrm{~cm} ; \mathrm{D}_{\mathrm{b}}=6.9 \mathrm{~cm}$

Fabric: ZF1

Hardness: 3

Firing: oxidized

Surface treatment: slipped on both surfaces and polished

Surface colour: external—1oR 5/8 red, slip-10R 4/6 red; internal, slip-10R $4 / 6$ red

Colour of break: 5 YR $5 / 6$ yellowish red

Decoration: incised-grooves on the external surface
Cat. 147

Bowl

Inv. no: $\mathrm{Z}_{\mathbf{1 5}} / \mathbf{2 0}$

Provenance: Tumulus 15, shaft (southern part), near blocking wall of main burial chamber

Construction: wheel-made (2)

State of preservation: incomplete, $96 \%$ of rim, complete base

Measurements: $\mathrm{Dr}=13.8 \mathrm{~cm} ; \mathrm{H}=8 \mathrm{~cm} ; \mathrm{D}_{\mathrm{b}}=6.2 \mathrm{~cm}$

Fabric: ZF1

Hardness: 3

Firing: oxidized

Surface treatment: slipped on both surfaces and polished

Surface colour: external-1oR 5/8 red, slip-10R 4/6 red; internal, slip-10R $4 / 6$ red

Colour of break: 5YR 5/4 reddish brown

Cat. 148

Bowl

Inv. no: $\mathrm{Z}_{15} / 45$

Provenance: Tumulus 15, main burial chamber, eastern part Construction: wheel-made (2)

State of preservation: incomplete, $88 \%$ of rim, complete base 
Measurements: $\mathrm{Dr}=13.7 \mathrm{~cm} ; \mathrm{H}=8.9 \mathrm{~cm} ; \mathrm{D}_{\mathrm{b}}=6.1 \mathrm{~cm}$

Fabric: $\mathrm{ZF1}$

Hardness: 3

Firing: oxidized

Surface treatment: slipped on both surfaces and polished

Surface colour: external, slip_-10R 4/6 red; internal, slip$\mathrm{1OR}_{4} / 6$ red

Colour of break: 2.5 YR $5 / 4$ reddish brown

Decoration: incised-grooves on external surface

References: (Czyżewska-Zalewska 2016, 728-730)

\section{Cat. 149}

Bowl

Inv. no: $\mathbf{Z 1 5} / 61$

Provenance: Tumulus 15, main burial chamber, eastern part Construction: wheel-made (2)

State of preservation: fragmentary, rim and wall fragments missing, $42 \%$ of rim, complete base

Measurements: $\mathrm{Dr}=13.6 \mathrm{~cm} ; \mathrm{H}=7.8 \mathrm{~cm} ; \mathrm{D}_{\mathrm{b}}=6.9 \mathrm{~cm}$

Fabric: ZF1

Hardness: $3 / 4$

Firing: oxidized

Surface treatment: slipped on both surfaces and polished

Surface colour: external, slip $-10 \mathrm{R} 5 / 6$ red; internal, slip$10 \mathrm{R}_{4} / 6$ red

Colour of break: 5 YR $5 / 6$ yellowish red

Decoration: incised-grooves on external surface

\subsection{Bowls Type II.6}

[Fig. 7.100]

\section{Cat. 150}

Bowl

Inv. no: $\mathbf{Z 1 3} / \mathbf{2 8}$

Provenance: Tumulus 13, chamber 3, central part

Construction: wheel-made (2)

State of preservation: complete

Measurements: $\mathrm{Dr}=13 \mathrm{~cm} ; \mathrm{H}=8.4 \mathrm{~cm}$

Fabric: not possible to determine

Hardness: 4

Firing: not possible to determine

Surface treatment: slipped and polished on both surfaces

Surface colour: slip-1oR 5/6 red

Colour of break: not possible to determine

Decoration: on external surface; incised-three grooves below rim

References: (Klimaszewska-Drabot and Czyżewska 2012, 365-367; Mahmoud El-Tayeb and Czyżewska 2011, $115-117$ )

\section{Cat. 151}

Bowl

Inv. no: $\mathrm{Z}_{7} / \mathbf{2 1}$
Provenance: Tumulus 7, superstructure

Construction: wheel-made (2)

State of preservation: fragmentary, $11 \%$ of rim, wall fragment

Measurements: $\mathrm{Dr}=13.2 \mathrm{~cm} ; \mathrm{H}=7 \mathrm{~cm}$

Fabric: ZF4

Hardness: 4

Firing: oxidized

Surface treatment: slipped on both surfaces

Surface colour: external natural surface -5 Y R 6/3 light reddish brown, slip —-1oR 4/6 red; internal—slip-10R 4/6 red

Colour of break: 1OYR 7/3 very pale brown

Decoration: on external surface; incised-six grooves below rim

Cat. $15^{2}$

Bowl

Inv. no: $\mathrm{Z14} / 26$

Provenance: Tumulus 14, chamber 2, western part

Construction: wheel-made (2)

State of preservation: incomplete, $95 \%$ of rim, body sherd missing

Measurements: $\mathrm{Dr}=11.6 \mathrm{~cm} ; \mathrm{H}=7.4 \mathrm{~cm}$

Fabric: Lower Nubian?

Hardness: 4

Firing: oxidized

Surface treatment: slipped and polished on both surfaces

Surface colour: slip-10R 4/6 red

Colour of break: $5^{\text {Y R } 6 / 4 ~ l i g h t ~ r e d d i s h ~ b r o w n ~}$

Decoration: on external surface; incised-three grooves below rim and two grooves on lower part of body

Cat. 153

Bowl

Inv. no: $\mathrm{Z14} / 28$

Provenance: Tumulus 14, chamber 2, southern part

Construction: wheel-made (2)

State of preservation: incomplete, body-sherd fragment missing

Measurements: $\mathrm{Dr}=12.5 \mathrm{~cm} ; \mathrm{H}=8.3 \mathrm{~cm}$

Fabric: Lower Nubian?

Hardness: 4

Firing: oxidized

Surface treatment: slipped on both surfaces

Surface colour: external, slip_-10R 5/6 red; internal, slip10R 4/6 red

Colour of break: 7.5YR 7/4 pink

Decoration: on external surface; incised-six grooves on body 


\section{Bowls Type II.6}

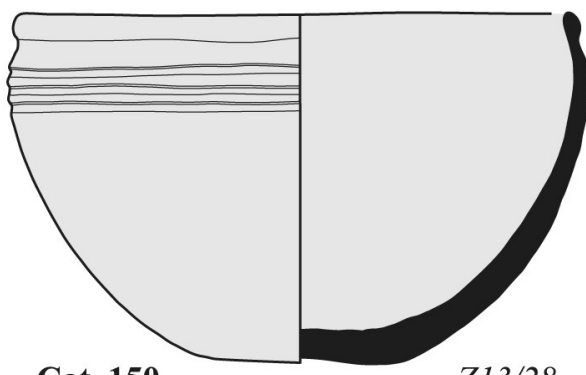

Cat. 150

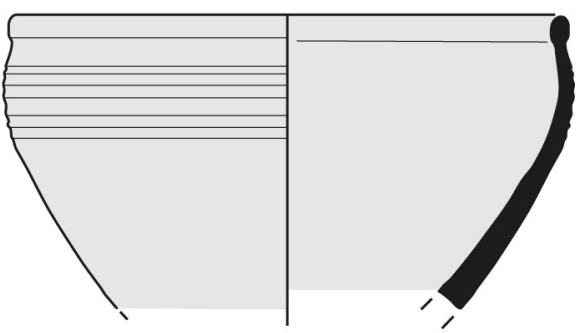

Cat. 151
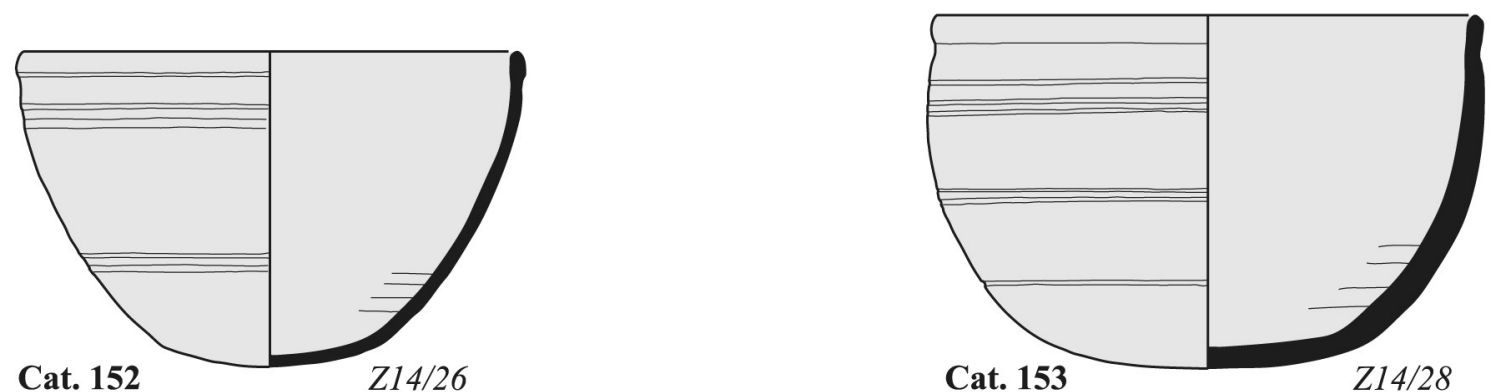

0 $5 \mathrm{~cm}$

FIGURE 7.30 Bowls of type II.6 with modelled rims and grooved decoration

\subsection{Bowls Type II.7}

Variant II.7a

\section{Cat. 154}

Bowl

Inv. no: $\mathbf{Z 1 1} / 3^{\circ}$

Provenance: Tumulus 11, chamber 2, central part

Construction: wheel-made (1)

State of preservation: incomplete, rim and fragment missing, $90 \%$ of rim

Measurements: $\mathrm{Dr}=12 \mathrm{~cm} ; \mathrm{H}=8.4 \mathrm{~cm}$

Fabric: ZF3

Hardness: 3

Firing: oxidized/reduced

Surface treatment: slipped on both surfaces

Surface colour: external, slip-2.5YR 5/6 red; internal, slip-10YR 8/1 white

Colour of break: 5 YR 3/2 dark reddish brown

References: (Klimaszewska-Drabot and Czyżewska 2012, 368-372; Mahmoud El-Tayeb and Czyżewska 2011, $115^{-117)}$

Variant II.7b

Cat. 155

Bowl

Inv. no: $\mathrm{Z11} / 34$
[Fig. 7.101] Provenance: Tumulus 11, chamber 1, southern part Construction: wheel-made (1) State of preservation: incomplete, $93 \%$ of rim Measurements: $\mathrm{Dr}=18.5 \mathrm{~cm} ; \mathrm{H}=13 \mathrm{~cm}$

Fabric: $\mathrm{ZF} 3$

Hardness: 3

Firing: oxidized

Surface treatment: slipped on both surfaces

Surface colour: external, slip-10R 4/6 red; internal, slip5 YR 8/2 pinkish white

Colour of break: 2.5 YR 3/6 dark red

Cat. 156

Bowl

Inv. no: Z6/12.1

Provenance: Tumulus 12, tunnel, blocking wall, central part near $\mathrm{N}$ wall

Construction: wheel-made (1)

State of preservation: fragmentary, restored from sherds, rim and wall fragments missing, $34 \%$ of rim, complete base

Measurements: $\mathrm{Dr}=2 \mathrm{O} \mathrm{cm} ; \mathrm{H}=12.6 \mathrm{~cm}$

Fabric: $\mathrm{ZF} 3$

Hardness: 4

Firing: oxidized 


\section{Bowls Type II.7}

variant II.7a

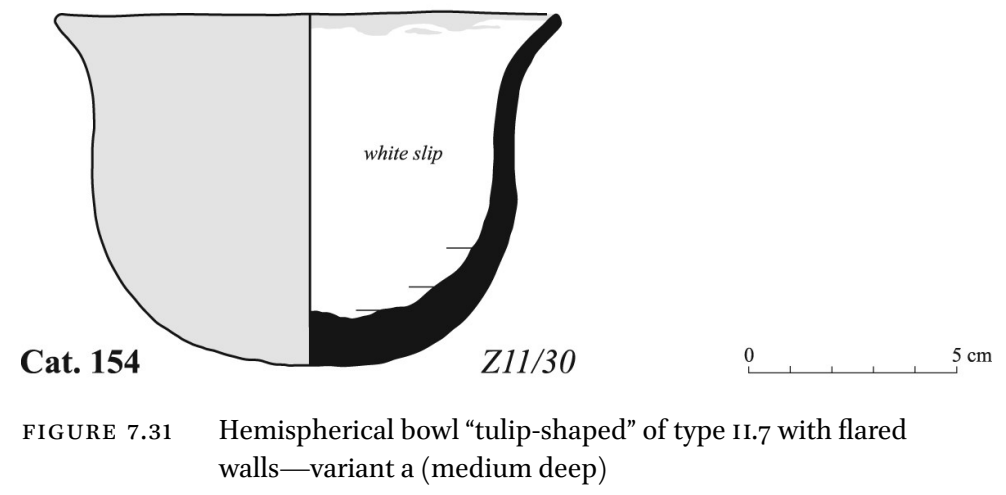

Surface treatment: slipped on both surfaces

Surface colour: external, slip- $7.5 \mathrm{R} 5 / 8$ red; internal7.5 Y $6 / 6$ reddish yellow, slip- 7.5 Y R $8 / 1$ white

Colour of break: 5 Y $6 / 8$ reddish yellow

\section{Cat. 157}

Bowl

Inv. no: Z6/12.2

Provenance: Tumulus 12, tunnel, blocking wall, central part near $\mathrm{N}$ wall

Construction: wheel-made (1)

State of preservation: incomplete, restored from sherds, rim and wall fragments missing, $62 \%$ of rim, complete base

Measurements: $\mathrm{Dr}=29 \mathrm{~cm} ; \mathrm{H}=19 \mathrm{~cm}$

Fabric: $\mathrm{ZF} 3$

Hardness: 3

Firing: reduced

Surface treatment: slipped on both surfaces

Surface colour: external-7.5YR 5/6 strong brown, slip$10 \mathrm{R} 5 / 8$ red; internal, slip- 7.5 YR $8 / 2$ pinkish white

Colour of break: 7.5 Y R $6 / 6$ reddish yellow

\subsection{Bowls Type II.8}

Variant II.8a

[Fig. 7.102]

Cat. 158

Doka bowl

Inv. no: $\mathbf{Z 6} / \mathbf{3} \cdot 3+\mathrm{Z} 28.1+Z_{35 \cdot 4}+Z_{39}+Z_{41.2}+Z_{5} 6+Z_{5} 8.2$

Provenance: Tumulus 6, fragments of vessel scattered inside tunnel

Construction: handmade (1) and (2)

State of preservation: fragmentary, $42 \%$ of rim, base missing

Measurements: $\mathrm{Dr}=54 \mathrm{~cm} ;{ }_{\mathrm{p}} \mathrm{H}=13.4 \mathrm{~cm}$

Fabric: ZF1O
Hardness: 3

Firing: reduced

Surface treatment: uncoated on both surfaces

Surface colour: external-2.5YR 6/4 light yellow-brown; internal-2.5YR 6/4 light yellow brown

Colour of break: Gley1 2.5/N black

Notes: mat impression barely visible on external surface; sherds of this vessel were used as digging tools

\section{Cat. 159}

Bowl

Inv. no: $\mathbf{Z 6 / 2 2 . 1}$

Provenance: Tumulus 6, fill of tunnel

Construction: handmade (1) and/or (2)

State of preservation: fragmentary, $20 \%$ of rim

Measurements: $\mathrm{Dr}=5^{2} \mathrm{~cm} ;{ }_{\mathrm{p}} \mathrm{H}=8 \mathrm{~cm}$

Fabric: $\mathrm{ZF10}$

Hardness: $2 / 3$

Firing: reduced

Surface treatment: slipped on internal surface

Surface colour: external-7.5R 4/4 weak red; internal, slip- $7 . \mathrm{S}^{\mathrm{R}} 5 / 4$ weak red

Colour of break: $5 \mathrm{Y} 2.5 / 1$ black

\section{Cat. 16o}

Bowl

Inv. no: $\mathbf{Z}_{\mathbf{4}} / \mathbf{1 . 3}+\mathbf{Z}_{\mathbf{4}} / \mathbf{4}$

Provenance: Tumulus 4, tunnel, ahead of entrance

Construction: handmade (1) and/or (2)

State of preservation: fragmentary, $30 \%$ of rim

Measurements: $\mathrm{Dr}=57 \mathrm{~cm} ; \mathrm{p} H=6.7 \mathrm{~cm}$

Fabric: ZF9

Hardness: 3

Firing: reduced

Surface treatment: slipped on both surfaces 
variant II.7b
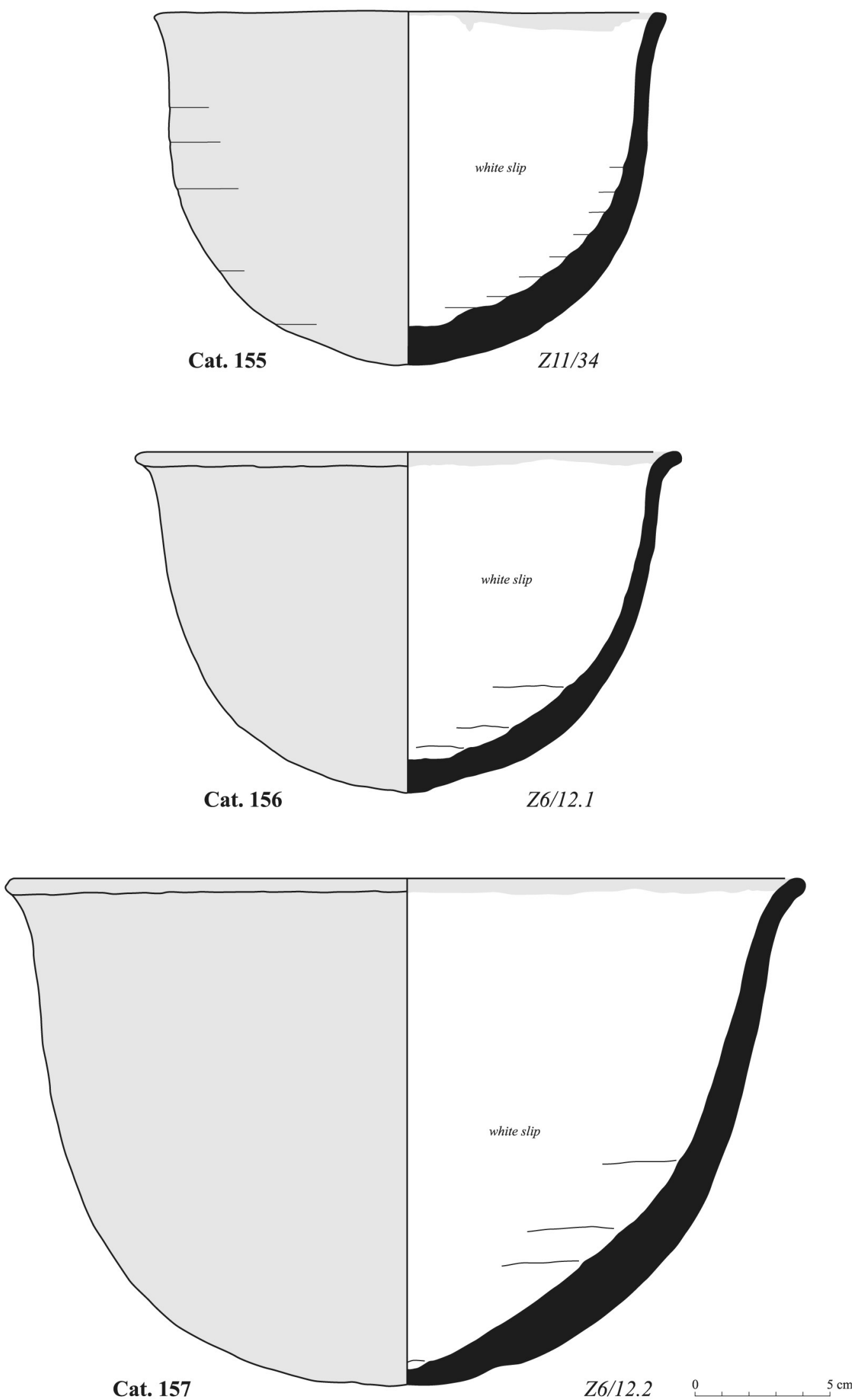

FIGURE 7.32 Hemispherical bowl "tulip-shaped" of type II.7 with flared walls—variant b (deep) 


\section{Bowls Type II.8}

variant II.8a

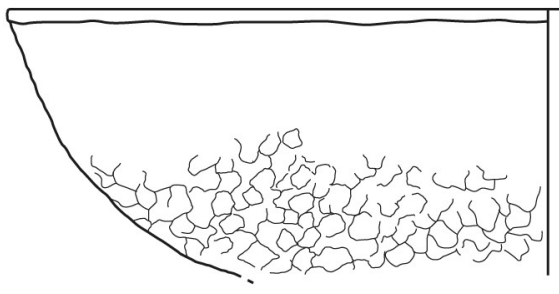

Cat. 158

Cat. 159

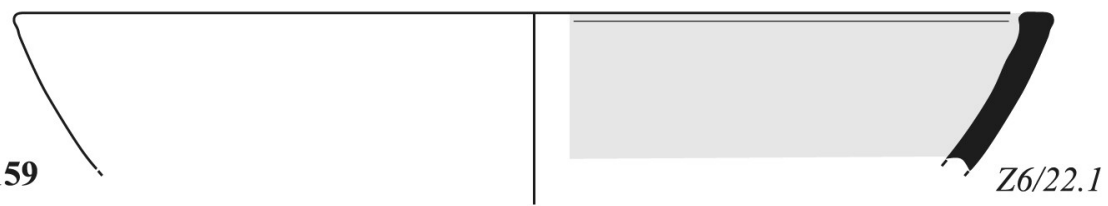

Cat. 160

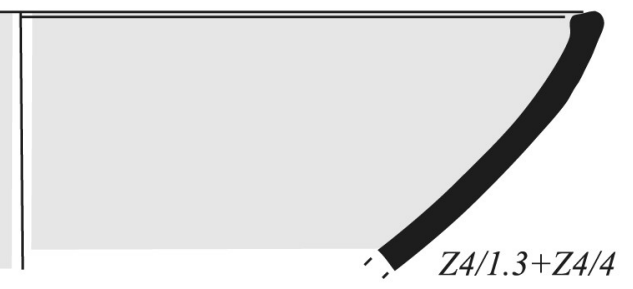

Cat. 161

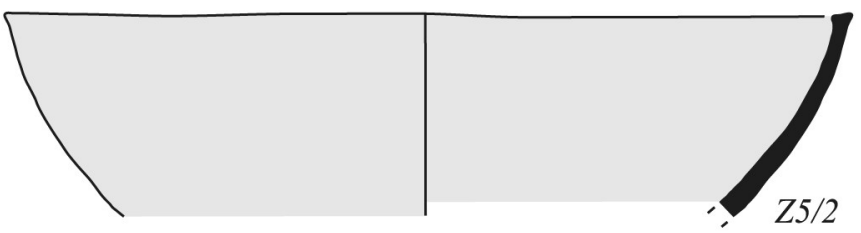

Cat. 162
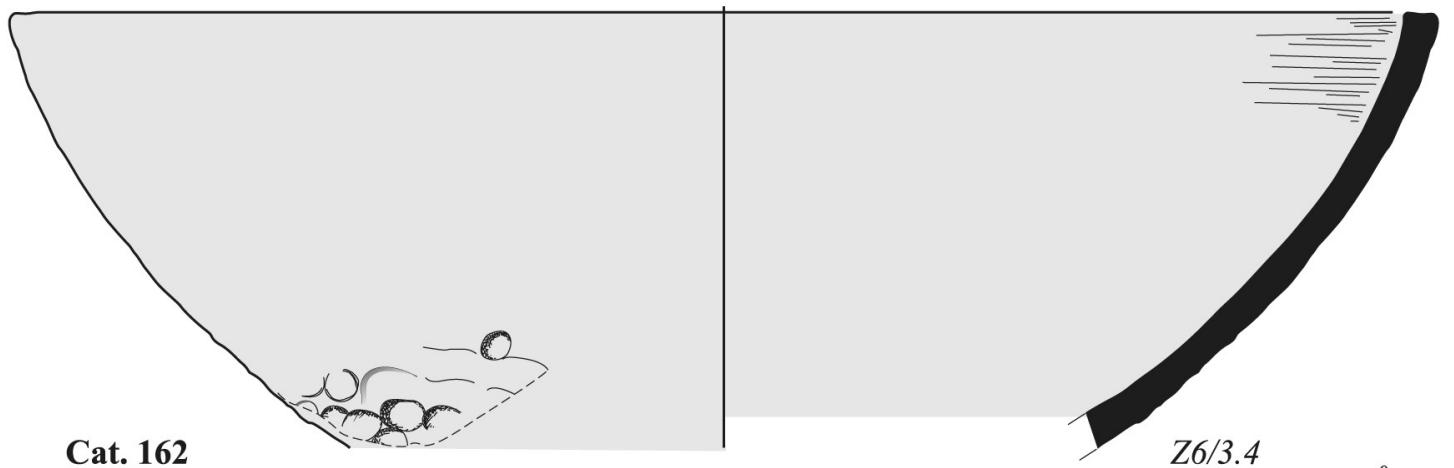

$Z 6 / 3.4$

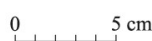

FIGURE 7.33 Large, shallow bowls of type II.8, variant a 


\section{variant II.8b}

Cat. 163

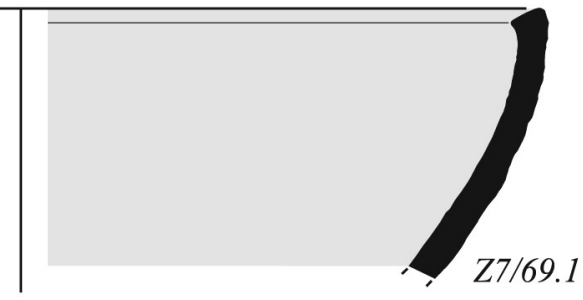

Cat. 164

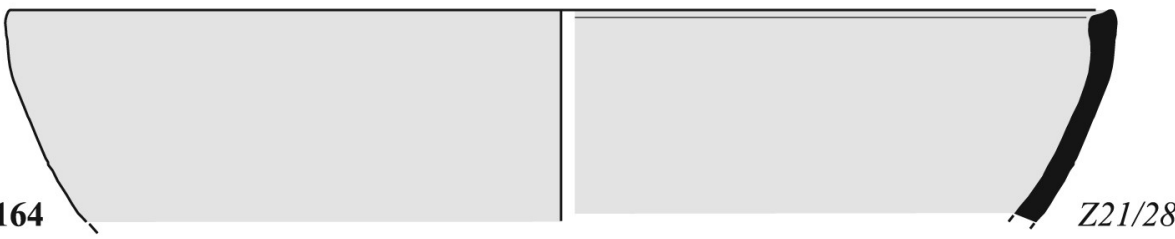

Cat. 165

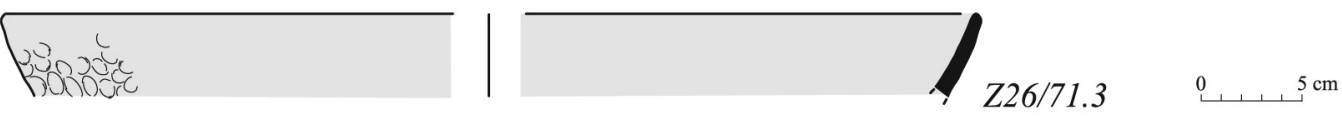

FIGURE 7.34 Large, shallow bowls of type II.8, variant b

Surface colour: external natural surface-1oR 5/4 weak red, slip $-7.5^{\mathrm{R}} 5 / 6$ red; internal natural surface2.5 YR $5 / 4$ reddish brown, slip $-7.5 \mathrm{R}$ 5/6 red

Colour of break: Gley1 2.5/N black

Notes: sherds of this vessel were used as digging tools

\section{Cat. 161}

Bowl

Inv. no: $\mathrm{Z}_{5} / \mathbf{2}$

Provenance: Tumulus 5, superstructure

Construction: handmade (1) and/or (2)

State of preservation: fragmentary, $6 \%$ of rim

Measurements: $\mathrm{Dr}=42 \mathrm{~cm} ;{ }_{\mathrm{p}} \mathrm{H}=10 \mathrm{~cm}$

Fabric: ZF10

Hardness: 3

Firing: reduced

Surface treatment: slipped on both surfaces

Surface colour: external natural surface-2.5YR 6/6 light red, slip-10R 5/6 red; internal natural surface2.5YR 6/6 light red, slip-10R 5/6 red

Colour of break: $2.5 \mathrm{Y} 4 / 1$ dark grey

Notes: sherds of this vessel were used as digging tools

Cat. 162

Bowl

Inv. no: $\mathrm{Z6} / 3 \cdot 4$

Provenance: Tumulus 6, E Tunnel

Construction: handmade (1) and/or (2)

State of preservation: fragmentary, $6 \%$ of rim
Measurements: $\mathrm{Dr}=70 \mathrm{~cm} ;{ }_{\mathrm{p}} \mathrm{H}=21.6 \mathrm{~cm}$

Fabric: ZF10

Hardness: 3

Firing: reduced

Surface treatment: slipped on both surfaces

Surface colour: external, slip-10R 4/6 red; internal, slip10R $5 / 6$ red

Colour of break: $5 \mathrm{Y} 4 / 2$ olive grey

Notes: sherds of this vessel were used as digging tools

Variant II.8b

Cat. 163

Bowl

Inv. $n o: \mathrm{Z}_{7} / \mathbf{6 9 . 1}$

Provenance: Tumulus 7, W tunnel

Construction: handmade (1) and/or (2)

State of preservation: fragmentary, $8 \%$ of rim

Measurements: $\mathrm{Dr}=c .43 \mathrm{~cm} ;{ }_{\mathrm{p}} \mathrm{H}=13.6 \mathrm{~cm}$

Fabric: ZF1O

Hardness: 3

Firing: reduced

Surface treatment: slipped on internal surface

Surface colour: external-7.5YR 5/4 brown; internal natural surface -7.5 YR $5 / 4$ brown, slip-10R 4/6 red

Colour of break: deep black

Notes: sherds of this vessel were used as digging tools 
Cat. 164

Bowl

Inv. no: $\mathbf{Z 2 1} / \mathbf{2 8}$

Provenance: Tumulus 21, superstructure

Construction: handmade (1) and/or (2)

State of preservation: fragmentary, $6 \%$ of rim

Measurements: $\mathrm{Dr}=55 \mathrm{~cm} ;{ }_{\mathrm{p}} \mathrm{H}=10.6 \mathrm{~cm}$

Fabric: ZF10

Hardness: 3

Firing: reduced

Surface treatment: slipped on both surfaces

Surface colour: external natural surface-7.5YR 4/3 brown, slip-10R 4/4 weak red; internal natural surface7.5YR 3/1 very dark grey, slip-10R 4/4 weak red

Colour of break: 7.5YR 3/2 dark brown

Cat. 165

Bowl

Inv. no: $\mathrm{Z26} / 71.3$

Provenance: Tumulus 26, fill of shaft (o.3 m below surface level)

Construction: handmade (1) and/or (2)

State of preservation: fragmentary, rim fragment

Measurements: $\mathrm{Dr}=$ ? cm; $\mathrm{p}=4 \mathrm{~cm}$

Fabric: ZF9

Hardness: 3

Firing: reduced

Surface treatment: slipped on both surfaces

Surface colour: slip-10R 4/6 red on both surfaces

Colour of break: 5 Y 2.5/1 black

\subsection{Bowls Type II.9}

Variant II.9a

Cat. 166

Bowl

Inv. no: $\mathbf{Z} \mathbf{2 4} / \mathbf{1 1}+\mathrm{Z}_{24} / 10$

Provenance: Tumulus 24, shaft

Construction: handmade (1) and (2)

State of preservation: fragmentary, 30\% of rim

Measurements: $\mathrm{Dr}=c .70 \mathrm{~cm} ;{ }_{\mathrm{p}} \mathrm{H}=27.4 \mathrm{~cm}$

Fabric: ZF9

Hardness: 3

Firing: reduced

Surface treatment: slipped on both surfaces

Surface colour: external, slip $-5^{\mathrm{R}} 5 / 4$ weak red; internal, slip-10R $5 / 6$ red

Colour of break: $5 \mathrm{Y}$ 5/1 grey

Notes: sherds of this vessel were used as digging tools
Cat. 167

Bowl

Inv. no: Z11/16

Provenance: Tumulus 11, chamber 2, northern part

Construction: handmade (1) and (2)

State of preservation: incomplete, restored from sherds, rim and base complete

Measurements: $\mathrm{Dr}=46.4 \mathrm{~cm} ; \mathrm{H}=25.2 \mathrm{~cm}$

Fabric: ZF1O

Hardness: 3

Firing: reduced

Surface treatment: slipped on both surfaces

Surface colour: external natural surface-7.5YR 6/4 light brown, slip-10R 5/6 red; internal natural surface$7 \cdot 5^{\mathrm{R}} 6 / 4$ pale red, slip-10R $5 / 6 \mathrm{red}$

Colour of break: deep black

Decoration: incised-strokes on rim

Notes: evidence of repairs to vessel-holes drilled (after firing) in vessel walls

References: (Klimaszewska-Drabot and Czyżewska 2012, 368-372; Mahmoud El-Tayeb and Czyżewska 2o11, $115^{-117)}$

Cat. 168

Bowl

Inv. no: $\mathbf{Z} 1 / 21+Z 1 / 22$

Provenance: Tumulus 1, fill of shaft

Construction: handmade (1) and/or (2)

State of preservation: fragmentary, $7 \%$ of rim

Measurements: $\mathrm{Dr}=59 \mathrm{~cm} ;{ }_{\mathrm{p}} \mathrm{H}=24 \mathrm{~cm}$

Fabric: ZF9

[Fig. 7.103] Hardness: 3

Firing: reduced

Surface treatment: slipped on both surfaces

Surface colour: slip-10R 4/6 red on both surfaces

Colour of break: deep black

Cat. 169

Bowl

Inv. no: $\mathrm{Z}_{7} / 5 \mathrm{O}+\mathrm{Z}_{7} / 69$

Provenance: Tumulus $7, \mathrm{~W}$ tunnel

Construction: handmade (1) and/or (2)

State of preservation: fragmentary, $27 \%$ of rim

Measurements: $\mathrm{Dr}=c .46 \mathrm{~cm} ;{ }_{\mathrm{p}} \mathrm{H}=23.4 \mathrm{~cm}$

Fabric: ZF10

Hardness: 3

Firing: reduced

Surface treatment: slipped on both surfaces

Surface colour: external natural surface $-5 \mathrm{YR} 5 / 3$ reddish brown, slip-10R 4/6 red; internal natural surface5YR 5/4 reddish brown, slip-10R 4/4 weak red 


\section{Bowls Type II.9}

variant II.9a
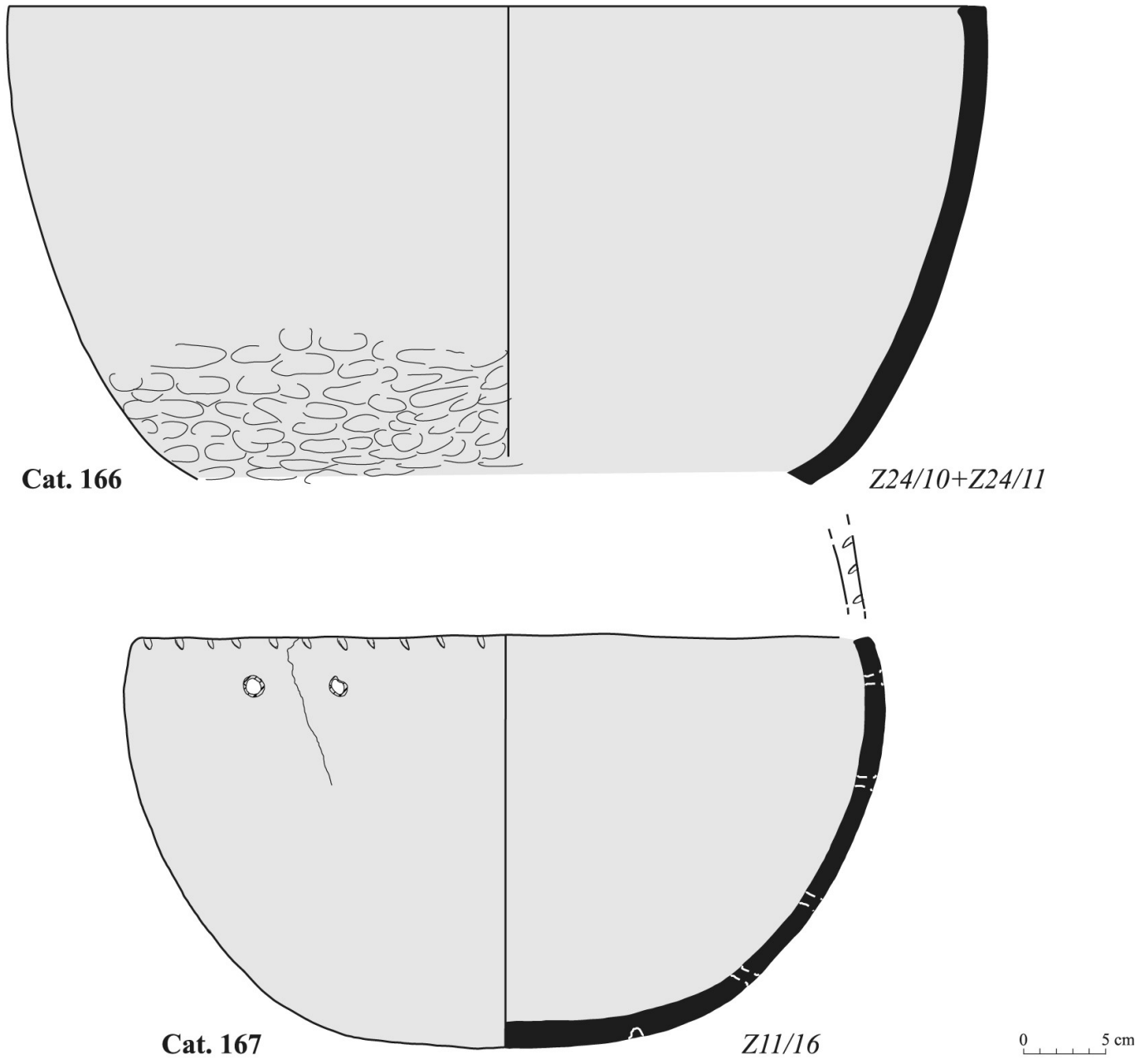

FIGURE 7.35 Large, deep bowls of type II.9, variant a

Colour of break: Gley1 2.5/N black

Decoration: incised-strokes on rim

Notes: evidence of repairs to vessel-holes drilled (after firing) in vessel walls

\subsection{Bowls Varia}

Cat. 170

Bowl

Inv. no: $\mathbf{Z 1 4} / 27$

Provenance: Tumulus 14, chamber 2, southern part

Construction: wheel-made (2)

State of preservation: complete

Measurements: $\mathrm{Dr}=12 \mathrm{~cm} ; \mathrm{H}=7.5 \mathrm{~cm}$

Fabric: not possible to determine

Hardness: 4

Firing: oxidized

Surface treatment: slipped on both surfaces
Surface colour: slip-10R 4/6 red, on both surfaces Colour of break: not possible to determine

Decoration: on external surface; incised-nine grooves on body

[Figs 7.104-7.105] Cat. 171

Bowl

Inv. no: $\mathbf{Z 2} / \mathbf{2 9}$

Provenance: Tumulus 2, chamber 2

Construction: wheel-made (2)

State of preservation: complete

Measurements: $\mathrm{Dr}=11.5 \mathrm{~cm} ; \mathrm{H}=8.5 \mathrm{~cm}$

Fabric: not possible to determine

Hardness: $3 / 4$

Firing: oxidized

Surface treatment: slipped and polished on both surfaces Surface colour: external, slip_-10R 4/8 red; internal, slip$10 \mathrm{R}_{4} / 6 \mathrm{red}$ 
variant II.9b
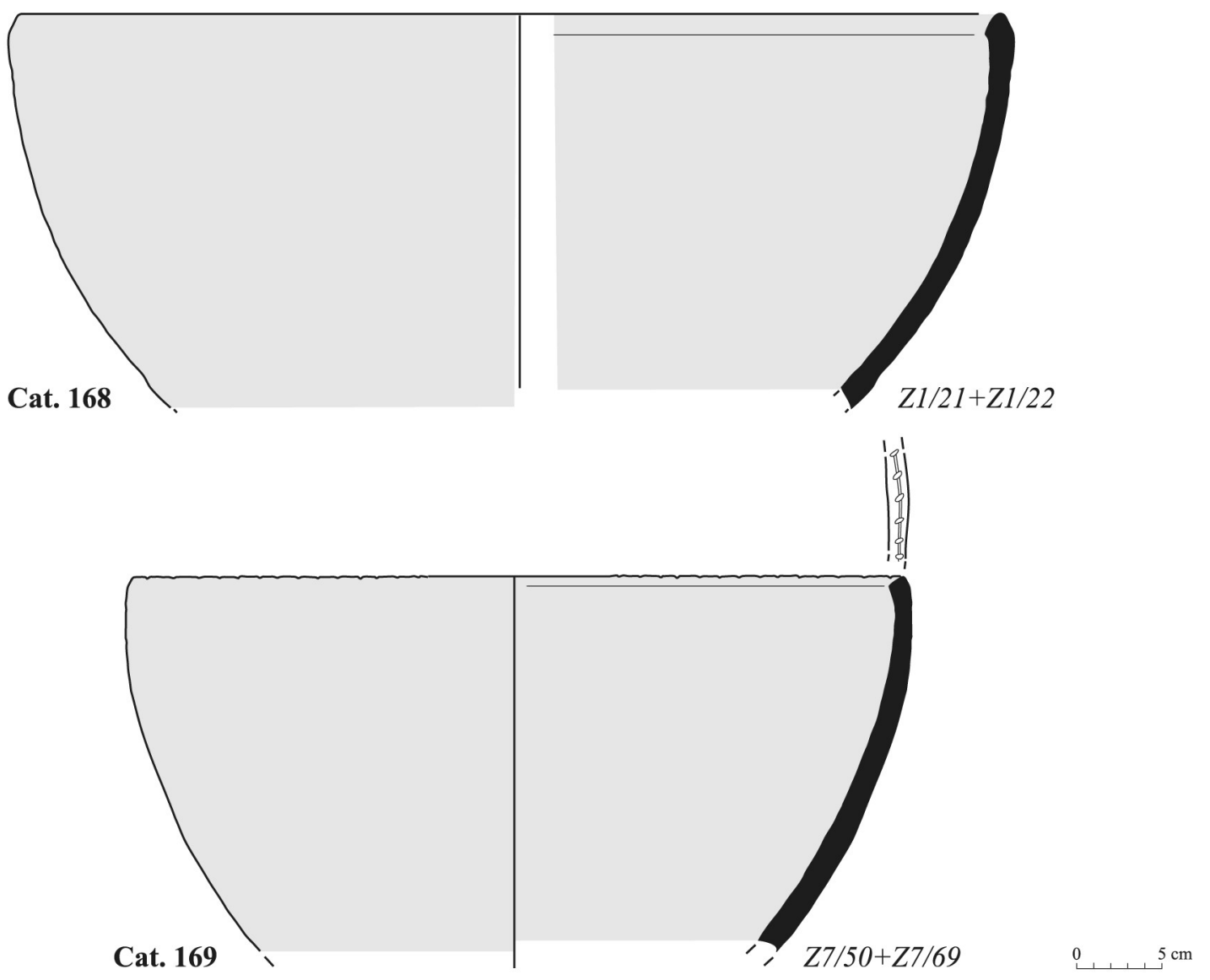

FIGURE 7.36 Large, deep bowls of type II.9, variant b

Colour of break: not possible to determine

Decoration: incised-two grooves below rim and two on lower part of vessel; painted-three vertical stripes composed of a white background with black outlines and filled with a zigzag design painted in black

References: (Klimaszewska-Drabot and Mahmoud ElTayeb 2014, 10-13)

\section{Cat. 172}

Bowl

Inv. no: $\mathrm{Z} 23 / 4$

Provenance: Tumulus 23, chamber 1

Construction: wheel-made (2)

State of preservation: complete

Measurements: $\mathrm{Dr}=10 \mathrm{~cm} ; \mathrm{H}=7.6 \mathrm{~cm}$

Fabric: not possible to determine

Hardness: $3 / 4$

Firing: not possible to determine

Surface treatment: slipped and polished on both surfaces Surface colour: slip_-1oR 4/8 red on both surface Colour of break: not possible to determine

Decoration: incised-two grooves below rim and two on lower part of vessel forming border for painted deco- ration; painted-below rim, one white band as background for black circles with dots; lower part of vessel, one white band as background for black crossed lines References: (Klimaszewska-Drabot and Mahmoud ElTayeb 2014, 10-13)

\section{Cat. 173}

Bowl

Inv. no: $\mathrm{Z}_{\mathbf{3}} / 7.2$

Provenance: Tumulus 3, fill of external shaft

Construction: wheel made (2)

State of preservation: fragmentary, $6 \%$ of rim

Measurements: $\mathrm{Dr}=10 \mathrm{~cm},{ }_{\mathrm{p}} \mathrm{H}=2.7 \mathrm{~cm}$

Fabric: Lower Nubian?

Hardness: $3 / 4$

Firing: oxidized

Surface treatment: slipped and polished on both surfaces Surface colour: external, slip-7.5YR 9/2 pale yellowish pink; internal, slip-7.5YR 9/1 white

Colour of break: $7.5 \mathrm{R}$ 2.5/1 reddish black

Decoration: painted brown bands ( 5 YR 3/2 dark reddish brown) on top of rim and on external surface 
Bowls Varia

(wheel-made)

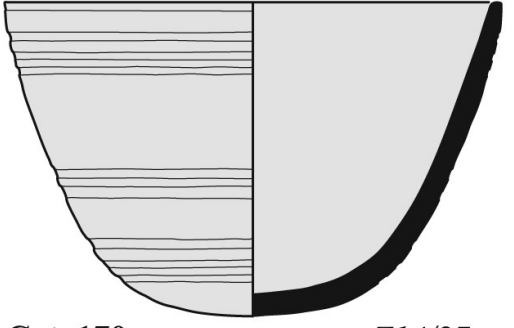

Cat. 170
Z14/27

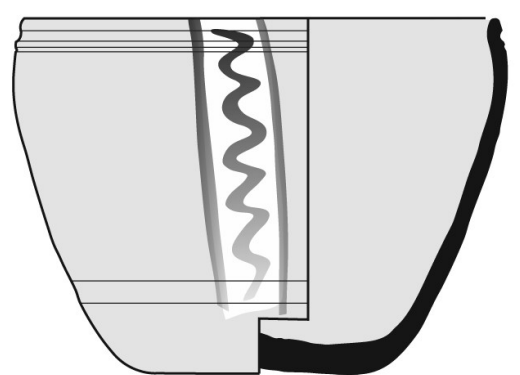

Cat. 171

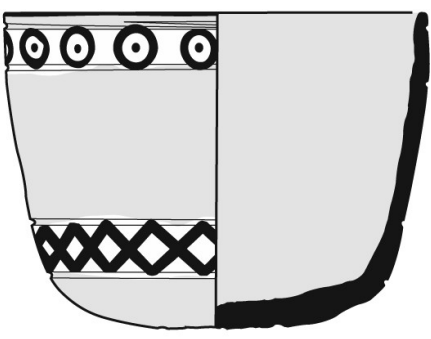

Cat. 172

$Z 23 / 4$

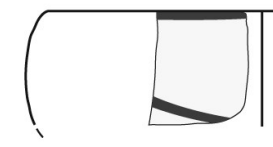

Cat. 173

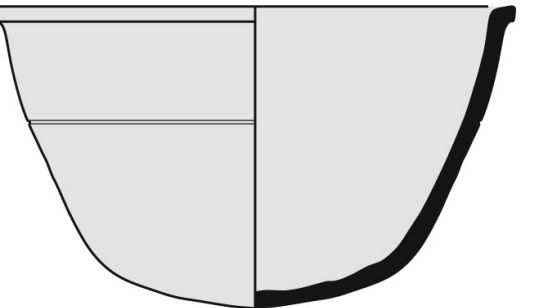

Cat. 175

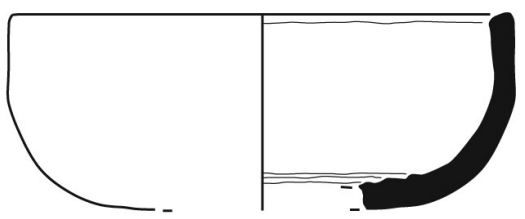

Cat. 177
Z4/219

Z12/84

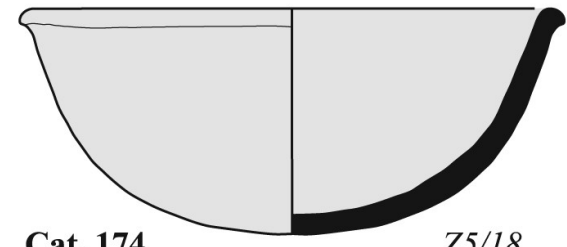

Cat. 174

Z5/18

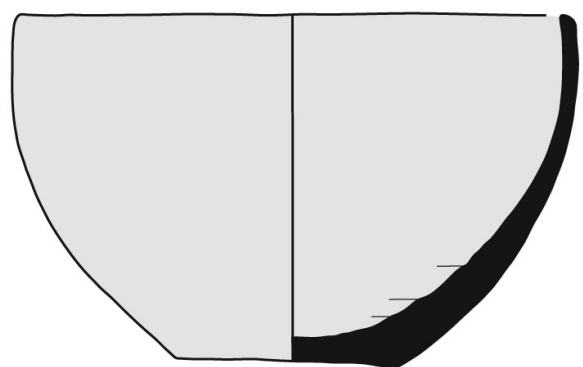

Cat. 176

Z11/21 $5 \mathrm{~cm}$

FIGURE 7.37 Bowls of different types

Cat. 174

Bowl

Inv. no: $\mathrm{Z}_{5} / \mathbf{1 8}$

Provenance: Tumulus 5, tunnel fill

Construction: wheel-made (1)

State of preservation: fragmentary, $15 \%$ of rim

Measurements: $\mathrm{Dr}=13 \mathrm{~cm} ; \mathrm{H}=5.5 \mathrm{~cm}$

Fabric: ZF6

Hardness: 4

Firing: reduced

Surface treatment: slipped on both surfaces

Surface colour: external natural surface-5YR 5/4 reddish brown; slip-10R $4 / 6$ red, on both surfaces

Colour of break: 7.5YR 4/1 dark grey
Cat. 175

Bowl

Inv. no: $\mathrm{Z}_{4 / 219}$

Provenance: Tumulus 4, E tunnel

Construction: wheel-made (2)

State of preservation: incomplete, $15 \%$ of rim, body sherd missing

Measurements: $\mathrm{Dr}=12.6 \mathrm{~cm} ; \mathrm{H}=7.3 \mathrm{~cm}$

Fabric: $\mathrm{ZF} 3$ ?

Hardness: 4

Firing: oxidized

Surface treatment: slipped and polished on both surfaces Surface colour: slip_-10R 4/6 red, on both surfaces 
Colour of break: 7.5YR 7/3 pink

Decoration: incised-one groove at mid-H

Cat. 176

Bowl

Inv. no: Z11/21

Provenance: Tumulus 11, chamber 2

Construction: wheel-made (1)

State of preservation: almost complete, $97 \%$ of rim, lip chipped off

Measurements: $\mathrm{Dr}=13.3^{-14.3} \mathrm{~cm} ; \mathrm{H}=8.5 \mathrm{~cm}$

Fabric: $\mathrm{ZF} 3$

Hardness: 4

Firing: oxidized

Surface treatment: slipped and burnished on both surfaces, burnish 2 on both surfaces, carefully executed

Surface colour: slip-1oR 4/6 red, on both surfaces

Colour of break: 5YR 4/6 yellowish red

References: (Klimaszewska-Drabot and Czyżewska 2012, 368-372)

Cat. 177

Bowl

Inv. no: $\mathbf{Z 1 2} / 84$

Provenance: Tumulus 12, fill of shaft

Construction: wheel-made (1)

State of preservation: fragmentary, $21 \%$ of rim

Measurements: $\mathrm{Dr}=12 \mathrm{~cm} ;{ }_{\mathrm{p}} \mathrm{H}=4 \mathrm{~cm}$

Fabric: ZF4

Hardness: 4

Firing: oxidized

Surface treatment: slipped and polished on both surfaces

Surface colour: slip-10R 4/6 red, on both surfaces

Colour of break: 5YR 5/4 reddish brown

Cat. 178

Bowl

Inv. no: $\mathrm{Z}_{5} / \mathbf{1 7}$

Provenance: Tumulus 5, sondage on S side of tumuli

Construction: wheel-made (2)

State of preservation: fragmentary, restored from sherds, $60 \%$ of rim, $27 \%$ of base, complete profile

Measurements: $\mathrm{Dr}=11 \mathrm{~cm} ; \mathrm{H}=7 \mathrm{~cm}$

Fabric: undefined

Hardness: $3 / 4$

Firing: oxidized

Surface treatment: slipped on both surfaces

Surface colour: slip-10R 4/6 red on both surfaces

Colour of break: 7.5YR 4/4 dark brown

Decoration: painted-dark dots (5YR 3/2 dark reddish brown) on top of rim and on external surface

\section{Cat. 179}

Bowl

Inv. no: $\mathrm{Z}_{5} / \mathbf{2 1}$

Provenance: Tumulus 5, tunnel fill

Construction: wheel-made (2)

State of preservation: fragmentary, restored from sherds, $27 \%$ of rim, rim and body fragment

Measurements: $\mathrm{Dr}=20 \mathrm{~cm} ;{ }_{\mathrm{p}} \mathrm{H}=12.8 \mathrm{~cm}$

Fabric: undefined

Hardness: 4

Firing: oxidized

Surface treatment: slipped on both surfaces

Surface colour: external, slip-10R 4/8 red; internal, slip1oR $5 / 6$ red

Colour of break: 1OYR 3/1 very dark grey

Decoration: painted — dark band (10R 2.5/2 very dusky) on top of rim

\section{Cat. 180}

Bowl

Inv. no: $\mathrm{Z6/2.1}+\mathrm{Z6} / 3 \cdot 5+\mathrm{Z6} / 55$

Provenance: Tumulus 6, sherds scattered in fill of external shaft and E tunnel

Construction: wheel-made (2)

State of preservation: fragmentary, restored from sherds, $39 \%$ of rim, rim and body fragments

Measurements: $\mathrm{Dr}=16.7 \mathrm{~cm} ;{ }_{\mathrm{p}} \mathrm{H}=9 \mathrm{~cm}$

Fabric: Egypt?

Hardness: $3 / 4$

Firing: oxidized

Surface treatment: slipped on both surfaces

Surface colour: external natural surface- 5 YR 4/4 reddish brown, slip-10R 4/8 red; internal natural surface5YR 4/4 reddish brown, slip- $5 \mathrm{R} 4 / 8$ red

Colour of break: 5YR 5/3 reddish brown

Cat. 181

Bowl

Inv. no: $\mathrm{Z}_{5} / 35$

Provenance: Tumulus 5, fill of superstructure

Construction: wheel-made (2)

State of preservation: fragmentary, $11 \%$ of rim

Measurements: $\mathrm{Dr}=20 \mathrm{~cm} ;{ }_{\mathrm{p}} \mathrm{H}=6 \mathrm{~cm}$

Fabric: ZF4

Hardness: 4

Firing: reduced

Surface treatment: slipped and burnished on both surfaces, burnish 1 on both surfaces

Surface colour: external, slip—-10R 4/4 weak red; internal, slip-10R $4 / 6$ red

Colour of break: Gley1 2.5/N black

Decoration: incised decoration on top of rim 


\section{Bowls Varia \\ (wheel-made)}

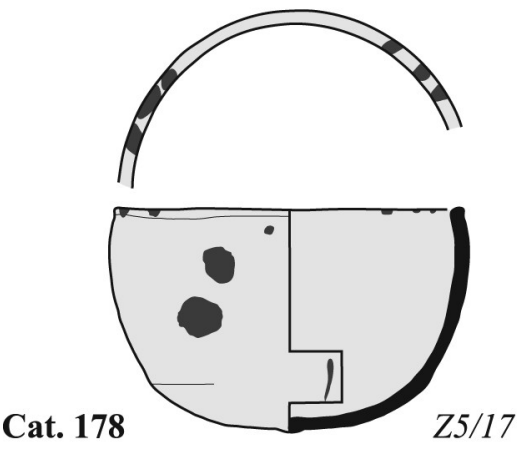

Cat. 178

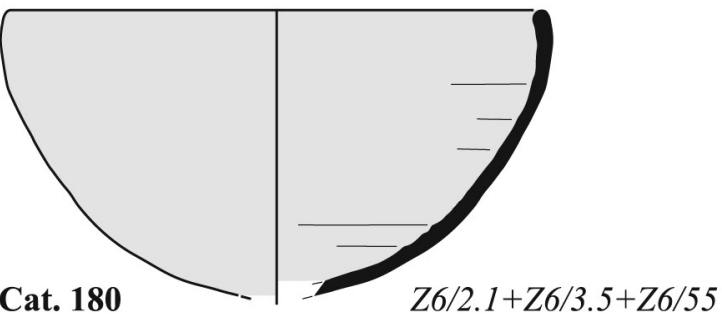

Cat. 180
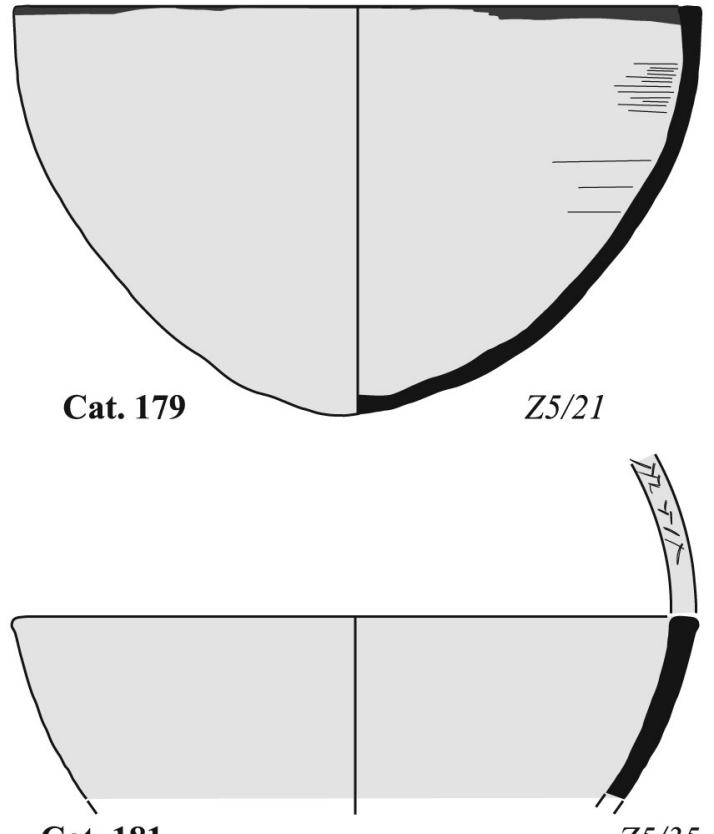

Cat. 181

Firing: reduced

Surface treatment: slipped on both surfaces

Surface colour: slip-10R $5 / 6$ red on both surfaces

Colour of break: 1OYR 3/2 very dark greyish brown

Cat. 184

Bowl

Inv. no: $\mathrm{Z}_{7} / 68$

Provenance: Tumulus 7, W tunnel, close to fifth pillar

Construction: handmade

State of preservation: fragmentary, $42 \%$ of rim

Measurements: $\mathrm{Dr}=20.6 \mathrm{~cm} ;{ }_{\mathrm{p}} \mathrm{H}=13.3 \mathrm{~cm}$

Fabric: ZF9

Hardness: 3

Firing: reduced

Surface treatment: slipped on both surfaces

Surface colour: external natural surface- 5 YR $4 / 4$ reddish brown, slip-1oR 4/6 red; internal, slip-10R 4/6 red

Inv. no: Z14/15

Provenance: Tumulus 14, chamber 1, south-eastern corner

Construction: handmade

State of preservation: incomplete, $83 \%$ of rim, lip chipped off

Colour of break: deep black

Cat. 185

Pot

Inv. no: $\mathbf{Z 2 6} / 87$

Provenance: Tumulus 26, chamber 2, southern part

Construction: handmade

\section{Fabric: $\mathrm{ZF} 3$}

Hardness: 3 


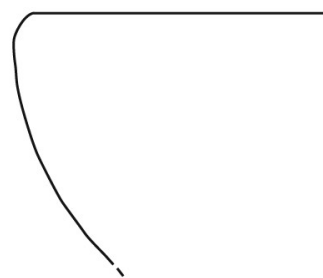

Cat. 182

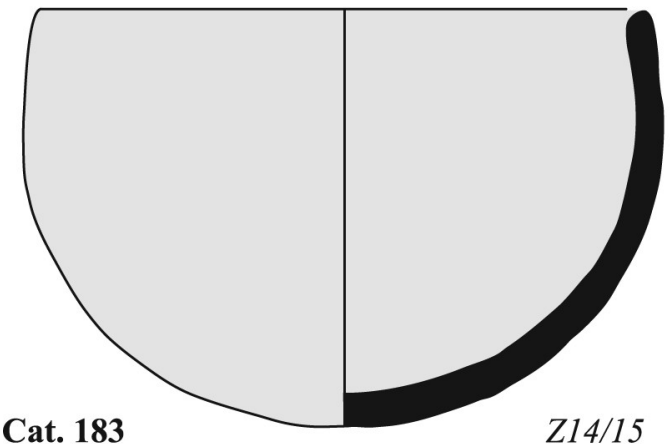

Cat. 184

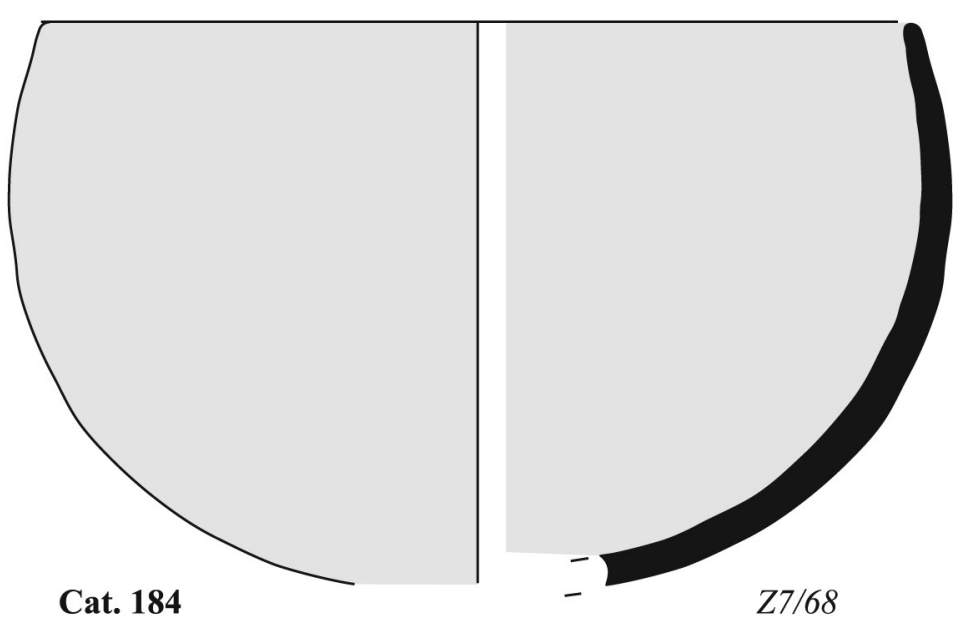

0 $5 \mathrm{~cm}$

FIGURE 7.39 Bowls of different types

State of preservation: fragmentary, $69 \%$ of rim, upper part of vessel

Measurements: $\mathrm{Dr}=20 \mathrm{~cm} ;{ }_{\mathrm{p}} \mathrm{H}=7.5 \mathrm{~cm}$

Fabric: ZF9

Hardness: 3

Firing: reduced

Surface treatment: slipped on both surfaces

Surface colour: external natural surface -5 YR 5/6 yellowish red, slip- $-7.5^{\mathrm{R}} 5 / 8 \mathrm{red}$; internal natural surface$5^{\text {YR }} 5 / 4$ reddish brown, slip $-7.5^{\mathrm{R}} 5 / 8$ red

Colour of break: Gley2 2.5/5P B bluish black

Cat. 186

Pot

Inv. no: $\mathbf{Z 1 0} / 28$

Provenance: Tumulus 10, burial chamber, under vessel $\mathrm{Z1O} / 11$

Construction: handmade

State of preservation: fragmentary, $12 \%$ of rim

Measurements: $\mathrm{Dr}=c .24 \mathrm{~cm} ;{ }_{\mathrm{p}} \mathrm{H}=8.5 \mathrm{~cm}$ ?

Fabric: ZF1O

Hardness: $2 / 3$

Firing: reduced

Surface treatment: uncoated

Surface colour: external-5YR 5/4 reddish brown; internal-1OYR 4/2 dark greyish brown

Colour of break: Gley1 2.5/N black
Cat. 187

Bowl with horizontal handle on rim

Inv. no: $\mathbf{Z 2 6 / 6 5 . 1}$

Provenance: Tumulus 26, shaft

Construction: handmade

State of preservation: fragmentary, $27 \%$ of rim, complete profile

Measurements: $\mathrm{Dr}=43 \mathrm{~cm} ; \mathrm{H}=15.5 \mathrm{~cm}$

Fabric: ZF9

Hardness: 3

Firing: reduced

Surface treatment: slipped and burnished on both surfaces; burnish 3 , badly executed on both surfaces

Surface colour: slip-1oR 4/6 red on both surfaces

Colour of break: deep black

\section{Group III-Spouted Bowls}

4.1 Spouted Bowls Type III.1

Cat. 188

[Fig. 7.106]

Spouted bowl

Inv. no: Z19/4

Provenance: Tumulus 19, burial chamber and bottom of shaft under destroyed blocking wall

Construction: handmade (1)

State of preservation: incomplete, restored from sherds, $90 \%$ of rim, complete base 


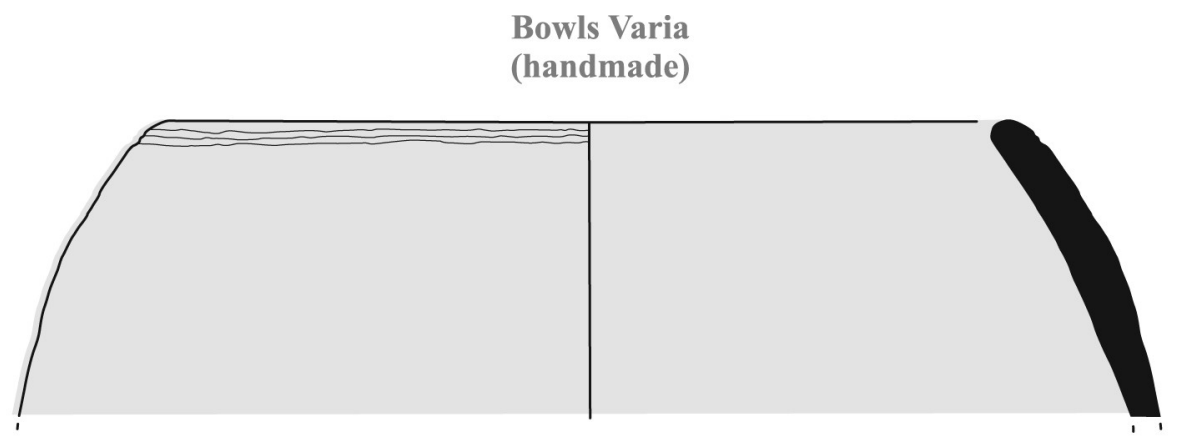

$\begin{array}{ll}\text { Cat. } 185 & Z 26 / 87\end{array}$

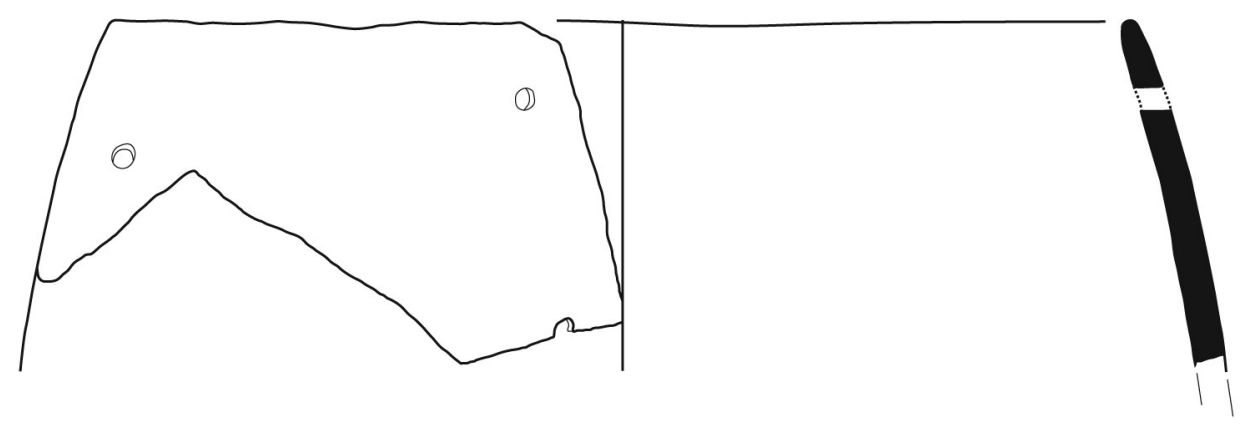

Cat. 186

Z10/28

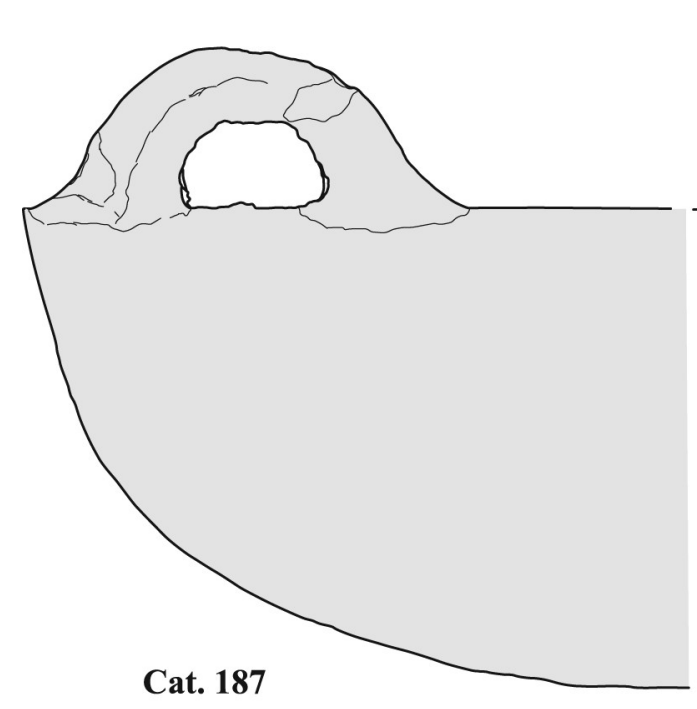

Cat. 187

FIGURE 7.40 Bowls of different types

Measurements: $\mathrm{Dr}=18.2 \mathrm{~cm} ; \mathrm{H}=16.5 \mathrm{~cm}$

Fabric: ZF10

Hardness: 3

Firing: reduced

Surface treatment: slipped on both surfaces

Surface colour: external, slip-10R 4/6 red; internal, slip10R $4 / 6$ red

Colour of break: Gley1 2.5/10Y greyish black

References: (Klimaszewska-Drabot 2010, 482)

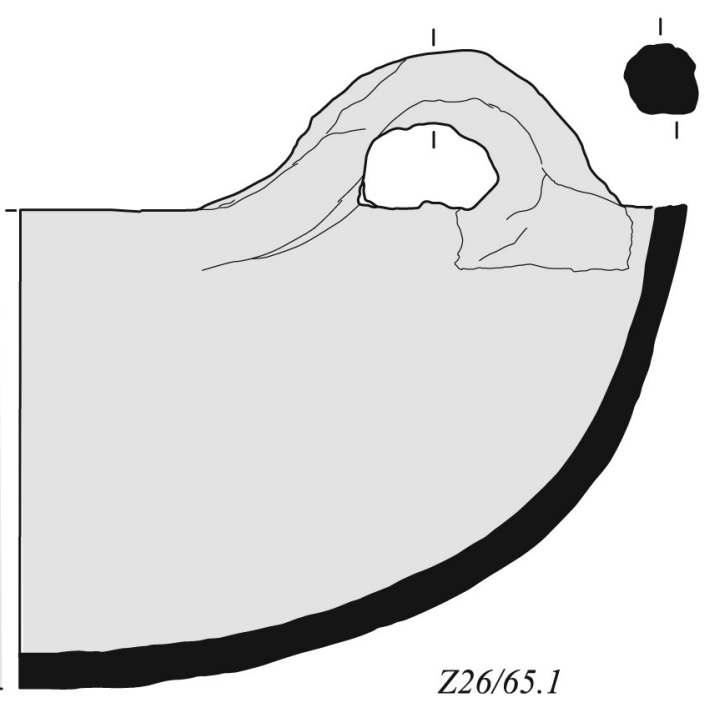

0 $5 \mathrm{~cm}$ 
Hardness: 3

Firing: reduced

Surface treatment: untreated

Surface colour: external—2.5YR 5/6 red; internal— 5YR 5/4 reddish brown

Colour of break: 1OYR 2/1 black

Decoration: incised geometrical decoration below rim and on belly: intertwined lines, horizontal strokes and triangles; red slip used as part of decoration: red slipped triangles combined with incised lines (on belly) and slipped band below rim

\subsection{Spouted Bowl Type III.2}

Cat. 19 o

Spouted bowl

Inv. no: $\mathrm{Z6} / 13$

Provenance: Tumulus 6, shaft

Construction: wheel-made (2)

State of preservation: incomplete, restored from sherds, $94 \%$ of rim

Measurements: $\mathrm{Dr}=18.5 \mathrm{~cm} ; \mathrm{H}=20.5 \mathrm{~cm}$

Fabric: ZF6

Hardness: 3

Firing: reduced

Surface treatment: slipped on external surface

Surface colour: external, slip_-10R 6/8 light red; internal2.5YR 6/6 light red

Colour of break: Gley1 2.5/N black

Decoration: incised-one groove below rim, four-five grooves at mid-H

\section{Group IV-Bowls}

\subsection{Bowls Type IV.1}

Variant IV.1a

Cat. 191

Bowl

Inv. no: $\mathrm{Z11} / \mathbf{1}$

Provenance: Tumulus 11, bottom part of shaft, central part Construction: handmade (1)

State of preservation: incomplete, $80 \%$ of rim

Measurements: $\mathrm{Dr}=21.7-24.8 \mathrm{~cm} ; \mathrm{H}=9.2 \mathrm{~cm}$

Fabric: $\mathrm{ZF} 8$

Hardness: $2 / 3$

Firing: reduced

Surface treatment: smoothed on internal surfaces

Surface colour: external-5YR 6/4 light reddish brown; internal-5YR 6/4 light reddish brown

Colour of break: deep black

Notes: clay added to base and modelled by hand
References: (Klimaszewska-Drabot and Czyżewska 2012, $369-372)$

Cat. 192

Bowl

Inv. no: Z11/2

Provenance: Tumulus 11, bottom part of shaft, central part Construction: handmade (1)

State of preservation: incomplete, $80 \%$ of rim

Measurements: $\mathrm{Dr}=22 \mathrm{~cm} ; \mathrm{H}=8.7 \mathrm{~cm}$

Fabric: $\mathrm{zF} 8$

Hardness: $2 / 3$

Firing: reduced

Surface treatment: smoothed on internal surfaces

Surface colour: external-7.5YR 6/4 light brown; internal-7.5YR 6/4 light brown

Colour of break: deep black

Notes: clay added to base and modelled by hand

References: (Klimaszewska-Drabot and Czyżewska 2012, $369-372)$

Cat. 193

Bowl

Inv. no: Z1o/13

Provenance: Tumulus 10, burial chamber, central part

Construction: handmade (1)

State of preservation: incomplete, $55 \%$ of rim, fragments of upper part of vessels missing

Measurements: $\mathrm{Dr}=17.4 \mathrm{~cm} ; \mathrm{H}=6.6 \mathrm{~cm}$

Fabric: ZF8

Hardness: $2 / 3$

Firing: reduced

Surface treatment: smoothed on both surfaces; burnished and slipped inside

[Fig. 7.107] Surface colour: external—7.5YR 5/4 brown; internal, slip2.5YR 4/4 reddish brown

Colour of break: 5 YR 2.5/1 black

Notes: clay added to base and modelled by hand

References: (Klimaszewska-Drabot 2010, 482)

Variant IV.1b

Cat. 194

Bowl

Inv. no: $\mathbf{Z 1 8} / 3$

Provenance: Tumulus 18, burial chamber, central part

Construction: mould (handmade [3])

State of preservation: incomplete, $78 \%$ of rim

Measurements: $\mathrm{Dr}=19.4-19.7 \mathrm{~cm} ; \mathrm{H}=6.9 \mathrm{~cm}$

Fabric: ZF10

Hardness: $2 / 3$

Firing: reduced 
Spouted Bowls type III.1

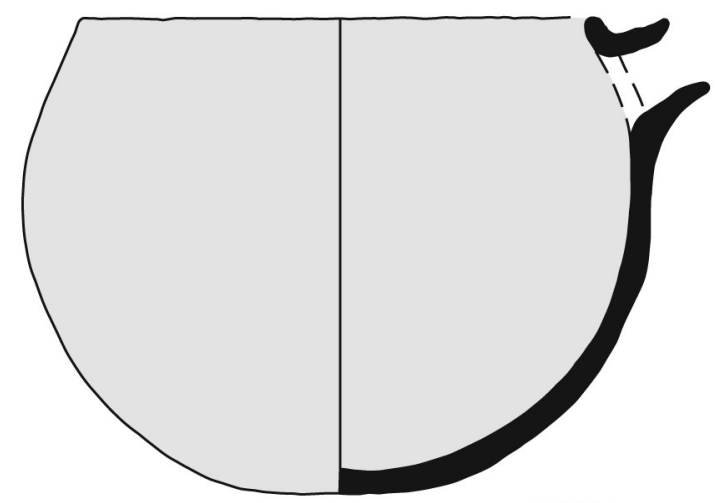

Cat. 188

$Z 19 / 4$

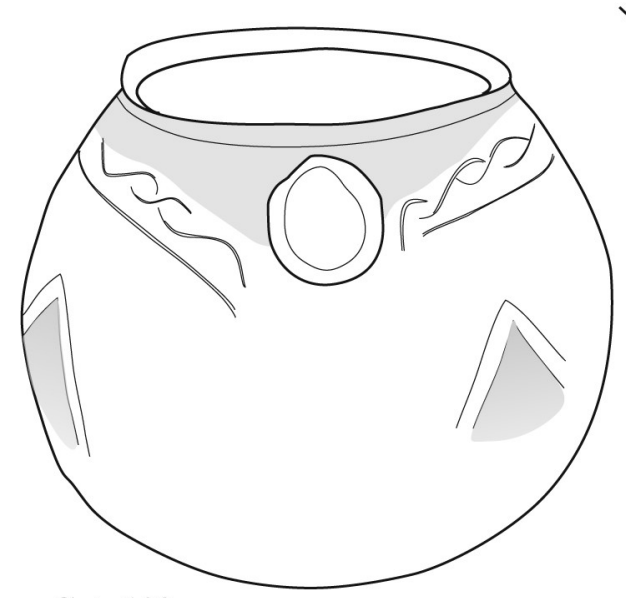

Cat. 189

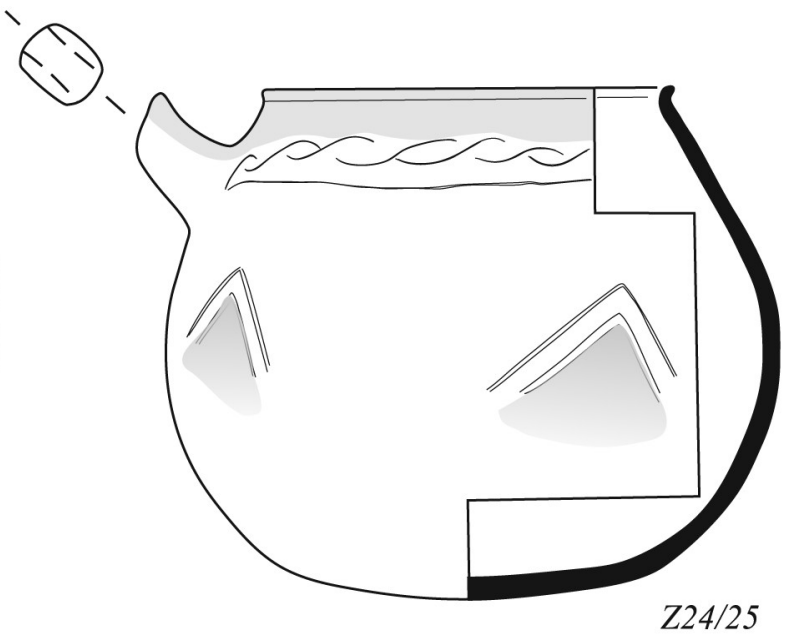

Spouted Bowls type III.2

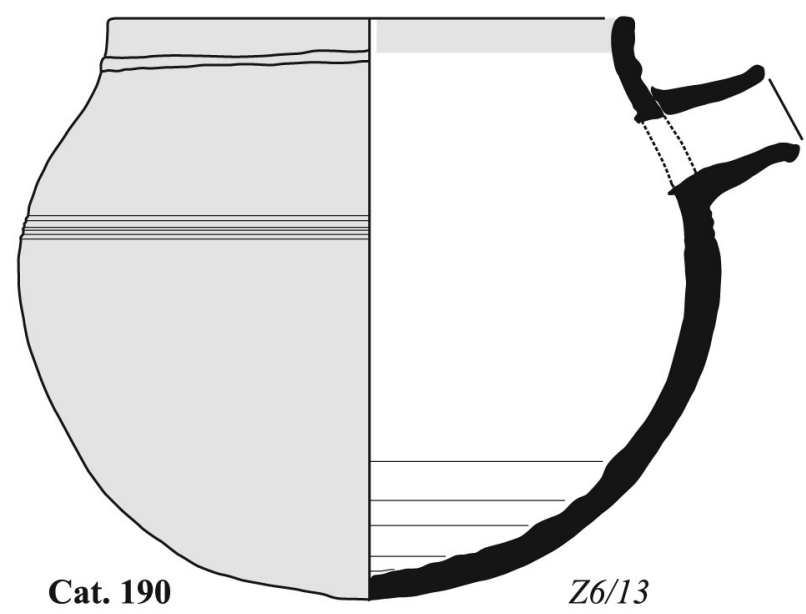

ㄴ, $, \quad, \quad 5 \mathrm{~cm}$

FIGURE 7.41 Spouted bowls of types III.1 and III.2 


\section{Bowls Type IV.1}

\section{variant IV.1a}
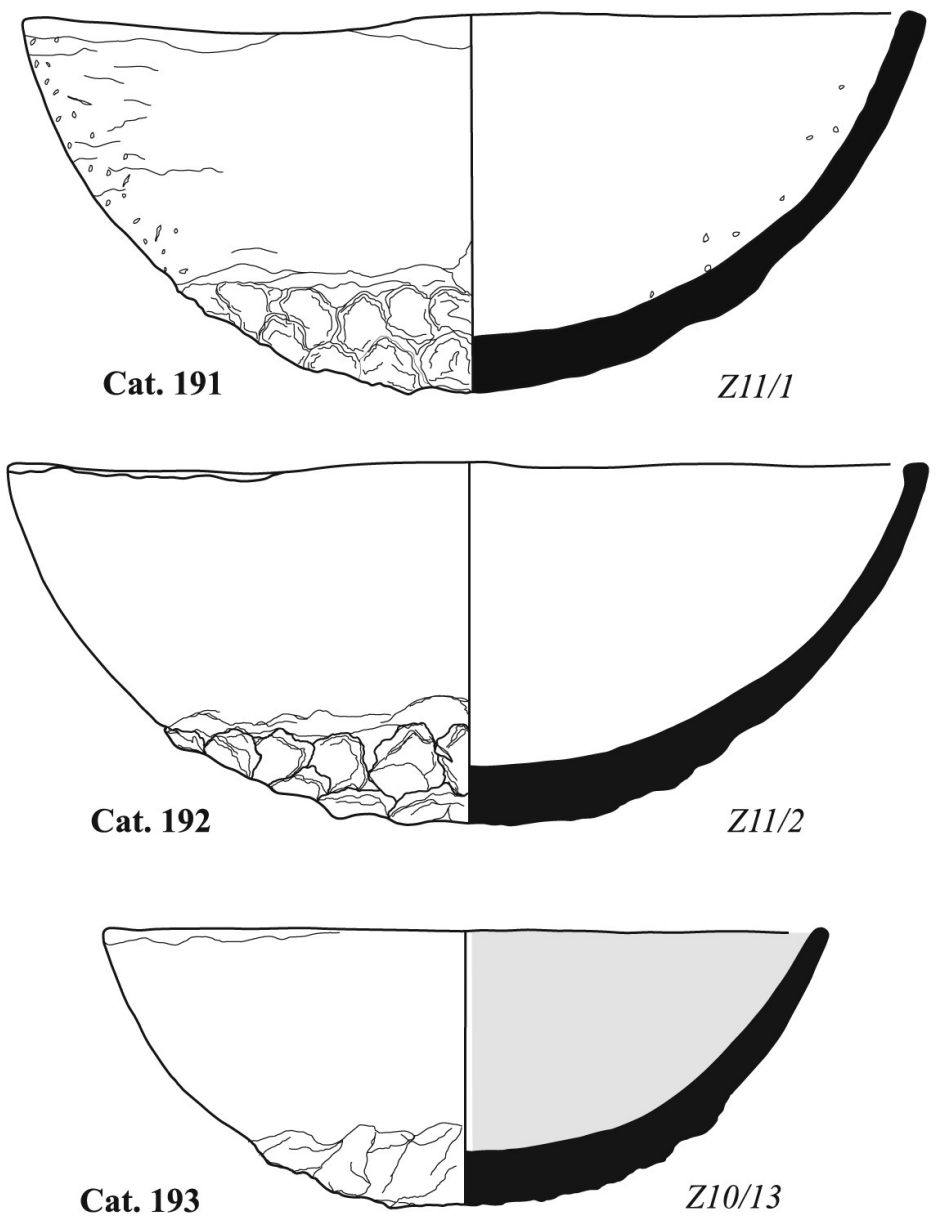

variant IV.1b

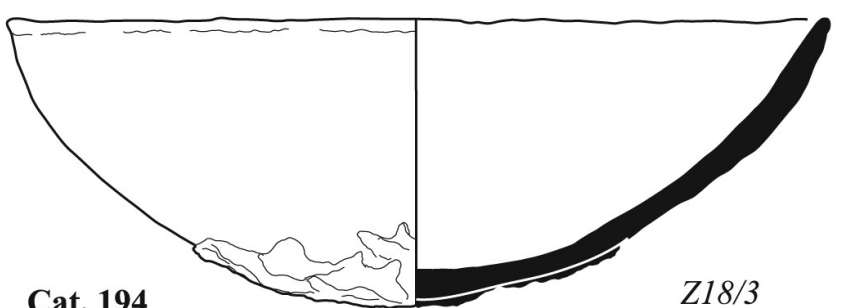

Cat. 194

$Z 18 / 3$

0 $5 \mathrm{~cm}$

FIGURE 7.42 Shallow bowls of type IV.1 with clay added to the base, variants a and b

Surface treatment: uncoated on both surfaces

Surface colour: external-7.5YR 6/4 light brown; internal-5YR 6/4 light reddish brown

Colour of break: 5 Y 2.5/1 black

Notes: clay added to base and modelled by hand

References: (Klimaszewska-Drabot 2010, 482)
5.2 Bowls Type IV.2

$[$ Fig. 7.107$]$
Cat. 195

Bowl

Inv. no: $\mathrm{Z} 25 / 9$

Provenance: Tumulus 25, chamber 2, central part Construction: handmade (1) 


\section{Bowls Type IV.2}

\section{variant IV.2a}

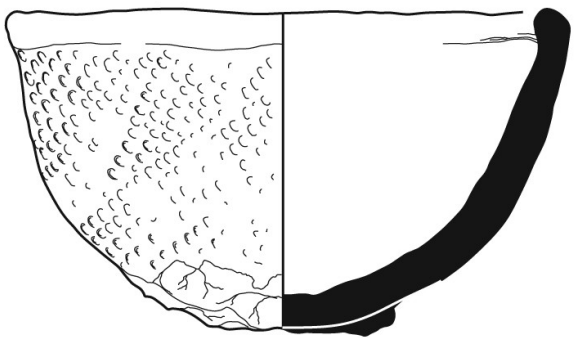

Cat. 195

Z25/9

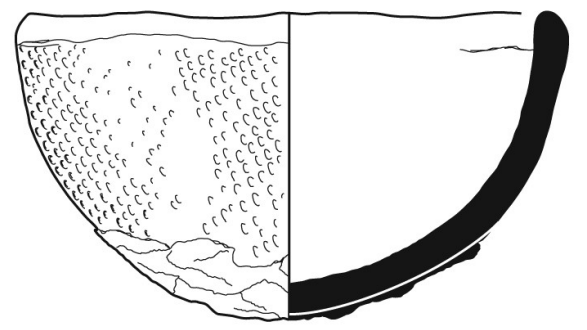

Cat. 196

$Z 25 / 10$

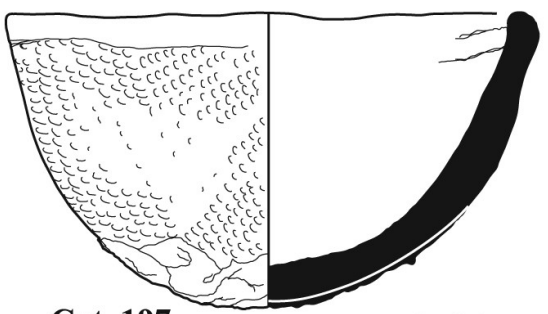

Cat. 197

$Z 25 / 20$ $5 \mathrm{~cm}$

FIGURE 7.43 Shallow bowls of type IV.2 with clay added to the base, variants a and b

State of preservation: complete

Measurements: $\mathrm{Dr}=12.9-13.5 \mathrm{~cm} ; \mathrm{H}=7.7 \mathrm{~cm}$

Fabric: not possible to determine

Hardness: $2 / 3$

Firing: not possible to determine

Surface treatment: external-mat-impressed, pattern 2; internal-uncoated

Surface colour: external-7.5YR 5/3 brown; internal$7 \cdot 5$ Y $6 / 3$ light brown

Colour of break: not possible to determine

Notes: clay added to base and modelled by hand

References: (Klimaszewska-Drabot 2010, 482)

\section{Cat. 196}

Bowl

Inv. no: $\mathbf{Z}_{25} / 10$

Provenance: Tumulus 25, chamber 2, central part

Construction: handmade (1)

State of preservation: incomplete, restored from sherds, body fragments missing

Measurements: $\mathrm{Dr}=13.3 \mathrm{~cm} ; \mathrm{H}=7.4 \mathrm{~cm}$

Fabric: ZF9

Hardness: $2 / 3$

Firing: reduced
Surface treatment: external-mat-impressed, pattern 2; internal-uncoated

Surface colour: external-7.5YR 5/4 brown; internal7.5YR $5 / 3$ brown

Colour of break: 5 YR 2.5/1 black

Notes: clay added to base and modelled by hand

References: (Klimaszewska-Drabot 2010, 482)

\section{Cat. 197}

Bowl

Inv. no: $\mathrm{Z} 25 / 20$

Provenance: Tumulus 25, chamber 3, central part

Construction: handmade (1)

State of preservation: complete

Measurements: $\mathrm{Dr}=11.8-12.5 \mathrm{~cm} ; \mathrm{H}=7.2 \mathrm{~cm}$

Fabric: not possible to determine

Hardness: $2 / 3$

Firing: not possible to determine

Surface treatment: external-mat-impressed, pattern 2; internal-uncoated

Surface colour: external-5YR 6/4 light reddish brown; internal- 7.5 YR $5 / 3$ brown

Colour of break: not possible to determine

Notes: clay added to base and modelled by hand

References: (Klimaszewska-Drabot 2010, 482) 
Variant IV.2b

\section{Cat. 198}

Bowl

Inv. no: $\mathrm{Z24/42}$

Provenance: Tumulus 24, chamber 1

Construction: handmade (1)

State of preservation: incomplete, body fragments missing

Measurements: $\mathrm{Dr}=17.5^{-18.5} \mathrm{~cm} ; \mathrm{H}=8.5 \mathrm{~cm}$

Fabric: ZF10

Hardness: $2 / 3$

Firing: reduced

Surface treatment: smoothed on both surfaces; burnished and slipped inside

Surface colour: external-7.5YR 7/4 pink; internal, slip10R $5 / 6$ red

Colour of break: deep black

Notes: clay added to base and modelled by hand

\subsection{Bowls Type IV.3}

[Fig. 7.107$]$

Variant IV.3a

Cat. 199

Bowl

Inv. no: Z26/21

Provenance: Tumulus 26, chamber 2, central part

Construction: handmade (1)

State of preservation: complete

Measurements: $\mathrm{Dr}=14.5 \mathrm{~cm} ; \mathrm{H}=7.3 \mathrm{~cm}$

Fabric: not possible to determine

Hardness: 3

Firing: not possible to determine

Surface treatment: external-mat-impressed, pattern 1; internal-smoothed

Surface colour: external-5YR 6/4 light reddish brown; internal-10R $5 / 6$ red

Colour of break: not possible to determine

Decoration: incised decoration on rim almost indiscernible

Notes: clay added to base and modelled by hand

Cat. 200

Bowl

Inv. no: $\mathrm{Z26} / 78$

Provenance: Tumulus 26, chamber 4, southern part

Construction: handmade (1)

State of preservation: complete

Measurements: $\mathrm{Dr}=13.6 \mathrm{~cm} ; \mathrm{H}=6.8 \mathrm{~cm}$

Fabric: not possible to determine

Hardness: 3

Firing: not possible to determine

Surface treatment: external-mat-impressed, pattern 1; internal-smoothed
Surface colour: external-2.5YR 4/4 reddish brown; internal- 5 Y $5 / 3$ reddish brown

Colour of break: not possible to determine

Decoration: incised decoration on rim

Notes: clay added to base and modelled by hand

Cat. 201

Bowl

Inv. no: $\mathrm{Z} 23 / 30$

Provenance: Tumulus 23, chamber 2

Construction: handmade (1)

State of preservation: complete

Measurements: $\mathrm{Dr}=15.7 \mathrm{~cm} ; \mathrm{H}=6.8 \mathrm{~cm}$

Fabric: not possible to determine

Hardness: 3

Firing: not possible to determine

Surface treatment: external-mat-impressed, pattern 1; internal-smoothed

Surface colour: external-7.5YR 5/4 brown; internal5 YR 5/4 reddish brown

Colour of break: not possible to determine

Decoration: incised decoration on rim

Notes: clay added to base and modelled by hand

Cat. 202

Bowl

Inv. no: $\mathrm{Z} 26 / 49$

Provenance: Tumulus 26, chamber 3, south-western part

Construction: handmade (1)

State of preservation: complete

Measurements: $\mathrm{Dr}=13.3 \mathrm{~cm} ; \mathrm{H}=6.4 \mathrm{~cm}$

Fabric: not possible to determine

Hardness: 3

Firing: not possible to determine

Surface treatment: external-mat-impressed, pattern 1; internal—smoothed and burnished

Surface colour: external-2.5YR 4/4 reddish brown; internal-5YR $4 / 3$ reddish brown

Colour of break: not possible to determine

Decoration: incised decoration on rim

Notes: clay added to base and modelled by hand

Cat. 203

Bowl

Inv. no: $\mathbf{Z 2 6 / 7 3}$

Provenance: Tumulus 26, chamber 4, southern part

Construction: handmade (1)

State of preservation: incomplete, body fragment missing

Measurements: $\mathrm{Dr}=16 \mathrm{~cm} ; \mathrm{H}=6.1 \mathrm{~cm}$

Fabric: ZF1O

Hardness: 3 
Firing: reduced

Surface treatment: external-mat-impressed, pattern 1; internal-smoothed and burnished

Surface colour: external-2.5YR 2.5/4 dark reddish brown; internal-1oR $4 / 4$ weak red

Colour of break: 1OYR 3/2 very dark greyish brown

Decoration: incised decoration on rim

Notes: clay added to base and modelled by hand

\section{Cat. 204}

Bowl

Inv. no: $\mathbf{Z 2 6 / 7 9}$

Provenance: Tumulus 26, chamber 2, northern part

Construction: handmade (1)

State of preservation: complete

Measurements: $\mathrm{Dr}=11.7 \mathrm{~cm} ; \mathrm{H}=6.8 \mathrm{~cm}$

Fabric: not possible to determine

Hardness: 3

Firing: not possible to determine

Surface treatment: external-mat-impressed, pattern 1; internal-smoothed and burnished

Surface colour: external-7.5YR 5/3 brown; internal2.5 YR $5 / 6$ red

Colour of break: not possible to determine

Decoration: incised decoration on rim

Notes: clay added to base and modelled by hand

Variant IV.3b

Cat. 205

Bowl

Inv. no: $\mathbf{Z 2 6 / 1 8}$

Provenance: Tumulus 26, chamber 4, central part

Construction: handmade (1)

State of preservation: complete

Measurements: $\mathrm{Dr}=14.5 \mathrm{~cm} ; \mathrm{H}=7 \mathrm{~cm}$

Fabric: not possible to determine

Hardness: 3

Firing: not possible to determine

Surface treatment: external-mat-impressed, pattern 1; internal-smoothed

Surface colour: external-7.5YR 5/4 brown; internal2.5 YR $5 / 4$ reddish brown

Colour of break: not possible to determine

Decoration: incised decoration on rim

Notes: clay added to base and modelled using a tool

Cat. 206

Bowl

Inv. no: Z26/19

Provenance: Tumulus 26, chamber 2, southern part

Construction: handmade (1)
State of preservation: complete

Measurements: $\mathrm{Dr}=14.7 \mathrm{~cm} ; \mathrm{H}=6.5 \mathrm{~cm}$

Fabric: not possible to determine

Hardness: $3 / 4$

Firing: not possible to determine

Surface treatment: external-mat-impressed, pattern 1; internal-smoothed

Surface colour: external-7.5YR 5/4 brown; internal2.5 YR $5 / 4$ reddish brown

Colour of break: not possible to determine

Decoration: incised decoration on rim

Notes: clay added to base and modelled using a tool

5.4 Bowls Type IV.4

Variant IV.4a

Cat. 207

Bowl

Inv. no: Z12/125

Provenance: Tumulus 12, chamber 3

Construction: handmade (1)

State of preservation: complete

Measurements: $\mathrm{Dr}=15.2-15.6 \mathrm{~cm} ; \mathrm{H}=7 \mathrm{~cm}$

Fabric: not possible to determine

Hardness: $3 / 4$

Firing: not possible to determine

Surface treatment: external-uncoated, pattern 1; internal-smoothed, burnished and slipped

Surface colour: external-1oR 5/6 red; internal, slip10R $4 / 6$ red

Colour of break: not possible to determine

Notes: clay added to base and modelled using a tool

Variant IV.4b

Cat. 208

Bowl

Inv. no: $\mathrm{Z26/55}$

Provenance: Tumulus 26, chamber 2, northern part

Construction: mould (handmade [3])

State of preservation: incomplete, $91 \%$ of rim

Measurements: $\mathrm{Dr}=17 \mathrm{~cm} ; \mathrm{H}=7.4 \mathrm{~cm}$

Fabric: $\mathrm{ZF} 5$

Hardness: 4

Firing: oxidized

Surface treatment: uncoated on both surfaces

Surface colour: external-5YR 5/4 reddish brown; internal-2.5YR $4 / 4$ reddish brown

Colour of break: $2.5 \mathrm{Y}$ 4/2 dark greyish brown

Decoration: incised decoration on rim

Notes: clay added to base and modelled by hand 


\section{Bowls Type IV.3}

\section{variant IV.3a}

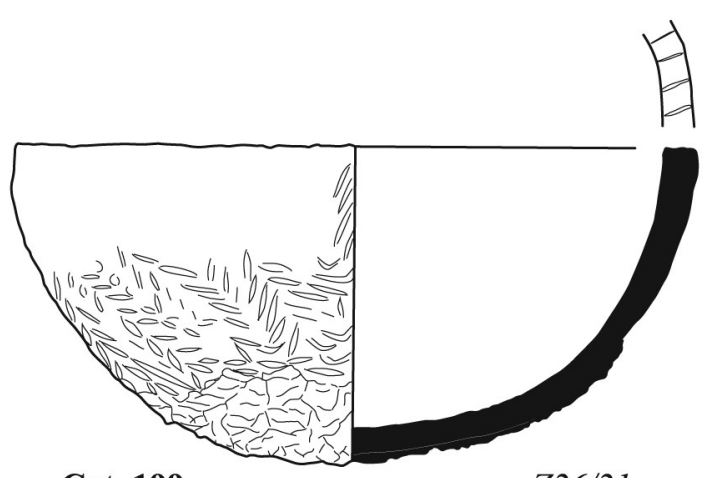

Cat. 199

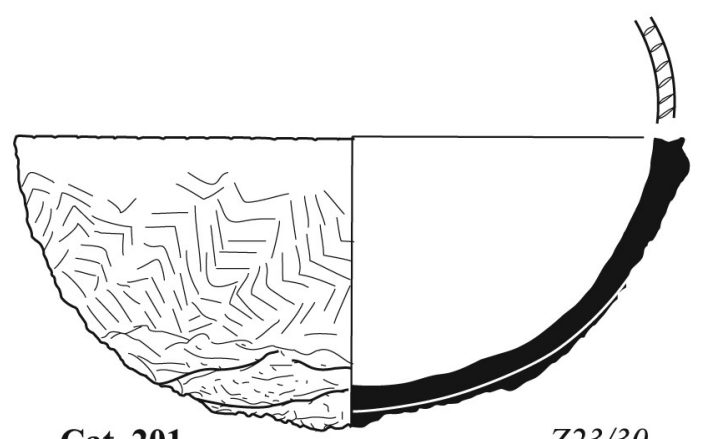

Cat. 201

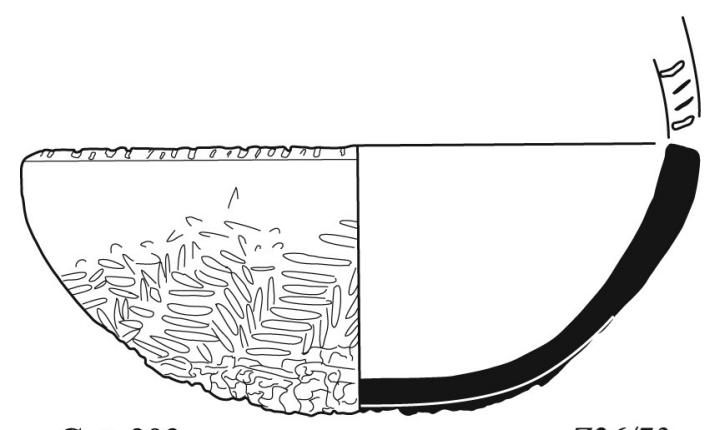

Cat. 203

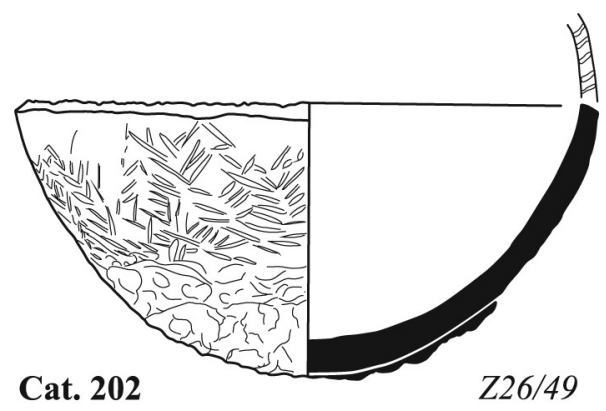

variant IV.3b
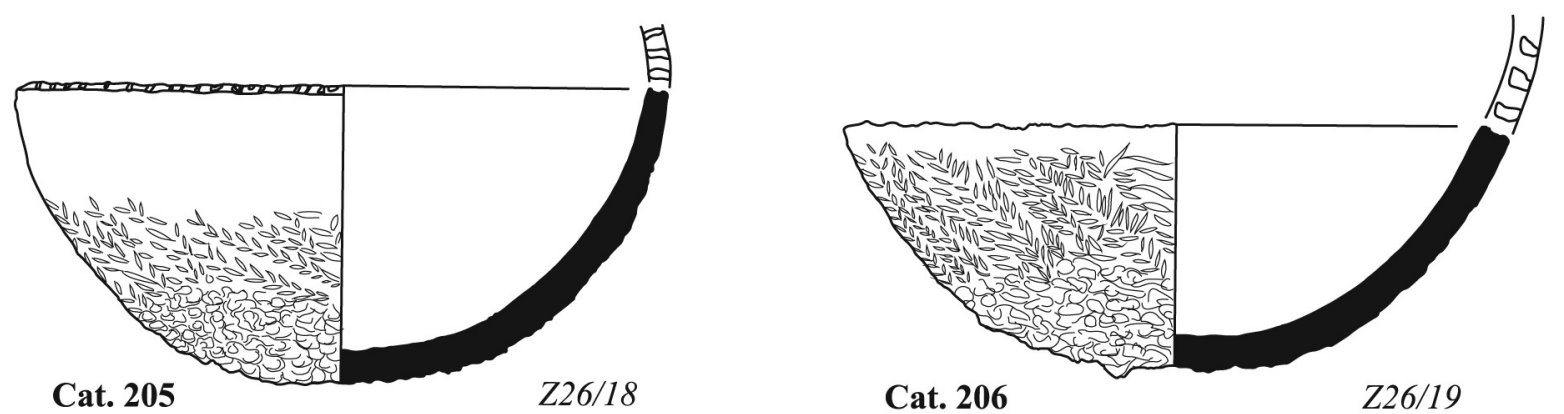

Cat. 206

Z26/19

0 $5 \mathrm{~cm}$

Shallow bowls of type IV.3 with clay added to the base, variants a and b 


\section{Bowls Type IV.4}

variant IV.4a

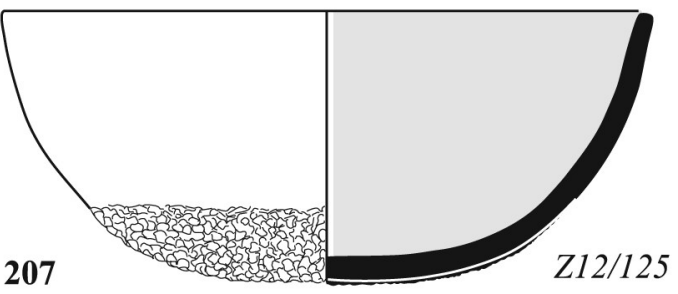

variant IV.4b
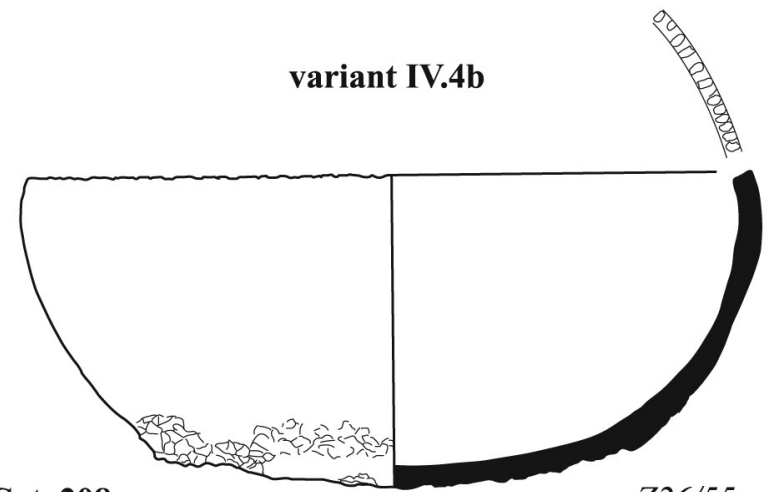

Cat. 208

$Z 26 / 55$

variant IV.4c
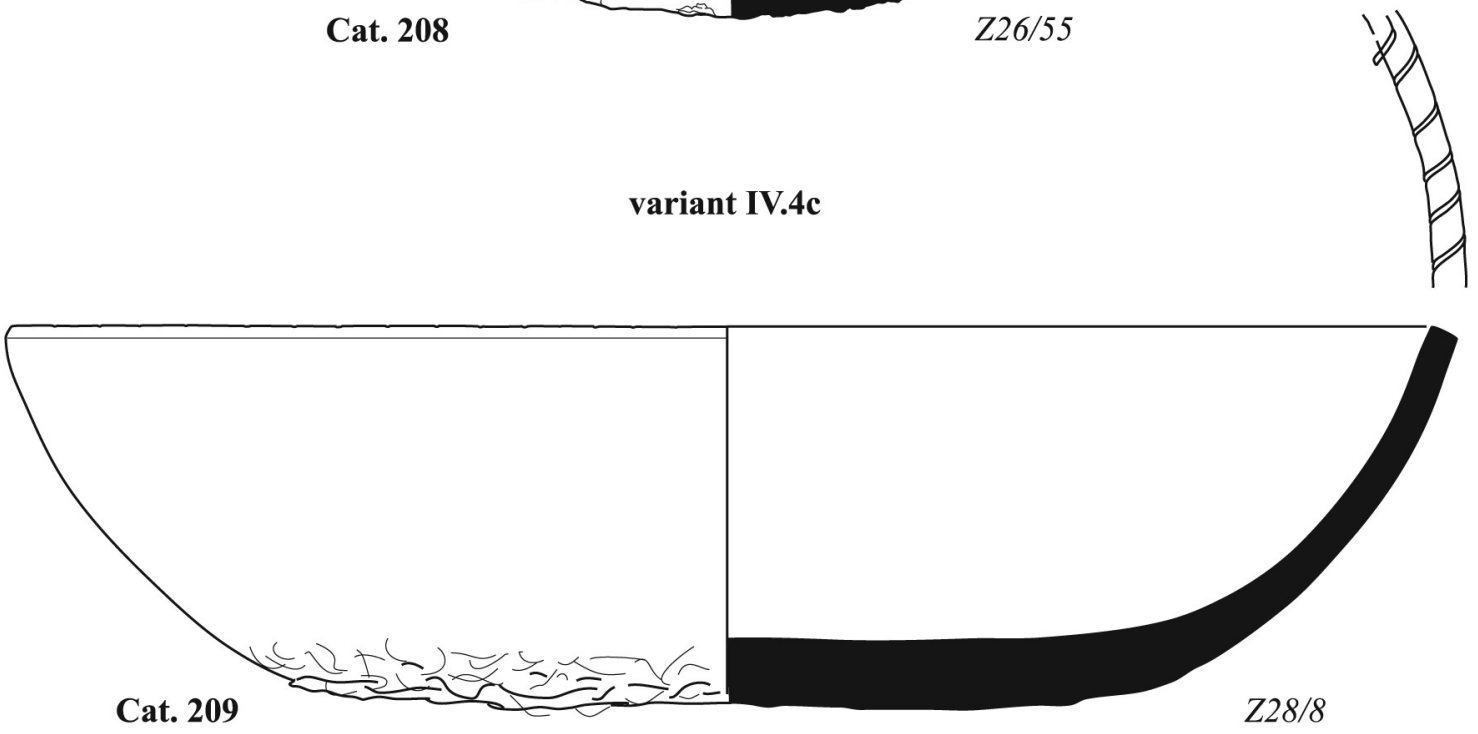

0 $5 \mathrm{~cm}$

FIGURE 7.45 Shallow bowls of type IV.4 with clay added to the base, variants a, b and c

Variant IV.4C

Cat. 209

Bowl

Inv. no: $\mathrm{Z} 28 / 8$

Provenance: Tumulus 28, burial chamber, central part Construction: mould (handmade [3])

State of preservation: complete

Measurements: $\mathrm{Dr}=24 \mathrm{~cm} ; \mathrm{H}=8.8 \mathrm{~cm}$

Fabric: not possible to determine

Hardness: 3
Firing: not possible to determine

Surface treatment: uncoated on both surfaces

Surface colour: external-2.5YR 5/6 red; internal— 5 YR 5/4 reddish brown

Colour of break: not possible to determine

Decoration: incised decoration on rim

Notes: clay added to base and modelled, probably using a tool 


\section{Group v-Pots}

\subsection{Pots Type V.1}

Cat. 210

Pot

Inv. no: Z18/1

Provenance: Tumulus 18, shaft, eastern part, near blocking wall of burial chamber

Construction: handmade (1)

State of preservation: incomplete, $84 \%$ of rim

Measurements: $\mathrm{Dr}=17-17.5 \mathrm{~cm} ; \mathrm{H}=14 \mathrm{~cm}$

Fabric: $\mathrm{ZF} 8$

Hardness: $2 / 3$

Firing: oxidized

Surface treatment: smoothed on both surfaces

Surface colour: external-7.5YR 6/3 light brown; internal -5 Y R $6 / 3$ light reddish brown

Colour of break: 7.5YR 6/3 light brown

Notes: clay added to base and modelled by hand

References: (Klimaszewska-Drabot 2010, 482)

Cat. 211

Pot

Inv. no: Z11/3

Provenance: Tumulus 11, bottom of shaft, central part

Construction: handmade (1)

State of preservation: incomplete, $86 \%$ of rim

Measurements: $\mathrm{Dr}=14 \mathrm{~cm} ; \mathrm{H}=11.9 \mathrm{~cm}$

Fabric: ZF8

Hardness: $2 / 3$

Firing: reduced

Surface treatment: smoothed on both surfaces

Surface colour: external-7.5YR 6/4 light brown; internal-7.5YR 6/4 light brown

Colour of break: deep black

Notes: clay added to base and modelled by hand

References: (Klimaszewska-Drabot and Czyżewska 2012, 369-372)

Cat. 212

Pot

Inv. no: Z11/23

Provenance: Tumulus 11, chamber 2, central part

Construction: handmade (1)

State of preservation: incomplete, $75 \%$ of rim, fragments of upper part of vessel missing

Measurements: $\mathrm{Dr}=14 \mathrm{~cm} ; \mathrm{H}=11.5 \mathrm{~cm}$

Fabric: ZF8

Hardness: $2 / 3$

Firing: reduced

Surface treatment: smoothed on both surfaces
Surface colour: external-7.5YR 6/4 light brown; internal-7.5YR 6/4 light brown

Colour of break: Gley1 2.5/N black

Notes: clay added to base and modelled by hand

References: (Klimaszewska-Drabot and Czyżewska 2012, 368-372)

Cat. 213

Pot

Inv. no: Z10/10

Provenance: Tumulus 10, burial chamber, central part

Construction: handmade (1)

State of preservation: incomplete, $58 \%$ of rim

Measurements: $\mathrm{Dr}=14 \mathrm{~cm} ; \mathrm{H}=11.5 \mathrm{~cm}$

Fabric: $\mathrm{ZF} 8$

Hardness: $2 / 3$

Firing: reduced

Surface treatment: smoothed on both surfaces; burnished and slipped inside

Surface colour: external-7.5YR 6/4 light brown; internal natural surface $-7.5^{\mathrm{YR}} 5 / 4$ brown, slip- $7.5 \mathrm{R} 5 / 8$ red

Colour of break: deep black

Notes: clay added to base and modelled by hand

References: (Klimaszewska-Drabot 2010, 482)

\subsection{Pots Type V.2}

[Fig. 7.109]

Variant v.2a

Cat. 214

Pot

Inv. no: Z24/17

Provenance: Tumulus 24, chamber 1, eastern part

Construction: handmade (1)

State of preservation: incomplete, $72 \%$ of rim, fragments of upper part of vessel missing

Measurements: $\mathrm{Dr}=10.7 \mathrm{~cm} ; \mathrm{H}=12.1 \mathrm{~cm}$

Fabric: ZF10

Hardness: 3

Firing: oxidized

Surface treatment: smoothed on both surfaces; burnished inside

Surface colour: external-5YR 6/6 yellowish red; internal-5YR $5 / 6$ yellowish red

Colour of break: $10 \mathrm{R} 4 / 8$ red

Notes: clay added to base and modelled by hand

Cat. 215

Pot

Inv. no: $\mathbf{Z} 24 / 21$

Provenance: Tumulus 24, chamber 1, eastern part

Construction: handmade (1)

State of preservation: complete 


\section{Pots Type V.1}
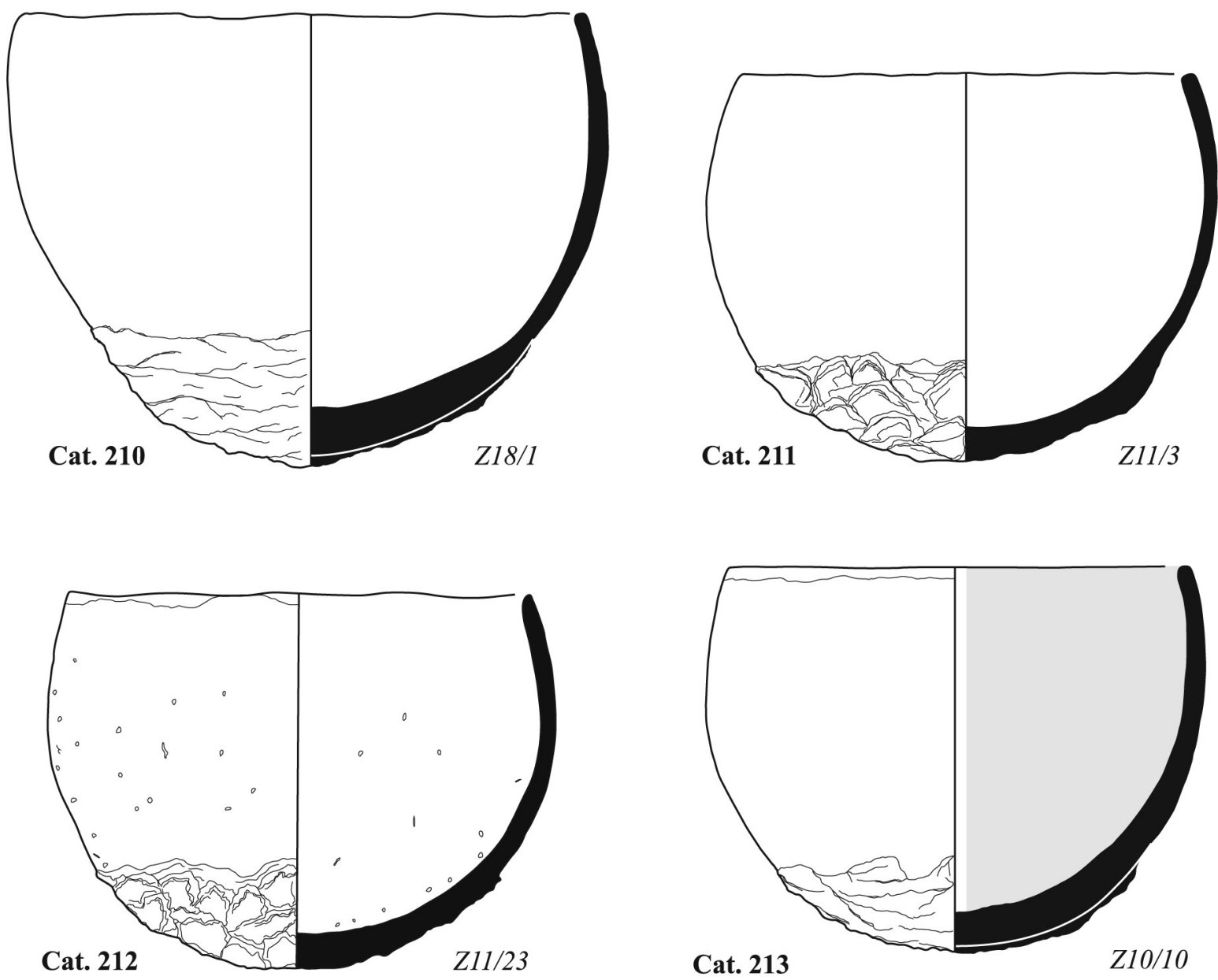

\section{Cat. 213}

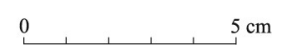

FIGURE 7.46 Deep globular pots of type v.1 with clay added to the base

Measurements: $\mathrm{Dr}=10.6 \mathrm{~cm} ; \mathrm{H}=12.1 \mathrm{~cm}$

Fabric: not possible to determine

Hardness: $2 / 3$

Firing: reduced

Surface treatment: smoothed on both surfaces; burnished inside

Surface colour: external-7.5YR 5/4 brown; internal7.5YR $5 / 4$ brown

Colour of break: not possible to determine

Notes: clay added to base and modelled by hand

Variant V.2b

Cat. 216

Pot

Inv. no: $\mathrm{Z25}_{2} / 16$

Provenance: Tumulus 25, chamber 3, central part

Construction: handmade (1)
State of preservation: complete

Measurements: $\mathrm{Dr}=8.8-9 \mathrm{~cm} ; \mathrm{H}=10.8 \mathrm{~cm}$

Fabric: not possible to determine

Hardness: $3 / 4$

Firing: not possible to determine

Surface treatment: smoothed and burnished on both surfaces

Surface colour: external-5YR 5/4 reddish brown; internal-5YR 4/4 reddish brown

Colour of break: not possible to determine

Notes: clay added to base and modelled using a tool

References: (Klimaszewska-Drabot 2010, 482) 


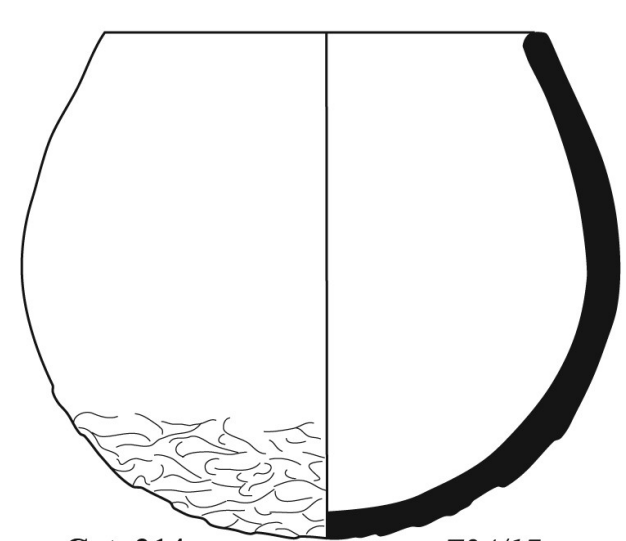

Cat. 214 variant V.2a

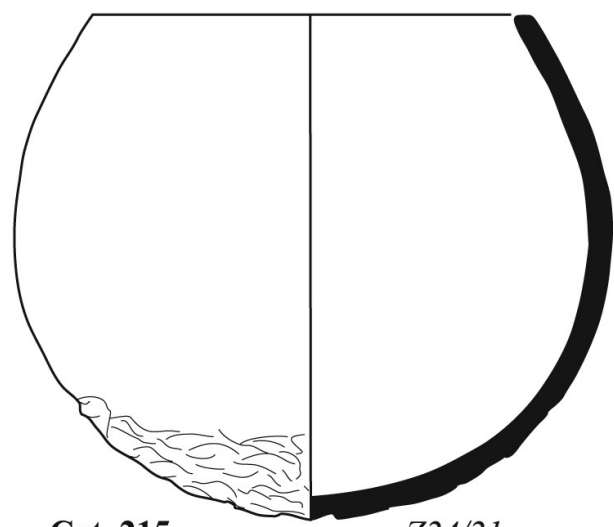

Cat. 215

variant V.2b
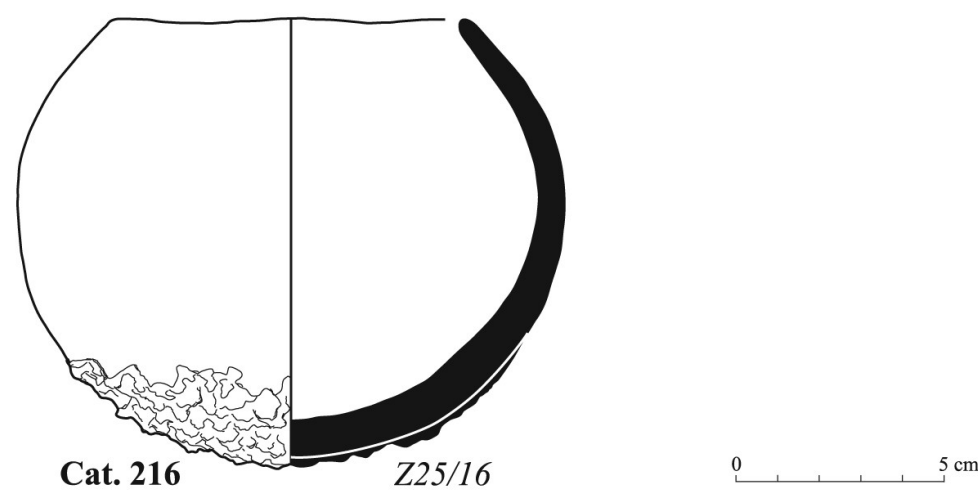

FIGURE 7.47 Deep globular pots of type v.2 with clay added to the base, variants a and b

6.3 Pots Type V.3

Variant v.3a

Cat. 217

Pot

Inv. no: $\mathrm{Z} 23 / 1$

Provenance: Tumulus 23, fill of shaft

Construction: handmade (1)

State of preservation: complete

Measurements: $\mathrm{Dr}=9.6 \mathrm{~cm} ; \mathrm{H}=12.2 \mathrm{~cm}$

Fabric: not possible to determine

Hardness: 3

Firing: not possible to determine

Surface treatment: external-mat-impressed, pattern 1; internal-smoothed

Surface colour: external-2.5YR 5/6 red; internal-2.5YR $4 / 6$ red

Colour of break: not possible to determine

Notes: mat impression poorly visible on surface

Notes: clay added to base and modelled by hand

References: (Klimaszewska-Drabot and Mahmoud El-

Tayeb 2014, 13)
[Figs 7.109-7.110] Cat. 218

Inv. no: $\mathbf{Z 2 5} / 3^{1}$

Provenance: Tumulus 25, chamber 3, southern part

Construction: handmade (1)

State of preservation: complete

Measurements: $\mathrm{Dr}=9.5 \mathrm{~cm} ; \mathrm{H}=10.3 \mathrm{~cm}$

Fabric: not possible to determine

Hardness: $2 / 3$

Firing: not possible to determine

Surface treatment: external-mat-impressed, pattern 2; internal-uncoated

Surface colour: external-7.5YR 6/4 light brown; internal-7.5YR $6 / 4$ light brown

Colour of break: not possible to determine

Notes: clay added to base and modelled by hand

References: (Klimaszewska-Drabot 2010, 482)

Cat. 219

Pot

Inv. no: $\mathrm{Z} 25 / 26$

Provenance: Tumulus 25, chamber 3, central part 


\section{Pots Type V.3}

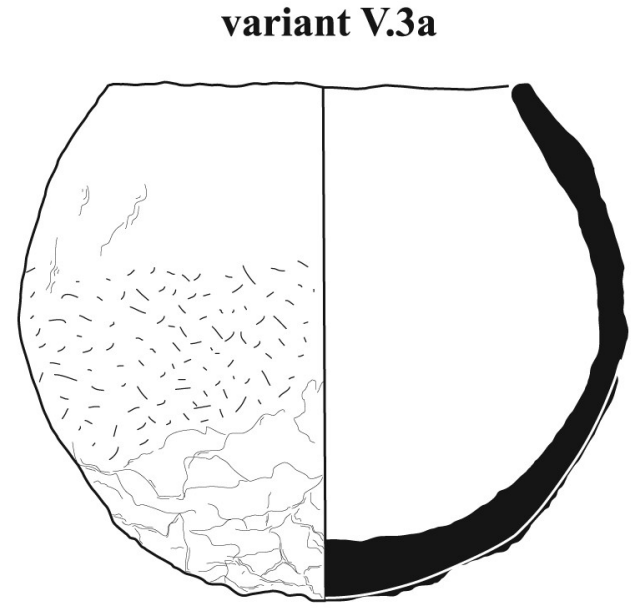

Cat. 217

$Z 23 / 1$

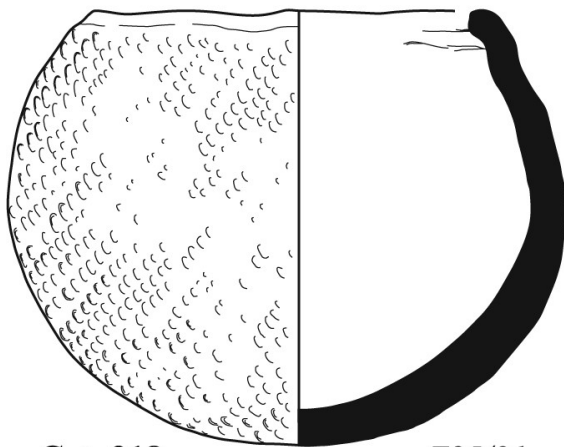

Cat. 218

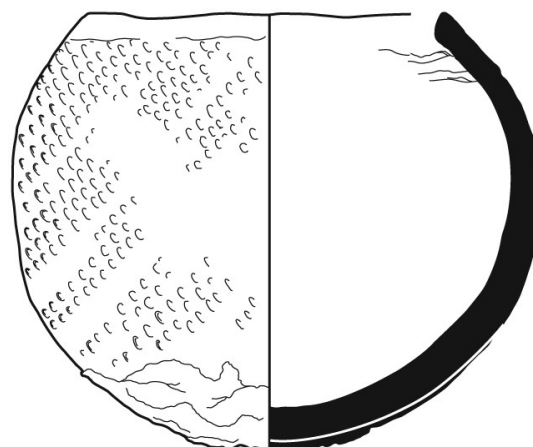

Cat. 219

$Z 25 / 26$

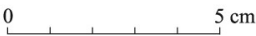

FIGURE 7.48 Deep globular pots of type v.3 with clay added to the base, variant a

Construction: handmade (1)

State of preservation: complete

Measurements: $\mathrm{Dr}=8.1-8.6 \mathrm{~cm} ; \mathrm{H}=10.3 \mathrm{~cm}$

Fabric: not possible to determine

Hardness: $2 / 3$

Firing: not possible to determine

Surface treatment: external-mat-impressed, pattern 2; internal-uncoated

Surface colour: external-7.5YR 6/3 light brown; internal-7.5YR $5 / 4$ brown

Colour of break: not possible to determine

Notes: clay added to base and modelled by hand

References: (Klimaszewska-Drabot 2010, 482)

Variant V.3b

Cat. 220

Pot

Inv. no: $\mathrm{Z}_{26 / 16}$

Provenance: Tumulus 26, chamber 1, south-western part

Construction: handmade (1)
State of preservation: complete

Measurements: $\mathrm{Dr}=10.8 \mathrm{~cm} ; \mathrm{H}=11.4 \mathrm{~cm}$

Fabric: not possible to determine

Hardness: 3

Firing: not possible to determine

Surface treatment: external-mat-impressed, pattern 2; internal-smoothed

Surface colour: external-5YR 4/4 reddish brown; internal-5YR $4 / 6$ yellowish red

Colour of break: not possible to determine

Notes: clay added to base and modelled using a tool

Cat. 221

Pot

Inv. no: $\mathbf{Z 2 6 / 2 2}$

Provenance: Tumulus 26, chamber 2, southern part

Construction: handmade (1)

State of preservation: complete

Measurements: $\mathrm{Dr}=11.7 \mathrm{~cm} ; \mathrm{H}=11 \mathrm{~cm}$

Fabric: not possible to determine 


\section{Hardness: 3}

Firing: not possible to determine

Surface treatment: external-mat-impressed, pattern 2; internal-smoothed

Surface colour: external-5YR 4/4 reddish brown; internal-5YR $4 / 4$ reddish brown

Colour of break: not possible to determine

Notes: clay added to base and modelled using a tool

\section{Cat. 222}

Pot

Inv. no: $\mathbf{Z 9 / 2 1}$

Provenance: Tumulus 9, chamber 1, western part

Construction: handmade (1)

State of preservation: incomplete, restored from sherds, body fragments missing

Measurements: $\mathrm{Dr}=11 \mathrm{~cm} ; \mathrm{H}=9.2 \mathrm{~cm}$

Fabric: ZF10

Hardness: 3

Firing: reduced

Surface treatment: external-mat-impressed, pattern 5; internal-smoothed

Surface colour: external-5YR 4/4 reddish brown; internal-5YR $4 / 4$ reddish brown

Colour of break: Gley1 2.5/N black

Notes: clay added to base and modelled using a tool

References: (Mahmoud El-Tayeb, Juszczyk-Futkowska, and Czyżewska 2014, 367, 369-371)

\section{Cat. 223}

Pot

Inv. no: $\mathrm{Z26/23}$

Provenance: Tumulus 26, chamber 2, southern part

Construction: handmade (1)

State of preservation: incomplete, $73 \%$ of rim

Measurements: $\mathrm{Dr}=11.5 \mathrm{~cm} ; \mathrm{H}=12.1 \mathrm{~cm}$

Fabric: ZF1O

Hardness: $2 / 3$

Firing: reduced

Surface treatment: external-mat-impressed, pattern 2; internal-smoothed and burnished

Surface colour: external-5YR 4/4 reddish brown; internal-5YR 4/6 yellowish red

Colour of break: deep black

Notes: clay added to base and modelled using a tool

Cat. 224

Pot

Inv. no: $\mathrm{Z14} / 20$

Provenance: Tumulus 14, chamber 1, central part

Construction: handmade (1)
State of preservation: complete

Measurements: $\mathrm{Dr}=9.8 \mathrm{~cm} ; \mathrm{H}=10 \mathrm{~cm}$

Fabric: ZF10

Hardness: 3

Firing: reduced

Surface treatment: external-mat-impressed, pattern 2; internal—smoothed and burnished

Surface colour: external-7.5YR 4/4 dark brown; internal-5YR 4/4 reddish brown

Colour of break: $2.5 \mathrm{Y}$ 3/1 very dark grey

6.4 Pots Type V.4

Variant V.4a

Cat. 225

Pot

Inv. no: $\mathrm{Z26/17}$

Provenance: Tumulus 26, chamber 3 , northern part

Construction: handmade (1)

State of preservation: complete

Measurements: $\mathrm{Dr}=11.5 \mathrm{~cm} ; \mathrm{H}=9.7 \mathrm{~cm}$

Fabric: not possible to determine

Hardness: 3

Firing: not possible to determine

Surface treatment: external-mat-impressed, pattern 1; internal-smoothed

Surface colour: external-5YR 4/4 reddish brown; internal-5YR 4/6 yellowish red

Colour of break: not possible to determine

Decoration: incised decoration on rim

Notes: clay added to base and modelled by hand

Cat. 226

Pot

Inv. no: Z12/96

Provenance: Tumulus 12, chamber 3, western part

Construction: handmade (1)

State of preservation: complete

Measurements: $\mathrm{Dr}=13 \mathrm{~cm} ; \mathrm{H}=10 \mathrm{~cm}$

Fabric: not possible to determine

Hardness: $3 / 4$

Firing: not possible to determine

Surface treatment: external-mat-impressed, pattern 1; internal-smoothed

Surface colour: external-2.5YR 5/6 red; internal-2.5YR 6/6 light red

Colour of break: not possible to determine

Decoration: incised decoration on rim

Notes: clay added to base and modelled by hand 


\section{variant V.3b}
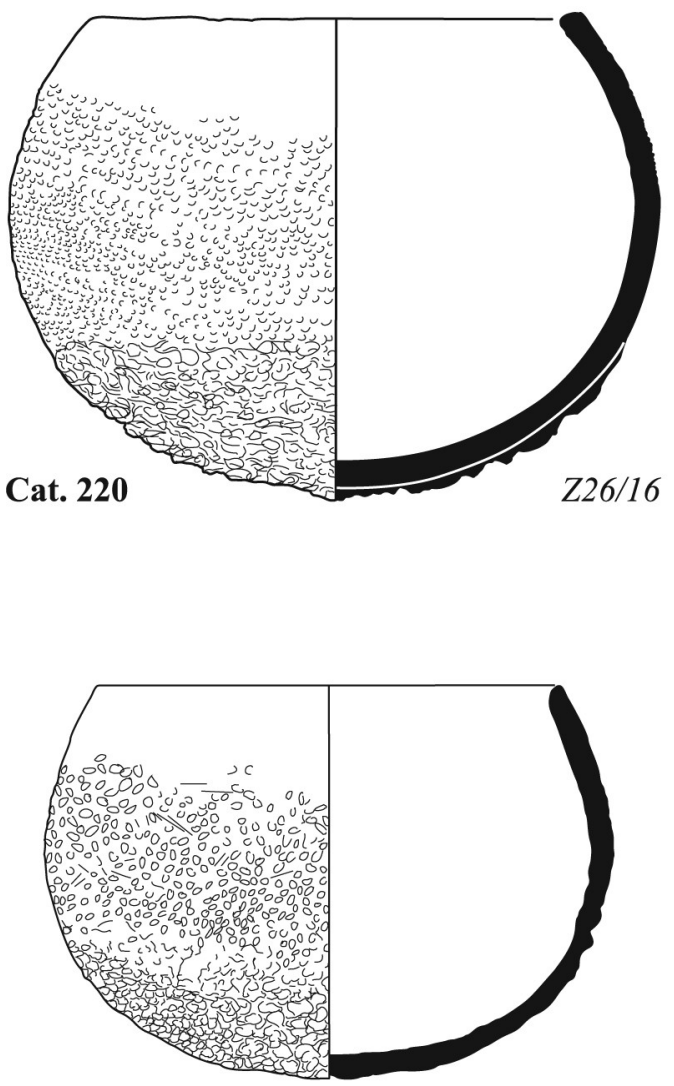

Cat. 222
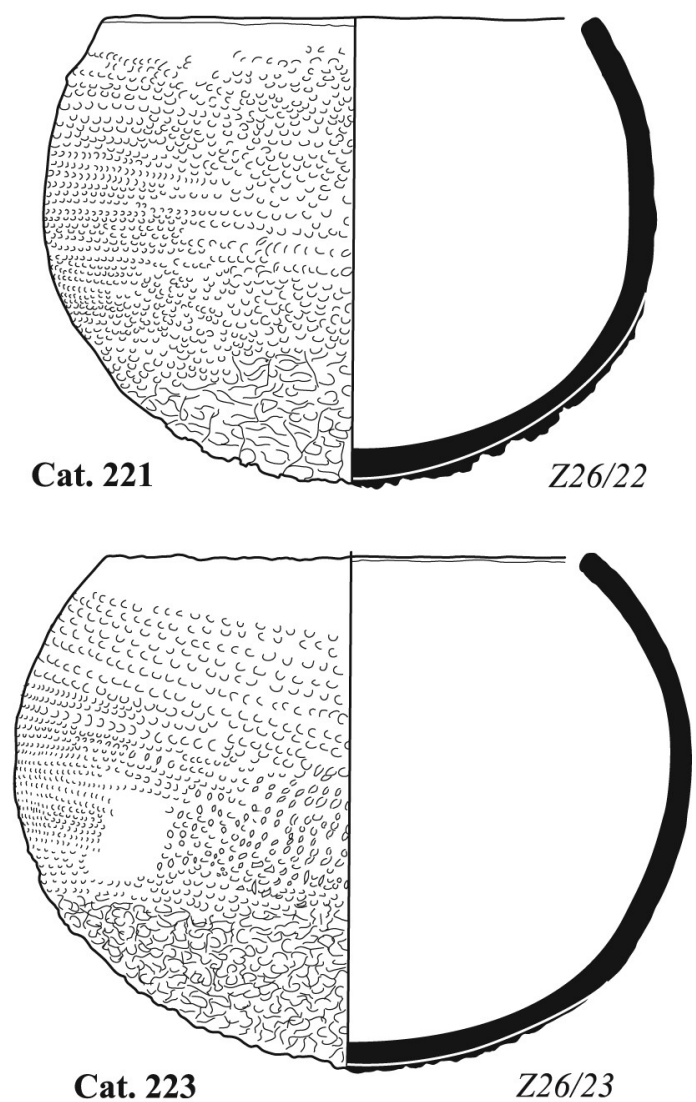

Z26/23
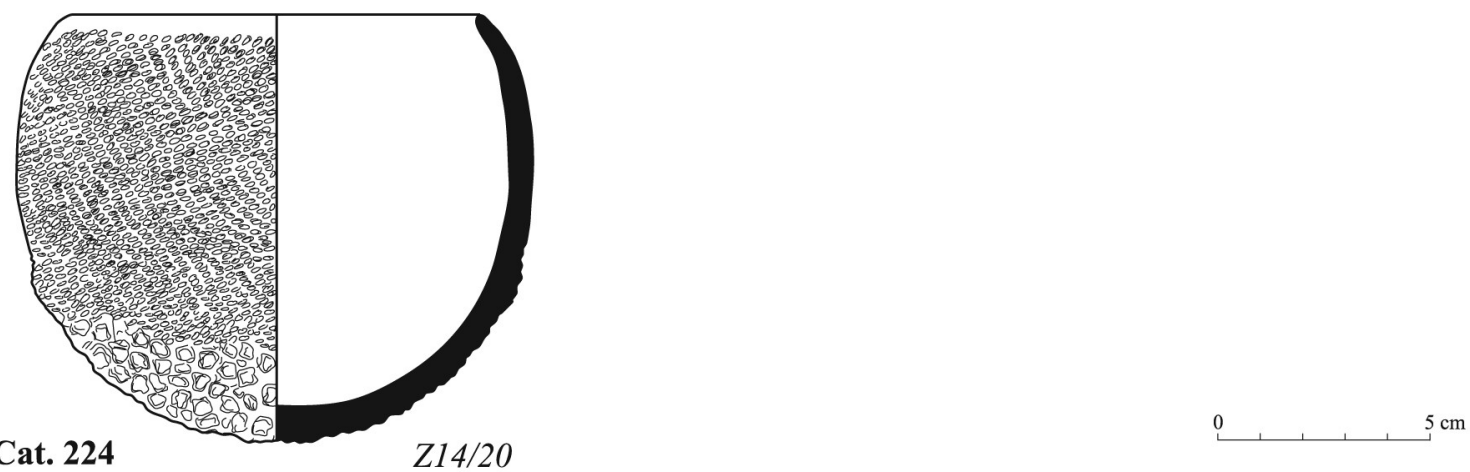

FIGURE 7.49 Deep globular pots of type v.3 with clay added to the base, variant b

Cat. 227

Pot

Inv. no: $\mathbf{Z 2 4 / 3 6}$

Provenance: Tumulus 24, chamber 1, eastern part Construction: handmade (1)

State of preservation: complete

Measurements: $\mathrm{Dr}=12.5 \mathrm{~cm} ; \mathrm{H}=11.2 \mathrm{~cm}$

Fabric: not possible to determine

Hardness: 3
Firing: not possible to determine

Surface treatment: external-mat-impressed, pattern 1; internal-smoothed and burnished

Surface colour: external-10YR 6/3 pale brown; internal1OYR 5/4 yellowish brown

Colour of break: not possible to determine

Decoration: incised decoration on rim

Notes: clay added to base and modelled by hand 


\section{Pots Type V.4}
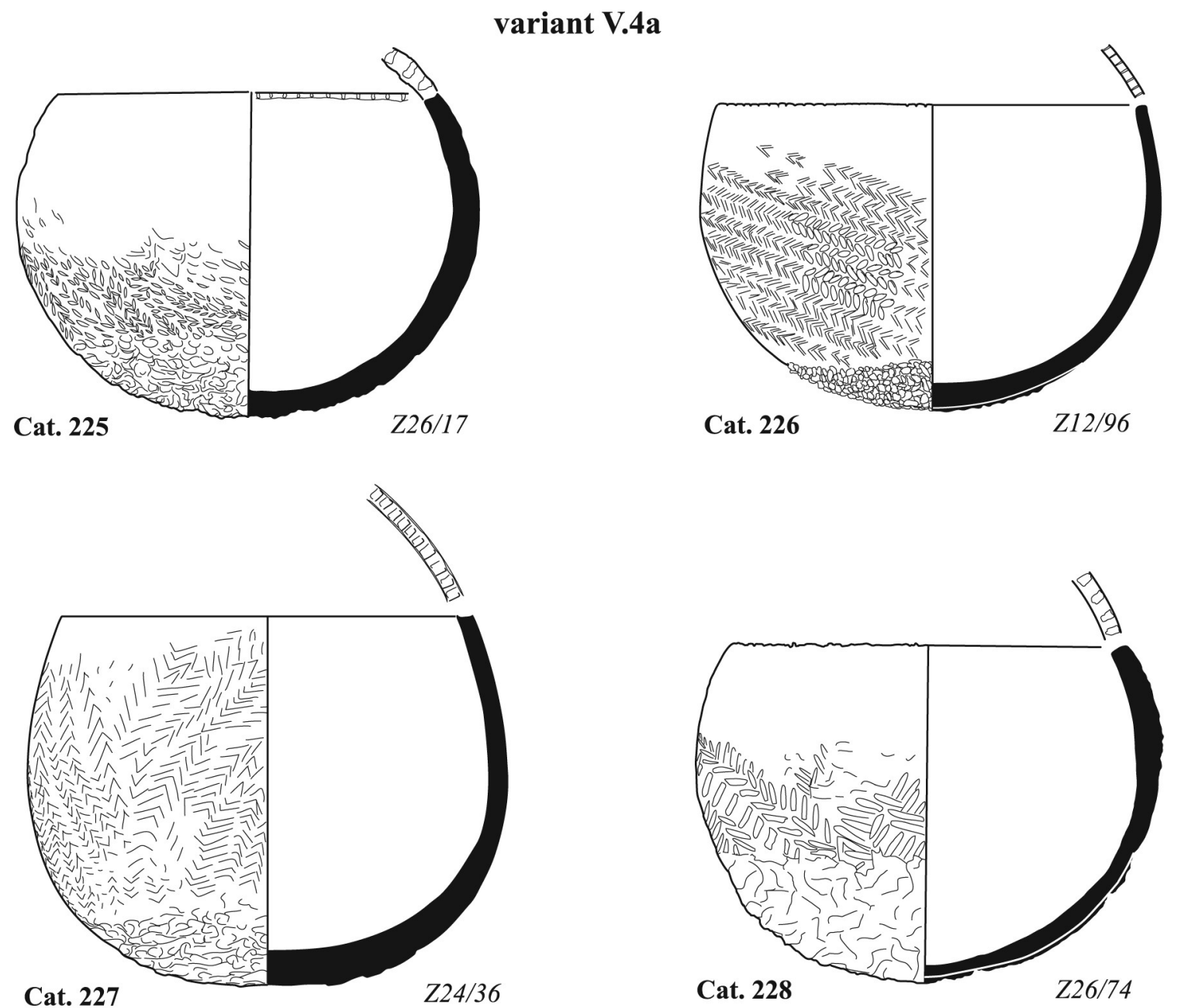

Cat. 227

$Z 24 / 36$

Cat. 228

0

FIGURE 7.50 Deep globular pots of type V.4 with clay added to the base, variant a

Cat. 228

Pot

Inv. no: $\mathbf{Z 2 6 / 7 4}$

Provenance: Tumulus 26, chamber 2, southern part

Construction: handmade (1)

State of preservation: incomplete, $92 \%$ of rim

Measurements: $\mathrm{Dr}=12 \mathrm{~cm} ; \mathrm{H}=10.3 \mathrm{~cm}$

Fabric: ZF1O

Hardness: $2 / 3$

Firing: not possible to determine

Surface treatment: external-mat-impressed, pattern 1; internal-smoothed and burnished

Surface colour: external-7.5YR 5/3 brown; internal7.5YR $5 / 4$ brown

Colour of break: 5 YR 2.5/1 black

Decoration: incised decoration on rim

Notes: clay added to base and modelled by hand
Variant V.4b

Cat. 229

Pot

Inv. no: Z26/59

Provenance: Tumulus 26, chamber 1, central part

Construction: handmade (1)

State of preservation: incomplete, restored from sherds, $98 \%$ of rim

Measurements: $\mathrm{Dr}=12 \mathrm{~cm} ; \mathrm{H}=9.7 \mathrm{~cm}$

Fabric: ZF1O

Hardness: 3

Firing: oxidized

Surface treatment: external-mat-impressed, pattern 1; internal-smoothed

Surface colour: external-5YR 5/6 yellowish red; internal- 5 YR $5 / 3$ reddish brown 
Colour of break: 2.5YR 4/8 red

Decoration: incised decoration on rim

Notes: clay added to base and modelled using a tool

Cat. 230

Pot

Inv. no: $\mathrm{Z26} / 82$

Provenance: Tumulus 26, chamber 4, southern part

Construction: handmade (1)

State of preservation: complete

Measurements: $\mathrm{Dr}=11 \mathrm{~cm} ; \mathrm{H}=9.7 \mathrm{~cm}$

Fabric: not possible to determine

Hardness: 3

Firing: not possible to determine

Surface treatment: external-mat-impressed, pattern 1; internal-smoothed

Surface colour: external-5YR 5/4 reddish brown; internal-5YR $4 / 3$ reddish brown

Colour of break: not possible to determine

Decoration: incised decoration on rim

Notes: clay added to base and modelled using a tool

Cat. 231

Pot

Inv. no: $\mathbf{Z 2 6 / 2 0}$

Provenance: Tumulus 26, chamber 4, the central part

Construction: handmade (1)

State of preservation: complete

Measurements: $\mathrm{Dr}=13 \mathrm{~cm} ; \mathrm{H}=10.4 \mathrm{~cm}$

Fabric: not possible to determine

Hardness: $3 / 4$

Firing: reduced

Surface treatment: external-mat-impressed, pattern 1; internal-smoothed and burnished

Surface colour: external-5YR 4/4 reddish brown; internal-5YR $4 / 6$ yellowish red

Colour of break: not possible to determine

Decoration: incised decoration on rim

Notes: clay added to base and modelled using a tool

Cat. $23^{2}$

Pot

Inv. no: $\mathrm{Z26} / 48$

Provenance: Tumulus 26, chamber 1, central part

Construction: handmade (1)

State of preservation: complete

Measurements: $\mathrm{Dr}=12 \mathrm{~cm} ; \mathrm{H}=11 \mathrm{~cm}$

Fabric: not possible to determine

Hardness: 3

Firing: not possible to determine

Surface treatment: external-mat-impressed, pattern 1; internal—smoothed and burnished
Surface colour: external-5YR 5/4 reddish brown; internal-5YR 4/4 reddish brown

Colour of break: not possible to determine

Decoration: incised decoration on rim

Notes: clay added to base and modelled using a tool

Cat. 233

Pot

Inv. no: $\mathrm{Z26} / 58$

Provenance: Tumulus 26, chamber 1, central part

Construction: handmade (1)

State of preservation: complete

Measurements: $\mathrm{Dr}=14 \mathrm{~cm} ; \mathrm{H}=9.8 \mathrm{~cm}$

Fabric: not possible to determine

Hardness: 3

Firing: not possible to determine

Surface treatment: external-mat-impressed, pattern 1; internal-smoothed and burnished

Surface colour: external-5YR 4/6 yellowish red; internal-5YR $4 / 6$ yellowish red

Colour of break: not possible to determine

Decoration: incised decoration on rim

Notes: clay added to base and modelled using a tool

Variant V.4C

Cat. 234

Pot

Inv. no: $\mathrm{Z28} / 7$

Provenance: Tumulus 28, burial chamber

Construction: handmade (1)

State of preservation: complete

Measurements: $\mathrm{Dr}=21.2 \mathrm{~cm} ; \mathrm{H}=17.8 \mathrm{~cm}$

Fabric: not possible to determine, probably zF1O

Hardness: 3

Firing: reduced

Surface treatment: external-mat-impressed, pattern 2; internal-smoothed

Surface colour: external-2.5YR 4/8 red; internal-2.5YR $4 / 6$ red

Colour of break: not possible to determine

Decoration: incised decoration on rim

Notes: clay added to base and modelled using a tool

7 Group VI-Pots

7.1 Pots Type VI.1

[Fig. 7.111]

Cat. 235

Pot with slipped rim and incised decoration

Inv. no: $\mathrm{Z}_{5} / \mathbf{1 9}$

Provenance: Tumulus 5, tunnel

Construction: handmade (1) 

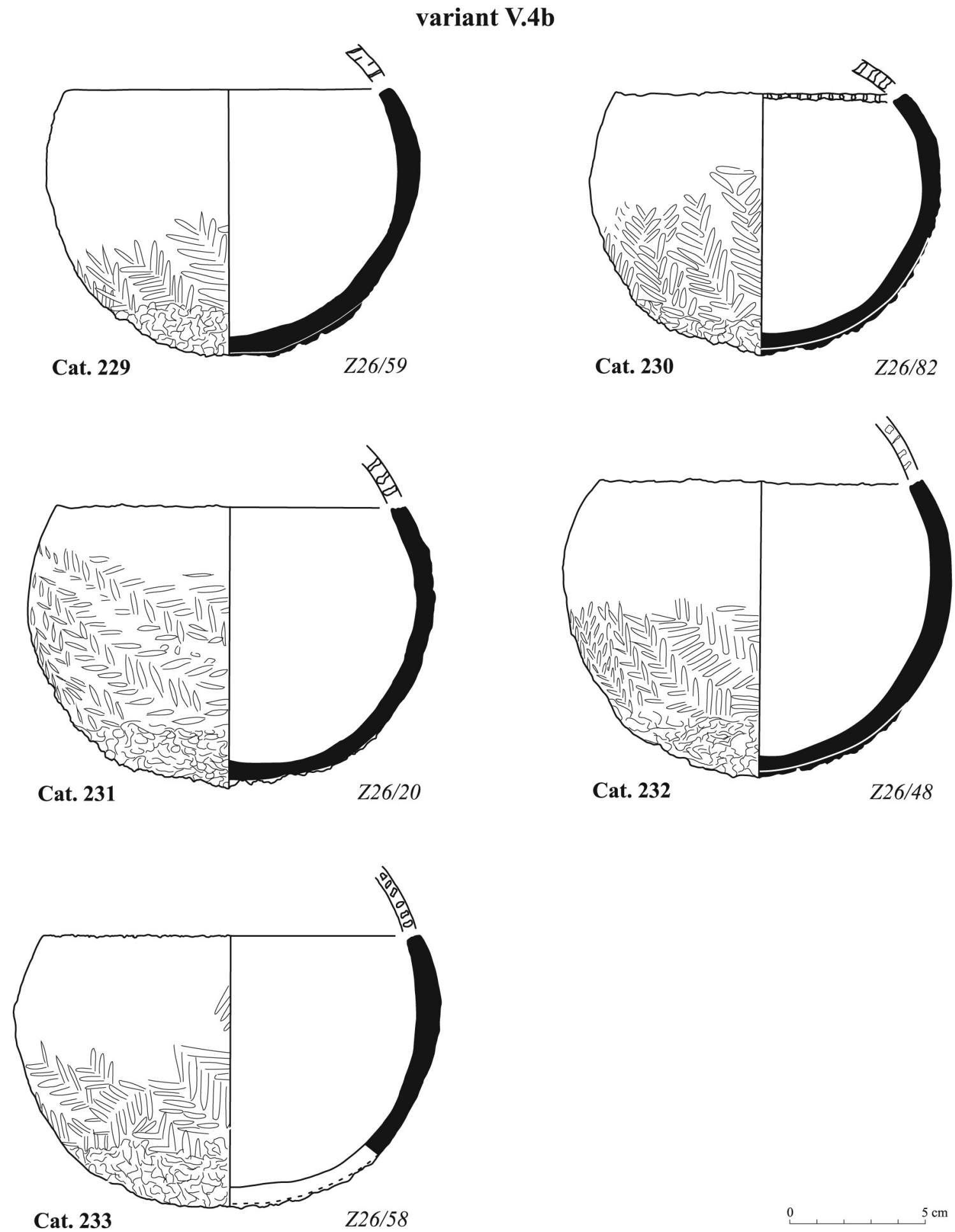

Cat. 232

Z26/48

Deep globular pots of type V.4 with clay added to the base, variant b 


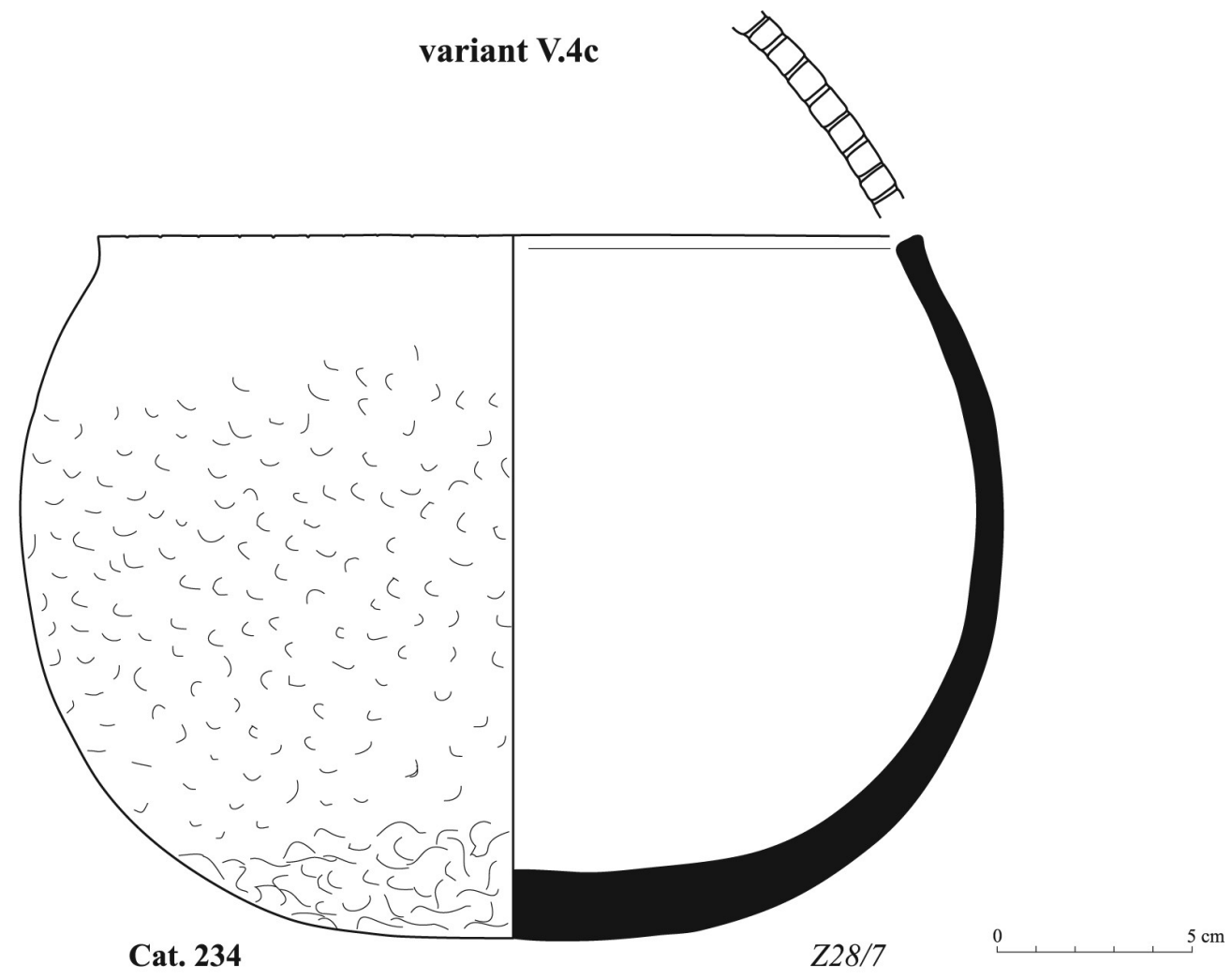

FIGURE 7.52 Deep globular pot of type V.4 with clay added to the base, variant c

State of preservation: fragmentary, $10 \%$ of rim, wall fragment

Measurements: $\mathrm{Dr}=13.2 \mathrm{~cm} ;{ }_{\mathrm{p}} \mathrm{H}=4.4 \mathrm{~cm}$

Fabric: ZF9

Hardness: 3

Firing: reduced

Surface treatment: slipped on rim

Surface colour: external natural surface-5YR 4/6 yellowish red, slip — 1OR 4/6 red; internal -5 YR 3/1 very dark grey

Colour of break: deep black

\section{Cat. 236}

Pot with slipped rim and mat impressions on body

Inv. no: $\mathrm{Z11} / 45$

Provenance: Tumulus 11, fill of shaft

Construction: handmade (1)

State of preservation: fragmentary, restored from pieces, fragments of rim and body, $91 \%$ of rim

Measurements: $\mathrm{Dr}=20.2 \mathrm{~cm} ;{ }_{\mathrm{p}} \mathrm{H}=16 \mathrm{~cm}$

Fabric: ZF9

Hardness: 3

Firing: reduced

Surface treatment: slipped on rim and mat-impressed on body

Surface colour: external natural surface -5 YR 5/4 reddish brown, slip —-10R 4/6 red; internal -7.5 Y $5 / 4$ brown
Colour of break: deep black

References: (Klimaszewska-Drabot and Czyżewska 2012, $368-372)$

\section{Cat. 237}

Pot with slipped rim, mat impressions on body and clay added to bottom

Inv. no: $\mathrm{Z11} / 46$

Provenance: Tumulus 11, fill of shaft

Construction: handmade (1)

State of preservation: fragmentary, restored from sherds, survives in four pieces, fragments of rim, body and base, $55 \%$ of rim

Measurements: $\mathrm{Dr}=20 \mathrm{~cm} ;{ }_{\mathrm{p}} \mathrm{H}=17.6 \mathrm{~cm}$ (upper part) + $7 \mathrm{~cm}$ (bottom part)

Fabric: $\mathrm{ZF9}$

Hardness: 3

Firing: reduced

Surface treatment: slipped on rim, mat-impressed on body

Surface colour: external natural surface $-5 \mathrm{YR} 5 / 4$ reddish brown, slip-10R 4/6 red; internal-7.5YR 5/4 brown

Colour of break: ${ }_{5} \mathrm{R}$ 2.5/1 reddish black

Notes: clay at bottom of vessel was added by pinching

References: (Klimaszewska-Drabot and Czyżewska 2012, 368-372) 


\section{Pots Type VI.1}

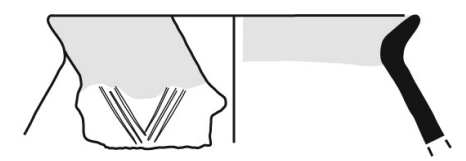

Cat. 235
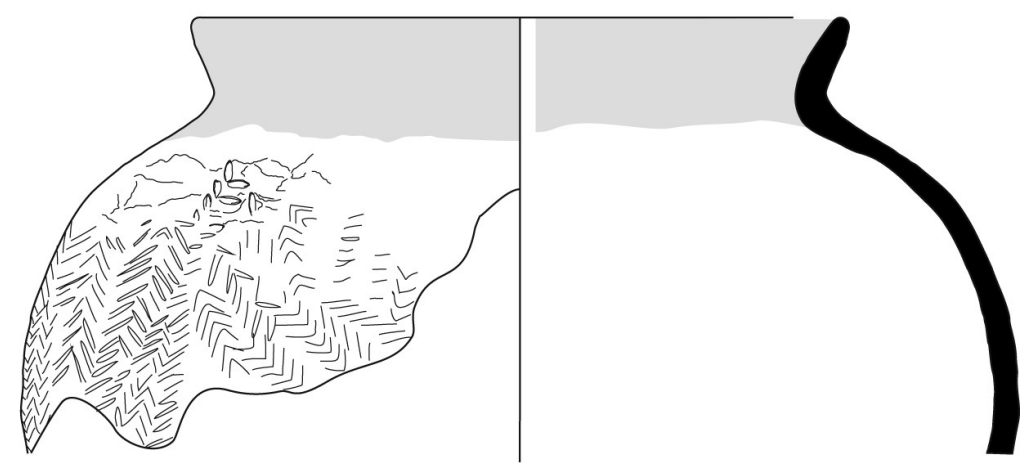

Cat. 236

Z11/45

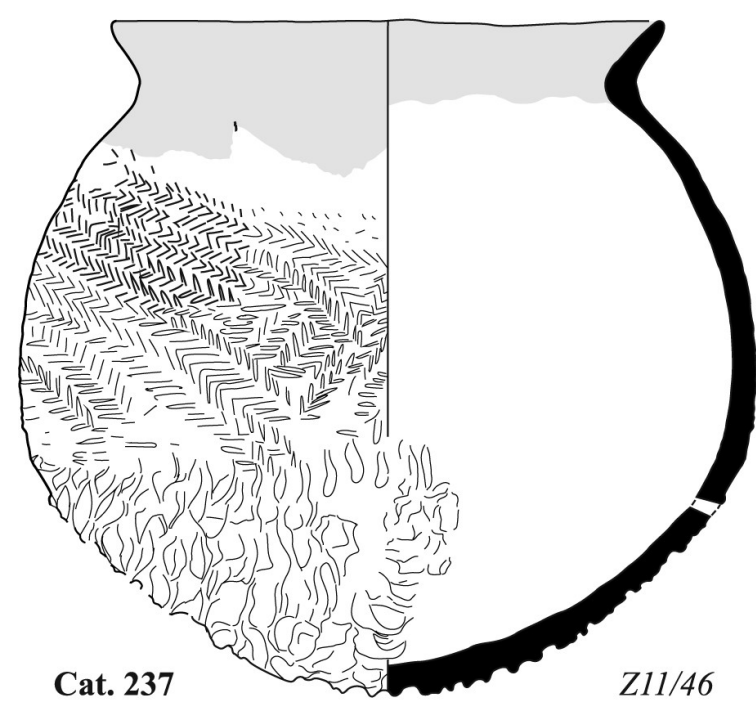

0 $5 \mathrm{~cm}$

FIGURE 7.53 Pots of type VI.1

7.2 Pots Type VI.2

Cat. 238

Pot

Inv. no: Z27/12

Provenance: Tumulus 27, superstructure

Construction: handmade (1)

State of preservation: fragmentary, sherds, $8 \%$ of rim

Measurements: $\mathrm{Dr}=16 \mathrm{~cm} ;{ }_{\mathrm{p}} \mathrm{H}=26.8 \mathrm{~cm}$

Fabric: ZF1O

Hardness: 3

Firing: reduced

Surface treatment: slipped external surface

Surface colour: external, slip_-10R 3/3 dusky red; internal-2.5YR 2.5/1 reddish black

Colour of break: deep black

\section{Cat. 239}

Pot

Inv. no: $\mathrm{Z11} / 48$
[Fig. 7.111] Provenance: Tumulus 11, fill of shaft Construction: handmade (1)

State of preservation: fragmentary, restored from sherds, rim and wall fragments, $69 \%$ of rim

Measurements: $\mathrm{Dr}=25.5 \mathrm{~cm} ;{ }_{\mathrm{p}} \mathrm{H}=16.3 \mathrm{~cm}$

Fabric: ZF9

Hardness: $2 / 3$

Firing: reduced

Surface treatment: slipped on both surfaces

Surface colour: external, slip-10R 5/6 red; internal1oR $5 / 6$ red

Colour of break: $5_{\mathrm{R}}$ 2.5/1 reddish black

References: (Klimaszewska-Drabot and Czyżewska 2012, $368-372)$

\subsection{Pots Type VI.3}

[Fig. 7.111]

\section{Cat. 240}

Pot

Inv. no: $\mathrm{Z11} / 44$ 
Provenance: Tumulus 11, fill of shaft

Construction: handmade (1)

State of preservation: fragmentary, restored from sherds, survives in three pieces, $75 \%$ of rim

Measurements: $\mathrm{Dr}=15.5 \mathrm{~cm} ; \mathrm{H}=24 \mathrm{~cm}$

Fabric: ZF9

Hardness: 3

Firing: reduced

Surface treatment: slipped on rim, mat-impressed body

Surface colour: external natural surface- 5 YR $4 / 4$ reddish brown, slip-10R 5/6 red; internal -5 Y R 5/4 reddish brown

Colour of break: deep black

Notes: four horizontal bosses added below rim; clay added to bottom of vessel by hand using different method to that noted on Z11/46

References: (Klimaszewska-Drabot and Czyżewska 2012, 368-372)

\section{Group VII-Jars/Bottles}

\subsection{Jars Type VII.1}

Variant VII.1a

Cat. 241

Jar (small, broad)

Inv. no: $\mathbf{Z 1 8} 8$

Provenance: Tumulus 18, burial chamber, southern part

Construction: handmade

State of preservation: incomplete, $77 \%$ of rim

Measurements: $\operatorname{Dr}=7.4 \mathrm{~cm} ; \max$ body $\operatorname{Dr}=19.4 \mathrm{~cm}$;

$$
\mathrm{H}=23.5 \mathrm{~cm}
$$

Fabric: ZF9

Hardness: $2 / 3$

Firing: reduced

Surface treatment: uncoated on both surfaces

Surface colour: external—5YR 6/4 light reddish brown

Colour of break: deep black

References: (Klimaszewska-Drabot 2010, 483-484)

\section{Cat. 242}

Jar (medium-sized, broad)

Inv. no: $\mathbf{Z} 20 / 5$

Provenance: Tumulus 20, burial chamber, central part

Construction: handmade

State of preservation: incomplete, $55 \%$ of rim, body sherds missing

Measurements: $\operatorname{Dr}=10.7 \mathrm{~cm} ; \max$ body diam. $=34 \mathrm{~cm}$; $\mathrm{H}=36.8 \mathrm{~cm}$

Fabric: ZF1O

Hardness: 3
Firing: reduced

Surface treatment: slipped and burnished on external surface

Surface colour: external, slip-10R 4/4 weak red

Colour of break: deep black

References: (Mahmoud El-Tayeb, Juszczyk-Futkowska, and Czyżewska 2014, 367-368)

Variant VII..1b

Cat. 243

Jar (large, broad)

Inv. no: $\mathbf{Z} \mathbf{9} / \mathbf{3}$

Provenance: Tumulus 9, chamber 3, southern part

Construction: handmade

State of preservation: incomplete, body sherd missing

Measurements: $\mathrm{Dr}=9.3 \mathrm{~cm} ; \max$ body diam. $=40 \mathrm{~cm}$; $\mathrm{H}=48 \mathrm{~cm}$

Fabric: ZF9

Hardness: 3

Firing: reduced

Surface treatment: slipped and burnished on external surface

Surface colour: external, slip-1oR 4/6 red; internal natural surface-deep black

Colour of break: deep black

Decoration: scratched—on belly and shoulder, one drawing, geometrical motif

References: (Mahmoud El-Tayeb, Juszczyk-Futkowska, and Czyżewska 2014, 367-368)

Cat. 244

Jar (medium-sized, broad)

Inv. no: $\mathrm{Z18} / 8$

Provenance: Tumulus 28 , burial chamber, southern part

Construction: handmade

State of preservation: complete

Measurements: $\mathrm{Dr}=5.2 \mathrm{~cm} ; \max$ body diam. $=30 \mathrm{~cm}$; $\mathrm{H}=34.1 \mathrm{~cm}$

Fabric: not possible to determine

Hardness: $2 / 3$

Firing: reduced

Surface treatment: slipped and burnished on external surface

Surface colour: external, slip $-2.5 \mathrm{Y} 3 / 1$ very dark grey

Colour of break: not possible to determine

Variant VII.1c

Cat. 245

Jar (medium-sized, broad)

Inv. no: Z28/9

Provenance: Tumulus 28, chamber 1, western part 


\section{Pots Type VI.2}
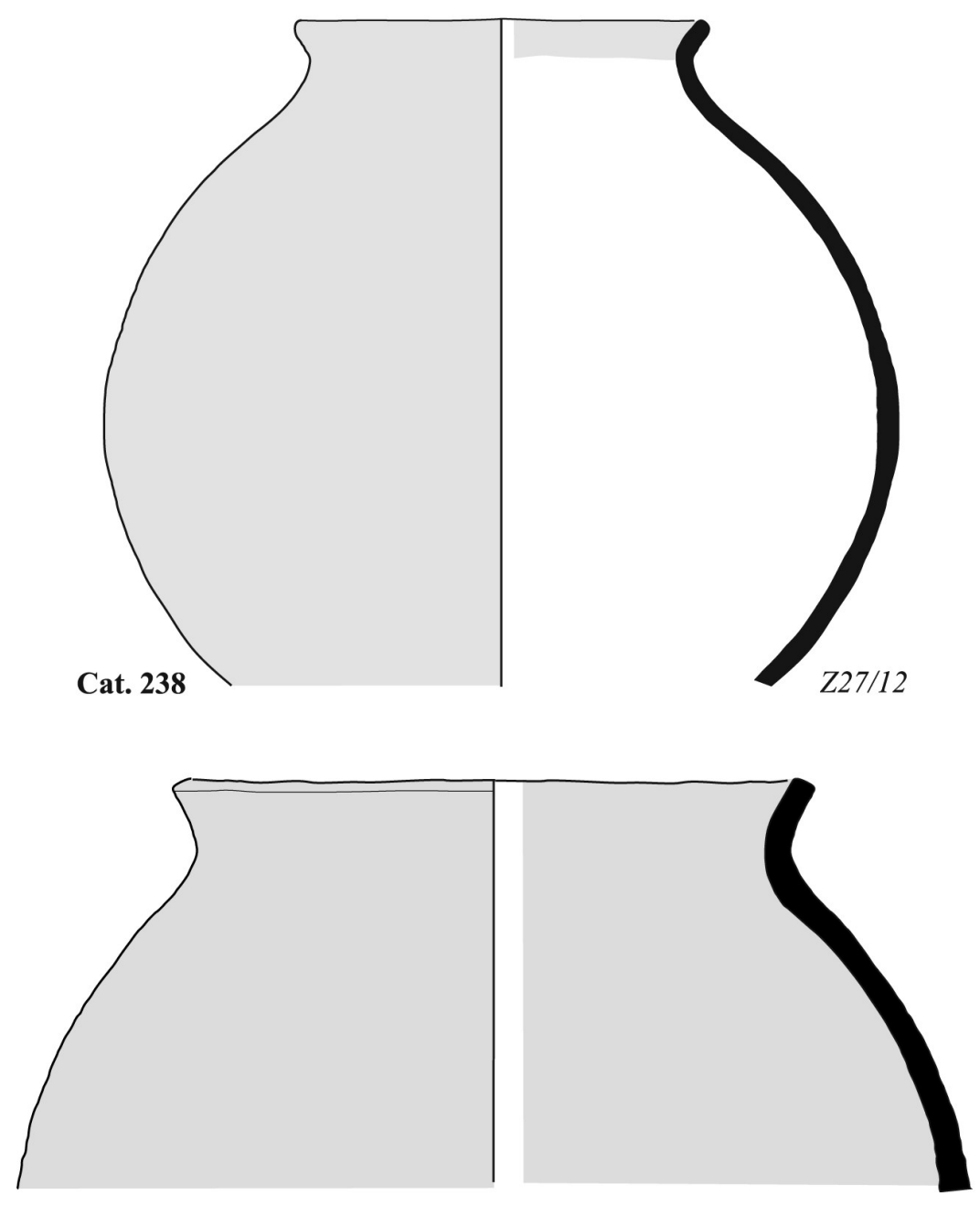

Cat. 239

$211 / 48$

Pots Type VI.3

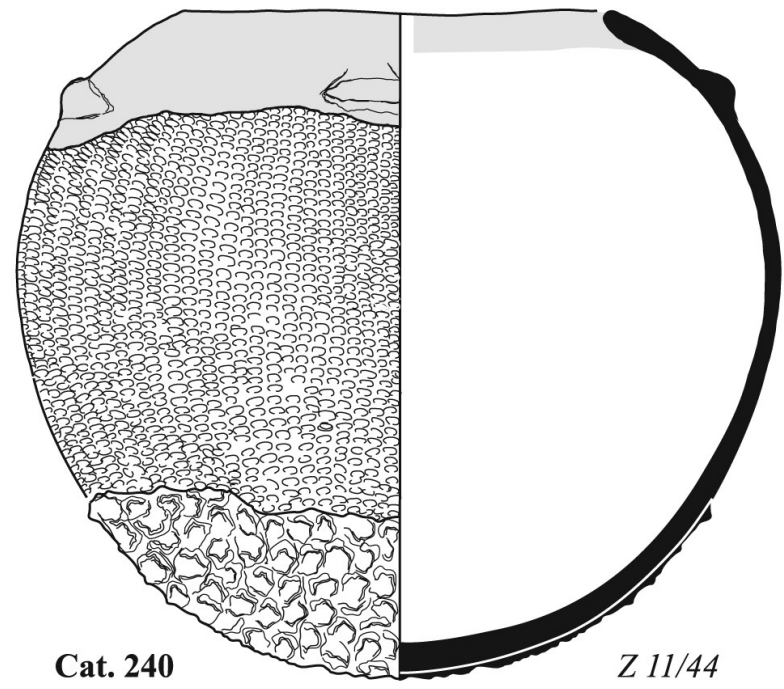

$\underbrace{0}$ $5 \mathrm{~cm}$

Pots of types VI.2 and VI.3 
240

CHAPTER 7

Jars Type VII.1
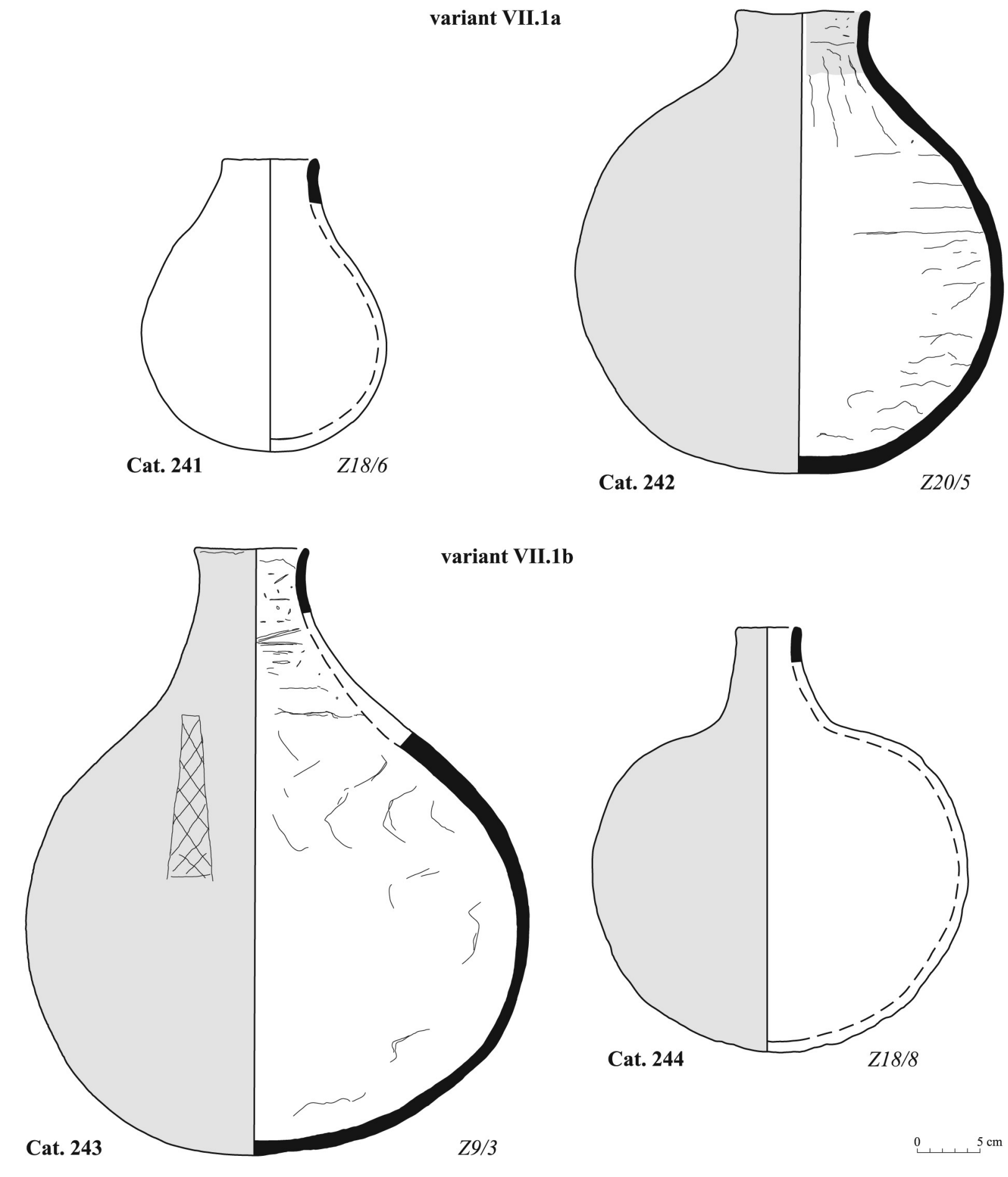

FIGURE 7.55 Bag-shaped jars of type VII.1, variants a and b

Mahmoud El-Tayeb and Eva Czyżewska-Zalewska - 9789004433755

Downloaded from Brill.com๑4/26/2023 01:09:34 PM

via free access 
Construction: handmade

State of preservation: complete

Measurements: $\operatorname{Dr}=5.3 \mathrm{~cm} ; \max$ body diam. $=27 \mathrm{~cm}$; $\mathrm{H}=31.8 \mathrm{~cm}$

Fabric: not possible to determine

Hardness: 3

Firing: reduced

Surface treatment: slipped and burnished on external surface

Surface colour: external, slip-10R $5 / 8 \mathrm{red}$

Colour of break: not possible to determine

Cat. 246

Jar (large, broad)

Inv. no: $\mathrm{Z12} / 71+\mathrm{Z12} / 78$

Provenance: Tumulus 12, chamber 1, central part

Construction: handmade

State of preservation: incomplete, restored from sherds, rim missing, body fragments missing

Measurements: max body diam. $=40 \mathrm{~cm} ;{ }_{\mathrm{p}} \mathrm{H}=45.5 \mathrm{~cm}$

Fabric: ZF11

Hardness: 3

Firing: reduced

Surface treatment: slipped, burnished on external surface and mat-impressed

Surface colour: external, slip-7.5YR 2.5/2 very dark brown

Colour of break: Gley 1 2.5/N black

Notes: mat impression visible beneath slip; evidence of repairs to vessel-holes executed in neck

References: (Czyżewska-Zalewska 2016, 735-737)

Cat. 247

Jar (small, broad)

Inv. no: $\mathbf{Z 2 8 / 6}$

Provenance: Tumulus 28, chamber 1, eastern part

Construction: handmade

State of preservation: almost complete, $95 \%$ of rim

Measurements: $\mathrm{Dr}=5.3 \mathrm{~cm} ; \max$ body diam. $=24 \mathrm{~cm}$; $\mathrm{H}=25.4 \mathrm{~cm}$

Fabric: ZF1O

Hardness: 3

Firing: reduced

Surface treatment: slipped and burnished on external surface

Surface colour: external, slip-10R $5 / 6$ red

Colour of break: not possible to determine

Decoration: applied-four small bosses on shoulder

Variant VII.1d

Cat. 248

Jar (medium-sized, broad)
Inv. no: $\mathrm{Z} 24 / 39$

Provenance: Tumulus 24, chamber 3, southern part

Construction: handmade

State of preservation: almost complete, $75 \%$ of rim

Measurements: $\mathrm{Dr}=8 \mathrm{~cm} ; \max$ body diam. $=29 \mathrm{~cm}$;

$$
\mathrm{H}=35 \mathrm{~cm}
$$

Fabric: ZF1O

Hardness: $3 / 4$

Firing: reduced

Surface treatment: slipped and burnished neck; matimpressed, pattern 1

Surface colour: external natural surface -7.5 YR 6/4 light brown, slip- $7.5 \mathrm{R} 4 / 6$ red

Colour of break: Gleyı 2.5/N black

Decoration: slip on neck and band of slip on shoulder with mat-impressed pattern on body

Cat. 249

Jar (large, broad)

Inv. no: $\mathbf{Z 1 2} / 72$

Provenance: Tumulus 12, chamber 1, eastern part

Construction: handmade

State of preservation: fragmentary, restored from sherds, upper part of vessel, rim missing

Measurements: max body diam. $=49 \mathrm{~cm} ;{ }_{\mathrm{p}} \mathrm{H}=42 \mathrm{~cm}$

Fabric: ZF9

Hardness: $3 / 4$

Firing: reduced

Surface treatment: slipped and burnished neck; matimpressed, pattern 3

Surface colour: external natural surface-2.5YR 6/8 light red, slip $-7.5 \mathrm{R}$ 4/4 weak red; internal-1OYR $7 / 3$ very pale brown

Colour of break: deep black

Decoration: slip on neck and band of slip on shoulder with mat-impressed pattern on body

References: (Czyżewska-Zalewska 2016, 735-737)

8.2 Jars Type VII.2

[Figs 7.114-7.115]

Variant VII.2a

Cat. $25^{\circ}$

Jar (small, broad)

Inv. no: $\mathbf{Z 1 8} / 7$

Provenance: Tumulus 18, burial chamber, northern part

Construction: handmade

State of preservation: incomplete, $50 \%$ of rim, fragments of bottom part of vessel missing

Measurements: Dr $=5.2 \mathrm{~cm} ;$ max body diam. $=16.6 \mathrm{~cm}$; $\mathrm{H}=19.1 \mathrm{~cm}$

Fabric: ZF9

Hardness: soft (2) 

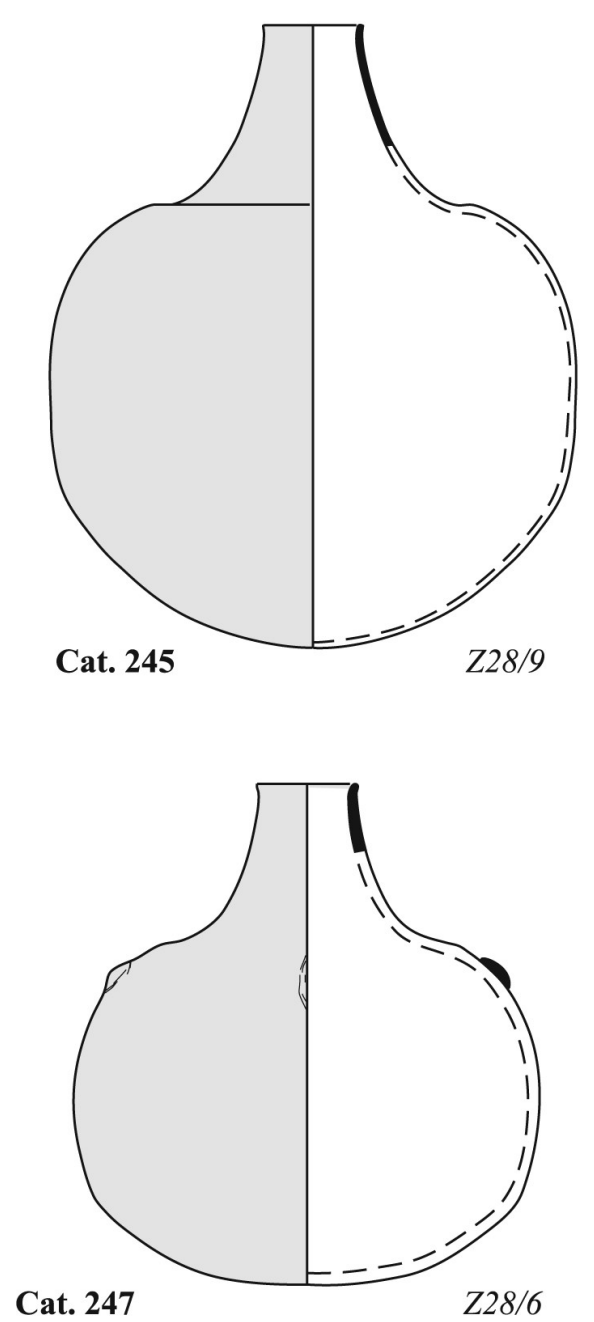

\section{Cat. 248}

Z24/39

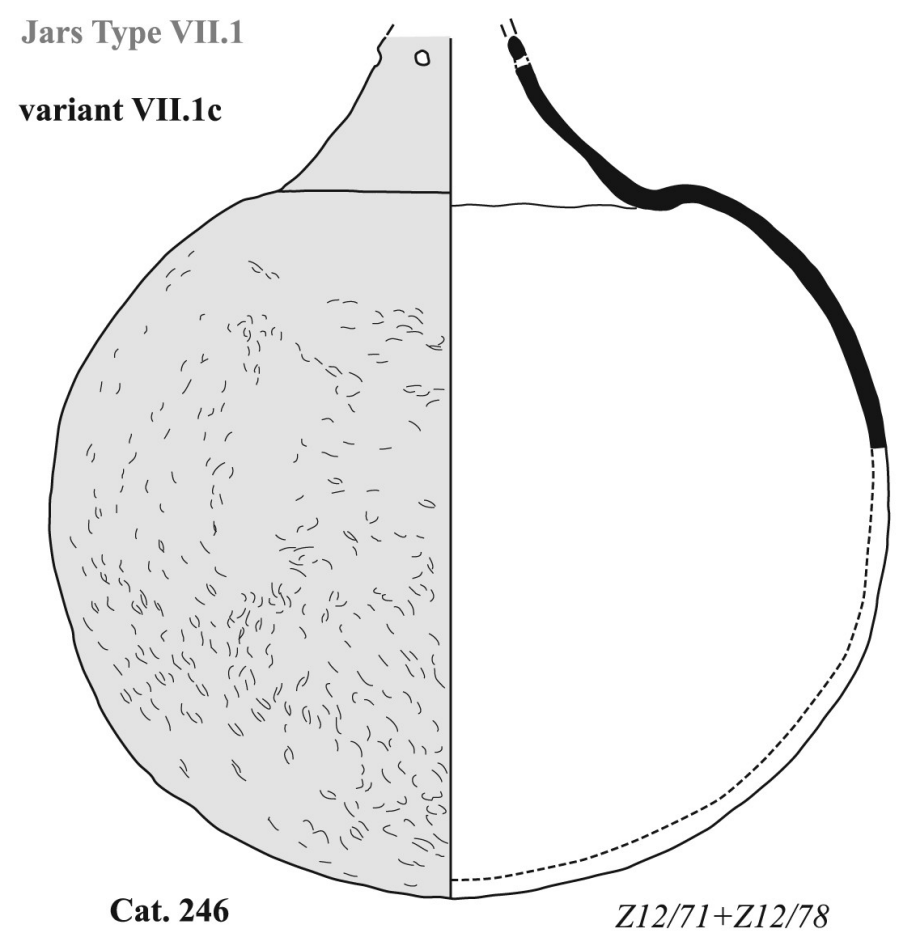

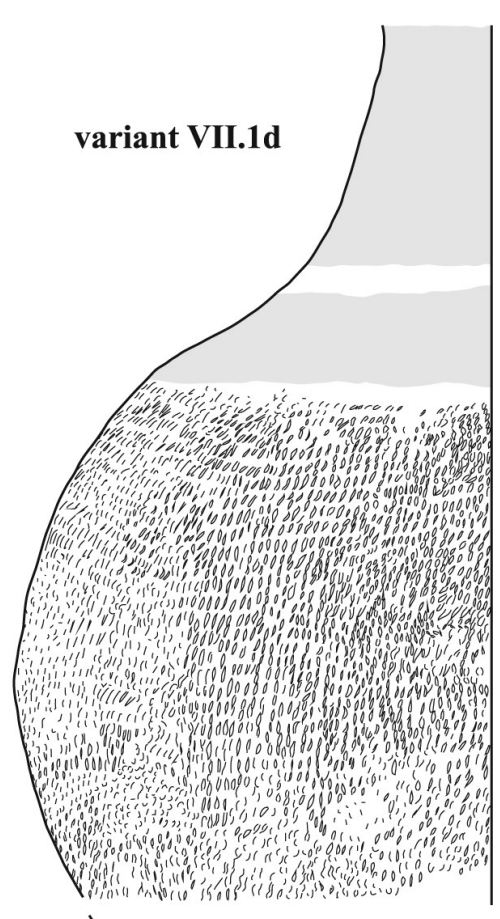

Cat. 249
Z12/72

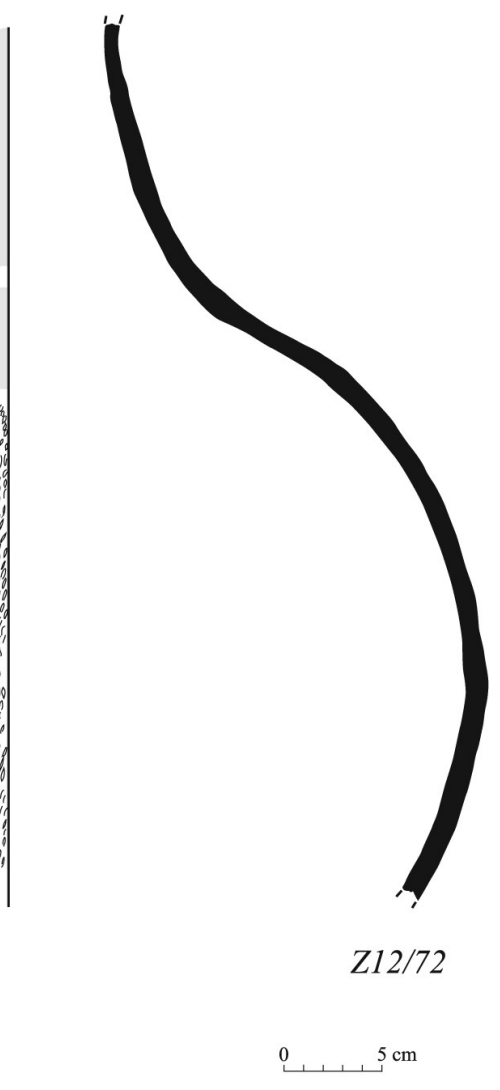

FIGURE 7.56

Bag-shaped jars of type viI.1, variants $\mathrm{c}$ and d 
Firing: reduced

Surface treatment: uncoated on both surfaces

Surface colour: external-1OYR 5/3 brown

Colour of break: deep black

References: (Klimaszewska-Drabot 2010, 483-484)

Cat. 251

Jar (large, broad)

Inv. no: $\mathbf{Z 1 3} / \mathbf{2 1}$

Provenance: Tumulus 13, chamber 2, northern part

Construction: handmade

State of preservation: complete

Measurements: $\mathrm{Dr}=6 \mathrm{~cm} ; \max$ body diam. $=30.5 \mathrm{~cm}$; $\mathrm{H}=40 \mathrm{~cm}$

Fabric: not possible to determine

Hardness: 3

Firing: reduced

Surface treatment: uncoated on both surfaces

Surface colour: external-1oR $5 / 6$ red

Colour of break: not possible to determine

References: (Klimaszewska-Drabot and Czyżewska 2012, $368-372)$

Cat. $25^{2}$

Jar (medium-sized/large, broad)

Inv. no: Z12/135

Provenance: Tumulus 12, shaft

Construction: handmade

State of preservation: incomplete, $98 \%$ of rim, restored from sherds, some body sherds missing

Measurements: Dr $=7 \mathrm{~cm} ; \max$ body diam. $=33 \mathrm{~cm}$; $\mathrm{H}=39.5 \mathrm{~cm}$

Fabric: ZF9

Hardness: 3

Firing: reduced

Surface treatment: uncoated on both surfaces

Surface colour: external-5YR 6/6 reddish yellow-2.5YR $5.6 \mathrm{red}-2.5 \mathrm{YR} 5 / 8 \mathrm{red}$

Colour of break: deep black

Cat. 253

Jar (small, broad)

Inv. no: Z21/9

Provenance: Tumulus 21, burial chamber, western part

Construction: handmade

State of preservation: complete

Measurements: Dr $=5 \mathrm{~cm} ;$ max body diam. $=17.3 \mathrm{~cm}$; $\mathrm{H}=21 \mathrm{~cm}$

Fabric: not possible to determine

Hardness: $3 / 4$

Firing: reduced
Surface treatment: slipped and burnished on external surface

Surface colour: external, slip -5 Y 2.5/1 black

Colour of break: not possible to determine

\section{Cat. 254}

Jar (small, broad)

Inv. no: $\mathrm{Z} 17 / 2$

Provenance: Tumulus 17, burial chamber, southern part

Construction: handmade

State of preservation: complete

Measurements: $\mathrm{Dr}=4.5 \mathrm{~cm} ; \max$ body diam. $=19 \mathrm{~cm}$; $\mathrm{H}=22.2 \mathrm{~cm}$

Fabric: not possible to determine

Hardness: 3

Firing: reduced

Surface treatment: slipped and burnished on external surface

Surface colour: external, slip-Gleyı 2.5/N black

Colour of break: not possible to determine

Decoration: scratched-on belly and shoulder, two drawings, floral and geometrical motifs

References: (Klimaszewska-Drabot and Czyżewska 2012, $362-363)$

Variant ViI.2b

Cat. 255

Jar (large, broad)

Inv. no: Z12/134

Provenance: Tumulus 12, shaft

Construction: handmade

State of preservation: incomplete, body fragments missing

Measurements: Dr $=7.3 \mathrm{~cm} ;$ max body diam. $=45 \mathrm{~cm}$; $\mathrm{H}=44 \mathrm{~cm}$

Fabric: ZF9

Hardness: 3

Firing: reduced

Surface treatment: slipped and burnished on the external surface

Surface colour: external natural surface-2.5YR 5/6 red, slip $-7.5^{\mathrm{R}}$ 5/6 red; internal-Gley 1 2.5/1 greenish black Colour of break: deep black

Decoration: applied - two small bosses on shoulder

References: (Czyżewska-Zalewska 2016, 730-733)

Cat. 256

Jar (large, broad)

Inv. no: $\mathrm{Z} 24 / 40$

Provenance: Tumulus 24, chamber 4, central part

Construction: handmade

State of preservation: complete 


\section{Jars Type VII.2}
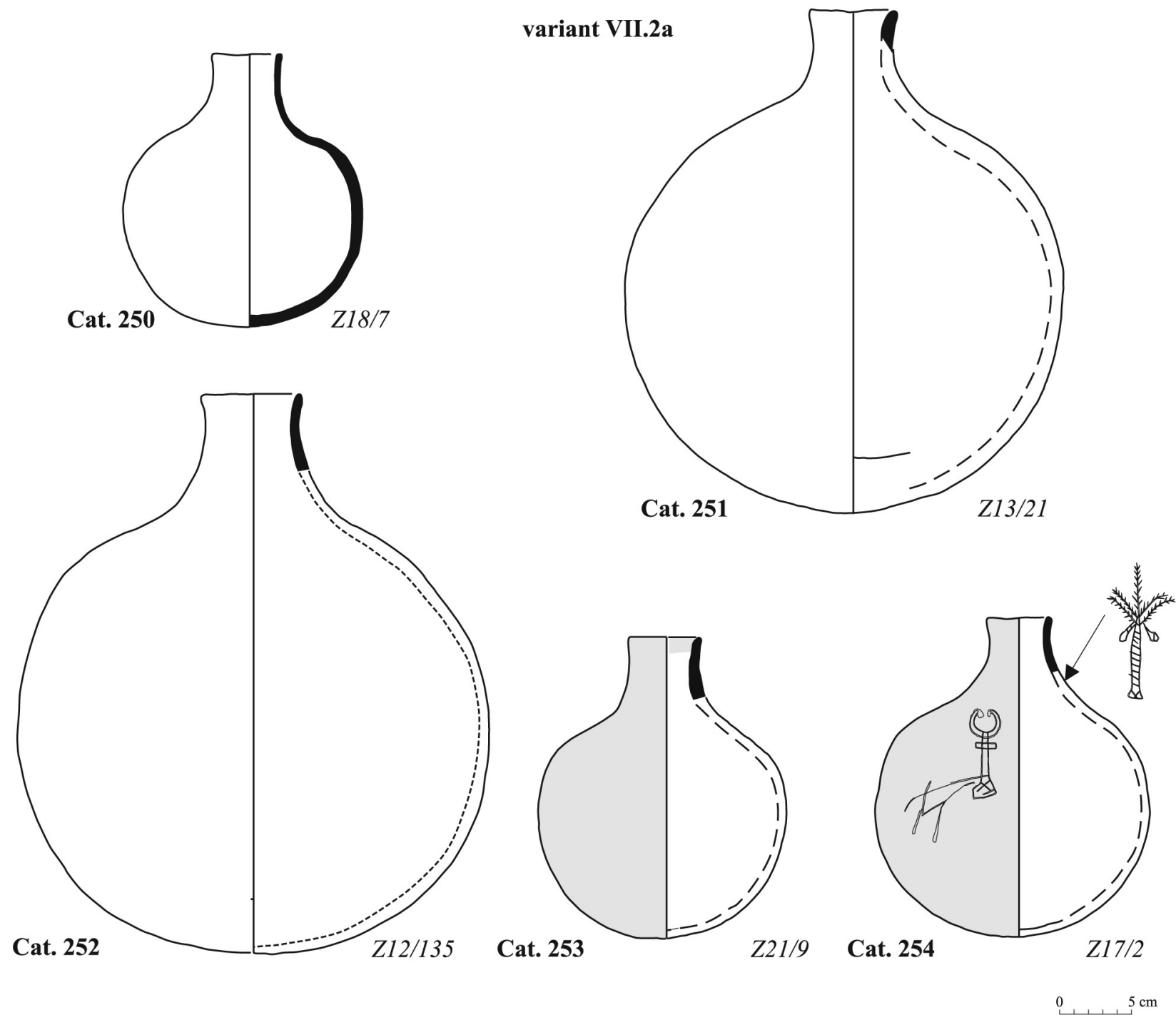

FIGURE 7.57 Globular jars of type viI.2, variant a

Measurements: $\operatorname{Dr}=8.5 \mathrm{~cm} ; \max$ body diam. $=40 \mathrm{~cm}$;

$$
\mathrm{H}=47 \mathrm{~cm}
$$

Fabric: not possible to determine

Hardness: 3

Firing: reduced

Surface treatment: slipped and burnished on external surface

Surface colour: external, slip-10YR 4/6 dark yellowish brown

Colour of break: not possible to determine

Decoration: applied—two small bosses on shoulder

\section{Variant VII.2C}

Cat. 257

Jar (medium-sized, broad)

Inv. no: Z15/52
Provenance: Tumulus 15, chamber 1, eastern part Construction: handmade

State of preservation: incomplete, upper part of vessel Measurements: $\operatorname{Dr}=8 \mathrm{~cm}$; $\max$ body diam. $=26.5 \mathrm{~cm}$; ${ }_{\mathrm{p}} \mathrm{H}=33 \mathrm{~cm}$

Fabric: ZF7

Hardness: 3

Firing: reduced

Surface treatment: mat-impressed on external surface, pattern 7

Surface colour: external natural surface -5 YR $6 / 6$ reddish yellow

Colour of break: 5YR 3/1 very dark grey

Decoration: incised-geometrical motifs below rim and wavy lines on neck of vessel 


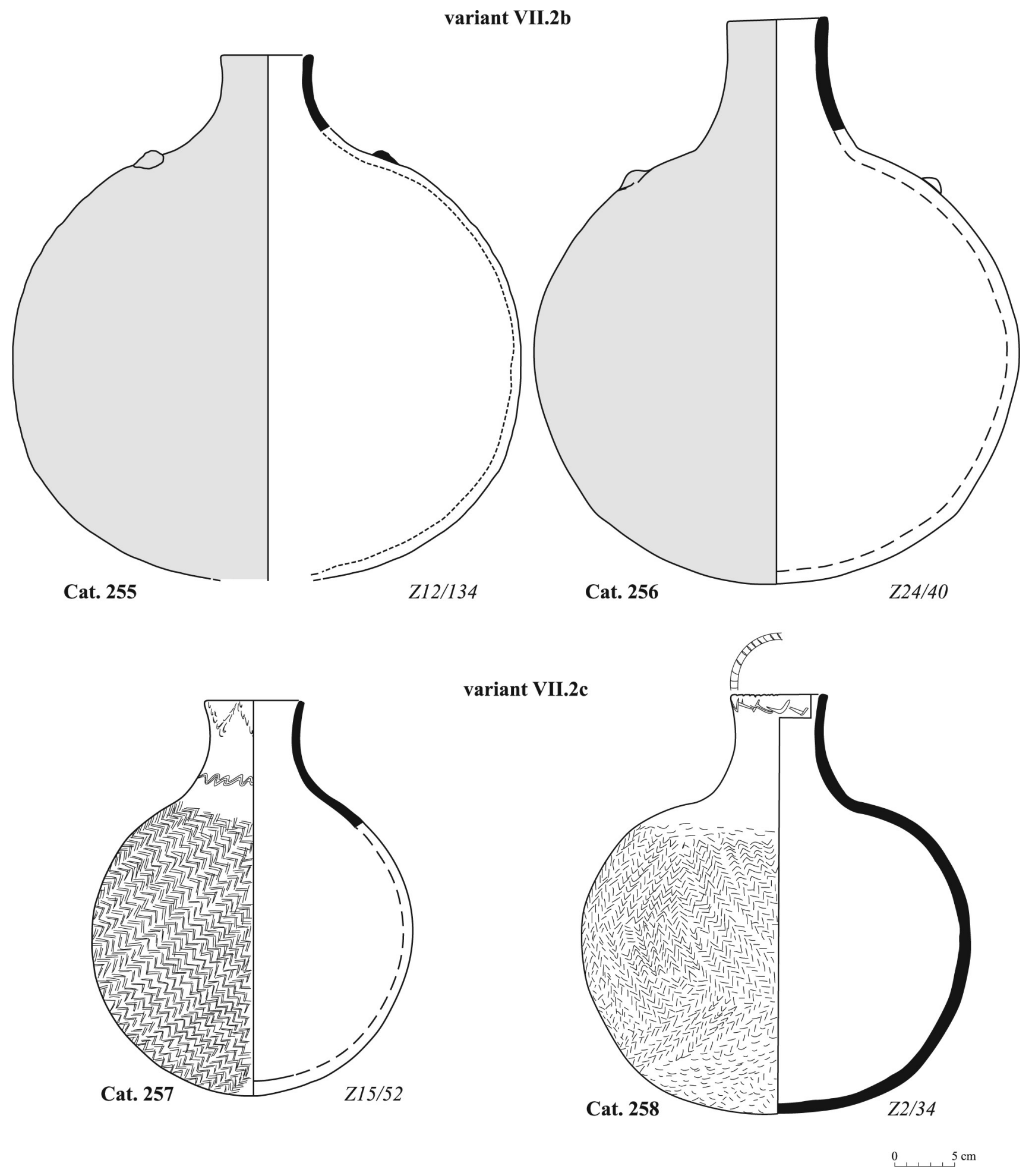

FIGURE 7.58 Globular jars of type viI.2, variants b and c

\section{Cat. 258}

Jar (medium-sized, medium broad)

Inv. no: $\mathrm{Z2} / 34$

Provenance: Tumulus 2, chamber 2

Construction: handmade

State of preservation: complete
Measurements: $\operatorname{Dr}=8 \mathrm{~cm} ; \max$ body diam. $=3^{2 \mathrm{~cm}}$; $\mathrm{H}=35 \mathrm{~cm}$

Fabric: not possible to determine

Hardness: 3

Firing: reduced

Surface treatment: mat-impressed on external surface, pattern 1 
Surface colour: external natural surface-7.5YR 6/4 light brown

Colour of break: not possible to determine

Decoration: incised-geometrical motifs below rim and strokes on rim of vessel

References: (Klimaszewska-Drabot and Mahmoud ElTayeb 2014, 13-15)

\subsection{Jars Type VII.3}

Variant viI.3a

\section{Cat. 259}

Jar (large, broad)

Inv. no: Z12/24.1

Provenance: Tumulus 24, fill of shaft, south-eastern part

Construction: handmade

State of preservation: fragmentary, $24 \%$ of rim, upper part of vessel

Measurements: $\mathrm{Dr}=6.5 \mathrm{~cm} ; \max$ body diam. $=44.5 \mathrm{~cm}$;

$$
{ }_{\mathrm{p}} \mathrm{H}=28.5 \mathrm{~cm}
$$

Fabric: ZF9

Hardness: 3

Firing: reduced

Surface treatment: slipped and burnished on external surface

Surface colour: external natural surface-5YR 6/6 reddish yellow, slip- $10 \mathrm{R} 5 / 6 \mathrm{red}-1 \mathrm{oR} 4 / 6 \mathrm{red}-1 \mathrm{OR} 4 / 8$ red; internal natural surface -5 YR $5 / 6$ yellowish red$2.5 \mathrm{Y} 2.5 / 1$ black

Colour of break: deep black

Variant VII.3b

\section{Cat. 26o}

Jar (large, broad)

Inv. no: Z2/39

Provenance: Tumulus 2, chamber 3

Construction: handmade

State of preservation: almost complete, $94 \%$ of rim

Measurements: $\mathrm{Dr}=5.5 \mathrm{~cm} ;$ max body diam. $=36.4 \mathrm{~cm}$; $\mathrm{H}=42.8 \mathrm{~cm}$

Fabric: not possible to determine

Hardness: $3 / 4$

Firing: reduced

Surface treatment: mat-impressed on external surface, pattern 1

Surface colour: external natural surface-7.5YR 6/4 light brown

Colour of break: not possible to determine

Decoration: incised-herringbone pattern below rim; punctuated - oval shapes on shoulder

References: (Klimaszewska-Drabot and Mahmoud ElTayeb 2014, 13-15)
Cat. 261

Jar (large, broad)

Inv. no: Z14/9

Provenance: Tumulus 14, chamber 3, central part

Construction: handmade

State of preservation: complete

Measurements: $\mathrm{Dr}=6.8 \mathrm{~cm} ; \max$ body diam. $=46 \mathrm{~cm}$; $\mathrm{H}=49 \mathrm{~cm}$

Fabric: not possible to determine

Hardness: 3

Firing: not possible to determine

Surface treatment: mat-impressed on external surface, pattern 1

Surface colour: external natural surface -2.5 YR 5/8 red

Colour of break: not possible to determine

\subsection{Bottles Type VII.4}

[Figs 7.117-7.118]

Variant VII.4a

Cat. 262

Bottle (medium-sized, medium broad)

Inv. no: $\mathrm{Z} 2 / 36$

Provenance: Tumulus 2, chamber 3

Construction: handmade

State of preservation: almost complete, $5 \%$ of rim

Measurements: $\mathrm{Dr}=7.6 \mathrm{~cm} ; \max$ body diam. $=27 \mathrm{~cm}$; $\mathrm{H}=34.5 \mathrm{~cm}$

Fabric: not possible to determine

Hardness: 3

Firing: not possible to determine

Surface treatment: mat-impressed on external surface, pattern 4

Surface colour: external natural surface -5 YR 5/4 reddish brown

Colour of break: not possible to determine

References: (Klimaszewska-Drabot and Mahmoud ElTayeb 2014, 13-15)

Cat. 263

Bottle (large, broad)

Inv. no: $\mathbf{Z 2} / 38$

Provenance: Tumulus 2, chamber 3

Construction: handmade

State of preservation: complete

Measurements: $\mathrm{Dr}=8.6 \mathrm{~cm} ; \max$ body diam. $=40 \mathrm{~cm}$; $\mathrm{H}=48.5 \mathrm{~cm}$

Fabric: not possible to determine

Hardness: 3

Firing: not possible to determine

Surface treatment: mat-impressed on external surface, pattern 4

Surface colour: external natural surface -5 YR $5 / 3$ reddish brown 


\section{Jars Type VII.3}

variant VII.3a

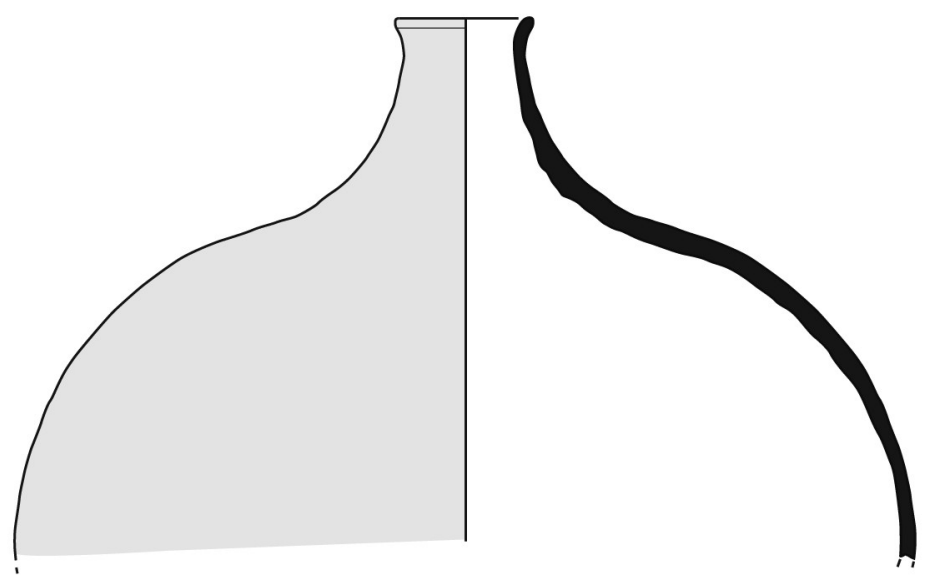

Cat. 259

Z12/24.1

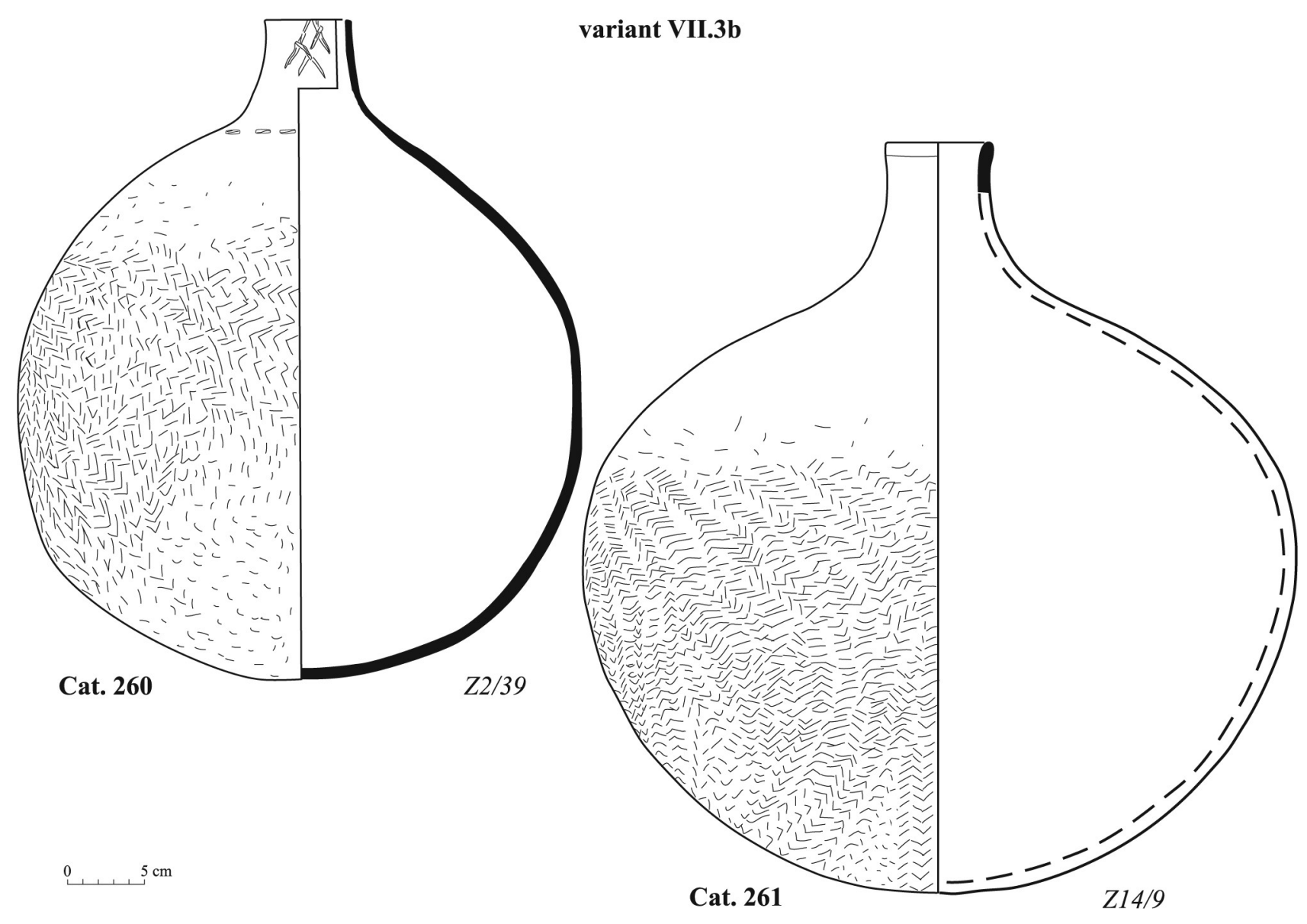

FIGURE 7.59 Globular jars of type viI.3, variants a and b 
Colour of break: not possible to determine

Decoration: incised-strokes below rim

References: (Klimaszewska-Drabot and Mahmoud ElTayeb 2014, 13-15)

\section{Cat. 264}

Bottle (large, broad)

Inv. no: $\mathbf{Z 1 5} / \mathbf{2 7}$

Provenance: Tumulus 15, chamber 2, southern part

Construction: handmade

State of preservation: complete

Measurements: $\mathrm{Dr}=8.7 \mathrm{~cm} ; \max$ body diam. $=43 \mathrm{~cm}$; $\mathrm{H}=55 \mathrm{~cm}$

Fabric: not possible to determine

Hardness: 3

Firing: reduced

Surface treatment: slipped and burnished neck; matimpressed, pattern 1

Surface colour: external natural surface-5YR 5/6 yellowish red, slip-2.5YR 4/6 red

Colour of break: not possible to determine

Decoration: slip on rim and neck with mat-impressed pattern on body; punctuated-oval shapes on shoulder

References: (Czyżewska-Zalewska 2016, 735-737)

\section{Cat. 265}

Bottle (large, medium broad)

Inv. no: $\mathrm{Z}_{15} / 59$

Provenance: Tumulus 15, chamber 1, eastern part

Construction: handmade

State of preservation: incomplete, $44 \%$ of rim

Measurements: Dr $=7.4 \mathrm{~cm} ; \max$ body diam. $=43.5 \mathrm{~cm}$; $\mathrm{H}=56 \mathrm{~cm}$

Fabric: ZF9

Hardness: 3

Firing: reduced

Surface treatment: slipped and burnished neck; matimpressed, pattern 1

Surface colour: external natural surface-5YR 5/6 yellowish red, slip-10R $5 / 6$ red

Colour of break: deep black

Decoration: slip on rim and neck with mat-impressed pattern on body; punctuated - oval shapes on shoulder References: (Czyżewska-Zalewska 2016, 730-733)

\section{Variant VII.4b}

Cat. 266

Bottle (large, broad)

Inv. no: $\mathbf{Z} 2 / 37$

Provenance: Tumulus 2, chamber 2

Construction: handmade
State of preservation: complete

Measurements: Dr $=7.6 \mathrm{~cm} ; \max$ body diam. $=42.5 \mathrm{~cm}$; $\mathrm{H}=51.5 \mathrm{~cm}$

Fabric: not possible to determine

Hardness: $3 / 4$

Firing: not possible to determine

Surface treatment: mat-impressed on external surface, pattern 1

Surface colour: external natural surface -5 YR 5/4 reddish brown

Colour of break: not possible to determine

Decoration: incised-strokes below rim; punctuatedoval shapes on shoulder

References: (Klimaszewska-Drabot and Mahmoud ElTayeb 2014, 13-15)

Cat. 267

Bottle (large, broad)

Inv. no: $\mathrm{Z} 9 / 4$

Provenance: Tumulus 9, chamber 2, central part

Construction: handmade

State of preservation: almost complete, $50 \%$ of rim

Measurements: $\mathrm{Dr}=8.2 \mathrm{~cm} ; \max$ body diam. $=39 \mathrm{~cm}$; $\mathrm{H}=48.5 \mathrm{~cm}$

Fabric: ZF10

Hardness: 3

Firing: reduced

Surface treatment: slipped and burnished neck and base; mat-impressed, pattern 2

Surface colour: external natural surface -5 YR 5/4 reddish brown, slip-10R 4/6 red; internal natural surface7.5YR $5 / 2$ brown

Colour of break: deep black

Decoration: slip on rim and neck with mat-impressed pattern on body

References: (Mahmoud El-Tayeb, Juszczyk-Futkowska, and Czyżewska 2014, 367-368)

\section{Cat. 268}

Bottle (large, broad)

Inv. no: Z13/16

Provenance: Tumulus 13, chamber 2, central part

Construction: handmade

State of preservation: almost complete, $75 \%$ of rim

Measurements: Dr $=8.1 \mathrm{~cm} ; \max$ body diam. $=37 \mathrm{~cm}$; $\mathrm{H}=45 \cdot 7 \mathrm{~cm}$

Fabric: ZF1O

Hardness: 3

Firing: reduced

Surface treatment: slipped and burnished neck and base; mat-impressed, pattern 1 
POTTERY PRESENTATION

249

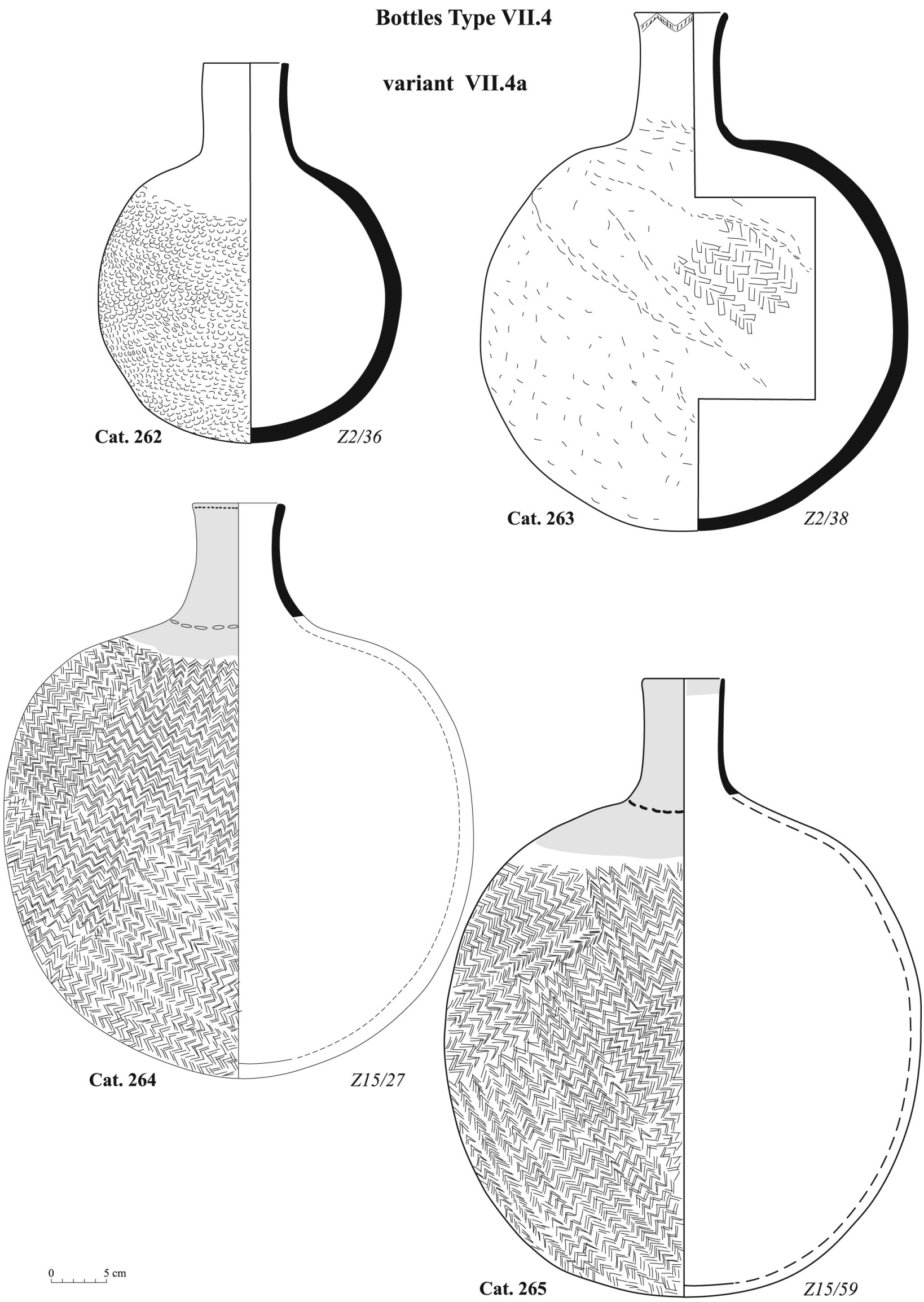

FIGURE 7.60 Globular bottles of type viI.4, variant a

Mahmoud El-Tayeb and Eva Czyżewska-Zalewska - 9789004433755

Downloaded from Brill.com04/26/2023 01:09:34 PM 


\section{Bottles Type VII.4}

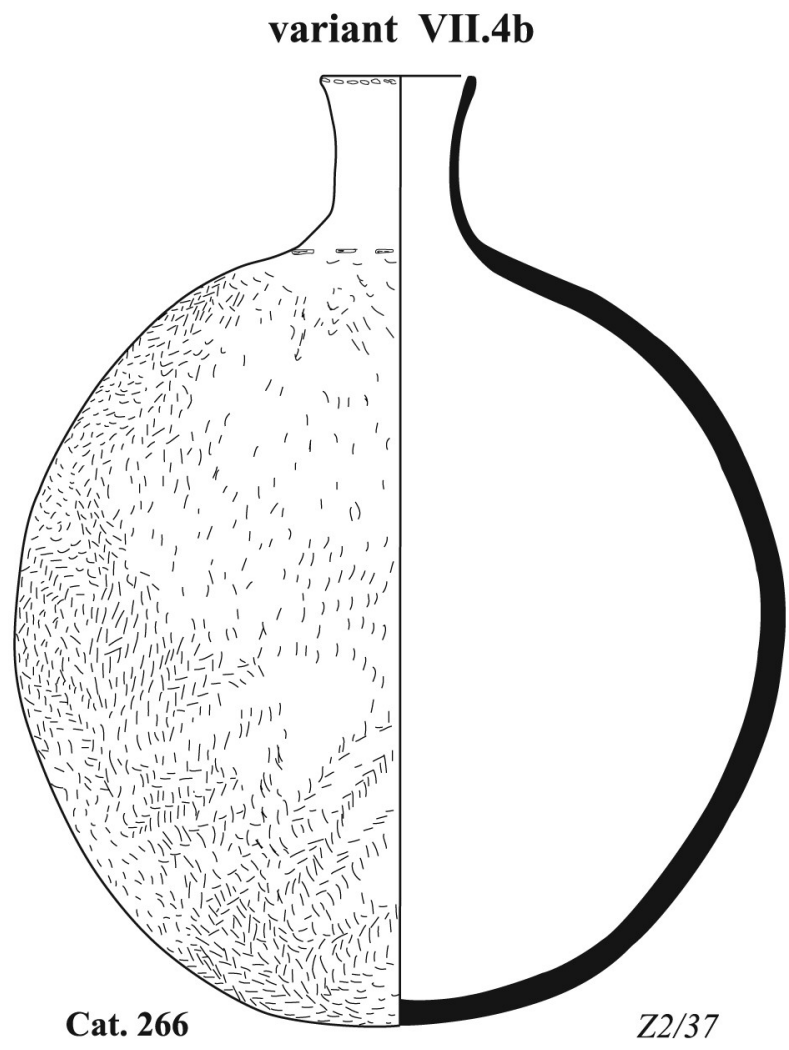

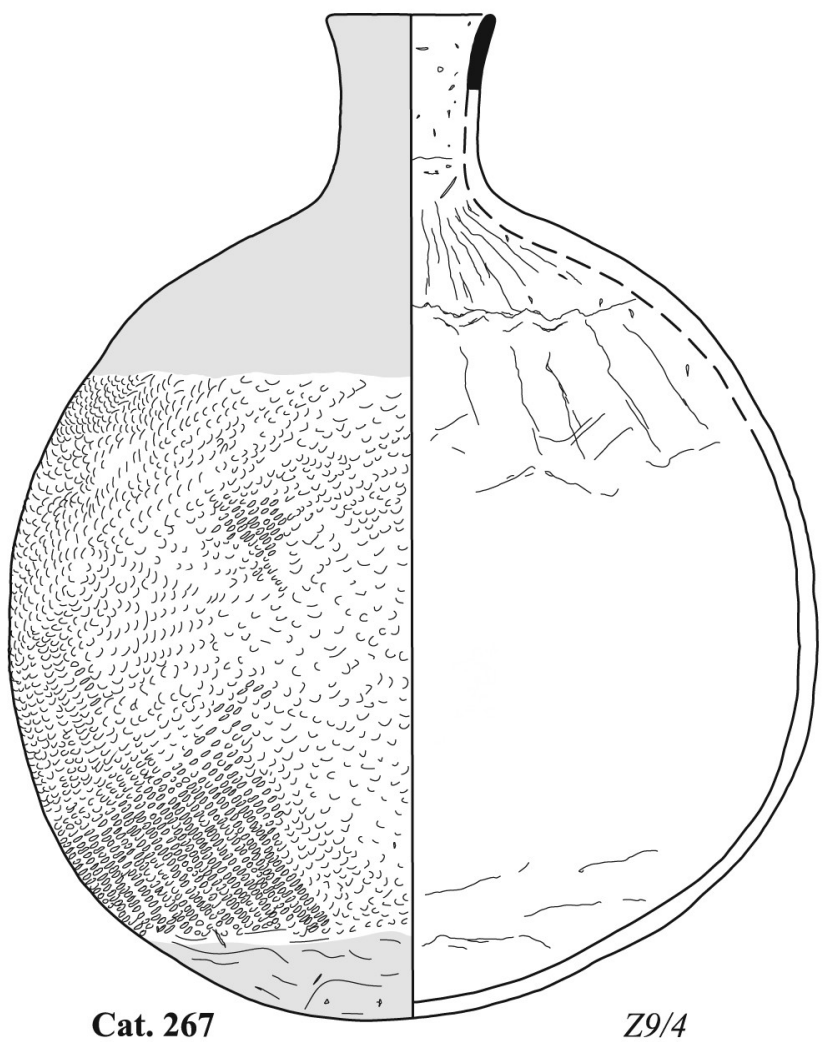

Cat. 267
$Z 9 / 4$

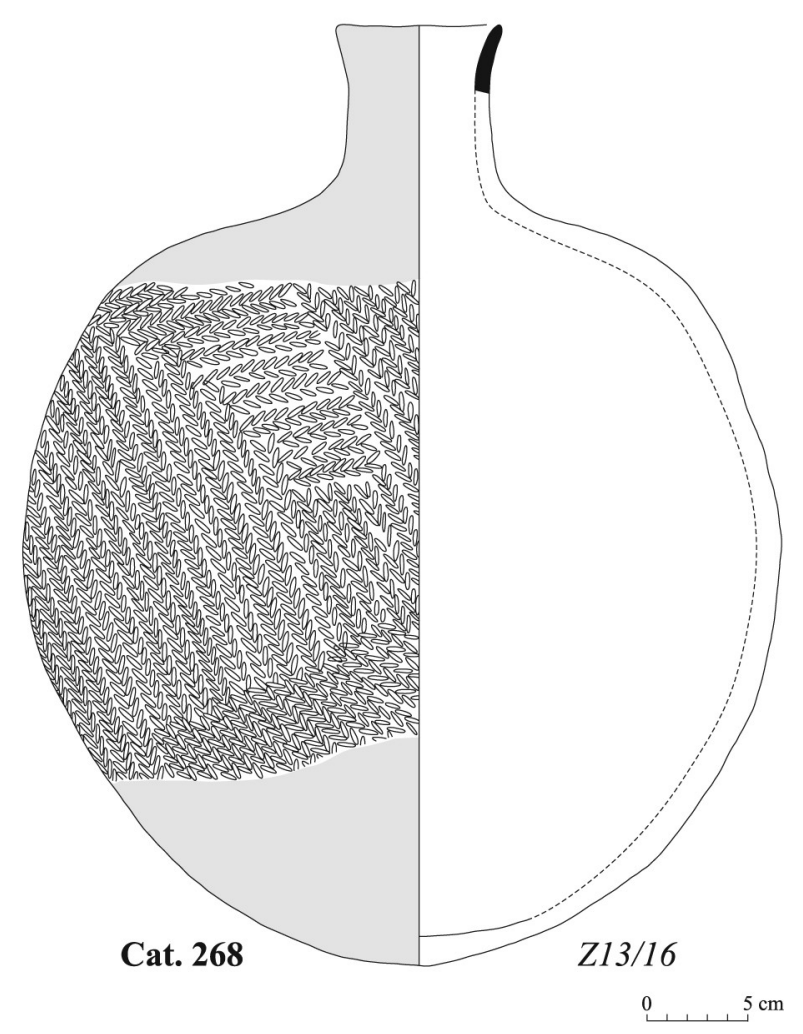

FIGURE 7.61 Globular bottles of type viI.4, variant b 
Surface colour: external natural surface- -5 YR $5 / 4$ reddish brown, slip-10R $4 / 6$ red

Colour of break: Gley1 2.5/N black

Decoration: slip on rim and neck with mat-impressed pattern on body

References: (Klimaszewska-Drabot and Czyżewska 2012, $365-367)$

\subsection{Bottles Type VII.5}

\section{Variant viI.5a}

\section{Cat. 269}

Bottle (medium-sized, medium broad)

Inv. no: $\mathrm{Z12} / 75$

Provenance: Tumulus 12, chamber 1, eastern part

Construction: handmade

State of preservation: almost complete, $92 \%$ of rim

Measurements: $\mathrm{Dr}=4.5 \mathrm{~cm} ; \max$ body diam. $=16.6 \mathrm{~cm}$;

$$
\mathrm{H}=22 \mathrm{~cm}
$$

Fabric: not possible to determine

Hardness: $3 / 4$

Firing: reduced

Surface treatment: slipped and burnished on external surface

Surface colour: external, slip—7.5YR 2.5/2 very dark brown

Colour of break: Gley 1 2.5/N black

Cat. 270

Bottle (medium-sized, broad)

Inv. no: $\mathrm{Z} 22 / 5_{5}$

Provenance: Tumulus 22, burial chamber

Construction: handmade

State of preservation: incomplete, $97 \%$ of rim

Measurements: $\mathrm{Dr}=6.3 \mathrm{~cm}$; $\max$ body diam. $=23.5 \mathrm{~cm}$; $\mathrm{H}=29 \mathrm{~cm}$

Fabric: not possible to determine

Hardness: 3

Firing: reduced

Surface treatment: slipped and burnished on external surface

Surface colour: external, slip-10R 4/6 red

Colour of break: not possible to determine

References: (Klimaszewska-Drabot and Mahmoud El-Tayeb 2014, 13-15)

\section{Variant VII.5b}

Cat. 271

Bottle (small, broad)

Inv. no: $\mathrm{Z1o} / \mathbf{2 2}$

Provenance: Tumulus 10, burial chamber, northern part

Construction: handmade

State of preservation: complete
Measurements: $\mathrm{Dr}=3.8 \mathrm{~cm} ; \max$ body diam. $=14.7 \mathrm{~cm}$; $\mathrm{H}=17.2 \mathrm{~cm}$

Fabric: not possible to determine

Hardness: 3

Firing: reduced

Surface treatment: uncoated

Surface colour: external—1oR 5/4 weak red

Colour of break: not possible to determine

Decoration: applied - two small bosses on shoulder

References: (Klimaszewska-Drabot 2010, 483-484)

\section{Cat. 272}

Bottle (large, medium broad)

Inv. no: $\mathbf{Z} 2 / 35$

Provenance: Tumulus 2, chamber 2

Construction: handmade

State of preservation: complete

Measurements: $\mathrm{Dr}=7.1 \mathrm{~cm} ; \max$ body diam. $=33 \mathrm{~cm}$; $\mathrm{H}=43 \mathrm{~cm}$

Fabric: not possible to determine

Hardness: $3 / 4$

Firing: reduced

Surface treatment: uncoated

Surface colour: external-2.5YR 5/6 red

Colour of break: not possible to determine

Decoration: applied - two small bosses on shoulder

References: (Klimaszewska-Drabot and Mahmoud El-Tayeb 2014, 13-15)

\section{Cat. 273}

Bottle (medium-sized, medium broad)

Inv. no: $\mathbf{Z 2 5} / 3$

Provenance: Tumulus 25, chamber 1, western part

Construction: handmade

State of preservation: incomplete, $94 \%$ of rim, body fragment missing

Measurements: Dr $=5.4 \mathrm{~cm} ; \max$ body diam. $=22.7 \mathrm{~cm}$; $\mathrm{H}=30 \mathrm{~cm}$

Fabric: ZF9

Hardness: $2 / 3$

Firing: reduced

Surface treatment: slipped and burnished on external surface

Surface colour: external, slip-10R 4/6 red

Colour of break: deep black

Decoration: applied — two small bosses on shoulder

Variant VII.5c

Cat. 274

Bottle (medium-sized, broad)

Inv. no: $\mathbf{Z 1 5} / \mathbf{5}^{1}$ 


\section{Bottles Type VII.5}

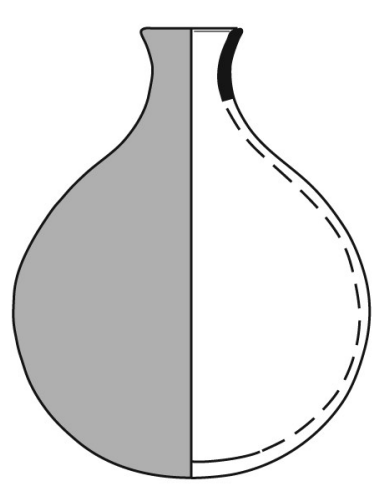

variant VII.5a

Cat. 269

Z12/75

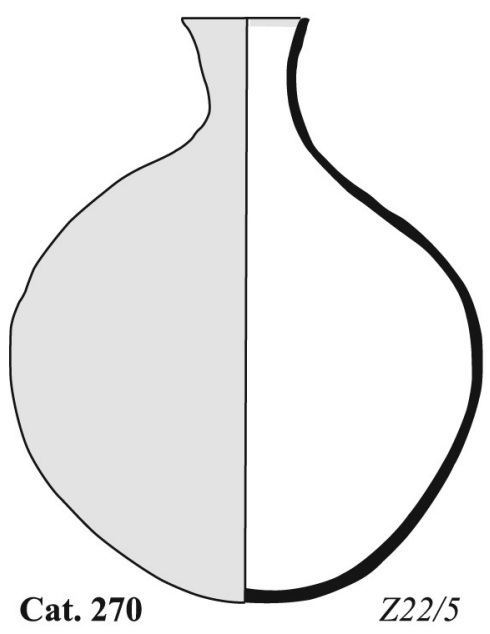

variant VII.5b

Cat. 270

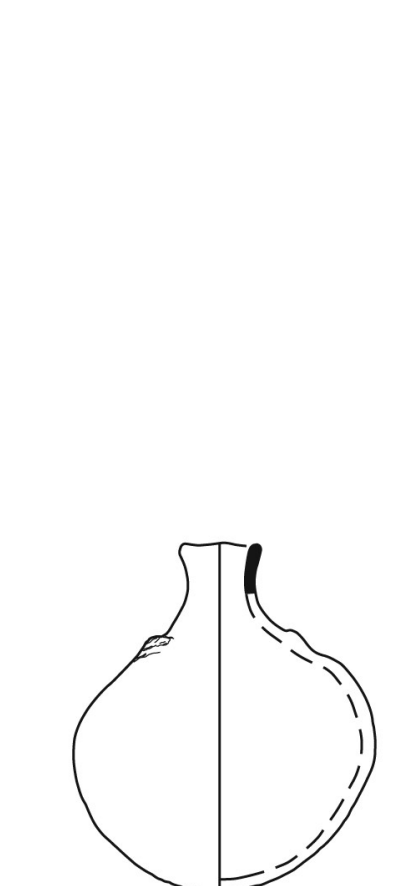

Cat. 271

$Z 10 / 22$

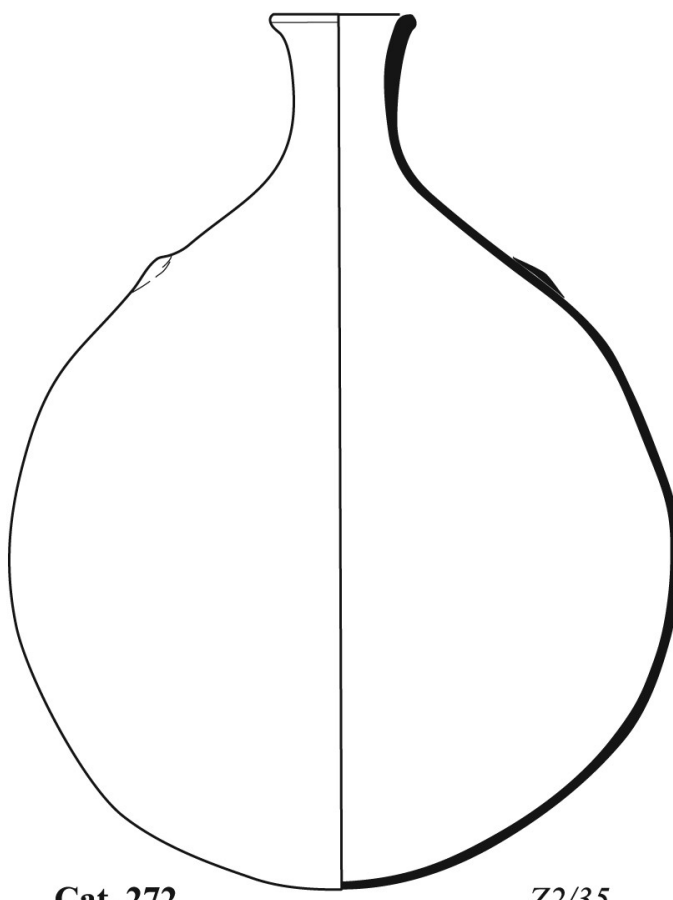

Z2/35

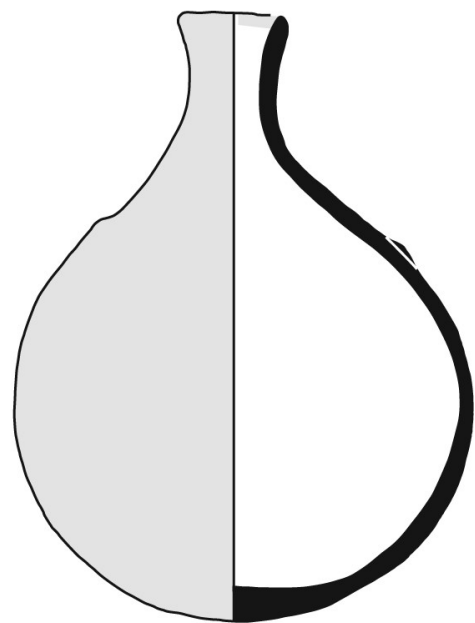

Cat. 273

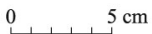

FIGURE 7.62 Bag-shaped bottles of type VII.5, variants a and b

Provenance: Tumulus 15, chamber 1

Construction: handmade

State of preservation: incomplete, body fragments missing Measurements: Dr $=7 \mathrm{~cm} ; \max$ body diam. $=27 \mathrm{~cm}$;

$$
\mathrm{H}=31 \mathrm{~cm}
$$

Fabric: ZF7

Hardness: $3 / 4$

Firing: reduced

Surface treatment: slipped and burnished neck; matimpressed, pattern 3

Surface colour: external natural surface -7.5 YR 5/4 brown, slip_-1OYR 4/6 dark yellowish brown
Colour of break: deep black

Decoration: slip on rim and neck with mat-impressed pattern on body

\section{Cat. 275}

Bottle (large, broad)

Inv. no: $\mathrm{Z26} / 35$

Provenance: Tumulus 26, chamber 3, eastern part

Construction: handmade

State of preservation: complete

Measurements: Dr $=9.6 \mathrm{~cm} ;$ max body diam. $=38 \mathrm{~cm}$; $\mathrm{H}=44.5 \mathrm{~cm}$ 
Fabric: not possible to determine

Hardness: $2 / 3$

Firing: reduced

Surface treatment: slipped and burnished neck; matimpressed, pattern 1

Surface colour: external natural surface-10YR 8/3 very pale brown, slip-7.5R 4/8 red

Colour of break: deep black

Decoration: slip on rim and neck with mat-impressed pattern on body

Cat. 276

Bottle (large, medium broad)

Inv. no: $\mathrm{Z14} / 33$

Provenance: Tumulus 14, shaft, southern part

Construction: handmade

State of preservation: fragmentary, restored from sherds, complete profile, body fragments missing

Measurements: Dr $=8 \mathrm{~cm} ; \max$ body diam. $=43 \mathrm{~cm}$; $\mathrm{H}=54 \mathrm{~cm}$

Fabric: ZF10

Hardness: $3 / 4$

Firing: reduced

Surface treatment: slipped and burnished neck; matimpressed, pattern 6

Surface colour: external natural surface-10R 6/6 light red, slip —1oR 5/6 red; internal natural surface_-Gleyı 3/N black

Colour of break: deep black

Decoration: slip on rim and neck with mat-impressed pattern on body

\subsection{Bottles Type VII.6}

Variant viı.6a

Cat. 277

Bottle (medium-sized, broad)

Inv. no: $\mathrm{Z}_{5} / 3$

Provenance: Tumulus 5, chamber 1, near southern wall of chamber

Construction: handmade

State of preservation: fragmentary, complete rim, upper part of vessel

Measurements: $\mathrm{Dr}=4.7 \mathrm{~cm} ; \max$ body diam. $=22.4 \mathrm{~cm}$; ${ }_{\mathrm{p}} \mathrm{H}=24.8 \mathrm{~cm}$

Fabric: ZF9

Hardness: 3

Firing: reduced

Surface treatment: slipped and burnished on external surface

Surface colour: external, slip-7.5YR 4/3 brown

Colour of break: deep black

References: (Klimaszewska-Drabot 2010, 483-484)
Variant VII.6b

Cat. 278

Bottle (medium-sized, medium broad)

Inv. no: $\mathrm{Z} 15 / 53$

Provenance: Tumulus 15, chamber 1, eastern part

Construction: handmade

State of preservation: complete

Measurements: $\operatorname{Dr}=5.5 \mathrm{~cm} ; \max$ body diam. $=23 \mathrm{~cm}$; $\mathrm{H}=30 \mathrm{~cm}$

Fabric: not possible to determine

Hardness: 3

Firing: reduced

Surface treatment: slipped and burnished neck; matimpressed, pattern 1

Surface colour: external natural surface-7.5YR 4/3 brown, slip-2.5YR 4/4 reddish brown

Colour of break: not possible to determine

Decoration: slip on neck and band of slip on shoulder with mat-impressed pattern on body; scratched-geometric pattern on shoulder

\section{Cat. 279}

Bottle (medium-sized, broad)

Inv. no: $\mathbf{Z 1 5} / 8$

Provenance: Tumulus 15, fill of shaft

Construction: handmade

State of preservation: incomplete, restored from sherds, $97 \%$ of rim, body fragments missing

Measurements: Dr $=6 \mathrm{~cm} ;$ max body diam. $=26.5 \mathrm{~cm}$; $\mathrm{H}=32.5 \mathrm{~cm}$

Fabric: $\mathrm{ZF} 8$

Hardness: 3

Firing: reduced

Surface treatment: slipped and burnished neck; matimpressed, pattern 1

Surface colour: external natural surface- -5 YR $5 / 6$ yellowish red, slip- $10 \mathrm{R} 5 / 6$ red

Colour of break: deep black

Decoration: slip on rim and neck with mat-impressed pattern on body

\section{Variant ViI.6c}

Cat. 28 o

Bottle (medium-sized, broad)

Inv. no: Z12/12o

Provenance: Tumulus 12, chamber 3, eastern part

Construction: handmade

State of preservation: complete

Measurements: Dr $=7.5 \mathrm{~cm} ; \max$ body diam. $=31 \mathrm{~cm}$; $\mathrm{H}=36.5 \mathrm{~cm}$

Fabric: not possible to determine

Hardness: 3 
Bottles Type VII.5

variant VII.5c

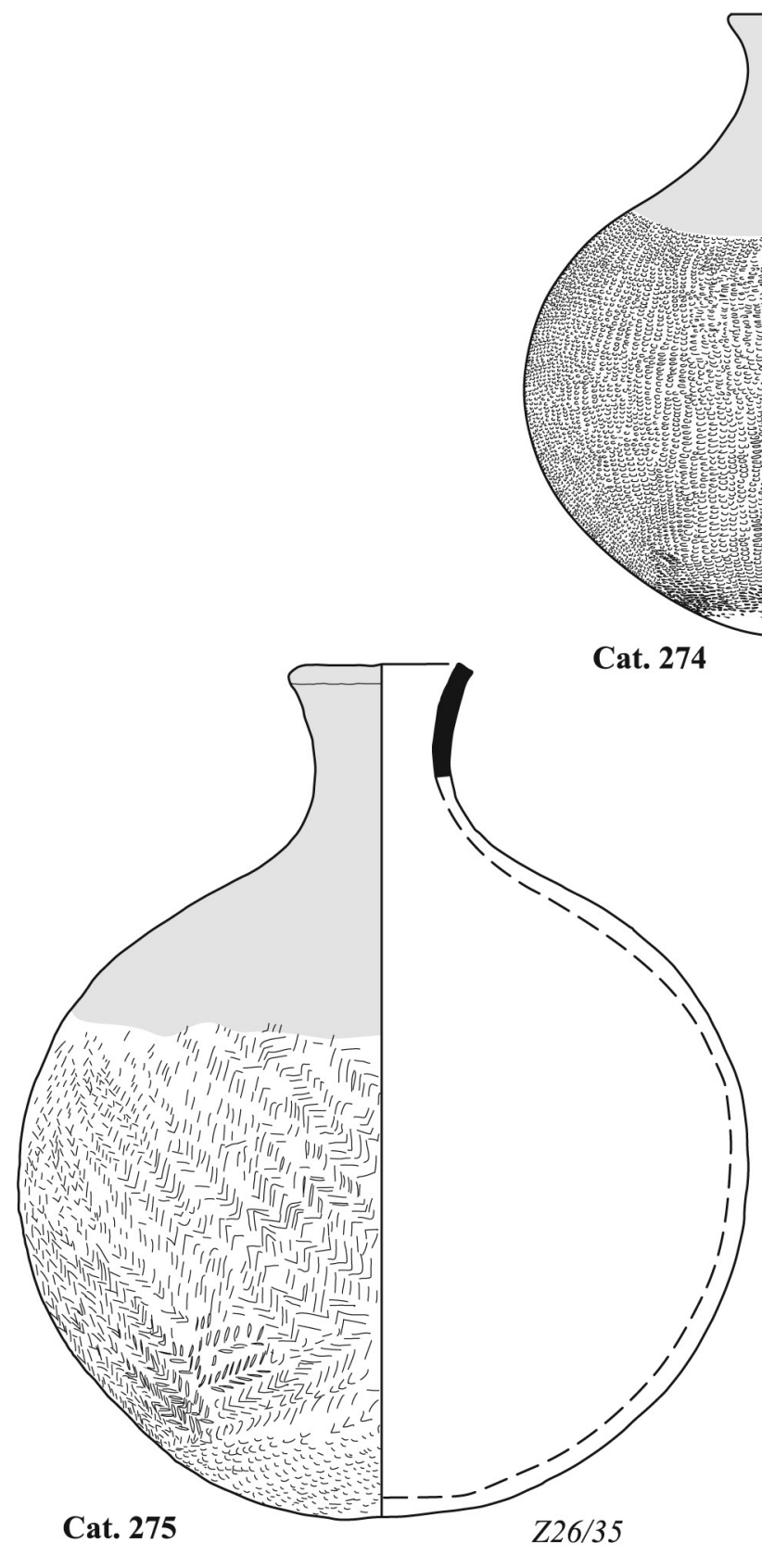

Cat. 275

FIGURE 7.63

Bag-shaped bottles of type viI.5, variant c
Firing: reduced

Surface treatment: uncoated

Surface colour: external-2.5YR 5/6 red

Colour of break: not possible to determine

Decoration: applied-two small bosses on shoulder; incised-five sets of two vertical strokes below rim

\section{Cat. 281}

Bottle (small, broad)

Inv. no: $\mathbf{Z} 2 \mathrm{O} / 3$

Provenance: Tumulus 20, burial chamber, central part Construction: handmade

State of preservation: incomplete, restored from sherds, body fragment missing 
Measurements: $\operatorname{Dr}=5.5 \mathrm{~cm} ; \max$ body diam. $=21 \mathrm{~cm} ;$ $\mathrm{H}=25.7 \mathrm{~cm}$

Fabric: ZF9

Hardness: 3

Firing: reduced

Surface treatment: slipped and burnished on external surface

Surface colour: external, slip—deep black

Colour of break: deep black

Decoration: applied-two small bosses on shoulder

References: (Mahmoud El-Tayeb, Juszczyk-Futkowska, and

Czyżewska 2014, 367-368)

Cat. 282

Bottle (medium-sized, broad)

Inv. no: $\mathbf{Z 2 O} / 4$

Provenance: Tumulus 20, burial chamber, central part

Construction: handmade

State of preservation: complete

Measurements: $\mathrm{Dr}=4.7 \mathrm{~cm} ; \max$ body diam. $=25 \mathrm{~cm}$; $\mathrm{H}=28.5 \mathrm{~cm}$

Fabric: not possible to determine

Hardness: $2 / 3$

Firing: reduced

Surface treatment: slipped and burnished on external surface

Surface colour: external, slip-1oR 4/8 red

Colour of break: not possible to determine

Decoration: applied—two small bosses on shoulder

Variant viı.6d

\section{Cat. 283}

Bottle (small, broad)

Inv. no: Z11/26

Provenance: Tumulus 11, chamber 2, central part

Construction: handmade

State of preservation: incomplete, restored from sherds, $75 \%$ of rim, body fragments missing

Measurements: $\operatorname{Dr}=3.5 \mathrm{~cm} ; \max$ body diam. $=12.3 \mathrm{~cm}$; $\mathrm{H}=14.4 \mathrm{~cm}$

Fabric: ZF9

Hardness: $3 / 4$

Firing: reduced

Surface treatment: slipped and burnished on external surface

Surface colour: external, slip-10R $2.5 / 2$ very dusky red; internal natural surface-10R 2.5/1 reddish black

Colour of break: deep black

Decoration: applied-two lugs on shoulder

References: (Klimaszewska-Drabot and Czyżewska 2012, 368-372)
Cat. 284

Bottle (large, broad)

Inv. no: Z12/121

Provenance: Tumulus 12, chamber 3, eastern part

Construction: handmade

State of preservation: incomplete, rim missing

Measurements: max body diam. $=36 \mathrm{~cm} ;{ }_{\mathrm{p}} \mathrm{H}=43 \mathrm{~cm}$

Fabric: not possible to determine

Hardness: 3

Firing: reduced

Surface treatment: slipped and burnished on external surface

Surface colour: external natural surface-5YR 5/6 yellowish red, slip-10R $4 / 8$ red

Colour of break: not possible to determine

Decoration: applied - two lugs on shoulder

Notes: evidence of repairs to vessel-holes executed in neck

Cat. 285

Bottle (large, broad)

Inv. no: $\mathrm{Z} \mathbf{9} / \mathbf{5}$

Provenance: Tumulus 9, chamber 2, northern part

Construction: handmade

State of preservation: incomplete, one lug missing

Measurements: Dr $=8.7 \mathrm{~cm} ; \max$ body diam. $=37.5 \mathrm{~cm}$; $\mathrm{H}=46 \mathrm{~cm}$

Fabric: not possible to determine

Hardness: $3 / 4$

Firing: not possible to determine

Surface treatment: slipped and burnished on external surface

Surface colour: external, slip_-10R 4/6 red

Colour of break: not possible to determine

Decoration: applied-two lugs on shoulder

Notes: evidence of repairs to vessel-holes executed in neck

Cat. 286

Bottle (large, broad)

Inv. no: Z12/21

Provenance: Tumulus 12, fill of shaft

Construction: handmade

State of preservation: incomplete, restored from sherds, $80 \%$ of rim, bottom part missing

Measurements: $\mathrm{Dr}=10 \mathrm{~cm} ; \max$ body diam. $=39 \mathrm{~cm}$;

$$
{ }_{\mathrm{p}} \mathrm{H}=46 \mathrm{~cm}
$$

Fabric: ZF9

Hardness: 3

Firing: reduced

Surface treatment: slipped and burnished on external surface 


\section{Bottles Type VII.6}

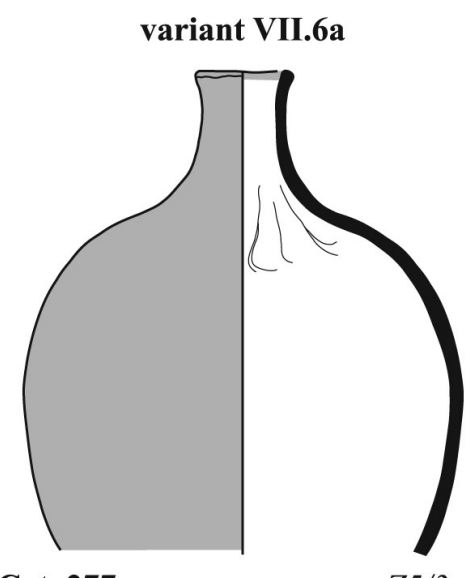

Cat. 277

$x$

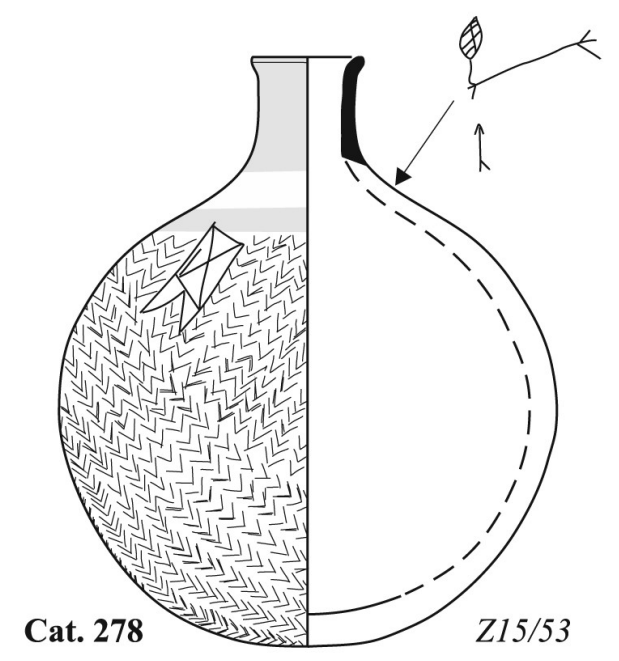

variant VII.6b
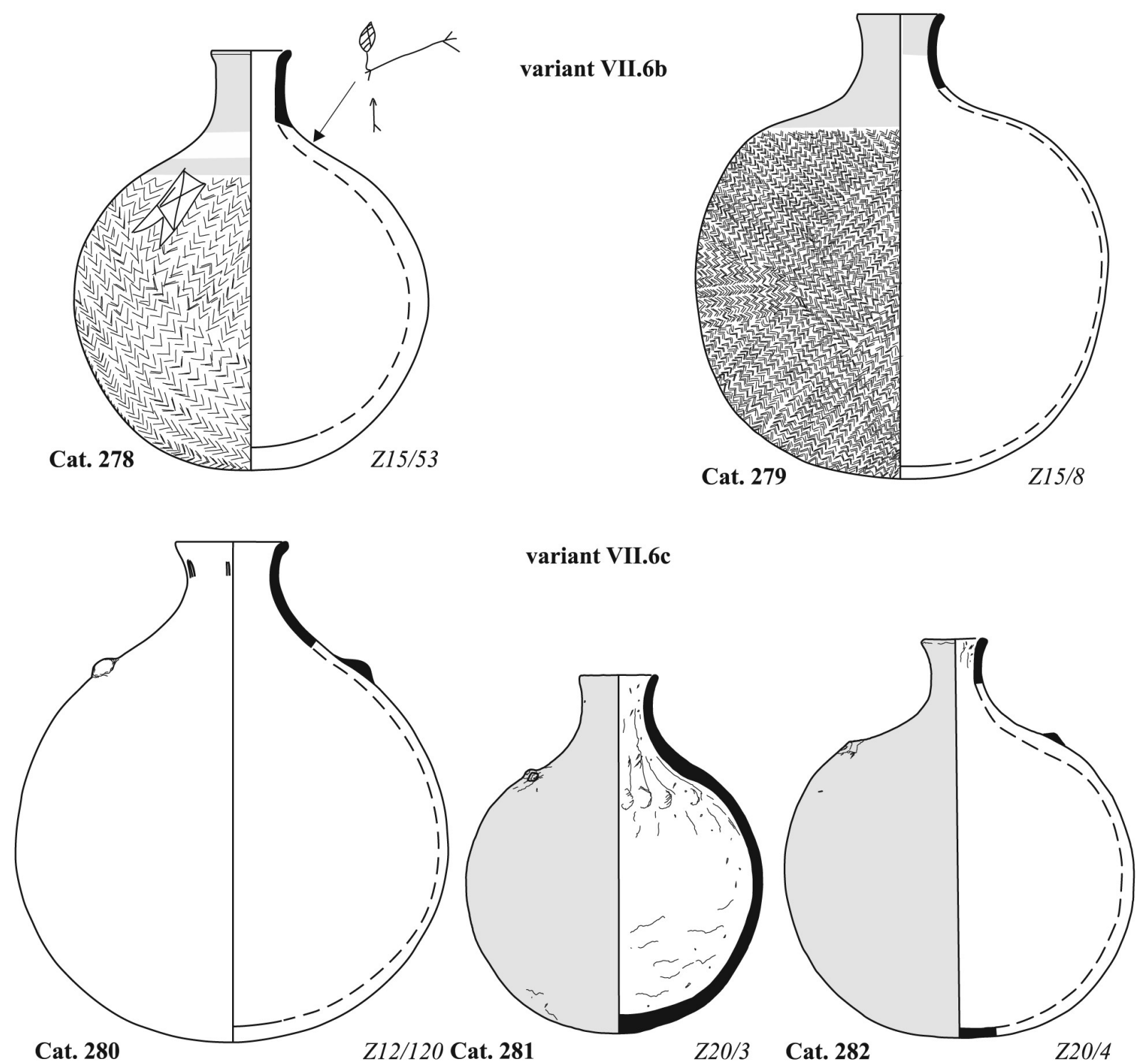

Z12/120 Cat. 281

Z20/3

Cat. 282

Z20/4

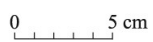

FIGURE 7.64 Globular bottles of type viI.6, variants a, b and c 


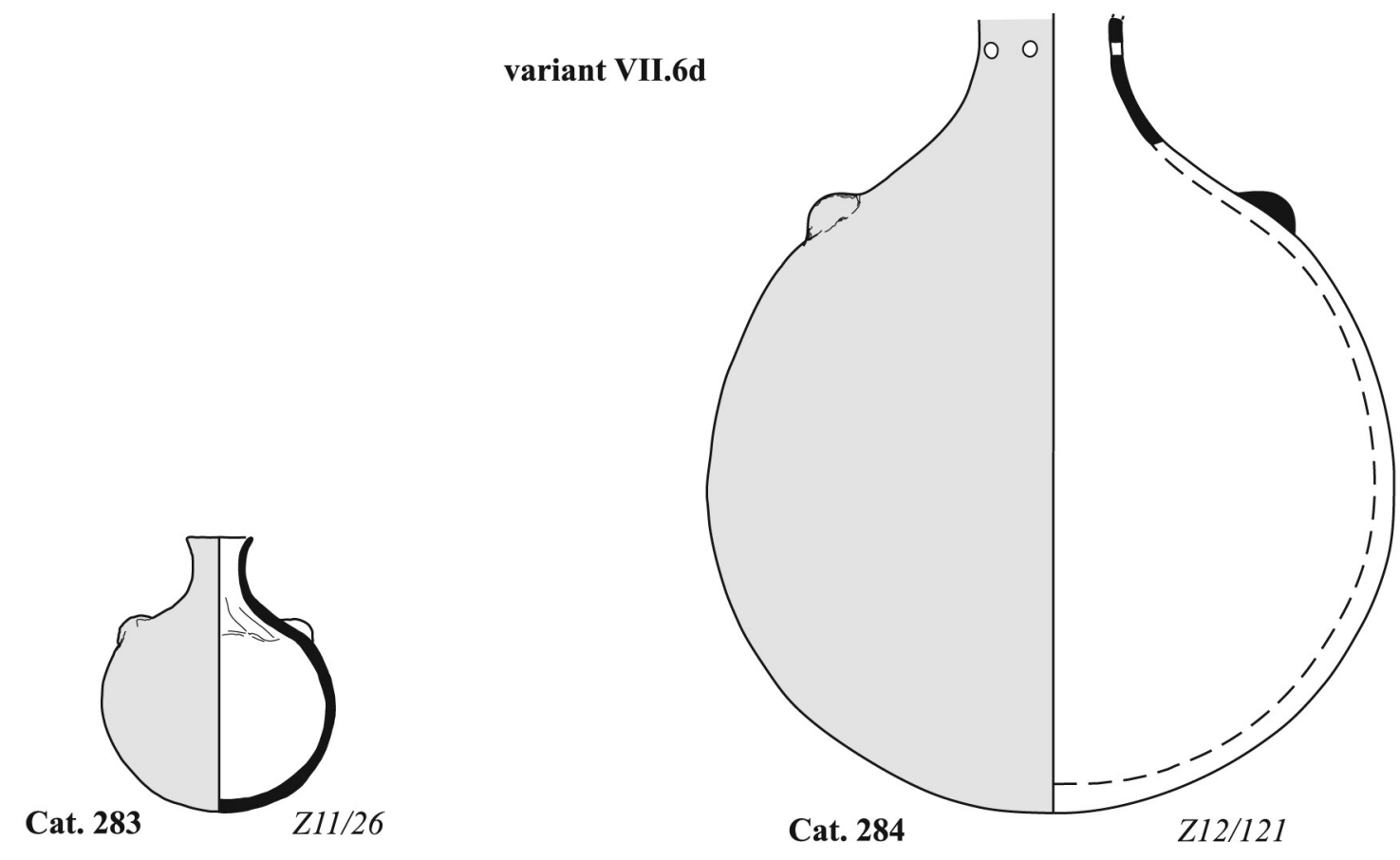

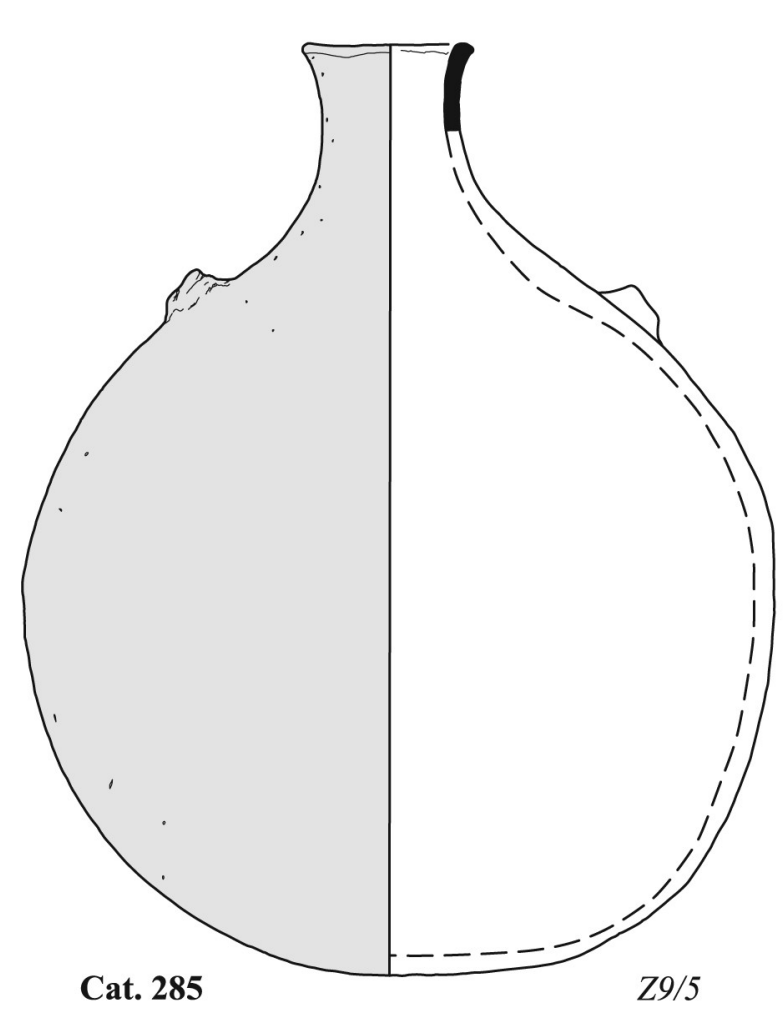

FIGURE 7.65

Globular bottles of type viI.6, variant $d$

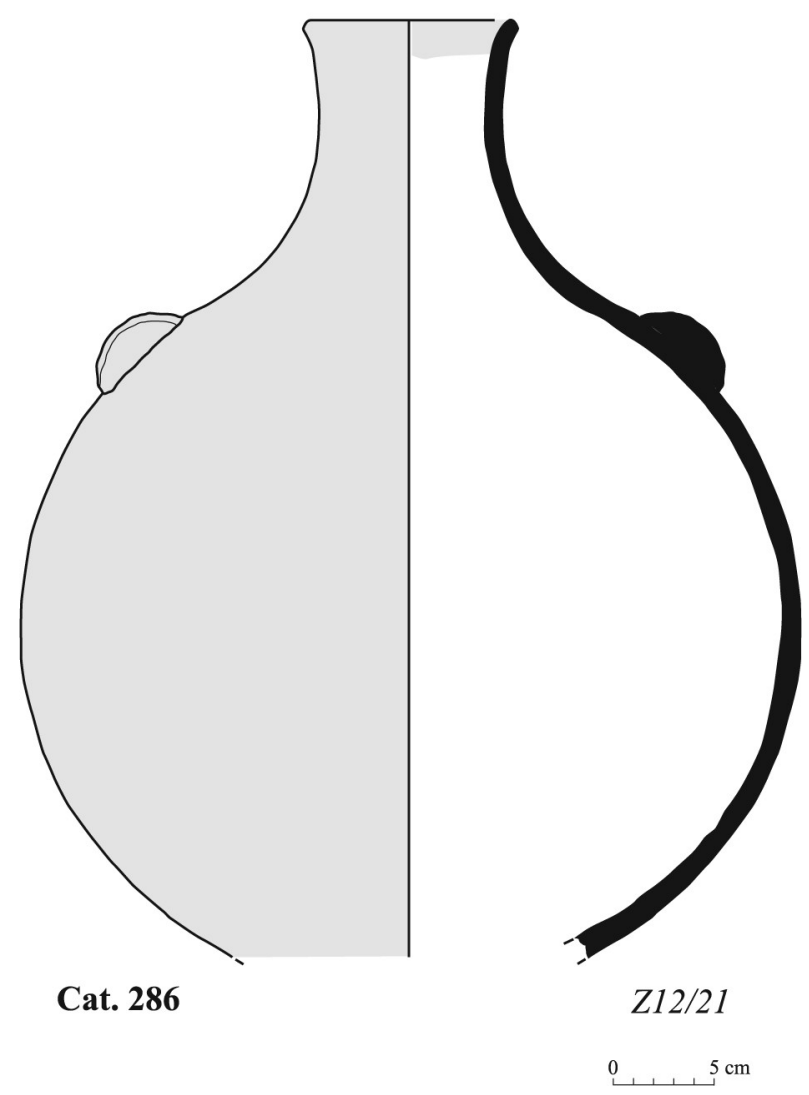


Surface colour: external natural surface-2.5YR 5/6 red, slip-10R 5/6 red; internal natural surface-2.5YR 3/1 dark reddish grey

Colour of break: deep black

Decoration: applied - two lugs on shoulder

\section{$9 \quad$ Jars Varia}

[Fig. 7.123]

Cat. 287

Jar (large, broad)

Inv. no: Z12/51

Provenance: Tumulus 12, fill of shaft

Construction: handmade

State of preservation: fragmentary, restored from sherds

Measurements: Dr $=13.5 \mathrm{~cm}$

Fabric: ZF11

Hardness: $3 / 4$

Firing: reduced

Surface treatment: slip on external surface

Surface colour: external natural surface -2.5 YR 5/8 red, slip-7.5YR 8/2 pinkish white; internal-2.5YR 5/8 red (upper part), $2.5 \mathrm{Y}_{3} / 1$ very dark grey

Colour of break: 5 Y 2.5/1 black

Decoration: incised-lines on shoulders and neck

Cat. 288

Jar (large, broad)

Inv. no: $\mathrm{Z}_{25} / \mathbf{2}$

Provenance: Tumulus 25, upper part of shaft

Construction: handmade

State of preservation: fragmentary, $25 \%$ of rim, upper part of vessel

Measurements: $\mathrm{Dr}=18.5 \mathrm{~cm} ;{ }_{\mathrm{p}} \mathrm{H}=26 \mathrm{~cm}$

Fabric: ZF9

Hardness: $3 / 4$

Firing: reduced

Surface treatment: slipped on external surface

Surface colour: external, slip-2.5YR 5/4 reddish brown; internal-5YR 6/4 light reddish brown

Colour of break: deep black

Decoration: painted-white strip on wall; incised-below rim

\section{Cat. 289}

Jar

Inv. no: $\mathrm{Z11} / \mathbf{4 2}^{2}$

Provenance: Tumulus 11, robber shaft

Construction: handmade

State of preservation: fragmentary, $72 \%$ of rim, neck and shoulder fragment with one boss
Measurements: $\mathrm{Dr}=9 \cdot 3 \mathrm{~cm} ;{ }_{\mathrm{p}} \mathrm{H}=10 \mathrm{~cm}$

Fabric: ZF1O

Hardness: 3

Firing: reduced

Surface treatment: slipped and burnished neck, and matimpressed body, pattern 1

Surface colour: external, slip-10R 5/8 red; internal1OYR 5/4 yellowish brown

Colour of break: deep black

Decoration: slip on rim and neck with mat-impressed pattern on body; applied-one boss extant on shoulder

References: (Klimaszewska-Drabot and Czyżewska 2012, 372-377)

Cat. 290

Jar (large, broad)

Inv. no: Z12/133.1

Provenance: Tumulus 12, chamber 2, southern part

Construction: handmade

State of preservation: fragmentary, restored from sherds, rim missing

Measurements: max body diam. $=56 \mathrm{~cm} ;{ }_{\mathrm{p}} \mathrm{H}=55 \mathrm{~cm}$

Fabric: $\mathrm{ZF} 7$

Hardness: 3

Firing: reduced

Surface treatment: slipped on external surface

Surface colour: external, slip-10R 5/8 red; internal1OYR 5/4 yellowish brown

Colour of break: deep black

Decoration: slip on rim and neck with mat-impressed pattern on body; applied-one boss extant on shoulder

Cat. 291

Jar (small, broad)

Inv. no: $\mathbf{Z 1 2} / 5^{2}$

Provenance: Tumulus 12, fill of shaft

Construction: wheel-made (2)

State of preservation: fragmentary, restored from sherds, rim and base missing

Measurements: max body diam. $=c .55 \cdot 5 \mathrm{~cm} ;{ }_{\mathrm{p}} \mathrm{H}=65 \mathrm{~cm}$

Fabric: ZF4

Hardness: 4

Firing: oxidized

Surface treatment: slipped and burnished on external surface

Surface colour: external natural surface-2.5YR 6/6 light red, slip-1oR $5 / 6$ red-1oR 4/6 red; internal -5 YR 6/6 reddish yellow (upper part), 7.5YR 6/3-6/4 light brown (lower part)

Colour of break: 5 YR $5 / 6$ yellowish red 


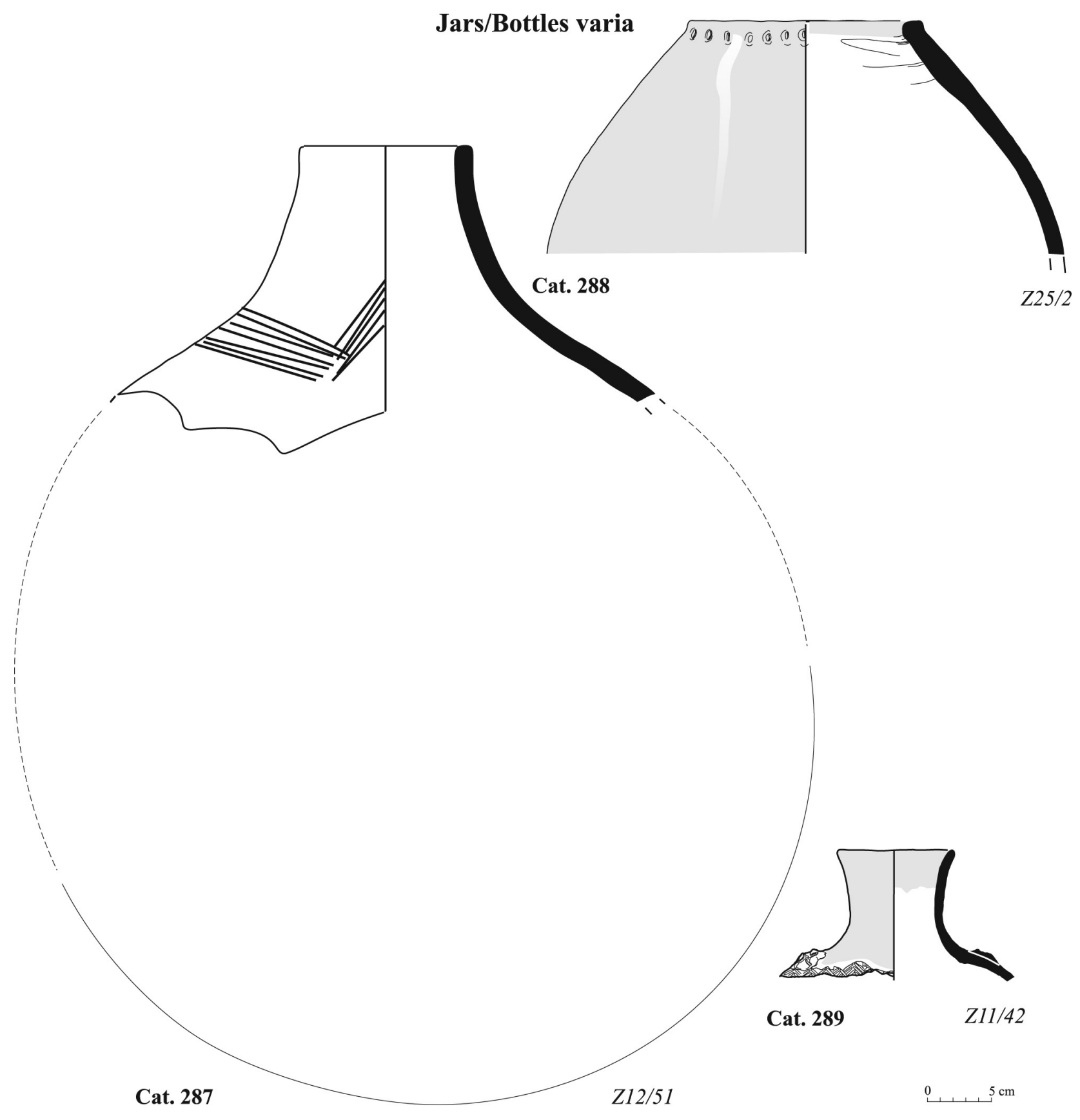

FIGURE 7.66 Globular jars of different types

\section{Cat. 292}

Bottle

Inv. no: $\mathrm{Z1o} / 25$

Provenance: Tumulus 10, burial chamber, northern part Construction: wheel-made (2)

State of preservation: complete

Measurements: $\mathrm{Dr}=6.1 \mathrm{~cm}, \max$ body diam. $=33.9 \mathrm{~cm}$,

$$
\mathrm{H}=42.9 \mathrm{~cm}
$$

Fabric: not possible to determine

Hardness: 2

Firing: oxidized
Surface treatment: uncoated

Surface colour: 1OYR 6/2 light brownish grey on both surfaces

Colour of break: 1OYR 6/2 light brownish grey

Decoration: incised-star pattern inside stopper, badly executed; stamped decoration on external wall and top of stopper

References: (Klimaszewska-Drabot 2010, 484-485) 


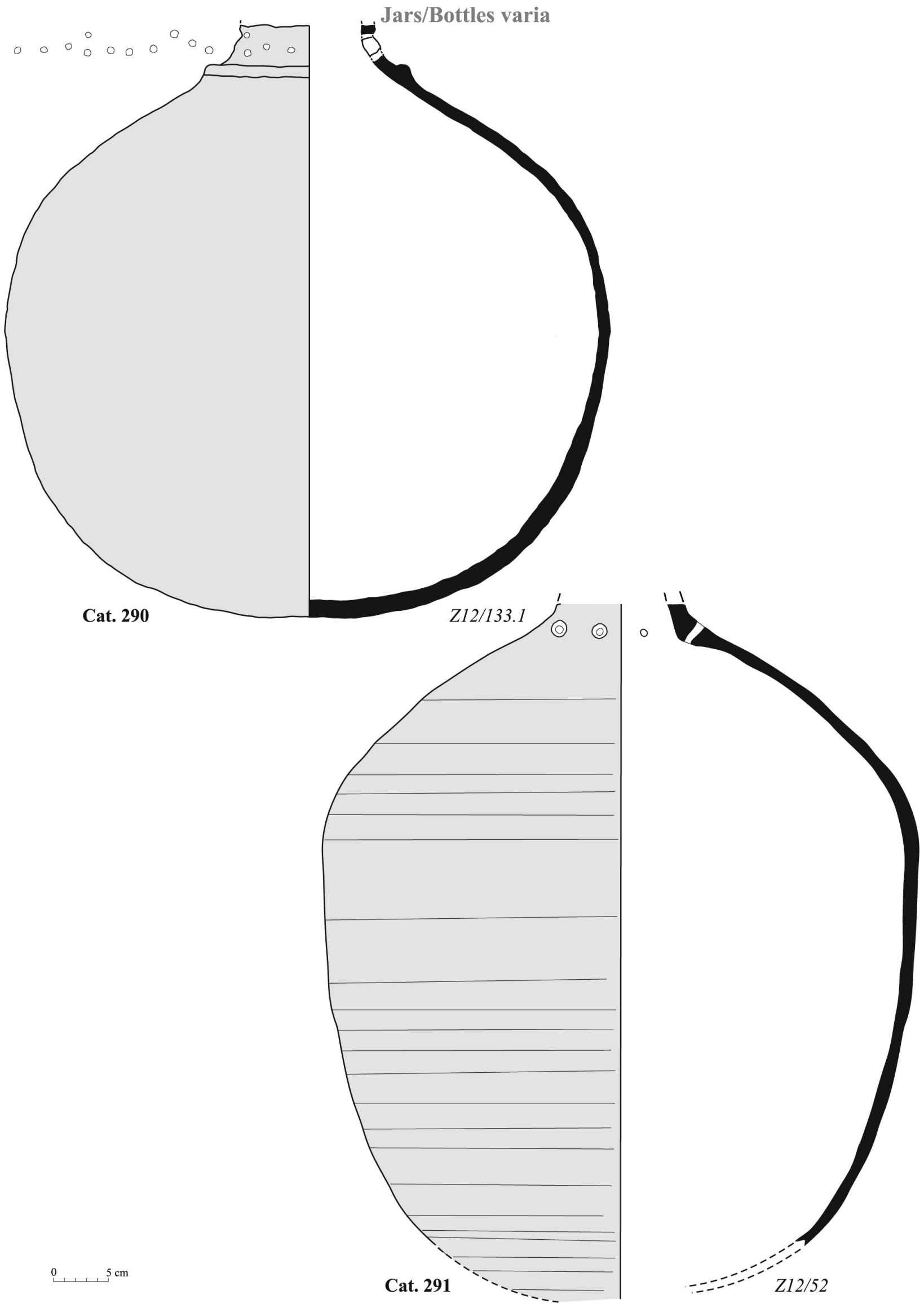

FIGURE 7.67 Globular jars of different types 


\section{Jars/Bottles varia}

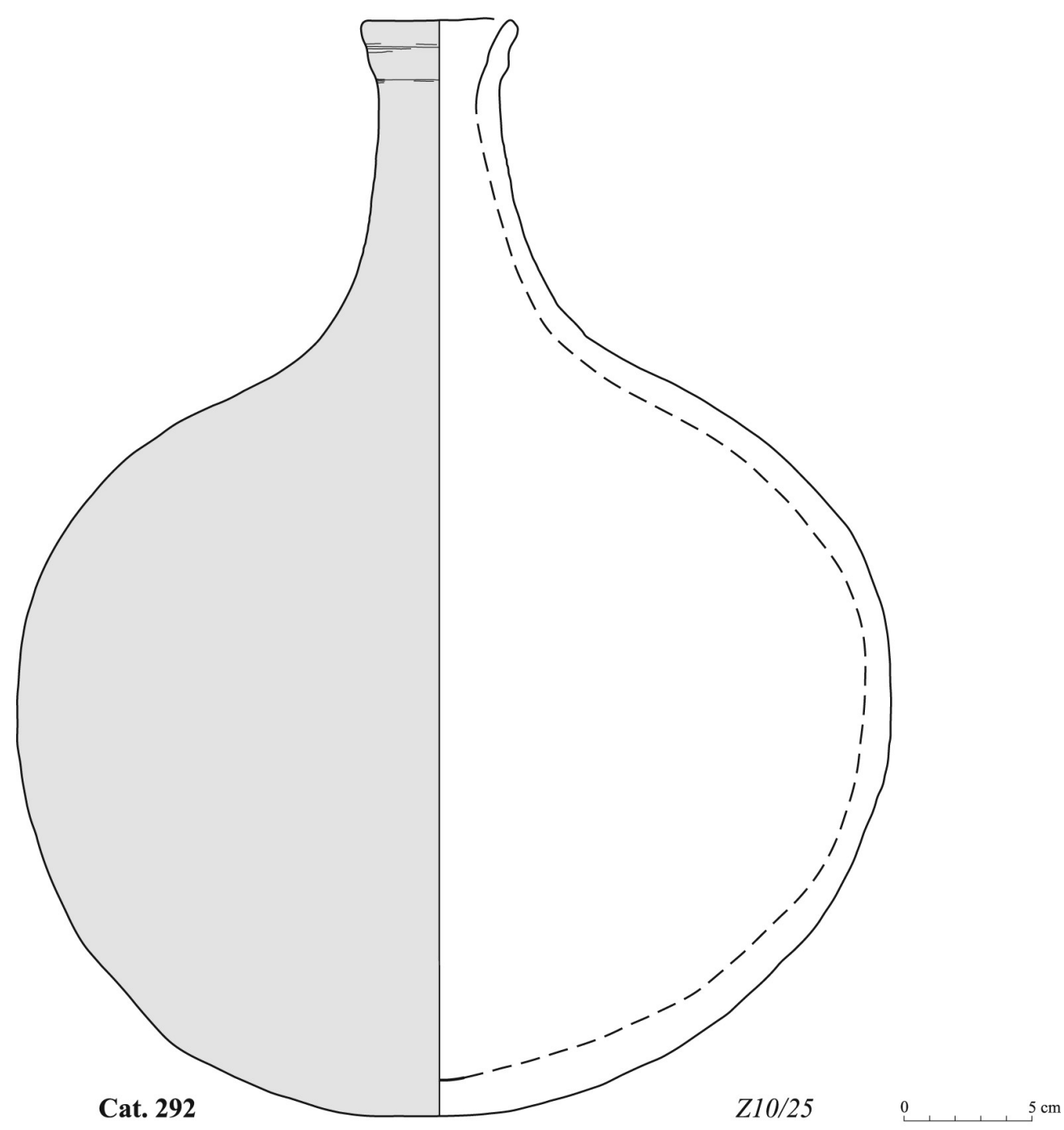

FIGURE 7.68 Globular jars of different types

10 Group VIII-Stands

\section{Cat. 293}

Stand

Inv. no: $\mathrm{Z} 23 / 40$

Provenance: Tumulus 23, chamber 1

Construction: wheel-made (2)

State of preservation: incomplete, $75 \%$ of rim, complete base

Measurements: $\mathrm{Dr}=9.5 \mathrm{~cm} ; \mathrm{D}_{\mathrm{b}}=10.6 \mathrm{~cm} ; \mathrm{H}=21.5 \mathrm{~cm}$

Fabric: not possible to determine

Hardness: $3 / 4$

Firing: oxidized

Surface treatment: slipped and polished on external surface; internal surface partially slipped

Surface colour: slip-2.5YR 5/6 red on both surfaces
[Fig. 7.124] Colour of break: not possible to determine

Decoration: incised-one set of five grooves below rim, and second set above base; three sets of two grooves on body forming border for painted decoration; paintedfive sets (one on rim, three below body, one on base) of white background bands with geometrical black decoration

References: (Klimaszewska-Drabot and Mahmoud El-Tayeb 2014, 10-13)

\section{Cat. 294}

Stand?

Inv. no: $\mathrm{Z}_{7} / 58.1$

Provenance: Tumulus 7, W tunnel

Construction: wheel-made (2)

State of preservation: fragmentary, $14 \%$ of rim 


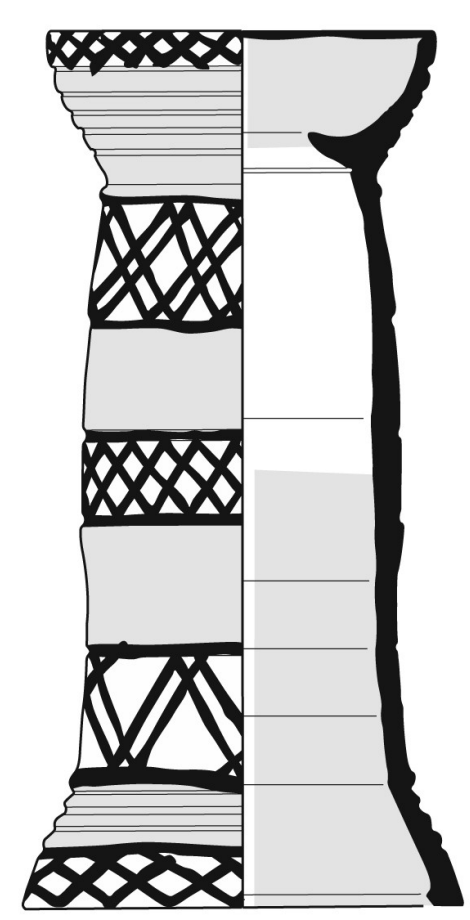

Cat. 293

Z23/40

FIGURE 7.69 Stands

Measurements: $\mathrm{Dr}=12.2 \mathrm{~cm} ; \mathrm{p}_{\mathrm{H}} \mathrm{H}=7.8 \mathrm{~cm}$

Fabric: ZF1O

Hardness: $3 / 4$

Firing: oxidized

Surface treatment: uncoated

Surface colour: external— 5 YR 5/4 reddish brown; internal- 5 Y $5 / 4$ reddish brown

Colour of break: 1OYR 4/2 dark greyish brown

11

\section{Group IX-Goblets}

11.1 Goblet Type IX.1

[Fig. 7.125]

\section{Cat. 295}

Goblet

Inv. no: Z24/20

Provenance: Tumulus 24, chamber 1, eastern part

Construction: wheel-made (2)

State of preservation: incomplete, restored from sherds, $66 \%$ of rim, foot missing

Measurements: $\operatorname{Dr}=7.6 \mathrm{~cm} ; \max$ body diam. $=10.7 \mathrm{~cm}$;

$$
{ }_{\mathrm{p}} \mathrm{H}=11 \mathrm{~cm}
$$

Fabric: ZF4

Hardness: $3 / 4$

Firing: oxidized

Surface treatment: slipped and polished on both surfaces

Surface colour: slip—1oR 4/6 red, on both surfaces

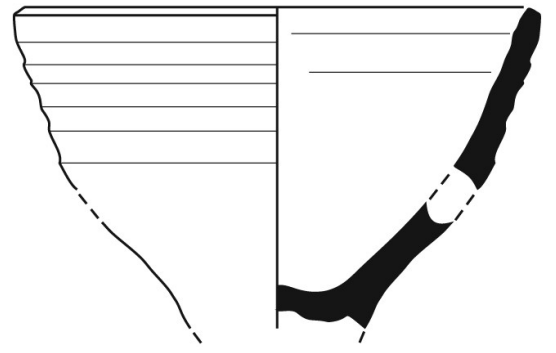

Cat. 294

Z7/58.1
Colour of break: 5YR 5/6 yellowish red

Decoration: on external surface; incised-four grooves below rim and seven on belly; painted — two dark bands and zigzag lines on belly, badly preserved

11.2 Goblet Type IX.2

[Fig. 7.125]

\section{Cat. 296}

Goblet

Inv. no: $\mathrm{Z} 10 / 7$

Provenance: Tumulus 10, burial chamber, southern part

Construction: wheel-made (2)

State of preservation: complete

Measurements: $\operatorname{Dr}=9.3 \mathrm{~cm} ; \max$ body diam. $=11.5 \mathrm{~cm}$;

$$
\mathrm{H}=9 \mathrm{~cm}
$$

Fabric: not possible to determine

Hardness: $3 / 4$

Firing: not possible to determine

Surface treatment: slipped on both surfaces

Surface colour: slip—1oR 4/6 red, on both surfaces

Colour of break: not possible to determine

Decoration: on external surface; incised-one groove at mid-H; painted-groups of white vertical and horizontal lines arranged alternately, black vertical wavy lines

References: (Klimaszewska-Drabot 2010, 484-485; Mahmoud El-Tayeb 2010, 214-216) incorrectly published as cup from tumulus 25 


\section{Goblets Type IX}

Goblet Type IX.1

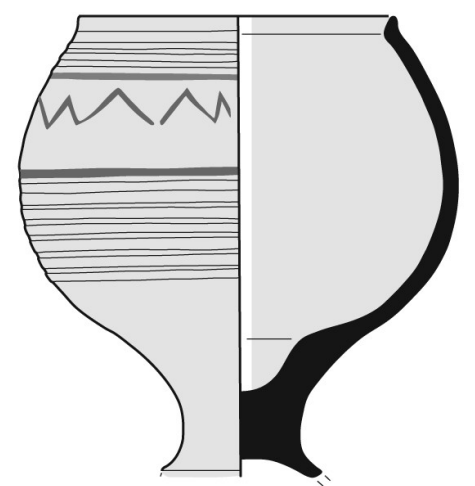

Cat. 295
$Z 24 / 20$
Goblet Type IX.2

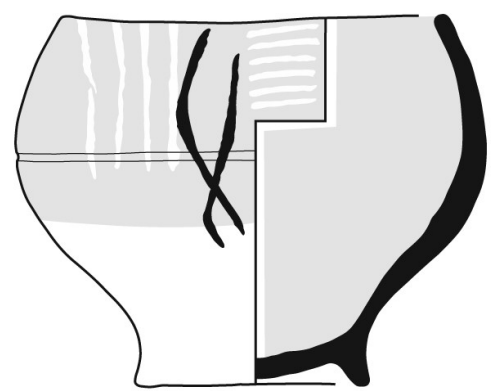

Cat. 296

Z10/7
Goblet Type IX.3

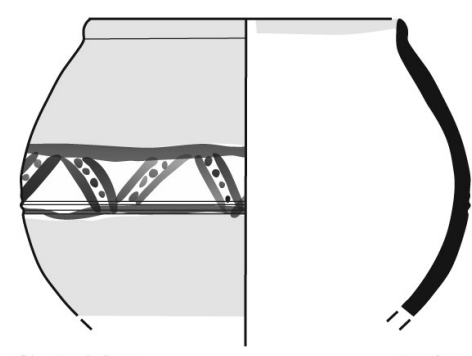

Cat. 297

FIGURE 7.70 Goblets of types IX.1, IX.2 and IX.3

\subsection{Goblet Type IX.3}

\section{Cat. 297}

Cup?

Inv. no: $\mathrm{Z} 4 / 202$

Provenance: Tumulus 4, E tunnel

Construction: wheel-made (2)

State of preservation: fragmentary, $21 \%$ of rim, body sherds Measurements: $\mathrm{Dr}=7.6 \mathrm{~cm} ; \max$ body diam. $=11 \mathrm{~cm}$;

$$
{ }_{\mathrm{p}} \mathrm{H}=7.2 \mathrm{~cm}
$$

Fabric: ZF4

Hardness: 4

Firing: oxidized

Surface treatment: slipped and polished on both surfaces

Surface colour: external, slip_-10R 4/6 red; internal, slip$10 \mathrm{R}_{5} / 6$ red

Colour of break: $10 \mathrm{R} 5 / 6$ red

Decoration: on external surface; incised-two grooves at mid-H; painted - white background bands, two horizontal black outlines, dark zigzag strokes forming outlines with dark dots between them

12

$$
\text { Group X-Oil Lamps }
$$

[Fig. 7.126]

Cat. 298

Oil lamp

Inv. no: $\mathrm{Z6} / 21$

Provenance: Tumulus 6, E tunnel

Construction: wheel-made (2)

State of preservation: incomplete, chipped surface Measurements: $\mathrm{Dr}=2.3 \mathrm{~cm} ; \mathrm{D}_{\mathrm{b}}=4.1 \mathrm{~cm} ; \mathrm{H}=6.7 \mathrm{~cm}$ Fabric: not possible to determine
[Fig. 7.125] Hardness: 3/4

Firing: not possible to determine

Surface treatment: smoothed on external surface

Surface colour: external natural surface-2.5YR 5/6 red

Colour of break: not possible to determine

Decoration: incised — cross on upper part of the lamp

Notes: potmark on base

\section{Cat. 299}

Oil lamp

Inv. no: $\mathrm{Z}_{7} / 49$

Provenance: Tumulus 7, context 8

Construction: wheel-made (2)

State of preservation: incomplete, broken handle, lip chipped off

Measurements: $\mathrm{Dr}=2.3 \mathrm{~cm} ; \mathrm{D}_{\mathrm{b}}=3.8 \mathrm{~cm} ; \mathrm{H}=5.9 \mathrm{~cm}$

Fabric: similar to $\mathrm{ZF} 4$

Hardness: 4

Firing: oxidized

Surface treatment: slipped and polished on external surface

Surface colour: external, slip-10R $5 / 6$ red

Colour of break: 5 YR $5 / 6$ yellowish red

Cat. 300

Oil lamp

Inv. no: $\mathrm{Z}_{4} / \mathbf{6 1}$

Provenance: Tumulus 4, W tunnel

Construction: wheel-made (2)

State of preservation: incomplete, rim and body sherd missing

Measurements: $\mathrm{D}_{\mathrm{b}}=2.9 \mathrm{~cm} ; \mathrm{H}=4.5 \mathrm{~cm}$ 
Fabric: not possible to determine

Hardness: $3 / 4$

Firing: oxidized

Surface treatment: slipped and polished on external surface

Surface colour: external, slip-10R 4/6 red-2.5YR 4/6 red, internal natural surface -5 Y $6 / 3$ light reddish brown

Colour of break: not possible to determine

Decoration: incised-herringbone pattern on upper part of lamp

Notes: potmark on base

Cat. 301

Oil lamp

Inv. no: $\mathbf{Z} 4 / 200$

Provenance: Tumulus 4, E tunnel

Construction: wheel-made (2)

State of preservation: incomplete, handle and body sherds missing

Measurements: $\mathrm{Dr}=2.4 \mathrm{~cm} ; \mathrm{D}_{\mathrm{b}}=3 \mathrm{~cm} ; \mathrm{H}=6.6 \mathrm{~cm}$

Fabric: similar to ZF4

Hardness: 3

Firing: oxidized

Surface treatment: slipped on the external surface

Surface colour: external natural surface-5YR 7/4 pink, slip- $10 \mathrm{R} 5 / 8$ red

Colour of break: 5YR 6/6 reddish yellow

Cat. 3 o2

Oil lamp

Inv. no: $\mathbf{Z} 4 / 10.7$

Provenance: Tumulus 4, tunnel

Construction: wheel-made (2)

State of preservation: incomplete, $75 \%$ of rim, body sherds missing

Measurements: $\mathrm{Dr}=1.3 \mathrm{~cm} ;{ }_{\mathrm{p}} \mathrm{H}=3.6 \mathrm{~cm}$

Fabric: not possible to determine

Hardness: $3 / 4$

Firing: oxidized

Surface treatment: slipped and polished on external surface

Surface colour: external natural surface- 5 YR 5/4 reddish brown, slip-10R $5 / 6$ red, internal natural surface5Y $6 / 4$ light reddish brown

Colour of break: not possible to determine
13

Group XI-Qawadis Type XI

[Fig. 7.127]

Cat. 303

Qadus

Inv. no: $\mathbf{Z 1 1} / \mathbf{4 3}+\mathrm{Zn1} / \mathbf{4 7}$

Provenance: Tumulus 11, robber shaft

Construction: wheel-made (2)

State of preservation: fragmentary, restored from sherds, $50 \%$ of rim, upper part of body, $100 \%$ of toe, one lower body sherd

Measurements: $\mathrm{Dr}=16.6 \mathrm{~cm} ;{ }_{\mathrm{p}} \mathrm{H}=31 \mathrm{~cm}$

Fabric: ZF6

Hardness: 4

Firing: reduced

Surface treatment: uncoated

Surface colour: external—1OR 5/6 red; internal—2.5YR 5/6 red

Colour of break: Gley1 3/N black

References: (Klimaszewska-Drabot and Czyżewska 2012, 372-377)

Cat. 304

Qadus

Inv. no: Z6/19.1+Z6/19.2+Z6/38.1

Provenance: Tumulus 6, fill of robber shaft, tunnel and central part of external shaft $3 \mathrm{~m}$ below surface level

Construction: wheel-made (2)

State of preservation: fragmentary, rim and wall fragments, $82 \%$ of rim

Measurements: $\mathrm{Dr}=16 \mathrm{~cm} ;{ }_{\mathrm{p}} \mathrm{H}=8.5 \mathrm{~cm}$

Fabric: ZF6

Hardness: 4

Firing: reduced

Surface treatment: uncoated

Surface colour: external—-5YR 5/4 reddish brown; internal- 5 Y $6 / 3$ light reddish brown

Colour of break: $2.5 \mathrm{Y} 4 / 1$ dark grey

Cat. 305

Qadus

Inv. no: Z26/6o.2,3+Z26/63.3+Z26/62+Z26/69+Z26/67+ Z26/64

Provenance: Tumulus 26, shaft fill, robber hole

Construction: wheel-made (2)

State of preservation: fragmentary, restored from sherds, $38 \%$ of rim, fragments of upper part of vessel, sherds of lower part of vessel

Measurements: $\mathrm{Dr}=15.6 \mathrm{~cm} ;{ }_{\mathrm{p}} \mathrm{H}=15 \mathrm{~cm}$

Fabric: ZF6

Hardness: 4

Firing: reduced 


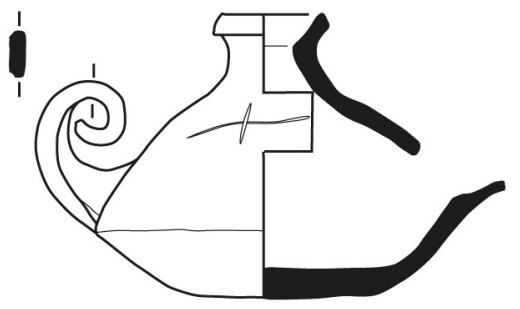

Cat. 298

Z6/21
GROUP X

Oil Lamps

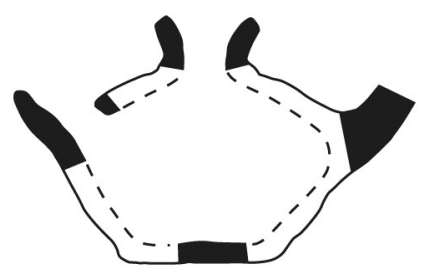

Cat. 299

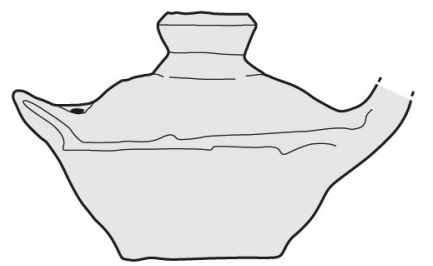

Z7/49
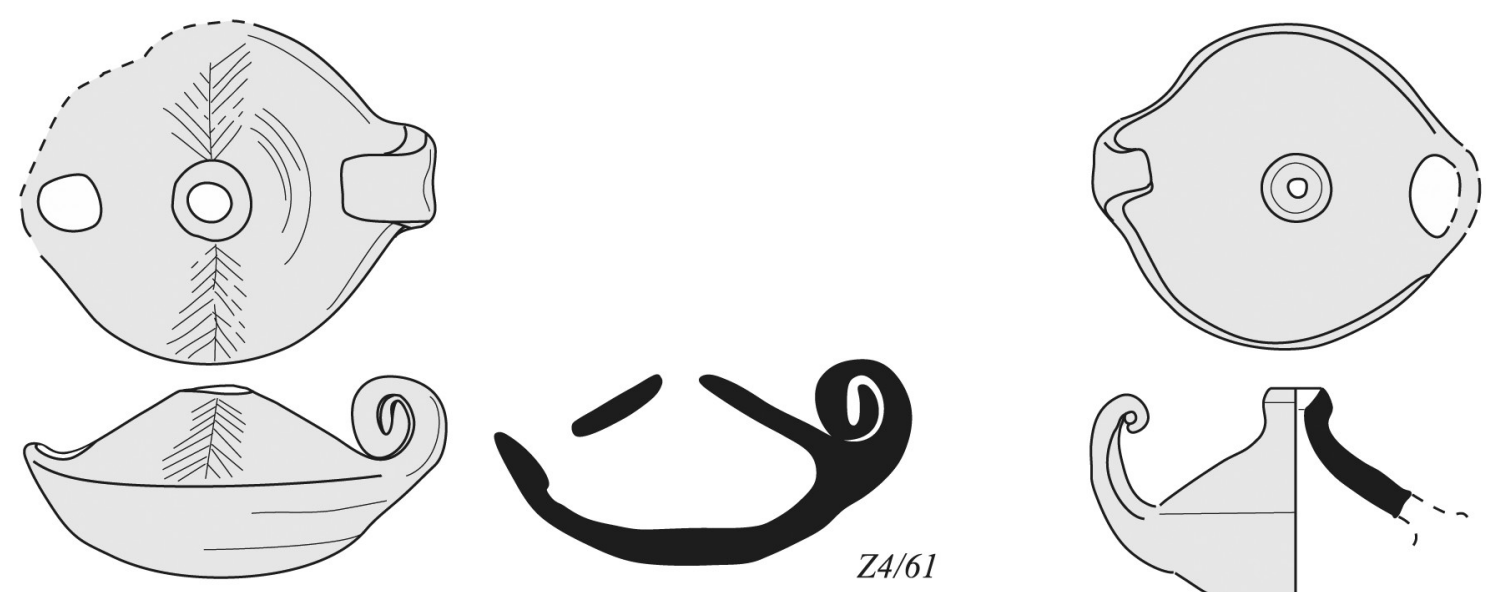

Z4/61

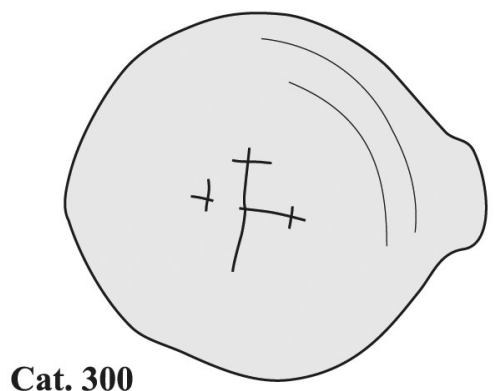

Cat. 300

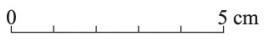

FIGURE 7.71 Oil lamps of group $\mathrm{X}$
Cat. $302 \quad Z 4 / 10.7$

Cat. $302 \quad Z 4 / 10.7$
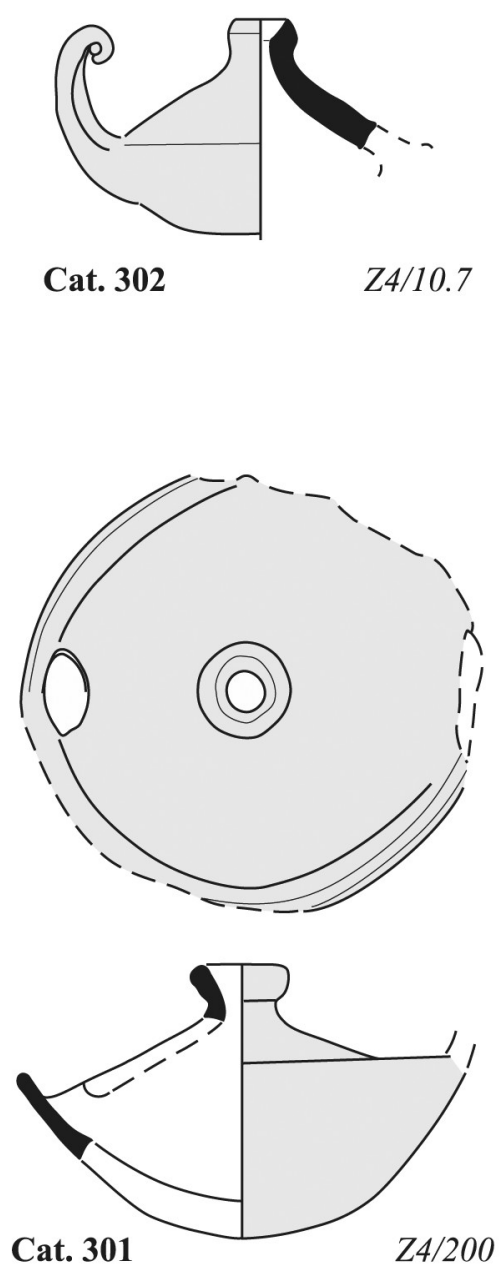


\section{Qawadis Type XI}

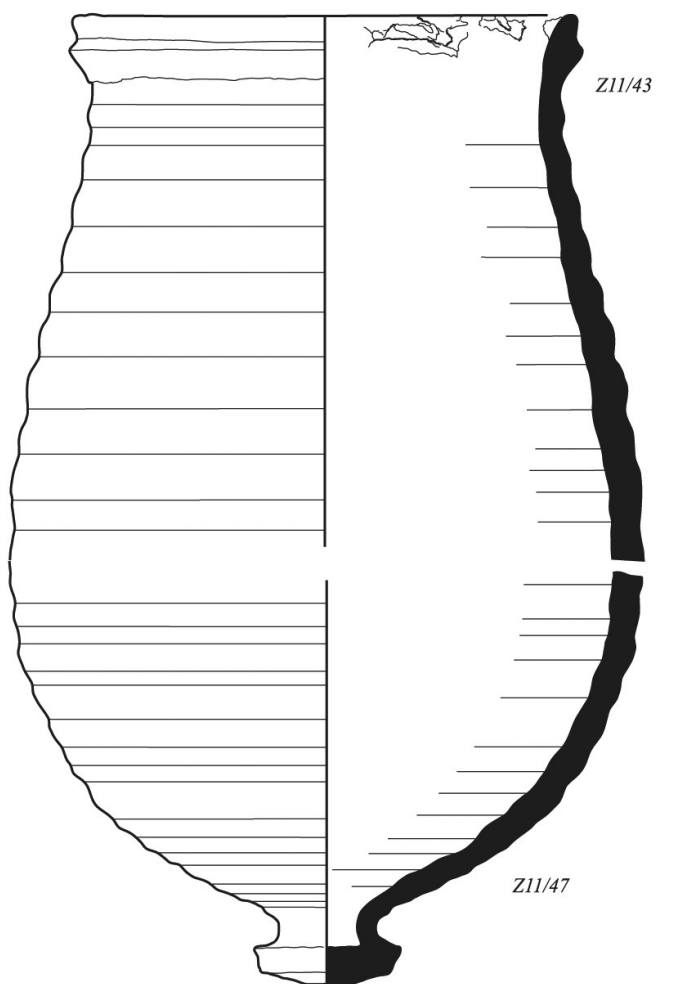

Cat. 303

$Z 11 / 43+Z 11 / 47$

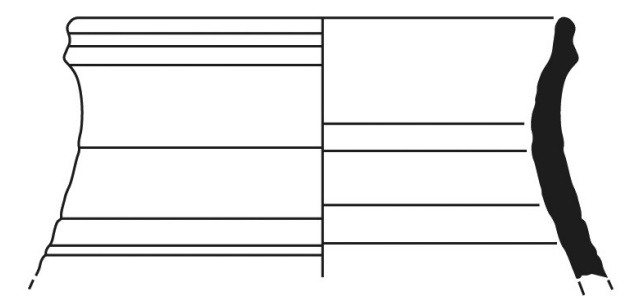

Cat. $304 \quad Z 6 / 19.1+Z 6 / 19.2+Z 6 / 38.1$

FIGURE 7.72 Qawadis of group XI

Surface treatment: uncoated

Surface colour: external-2.5YR 5/4 reddish brown; internal-2.5YR $5 / 6$ red

Colour of break: Gley1 2.5/N black

Notes: visible deformation of lower part of vessel

Cat. 306

Qadus

Inv. no: $\mathrm{Z} 26 / 6$ o.1 $+\mathrm{Z} 26 / 41+\mathrm{Z} 26 / 68+Z_{2} 6 / 71$

Provenance: Tumulus 26 , shaft fill

Construction: wheel-made (2)

State of preservation: fragmentary, restored from sherds, $93 \%$ of rim, fragments of upper part of vessel

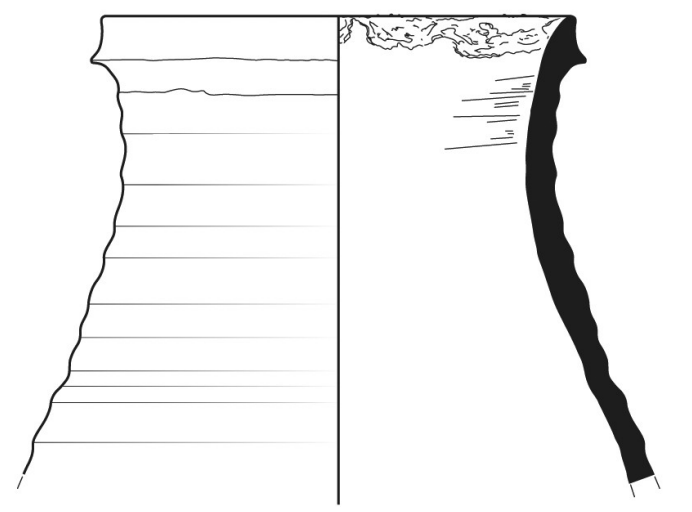

$Z 26 / 60.2+Z 26 / 60.3+Z 26 / 63.3+Z 26 / 62$

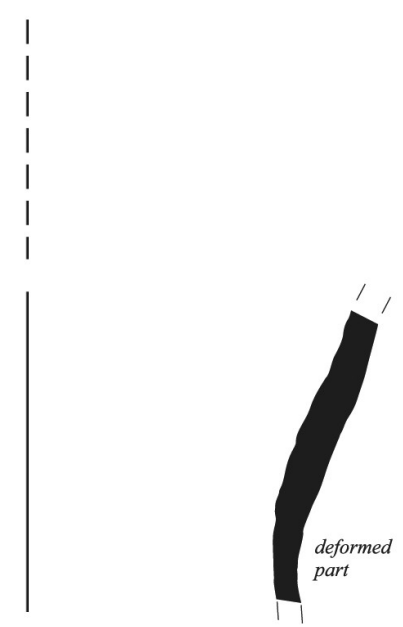

Cat. 305

$Z 26 / 69+Z 26 / 67+Z 26 / 64$ $5 \mathrm{~cm}$
Measurements: $\mathrm{Dr}=16.3 \mathrm{~cm} ;{ }_{\mathrm{p}} \mathrm{H}=16.4 \mathrm{~cm}$

Fabric: ZF6

Hardness: 4

Firing: reduced

Surface treatment: uncoated

Surface colour: external-5YR 6/4 light reddish brown; internal -5 YR $5 / 2$ reddish grey

Colour of break: 1OYR 3/1 very dark grey

Cat. 307

Qadus

Inv. no: $\mathbf{Z 2 6 / 6 8 . 2}$

Provenance: Tumulus 26, shaft, near S chamber

Construction: wheel-made (2) 
State of preservation: fragmentary, restored from sherds, upper part of vessel, $27 \%$ of rim

Measurements: $\mathrm{Dr}=17 \mathrm{~cm} ;{ }_{\mathrm{p}} \mathrm{H}=16.5 \mathrm{~cm}$

Fabric: ZF6

Hardness: 4

Firing: reduced

Surface treatment: uncoated

Surface colour: external-2.5YR 5/6 red; internal-2.5YR $5 / 8 \mathrm{red}$

Colour of break: $2.5 \mathrm{Y}$ 4/1 dark grey

Cat. 308

Qadus

Inv. no: Z26/65.2

Provenance: Tumulus 26, shaft fill, near $\mathrm{W}$ wall of shaft

Construction: wheel-made (2)

State of preservation: fragmentary, rim and wall fragments, $41 \%$ of rim

Measurements: $\mathrm{Dr}=15 \mathrm{~cm} ;{ }_{\mathrm{p}} \mathrm{H}=11 \mathrm{~cm}$

Fabric: $\mathrm{ZF} 6$

Hardness: 4

Firing: reduced

Surface treatment: uncoated

Surface colour: external—1OR 5/6 red; internal—1OR 5/4 weak red

Colour of break: 5YR 4/1 dark grey

Cat. 309

Qadus

Inv. no: Z26/70.1

Provenance: Tumulus 26, shaft fill, NW corner of shaft

Construction: wheel-made (2)

State of preservation: fragmentary, $20 \%$ of rim

Measurements: $\mathrm{Dr}=15 \mathrm{~cm} ;{ }_{\mathrm{p}} \mathrm{H}=4.5 \mathrm{~cm}$

Fabric: $\mathrm{ZF} 6$

Hardness: 4

Firing: reduced

Surface treatment: uncoated

Surface colour: external-2.5YR $5 / 6$ red; internal $-2.5 \mathrm{YR}$ $5 / 4$ reddish brown

Colour of break: Gleyı 3/N black

Cat. 310

Qadus

Inv. no: Z26/69.3

Provenance: Tumulus 26, shaft fill, robber shaft

Construction: wheel-made (2)

State of preservation: fragmentary, $22 \%$ of rim

Measurements: $\mathrm{Dr}=21 \mathrm{~cm} ;{ }_{\mathrm{p}} \mathrm{H}=6.5 \mathrm{~cm}$

Fabric: zF6
Hardness: 4

Firing: reduced

Surface treatment: uncoated

Surface colour: external-5YR 6/9 light reddish brown; internal-5YR 6/9 light reddish brown

Colour of break: $2.5 \mathrm{Y}$ 4/1 dark grey

Cat. 311

Qadus

Inv. no: $\mathrm{Z}_{25} / \mathbf{1}$

Provenance: Tumulus 26, shaft fill

Construction: wheel-made (2)

State of preservation: fragmentary, $100 \%$ of base

Measurements: $\mathrm{D}_{\mathrm{b}}=3.5 \mathrm{~cm} ;{ }_{\mathrm{p}} \mathrm{H}=3.4 \mathrm{~cm}$

Fabric: zF6

Hardness: 4

Firing: reduced

Surface treatment: uncoated

Surface colour: external-5YR 5/6 yellowish red; internal-2.5YR $5 / 6$ red

Colour of break: 2 Y 2.5/1 black

\section{Group XII-Amphorae}

14.1 Amphorae Type XII.1

[Fig. 7.128]

Cat. 312

Amphora ("Dongolan" amphora Pluskota type A)

Inv. no: $\mathrm{Z26} / \mathbf{3 2}^{2}$

Provenance: Tumulus 26, chamber 1, central part

Construction: wheel-made (2)

State of preservation: incomplete, restored from sherds, rim and base complete

Measurements: $\mathrm{Dr}=7.7 \mathrm{~cm} ; \mathrm{H}=54 \mathrm{~cm} ; \mathrm{D}_{\mathrm{b}}=2.5 \mathrm{~cm}$

Fabric: similar to the Dongolan ALF 1 fabric

Hardness: 4

Firing: oxidized

Surface treatment: uncoated

Surface colour: external-1oR 6/3 pale red, internal5 YR 6/4 light reddish brown

Colour of break: $10 \mathrm{R} 6 / 3$ pale red

Cat. 313

Amphora ("Dongolan" amphora Pluskota type A)

Inv. no: $\mathrm{Z} 4 / 14.6$

Provenance: Tumulus 4, tunnel

Construction: wheel-made (2)

State of preservation: incomplete, survives in two parts, $55 \%$ of rim, $35 \%$ of base

Measurements: $\mathrm{Dr}=8 \mathrm{~cm} ; \mathrm{H}=55 \mathrm{~cm} ; \mathrm{D}_{\mathrm{b}}=3.5 \mathrm{~cm}$

Fabric: similar to the Dongolan ALF 1 fabric 


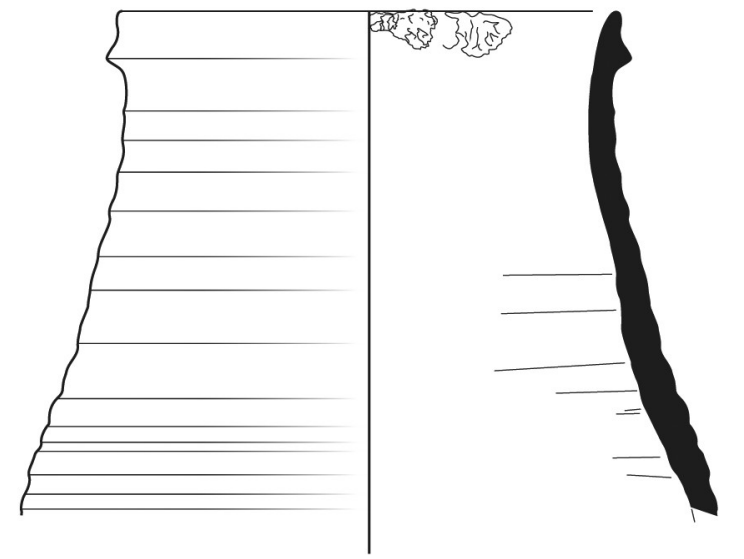

Cat. $306 \quad Z 26 / 60.1+Z 26 / 41+Z 26 / 68+Z 26 / 71$

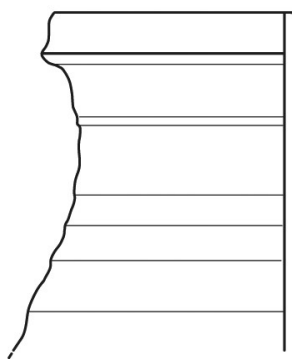

Cat. 308

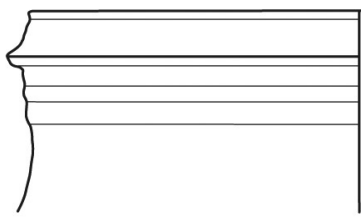

Cat. 310

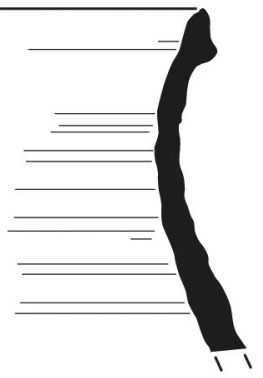

Z26/65.2
FIGURE 7.73

Qawadis of group XI

\section{Hardness: 4}

Firing: oxidized

Surface treatment: uncoated

Surface colour: external-10R 5/6 red, internal-10R 7/4 pale red

Colour of break: 7.5YR 8/4 pink

\section{Cat. 314}

Amphora ("Dongolan" amphora Pluskota type A)

Inv. no: Z24/29

Provenance: Tumulus 24, south-eastern part of shaft

Construction: wheel-made (2)

State of preservation: fragmentary, restored from sherds, rim and base missing

Measurements: ${ }_{\mathrm{p}} \mathrm{H}=50.6 \mathrm{~cm}$

Fabric: similar to the Dongolan ALF 1 fabric

Hardness: 4

\section{Qawadis Type XI}

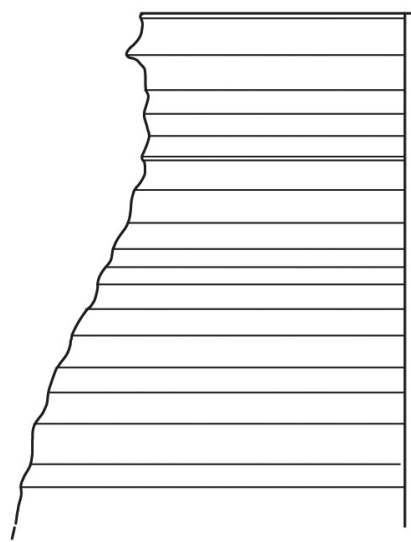

Cat. 307

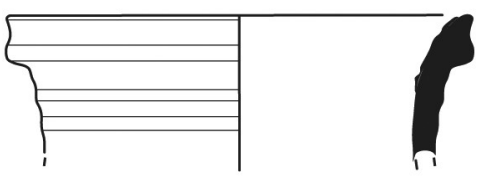

Cat. 309

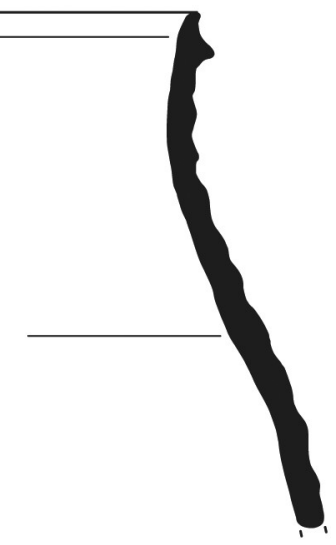

Z26/68.2

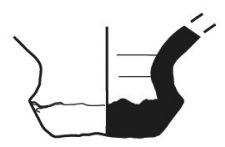

Cat. $311 \quad Z 25 / 1$

0 $5 \mathrm{~cm}$

Firing: oxidized

Surface treatment: uncoated

Surface colour: external-2.5YR 4/6 red, internal-2.5YR $5 / 6 \mathrm{red}$

Colour of break: 2.5 YR $5 / 6$ red

\section{Cat. 315}

Amphora ("Dongolan" amphora Pluskota type A)

Inv. no: $\mathrm{Z}_{7} / 25$

Provenance: Tumulus 7 , fragments scattered in different places: topsoil, probably modern dump; contexts 1, 2, 3, 5 , fill of external shaft

State of preservation: fragmentary, restored from sherds, survives in two pieces, rim and base missing

Construction: wheel-made (2)

Measurements: $\mathrm{H}$ : upper part $=24.6 \mathrm{~cm}$, lower part $=$ $12.7 \mathrm{~cm}$ 


\section{Amphorae Type XII.1}

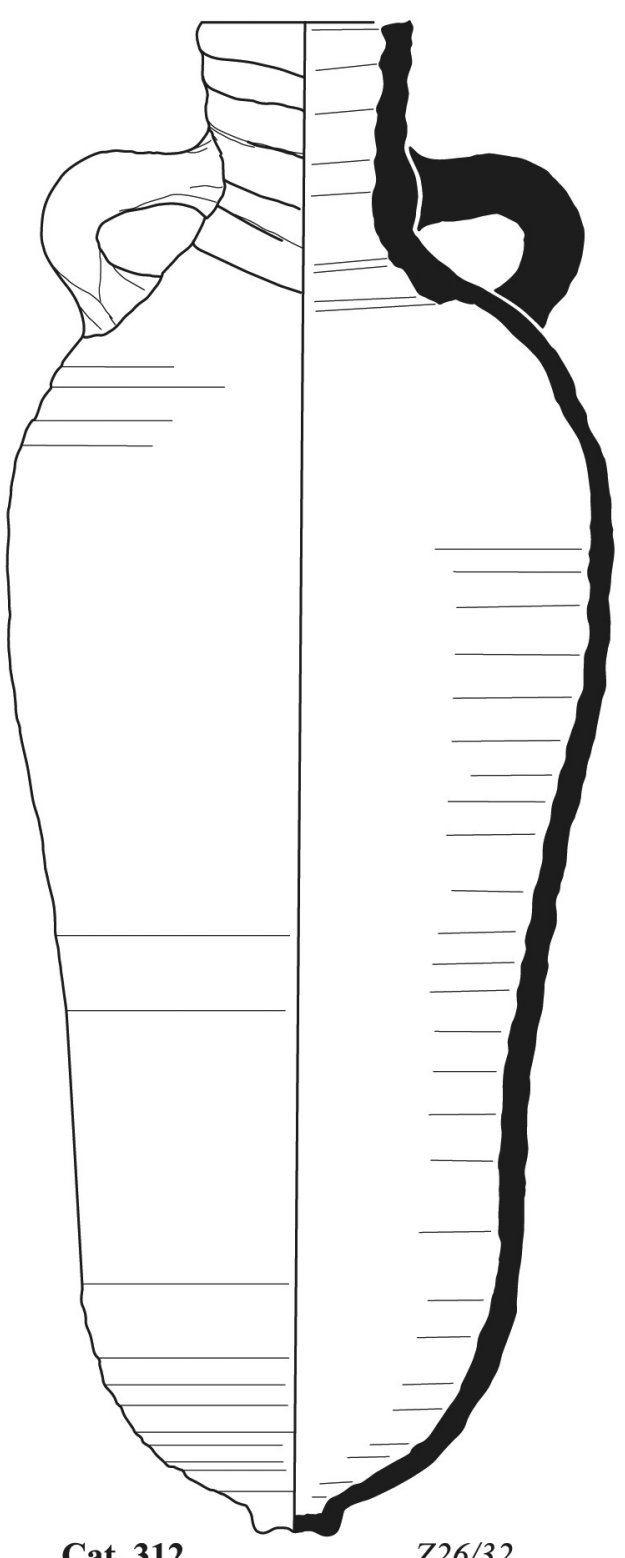

Cat. 312

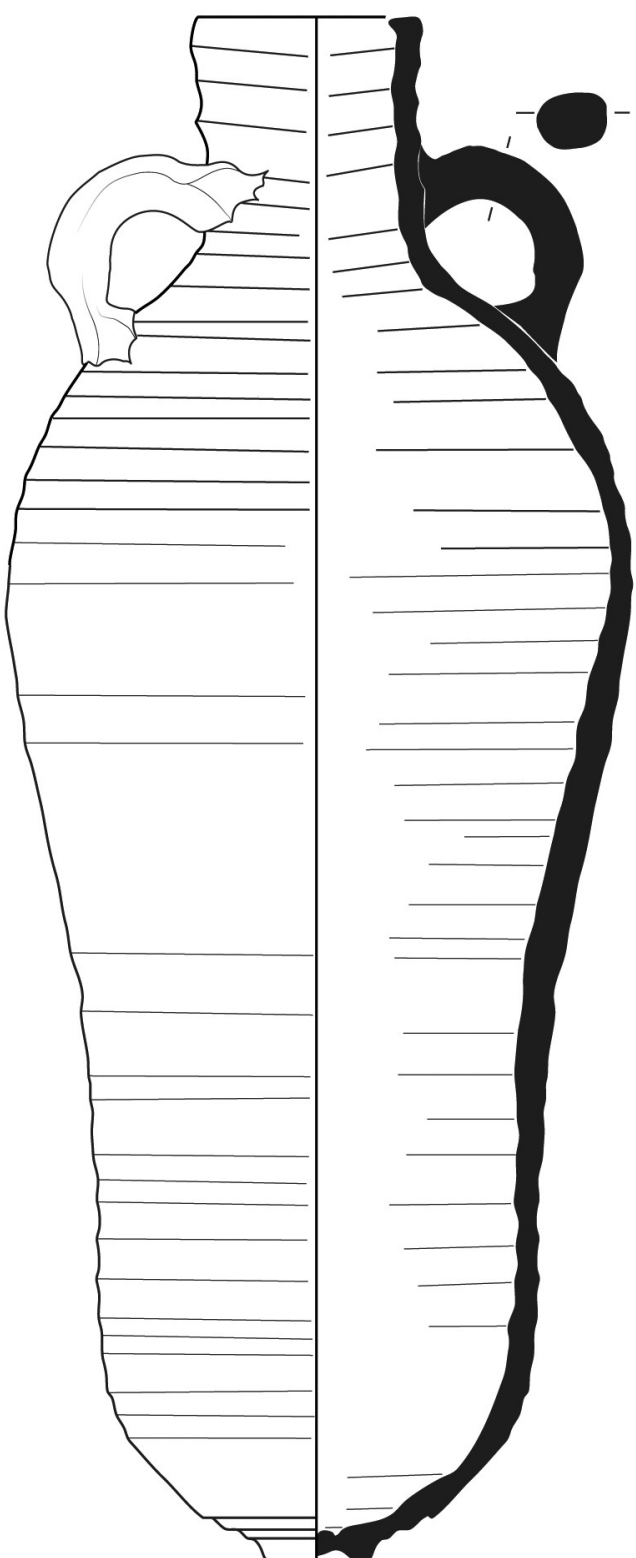

Cat. 313

$0,, 5 \mathrm{~cm}$

FIGURE 7.74 Transport amphorae of type XII.1, LRA “Dongola” (Pluskota A amphorae)

Fabric: similar to the Dongolan ALF 1 fabric

Hardness: 4

Firing: oxidized

Surface treatment: uncoated

Surface colour: external—1OR 5/6 red, internal $-5 \mathrm{YR} 8 / 3$ pink

Colour of break: 5YR 6/4 light reddish brown

Cat. 316

Amphora ("Dongolan" amphora Pluskota type A)

Inv. no: $\mathbf{Z} \mathbf{4} / \mathbf{3 1}$
Provenance: Tumulus 4, fragments scattered in different places: fill of external shaft; fill of W tunnel $(3.40-4.25 \mathrm{~m}$ from edge of shaft), layer 5

Construction: wheel-made (2)

State of preservation: fragmentary, $49 \%$ of rim, part of neck and shoulders, one handle

Measurements: $\mathrm{Dr}=9.4 \mathrm{~cm} ;{ }_{\mathrm{p}} \mathrm{H}=13.5 \mathrm{~cm}$

Fabric: similar to the Dongolan ALF 1 fabric

Hardness: 3

Firing: oxidized

Surface treatment: uncoated 
Cat. 314

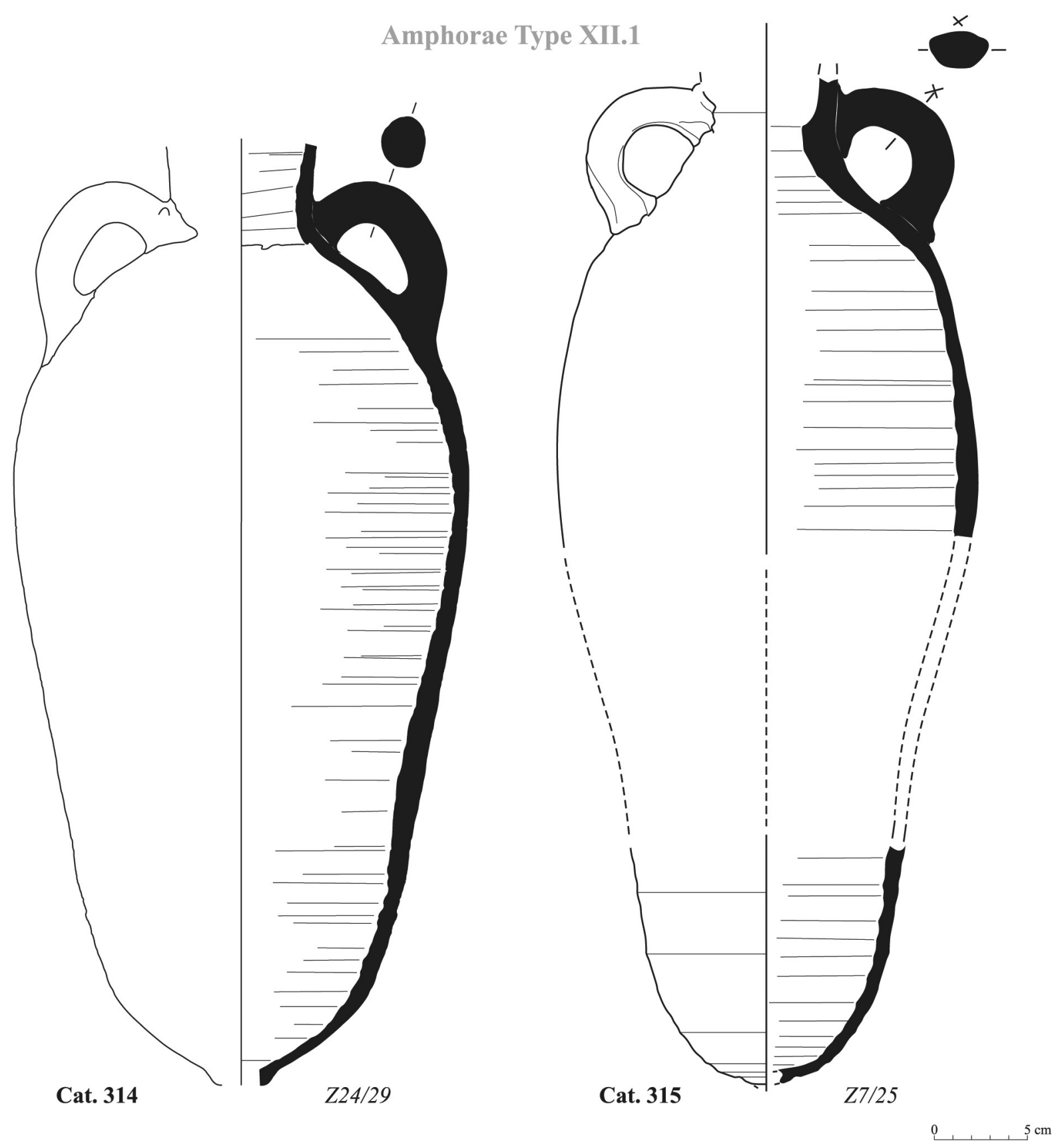

Z24/29

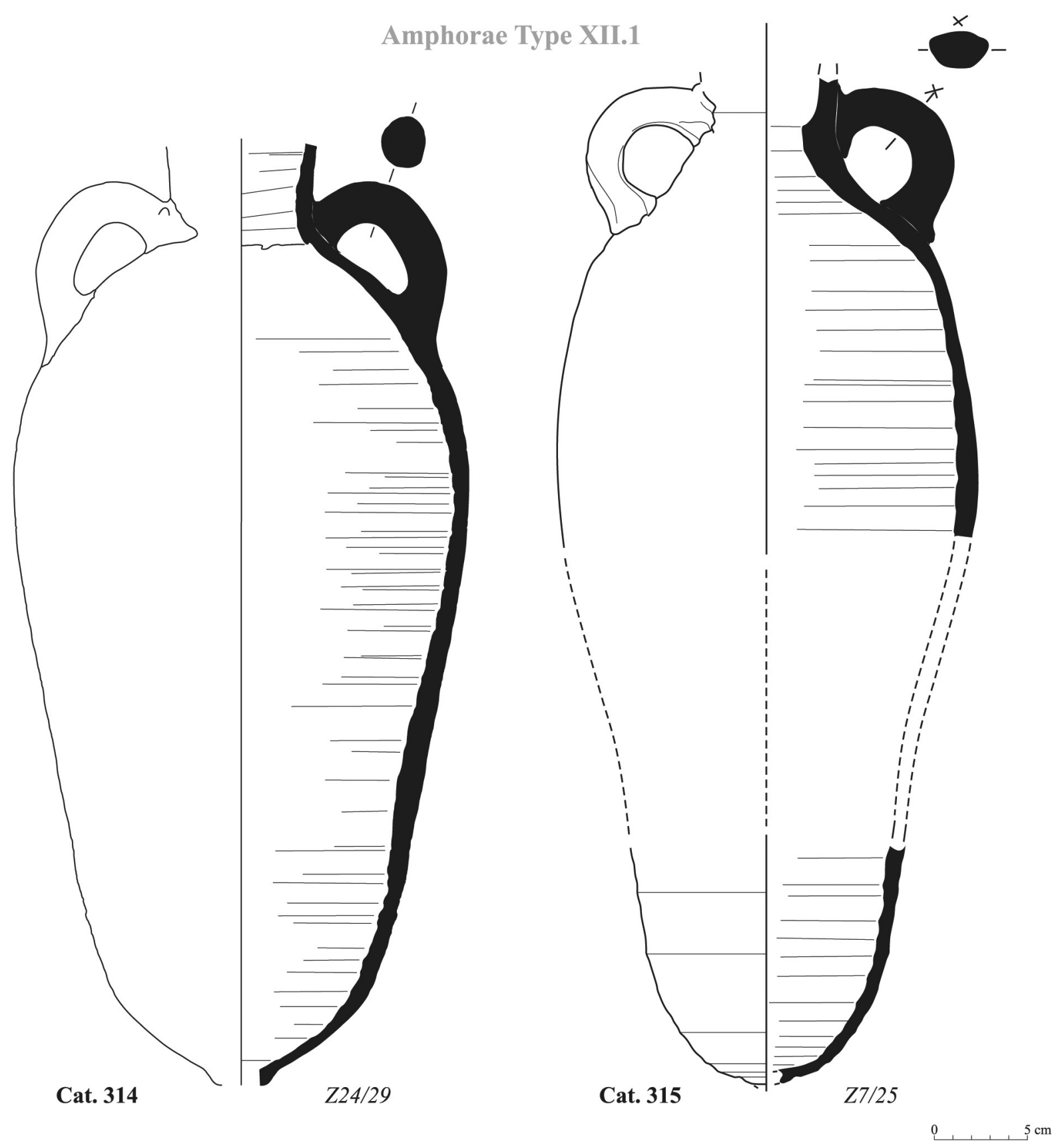

\section{Amphorae Type XIII.1}

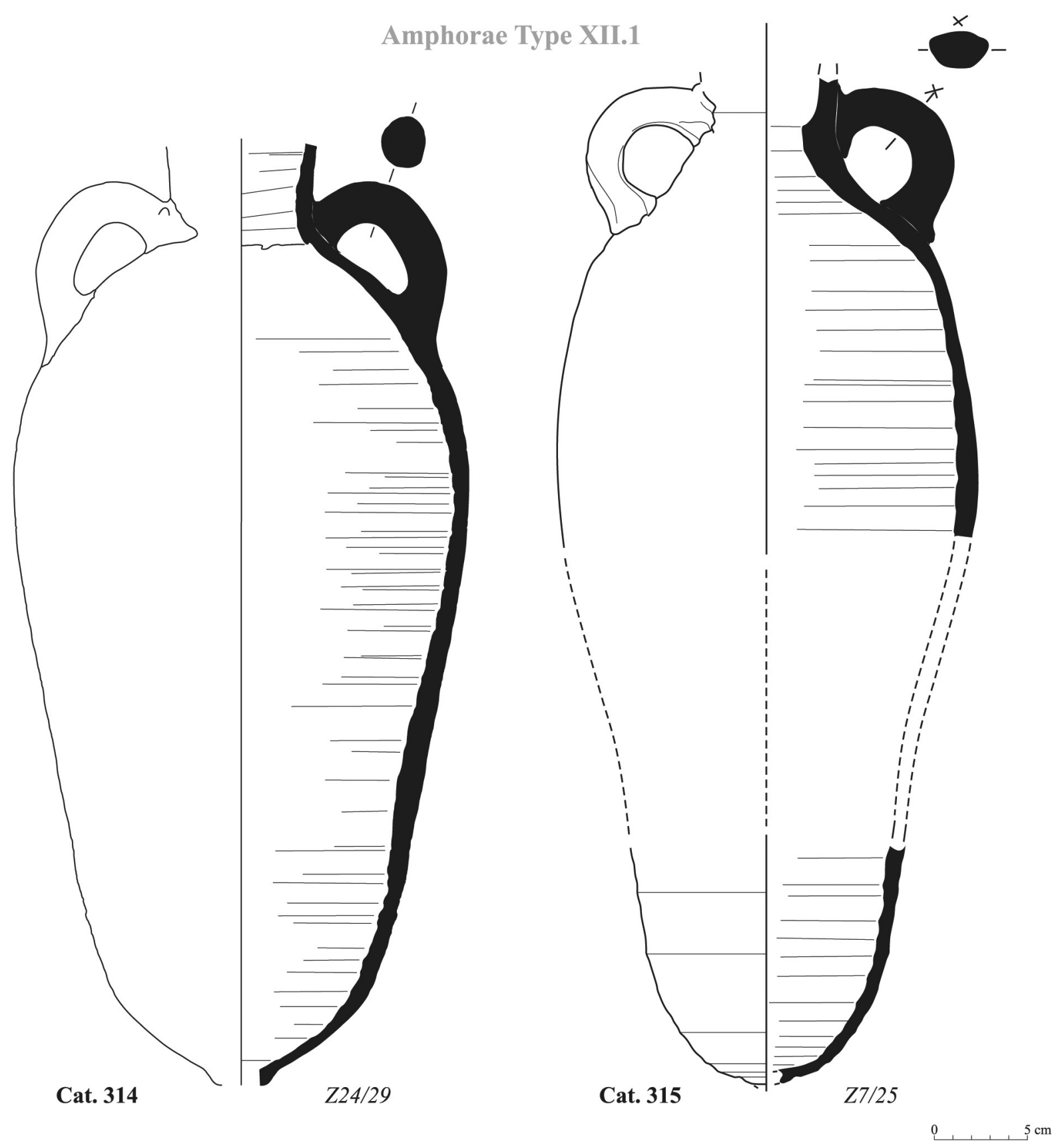

FIGURE 7.75 Transport amphorae of type XII.1, LRA “Dongola” (Pluskota A amphorae)

Surface colour: external—1oR 5/6 red, internal—10R 5/6 red

Colour of break: 2.5 YR $7 / 4$ pale yellow

\section{Cat. 317}

Amphora ("Dongolan" amphora Pluskota type A)

Inv. no: $\mathrm{Z}_{4} / \mathbf{2 2 5}$

Provenance: Tumulus 4, Eastern tunnel

State of preservation: fragmentary, $80 \%$ of rim

Construction: wheel-made (2)

Measurements: $\mathrm{Dr}=8.3 \mathrm{~cm} ;{ }_{\mathrm{p}} \mathrm{H}=6.5 \mathrm{~cm}$

Fabric: similar to the Dongolan ALF 2 fabric
Hardness: 3

Firing: oxidized

Surface treatment: uncoated

Surface colour: external-10R 5/4 weak red, internal1oR $5 / 6$ red

Colour of break: 2.5YR 6/6 light red

Cat. 318

Amphora ("Dongolan" amphora Pluskota type A)

Inv. no: $\mathrm{Z}_{4} / \mathbf{2 2 6}$

Provenance: Tumulus 4, E tunnel

State of preservation: fragmentary, $50 \%$ of base 
Construction: wheel-made (2)

Measurements: $\mathrm{D}_{\mathrm{b}}=3.6 \mathrm{~cm} ;{ }_{\mathrm{p}} \mathrm{H}=7 \mathrm{~cm}$

Fabric: similar to the Dongolan ALF 2 fabric

Hardness: 4

Firing: oxidized

Surface treatment: uncoated

Surface colour: external-7.5R 4/8 red; internal-10R 7/4 pale red

Colour of break: 10R 6/6 light red

\subsection{Amphora Type XII.2}

Cat. 319

Amphora LRA 3 with plug

Inv. no: $\mathrm{Z} 4 / 224$

Provenance: Tumulus 4, E tunnel

State of preservation: incomplete, survives in two pieces, complete rim, base missing

Construction: wheel-made (2)

Measurements: $\mathrm{Dr}=3.8 \mathrm{~cm} ;{ }_{\mathrm{p}} \mathrm{H}=48.7 \mathrm{~cm}$

Fabric: Asia Minor

Hardness: 4

Firing: oxidized

Surface treatment: uncoated

Surface colour: external—1oR 5/6 red, internal—2.5YR 4/6 red

Colour of break: 10R 4/8 red

\subsection{Amphorae Type XII.3}

[Fig. 7.129]

Cat. 320

Gazan Amphora (Majcherek Type 4)

Inv. no: $\mathrm{Z}_{7} / \mathbf{2 2}+\mathrm{Z}_{7} / 54$ (upper part) $+\mathrm{Z}_{7} / \mathbf{4 1}$ (body) $+\mathrm{Z}_{7} / 54$ (bottom)

Provenance: Tumulus 7 , fragments scattered in different places: topsoil, probably modern dump; contexts 1, 2, 3, $5,8,10$; fill of external shaft

State of preservation: fragmentary, restored from sherds, $100 \%$ of rim, $83 \%$ of base

Construction: wheel-made (2)

Measurements: $\mathrm{Dr}=11 \mathrm{~cm} ; \mathrm{D}_{\mathrm{b}}=3.6 \mathrm{~cm} ;{ }_{\mathrm{p}} \mathrm{H}=19.4 \mathrm{~cm}$ (upper part $)+30 \mathrm{~cm}$ (body) $+5 \mathrm{~cm}$ (bottom); $\mathrm{D}_{\mathrm{b}}=2.5 \mathrm{~cm}$

Fabric: Gaza

Hardness: 4

Firing: oxidized

Surface treatment: uncoated

Surface colour: external-5YR 6/4 light reddish brown; internal-5YR 6/4 light reddish brown

Colour of break: 5 YR $5 / 6$ yellowish red

Notes: upper part of vessel $\left(\mathrm{Z}_{7} / \mathbf{2 2}+\mathrm{Z}_{7} / 54\right)$ can be reassembled with body section $\left(\mathrm{Z}_{7} / 41\right)$ and bottom of vessel $\left(\mathrm{Z}_{7} / 54\right)$
Cat. $3^{21}$

Gazan Amphora (Majcherek Type 4)

Inv. no: $\mathrm{Z}_{7} / 93+\mathrm{Z}_{7} / 54$ (bottom)

Provenance: Tumulus 7, fill of external shaft

State of preservation: fragmentary, restored from sherds, $52 \%$ of rim, shoulders and handle fragments

Construction: wheel-made (2)

Measurements: $\mathrm{Dr}=11 \mathrm{~cm} ; \mathrm{D}_{\mathrm{b}}=3.6 \mathrm{~cm} ;{ }_{\mathrm{p}} \mathrm{H}=13.6 \mathrm{~cm}$ (upper part) $+5 \mathrm{~cm}$ (bottom)

Fabric: Gaza

Hardness: 4

Firing: oxidized

Surface treatment: uncoated

Surface colour: external-5YR 6/4 light reddish brown; internal-5YR 6/4 light reddish brown

Colour of break: 5 YR $5 / 6$ yellowish red

Notes: upper part of vessel $\left(\mathrm{Z}_{7} / 93\right)$ can be reassembled with bottom of vessel $\left(\mathrm{Z}_{7} / 54\right)$

\subsection{Amphorae Type XII.4}

[Fig. 7.129]

Cat. $3^{22}$

Amphora

Inv. no: $\mathbf{Z 2} / \mathbf{3}^{2}$

Provenance: Tumulus 2, chamber 2

State of preservation: complete

Construction: wheel-made (2)

Measurements: $\mathrm{Dr}=5.3 \mathrm{~cm} ; \mathrm{D}_{\mathrm{b}}=7.7 \mathrm{~cm} ; \mathrm{H}=30 \mathrm{~cm}$

Fabric: probably Aswan

Hardness: 4

Firing: oxidized

Surface treatment: uncoated

Surface colour: external-10 R 6/4 light red

Colour of break: 5 YR 7/4 pink

Decoration: dark spiral lines painted on body

Cat. 323

Amphora

Inv. no: $\mathrm{Z} \mathbf{1 5} / 5$

Provenance: Tumulus 15, fill of shaft, southern part, near E wall

State of preservation: complete

Construction: wheel-made (2)

Measurements: $\mathrm{Dr}=6.5 \mathrm{~cm} ; \mathrm{D}_{\mathrm{b}}=7 \mathrm{~cm} ; \mathrm{H}=23.5 \mathrm{~cm}$

Fabric: probably Aswan

Hardness: $3 / 4$

Firing: oxidized

Surface treatment: slip on external surface

Surface colour: external natural surface-5YR 7/4 pink, slip- 2.5 YR $5 / 4$ reddish brown

Colour of break: not possible to determine 
Amphorae Type XII.1

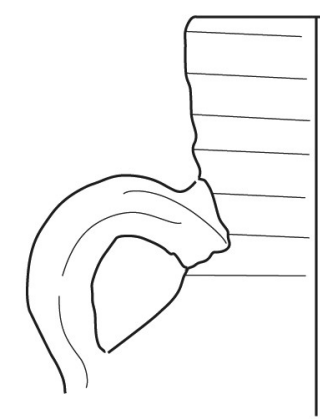

Cat. 316

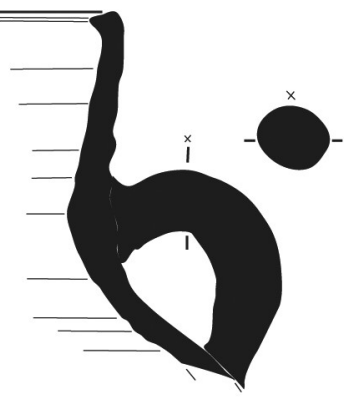

Z4/31

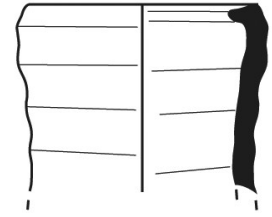

Cat. 317

$Z 4 / 225$

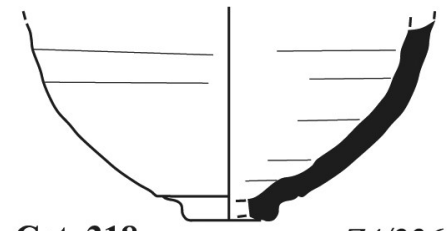

Cat. 318

$Z 4 / 226$
Amphorae Type XII.2

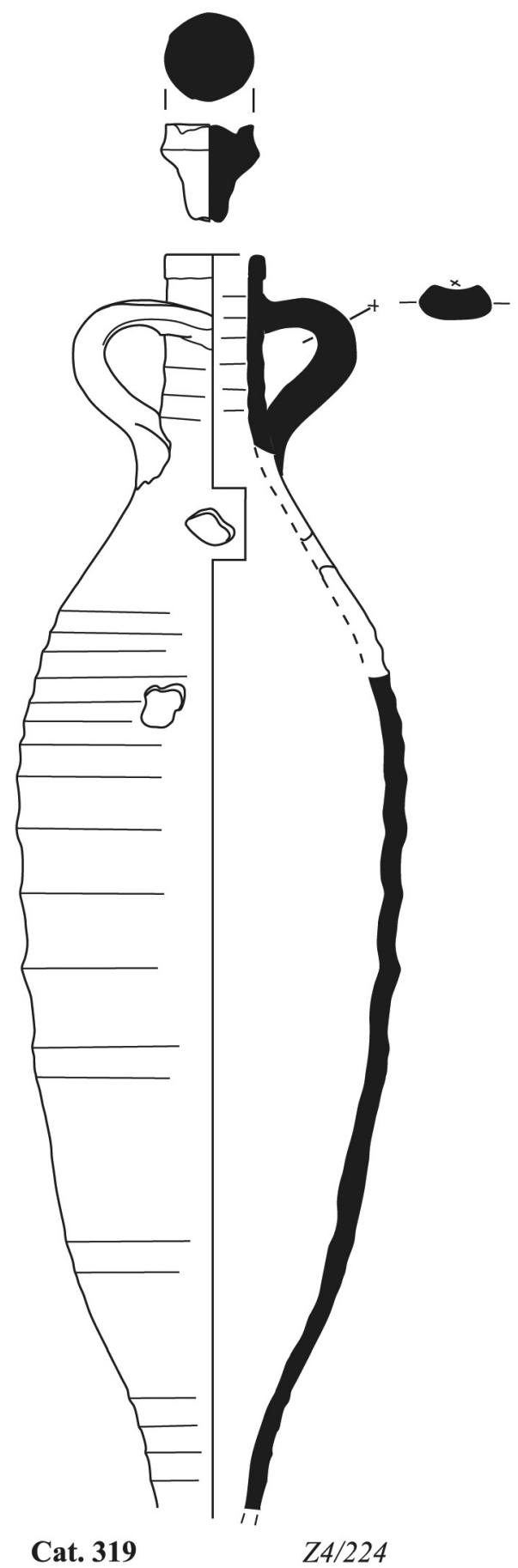

FIGURE 7.76 Transport amphorae of type XII.1, LRA "Dongola" (Pluskota A amphorae) and type XII.2, LRA 3 (Asia Minor) 
Amphorae Type XII.3

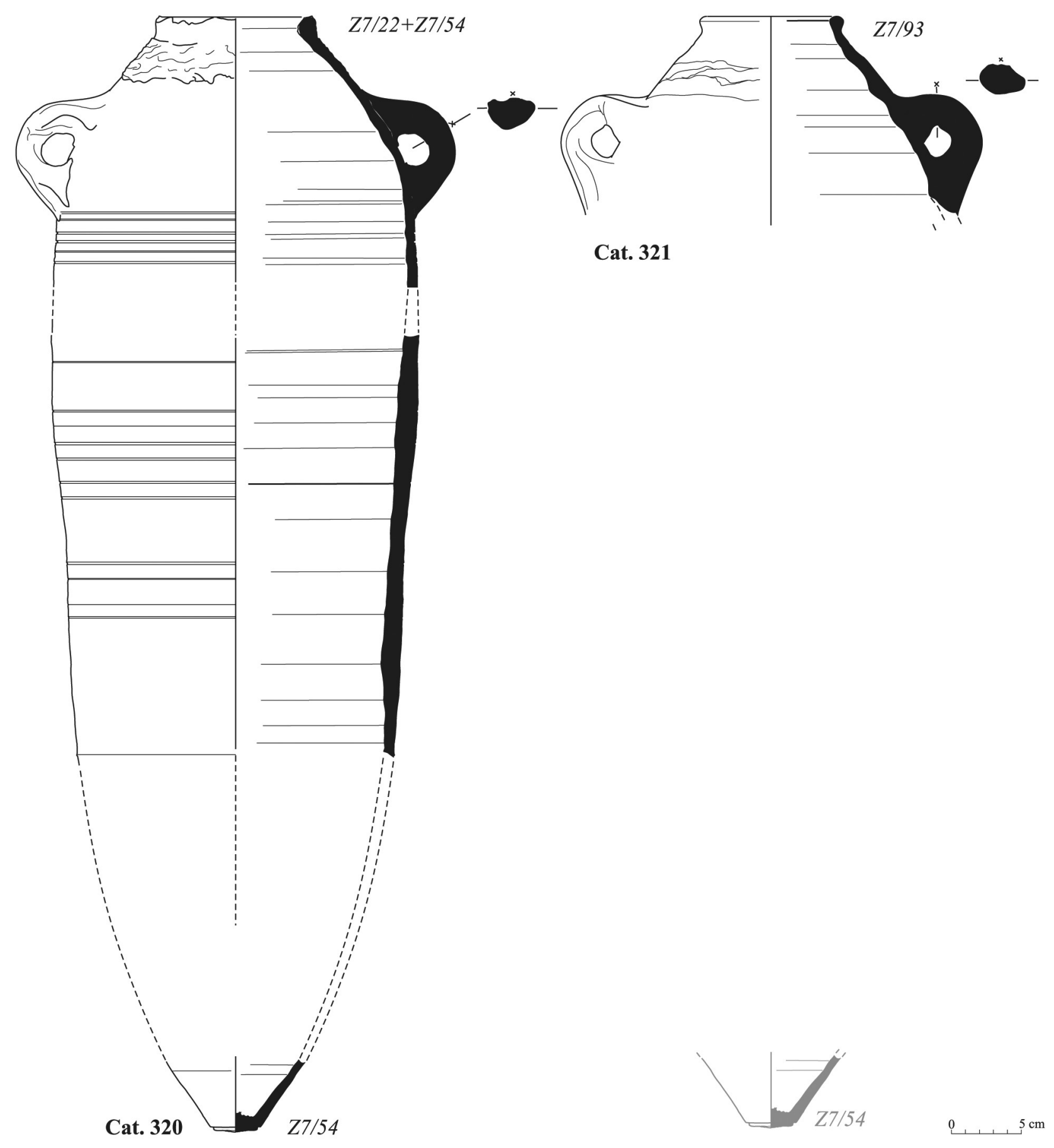

FIGURE 7.77 Transport amphorae of type XII.3, LRA 4 


\section{Amphorae Type XII.4}

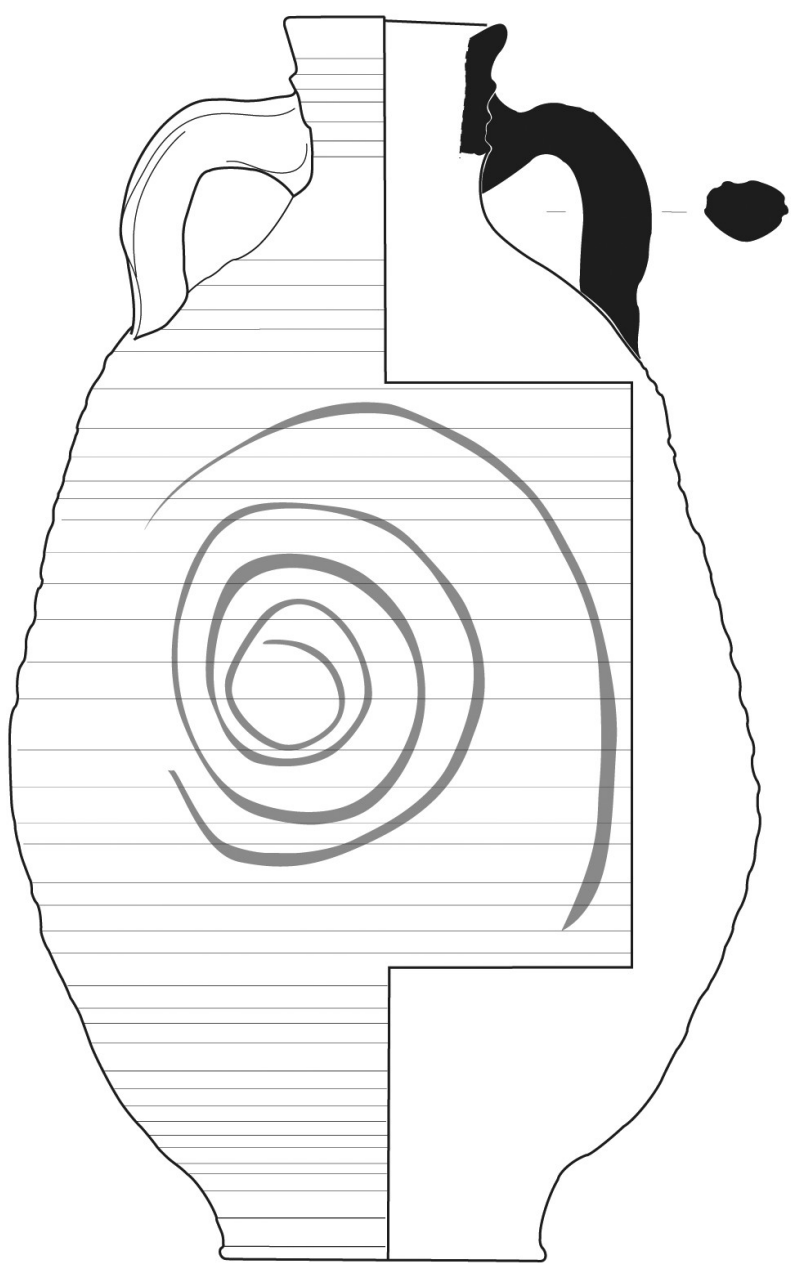

Cat. 322

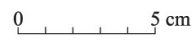

FIGURE 7.78

Small table amphorae of type XII.4

Cat. 324

Amphora

Inv. no: $\mathbf{Z} 15 / 56$

Provenance: Tumulus 15, main burial chamber, eastern part, near south wall

State of preservation: complete

Construction: wheel-made (2)

Measurements: $\mathrm{Dr}=4.4 \mathrm{~cm} ; \mathrm{D}_{\mathrm{b}}=6.8 \mathrm{~cm} ; \mathrm{H}=13.6 \mathrm{~cm}$

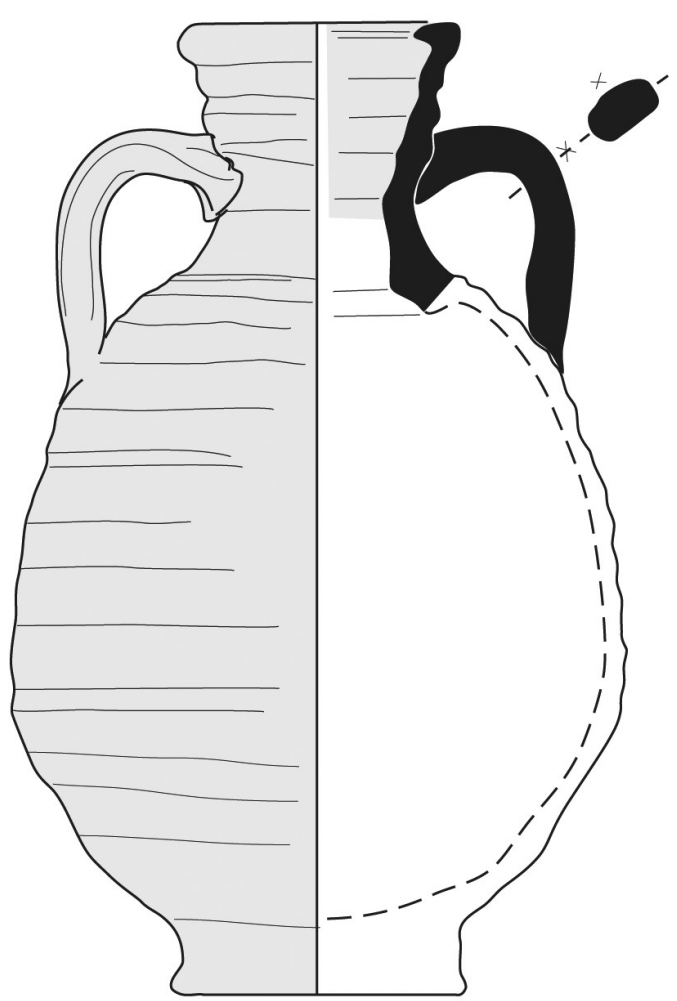

Cat. 323

$Z 15 / 5$

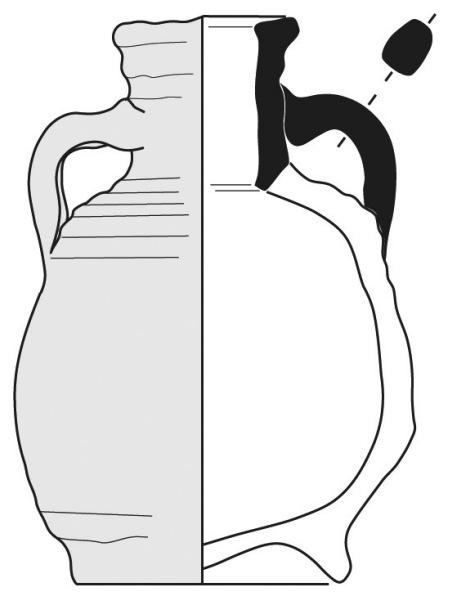

Cat. 324

$Z 15 / 56$

Fabric: Aswan area, different to fabric of $\mathrm{Z}_{15} / 5$ and $\mathrm{Z}_{2} / 3_{2}$; poor quality, more sandy and friable

Hardness: soft (2/3)

Firing: oxidized?

Surface treatment: slip on external surface

Surface colour: external natural surface-5YR 6/6 reddish yellow, slip-10R 6/6 light red

Colour of break: not possible to determine

Notes: surface very corroded 


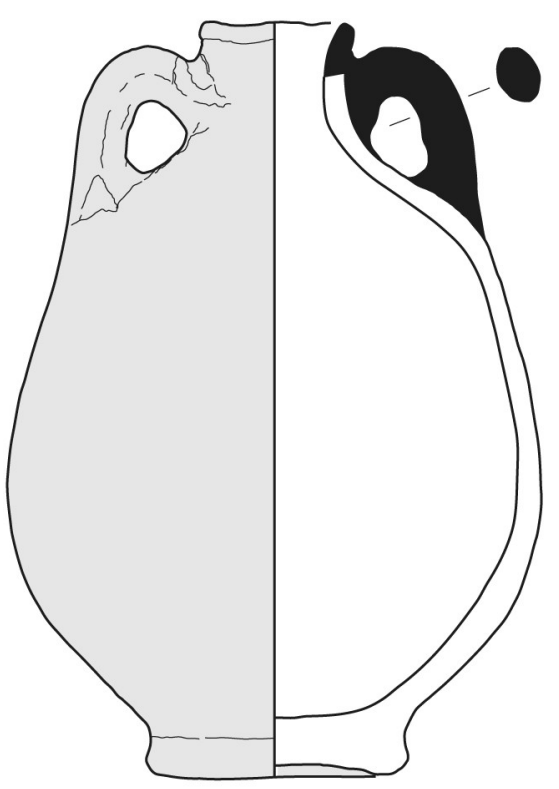

Cat. 325

Z25/6

FIGURE 7.79 Small table amphorae of type XII.5

\subsection{Amphorae Type XII.5}

Cat. 325

Amphora

Inv. no: $\mathrm{Z}_{25} / 6$

Provenance: Tumulus 25, chamber 2, central part

State of preservation: complete

Construction: wheel-made (2)

Measurements: $\mathrm{Dr}=3.4 \mathrm{~cm} ; \mathrm{D}_{\mathrm{b}}=6.1 \mathrm{~cm} ; \mathrm{H}=17.9 \mathrm{~cm}$

Fabric: not possible to determine

Hardness: $3 / 4$

Firing: not possible to determine

Surface treatment: slip on external surface

Surface colour: external natural surface-5YR 5/6 yellowish red, slip- $10 \mathrm{R} 4 / 8$ red

Colour of break: not possible to determine

References: (Klimaszewska-Drabot 2010, 484-485; Mahmoud El-Tayeb 2010, 214-216)

Cat. 326

Amphora

Inv. no: $\mathbf{Z 1 6} / \mathbf{1}$

Provenance: Tumulus 16, fill of shaft, north-eastern part

State of preservation: fragmentary, body sherds and one handle

Construction: wheel-made (2)

Measurements: ${ }_{\mathrm{p}} \mathrm{H}=10 \mathrm{~cm}$

Fabric: Lower Nubian?

Hardness: 4

Firing: oxidized

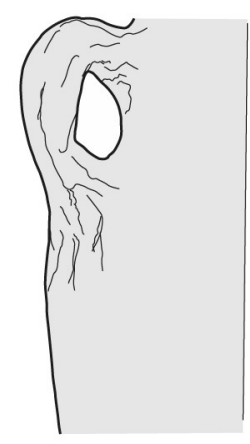

Cat. 326

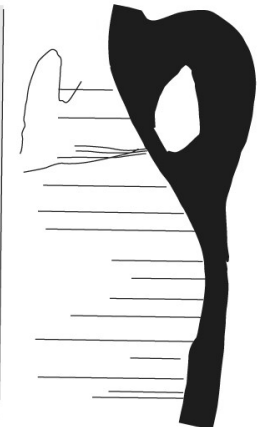

$Z 16 / 1$
[Fig. 7.129] Surface treatment: slip on external surface

Surface colour: external, slip-10R 4/6 red; internal2.5 Y $6 / 4$ light yellow brown

Colour of break: 2.5YR 6/8 light red

References: (Mahmoud El-Tayeb, Juszczyk-Futkowska, and Czyżewska 2014, 367, 369-371)

\subsection{Amphorae Type XII.6}

[Fig. 7.129]

\section{Cat. 327}

Amphora

Inv. no: $\mathbf{Z}_{\mathbf{4}} / \mathbf{2}+\mathbf{Z}_{\mathbf{4}} / \mathbf{1 0 . 1 1}$

Provenance: Tumulus 4, tunnel

State of preservation: fragmentary, rim complete, $36 \%$ of base, body sherds missing

Construction: wheel-made (2)

Measurements: $\mathrm{Dr}=4 \mathrm{~cm} ; \mathrm{D}_{\mathrm{b}}=8.5 \mathrm{~cm} ; \mathrm{H}=36 \mathrm{~cm}$

Fabric: ZF5

Hardness: 4

Firing: oxidized

Surface treatment: slipped on external surface

Surface colour: external natural surface -5 YR $6 / 6$ reddish yellow, slip-10R 5/4 weak red; internal 5YR 4/1 dark grey

Colour of break: Gley2 4/5Р В dark bluish grey

Decoration: incised-five grooves on upper part of body; painted-geometrical pattern, white bands and black dots on shoulder of vessel 


\section{Amphorae Type XII.6}

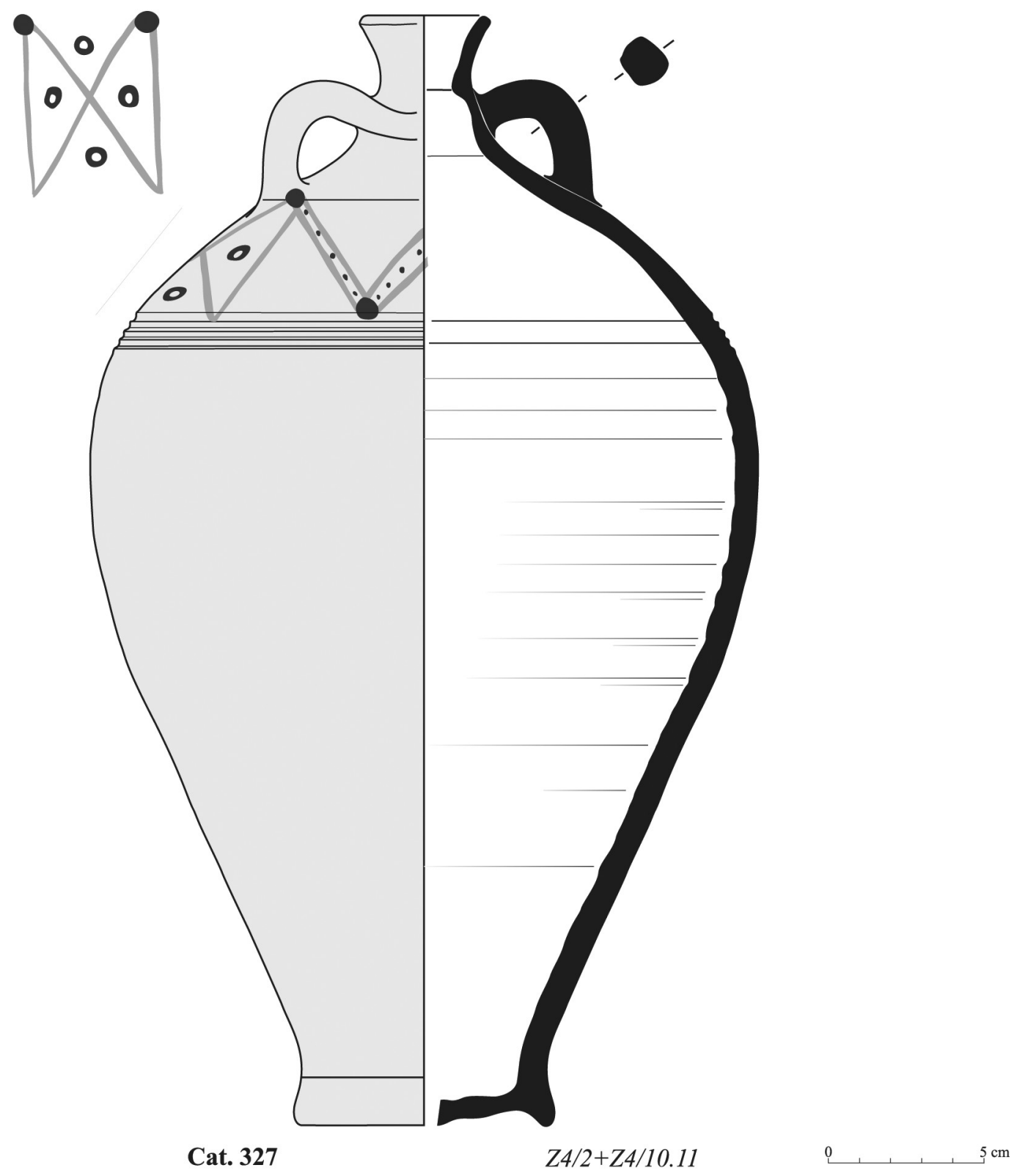

FIGURE 7.80 Amphora of type XII.6

\subsection{Amphorae Varia}

Cat. 328

Amphora

Inv. no: $\mathrm{Z} 4 / 10.9$

Provenance: Tumulus 4, tunnel

State of preservation: fragmentary, $16 \%$ of rim, neck fragment and one handle

Construction: wheel-made (2)

Measurements: $\mathrm{Dr}=8.6 \mathrm{~cm} ;{ }_{\mathrm{p}} \mathrm{H}=4.5 \mathrm{~cm}$

Fabric: Egyptian?

Hardness: medium hard/hard (3/4)

Firing: oxidized
[Fig. 7.129] Surface treatment: uncoated

Surface colour: external-2.5YR 6/6 light red; internal2.5 YR $7 / 6$ light red

Colour of break: 1OYR 5/4 yellowish brown

Cat. 329

Amphora

Inv. no: $\mathrm{Z}_{3} / 6.1+\mathrm{Z}_{3} / 7.1$

Provenance: Tumulus 3, fill of external shaft

State of preservation: fragmentary, neck and one handle Construction: wheel-made (2)

Measurements: ${ }_{\mathrm{p}} \mathrm{H}=3.8 \mathrm{~cm}$ 


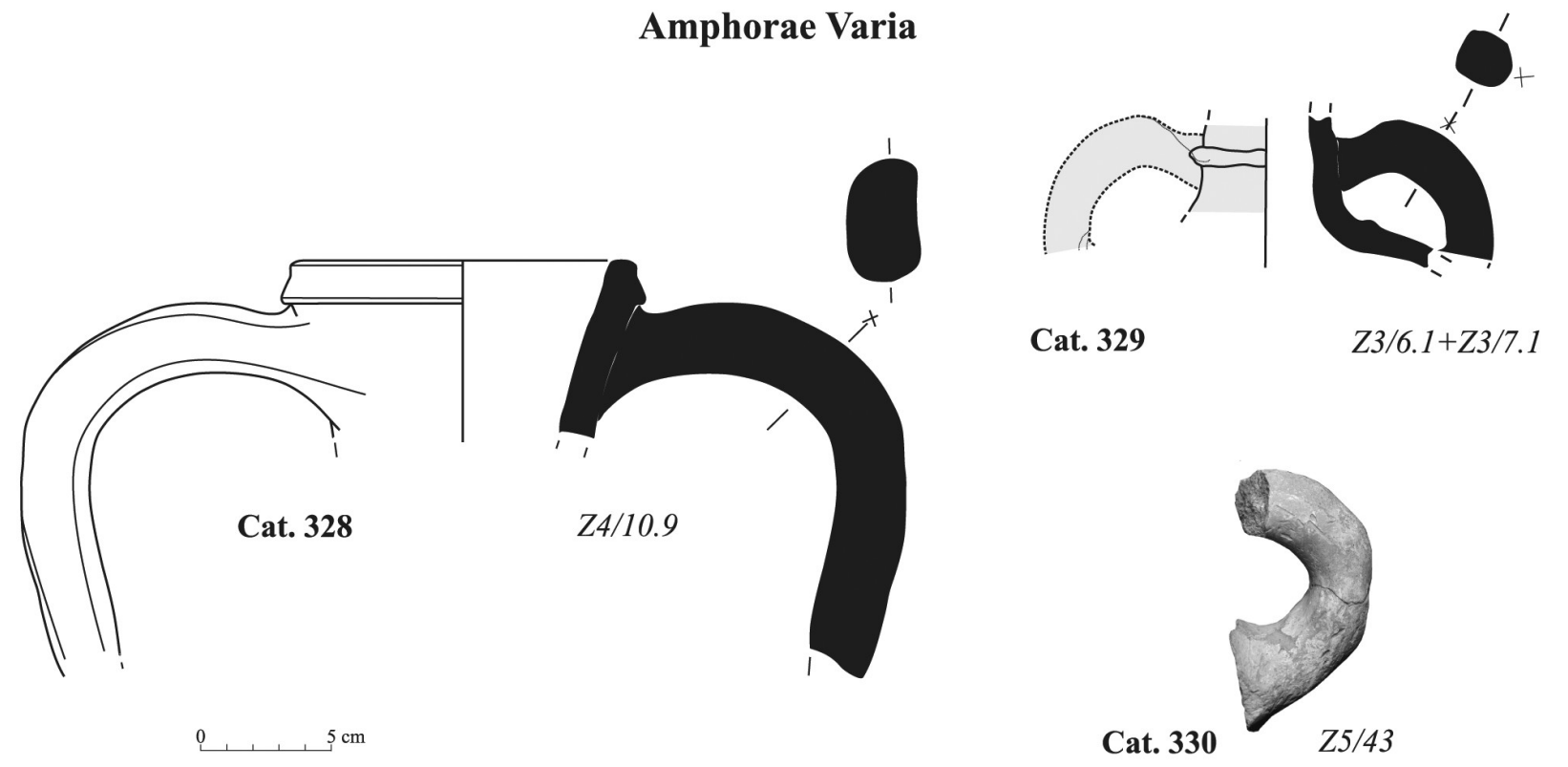

FIGURE 7.81 Small amphorae of different types

Fabric: Aswan area

Hardness: 4

Firing: oxidized

Surface treatment: slip on external surfaces

Surface colour: external natural surface-2.5YR 6/6 light red, slip-10R 5/6 red; internal-2.5YR 6/4 light yellow brown

Colour of break: 2.5YR 5/6 red

\section{Cat. 33 o}

Amphora

Inv. no: $\mathrm{Z}_{5} / 43$

Provenance: Tumulus 5, tunnel

State of preservation: fragmentary, one handle

Construction: handmade

Measurements: ${ }_{\mathrm{p}} \mathrm{H}=c .6 .5 \mathrm{~cm}$

Fabric: Aswan area

Hardness: $3 / 4$

Firing: oxidized

Surface treatment: slip on white background on external surface

Surface colour: external natural surface-2.5YR 6/4 light yellow-brown, slip-5YR 6/8 reddish yellow

Colour of break: 2.5 YR $5 / 6$ red

\section{Group XIII-Bottles}

15.1 Bottles Type XIII.1

Cat. 331

Small bottle
Inv. no: $\mathrm{Z6} 6 / 7$

Provenance: Tumulus 6, external shaft and tunnel Construction: wheel-made (2)

State of preservation: fragmentary

Measurements: $\mathrm{Dr}=2 \mathrm{~cm} ; \mathrm{H}=11.8 \mathrm{~cm}$

Fabric: Aswan area?

Hardness: 4

Firing: oxidized

Surface treatment: slip on external surface

Surface colour: external natural surface-7.5YR 8/4 pink, slip-7.5YR $7 / 6$ reddish yellow; internal-7.5YR $8 / 2$ pinkish white

Colour of break: 7.5YR 8/3 pink

Cat. $33^{2}$

Small bottle

Inv. no: $\mathbf{Z} 13 / 22$

Provenance: Tumulus 13, shaft, near blocking wall of chamber 1

Construction: wheel-made (2)

State of preservation: fragmentary, part of neck and body

Measurements: ${ }_{\mathrm{p}} \mathrm{H}=5.5 \mathrm{~cm}$

Fabric: Mediterranean?

Hardness: 4

Firing: oxidized

Surface treatment: uncoated

Surface colour: external-7.5YR 7/3 pink, internal-7.5YR $8 / 2$ pinkish white

[Fig. 7.130] Colour of break: 7.5YR 6/4 light brown 


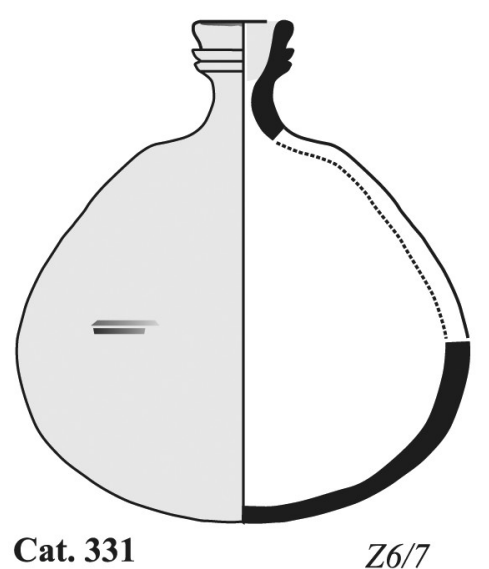

Bottles Type XIII.2

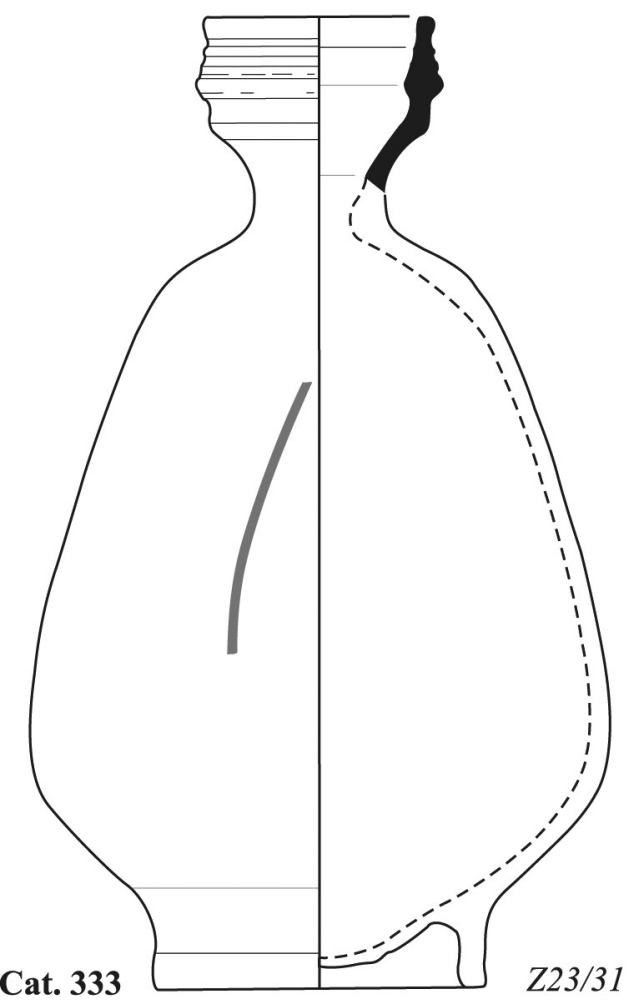

\section{Bottles Type XIII.1}

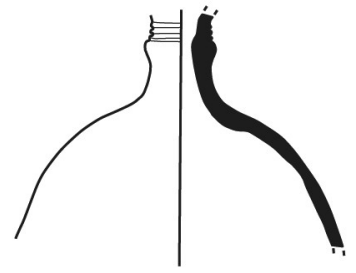

Cat. 332

\section{Bottles Type XIII.3}

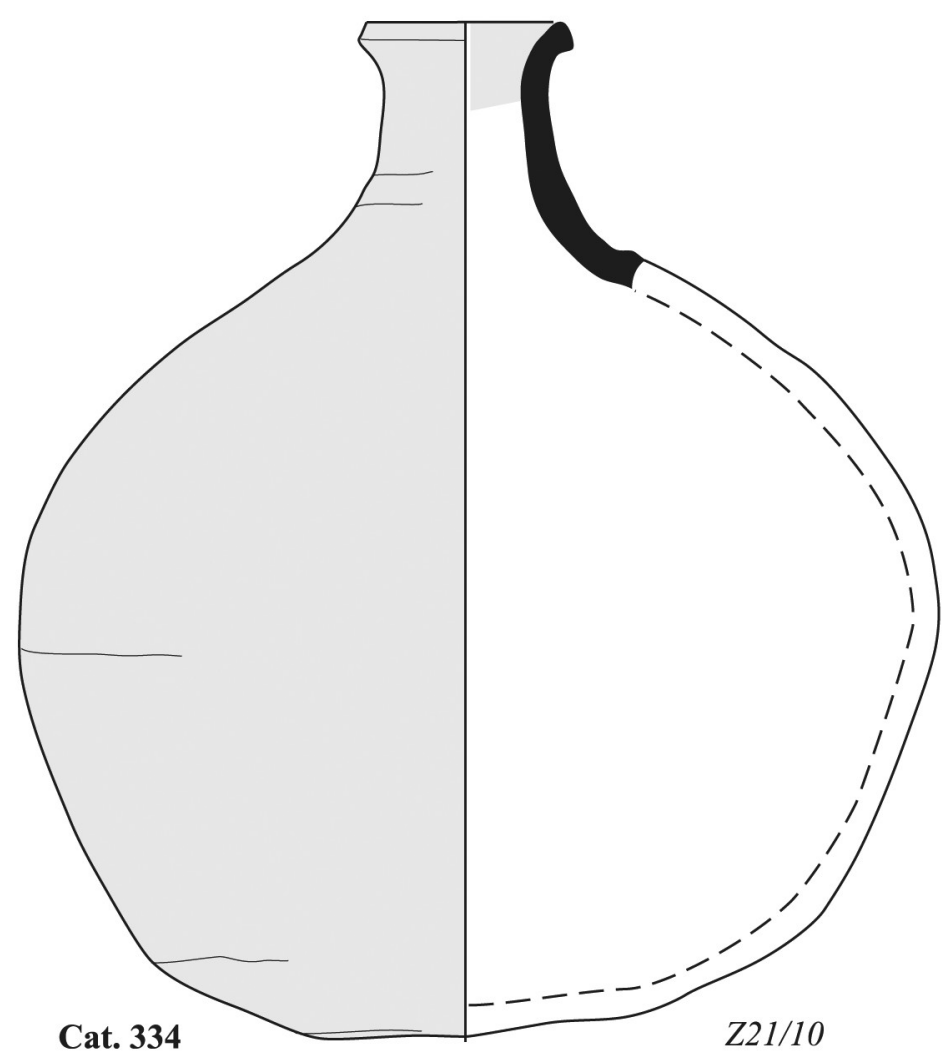

0 $5 \mathrm{~cm}$

FIGURE 7.82 Small bottles of types XIII.1, XIII.2 and XIII.3

\subsection{Bottles Type XIII.2}

Cat. 333

Medium bottle

Inv. no: $\mathbf{Z 2 3} / \mathbf{3}^{1}$

Provenance: Tumulus 23, chamber 1

Construction: wheel-made (2)

State of preservation: almost complete, complete rim, $77 \%$ of base

Measurements: $\mathrm{Dr}=5.2 \mathrm{~cm}, \mathrm{D}_{\mathrm{b}}=7.5 \mathrm{~cm}, \mathrm{H}=23 \mathrm{~cm}$
[Fig. 7.130] Fabric: Aswan area

Hardness: 4

Firing: oxidized

Surface treatment: uncoated

Surface colour: external-5YR 6/4 light reddish brown Colour of break: 5 YR $7 / 4$ pink

Decoration: traces of decoration visible on body-fragment of dark line 
Jug Type XIV.1

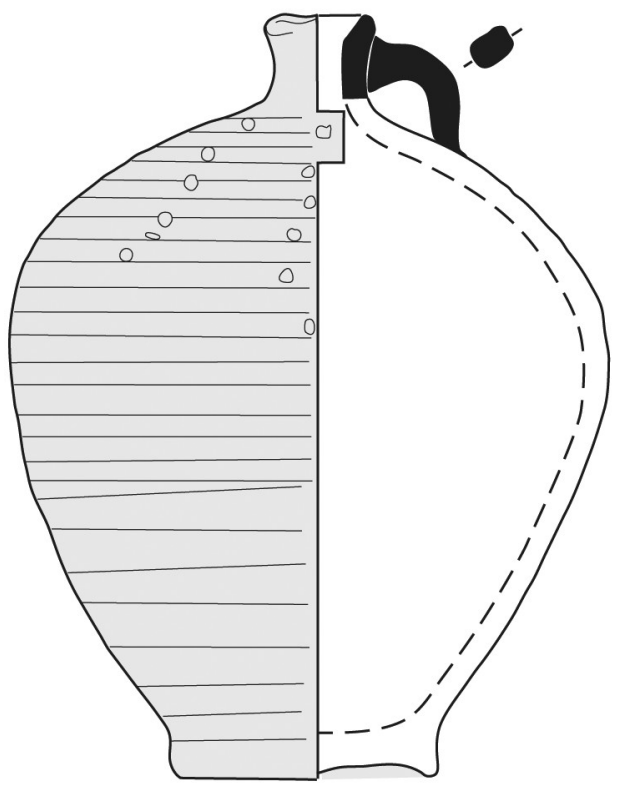

Cat. 335

$Z 15 / 6$
15.3 Bottles Type XIII.3

Cat. 334

Medium bottle

Inv. no: $\mathbf{Z 2 1} / \mathbf{1 0}$

Provenance: Tumulus 21, burial chamber, south-western part

Construction: wheel-made (2)

State of preservation: complete

Measurements: $\mathrm{Dr}=5 \mathrm{~cm}, \mathrm{H}=24 \mathrm{~cm}$

Fabric: not possible to determine

Hardness: 4

Firing: not possible to determine

Surface treatment: slip on external surface

Surface colour: external natural surface-5YR 5/6 yellowish red, slip-2.5YR 5/6 red

Colour of break: not possible to determine

16 Group XIV-Jugs

16.1 Jugs Type XIV.1

Cat. 335

Jug

Inv. no: $\mathrm{Z}_{15} / 6$

Provenance: Tumulus 15, fill of shaft, southern corner
[Fig. 7.130] Construction: wheel-made (2)

\section{Jug Type XIV.2}

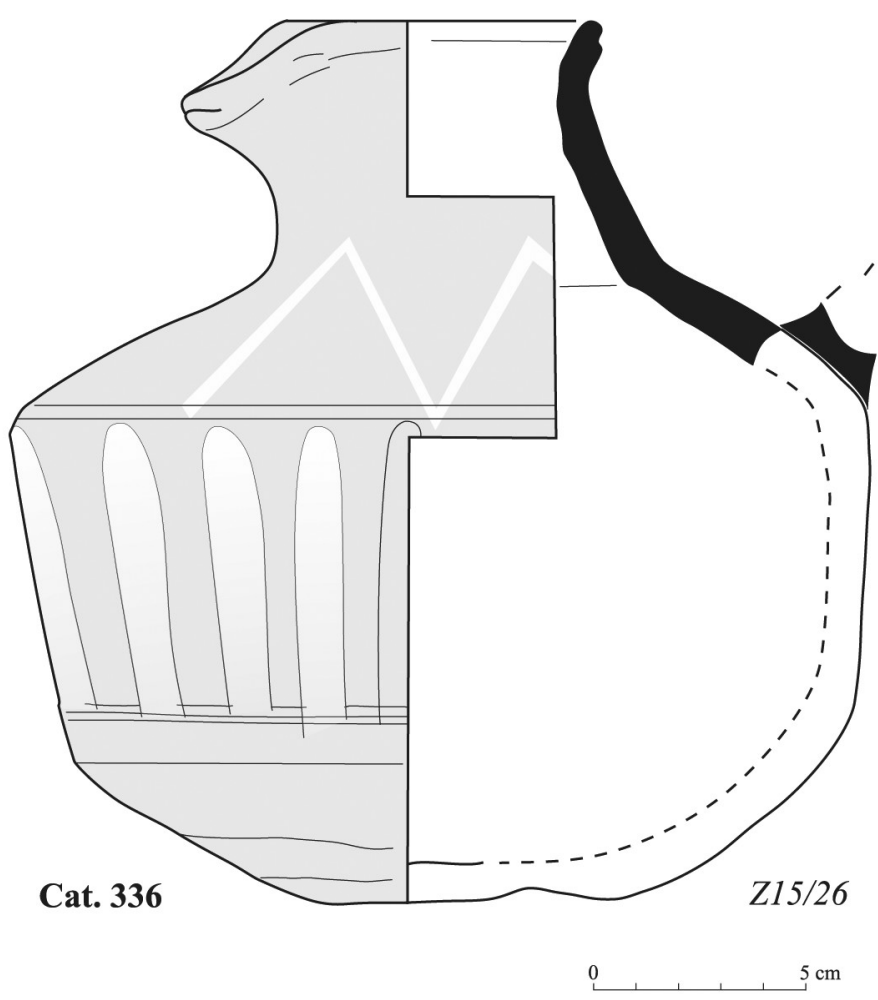

State of preservation: complete

Measurements: $\mathrm{Dr}=2.5 \mathrm{~cm}, \mathrm{D}_{\mathrm{b}}=6.2 \mathrm{~cm}, \mathrm{H}=18 \mathrm{~cm}$

Fabric: not possible to determine

Hardness: 3

Firing: not possible to determine

Surface treatment: slip on external surface

Surface colour: external natural surface -5 YR 6/6 reddish brown, slip- 2.5 Y $5 / 8$ red

Colour of break: not possible to determine

Decoration: four vertical lines of punctuated dots (probably executed using some kind of reed) on shoulder of vessel

16.2 Jugs Type XIV.2

[Fig. 7.131]

Cat. 336

Jug

Inv. no: $\mathrm{Z}_{\mathbf{1 5}} / \mathbf{2 6}$

Provenance: Tumulus 15, chamber 2, south part

Construction: wheel-made (2)

[Fig. 7.131] State of preservation: incomplete, $61 \%$ of rim, complete base, handle missing

Measurements: $\mathrm{Dr}=10 \times 7.2 \mathrm{~cm}, \mathrm{H}=21 \mathrm{~cm}$

Fabric: not possible to determine

Hardness: $3 / 4$ 


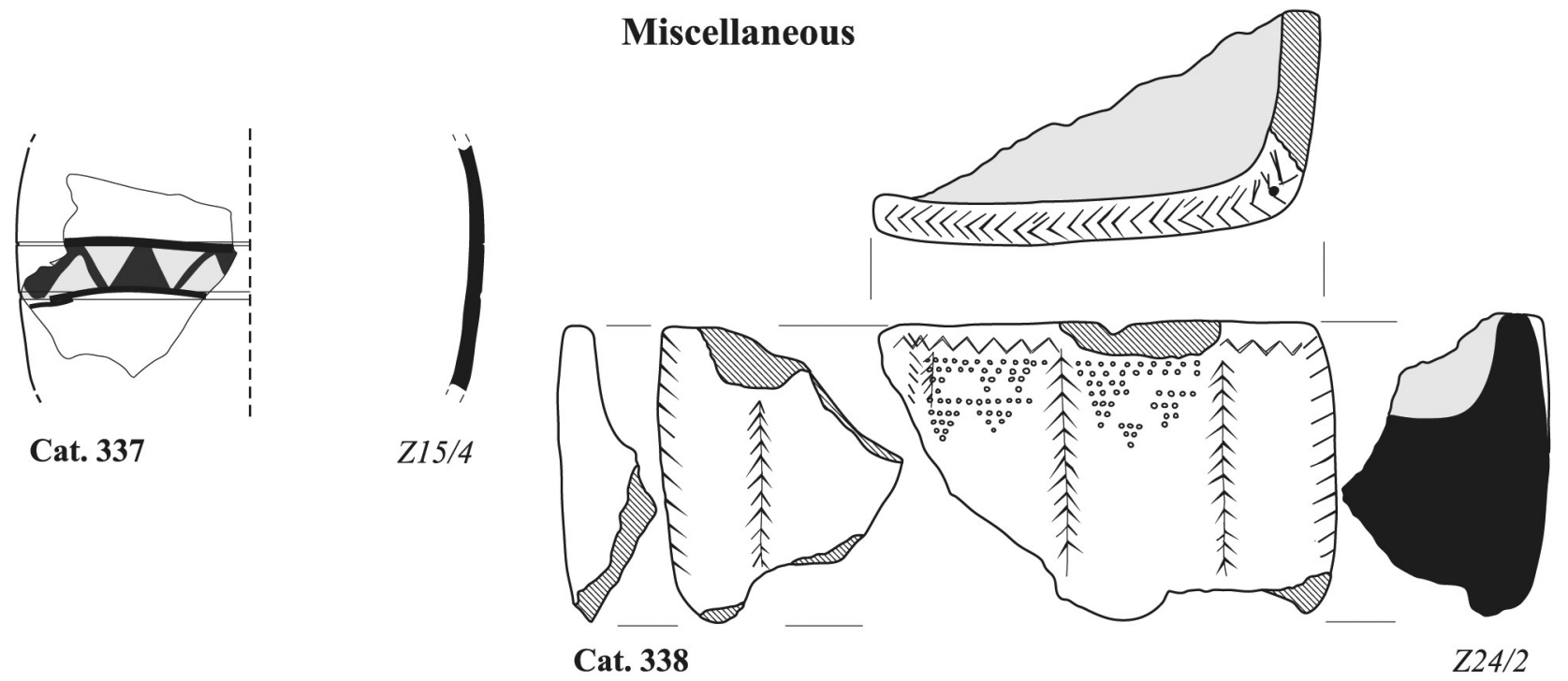

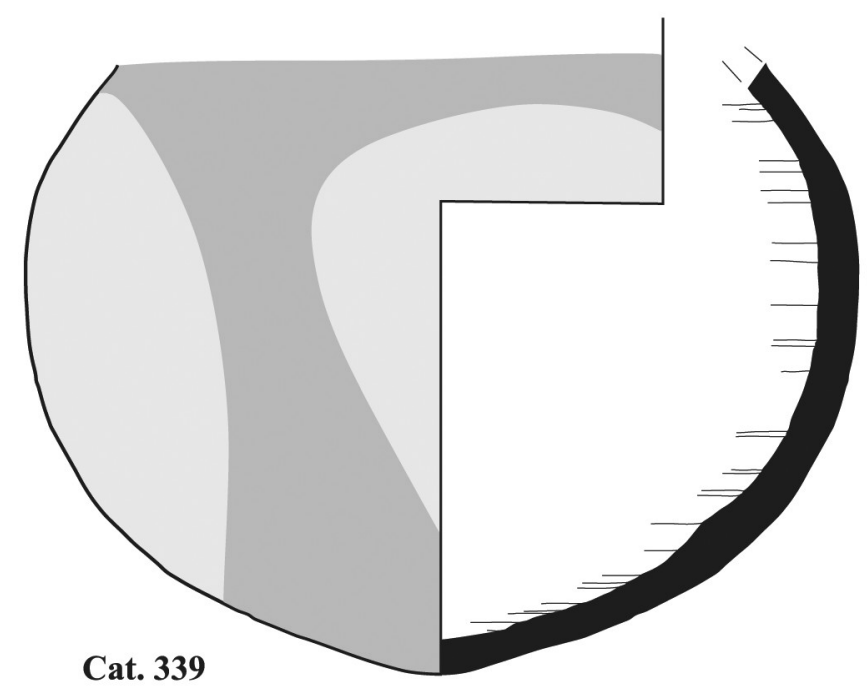

Cat. 339

FIGURE 7.84 Miscellaneous wheel-made and handmade vessels

Firing: not possible to determine

Surface treatment: slip on external surface

Surface colour: external natural surface- 5 YR $5 / 6$ yellowish red, slip-1oR $4 / 6$ red

Colour of break: not possible to determine

Decoration: painted decoration on shoulders-white strokes; belly accentuated with concave vertical lines filled with white

\section{Miscellaneous}

[Fig. 7.132]

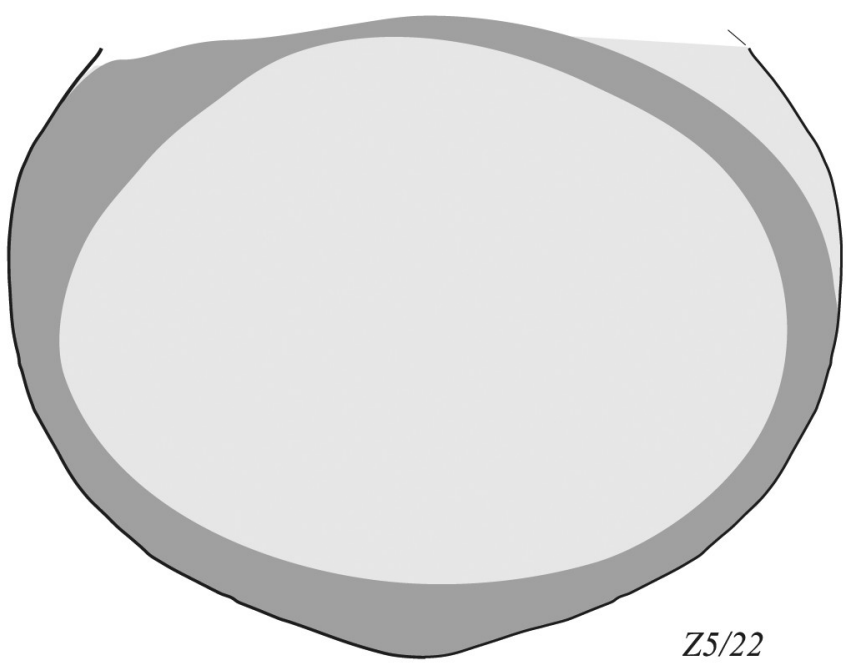

0 $5 \mathrm{~cm}$

Provenance: Tumulus 15, fill of shaft Construction: wheel-made State of preservation: single sherd

Fabric: ZF1

Hardness: 3

Firing: oxidized

Surface treatment: slipped on external surface

Surface colour: external, slip- $10 \mathrm{R} 5 / 8$ red

Colour of break: 7.5YR 4/4 dark brown

Decoration: incised - two poorly executed grooves forming border for decoration; painted-white band as background and black triangles as decorative pattern

Cat. 337

Body sherd

Inv. no: $\mathrm{Z15}_{5} / 4$ 

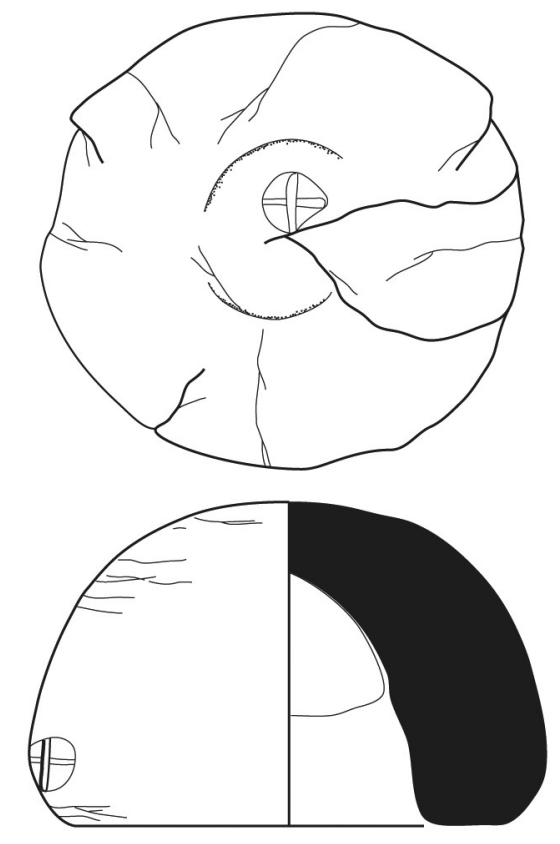

\section{Miscellaneous}
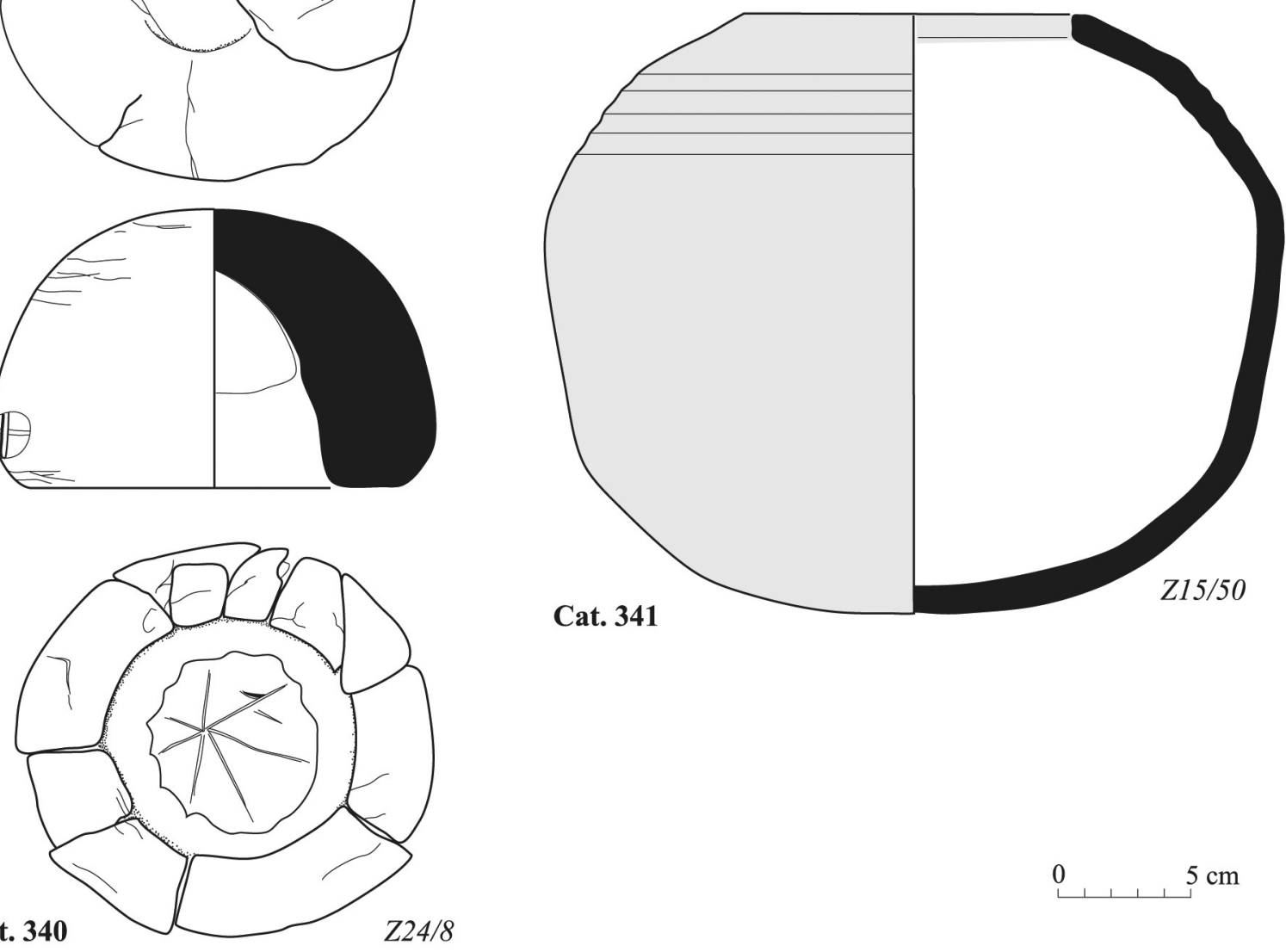

FIGURE 7.85 Miscellaneous wheel-made and handmade vessels

Cat. $33^{8}$

Basin?

Inv. no: $\mathrm{Z24} / 2$

Provenance: Tumulus 24, superstructure

Construction: handmade

State of preservation: fragmentary

Measurements: ${ }_{\mathrm{p}} \mathrm{H}=7.2 \mathrm{~cm}$

Fabric: similar to ZF9

Hardness: 4

Firing: reduced

Surface treatment: slipped on internal surface

Surface colour: external-7.5YR 5/4 brown; internal natural surface -7.5 YR $5 / 3$ brown, slip-10R 3/4 dusky red

Colour of break: 1OYR 2/1 black

Decoration: punctuated and incised decoration on external wall and top of rim; herringbone and dots arranged in triangles
Cat. 339

Pot?

Inv. no: $\mathrm{Z}_{\mathbf{5}} / \mathbf{2 2}$

Provenance: Tumulus 5, tunnel fill

Construction: wheel-made (2)

State of preservation: fragmentary, restored from sherds, lower part

Measurements: ${ }_{\mathrm{p}} \mathrm{H}=14 \mathrm{~cm}$

Fabric: similar to $\mathrm{ZF9}$

Hardness: 4

Firing: reduced

Surface treatment: slipped on external surface

Surface colour: external natural surface -5 YR 6/6 reddish yellow, slip $1-10 \mathrm{R} 5 / 4$ weak red, slip $2-7.5 \mathrm{R}$ 4/4 weak red; internal natural surface-1OYR $5 / 3$ brown

Colour of break: $2.5 Y$ 2.5/1 black

Decoration: painted-with slip, slip 1 as background, and slip 2 as top layer applied in circles 
Cat. 340

Stopper

Inv. no: $\mathbf{Z 2 4 / 8}$

Provenance: Tumulus 24, robber shaft

Construction: handmade

State of preservation: fragmentary, $50 \%$ of rim

Measurements: $\mathrm{Dr}=15 \mathrm{~cm}, \mathrm{H}=10.6 \mathrm{~cm}$

Fabric: not possible to determine, abundant in fine to large organics, medium quantity of small to medium-sized white particles and small stones

Hardness: 2

Firing: probably dried

Surface treatment: uncoated

Surface colour: 10YR 6/2 light brownish grey on both surfaces

Colour of break: 10YR 6/2 light brownish grey

Decoration: incised-star pattern inside stopper, badly executed; stamped decoration on external wall and top of stopper

\section{Cat. 341}

Jar (small, broad)

Inv. no: $\mathrm{Z}_{15} / 5^{\circ}$

Provenance: Tumulus 15, chamber 1, eastern part

Construction: wheel-made (2)

State of preservation: incomplete, $97 \%$ of rim, lip chipped off

Measurements: $\operatorname{Dr}=13.5 \mathrm{~cm} ; \max$ body $\operatorname{Dr}=26.5 \mathrm{~cm}$; $\mathrm{H}=22.5 \mathrm{~cm}$

Fabric: not possible to determine

Hardness: 3

Firing: reduced

Surface treatment: slipped on external surface

Surface colour: external natural surface- -5 YR 5/6 yellowish red, slip-2.5R 6/4 red light reddish brown

Colour of break: not possible to determine 


\section{Colour Plates}

I.1
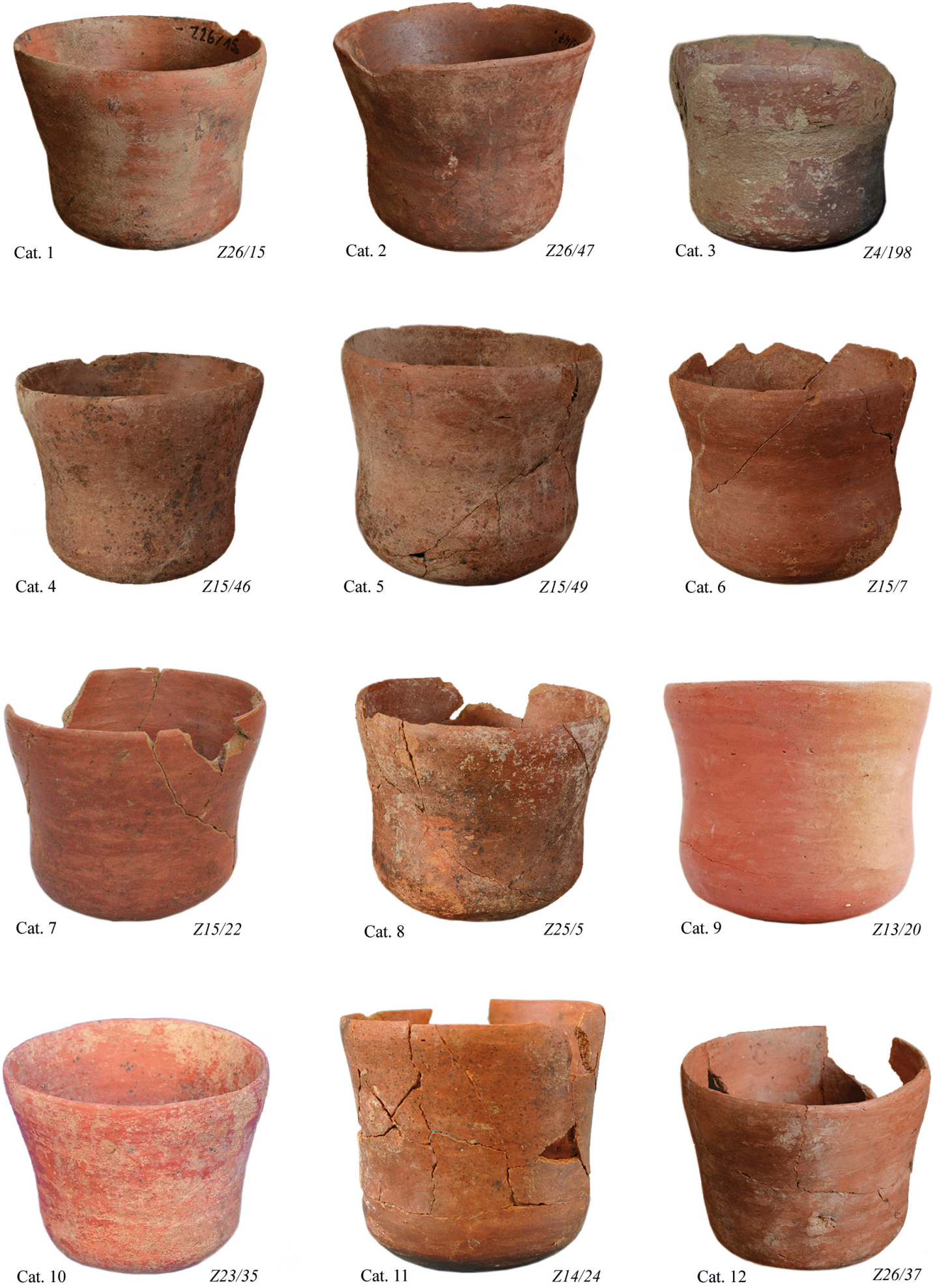

Cat. 12 Z26/37

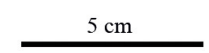

FIGURE 7.86 Cups of type I.1

Z26/15, Z26/47, Z4/198, z15/46, Z15/49, Z15/7, Z15/22 РнотоS ву А. кАмRоWSкі; Z25/5 РНОTO BY К. КОTLEWSKI; Z13/2O РНОTO вY О. ВІАŁOSTOCKA; Z23/35 РНОTO BY K. KOTLEWSKI; Z14/24 РНОто BY т. WOJTCZAK; Z26/37 Рното BY A. KAMROWSKI 
I.1
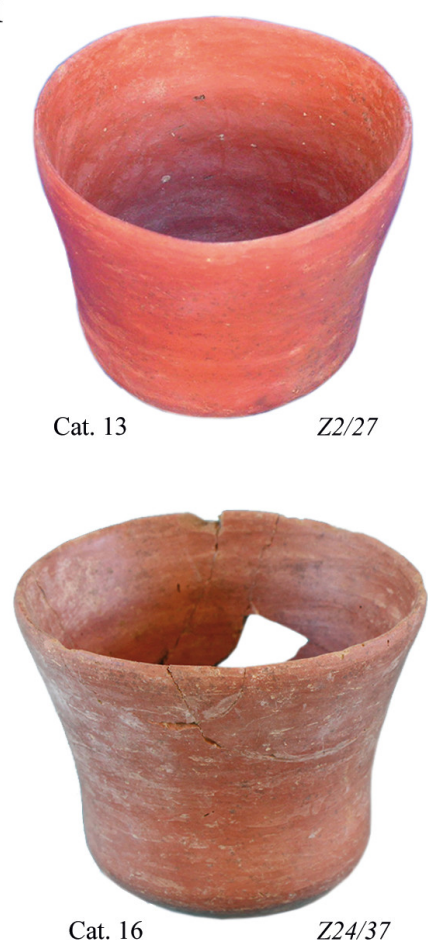

Z24/37
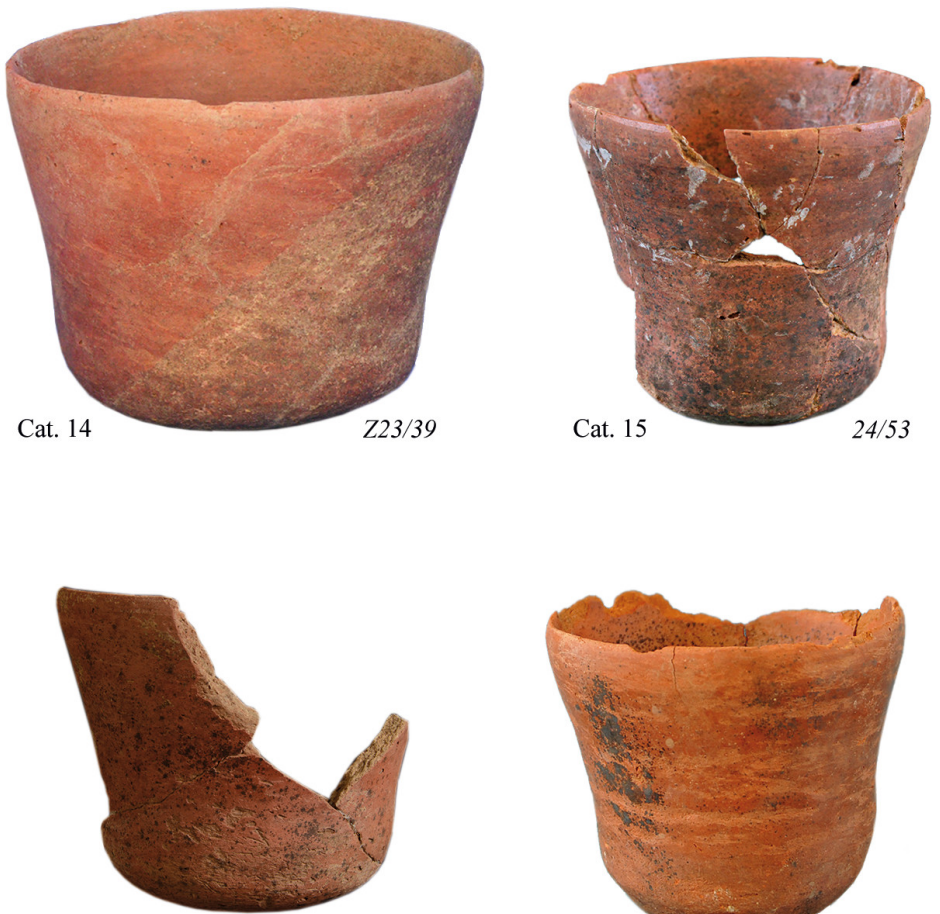

Cat. 17

226/71.1

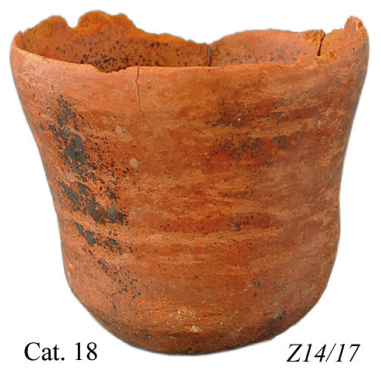

variant I. la

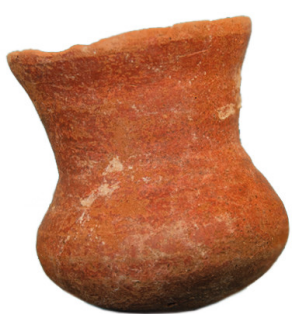

Cat. 19
Z21/6
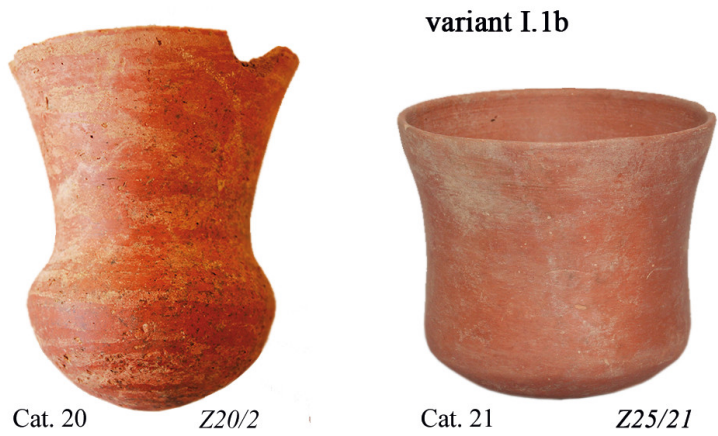

Cat. 21

Z25/21

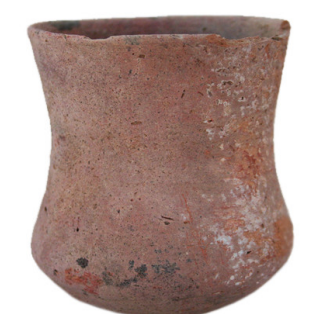

Cat. 22

I.2

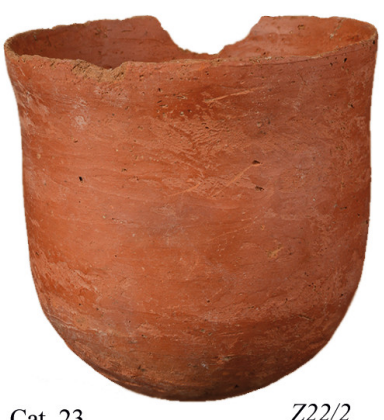

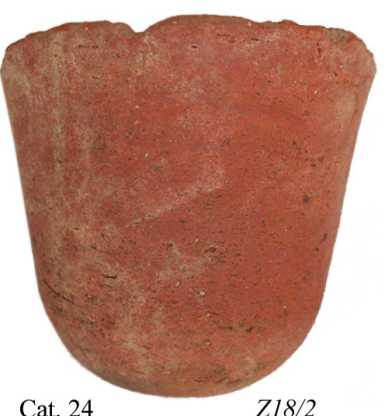

Cat. 24

$Z 18 / 2$

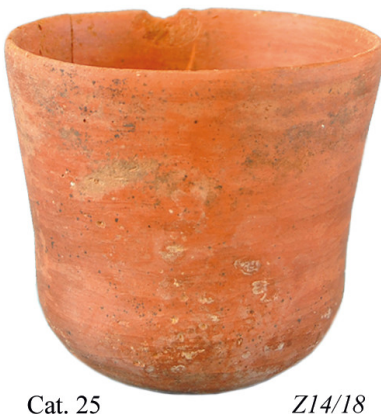

Cat. 25
Z14/18

Z22/2

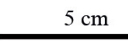

FIGURE 7.87 Cups of types I.1 and I.2

Z2/27, 23/39 PнотоS BY K. KOTLEWSKI; Z24/53, Z24/37 PHOTOS вY T. WOJTCZAK; Z26/71.1 РНОTO BY A. KAMROWSKI; Z14/17 Рното вY T. WOJTCZAK; Z21/6 Рното BY R. MAHLER; Z2O/2 PHOTO BY E. CZYŻEWSKA-ZALEWSKA; Z25/21, Z1O/9 PHOTOS BY K. KOTLEWSKI; Z22/2 PHOTO BY A. KAMROWSKI; Z18/2 PHOTO BY K. KOTLEWSKI; Z14/18 Рното ву T. WOJTCZAK 
I.2
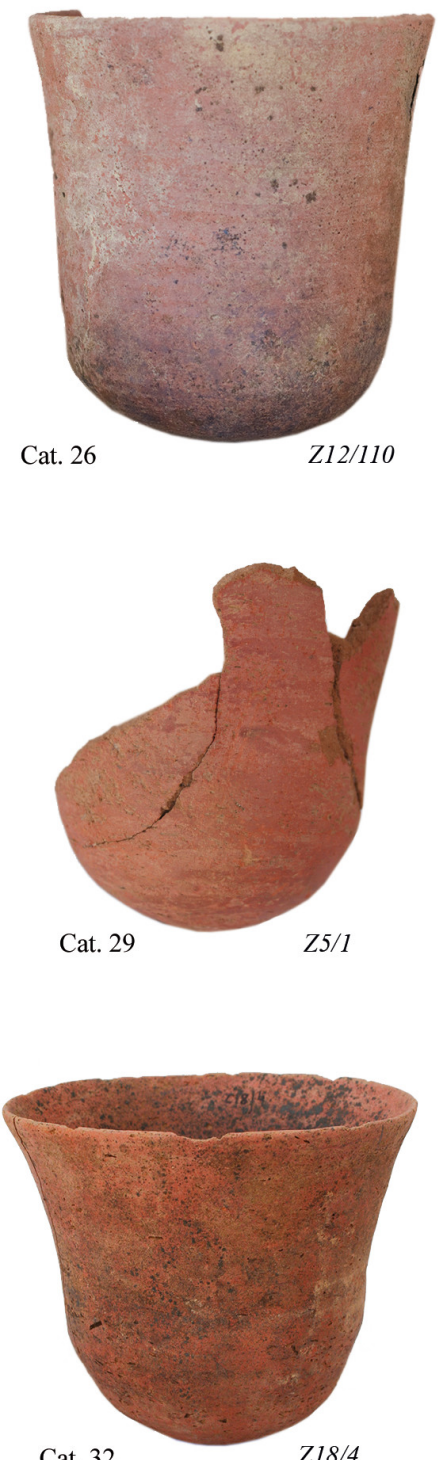

Cat. 32

Z18/4

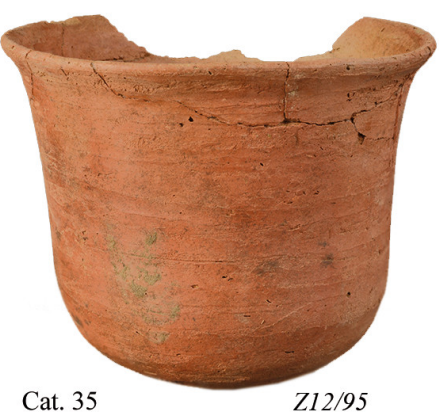

Cat. 35

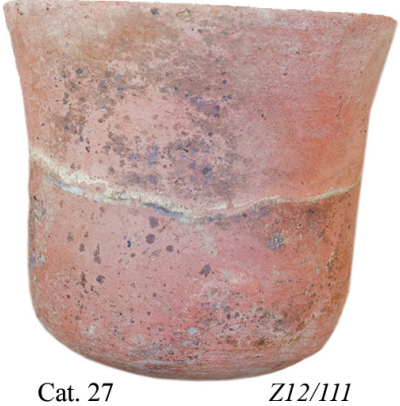

Cat. 27
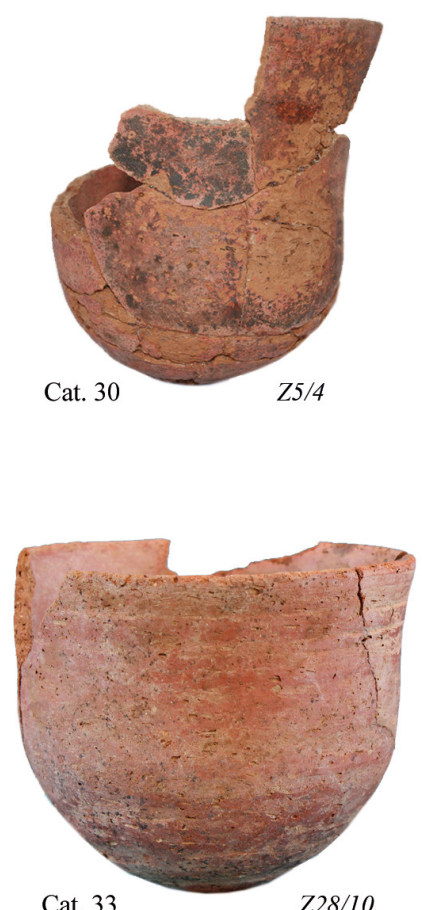

Cat. 33

$228 / 10$

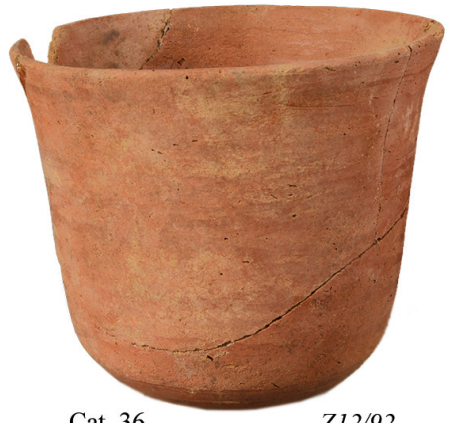

Cat. 36
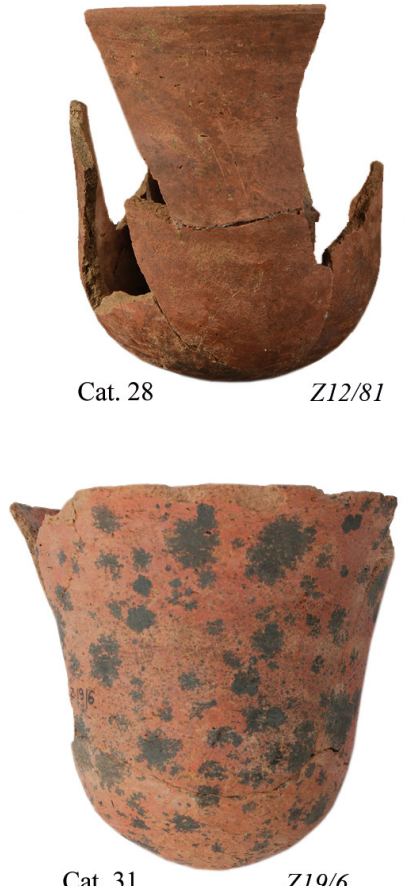

Cat. 31

Z19/6

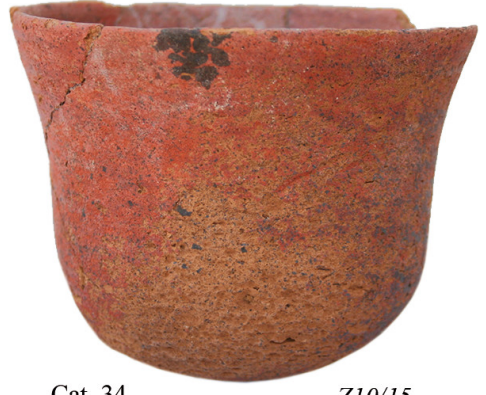

Cat. 34

210/15

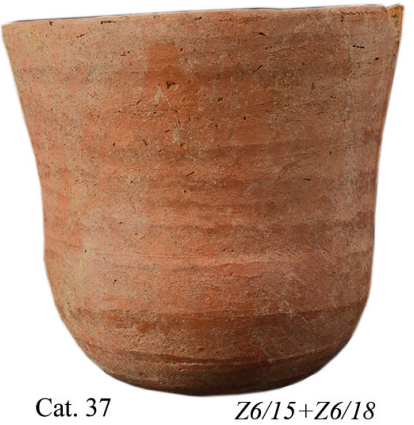

FIGURE 7.88 Cups of type I.2

Z12/110, Z12/111 PHOTOS BY E. SKOWROŃSKA; Z12/81 РнOTO BY A. KAMROWSKI;

Z5/1, Z5/4, Z19/6, Z18/4 РнотоS BY K. KOTLEWSKI; Z28/10 Рното BY T. WOJTCZAK;

Z10/15 РнОто ВY К. КоTLEWSKI; Z12/95, Z12/92, Z6/15+Z6/18 РНОтоS ВY А. КАМ-

ROWSKI 
I.2
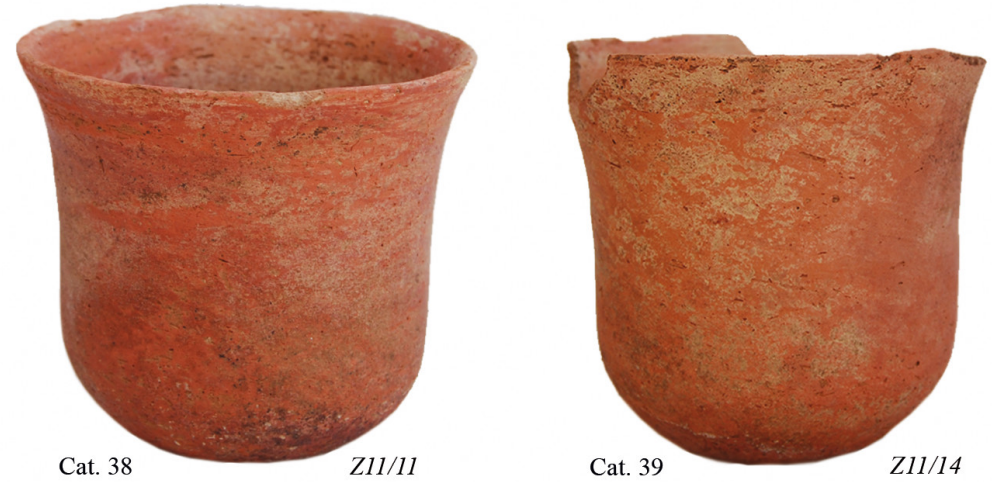

variant I.2a

Cat. 38

Cat. 39
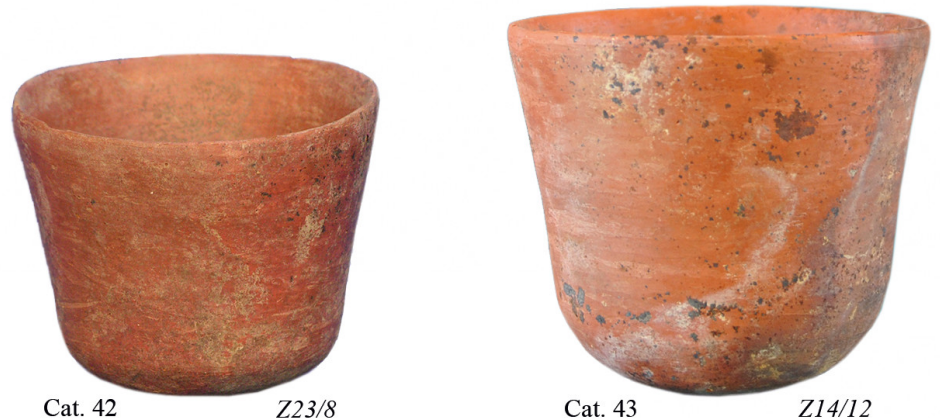

Cat. 41

Z23/7

Cat. 42

$Z 23 / 8$

Cat. 43

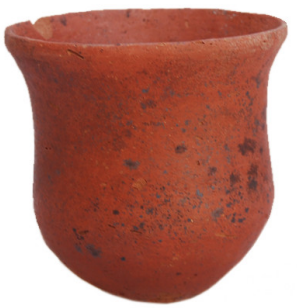

Cat. 40

Z11/19
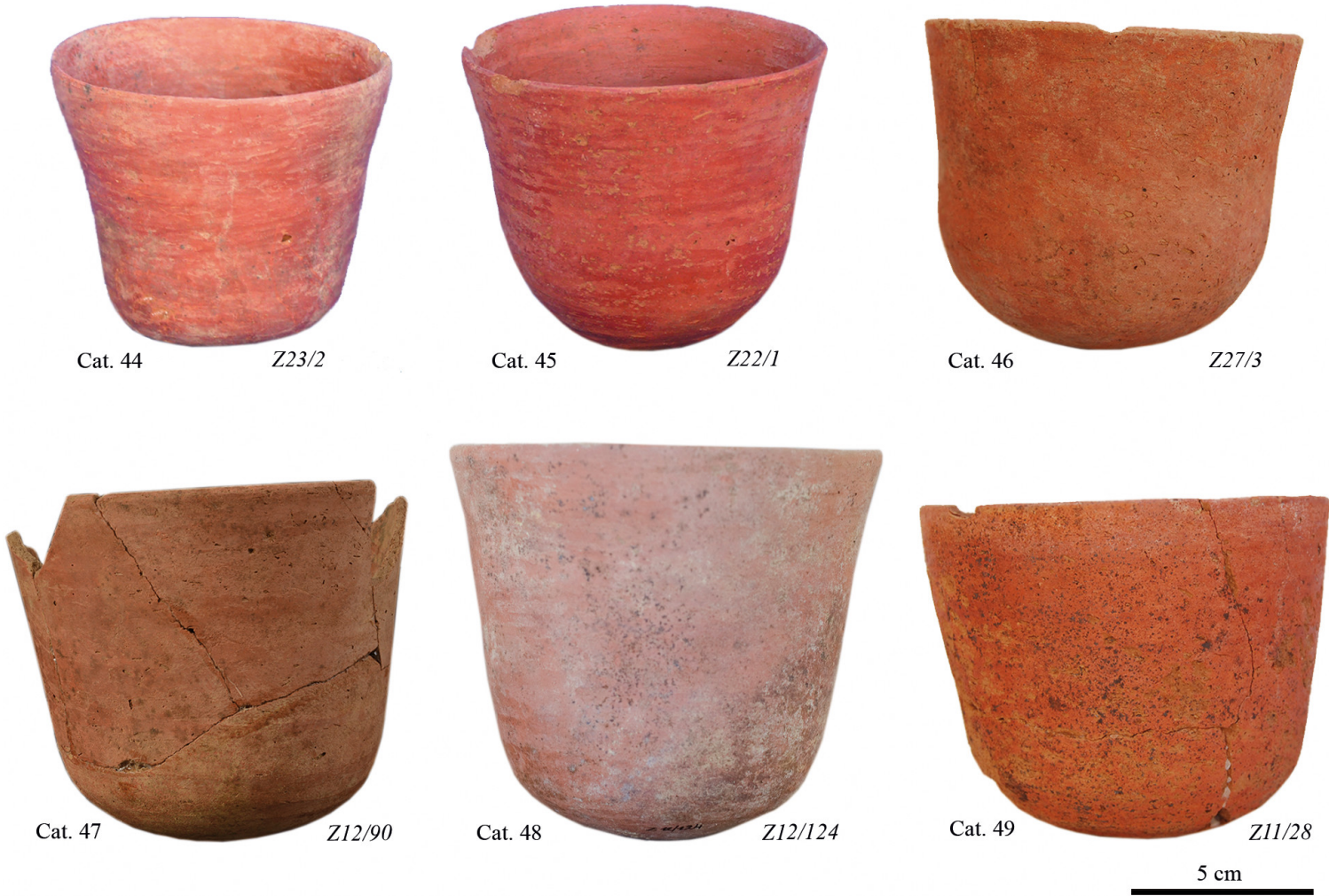

FIGURE 7.89 Cups of types I.2 and I.3

Z11/11, Z11/14, Z11/19 РнотоS ву о. вІаєоsтоска; Z23/7, z23/8 РнотоS

BY K. KOTLEWSKI; Z14/12 РнОтО вY т. WOJTCZAK; Z23/2, Z22/1 РноTOS BY

K. KOTLEWSKI; Z27/3 РНОTO BY О. ВIAŁOSTOCKA; Z12/9O РНОTO BY A. KAMROWSKI;

Z12/124 PHOTO BY Е. SKOWROŃSKA; Z11/28 РНОTO BY О. BIAŁOSTOCKA 
I.3
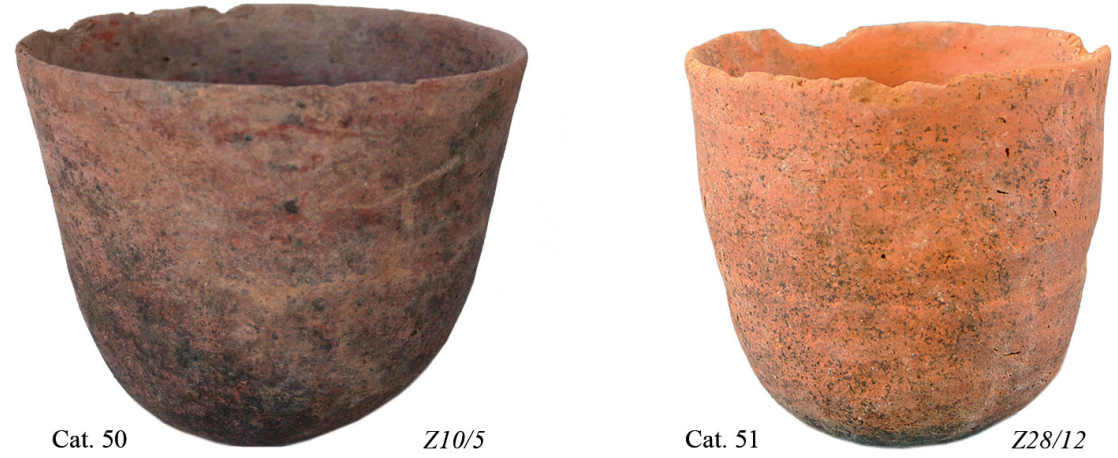

I.4

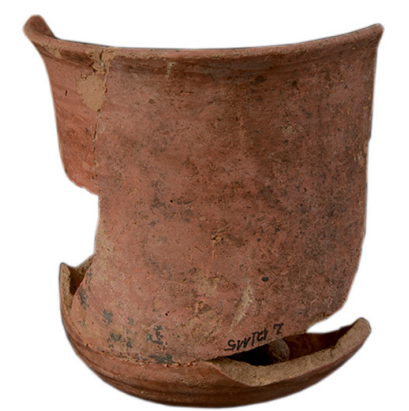

Cat. 52

$Z 12 / 98+Z 12 / 115$
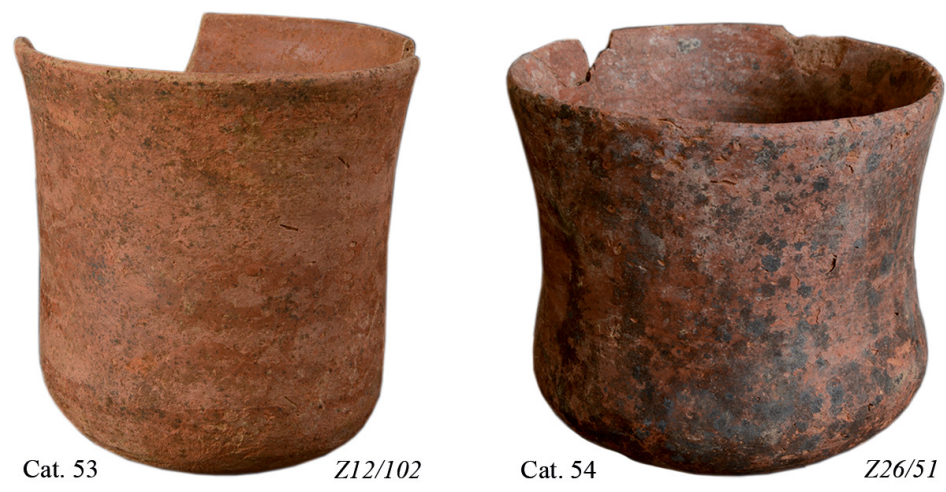

I.5
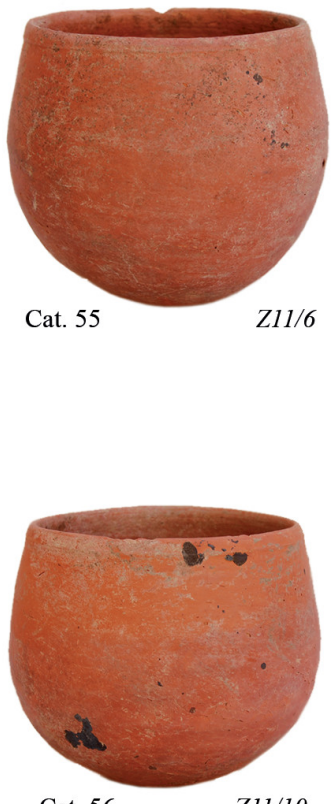

Cat. 56
Z11/10

\section{Cups varia}
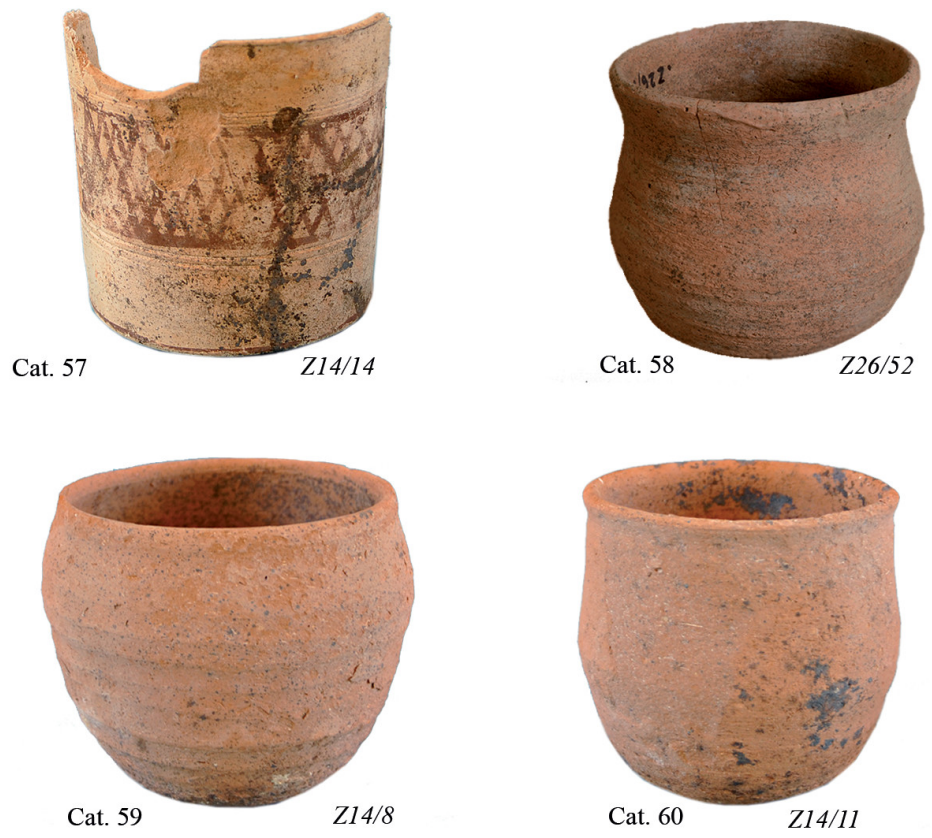

Cat. 60

Z14/11

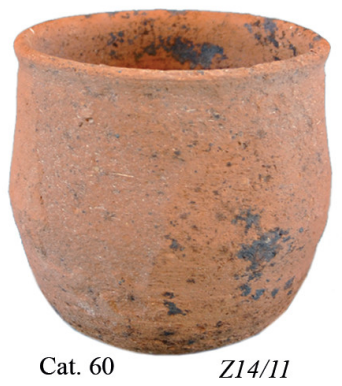

FIGURE 7.90 Cups of types I.3, I.4, I.5 and varia

Z1O/5 РНОто ВY К. котLEWSKI; Z28/12 РнОто вY T. WOJTCZAK; Z12/98+Z12/115,

Z12/102, Z26/51.1 РНОTOS BY A. KAMROWSKI; Z11/6, Z11/10 РНОTOS BY

O. BIAŁOSTOCKA; Z14/14 РНОTO BY т. WOJTCZAK; Z26/52 РнOTO BY A. KAMROWSKI;

Z14/8, Z14/11 РнотOS BY T. WOJTCZAK 


\section{Cups varia}
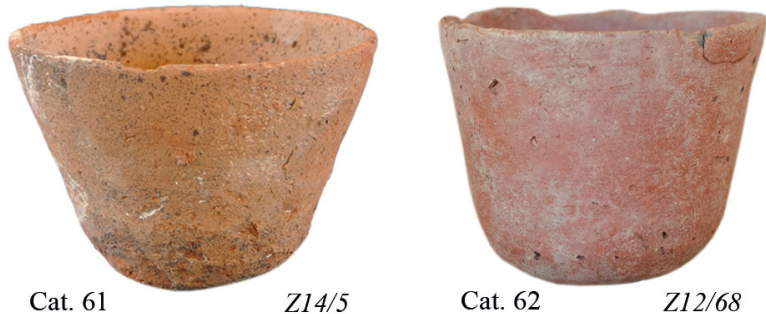

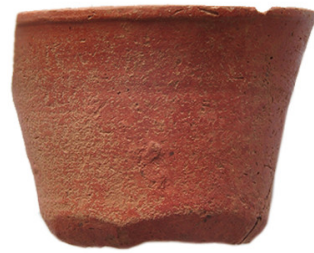

Cat. 63

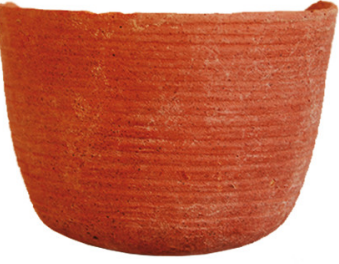

Cat. 64

$Z 9 / 2$

$5 \mathrm{~cm}$

FIGURE 7.91 Cups varia

Z14/5 РНОTO BY T. WOJTCZAK; Z12/68 PHOTO BY E. SKOWROŃSKA; Z7/2O PHOTO BY

A. KAMROWSKI; Z9/2 PHOTO BY E. CZYŻEWSKA-ZALEWSKA

II.1

variant II.1a
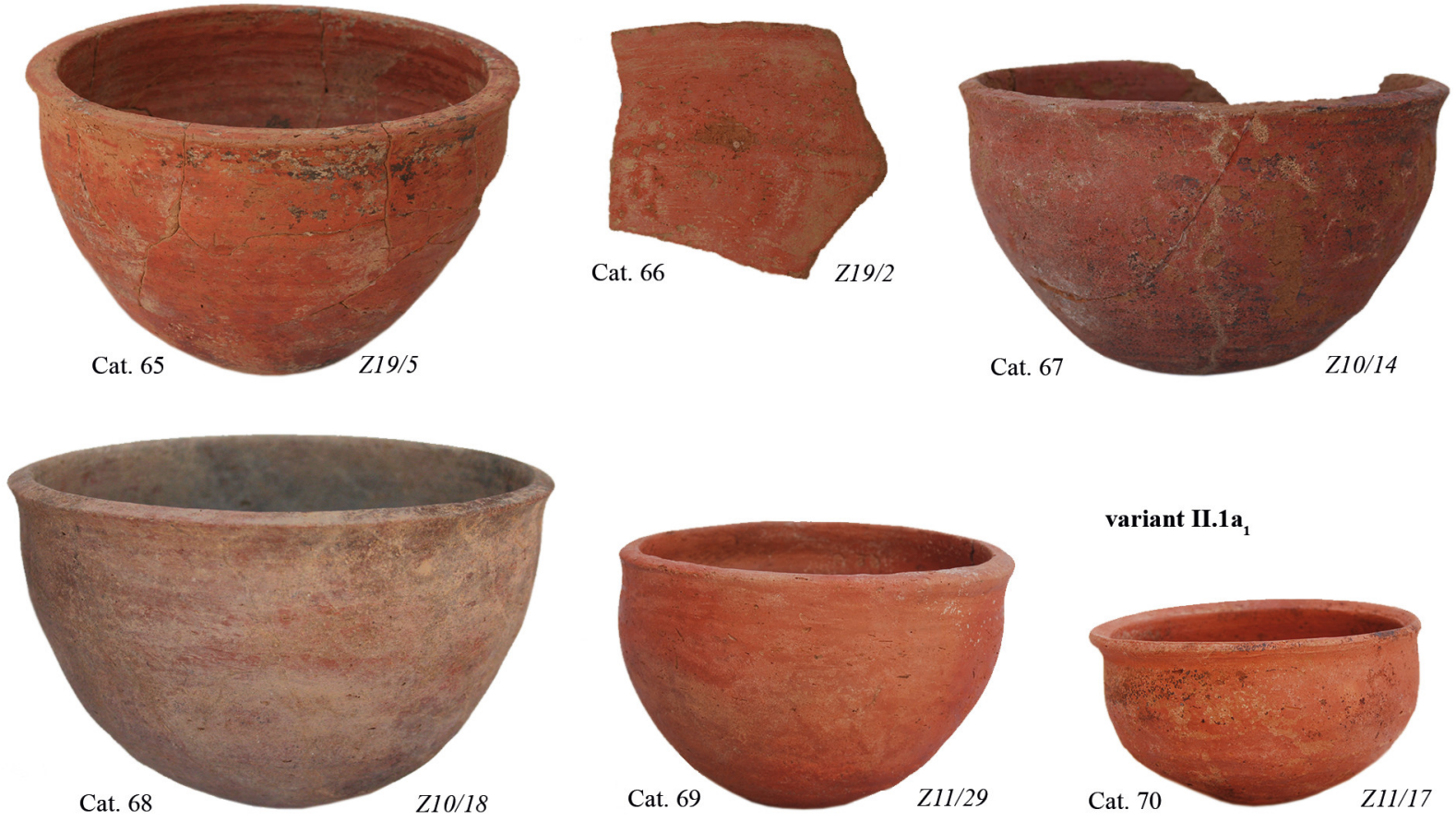

variant II.1a

variant II.1b
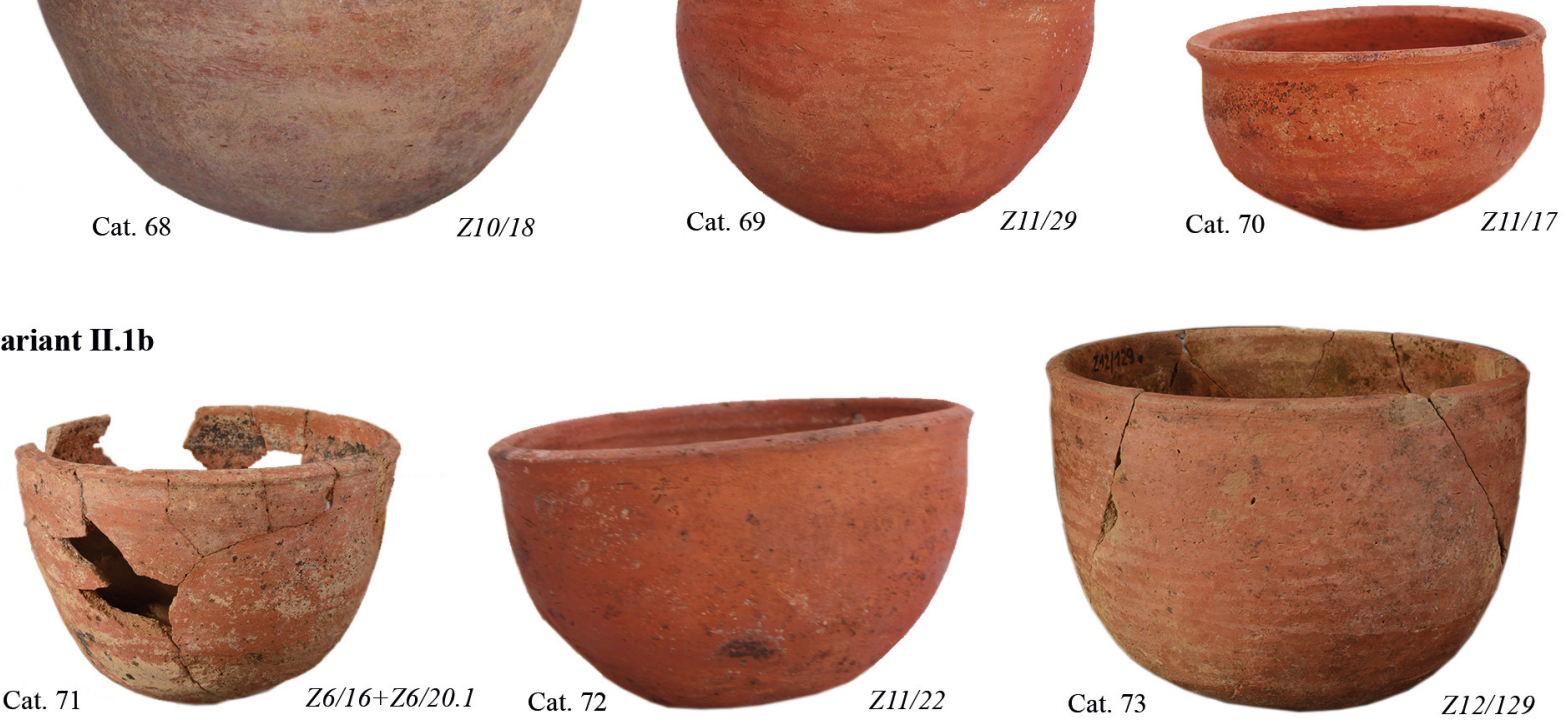

Cat. 71

$Z 6 / 16+Z 6 / 20.1$

Cat. 72

$Z 11 / 22$

Cat. 73

FIGURE 7.92 Bowls of type II.1

Z19/5, Z19/2, Z1O/14, Z1O/18 РнотоS BY K. KOTLEWSKI; Z11/29, Z11/17 РнотоS

BY о. вIAŁOSTOCKA; Z6/16+Z6/20.1 РнотO BY A. KAMROWSKI; Z11/22 Рното вY

O. BIAŁOSTOCKA; Z12/129 PHOTO BY A. KAMROWSKI 
II.2

variant II.2a
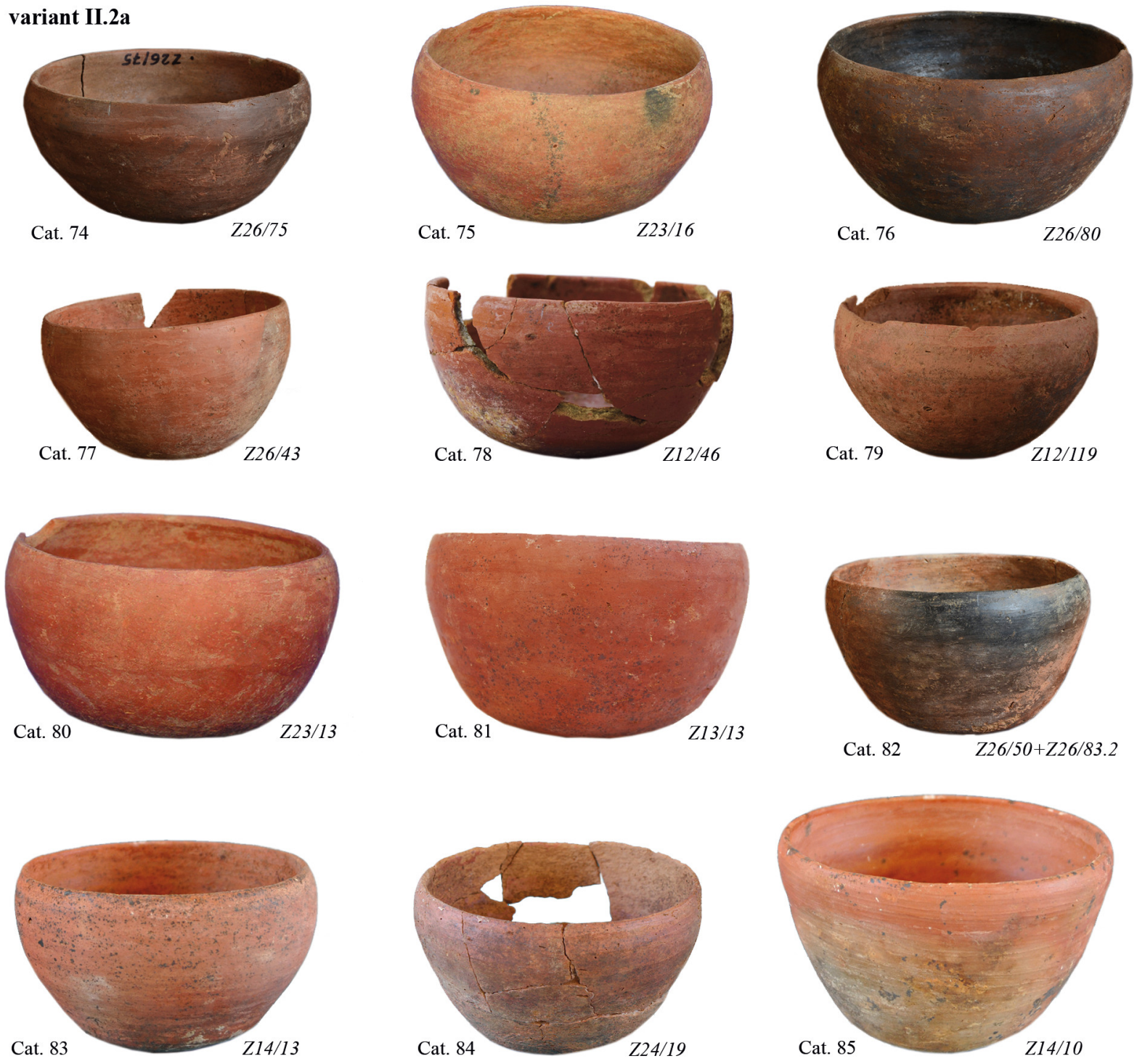

\section{variant II.2b}
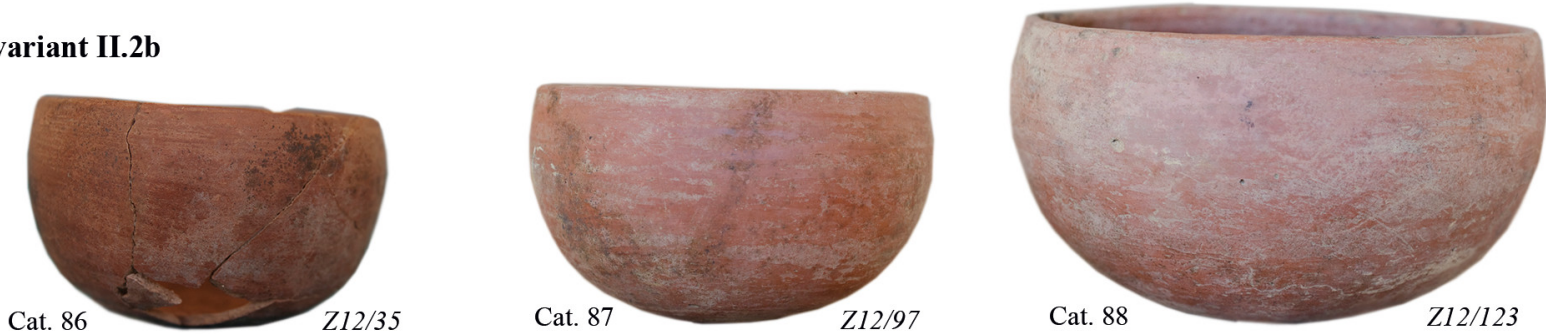

$5 \mathrm{~cm}$

FIGURE 7.93 Bowls of type II.2

Z26/75 РНОTO BY A. KAMROWSKI; Z23/16 РНОTO BY K. KOTLEWSKI; Z26/80,

Z26/43 РнотоS BY A. KAMROWSKI; Z12/46 РноTO BY Z. KOWARSKA; Z12/119

PHOTO BY A. KAMROWSKI; Z23/13 РНОTO BY K. KOTLEWSKI; Z13/13 РНOTO BY

O. BIAŁOSTOCKA; Z26/5O+Z26/83.2 Рното вY A. KAMROWSKI; Z14/13, Z24/19, Z14/10

PHOTOS BY T. WOJTCZAK; Z12/35 PHOTO BY R. MAHLER; Z12/97, Z12/123 PHOTOS BY

E. SKOWROŃSKA 


\section{variant II.2b}
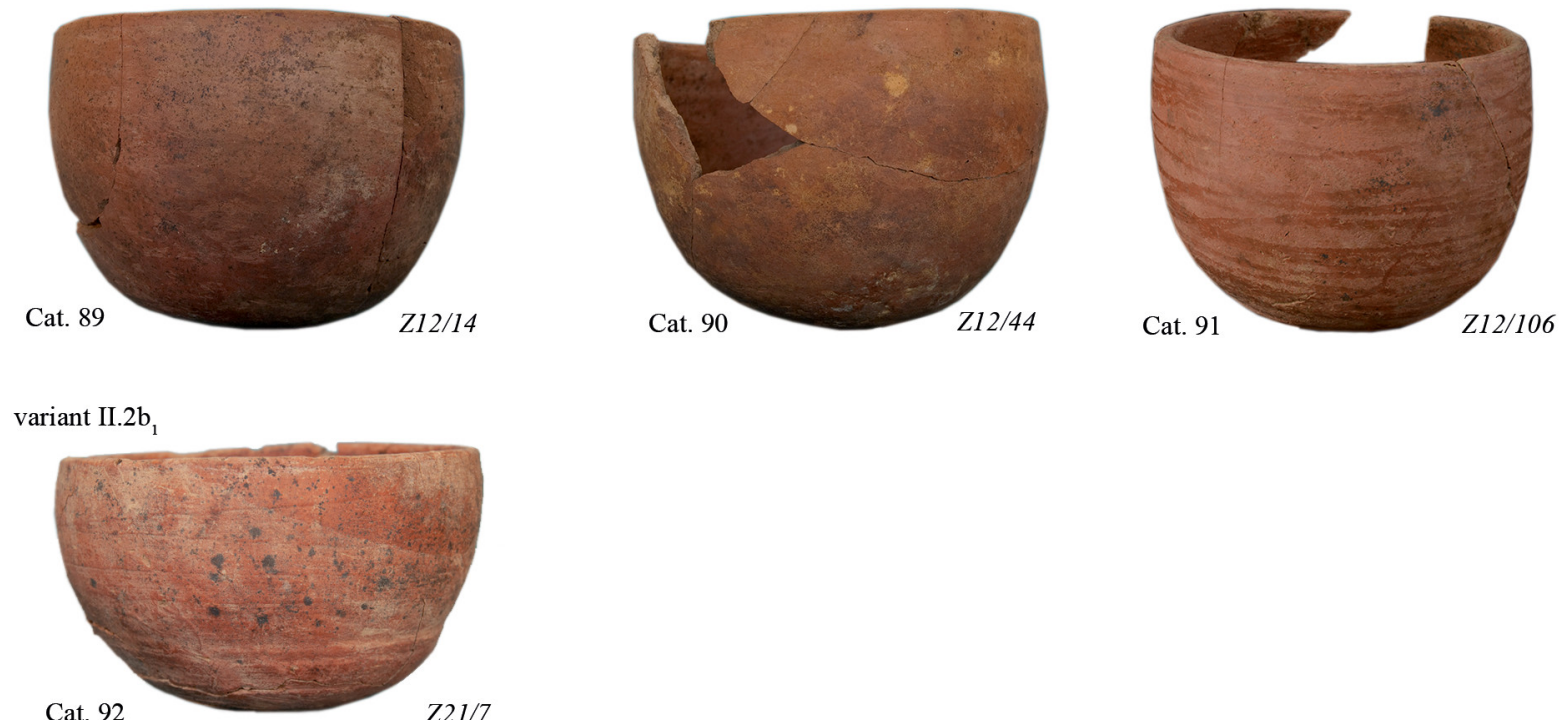

\section{variant II.2c}

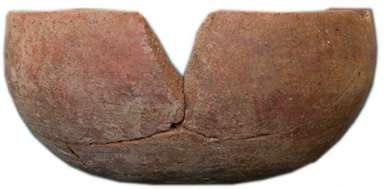

Cat. 93
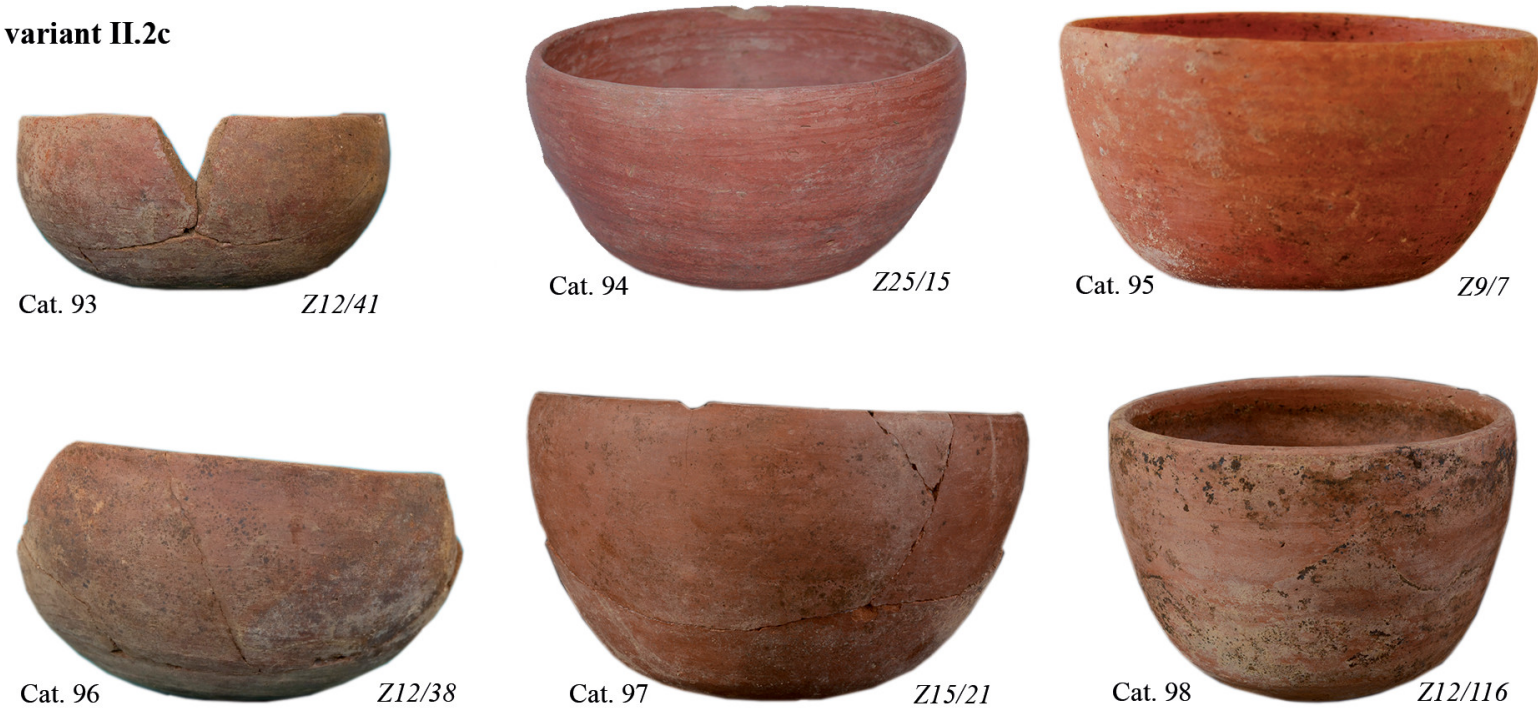

variant II.2d
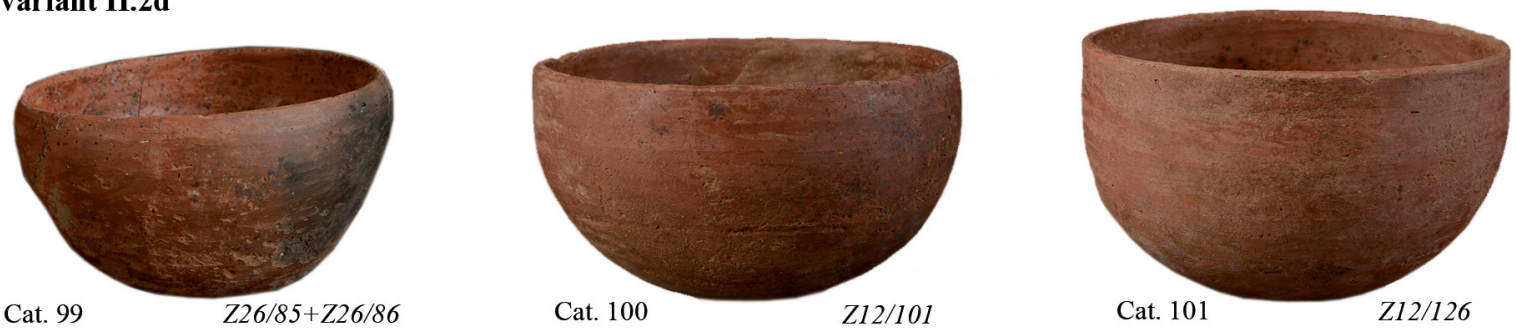

Cat. 99

Cat. 100

Z12/101

Cat. 101

Z12/126

FIGURE 7.94 Bowls of type II.2

Z12/14, Z12/44 PHOTOS BY R. MAHLER; Z12/106 PHOTO BY A. KAMROWSKI; Z21/7, Z12/41 PHOTOS BY R. MAHLER; Z25/15 PHOTO BY K. KOTLEWSKI; Z9/7 РНOTO BY

E. CZYŻEWSKA-ZALEWSKA; Z12/38 PHOTO BY R. MAHLER; Z15/21, Z12/116 РнOTOS BY

A. KAMROWSKI; Z26/85+Z26/86, Z12/101, Z12/126 РнотOS BY A. KAMROWSKI 


\section{variant II.2d}
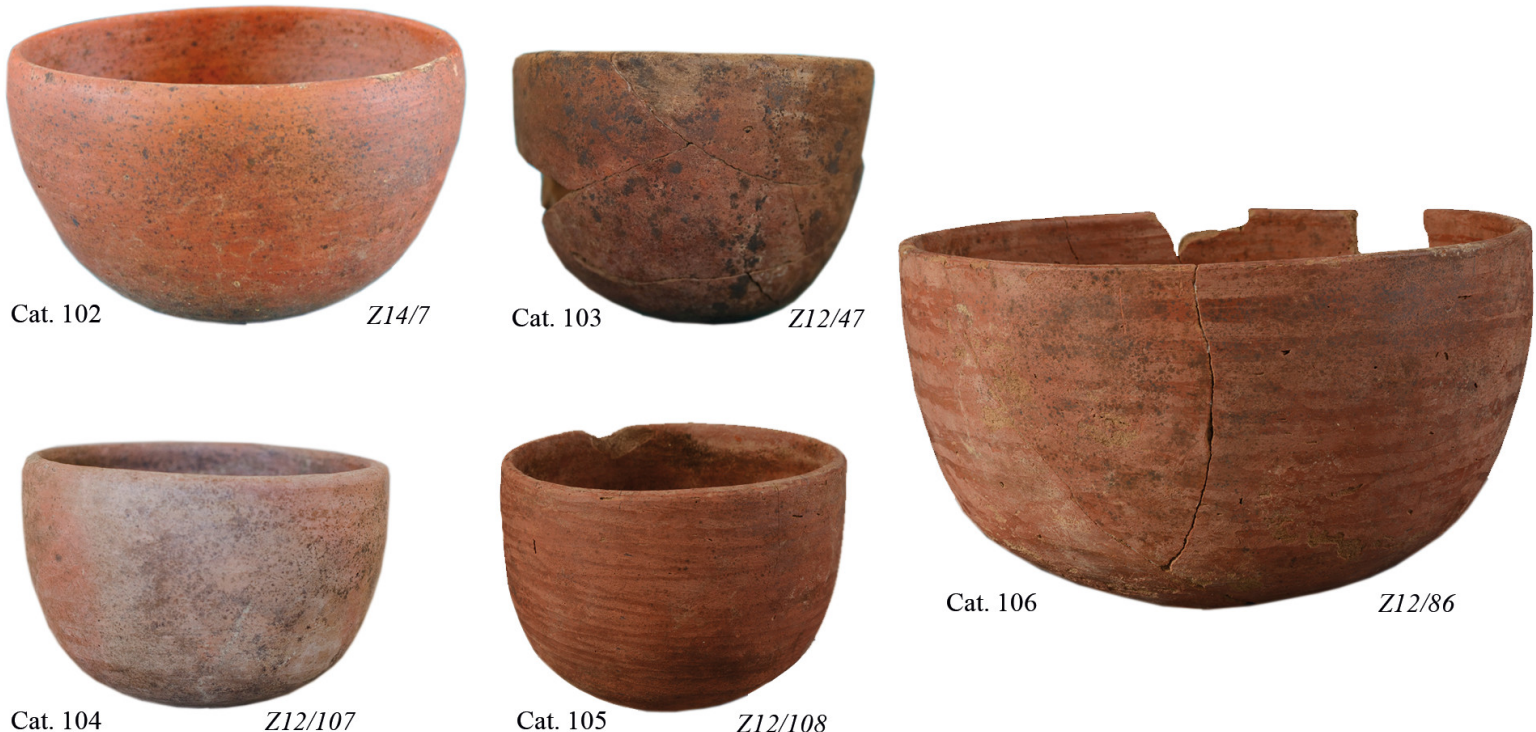

Cat. 104

Z12/107

Cat. 105

Z12/108

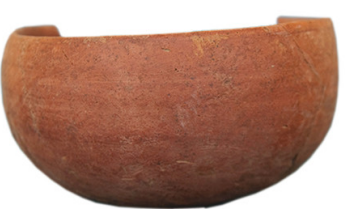

Cat. 108

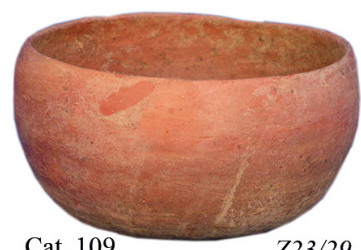

Cat. 109

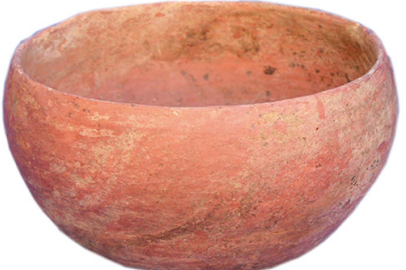

Cat. 110

\section{II.3}

\section{variant II.3a}
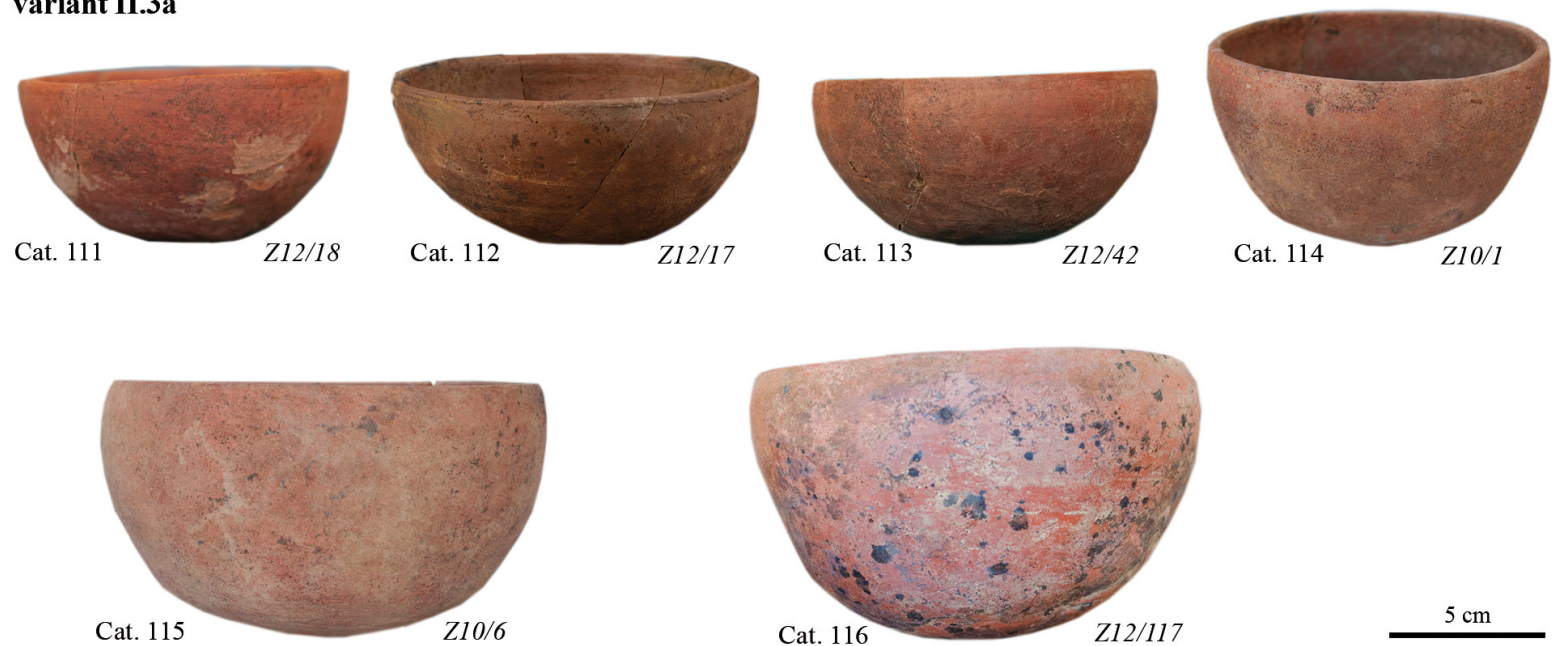

Cat. 114

FIGURE 7.95 Bowls of types II.2 and II.3

Z14/7 PнОTO BY T. WOJTCZAK; Z12/47 PHOTO BY R. MAHLER; Z12/1O7 PHOTO BY E. SKOWROŃSKA; Z12/108, Z12/86 РноTOS BY A. KAMROWSKI; Z25/11 РНОTO BY K. KOTLEWSKI; Z12/37 PHOTO BY R. MAHLER; Z23/29, Z23/22 PHOTOS BY K. KOTLEWSKI; Z12/18 PHOTO BY R. MAHLER; Z12/17 PHOTO BY A. KAMROWSKI; Z12/42 PHOTO BY R. MAHLER; Z1O/1, Z1O/6 PHOTOS BY K. KOTLEWSKI; Z12/117 PHOTO BY E. SKOWROŃSKA 


\section{variant II.3a}

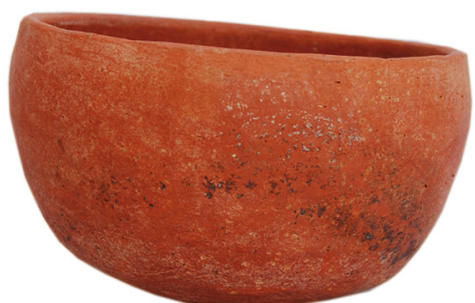

Cat. 117

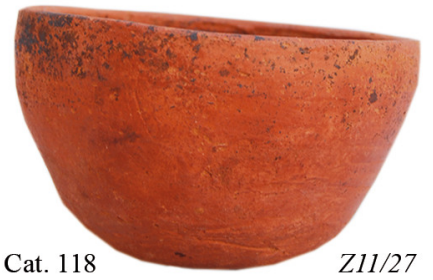

variant II.3b

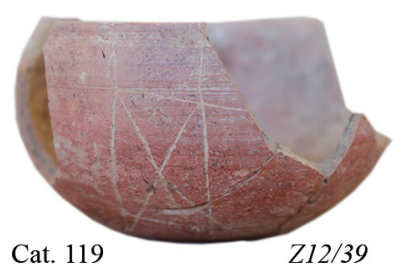

Cat. 119

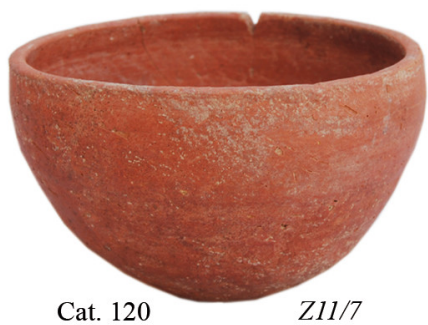

Cat. 120

$111 / 7$

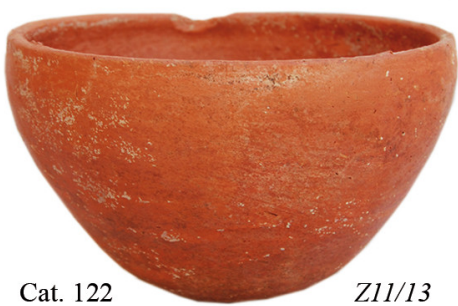

$5 \mathrm{~cm}$

FIGURE 7.96 Bowls of type II.3

Z11/25, Z11/27 РНОTOS BY O. BIAŁOSTOCKA; Z12/39 РнOTO BY E. SKORWOŃSKA;

Z11/7, Z11/9, Z11/13 РнотоS ву о. ВІАєOSTOCKA

II.4

variant II.4a
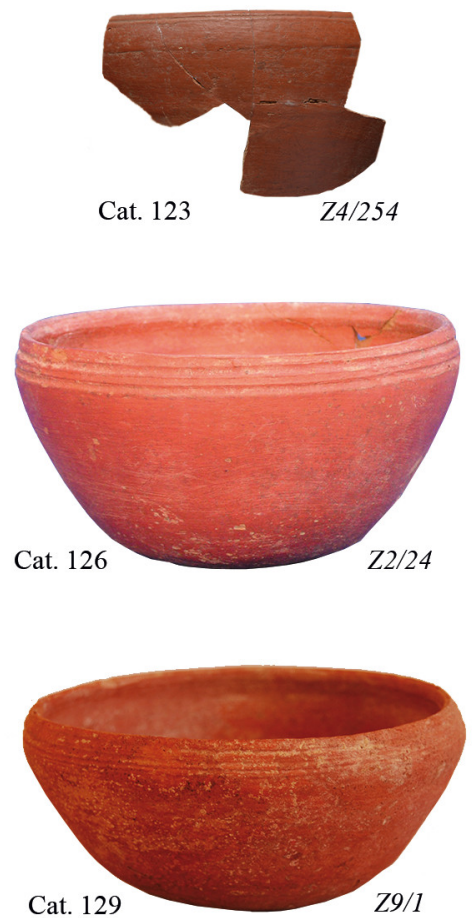
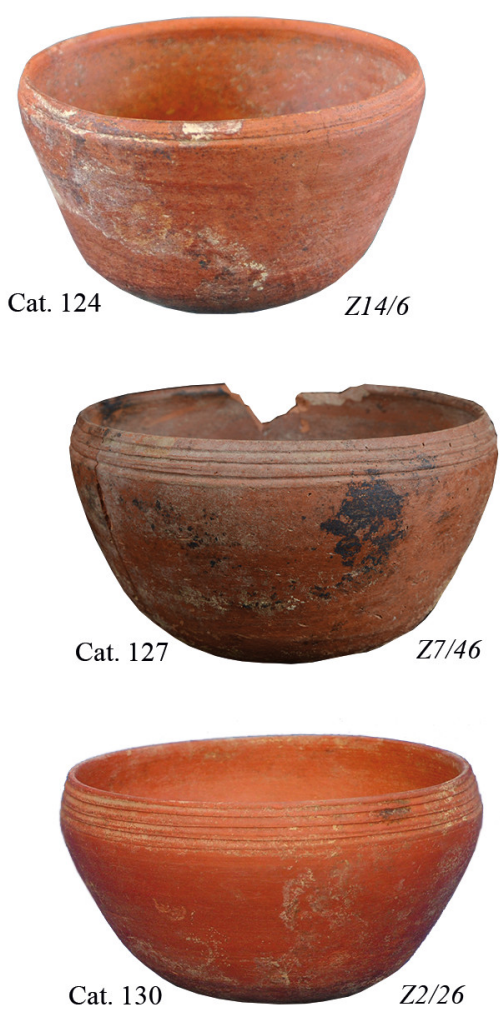
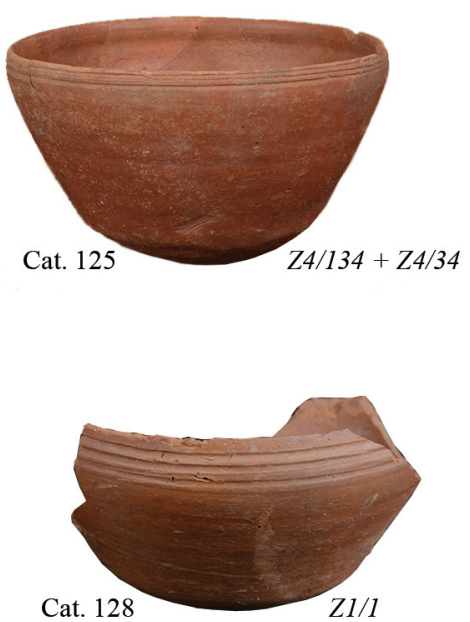

FIGURE 7.97 Bowls of type II.4

Z4/254 РнОто ву А. кАмRоWSKI; Z14/6 Рното ву т. WOJTCZAK; Z4/134+Z4/34

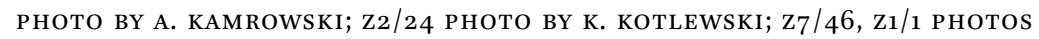
BY A. KAMROWSKI; Z9/1 Рното вY E. CZYŻEWSKA-ZALEWSKA; Z2/26 РнОто вY K. KOTLEWSKI 


\section{variant II.4a}
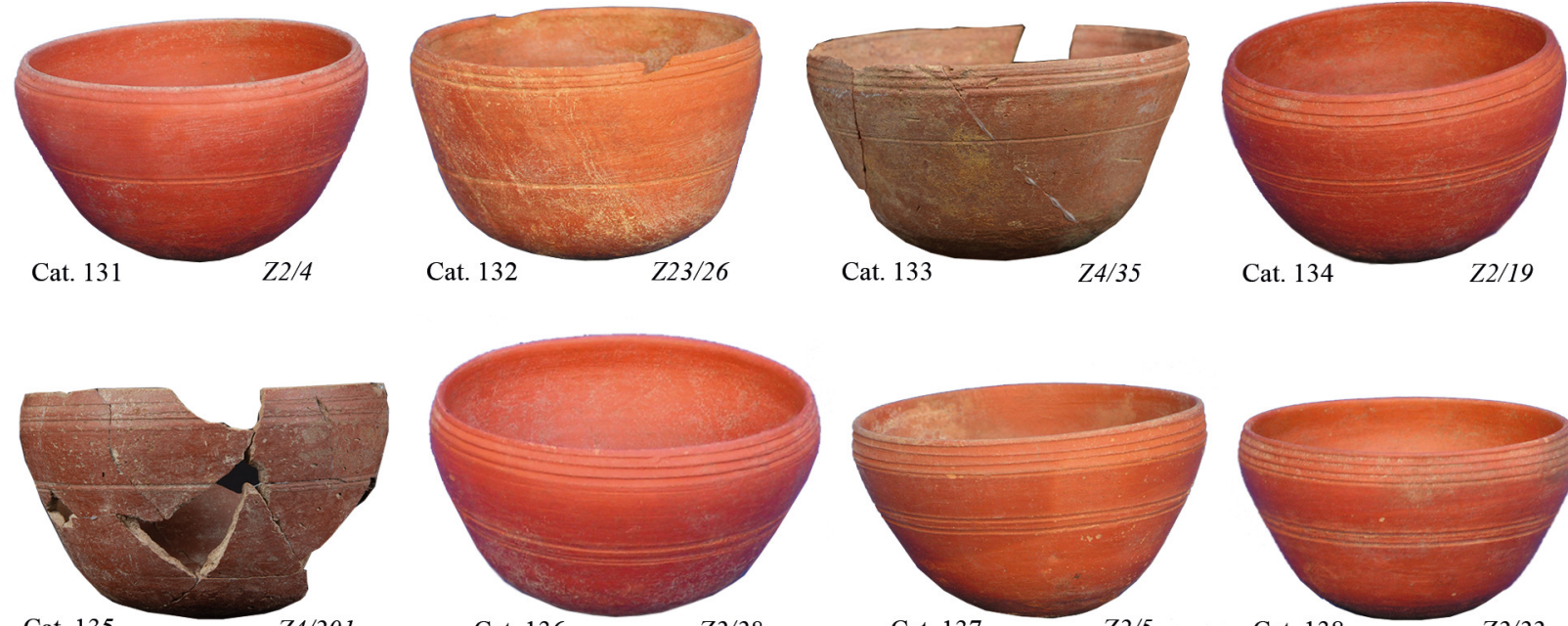

Cat. 136

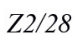

Cat. 137

Z2/5

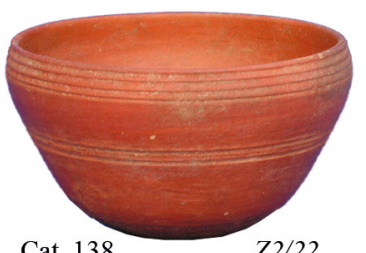

Cat. 138

variant II.4b
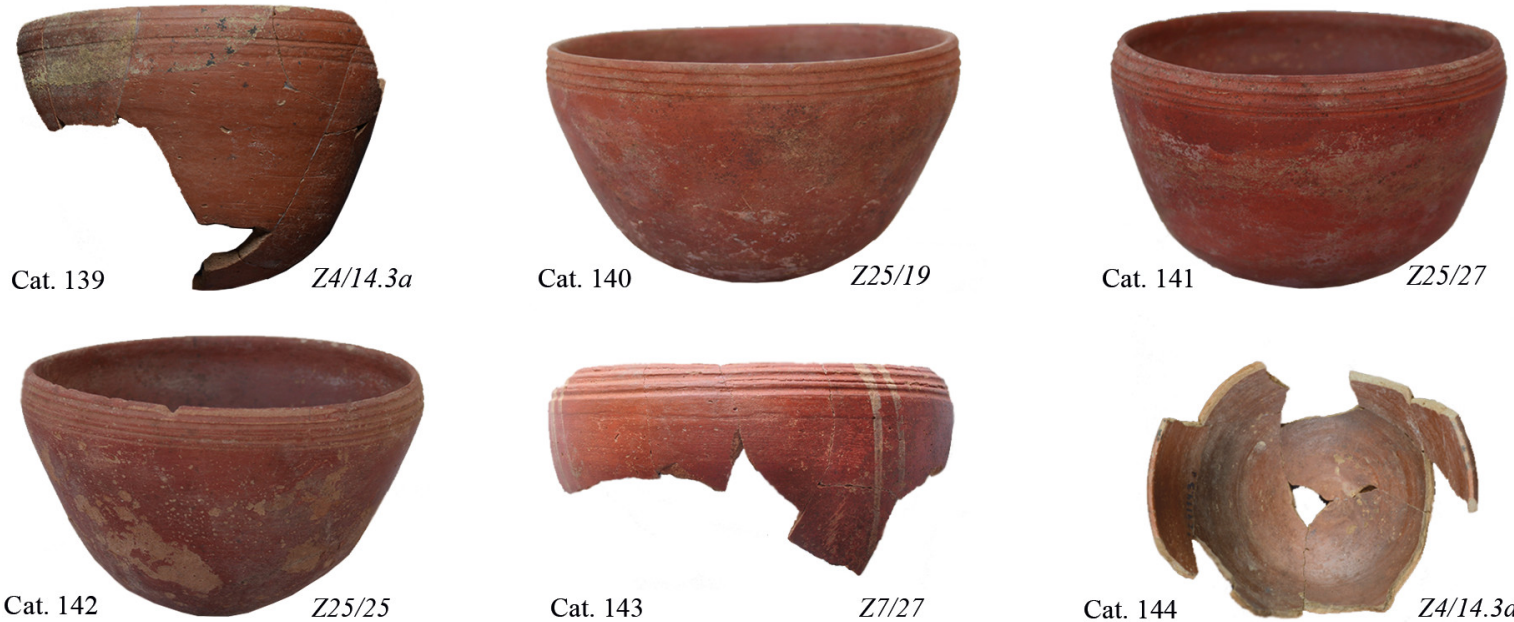

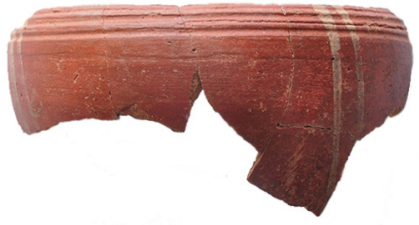

Cat. 143
Z7/27

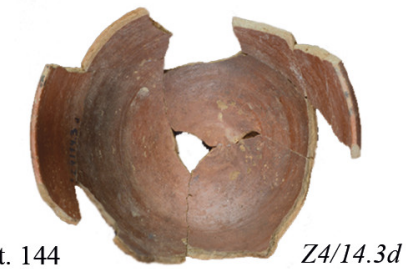

FIGURE 7.98 Bowls of type II.4

Z2/4, Z23/26 РНОтоS BY K. KOTLEWSKI; Z4/35 РНОTO ВY А. KAMROWSKI; Z2/19 Рното вY К. КотLEWSKI; Z4/2O1 Рното вY А. KAMROWSKI; Z2/28, Z2/5, Z2/22 PHOTOS BY K. KOTLEWSKI; Z4/14.3A PHOTO BY A. KAMROWSKI; Z25/19, Z25/27, Z25/25 PноTOS BY K. KOTLEWSKI; Z7/27, Z4/14.3D PHOTOS BY A. KAMROWSKI

\section{II.5}

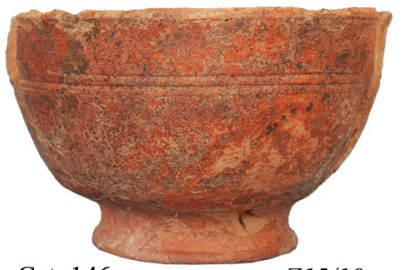

Cat. 146

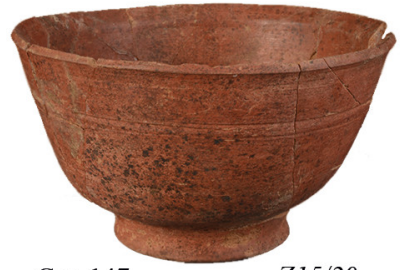

Cat. 147

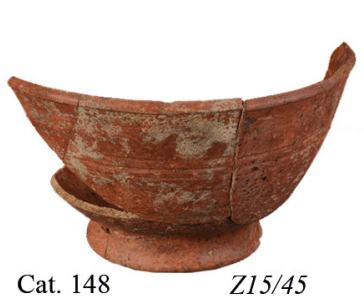

Cat. 148

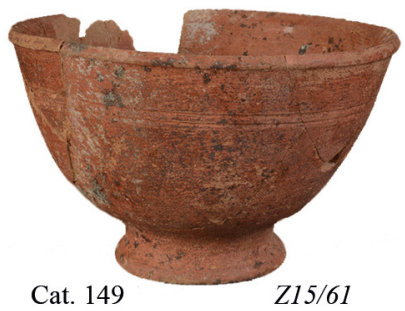

Cat. 149

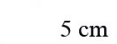

FIGURE 7.99 Bowls of type II.5

Z15/19 PHOTO BY R. MAHLER; Z15/2O, Z15/45, Z15/61 PHOTOS BY A. KAMROWSKI 
II.6

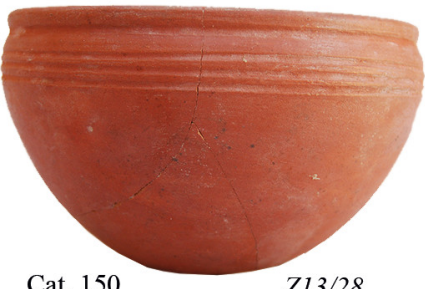

Cat. 150

Z13/28

Cat. 151

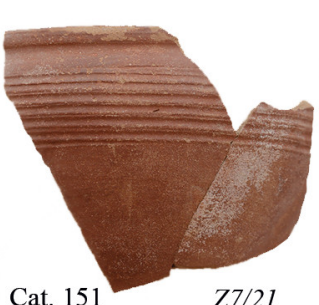

$77 / 21$
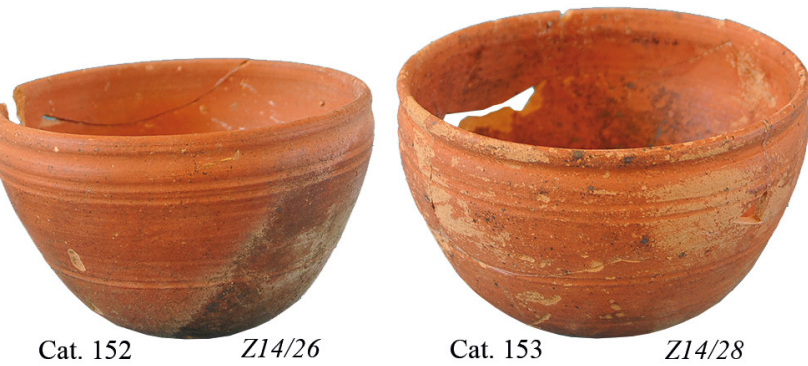

Z14/26

$5 \mathrm{~cm}$

FIGURE 7.100 Bowls of type II.6

Z13/28 рното ву о. віАŁOSTOCKA; Z7/21 Рното ву А. KАMROWSKI; Z14/26, Z14/28 PHOTOS BY T. WOJTCZAK

II.7

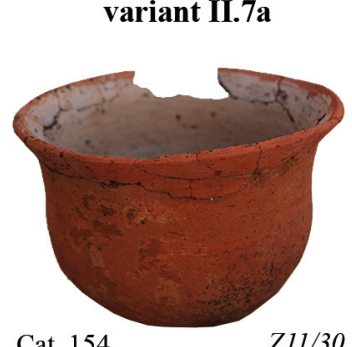

Cat. 154

\section{$211 / 30$}

variant II.7b
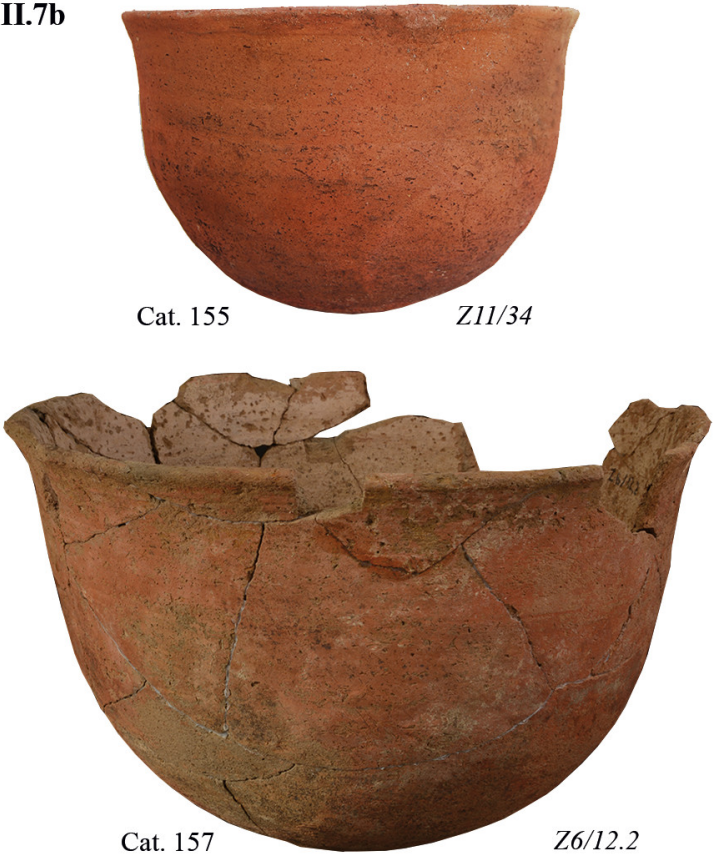

FIGURE 7.101 Bowls of type II.7

Z11/3о, Z11/34 РнотоS ву о. вІАєоSTосKA; z6/12.1. z6/12.2 РнотоS BY A. KAMROWSKI 


\section{II.8}

\section{variant II.8a}
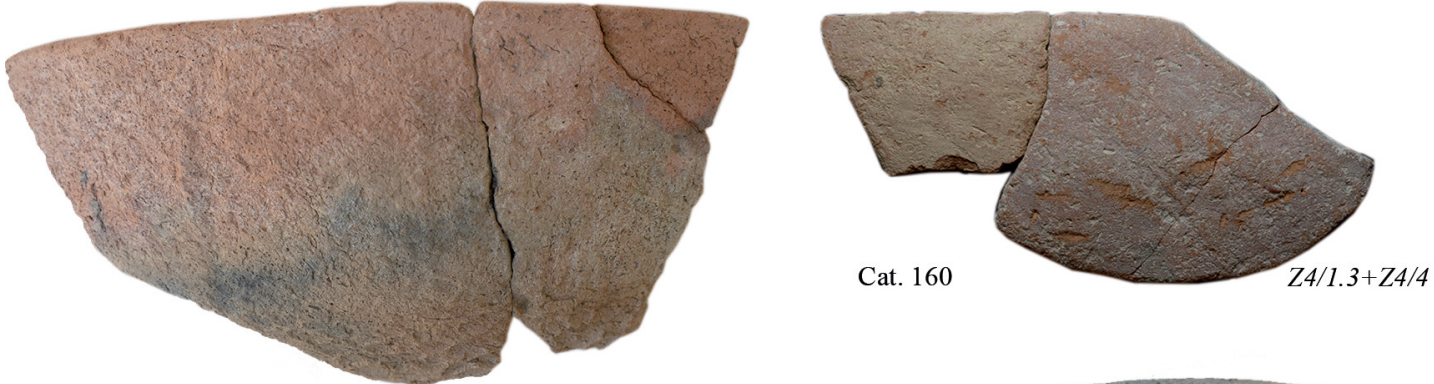

Cat. 158

$Z 6 / 3.3+Z 6 / 28.1+Z 6 / 35.4+Z 6 / 39+Z 6 / 56+Z 6 / 58.2$
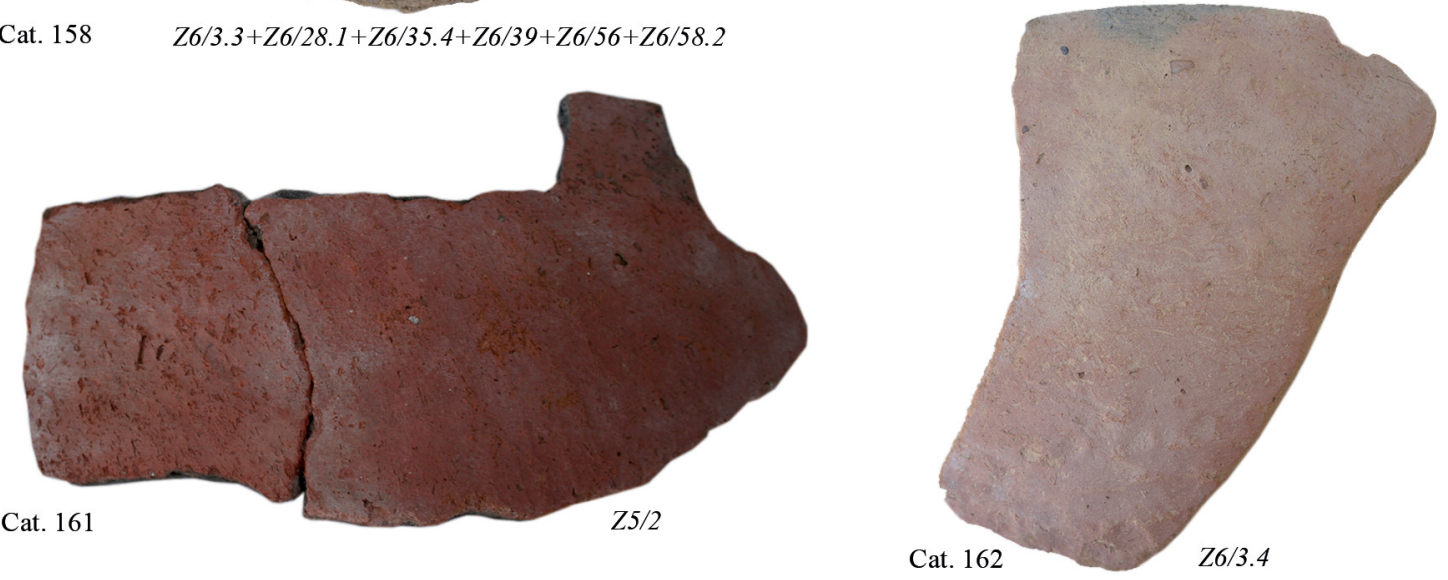

\section{variant II.8b}
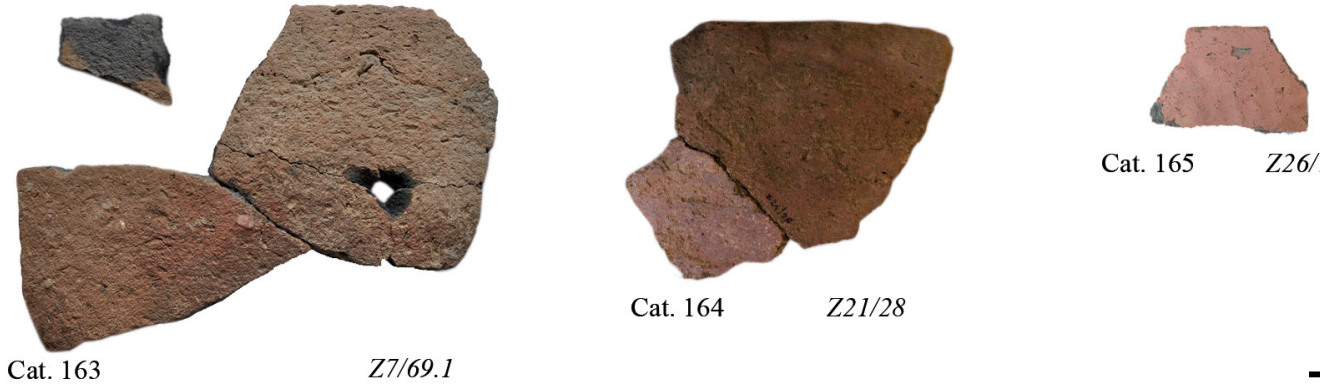

Cat. 165

Z26/71.3

Cat. 164

$Z 21 / 28$

Cat. 163

Z7/69.1

$5 \mathrm{~cm}$

FIGURE 7.102 Bowls of type II.8

$\mathrm{z} 6 / 3 \cdot 3+\mathrm{z} 6 / 28.1+\mathrm{z} 6 / 35 \cdot 4+\mathrm{z} 6 / 39+\mathrm{z} 6 / 56+\mathrm{z} 6 / 58.2, \mathrm{z} 4 / 1 \cdot 3+\mathrm{z} 4 / 4$ Рнотов ву А. кАм-

ROWSKI; Z5/2 РНОTO BY К. КОTLEWSKI; Z6/3.4, Z7/69.1 РНОTOS BY А. КАMROWSKI;

Z21/28 Рното BY Z. KOWARSKA; Z26/71.3 РНОTO BY A. KAMROWSKI 
II.9

\section{variant II.9a}
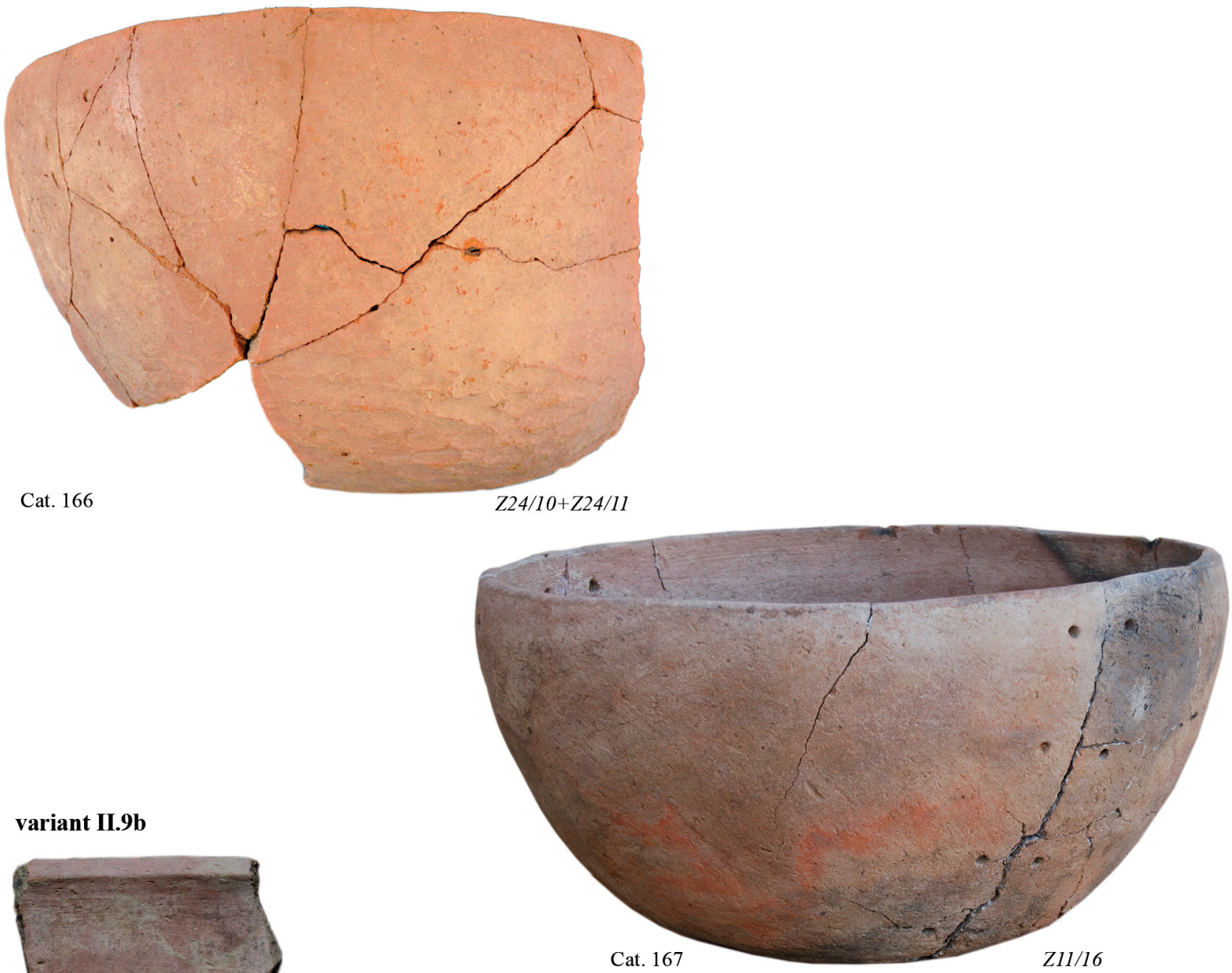

Cat. $168 \quad Z 1 / 21+Z 1 / 22$
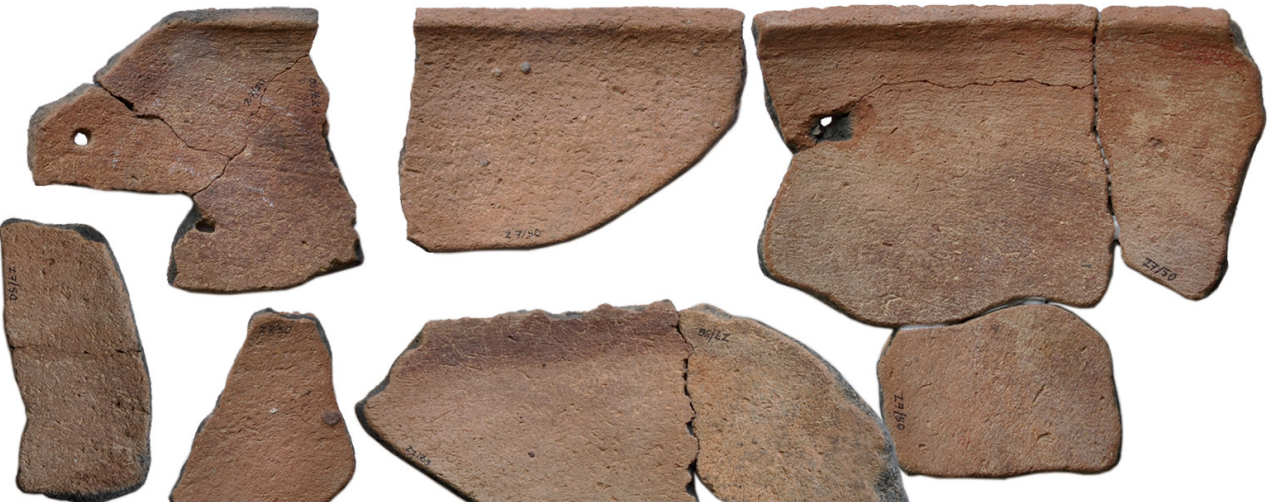

$5 \mathrm{~cm} \quad$ Cat. 169

$\begin{array}{ll}5 \mathrm{~cm} & \text { Cat. } 169\end{array}$
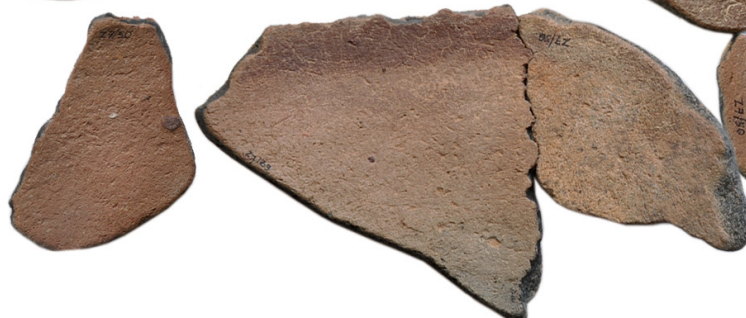

Z24/10+Z24/11 Рното вY T. WOJTCZAK; Z11/16 РНОTO вY О. ВIAŁOSTOCKA;

$\mathrm{Z1} / 21+\mathrm{Z1} / 22, \mathrm{z}_{7} / 5 \mathrm{O}+\mathrm{Z} 7 / 69$ PнотоS ву А. KАMROWSKI 

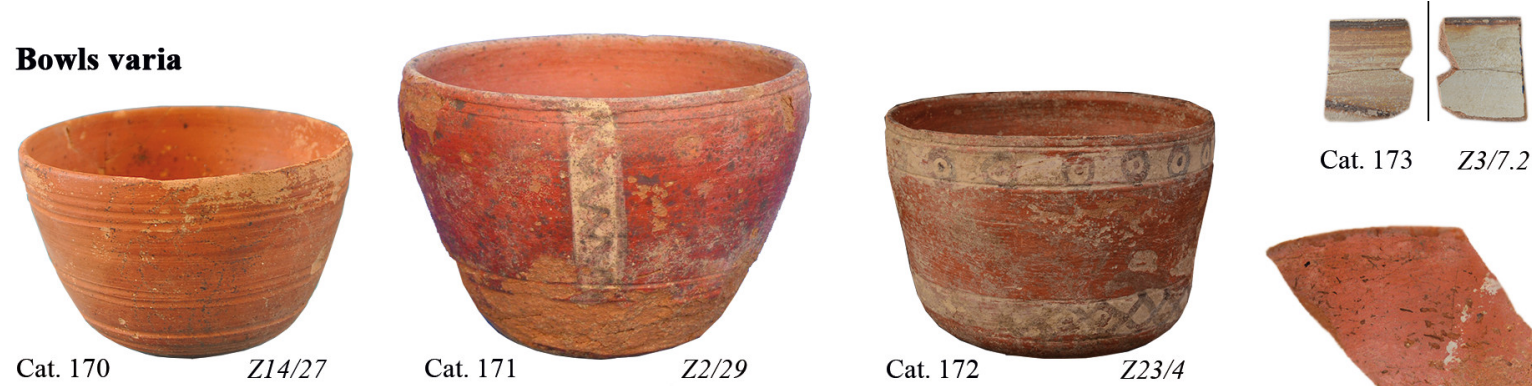

Cat. $173 \quad Z 3 / 7.2$

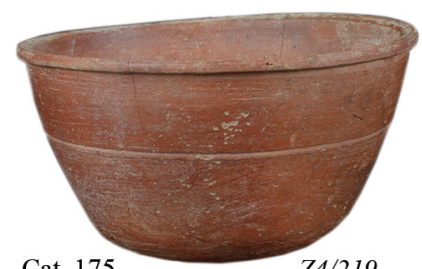

Cat. 175

24/219
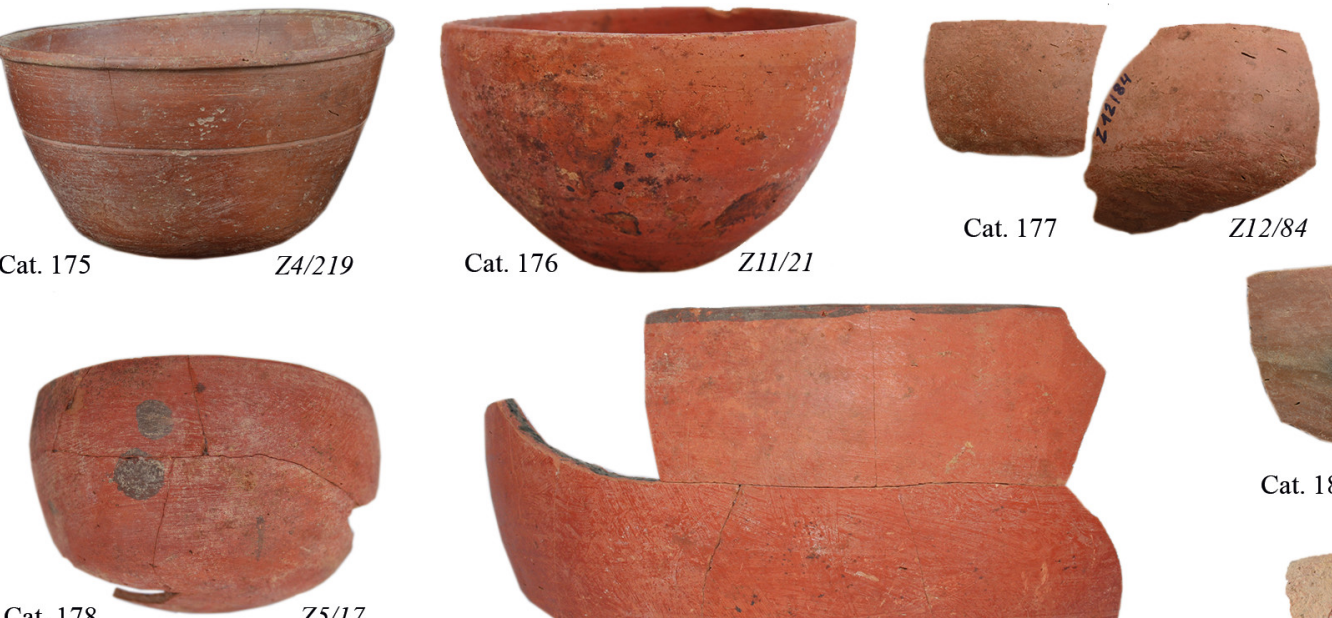

Cat. 174

Z5/18

Cat. 178 Z5/17
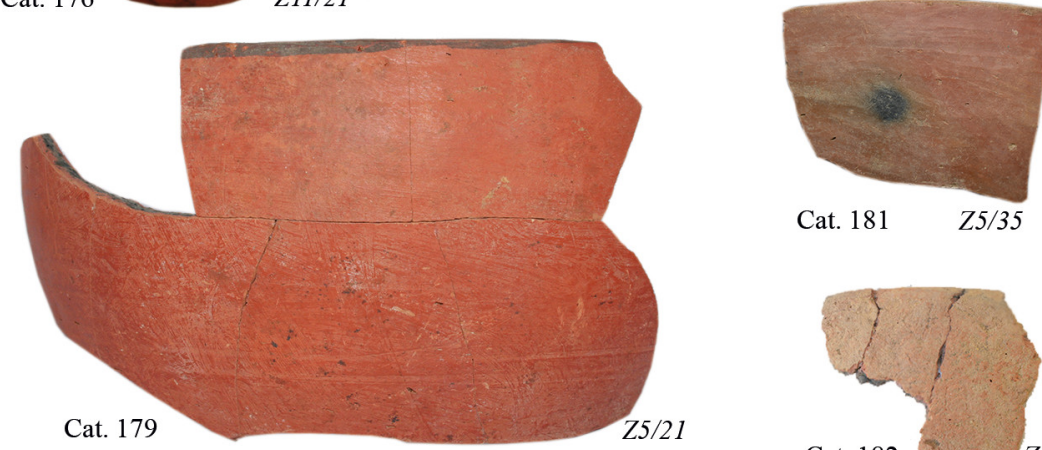

Cat. 181

$Z 5 / 35$

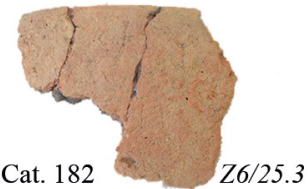

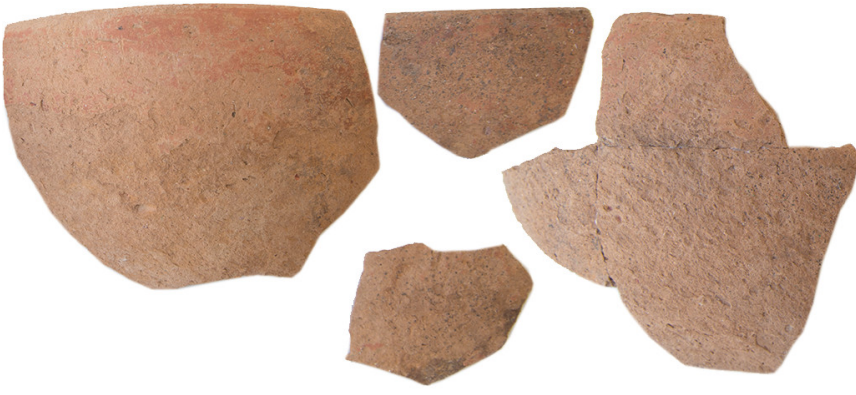

Cat. 180 $Z 6 / 2.1+Z 6 / 3.5+Z 6 / 55$
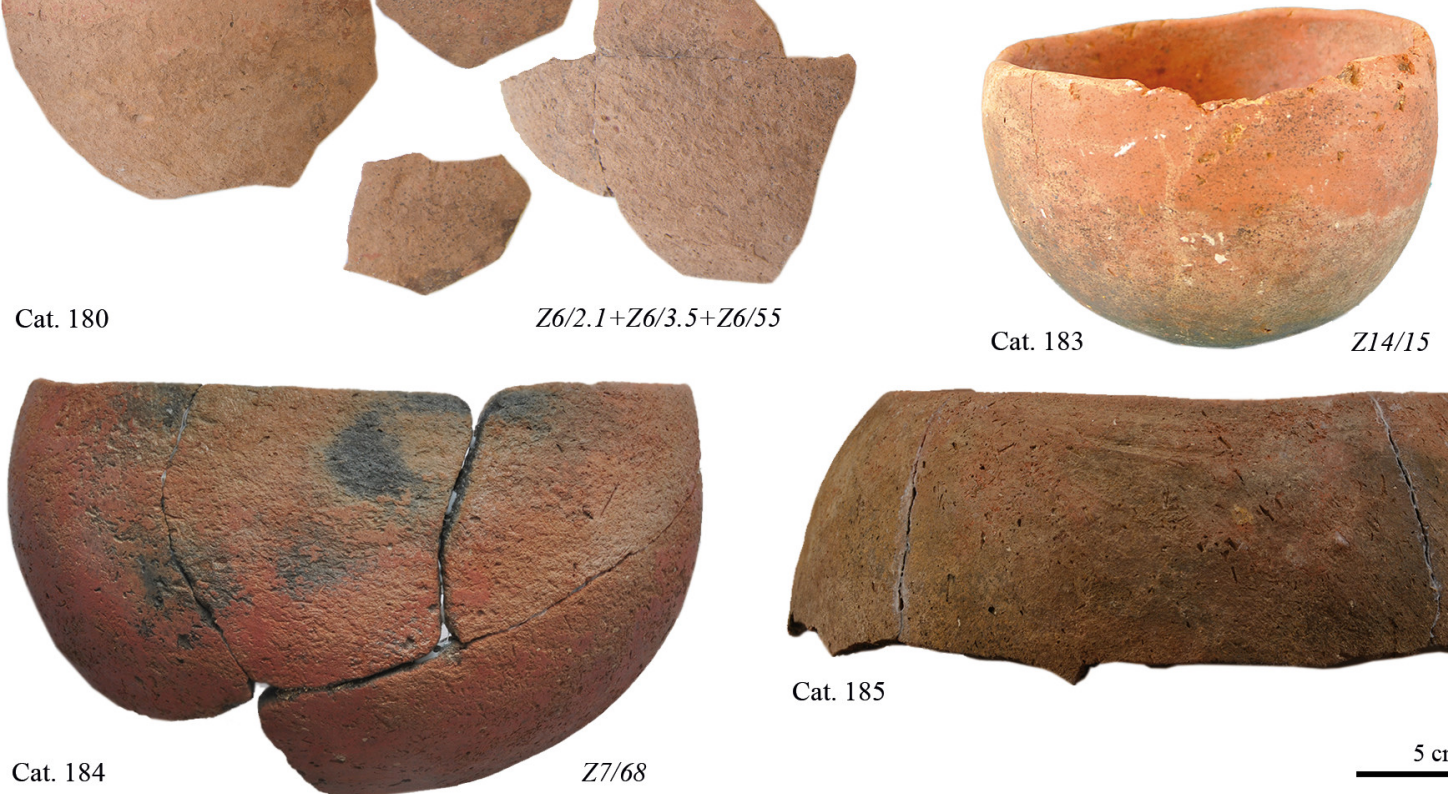

Cat. 183

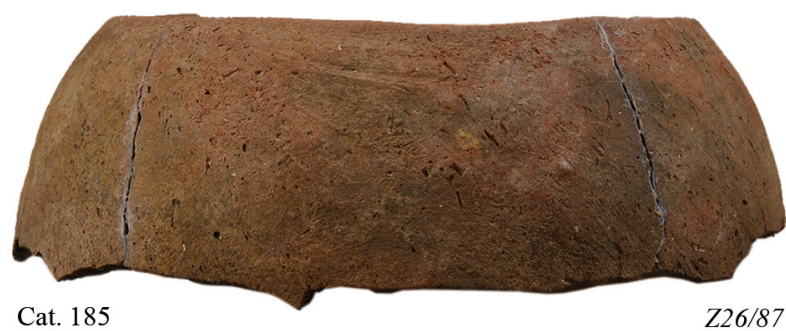

$5 \mathrm{~cm}$

FIGURE 7.104 Bowls varia

Z14/27 РНОто ВY т. WOJTCZAK; Z2/29 РНОTO ВY К. КОTLEWSKI; Z23/4, Z3/7.2 РноTOS BY А. KAMROWSKI; Z5/18 РнотО ВY К. КоTLEWSKI; Z4/219 РНОTO ВY

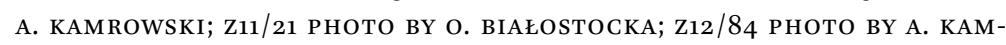
ROWSKI; Z5/17, Z5/21 РНОTOS BY К. КОTLEWSKI; Z6/2.1+Z6/3.5+Z6/55, Z5/35, z6/25.3 РнОтоS вY A. КАMROWSKI; Z14/15 Рното вY т. WOJTCZAK; z7/68, z26/87 PHOTOS BY A. KAMROWSKI 

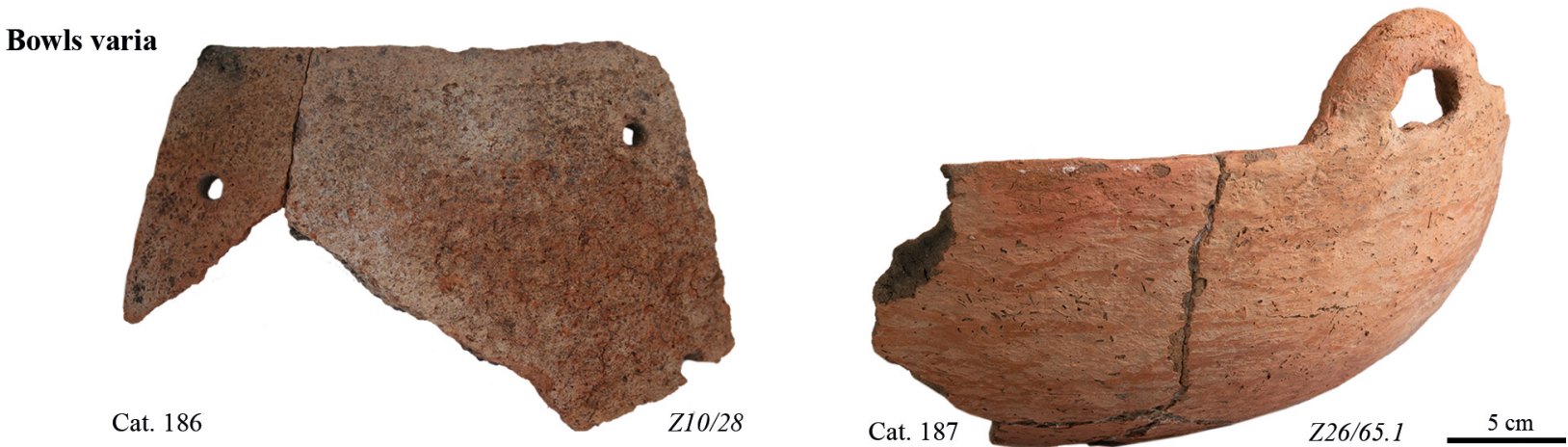

FIGURE 7.105 Bowls varia

Z10/28 Рното BY К. KOTLEWSKI; Z26/65.1 РНОTO BY A. KAMROWSKI

\section{III.1}
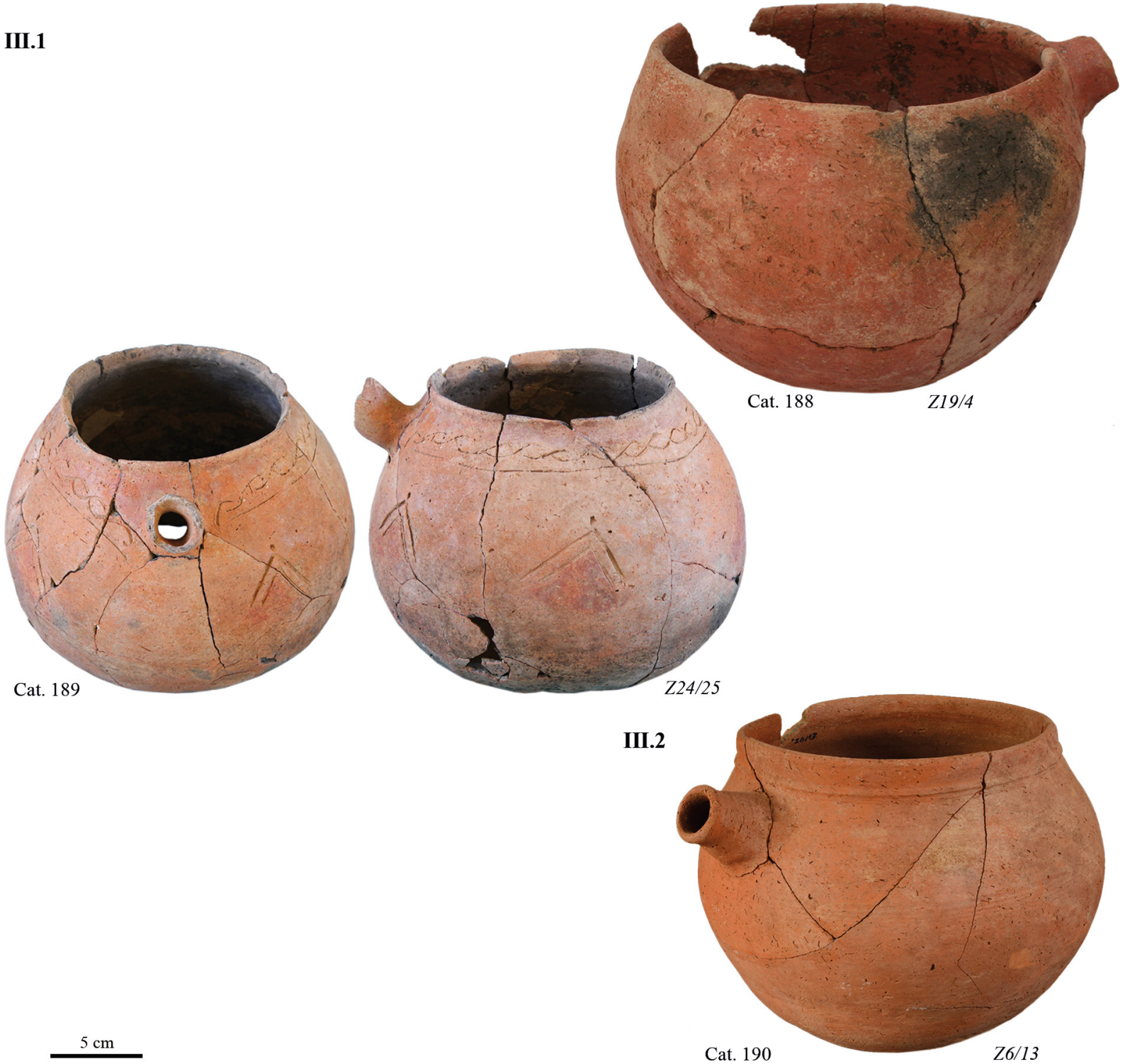

FIGURE 7.106 Spouted bowls of types III.1 and III.2

Z19/4 Рното BY K. KOTLEWSKi; Z24/25 Рното BY T. WOJTCZAK; Z6/13 Рното вY

A. KAMROWSKI 
IV.1
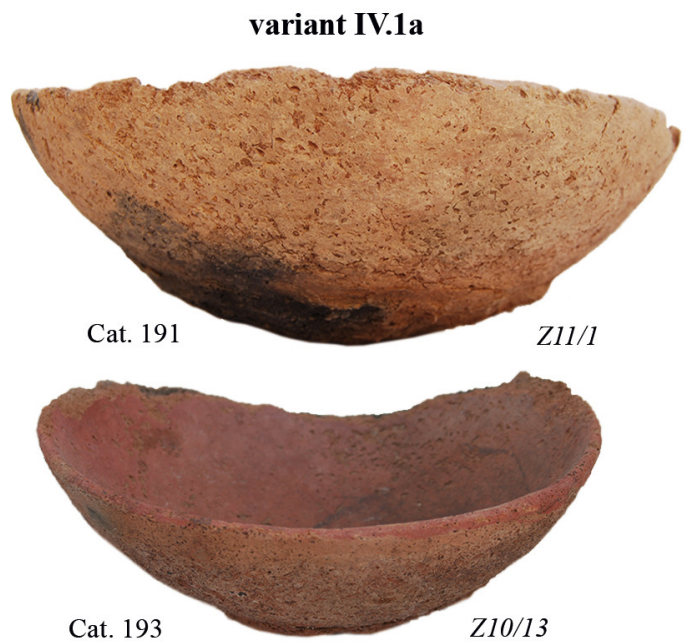

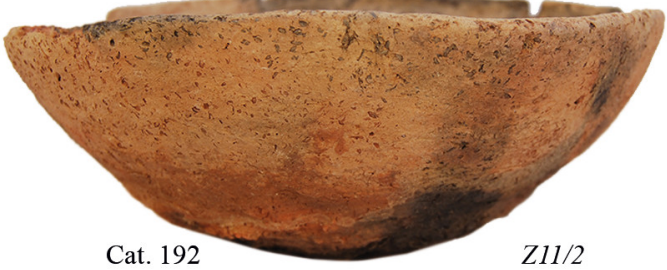

variant IV.1b

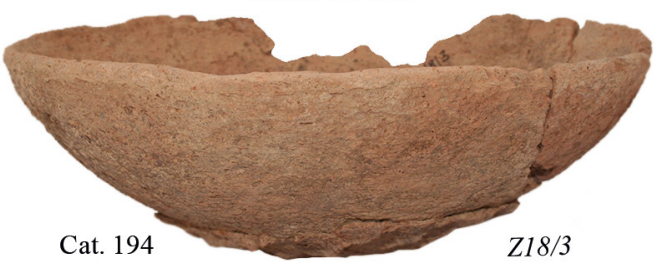

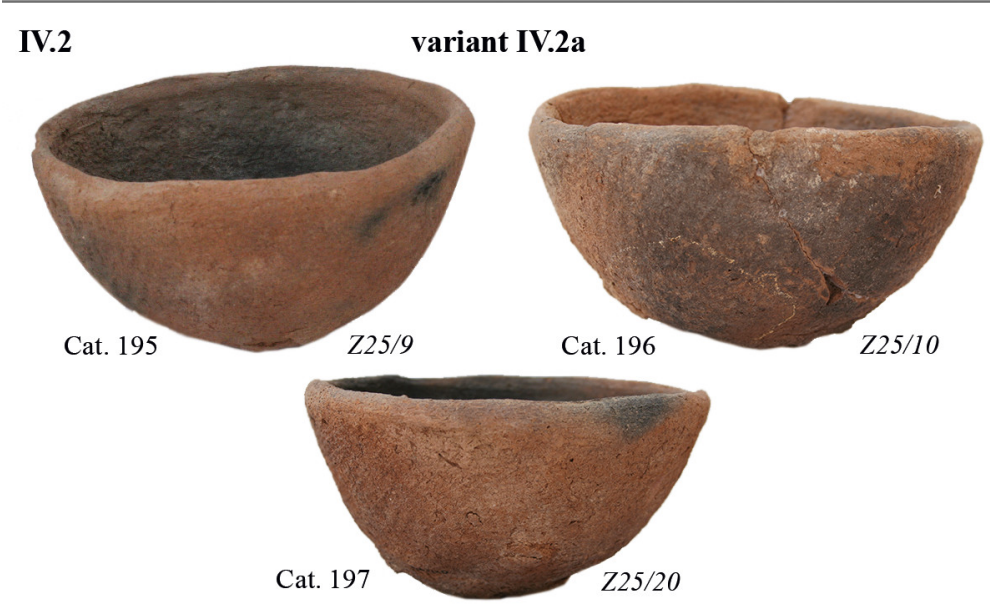

variant IV.2b
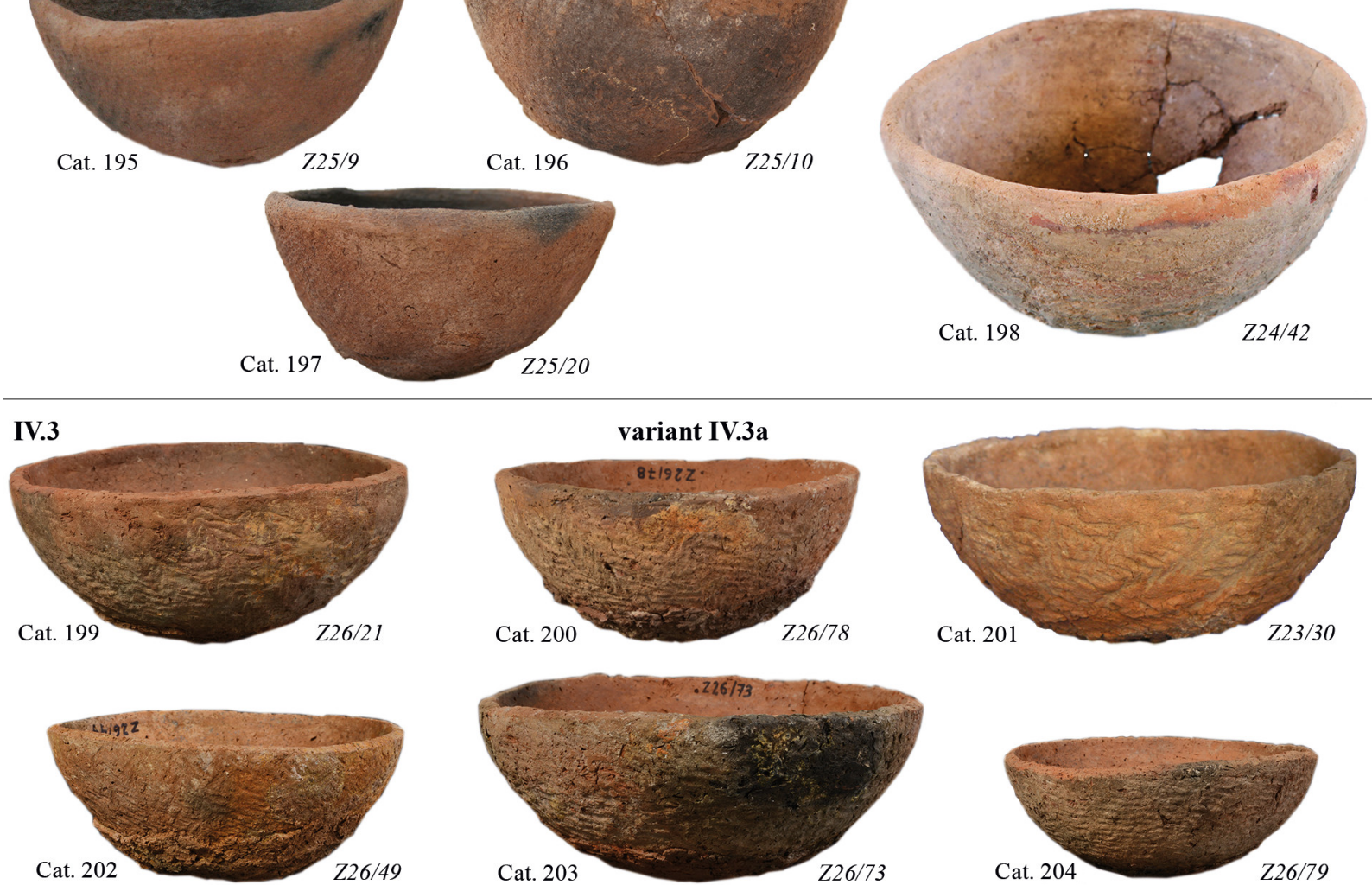

variant IV.3b
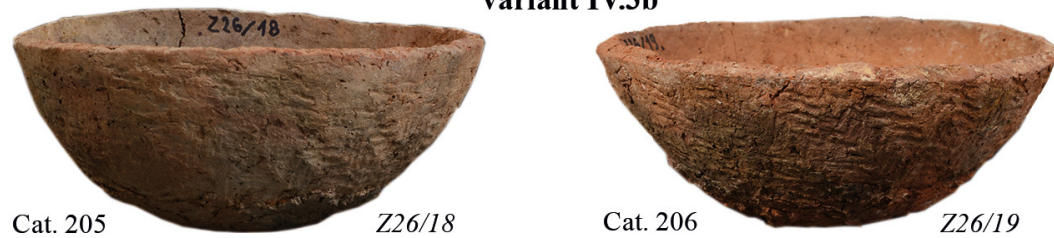

$26 / 79$

Cat 205

$Z 26 / 18$

FIGURE 7.107 Bowls of types IV.1, IV.2 and IV.3

Z11/1, Z11/2 РнотоS вY О. ВІАєOSTOCKA; Z1O/13, Z18/3, Z25/9, Z25/10, Z25/20 РHOтOS вY К. котLEWSKI; Z24/42 Рното вY т. WOJTCZAK; Z26/21, Z26/78 РнотоS ву A. KAMROWSKI; Z23/3о РНОто вY К. КотLEWSKI; Z26/49, Z26/73, Z26/79, Z26/18, Z26/19 РнотоS ву А. КAMROWSKI 
IV.4

variant IV.4a

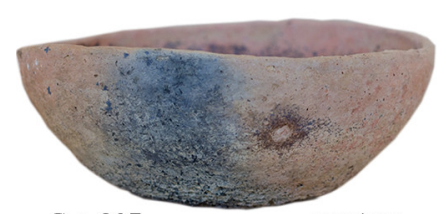

Cat. 207

Z12/125

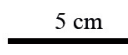

FIGURE 7.108 Bowls of type IV.4 Z12/125 PнОто вY E. SKOWROŃSKA; Z26/55 Рното вY A. KAMROWSKI; Z28/8 РнОто BY T. WOJTCZAK
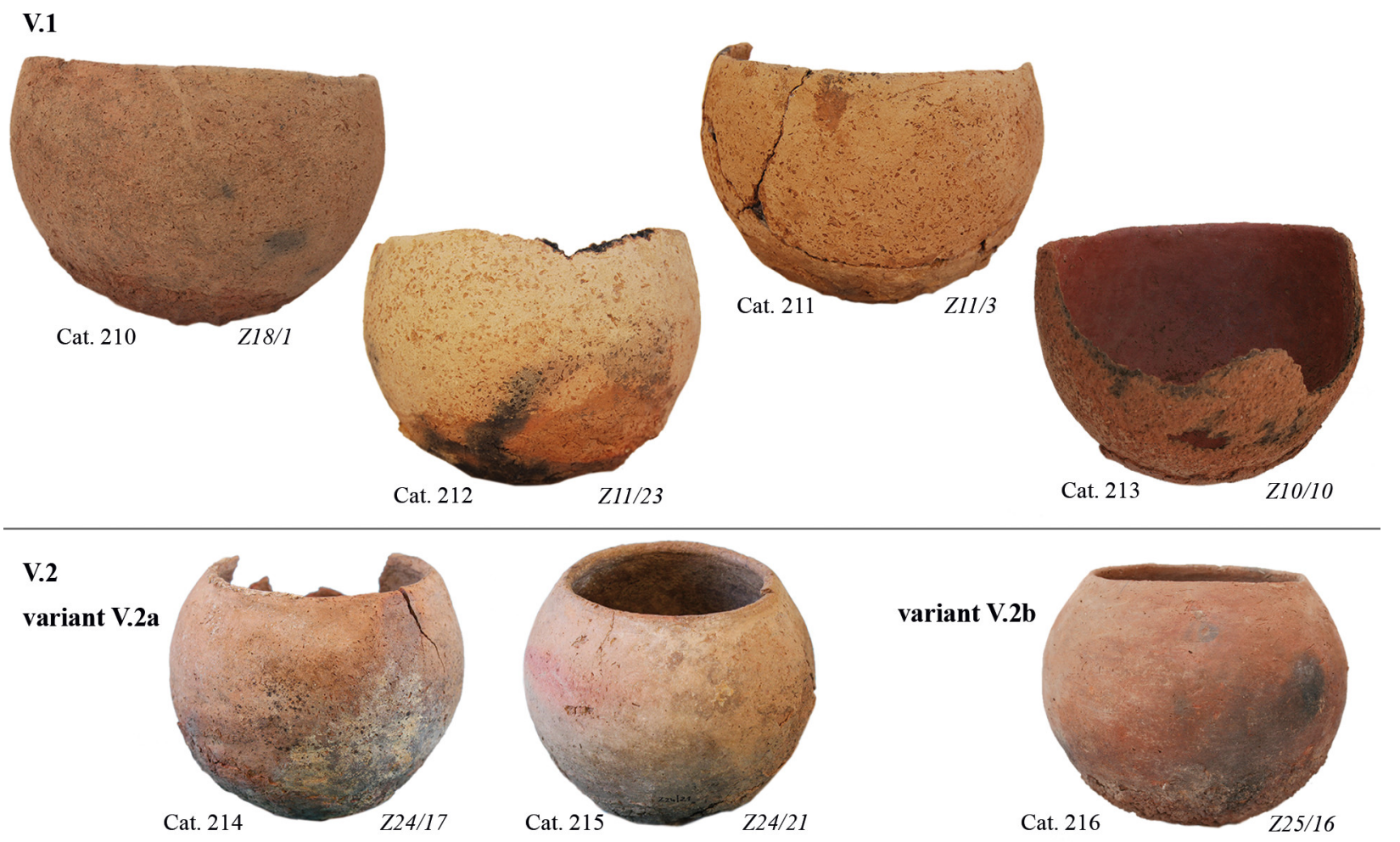

variant V.2b

Cat. 209

variant IV.4c

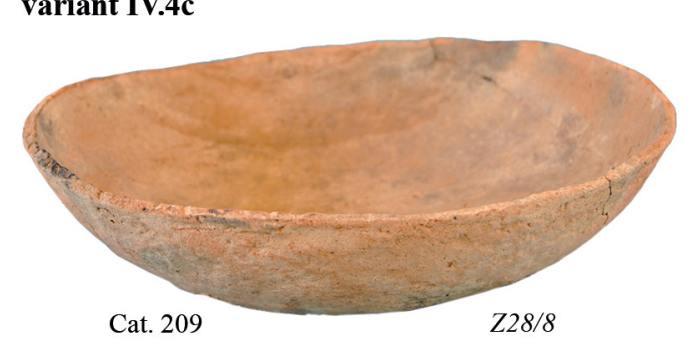

variant IV.4b

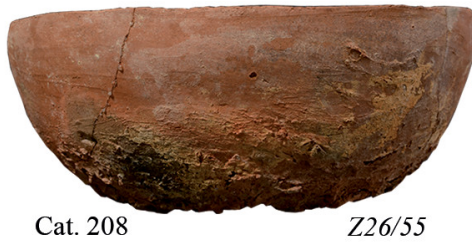

Cat. 208

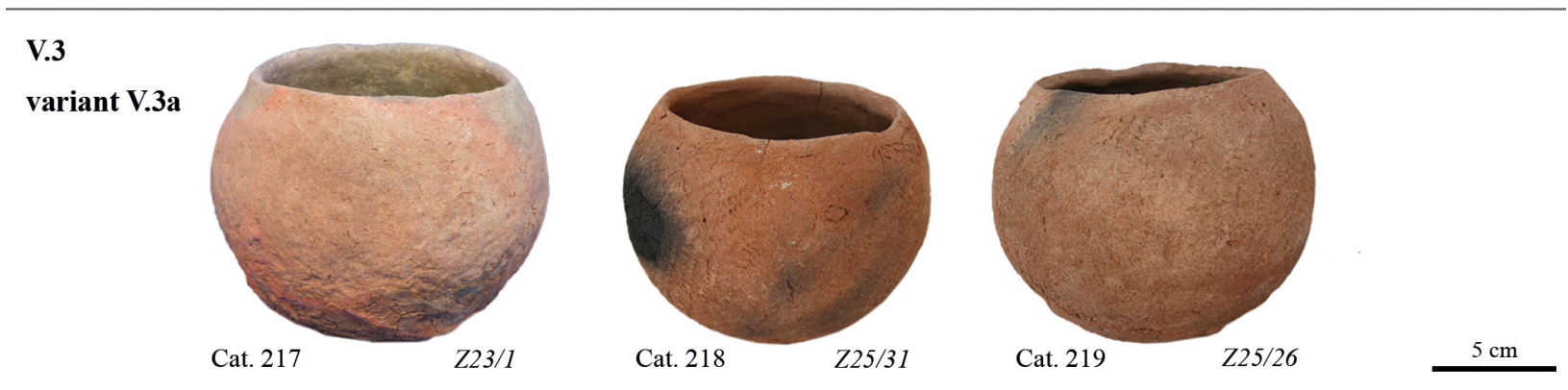

FIGURE 7.109 Pots of types V.1, V.2 and V.3

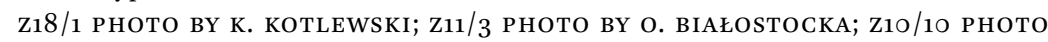
BY К. КотLEWSKI; Z11/23 РНОТО ВY О. ВІАєOSTOCKA; Z24/17, Z24/21 РНОТОS ВY т. WOJTCZAK; Z25/16, Z23/1, Z25/31, Z25/26 РнОTOS BY K. KOTLEWSKI 


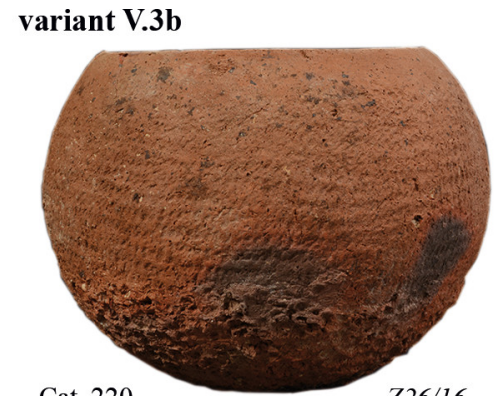

Cat. 220
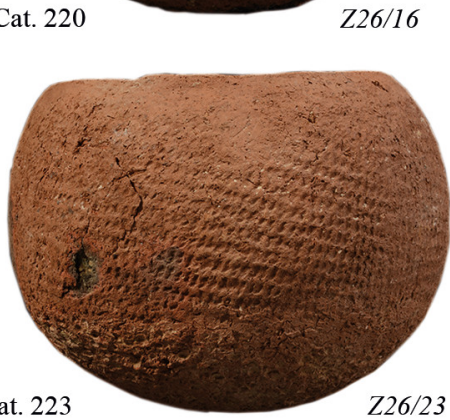

V.4 variant V.4a

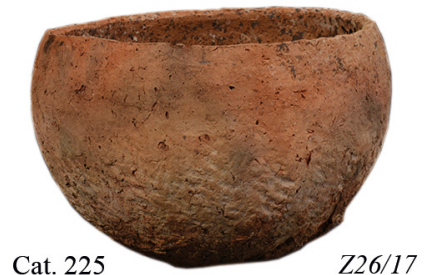

variant V.4b
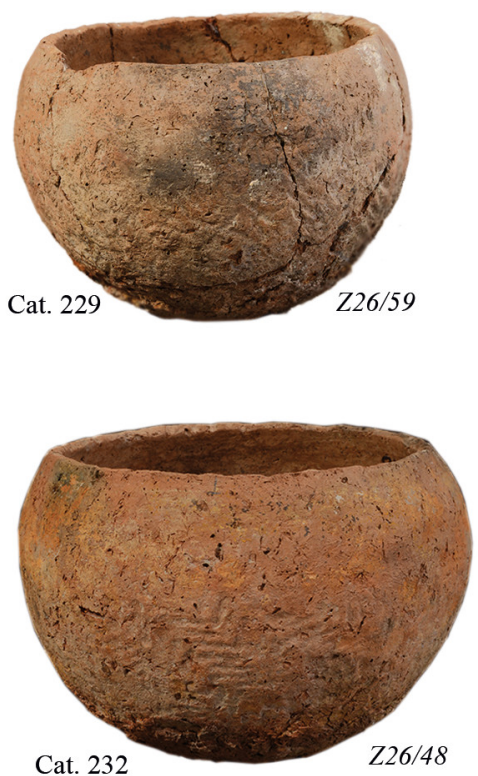

Cat. 232

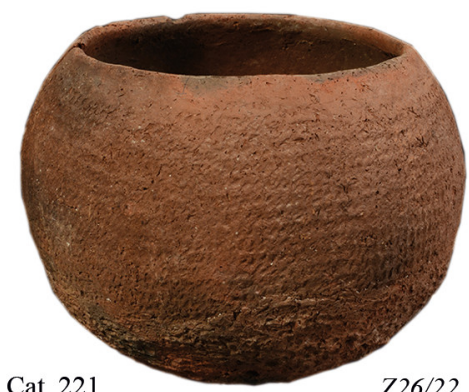

Cat. 221

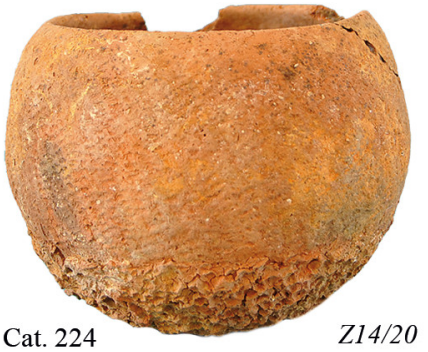

Cat. 224

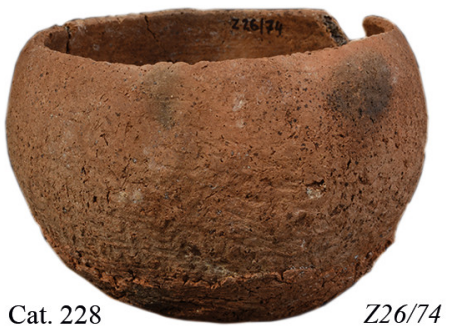

Cat. 228

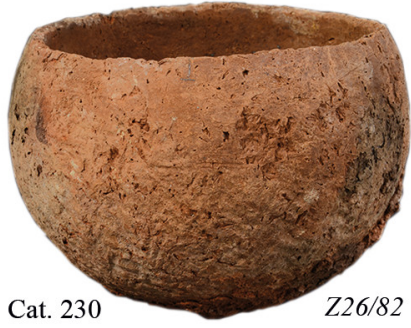

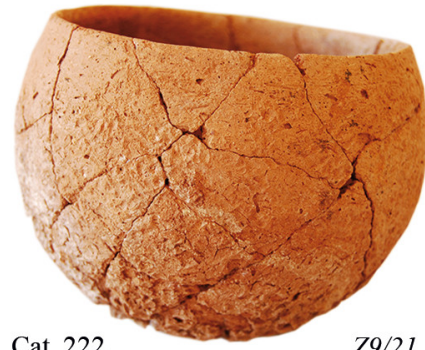

Z9/21
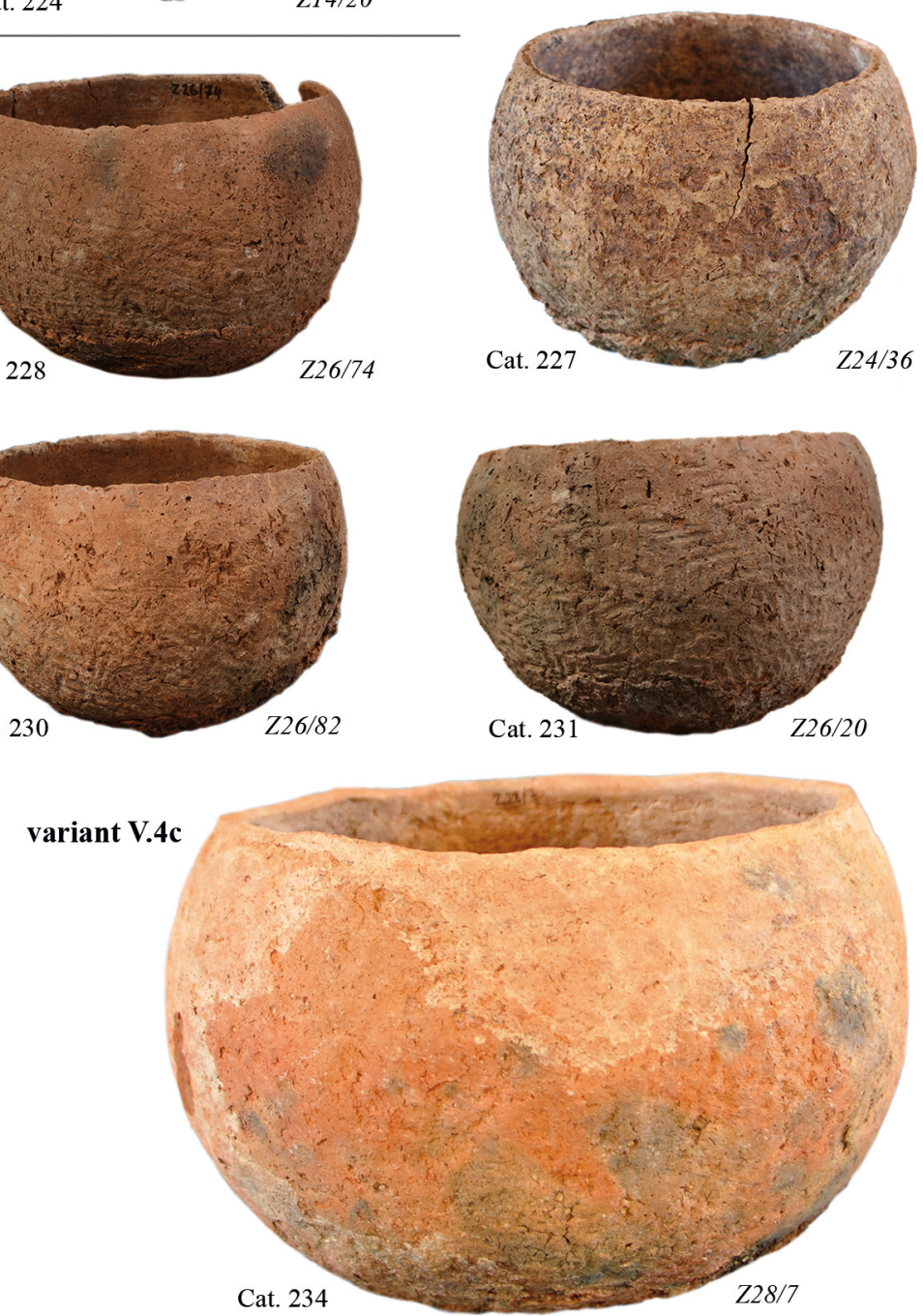

Cat. 234

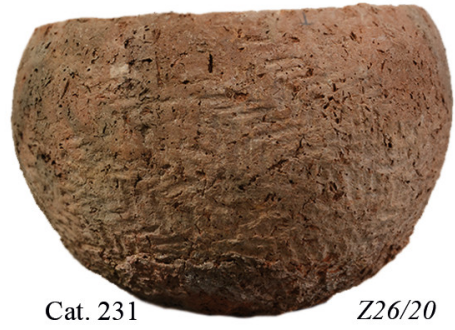

$5 \mathrm{~cm}$

FIGURE 7.110 Pots of types V.3 and V.4

Z26/16, Z26/22 PHOTOS BY A. KAMROWSKI; Z9/21 PHOTO BY E. CZYŻEWSKA-

ZALEWSKA; Z26/23 PHOTO BY A. KAMROWSKI; Z14/2O PHOTO BY T. WOJTCZAK;

Z26/17 Рното вY A. KAMROWSKI; Z24/36 Рното ву т. WOJTCZAK; Z26/74, Z26/59,

Z26/82, Z26/2O, Z26/48 PнотоS BY A. KAмRoWsKi; Z28/7 Рното ву т. WOJTCZAK 

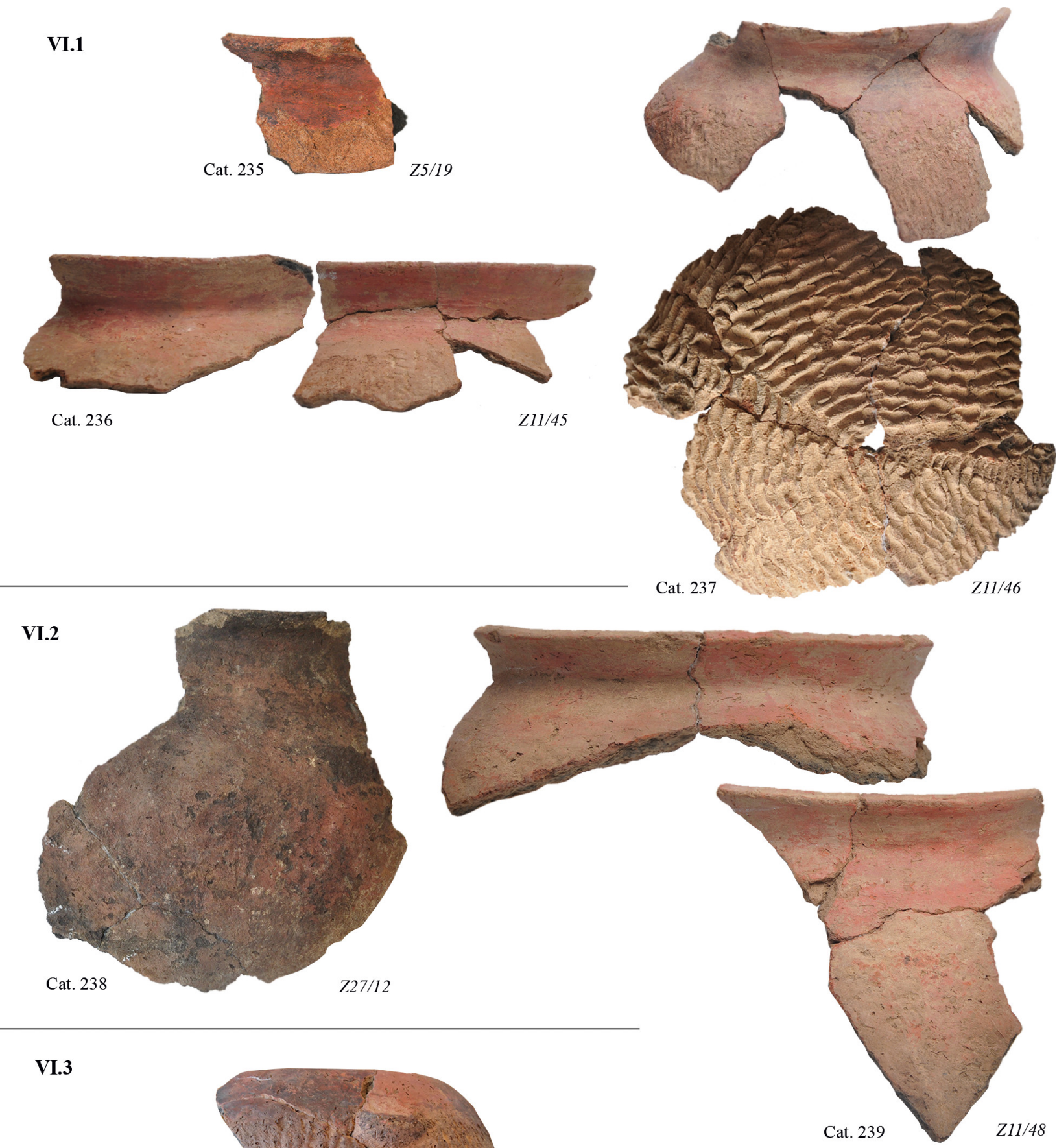

VI.3

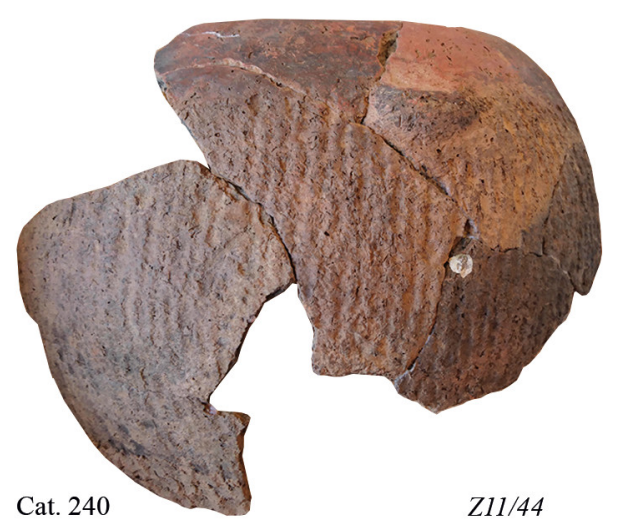

$Z 11 / 48$

FIGURE 7.111 Pots of type VI

Z5/19 РНОто ВY к. коLTEWSKI; Z11/45, Z11/46, Z27/12, Z11/48 РнотоS

BY A. KAMROWSKI; Z11/44 PнOTO BY E. CZYŻEWSKA-ZALEWSKA 


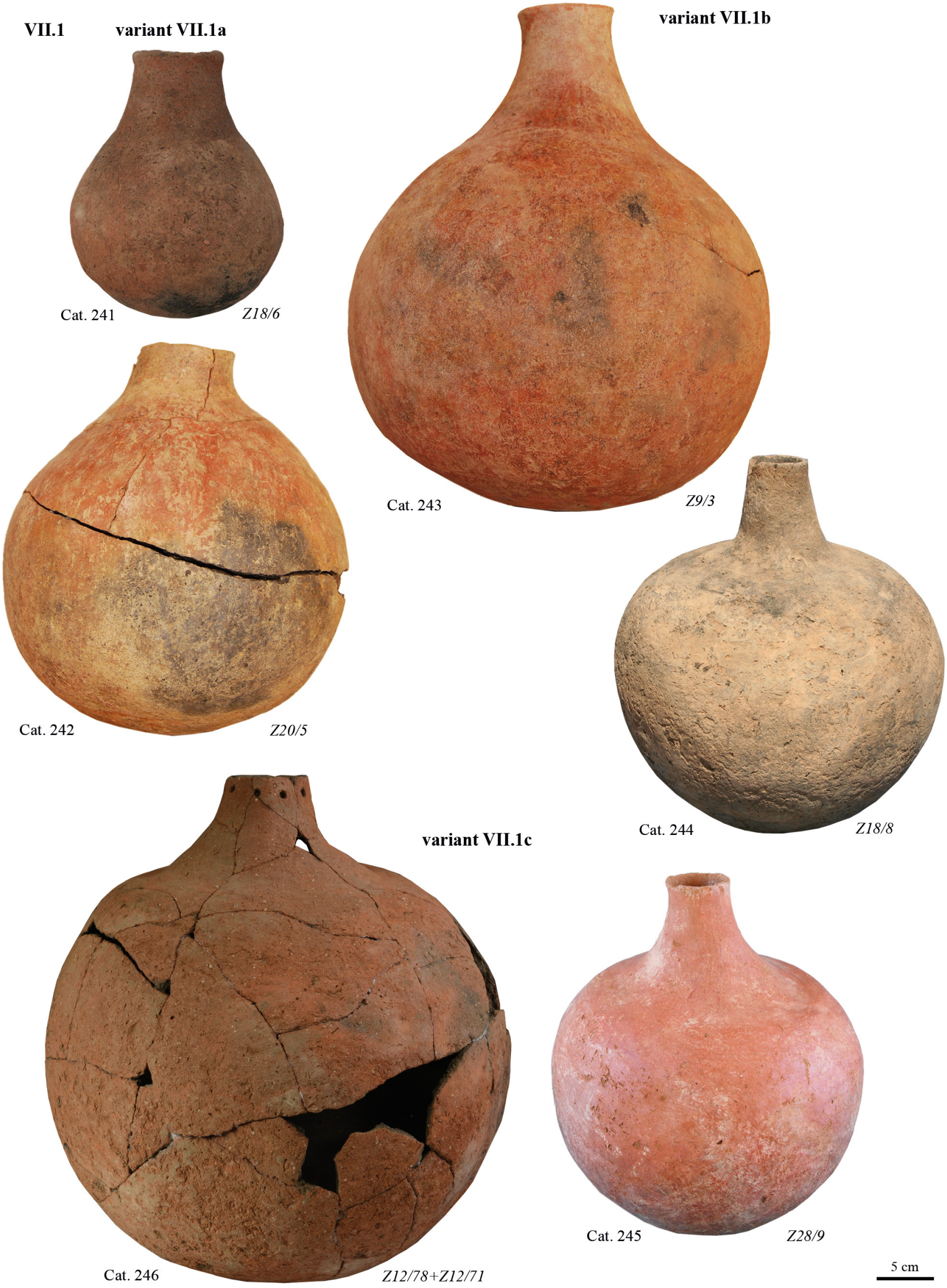

FIGURE 7.112 Jars of type VII.1

Z18/6 РНОTO BY K. KOTLEWSKI; Z2O/5, Z9/3 PHOTOS BY E. CZYŻEWSKA-ZALEWSKA; Z18/8 РНОтО ВY К. КотLEWSKI; Z12/78+Z12/71 РНОTO BY A. KAMROWSKI; Z28/9 Рното ву т. WOJTCZAK 


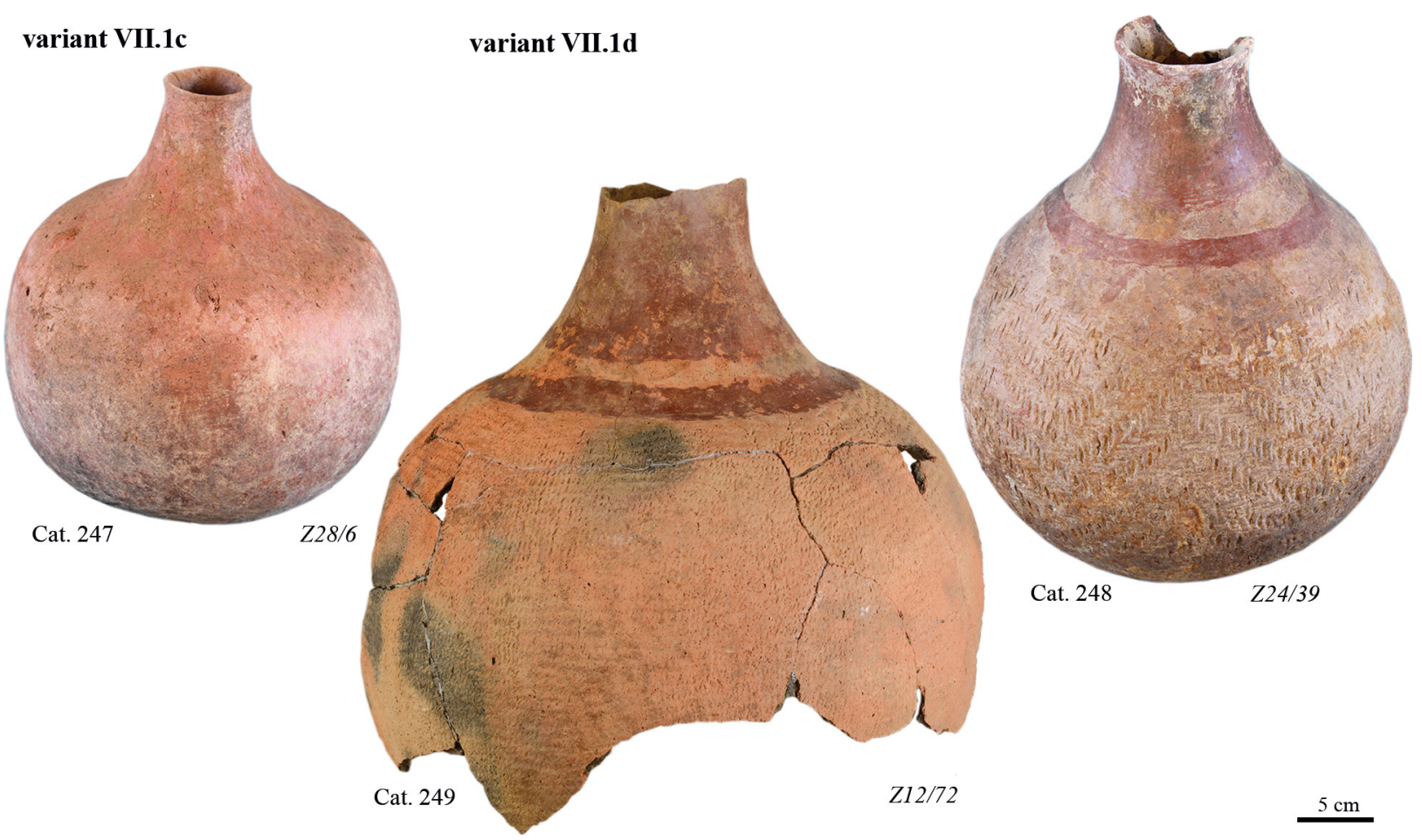

FIGURE $7.113 \quad$ Jars of type VII.1

Z28/6, Z24/39 РнотоS BY T. WOJTCZAK; Z12/72 Рното BY A. KAMROWSKI

VII.2

variant VII.2a

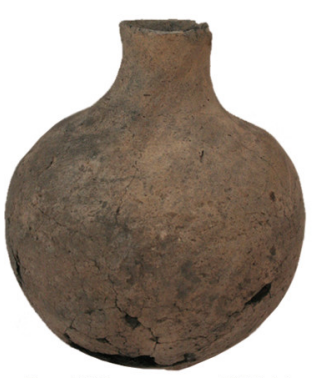

Cat. 250

$218 / 7$

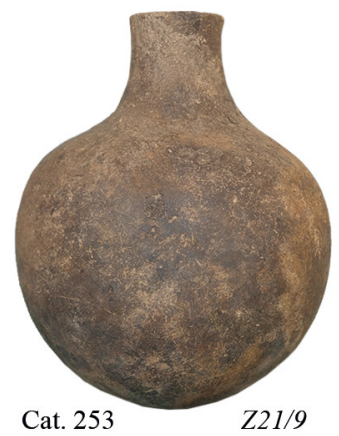

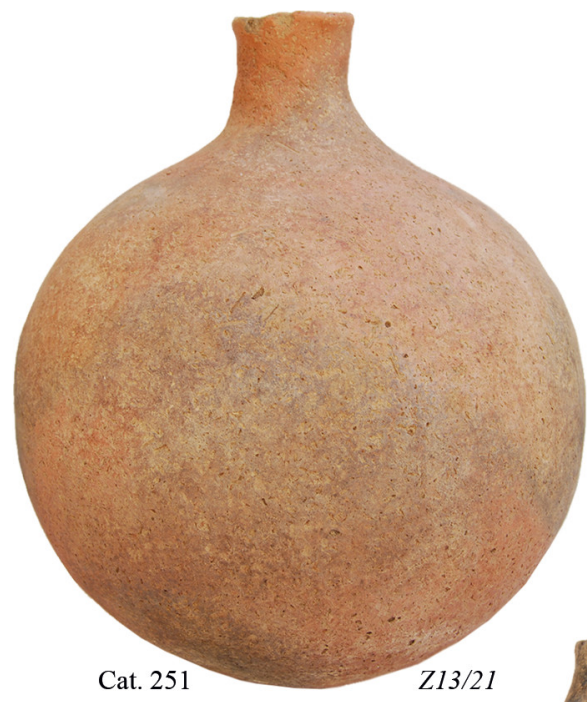

Cat. 251

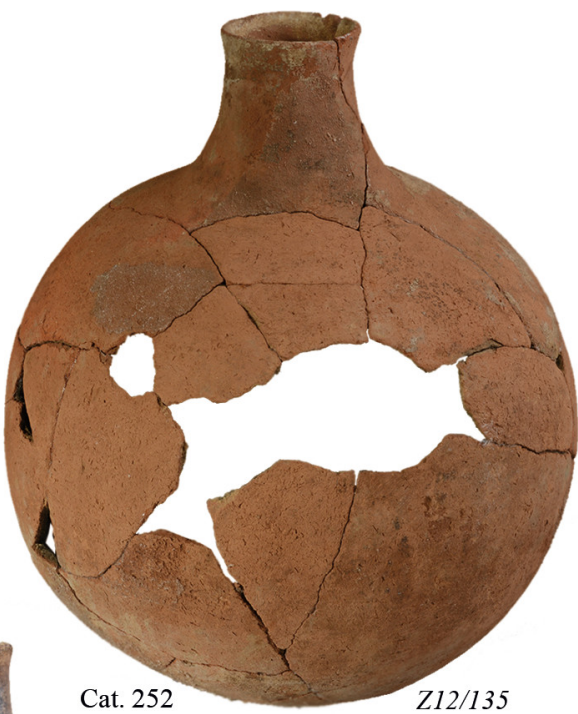

Cat. 252

FIGURE 7.114 Jars of type VII.2

Z18/7 РНОTO BY K. KOTLEWSKI; Z13/21 РНОTO BY O. ВIAŁOSTOCKA; Z12/135 РНОто

BY A. KAMROWSKI; Z21/9 PHOTO BY R. MAHLER; Z17/2 PHOTO BY O. BIAŁOSTOCKA 


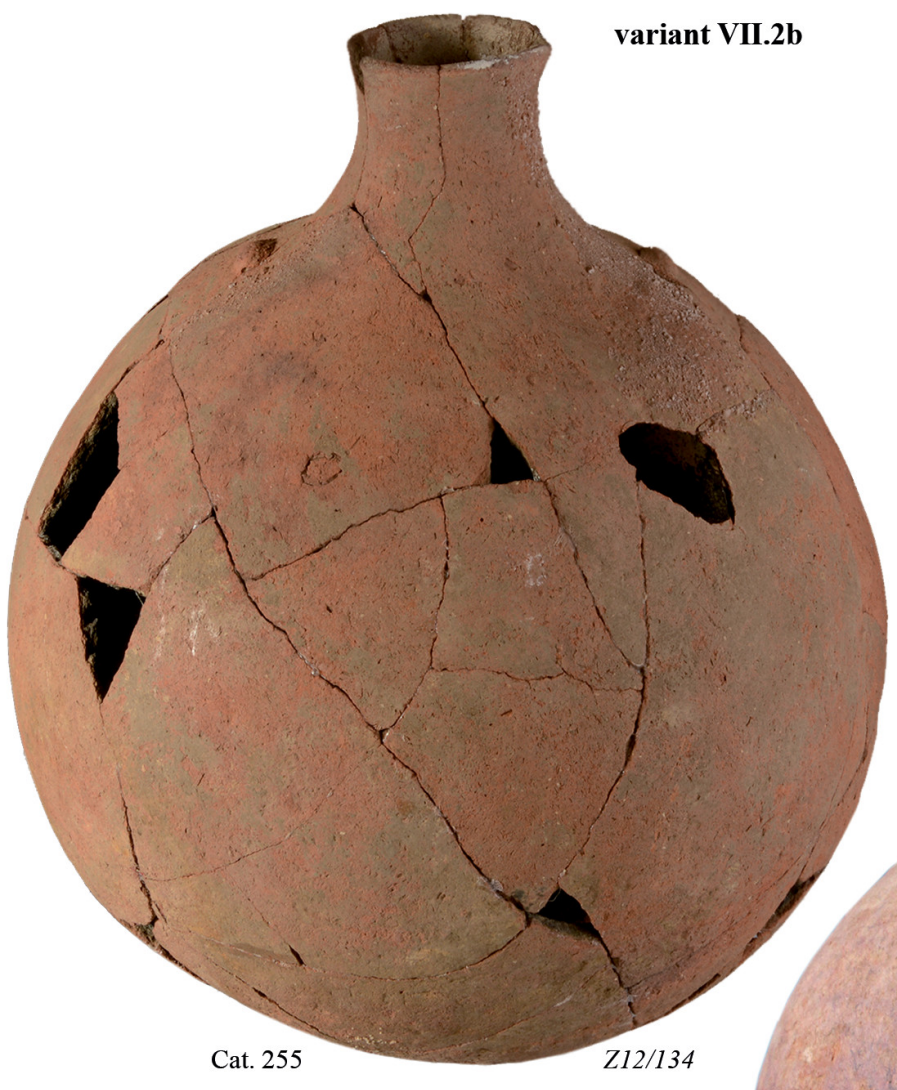

\section{variant VII.2c}

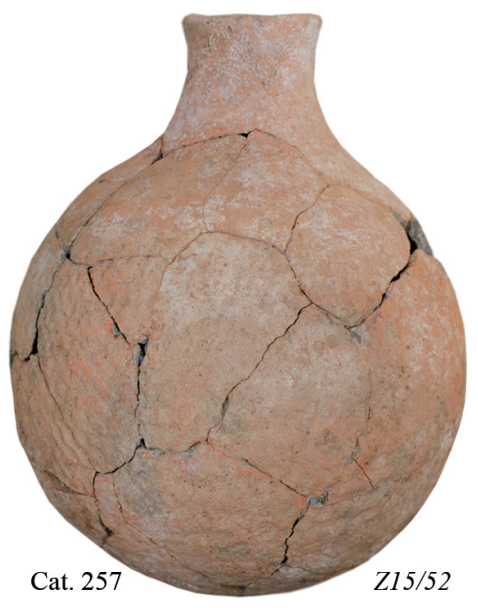

Cat. 256

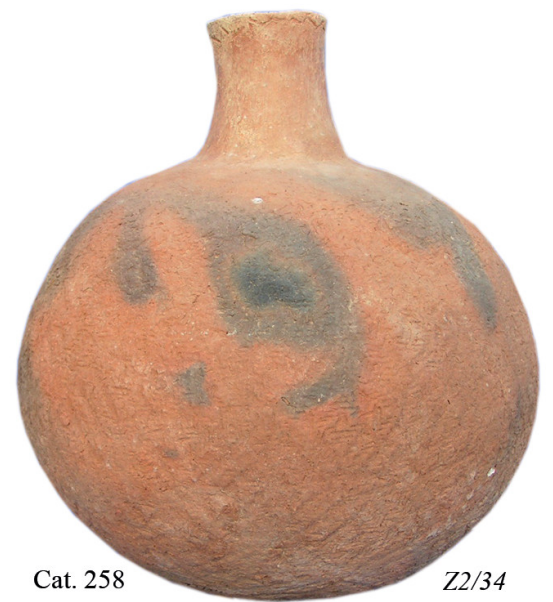

$Z 24 / 40$

FIGURE 7.115 Jars of type VII.2

Z12/134 PHOTO BY A. KAMROWSKI; Z24/40 РНОTO вY T. WOJTCZAK; Z15/52 РНОTO BY E. SKOWROŃSKA; Z2/34 PHOTO BY K. KOTLEWSKI 
VII.3
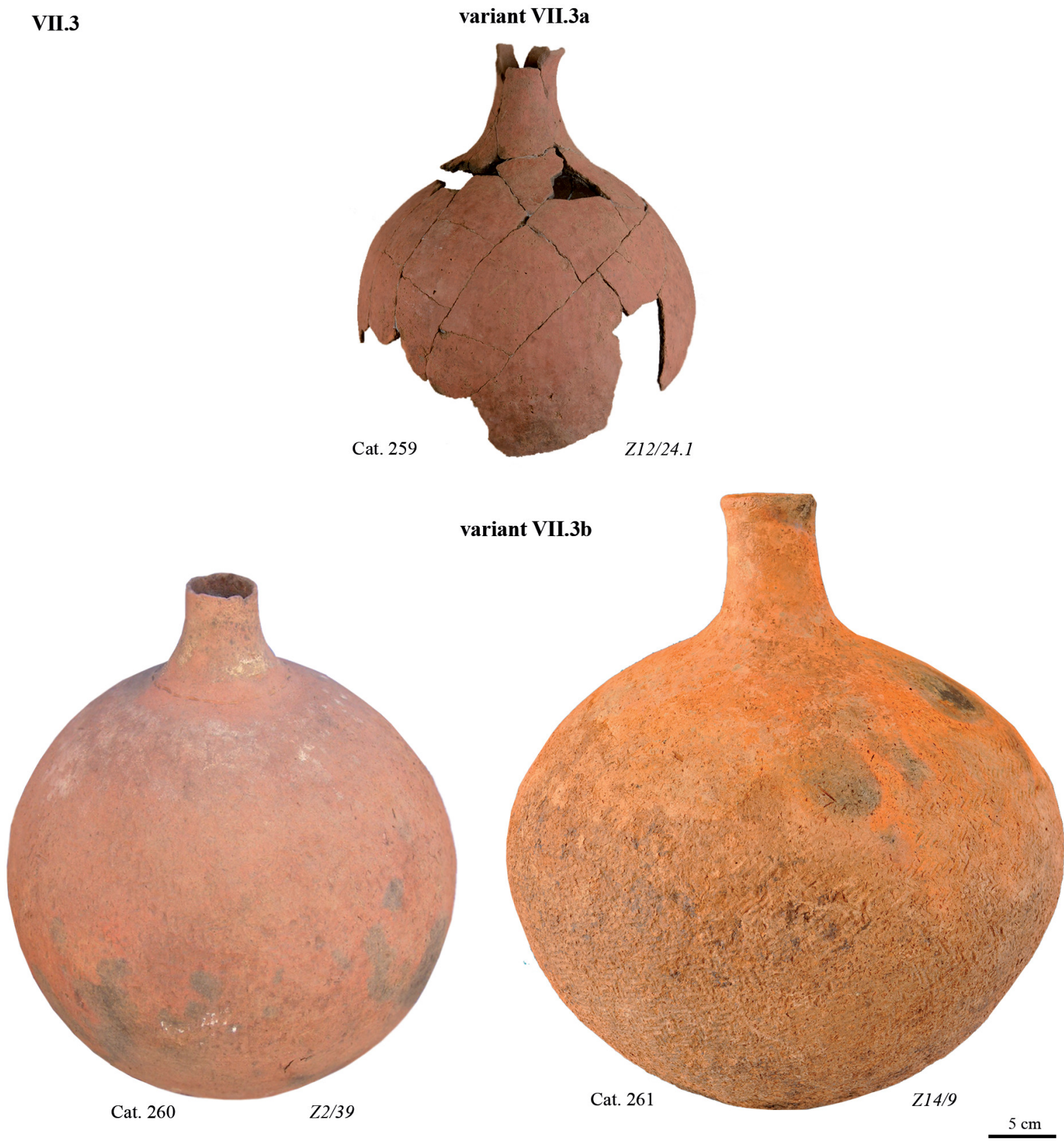

FIGURE 7.116 Jars of type VII.3

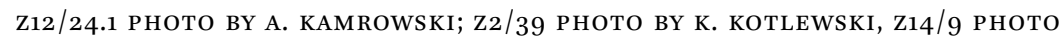
BY T. WOJTCZAK 
VII.4
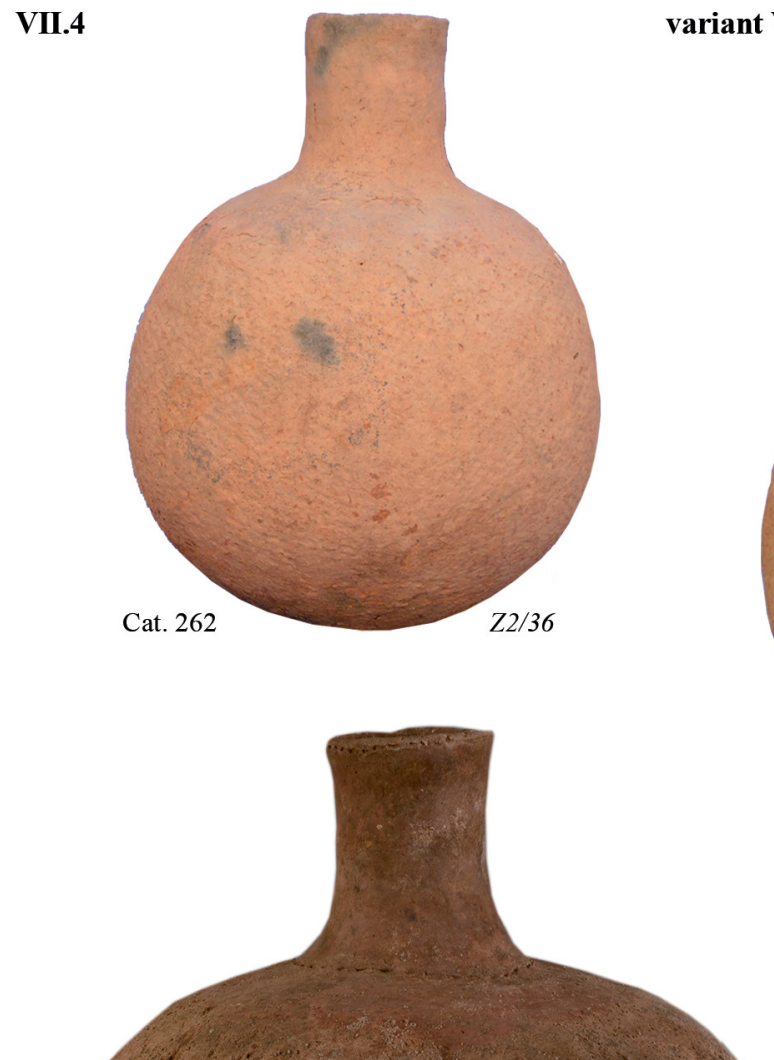

variant VII.4a
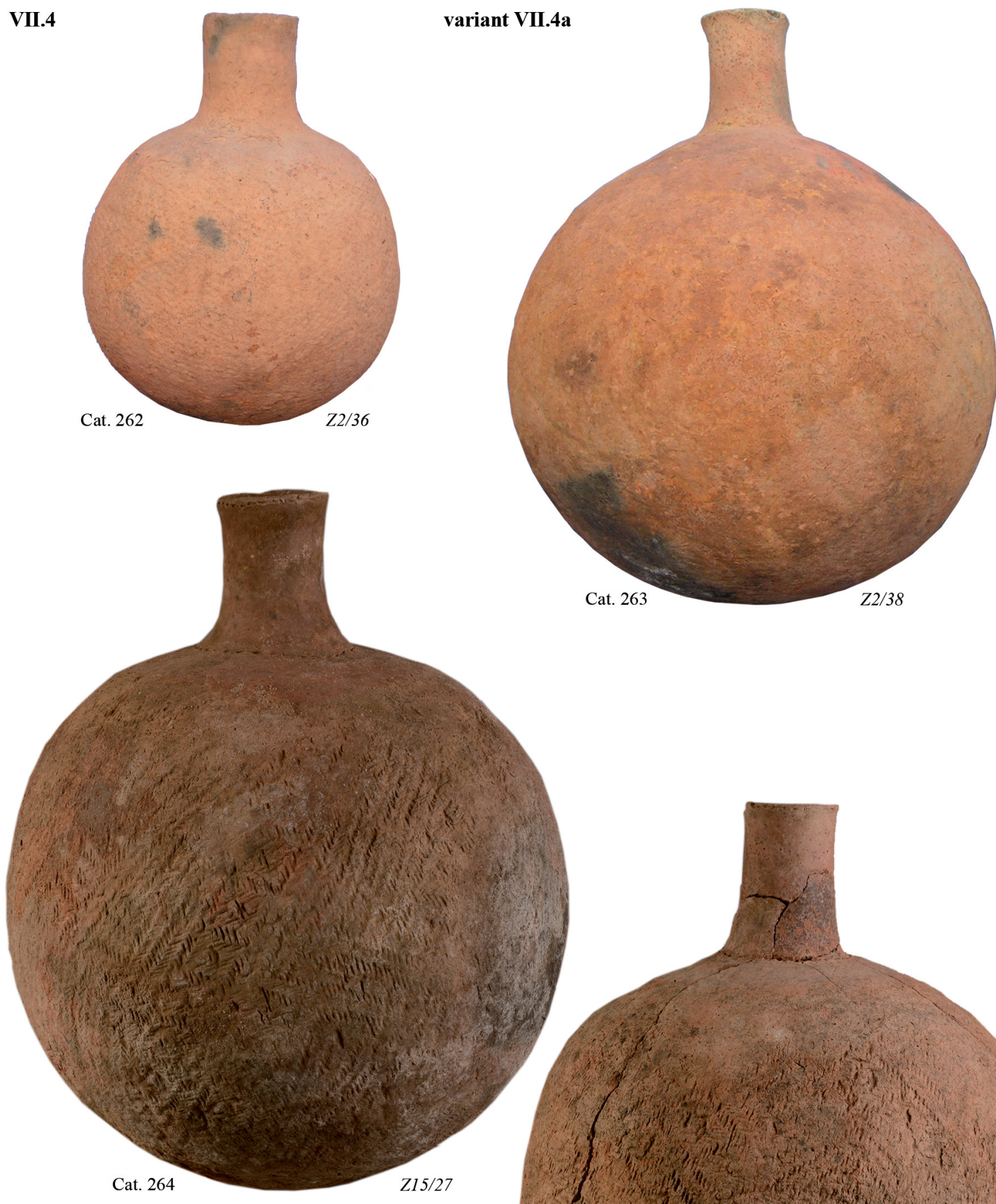

$5 \mathrm{~cm}$

FIGURE 7.117 Bottles of type VII.4

Z2/36, Z2/38 РнотоS вY K. коTLEWSKI; Z15/27, Z15/59 РнотOS BY A. KAMROWSKI 


\section{variant VII.4b}
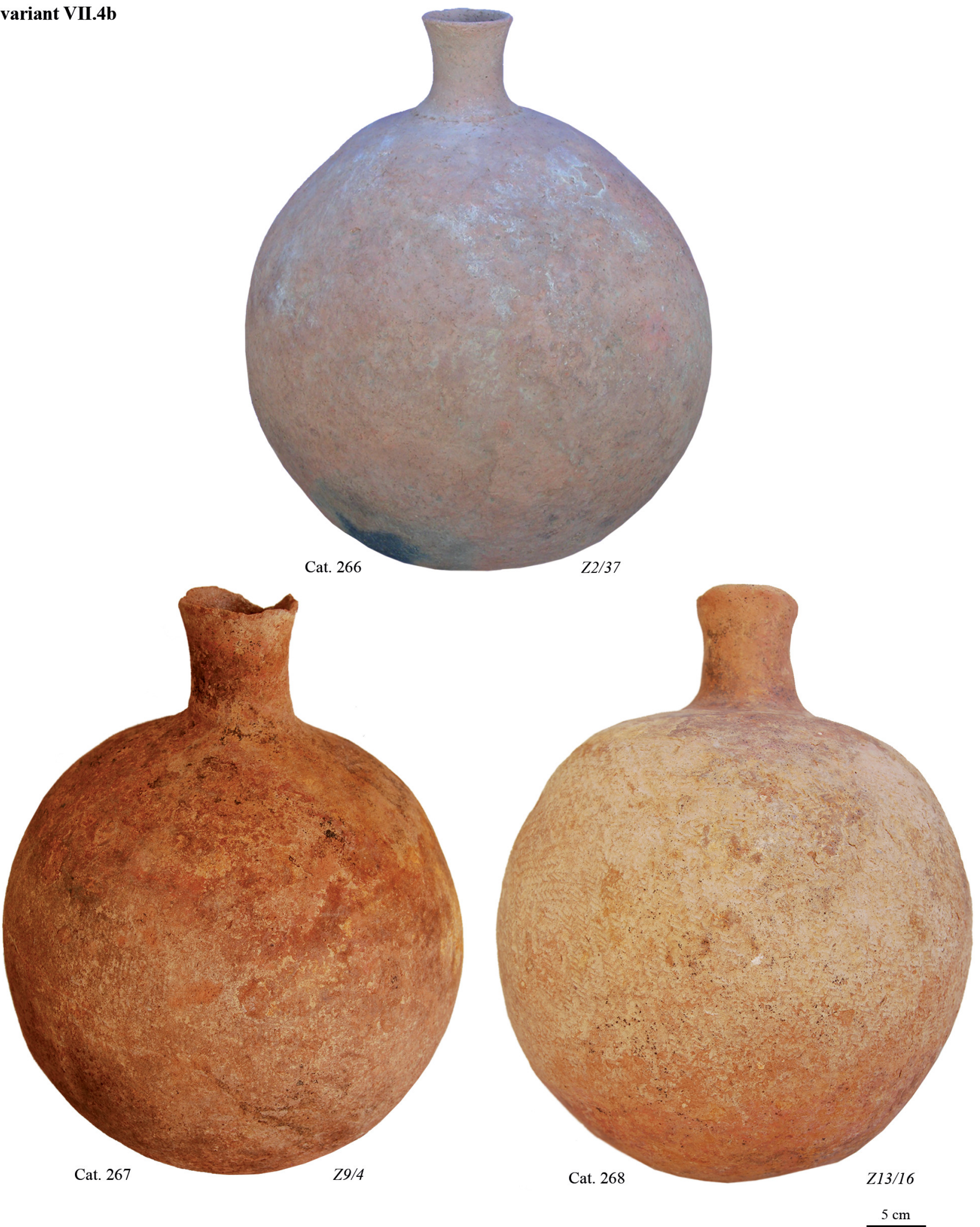

FIGURE 7.118 Bottles of type VII.4

Z2/37 РНОто вY К. КотLEWSKI; Z9/4 РНОто вY E. CZYŻEWSKA-ZALEWSKA; Z13/16 РНОТО BY О. BIAŁOSTOCKA 


\section{VII.5}

\section{variant VII.5a}

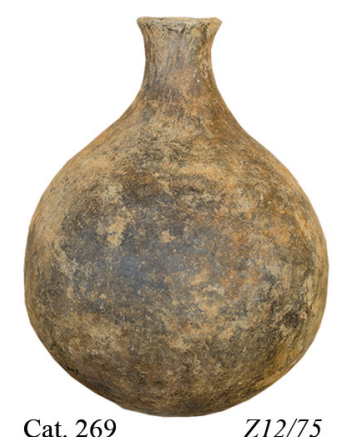

\section{variant VII.5b}

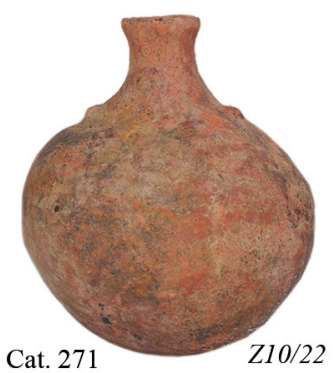

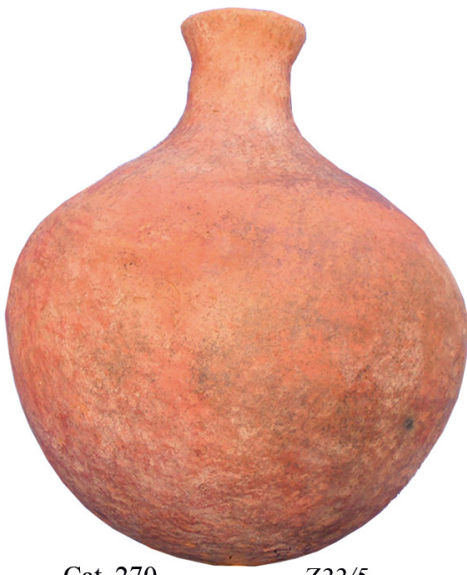

Cat. $270 \quad Z 22 / 5$

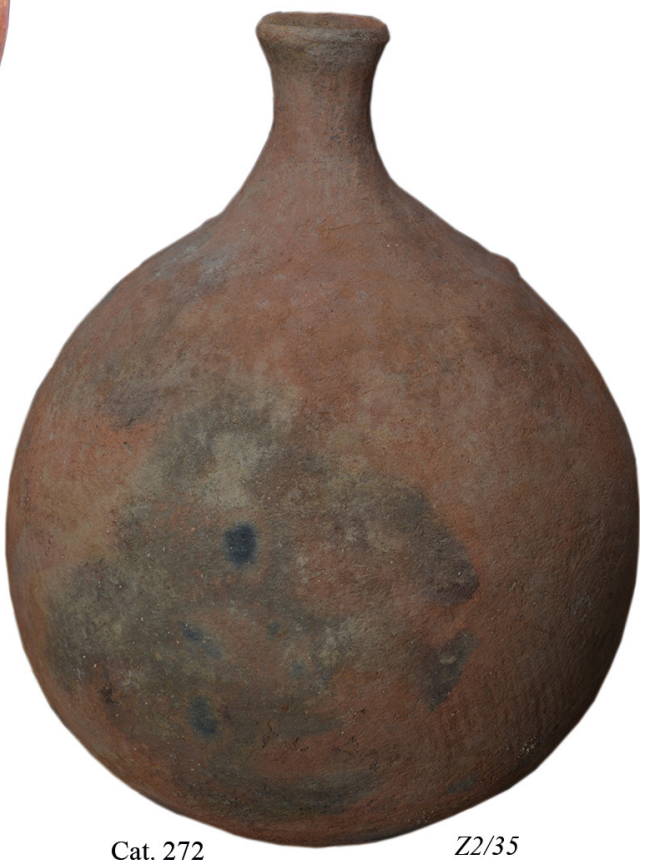

variant VII.5c

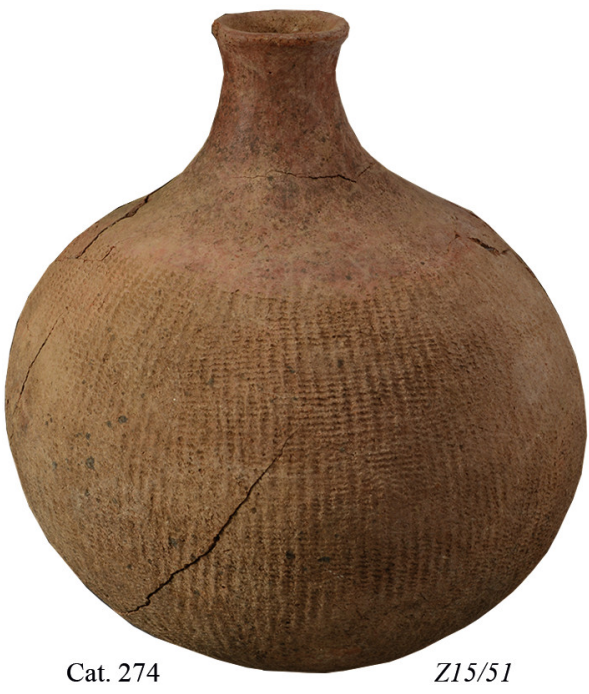

$5 \mathrm{~cm}$

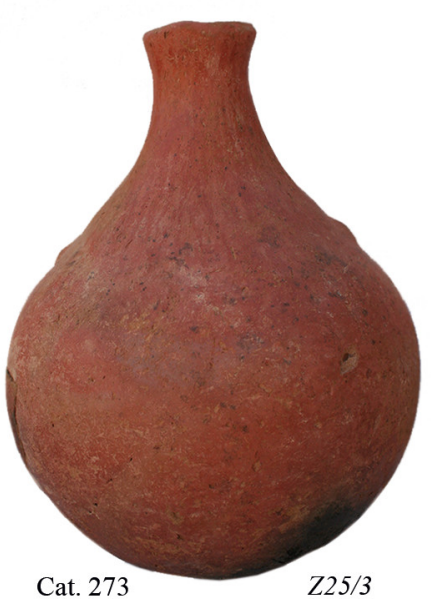

Cat. 272

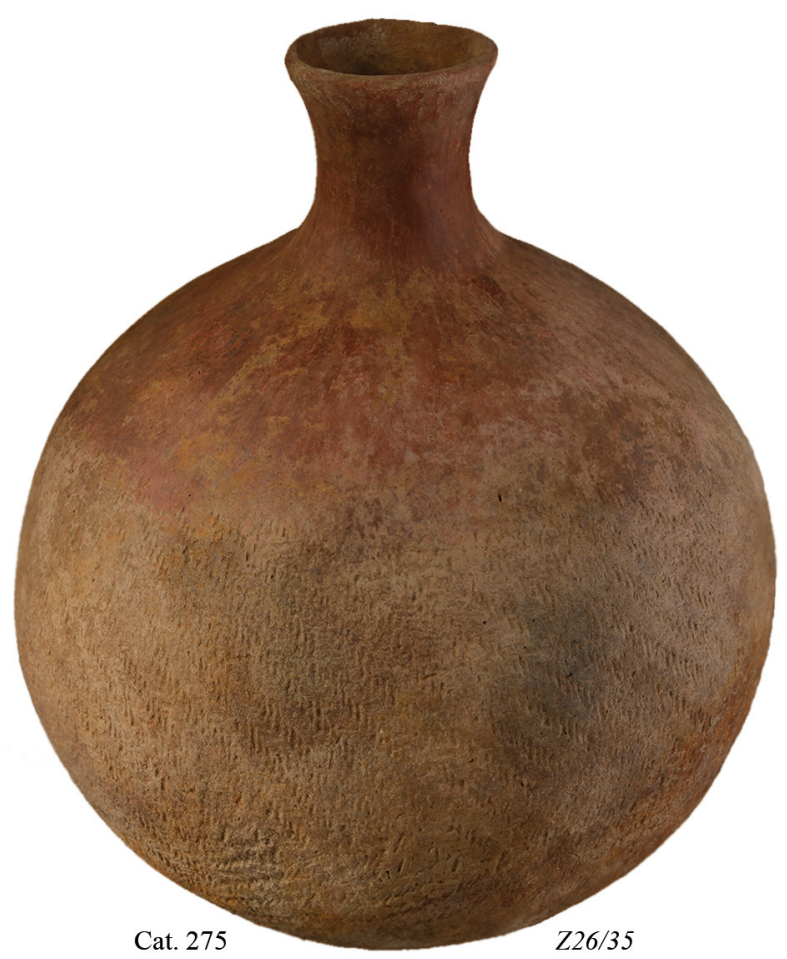

Cat. 275

FIGURE 7.119 Bottles of type VII.5

Z12/75 РнОтO BY E. SKOWROŃSKA; Z22/5, Z10/22, Z25/3 РНОTOS BY K. KOTLEWSKI;

Z2/35, Z15/51, Z26/35 РнотоS ву А. КАMROWSKI 


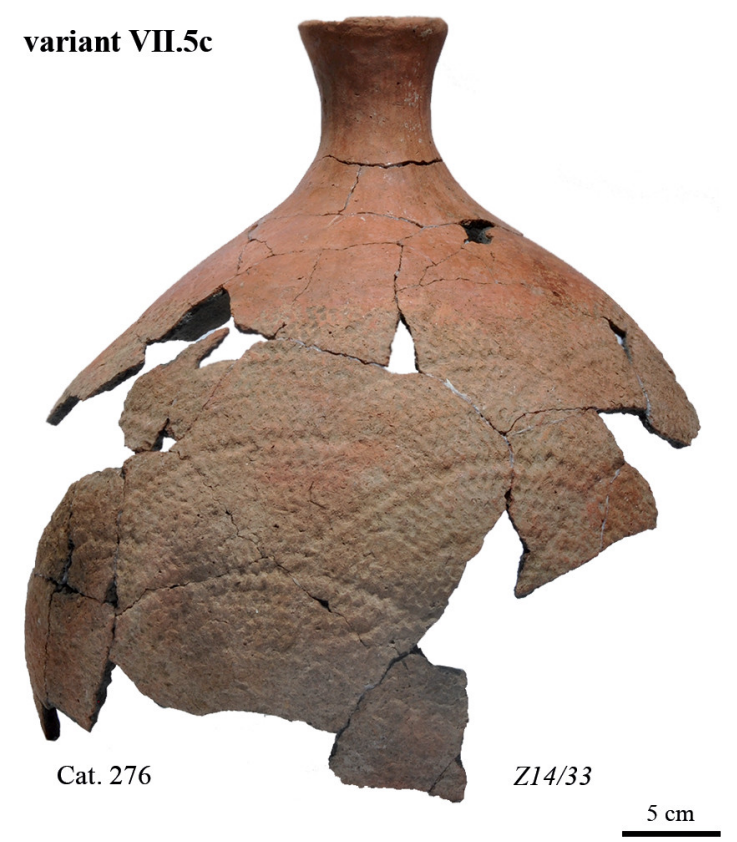

FIGURE 7.120 Bottles of type VII.5

Z14/33 РНОто ВY А. KАMROWSKI

VII.6

variant VII.6a

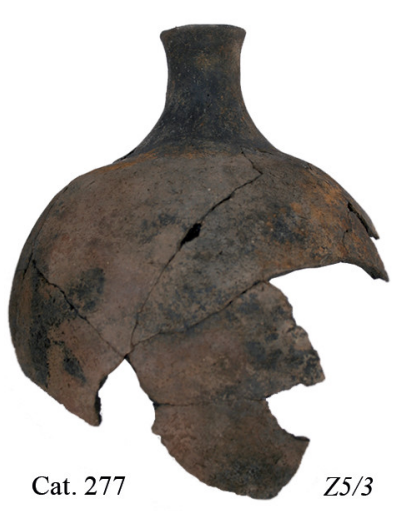

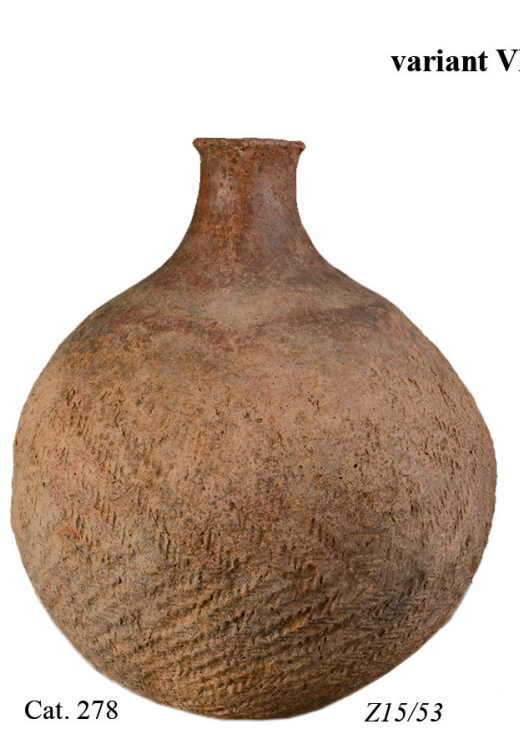

Z15/53

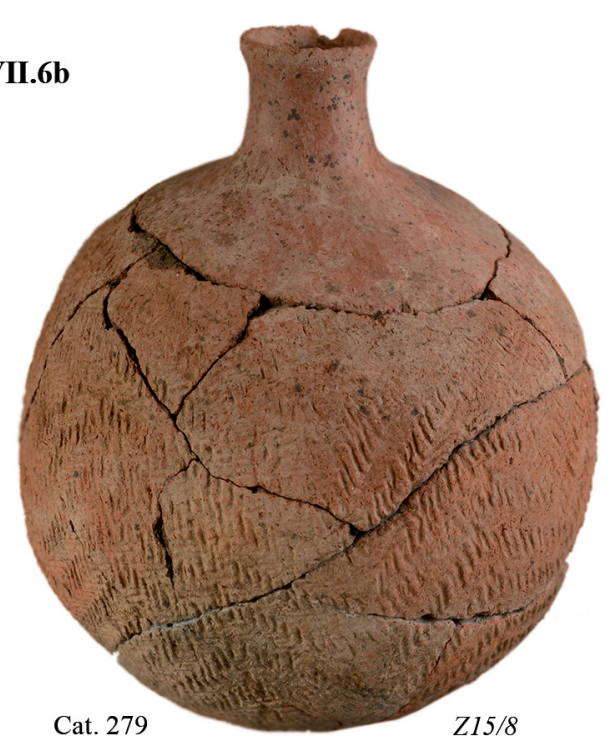

$5 \mathrm{~cm}$

FIGURE 7.121 Bottles of type VII.6

z5/3 РнОто вY к. коTLEWSKI; z15/53, z15/8 РнOTOS BY A. KAMROWSKI 


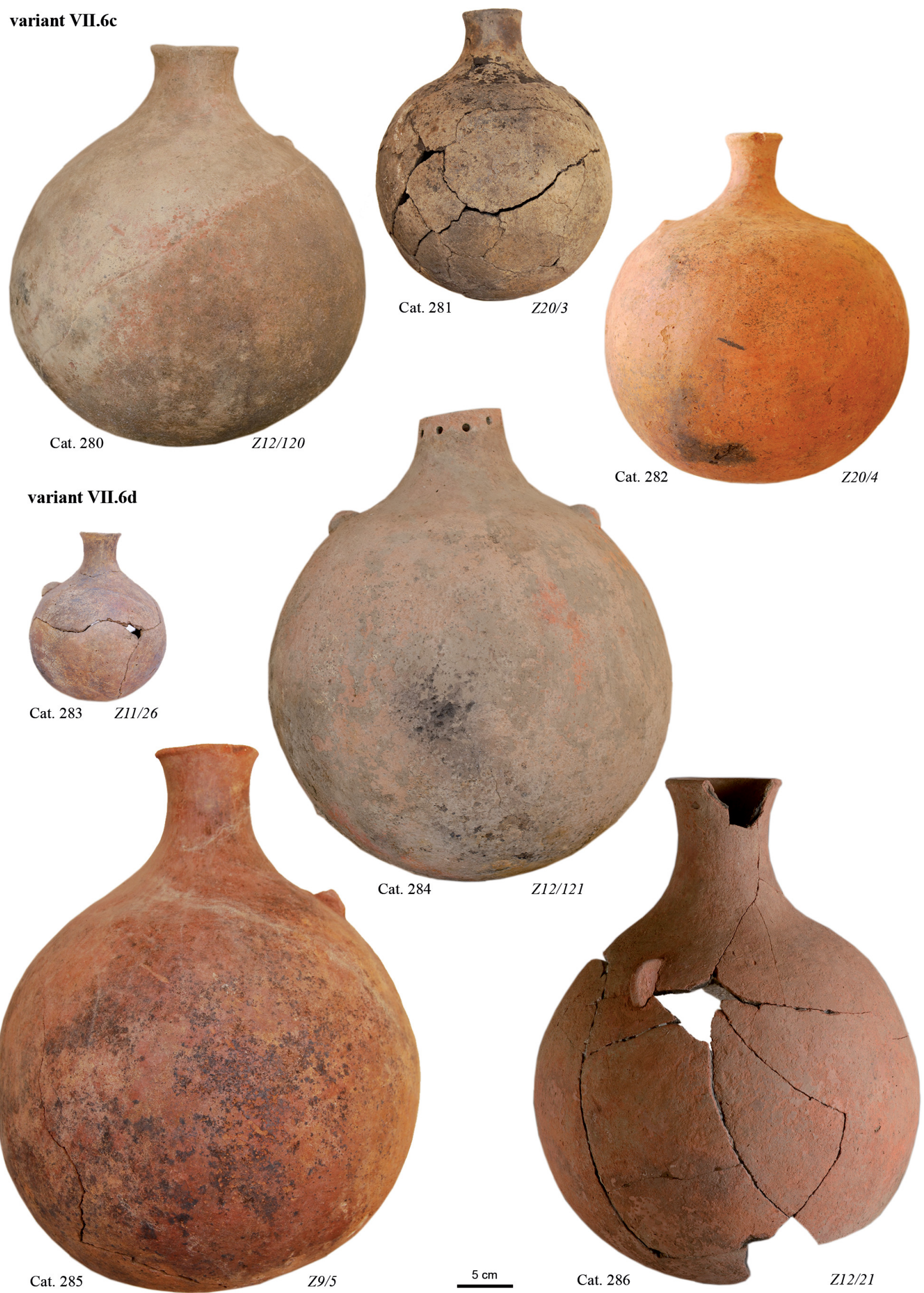

FIGURE 7.122 Bottles of type VII.6

Z12/12O PHOTO BY E. SKOWROŃSKA; Z2O/3, Z2O/4 PHOTOS BY E. CZYŻEWSKA-

ZALEWSKA; Z11/26 PHOTO BY O. BIAŁOSTOCKA; Z12/121 PHOTO BY E. SKOWROŃSKA;

Z9/5 РНОTO BY E. CZYŻEWSKA-ZALEWSKA; Z12/21 РНОTO BY A. KAMROWSKI 


\section{Jars varia}
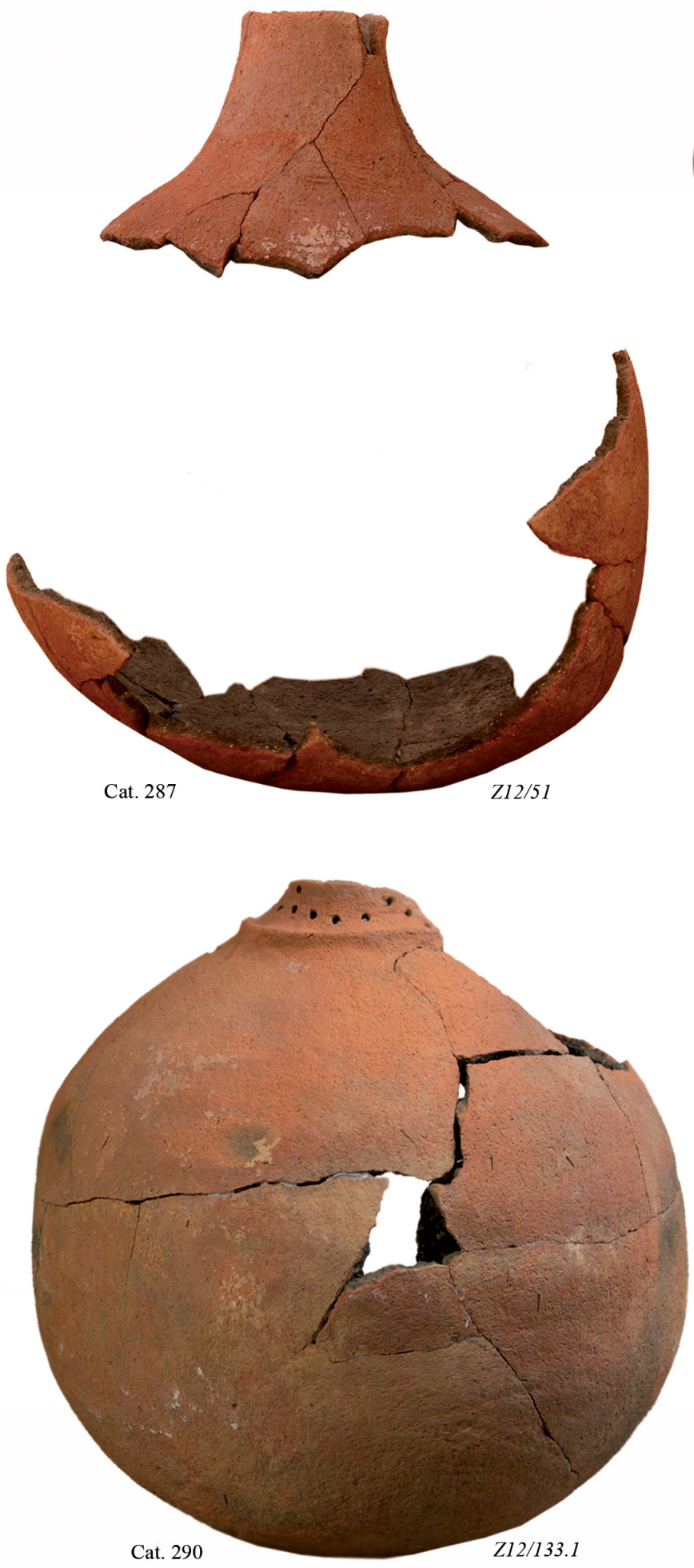

$5 \mathrm{~cm}$
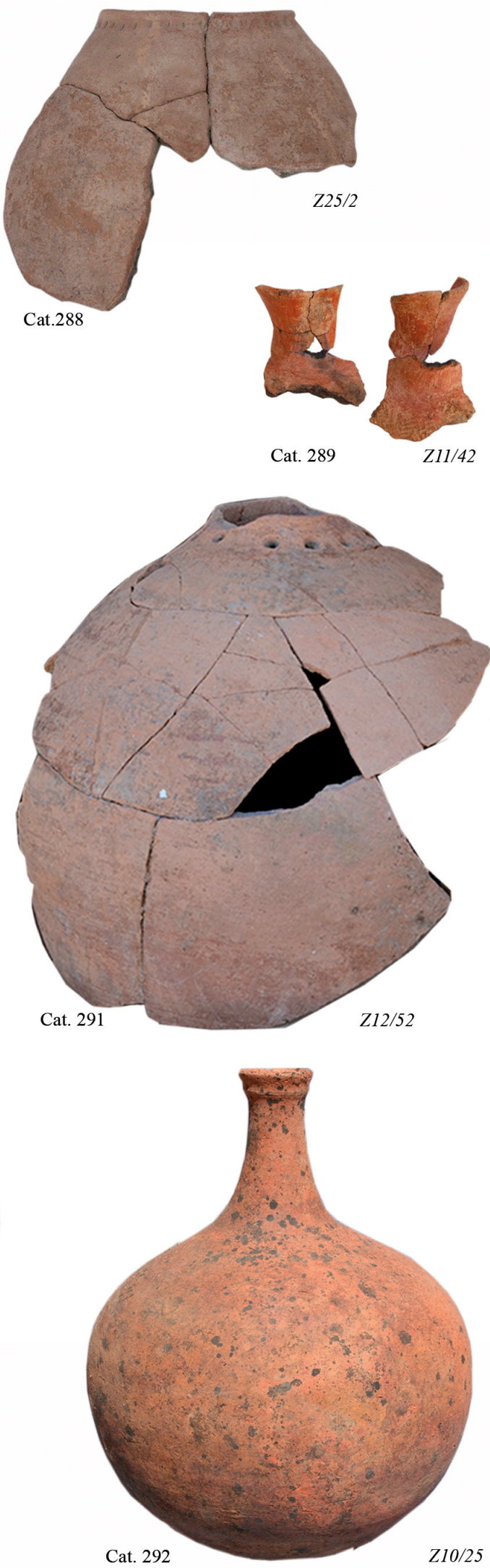

FIGURE 7.123 Jars of different types

Z12/51 РНОTO BY Z. KOWARSKA; Z25/2 РНОTO вY К. КOTLEWSKI; Z11/42 РНОTO

BY О. ВIAŁOSTOCKA; Z12/133.1 РНОTO ВY А. КАMROWSKI; Z12/52 РНОTO ВY

Z. KOWARSKA; Z1O/25 РНОTO BY K. KOTLEWSKI 

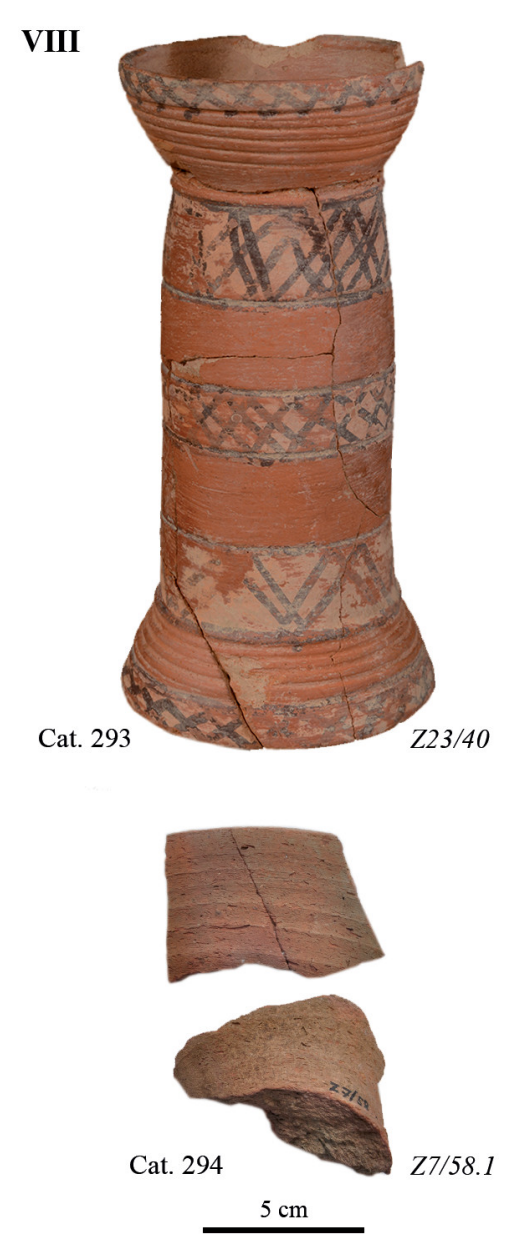

FIGURE 7.124

Stands of type IX

Z23/40, Z7/58.1 РнотоS BY

A. KAMROWSKI
IX.1

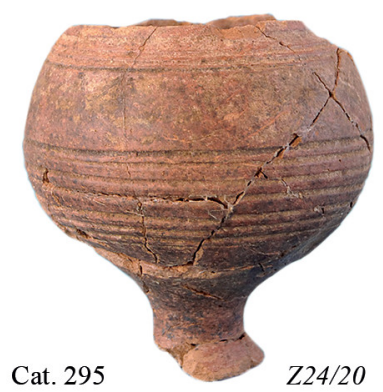

IX.2

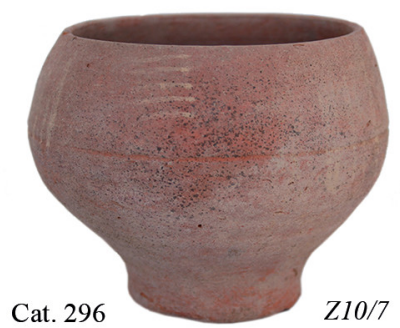

IX.3

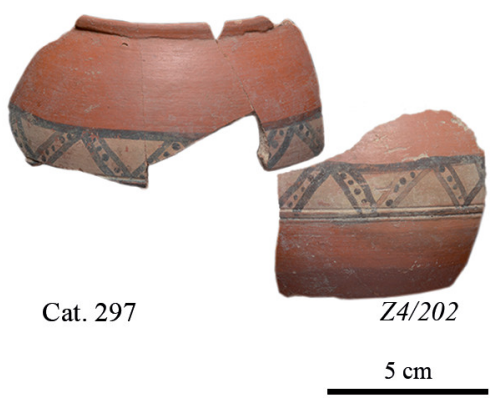

FIGURE 7.125

Goblets of types IX.1, IX.2 and IX.3

Z24/2O PнОто вY т. WOJTCZAK; Z1O/7 Рното BY К. KOTLEWSKI; Z4/2O2 РНОTO BY

A. KAMROWSKI

$\mathbf{x}$
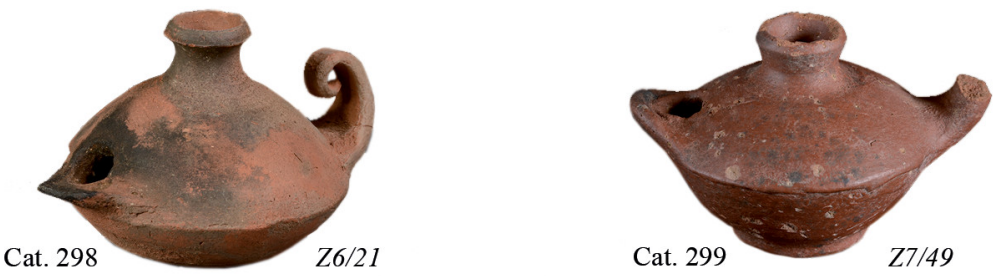

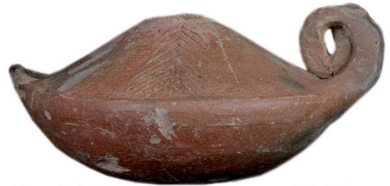

Cat. 300

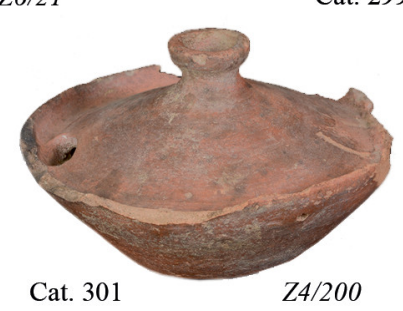

Cat. 301

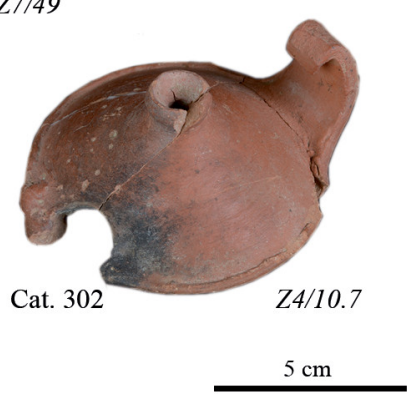

FIGURE 7.126 Oil lamps

z6/21, Z7/49, Z4/61, Z4/10.7, Z4/20о РнотоS вY A. KAMROWSKI 
XI

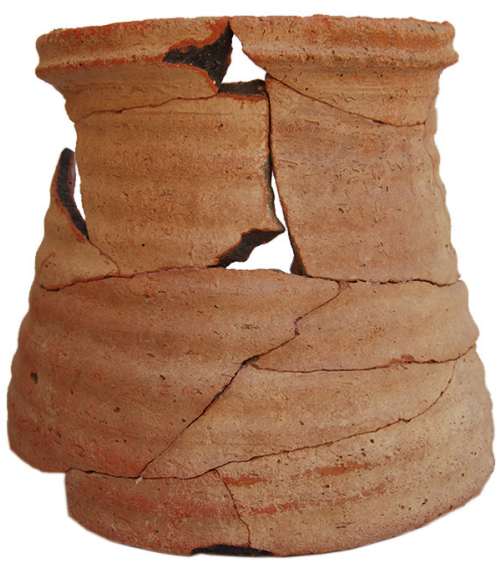

Cat. 303
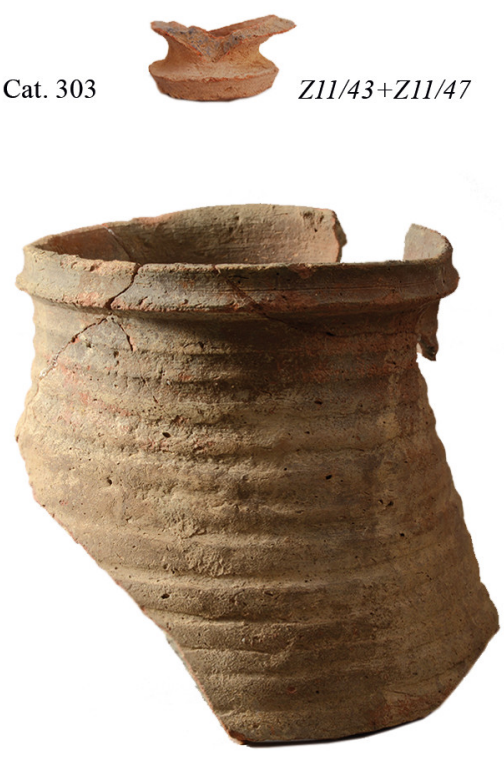

Cat. 306
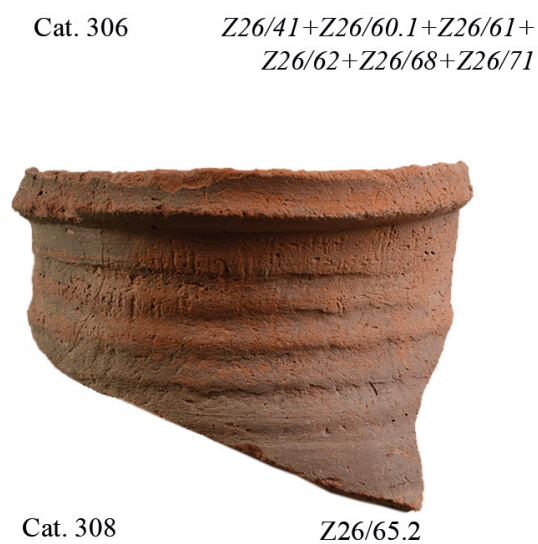

Cat. 308
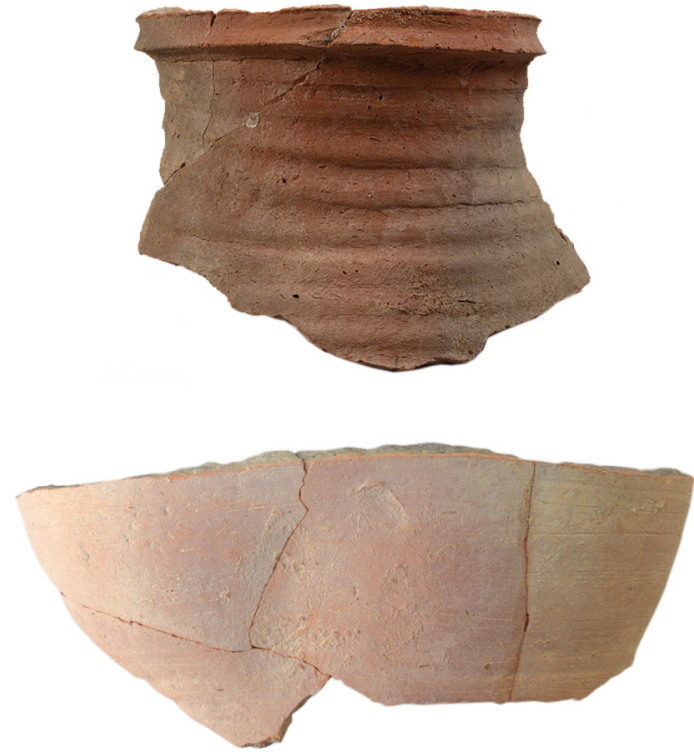

Cat. 305

$Z 26 / 60.2,3+Z 26 / 62+Z 26 / 63.3+$ $Z 26 / 64+Z 26 / 67+Z 26 / 69$
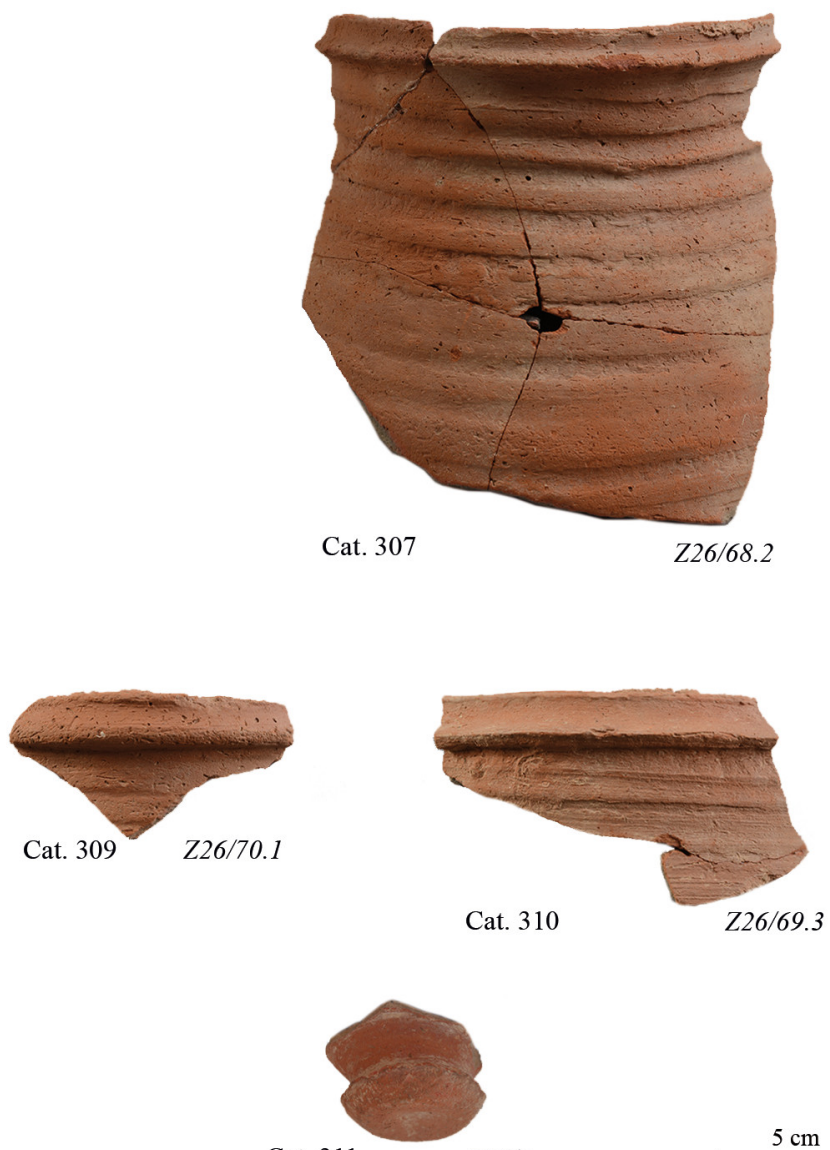

Cat. 311

$5 \mathrm{~cm}$

FIGURE 7.127 Qawadis of type XI

Z11/43+Z11/47 РнОто вY о. вІАєOSTоскA; z6/19.1+Z6/19.2+Z6/38.1;

$\mathrm{Z} 26 / 6$ o.2,3+Z26/62+Z26/63.3+Z26/64+Z26/69+Z26/67; Z26/41+Z26/6o.1+Z26/61+

Z26/62+Z26/68+Z26/71; z26/68.2, z26/65.2, z26/70.1, z26/69.3 РнотоS ву

A. KAMROWSKI; Z25/1 PHOTO BY K. KOTLEWSKI 

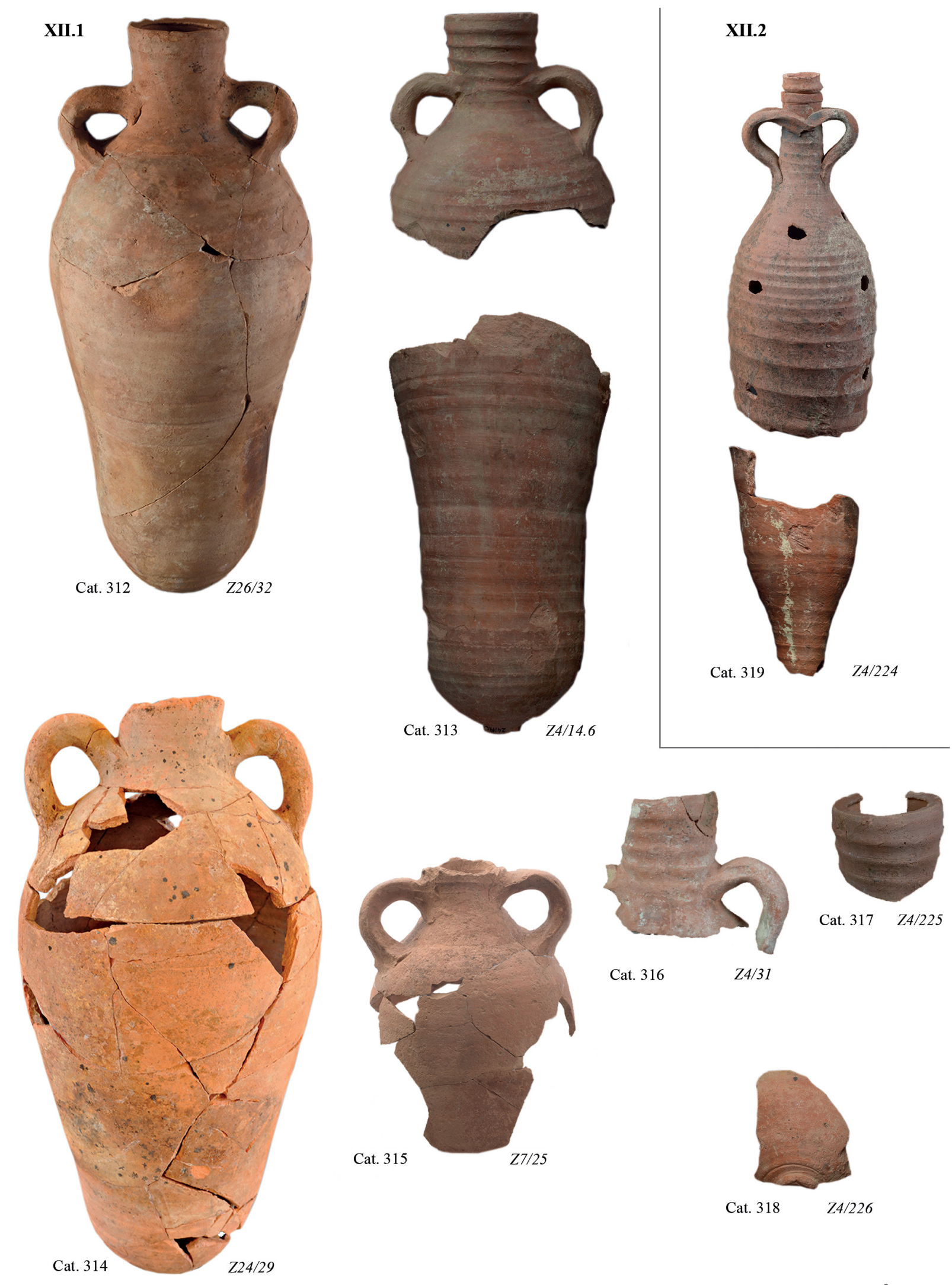

Cat. $317 \quad Z 4 / 225$

Cat. 316

$$
Z 4 / 31
$$

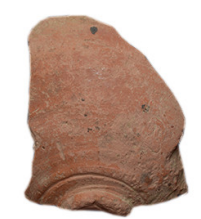

Cat. 318

$74 / 226$

FIGURE 7.128 Amphorae of types XII.1 and XII.2

Z26/32, Z4/14.6 рнотоs ву А. кAмRowski; Z24/29 Рното ву т. WоJтCZAK; Z7/25,

$\mathrm{Z} 4 / 31, \mathrm{Z} 4 / 225, \mathrm{Z} 4 / 226, \mathrm{Z} 4 / 224$ PнотоS BY A. KAMROWSKI 


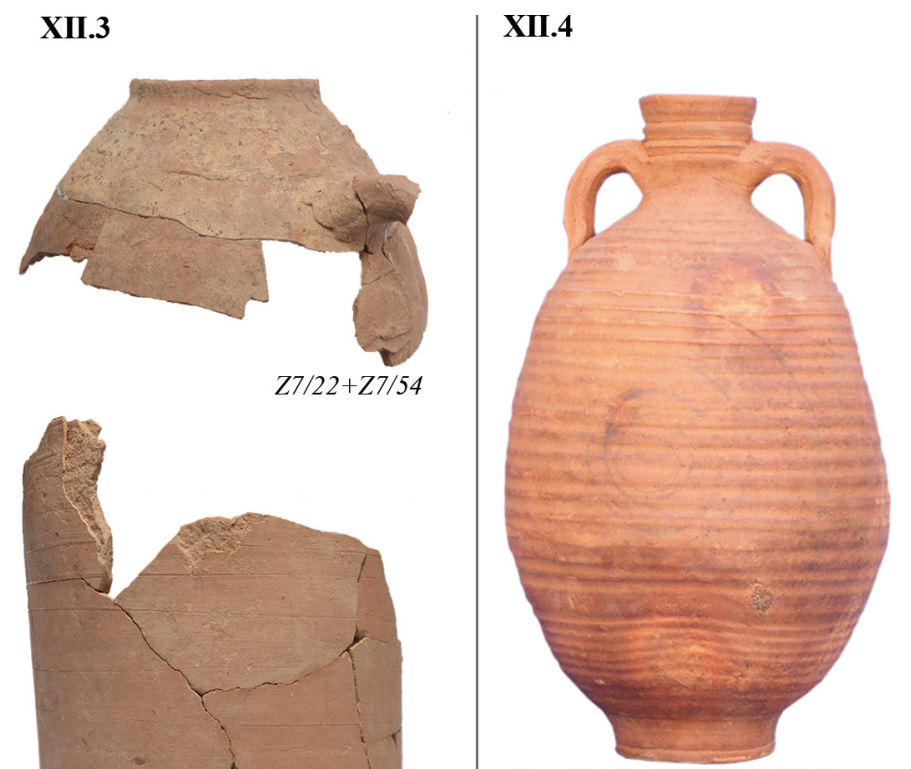

Cat. 322

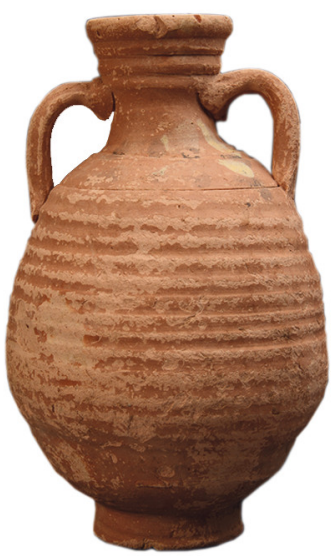

Cat. 323

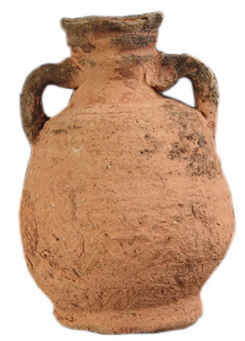

Cat. $324 \quad Z 15 / 56$

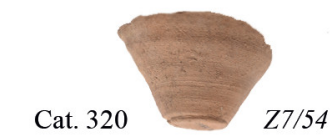

Cat. 321

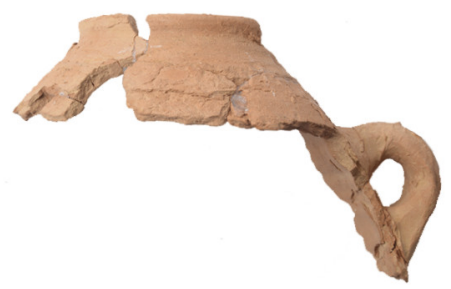

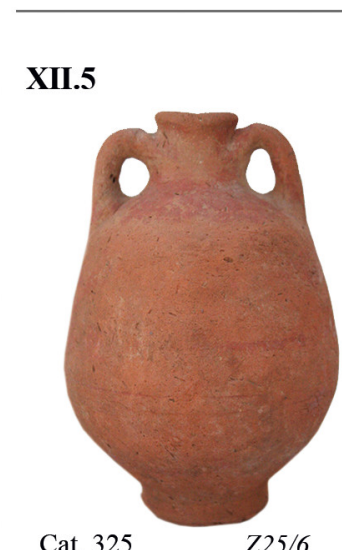

Cat. 325

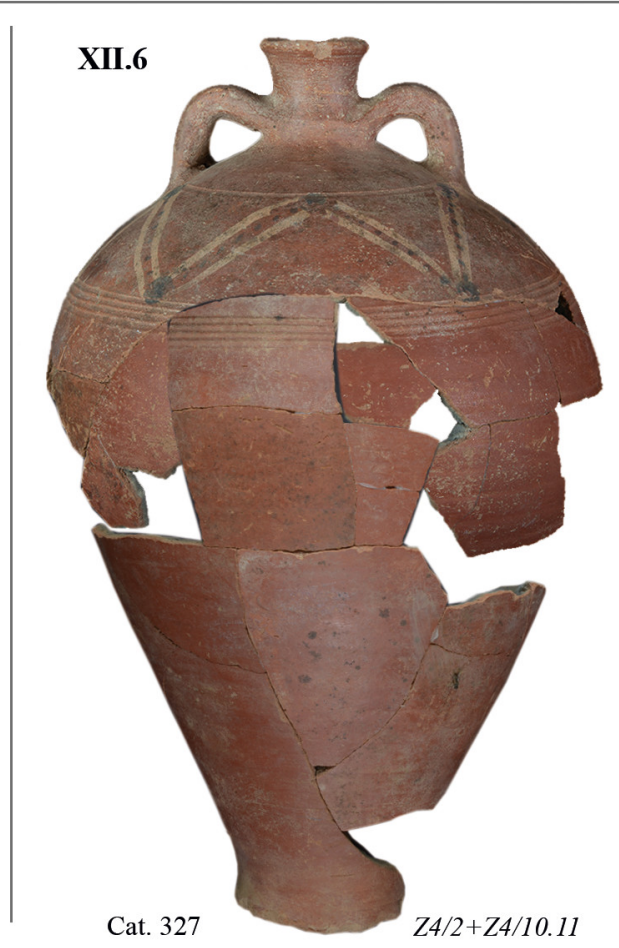

Cat. 326

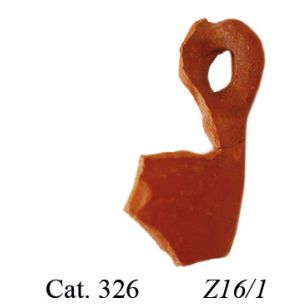

Cat. 32

\section{Amphorae varia}
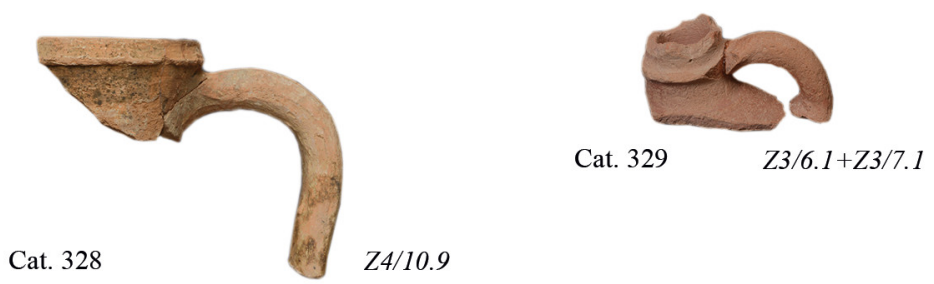

Z4/10.9

FIGURE 7.129 Amphorae of types XII.3, XII.4, XII.5, XII.6 and varia of different shapes $\mathrm{z}_{7} / 22+\mathrm{Z}_{7} / 41+\mathrm{Z}_{7} / 54, \mathrm{z}_{7} / 93+\mathrm{Z}_{7} / 54$ РнотоS ву А. КАмкоWSкі; $22 / 32$ Рното ву K. котLEWSKI; Z15/5, Z15/56, Z25/6, Z16/1, Z4/2+Z4/10.11, z3/6.1+Z3/7.1, Z5/43 PHOTOS BY A. KAMROWSKI 

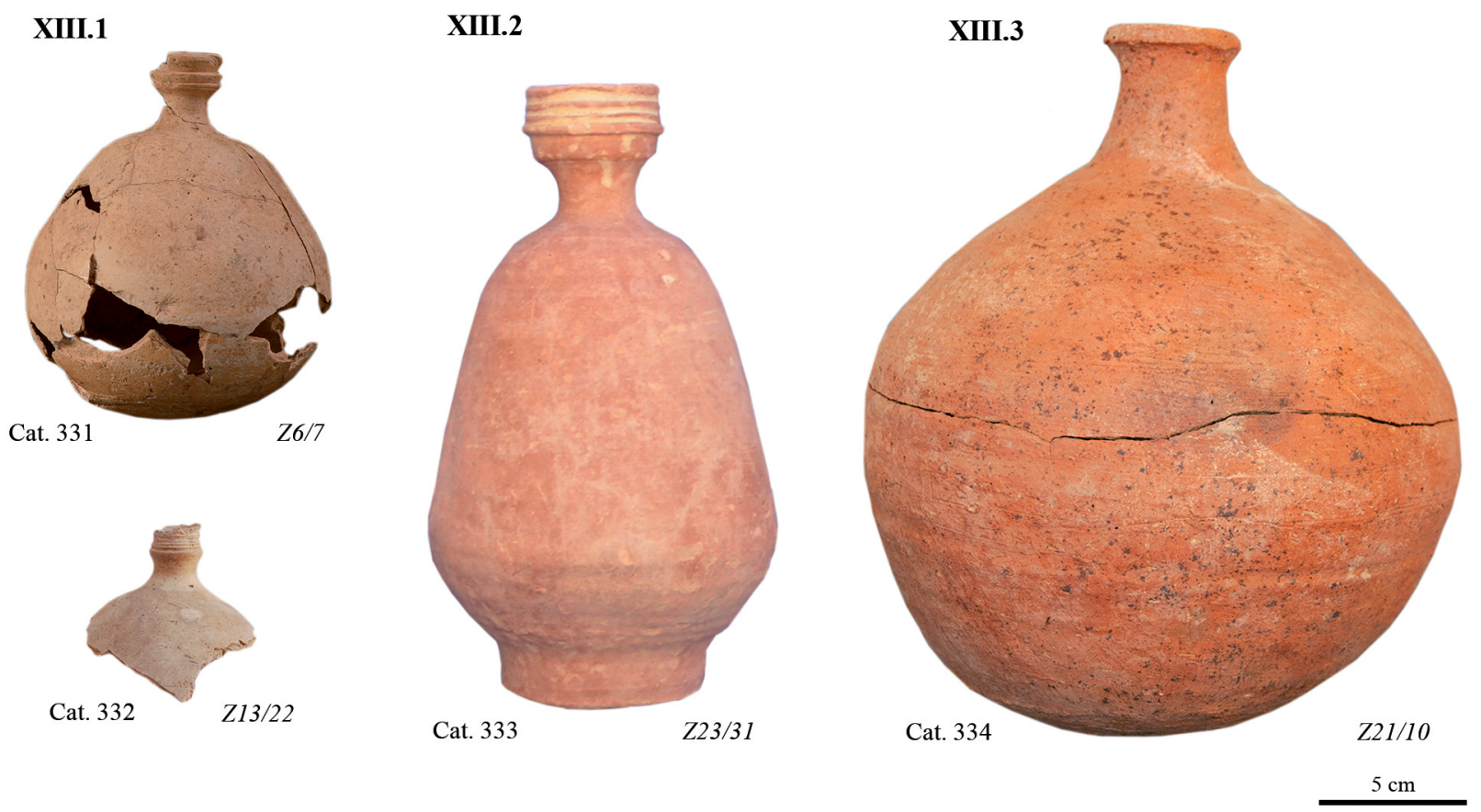

FIGURE 7.130 Bottles of type XIII.1, XIII.2 and XIII.3

Z6/7 PHOTO BY R. MAHLER; Z13/22 PHOTO BY O. BIAŁOSTOCKA; Z23/31 PHOTO BY

K. KOTLEWSKI; Z21/1O PHOTO BY R. MAHLER
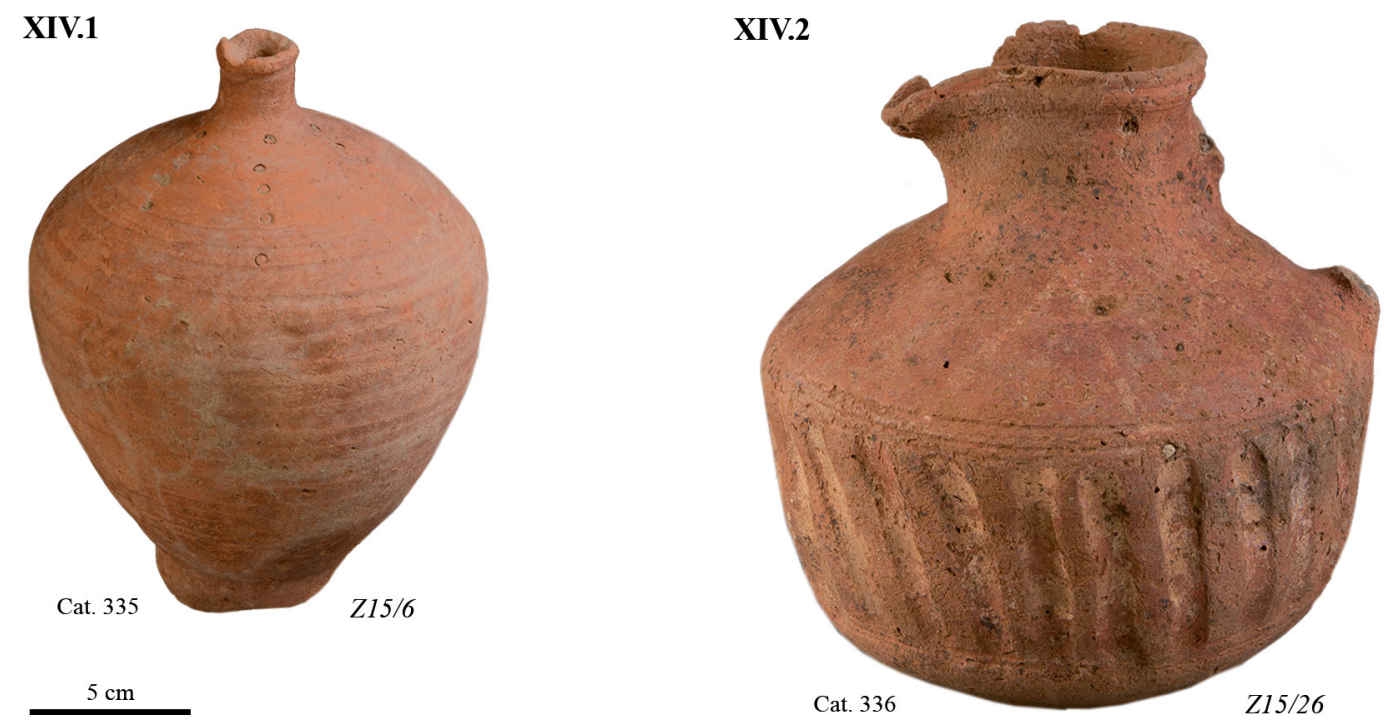

FIGURE 7.131 Jugs of types XIV.1 and XIV.2

Z15/6, Z15/26 PнOTOS BY A. KAMROWSKI 


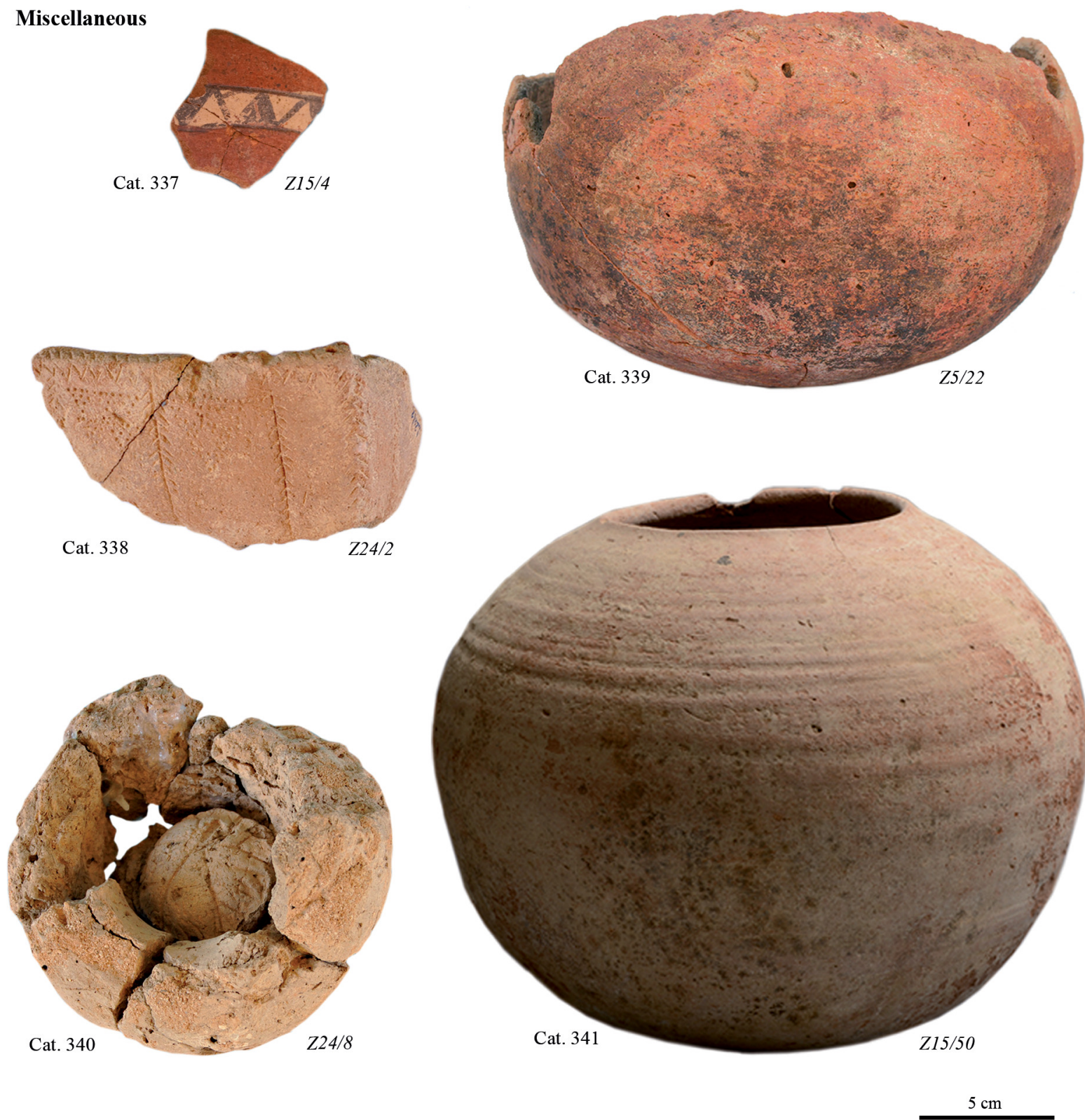

FIGURE 7.132 Miscellaneous vessels

Z15/4 PHOTO BY R. MAHLER; Z24/2 PHOTO BY T. WOJTCZAK; Z5/22 PHOTO BY

A. KAMROWSKI; Z24/8 РНОTO BY E. SKOWROŃSKA; Z15/50 PHOTO BY A. KAMROWSKI 


\section{Vessel Distribution inside Tumuli}

This chapter presents the distribution of all vessels and diagnostic fragments recorded during archaeological work. The main vessel groups presented in the catalogue according to typology are marked on the tomb plans in different colours:

Red Group I, cups

Green Group II, types II.1-II.7, small bowls

Dark blue Group II, types II.8-II.9, large bowls

Black Group III, spouted bowls

Purple Group IV, shallow cooking bowls

Light pink Group V, deep cooking bowls

Pink Group v, deep cooking bowls

Blue Group viI, jars/bottles

Grey Group VIII, pot stands

Dark brown Group IX, goblets

Yellow Group X, oil lamps

Violet Group XI, qawadis

Brown Group XII, amphorae

Light green Group XIII, bottles

Light blue Group XIV, jugs

Vessels, mostly complete or almost complete, marked on the plans were found inside the chambers or inside the shafts of the tumuli. Some vessels recorded in pieces or diagnostic fragments, mostly recovered from the shaft fill or the superstructure, are presented in the drawings but not on the plans of the tumuli. There are no grave plans with vessel distribution for type I tombs because in these instances the pottery was found in the form of sherds scattered in the fill of the tunnels. The order in which the tumuli are presented corresponds to the typology set out by Mahmoud El-Tayeb in Volume I (see Volume I, Chapter 3). 


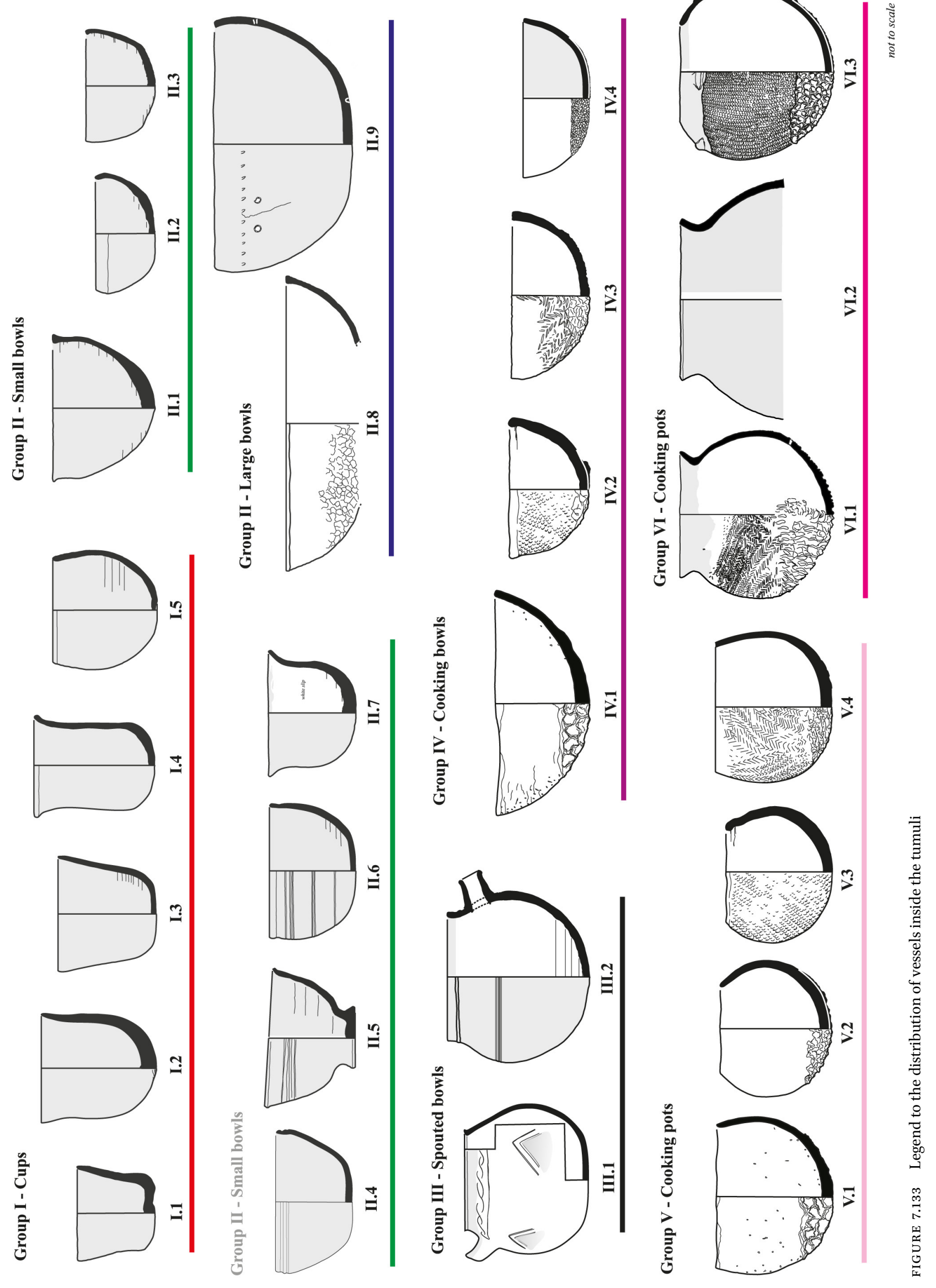


产
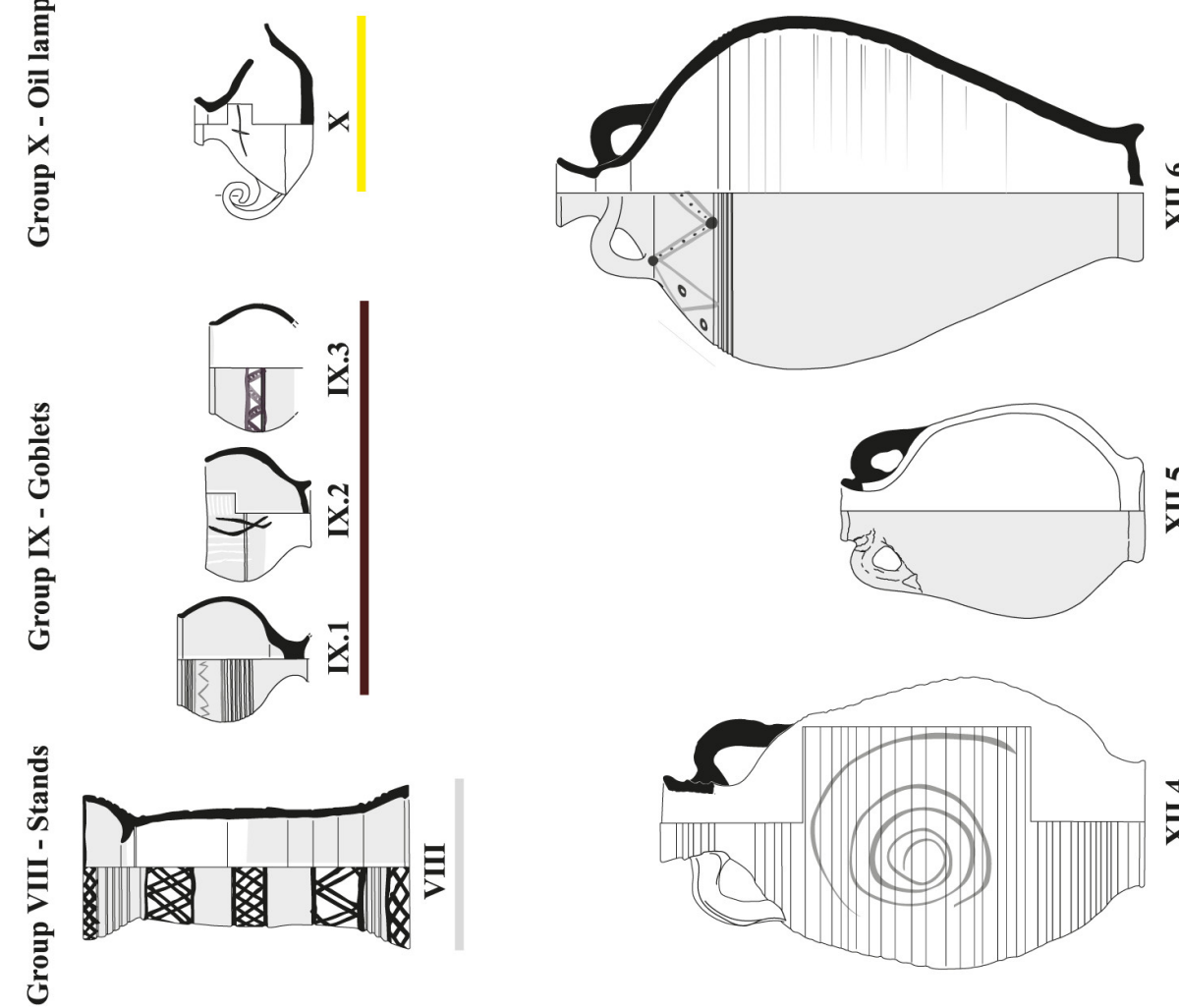

$\stackrel{n}{\bar{x}}$
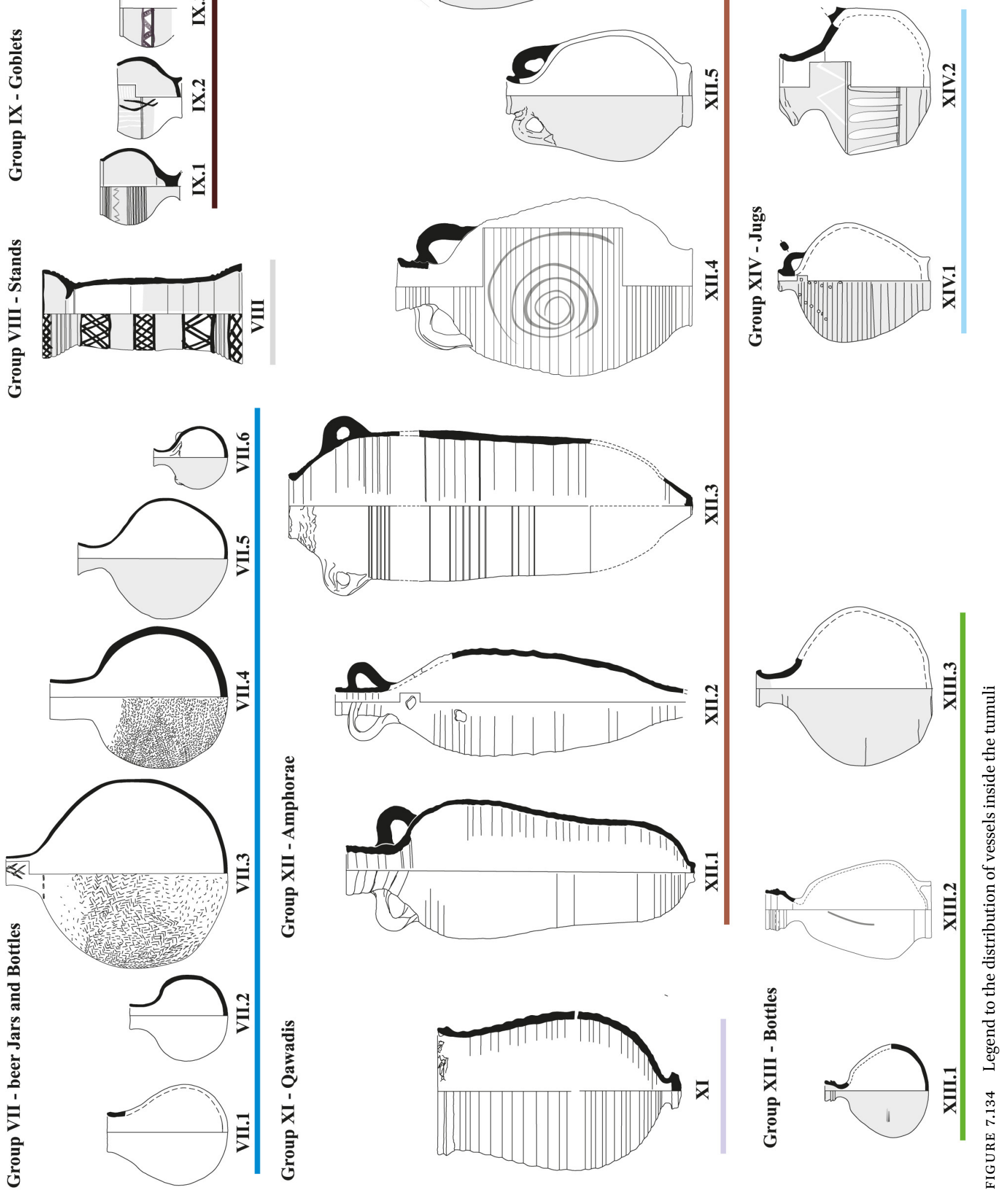


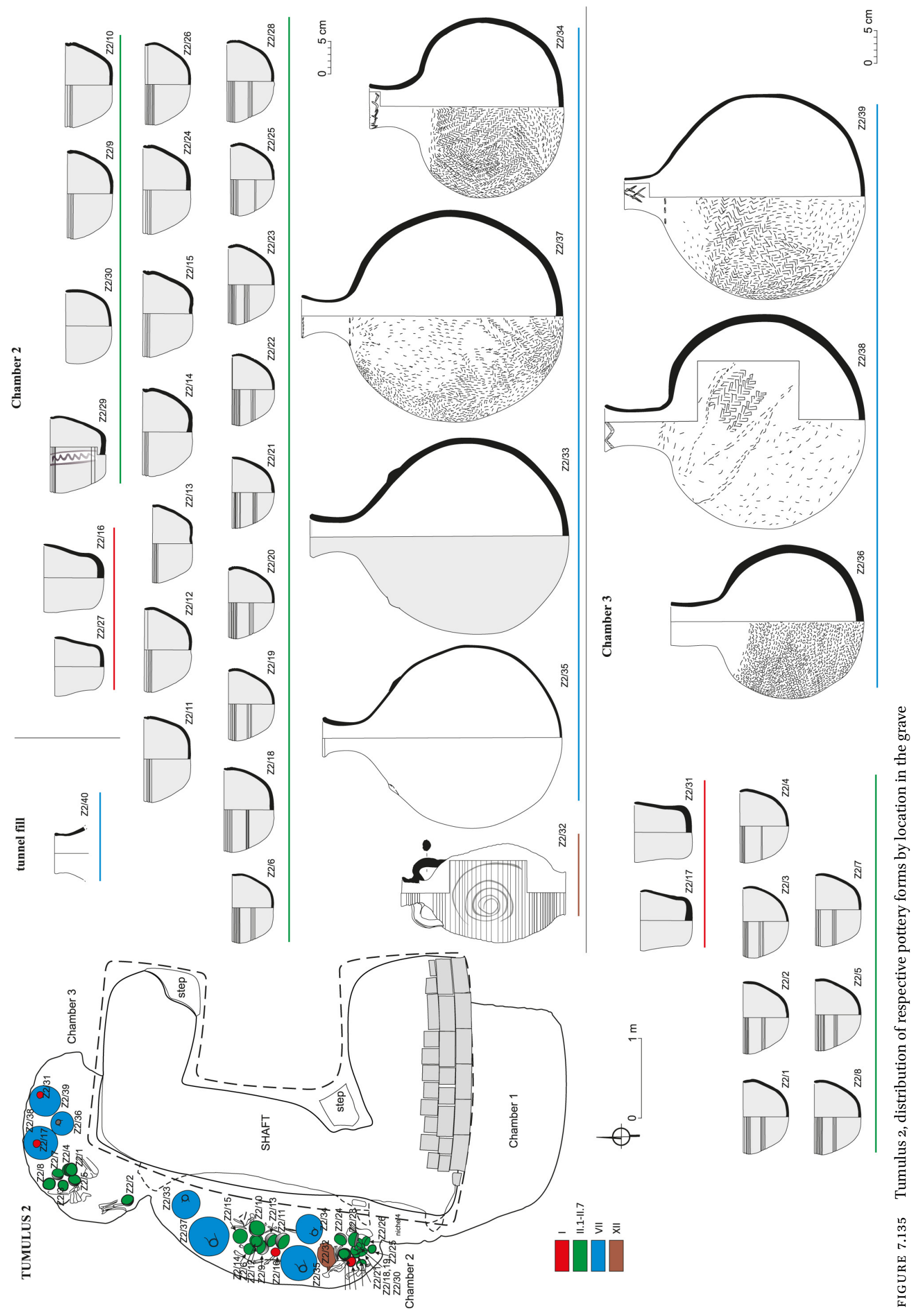



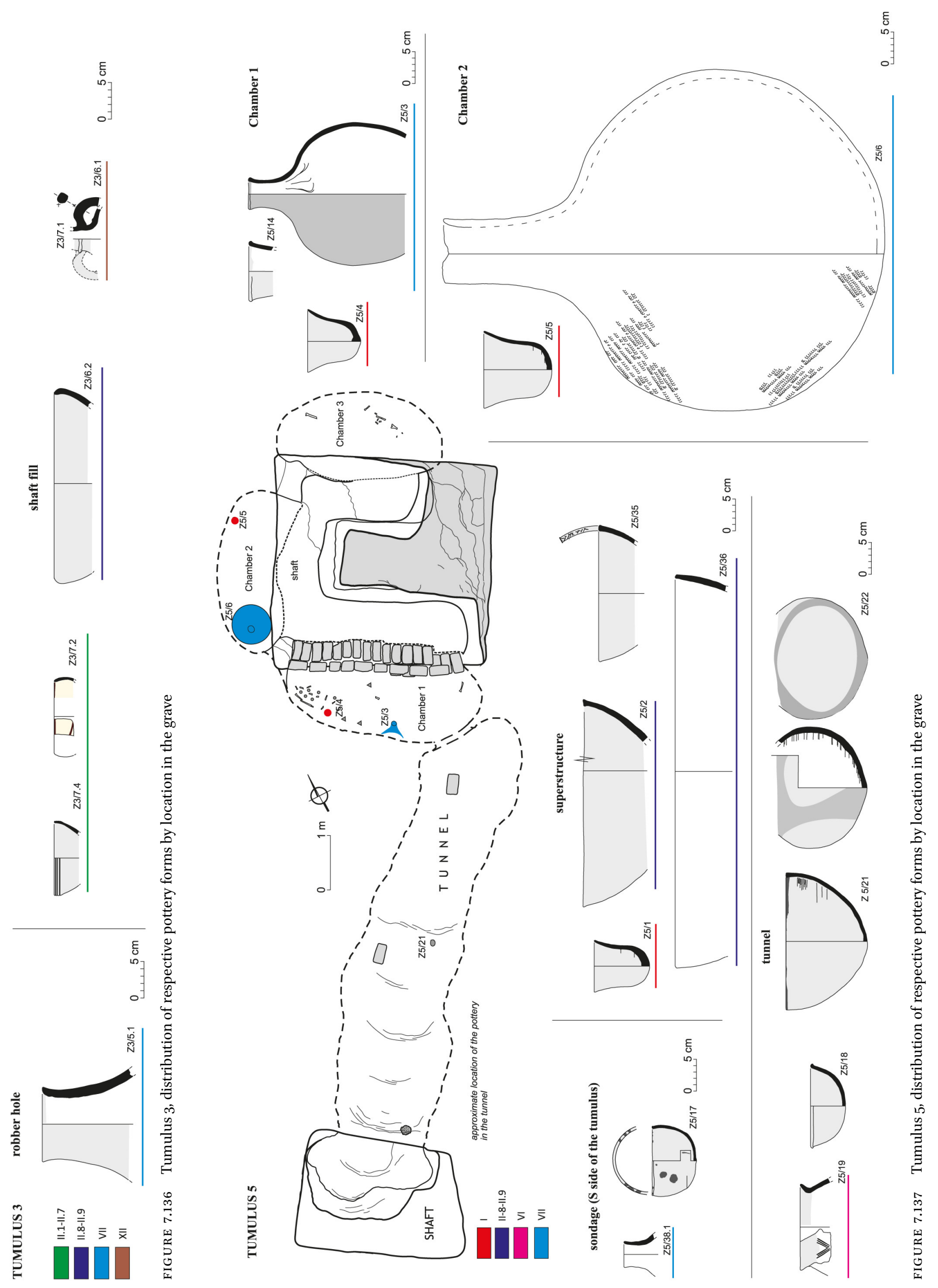


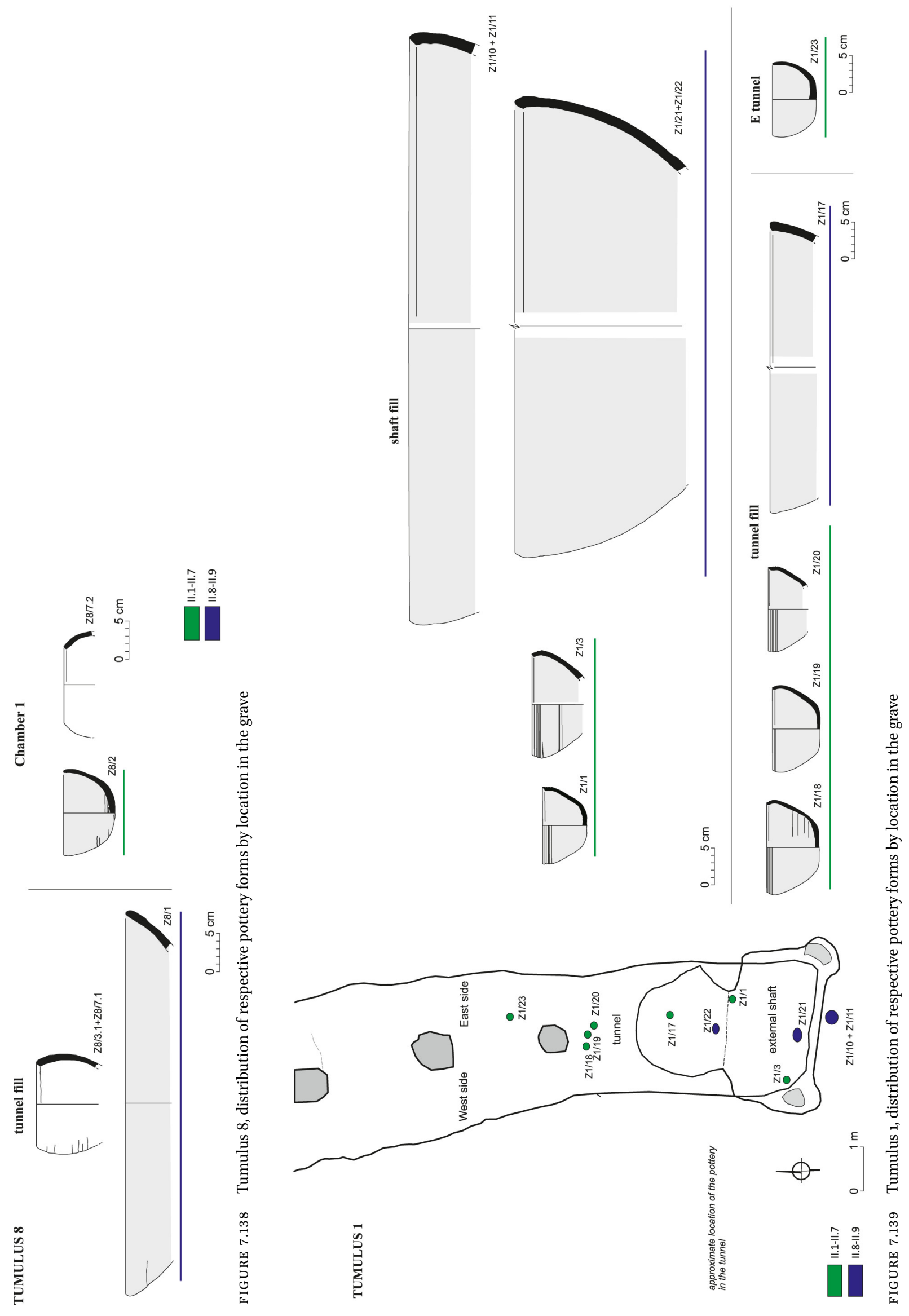




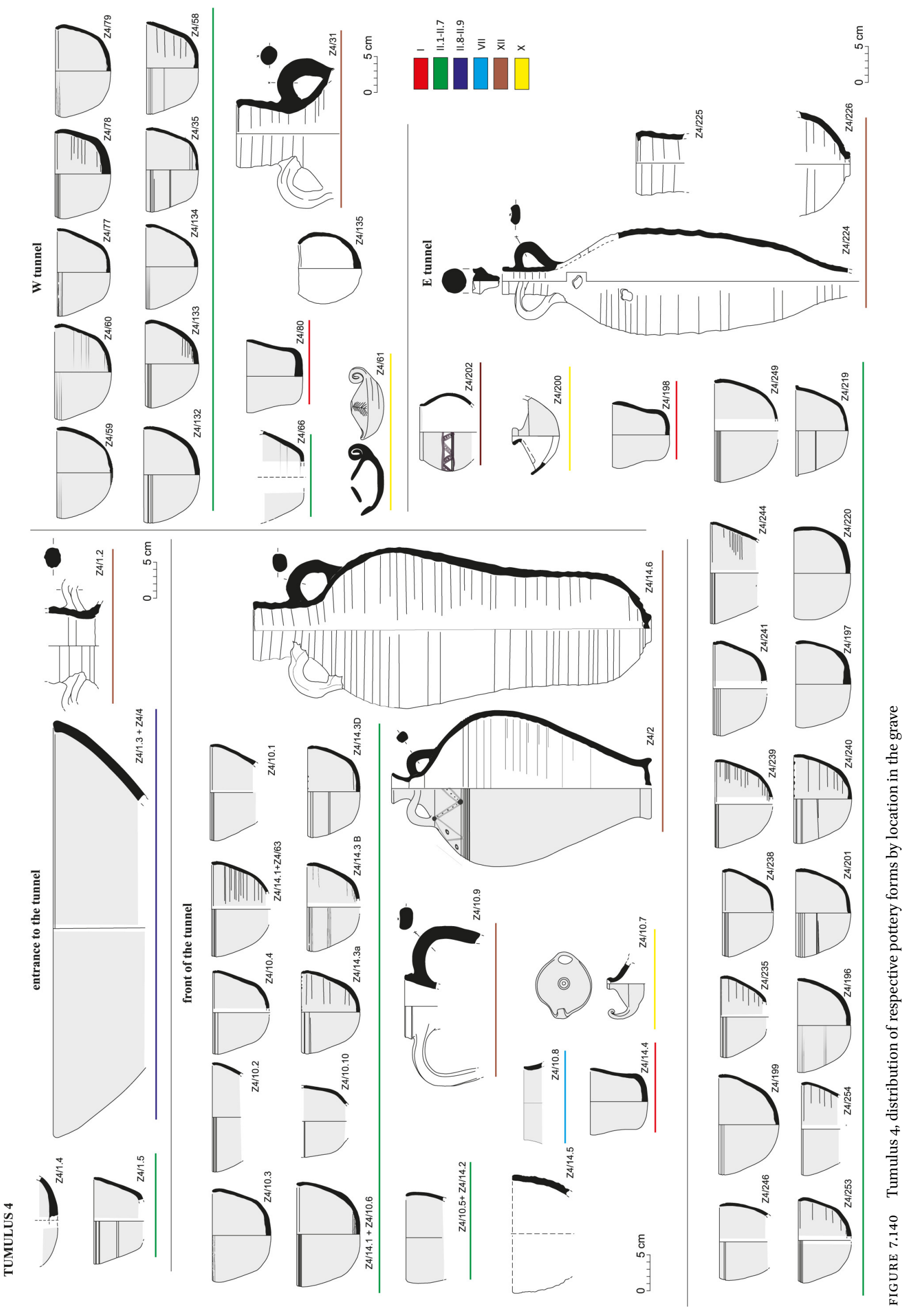




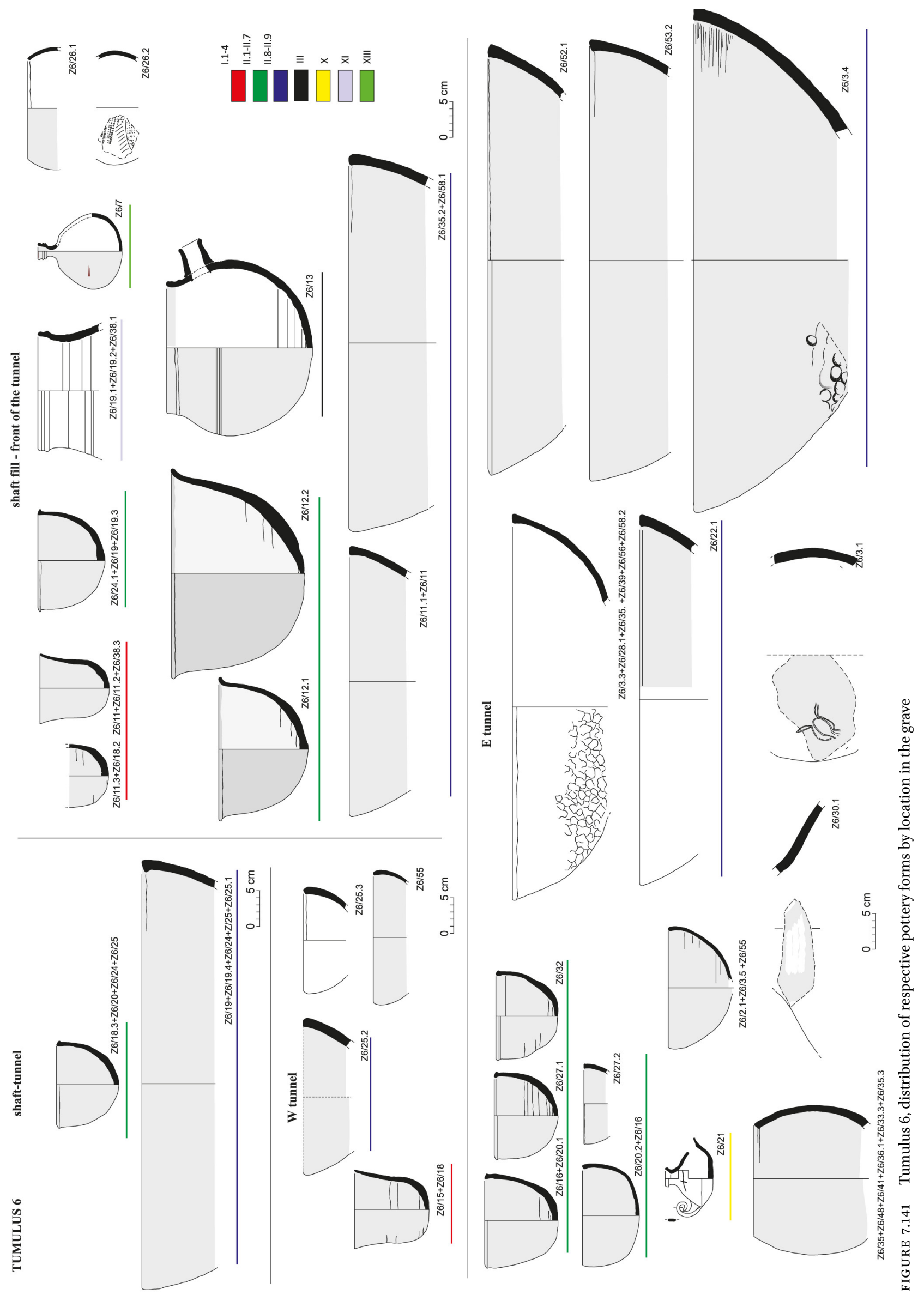



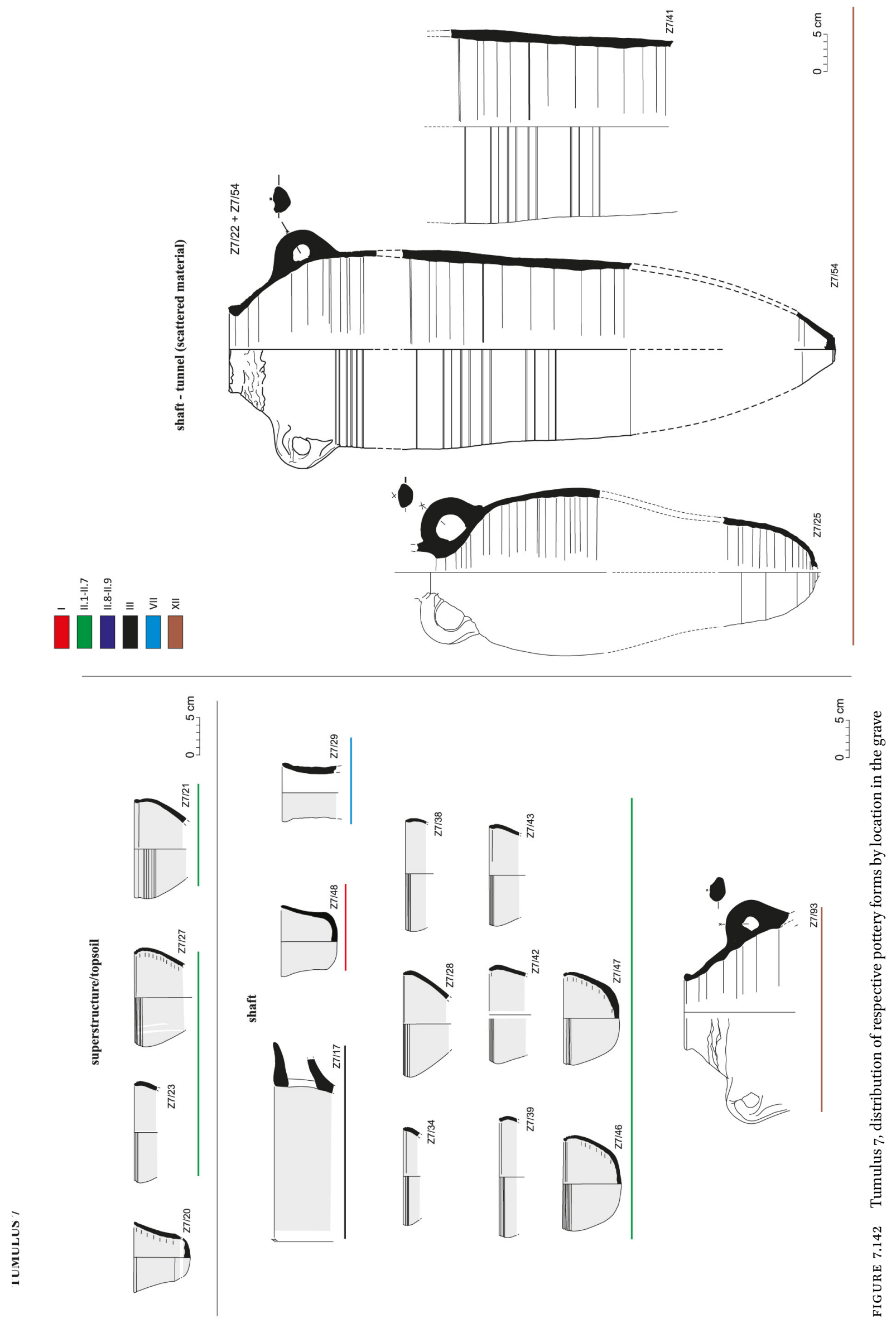


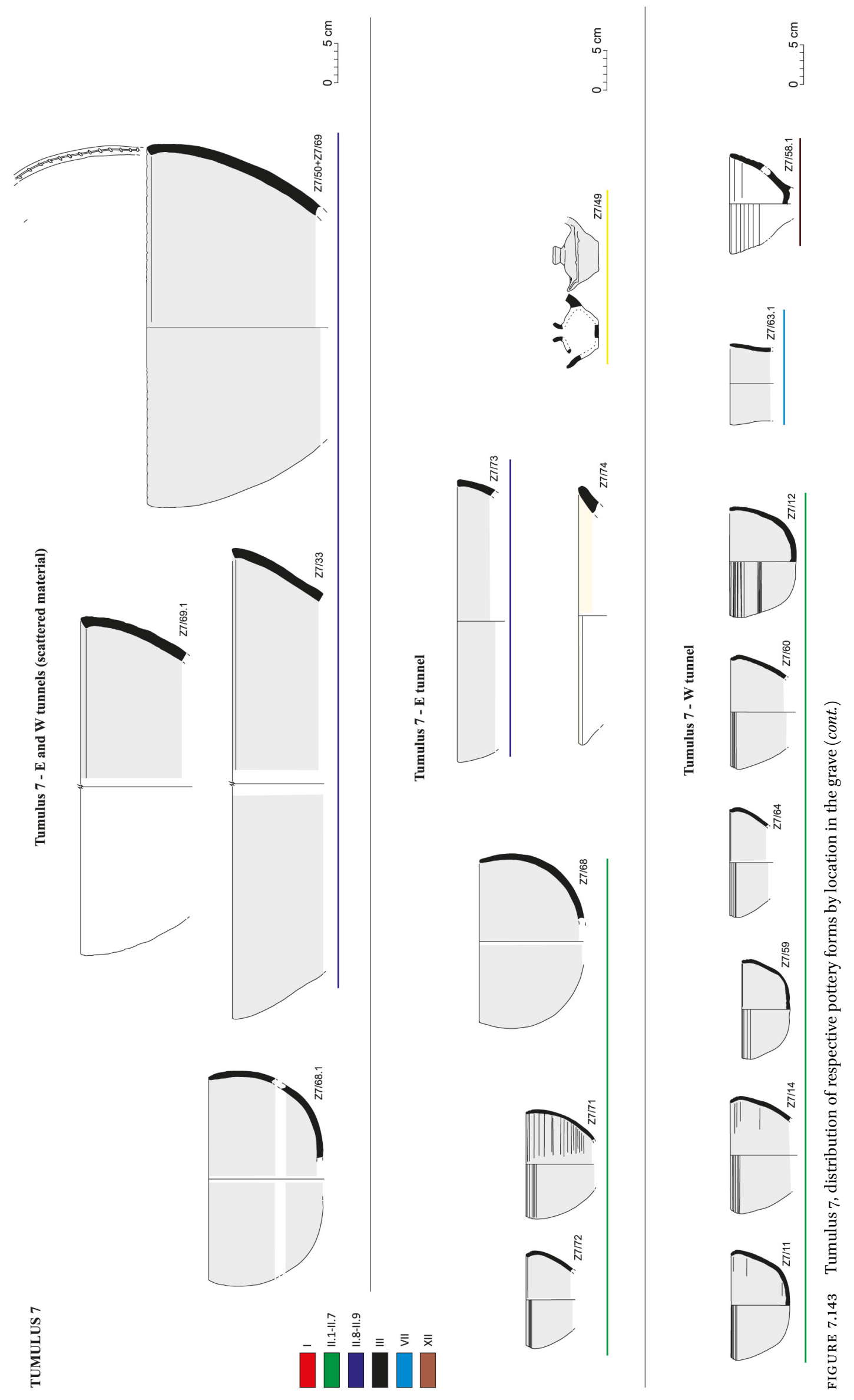




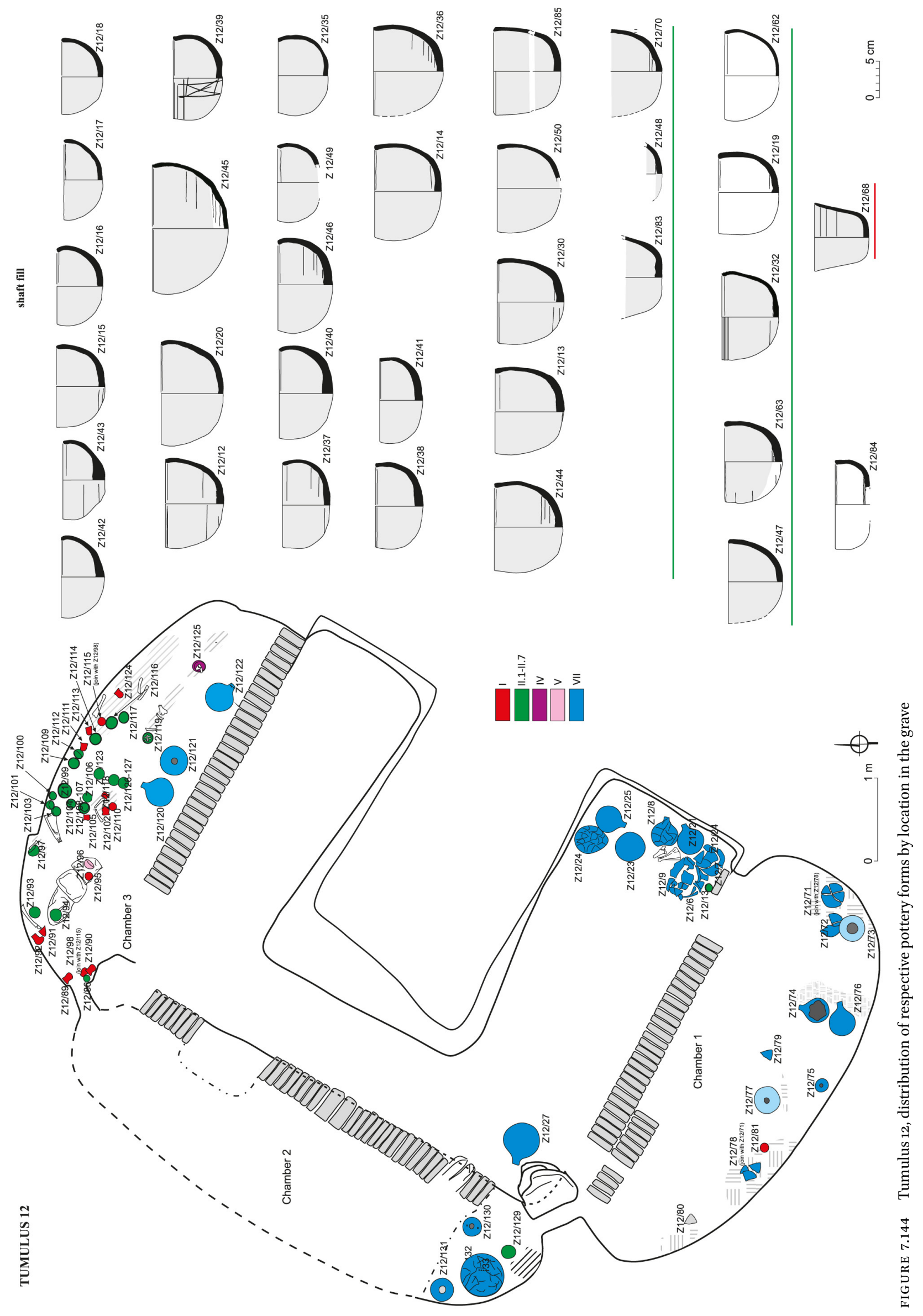




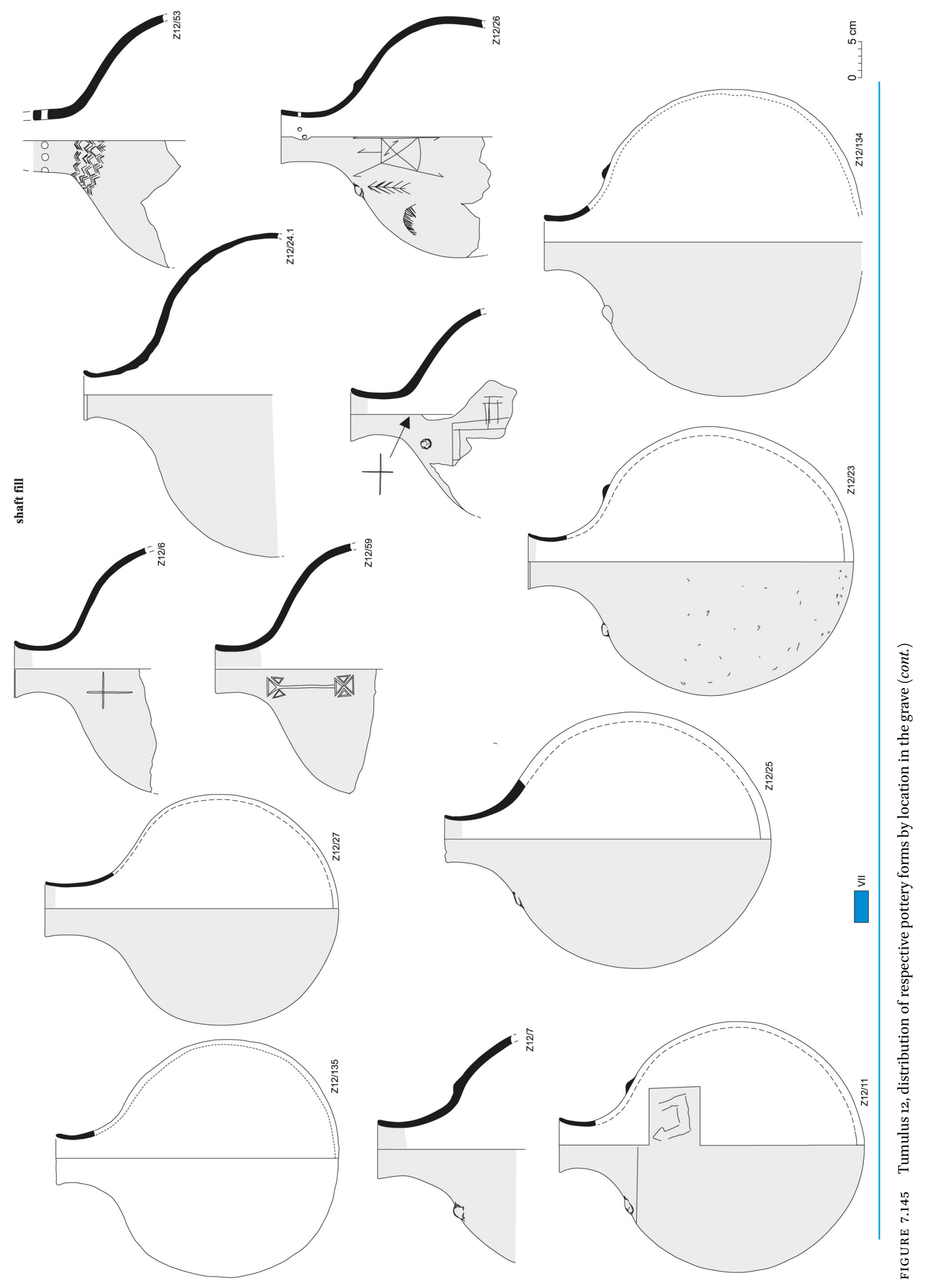




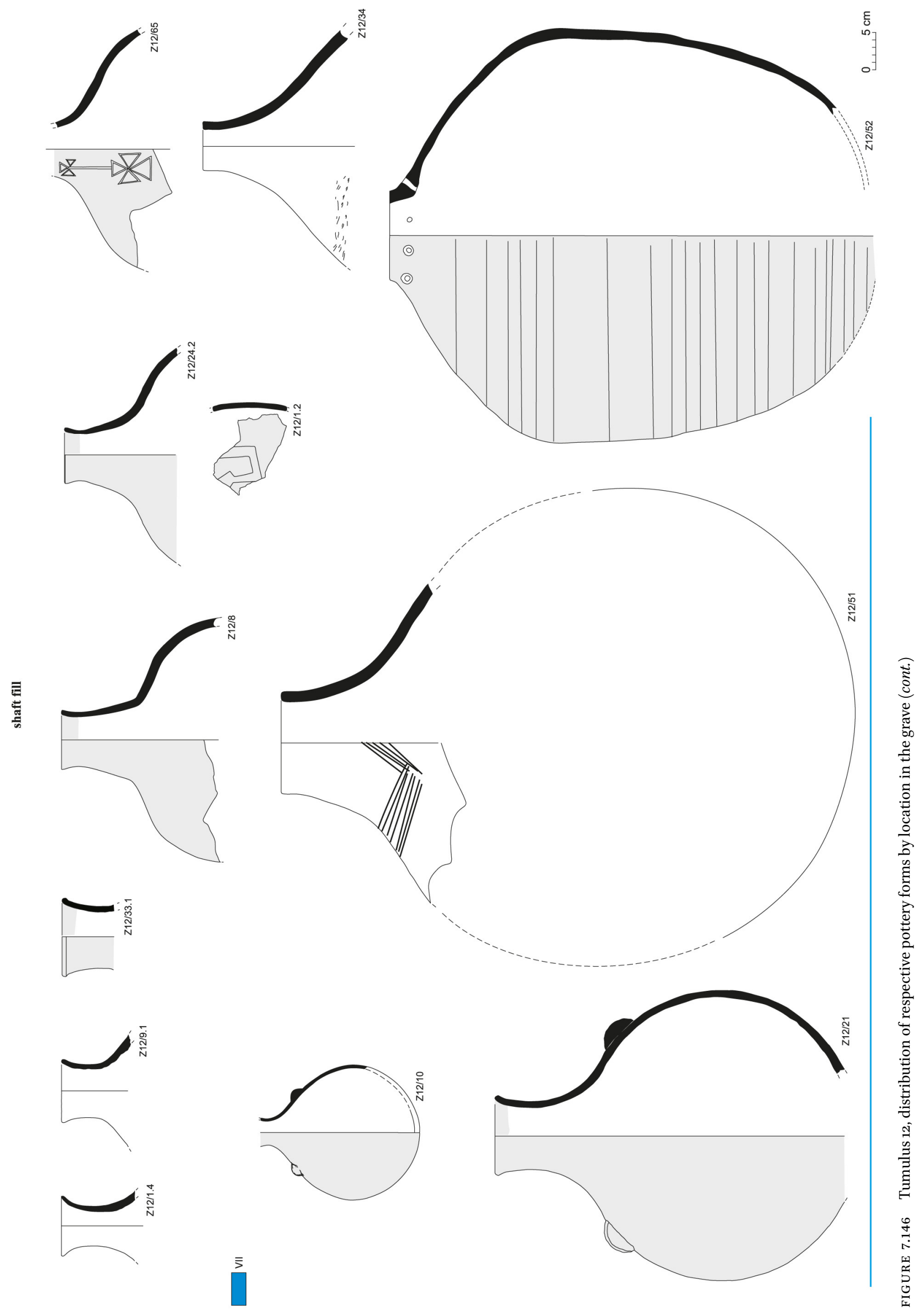




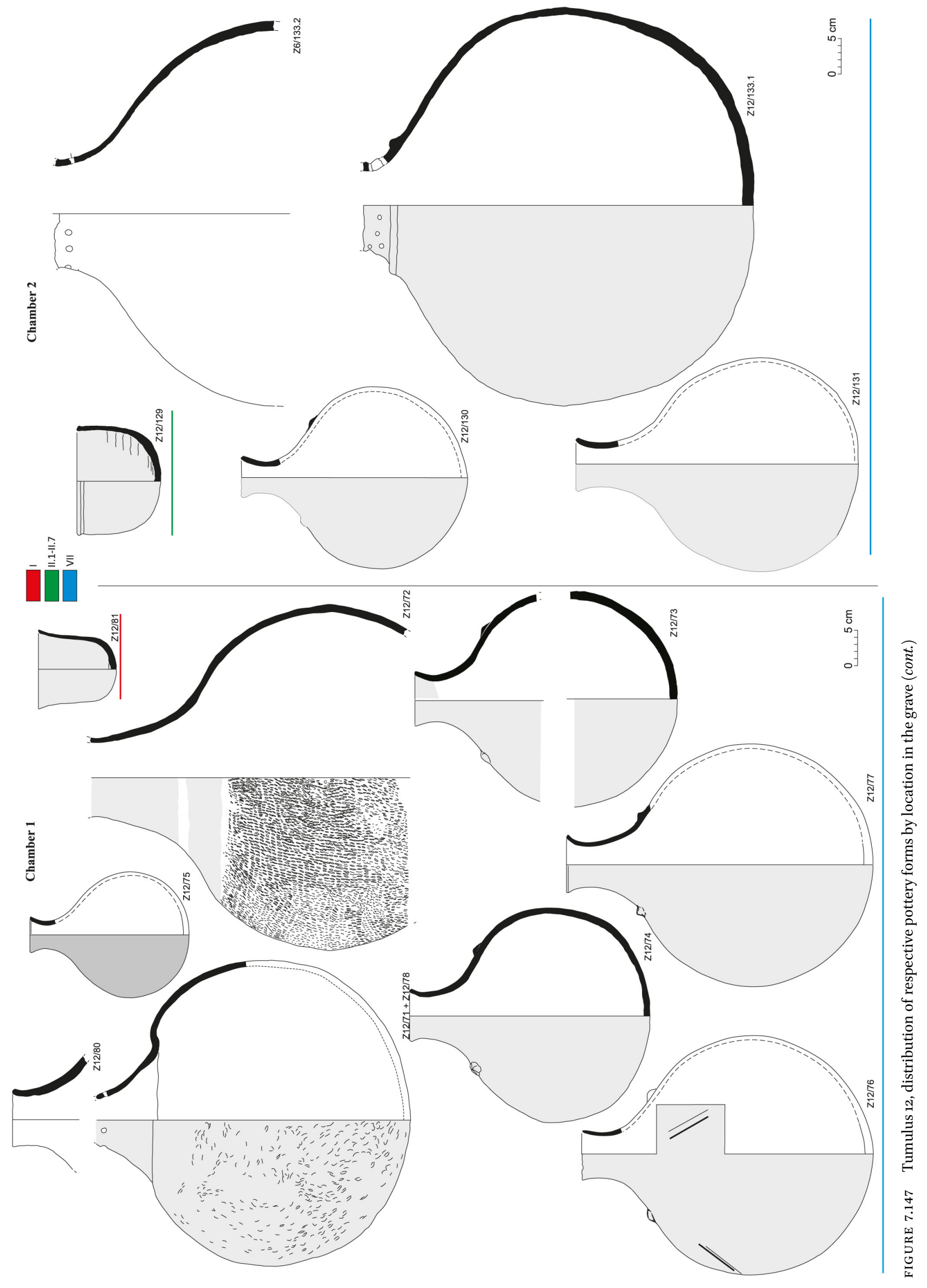




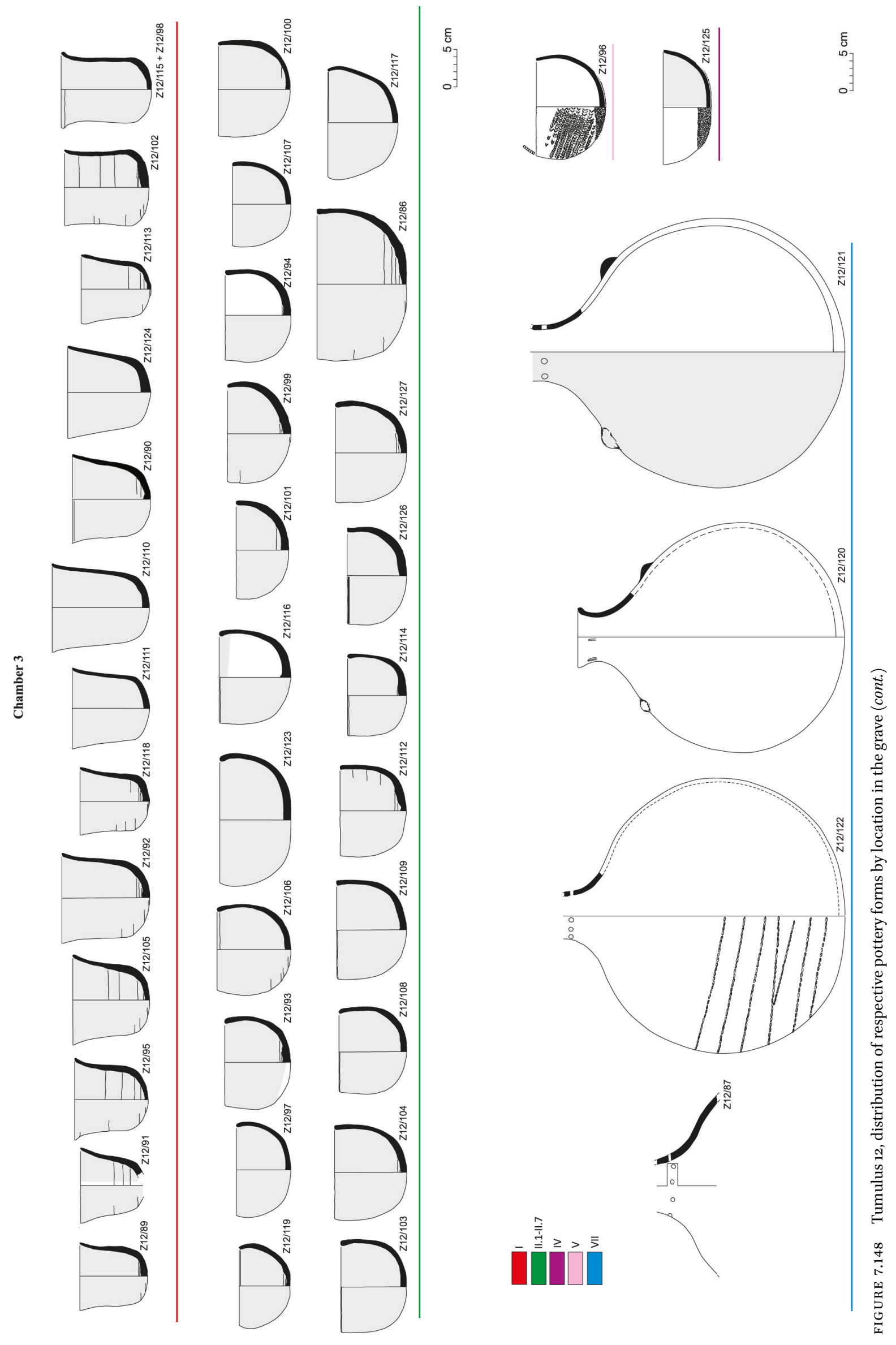




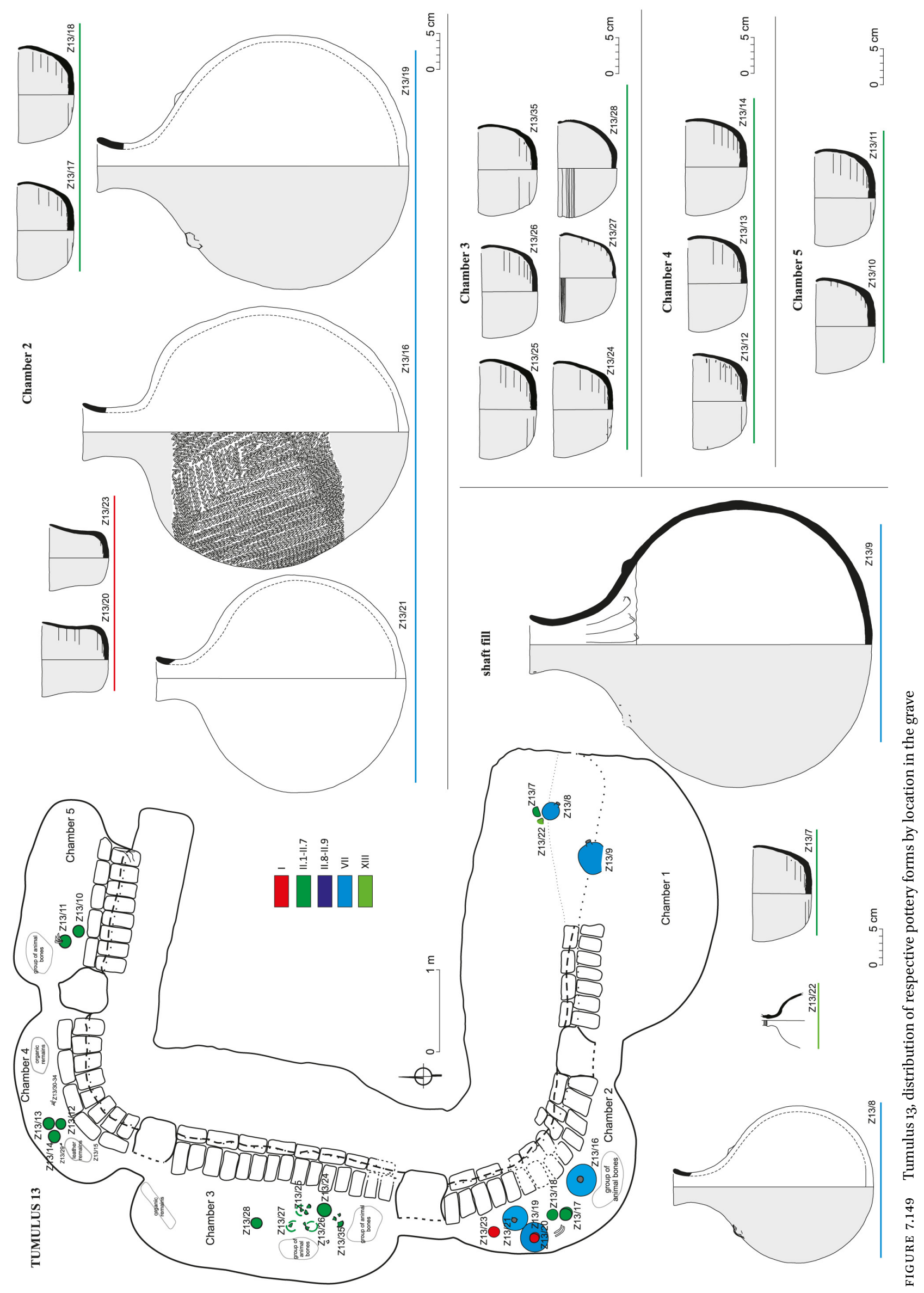




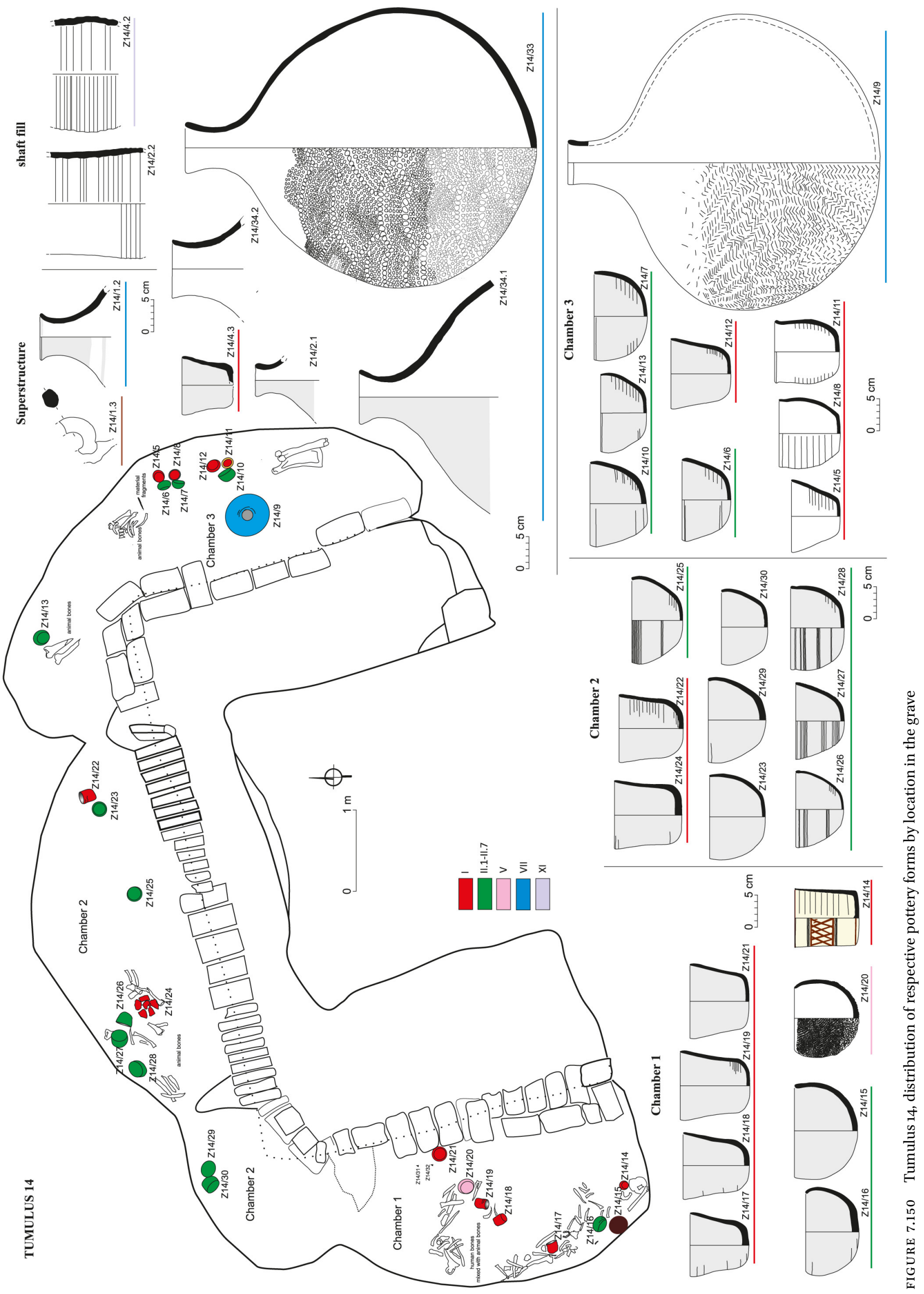




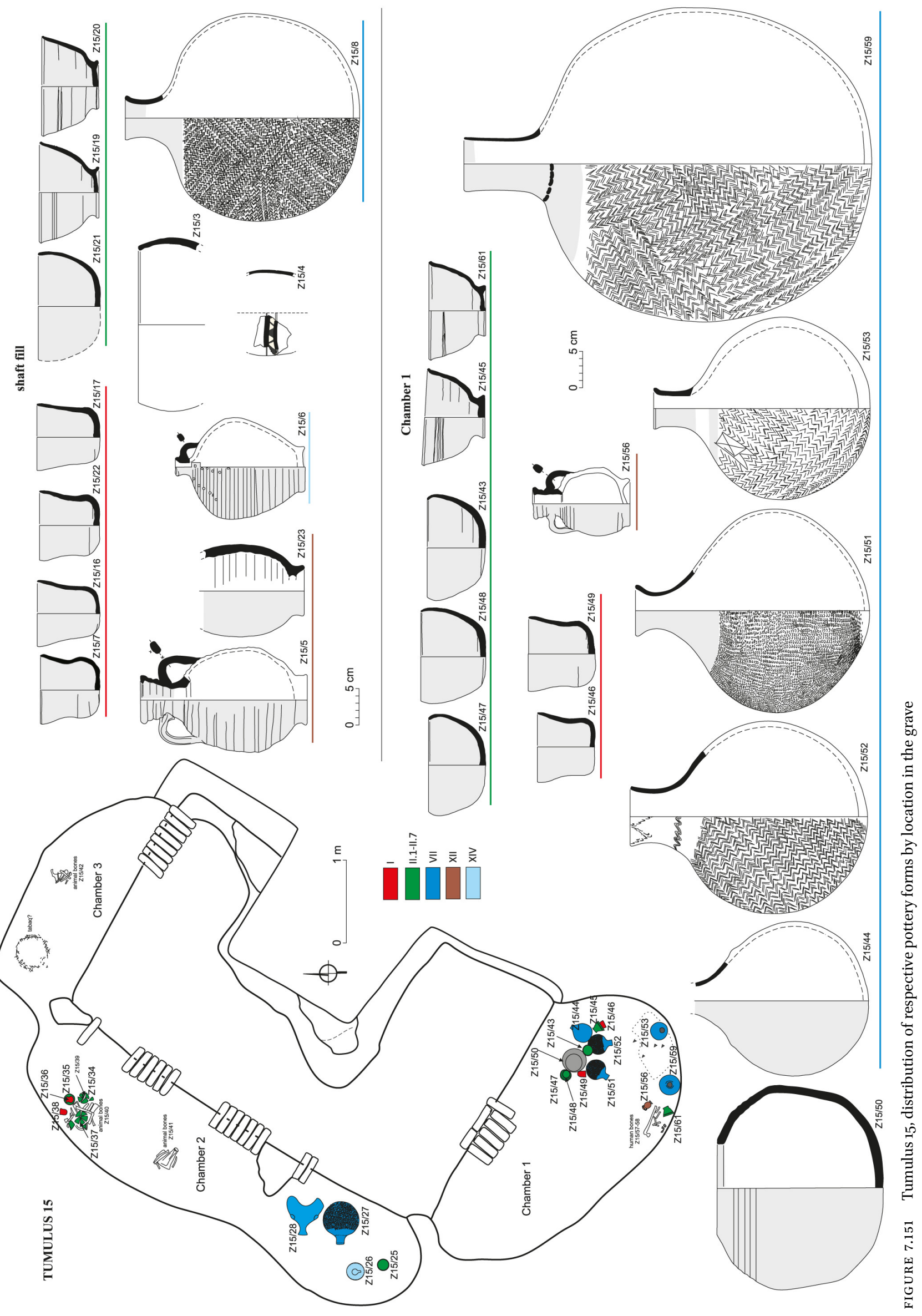



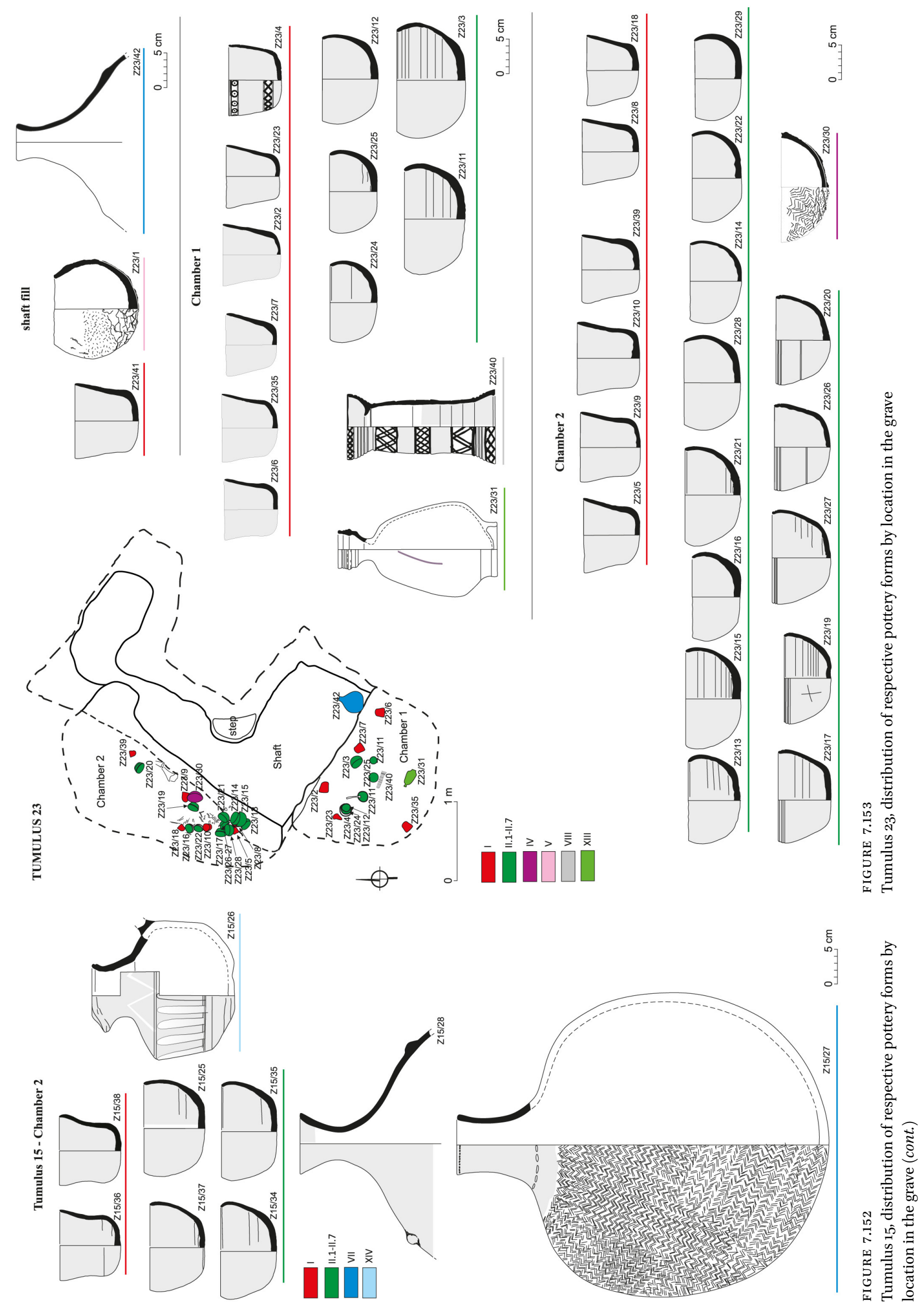


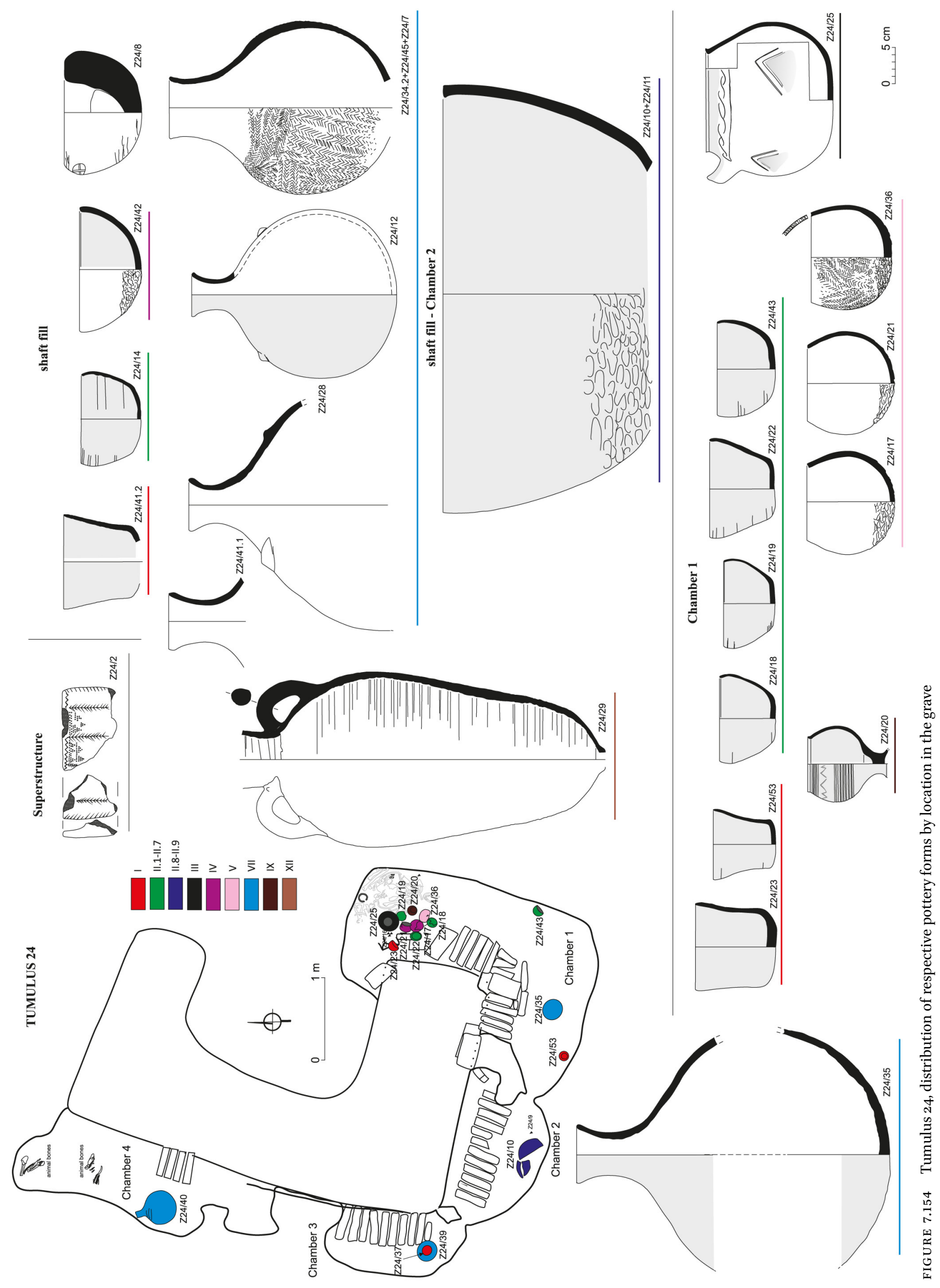



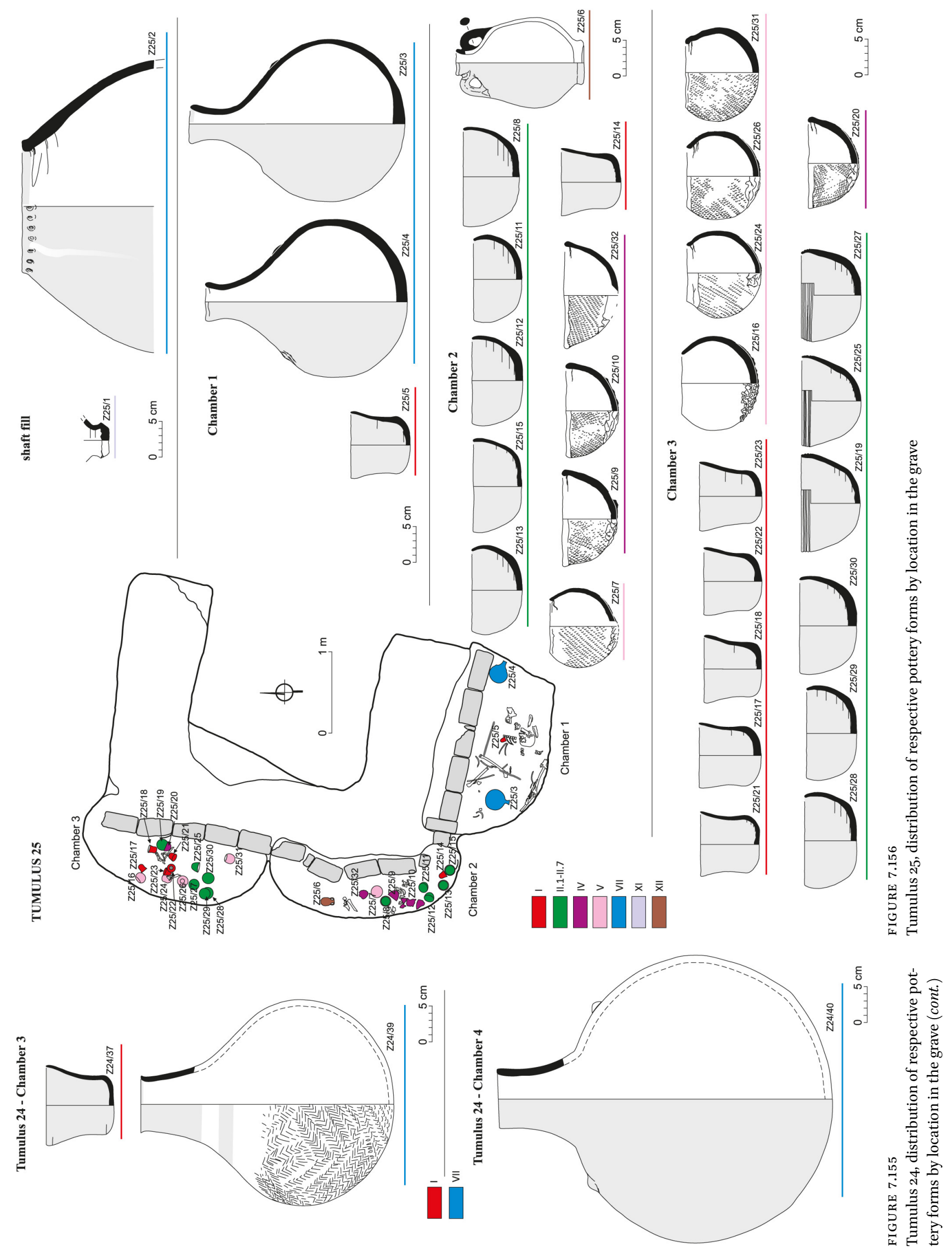


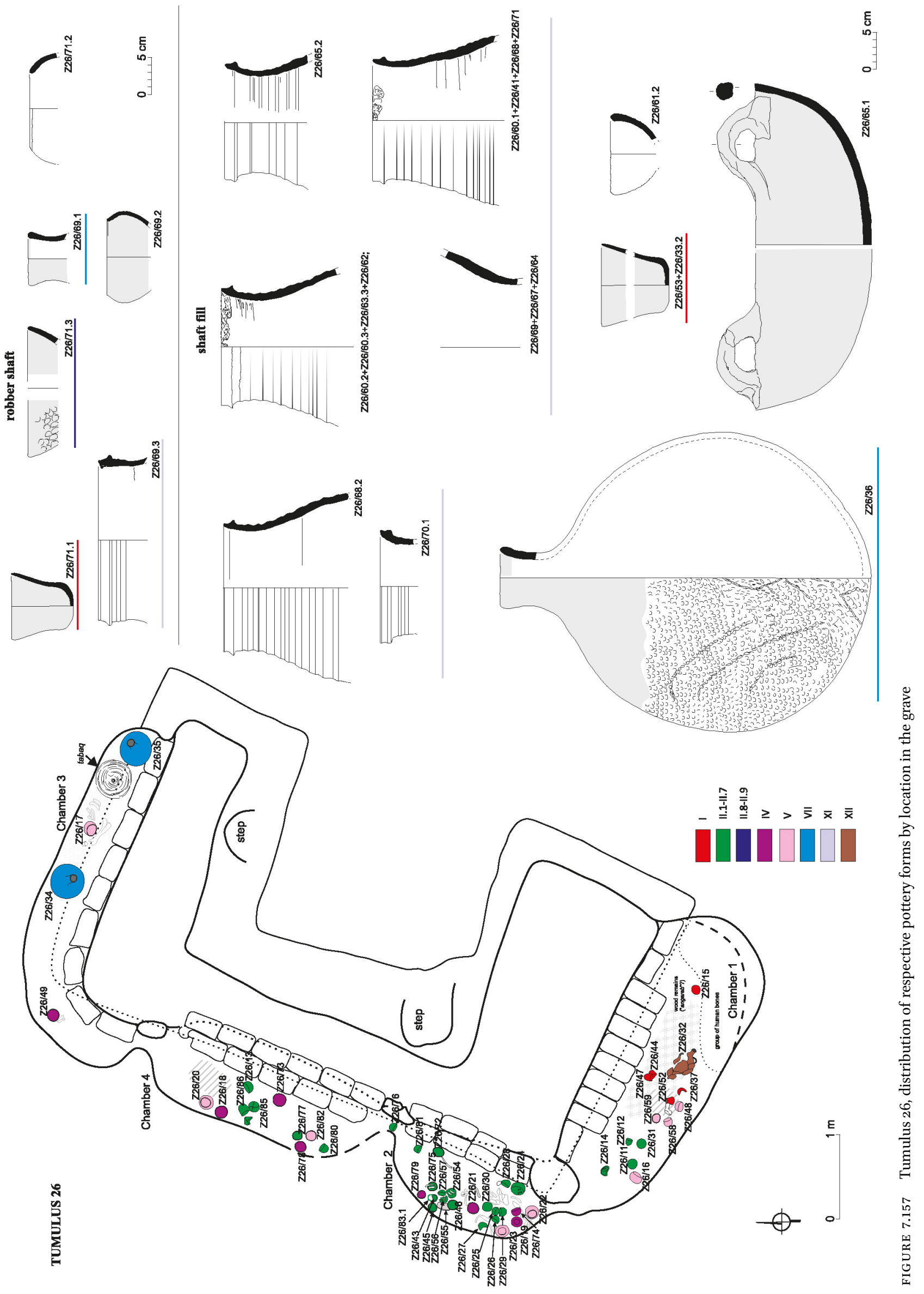




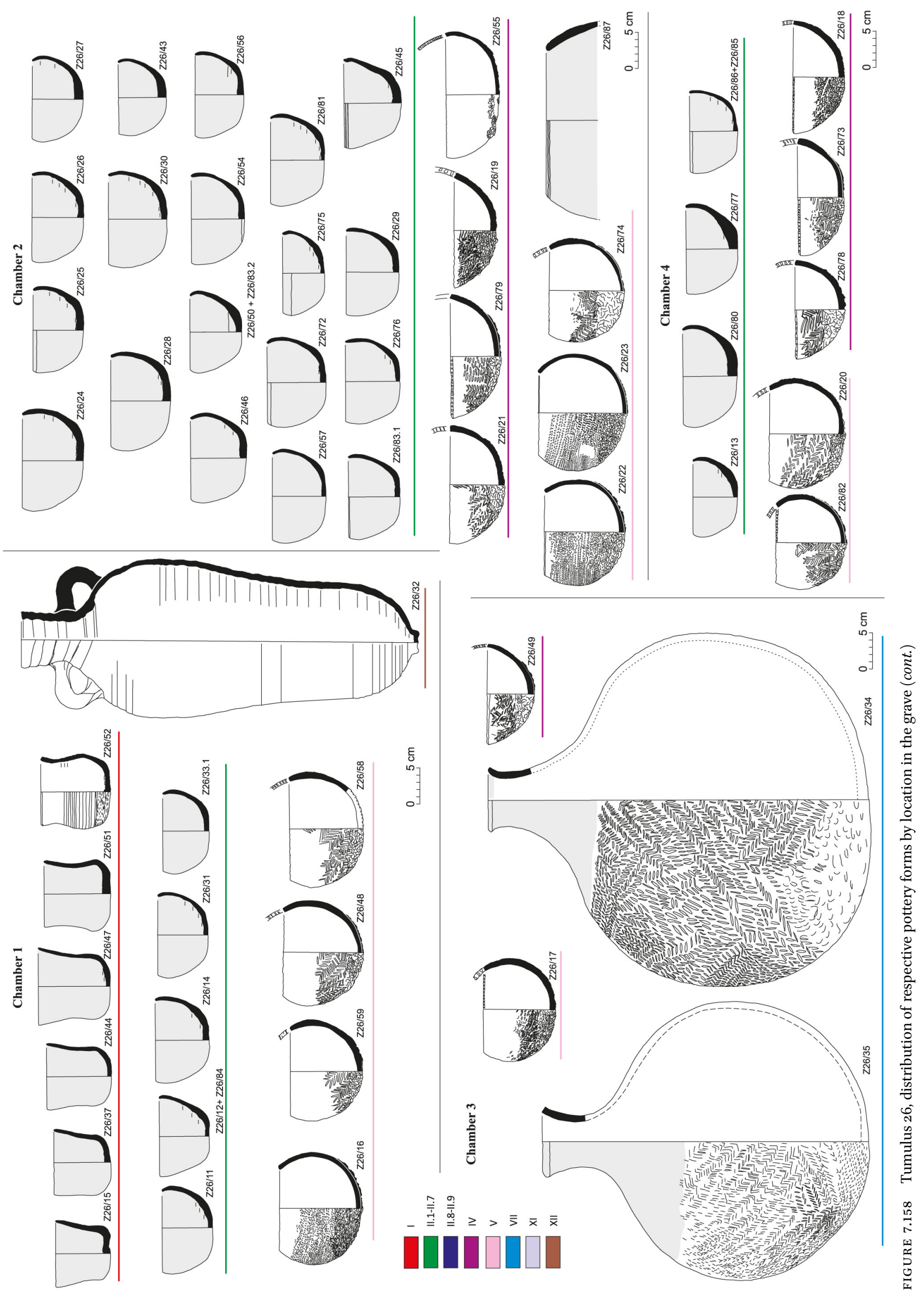




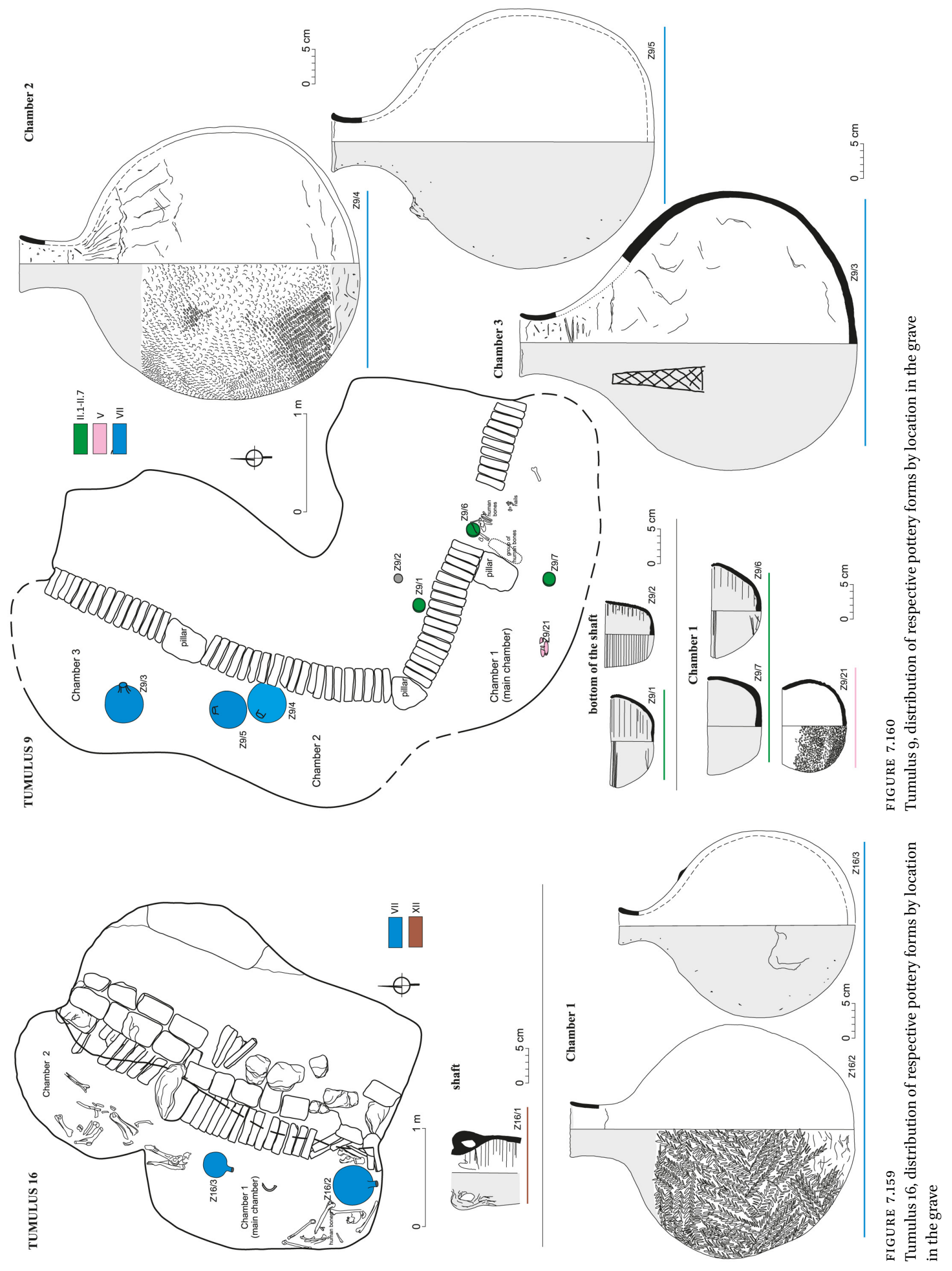




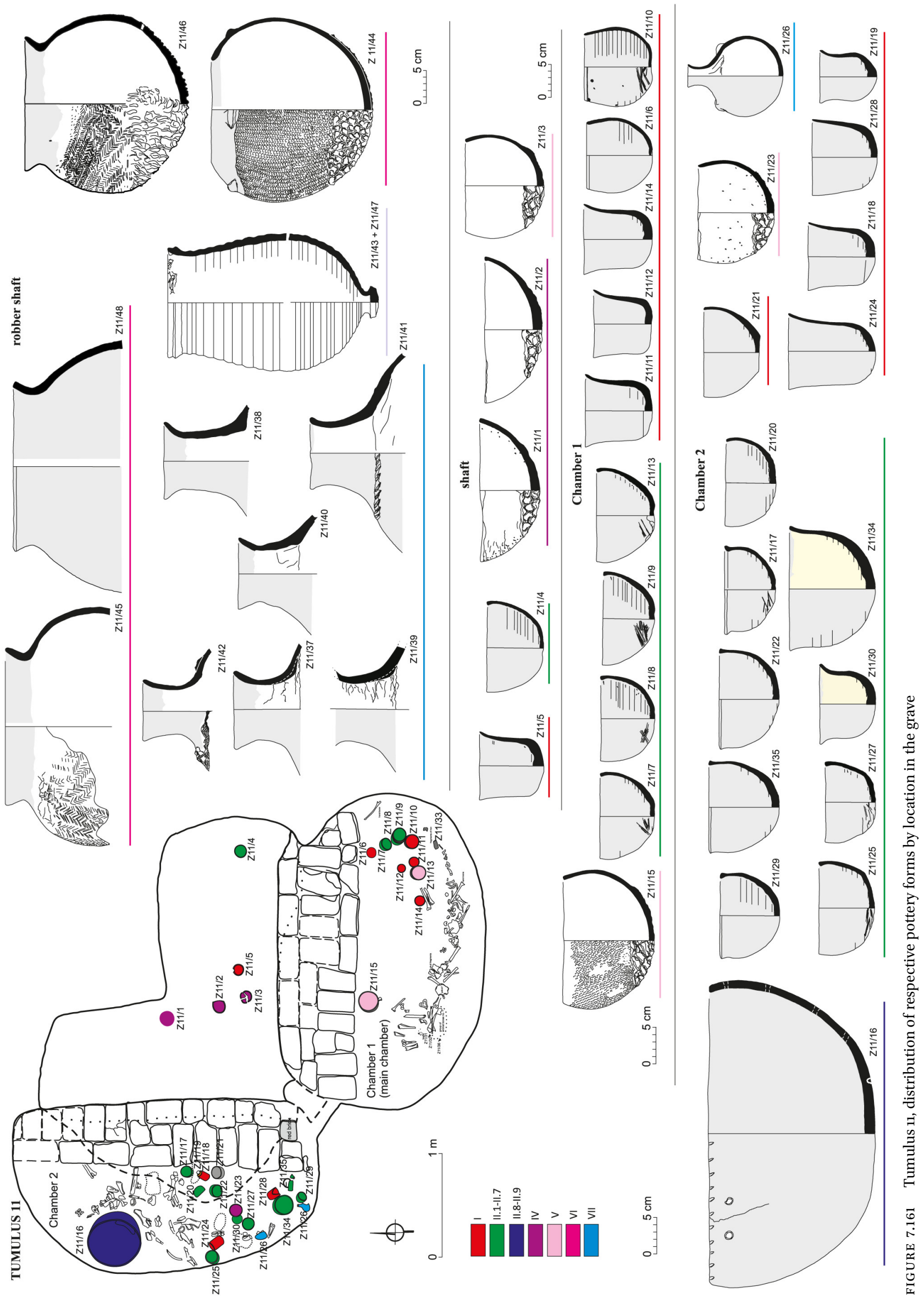



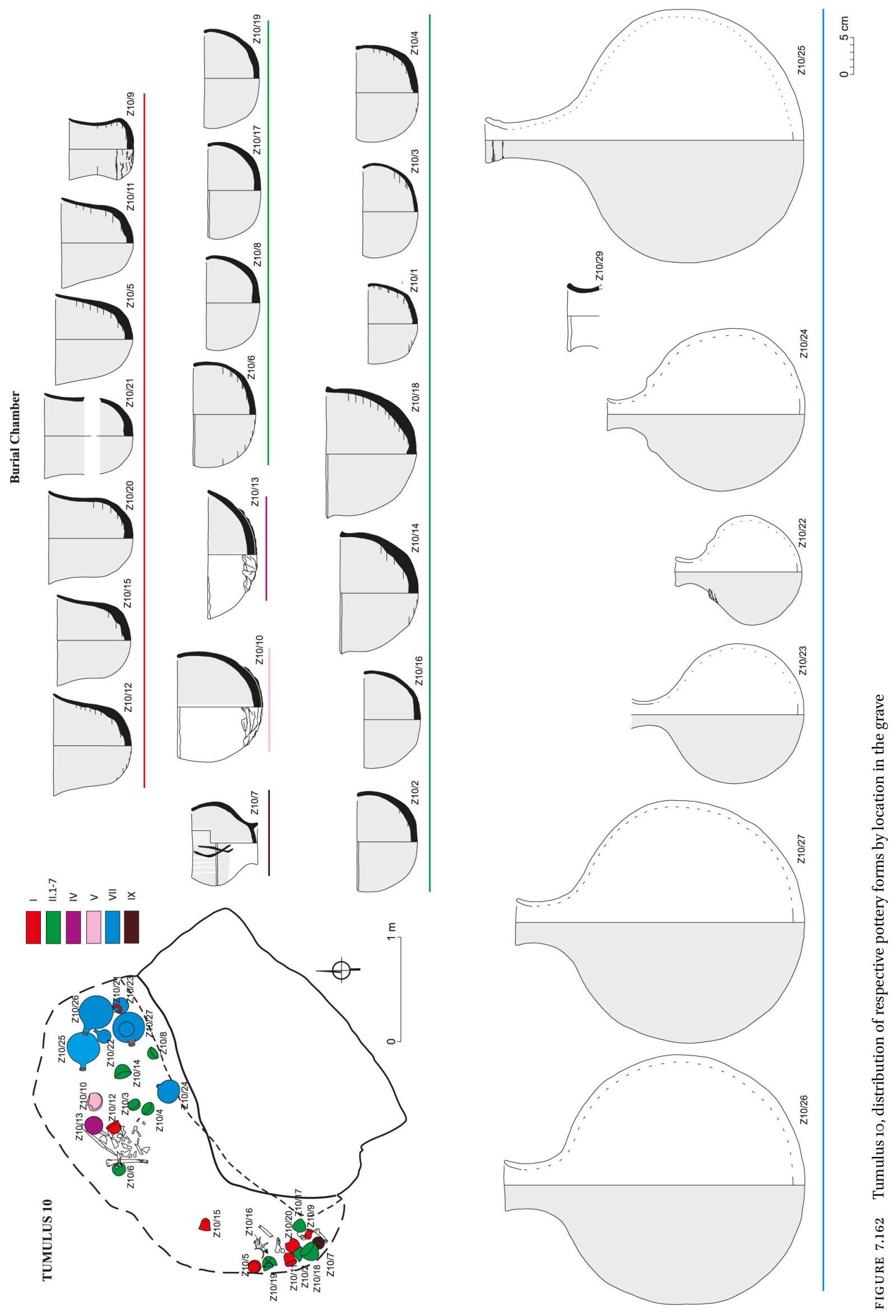

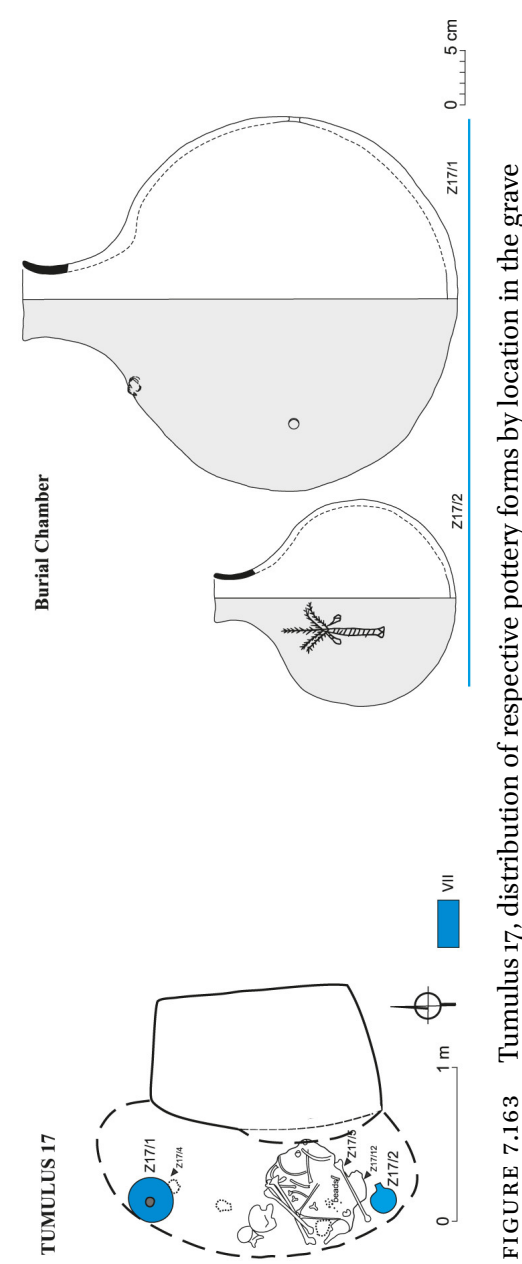
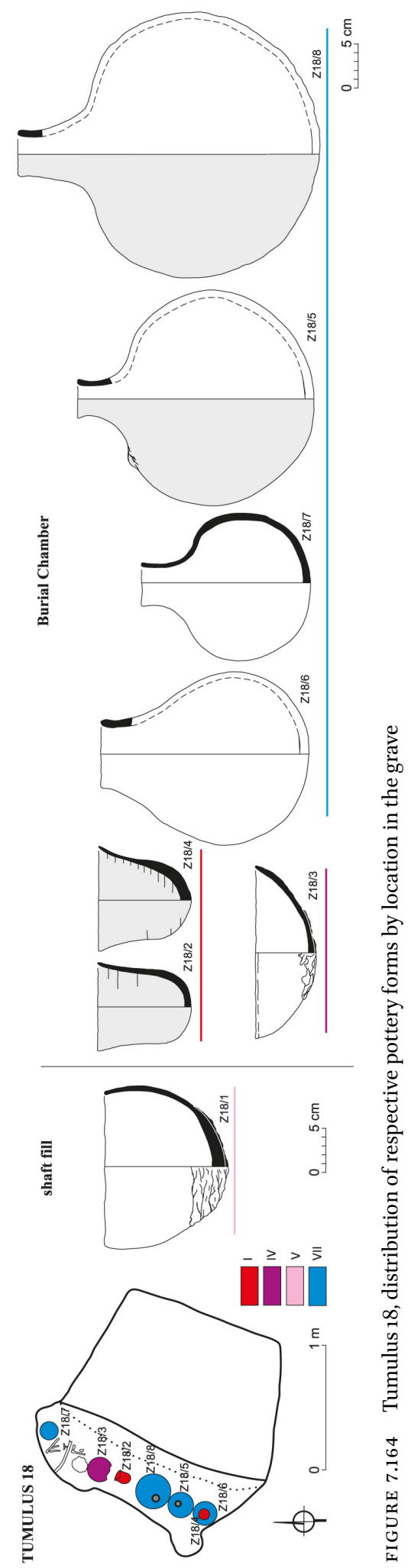
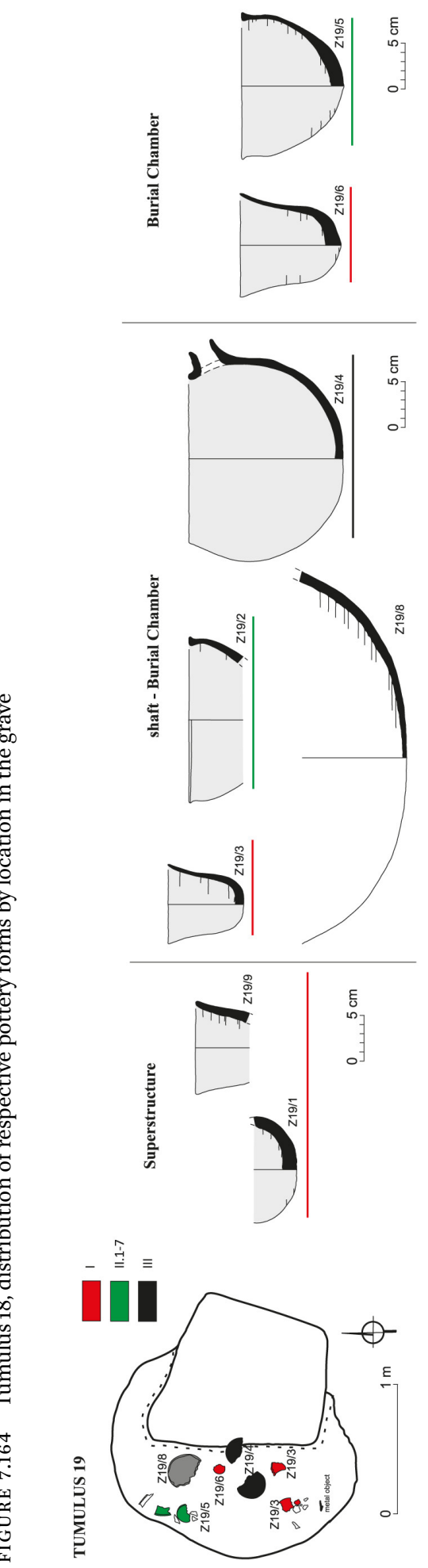

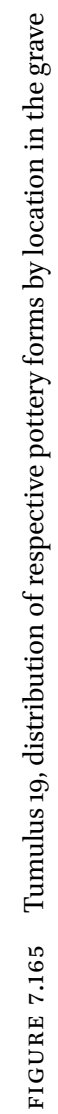



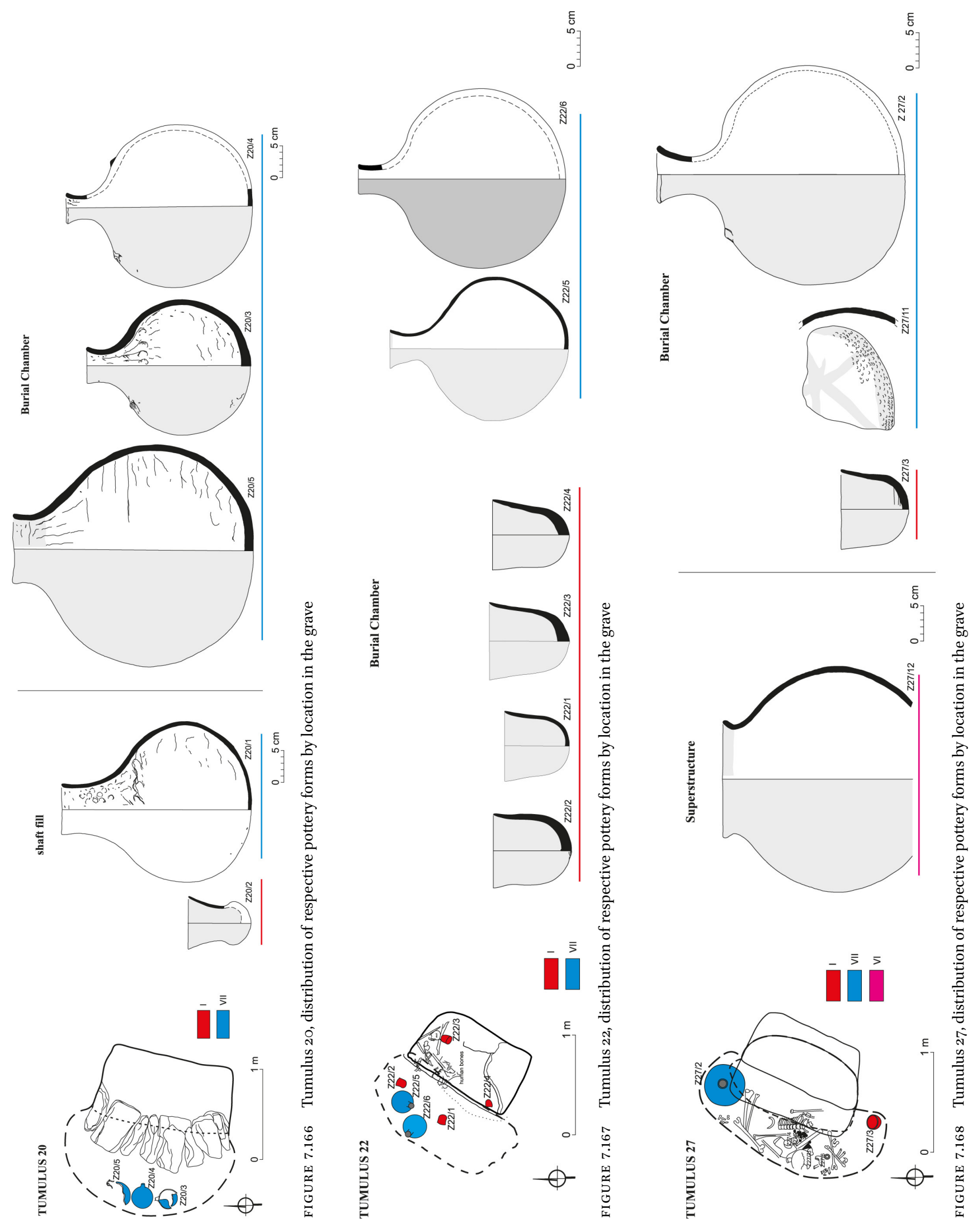


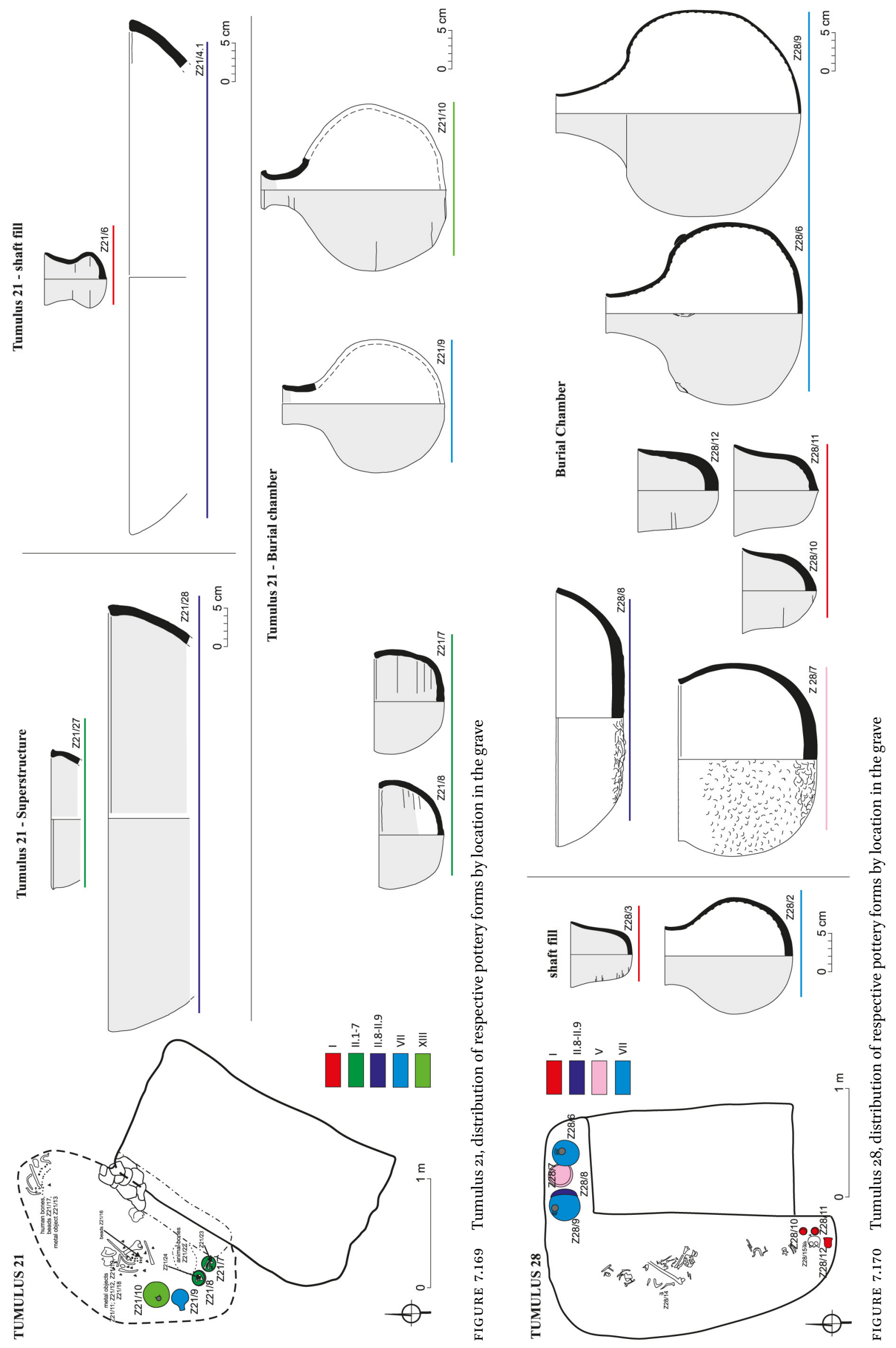

